University of Louisville

ThinkIR: The University of Louisville's Institutional Repository

Electronic Theses and Dissertations

$12-2019$

\title{
Stability and reactivity analysis of single metal and bimetallic nanostructures by anodic stripping voltammetry.
}

Dhruba Kumar Pattadar

University of Louisville

Follow this and additional works at: https://ir.library.louisville.edu/etd

Part of the Analytical Chemistry Commons

\section{Recommended Citation}

Pattadar, Dhruba Kumar, "Stability and reactivity analysis of single metal and bimetallic nanostructures by anodic stripping voltammetry." (2019). Electronic Theses and Dissertations. Paper 3341.

Retrieved from https://ir.library.louisville.edu/etd/3341

This Doctoral Dissertation is brought to you for free and open access by ThinkIR: The University of Louisville's Institutional Repository. It has been accepted for inclusion in Electronic Theses and Dissertations by an authorized administrator of ThinkIR: The University of Louisville's Institutional Repository. This title appears here courtesy of the author, who has retained all other copyrights. For more information, please contact thinkir@louisville.edu. 


\title{
STABILITY AND REACTIVITY ANALYSIS OF SINGLE METAL AND BIMETALLIC NANOSTRUCTURES BY ANODIC STRIPPING VOLTAMMETRY \\ By \\ Dhruba Kumar Pattadar
}

\author{
A Dissertation \\ Submitted to the Faculty of the \\ College of Arts and Sciences of the University of Louisville \\ in Partial Fulfillment of the Requirements \\ for the Degree of \\ Doctor of Philosophy in Chemistry \\ Department of Chemistry \\ University of Louisville \\ Louisville, Kentucky
}

December 2019 
Copyright 2019 by Dhruba K. Pattadar

All rights reserved 



\title{
STABILITY AND REACTIVITY ANALYSIS OF SINGLE METAL AND BIMETALLIC NANOSTRUCTURES BY ANODIC STRIPPING VOLTAMMETRY
}

\author{
Dhruba Kumar Pattadar \\ A Dissertation Approved on
}

July 23rd, 2019

by the following Dissertation Committee:

Dissertation Director - Dr. Francis P. Zamborini

Dr. Richard Baldwin

Dr. Craig Grapperhaus

Dr. Gamini Sumanasekera 
This Dissertation is dedicated to my sweet mom, Archana Saha and my lovely wife, Anamika Saha 


\section{ACKNOWLEDGEMENTS}

First, I would like to give special thanks to my supervisor, Dr. Francis P. Zamborini for his excellent guidance and support throughout my graduate studies. His time and patience were really helpful to achieve my goals. He always encouraged me to develop deep scientific knowledge in the field of electroanalytical chemistry. I am grateful to him for giving me the opportunity to work in his lab for my PhD studies.

Besides my advisor, I would like to give thanks to my dissertation committee members Dr. Craig A. Grapperhaus, Dr. Gamini Sumanesekera and Dr Richard P. Baldwin for providing me with valuable suggestions to develop my research projects and also to improve my dissertation write up. Also, I would like to thank all my lab mates specifically Jay N. Sharma, Badri P. Mainali and Hari K. Nambiar for their help and contribution towards the development and successful completion of my research projects.

Lastly, I am grateful to my parents, my family members and my beloved wife Anamika Saha for their constant encouragement and support to pursue PhD degree. 


\section{ABSTRACT}

\section{STABILITY AND REACTIVITY ANALYSIS OF SINGLE METAL AND BIMETALLIC NANOSTRUCTURES BY ANODIC STRIPPING VOLTAMMETRY}

Dhruba Kumar Pattadar

July 23, 2019

This dissertation has two main themes. The first involves studies aimed at developing anodic stripping voltammetry (ASV) as an analytical tool to characterize metal nanoparticles (NPs), with a focus on the size and aggregate structure of single metal NPs and the composition and atomic arrangement of bimetallic NPs. The second main theme involves studies that use ASV and electrochemical surface area-to-volume (SA/V) measurements to study the unique reactivity and transformations of single metal and bimetallic NPs. The transformations involve size and composition changes in response to ozone and electrochemical potential. Reactions involve size dependent galvanic exchange and electrocatalytic activity. 
The size of $1.6 \mathrm{~nm}$ diameter tetrakis(hydroxymethyl)phosphonium chloride (THPC)-stabilized and $0.9 \mathrm{~nm}$ diameter triphenylphosphine monosulfonate (TPPS)-stabilized Au NPs were characterized by ASV, which show $E_{p}$ (oxidation peak potential) values of 0.45 and $0.20 \mathrm{~V}$, respectively, vs. $\mathrm{Ag} / \mathrm{AgCl}$. The $\mathrm{E}_{\mathrm{p}}$ values followed the trend of decreasing values as the size of the Au NPs decrease and fit well with theory and the size determined by electron microscopy. $15 \mathrm{~nm}$ diameter Au NPs aggregated by addition of THPC displayed an $\mathrm{E}_{p}$ value of $0.77 \mathrm{~V}$, which is the same $E_{p}$ as that of isolated, non-aggregated $15 \mathrm{~nm}$ diameter Au NPs. Transmission electron microscopy (TEM) measurements and SA/V measurements showed that the aggregates were highly linear and the NPs were not well connected with THPC, which led to no change in SA/V between aggregated and non-aggregated Au NPs. The pH-induced Au NP aggregates, in contrast, were fused together, 3D in nature, and had lower SA/V. This explains the larger $E_{p}$ values for oxidation $(\sim 0.95 \mathrm{~V})$. ASV also provides the composition of $\mathrm{Cu}$ and $\mathrm{Au}$ in bimetallic $\mathrm{Cu}_{1} \mathrm{Aux}(\mathrm{x}=0.1-1) \mathrm{NPs}$ in one scan in $\mathrm{KCl}$ electrolyte. The ASV shows a different peak signature for different arrangements of $\mathrm{Cu}$ and $\mathrm{Au}$ in the NP, including Cu/Au core/shell, Au/Cu core/shell, and CuAu mixed alloy arrangements.

The second aim of this work involves the use of ASV and SA/V measurements to monitor the size-dependent reactivity of Au NPs and reactivity of bimetallic AuCu NPs. $1.6 \mathrm{~nm}$ THPC-stabilized and $0.9 \mathrm{~nm}$ diameter TPPSstabilized Au NPs increased in size to 4-10 nm diameter following 1-2 min of ozone treatment or one electrochemical oxidation-reduction cycle based on a positive shift in their Ep in ASV. $4 \mathrm{~nm}$ diameter Au NPs and larger are stable during these 
same treatments. $1.6 \mathrm{~nm}$ diameter $\mathrm{Au}$ NPs exchange with $\mathrm{Ag}^{+}$ions by galvanic exchange, whereas $4 \mathrm{~nm}$ diameter Au NPs and larger show no exchange with $\mathrm{Ag}^{+}$ ions. Finally, $1.6 \mathrm{~nm}$ diameter Au NPs show higher activity than 0.9 or $4 \mathrm{~nm}$ diameter Au NPs for $\mathrm{CO}_{2}$ reduction or the hydrogen evolution reaction (HER). The activity is related to the number of corner and edge sites available for the different sized NPs. 
TABLE OF CONTENTS

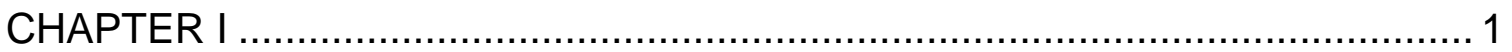

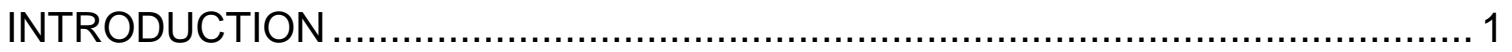

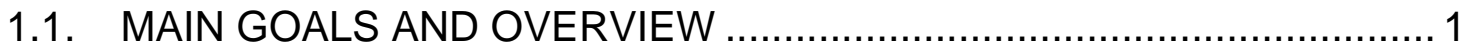

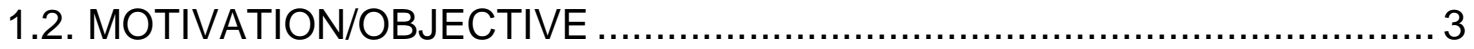

1.2. IMPORTANCE OF METAL NANOPARTICLES RESEARCH............... 6

1.4. SYNTHESIS OF METAL NANOPARTICLES .................................... 8

1.4.1. Synthesis of water-soluble colloidal $4-50 \mathrm{~nm}$ diameter Au nanoparticles.

1.4.2. Synthesis of strong ligand protected 1-2 $\mathrm{nm}$ diameter Au NPs........ 10

1.4.3. Synthesis of water-soluble weak ligand protected 1-2 nm diameter $\mathrm{Au}$

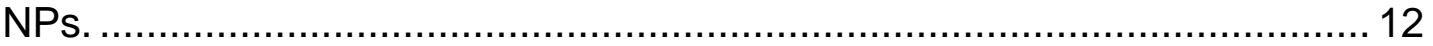

1.5. ELECTROCHEMICAL STUDIES OF METAL NANOPARTICLES ........... 13

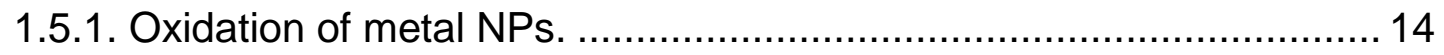

1.6. SIZE STABILITY STUDY OF METAL NPs...................................... 22

1.7. SIZE DEPENDENT GALVANIC EXCHANGE REACTION OF METAL NPS. 
1.8. BIMETALLIC NANOPARTICLES

CHAPTER II

2.3.1. Synthesis of $0.9 \mathrm{~nm}$ Diameter TPPS-Protected $\mathrm{Au}_{11}$ Clusters.

2.3.2. Synthesis of $1.6 \mathrm{~nm}$ Diameter THPC Au NPs.

2.3.3. Synthesis of $\sim 2 \mathrm{~nm}$ Diameter Glutathione-Capped Au NPs.

2.3.4. Synthesis of Dendrimer-Coated 1-2 nm Diameter Au NPs.

2.3.5. Synthesis of Citrate Stabilized $4 \mathrm{~nm}$ Diameter Au NPs.

2.3.6. Preparation of THPC 4 nm Diameter Au NPs.

2.3.7. Synthesis of $15 \mathrm{~nm}$ Average Diameter Citrate-Stabilized Au NPs. .... 49

2.3.8. Synthesis of Citrate-Stabilized $50 \mathrm{~nm}$ Diameter Au NPs. 51

2.3.9. Synthesis of Core/Shell Cu1/Aux NPs with Varied Au. 52

2.3.10. Synthesis of CuAu Mixed Alloy NPs. 52

2.3.11. Synthesis of Core/Shell Au0.33/Cu1 NPs. 53 
2.4.1. Functionalization of Glass/ITO Electrodes with 3-

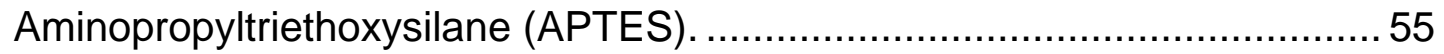

2.4.2. Attachment of Citrate-Coated Nanoparticles to Functionalized

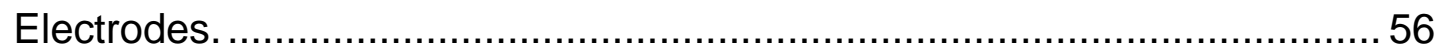

2.4.3. Galvanic replacement of Au NPs with $\mathrm{Ag}^{+}$ions................................ 56

2.5. CHARACTERIZATION AND INSTRUMENTATIONS ............................. 57

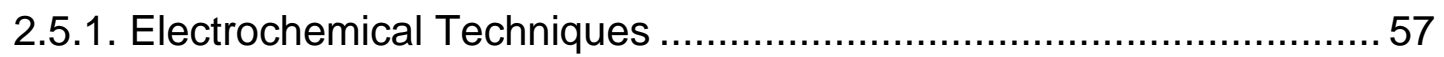

2.5.2. Ultraviolet-Visible Spectroscopy (UV-vis) ........................................ 61

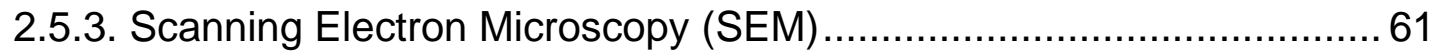

2.5.4. Transmission Electron Microscope (TEM) …................................. 63

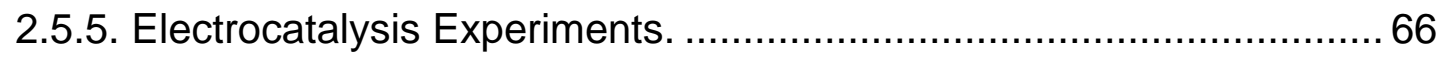

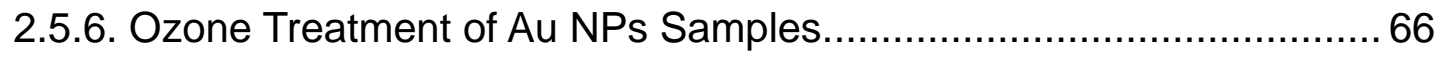

2.5.7. Thermal Treatment of glass/ITO Attached Au NPs............................6 66

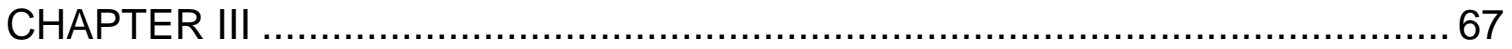

A SIZE STABILITY STUDY OF CATALYTICALLY-ACTIVE SUB-2 nm DIAMETER GOLD NANOPARTICLES SYNTHESIZED WITH WEAK STABILIZER ............ 67

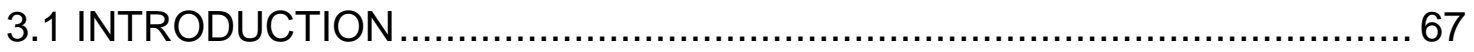

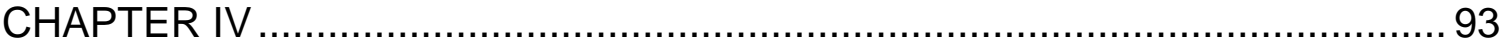

ELECTROXIDATION AND SIZE STABILITY OF AN 11-ATOM Au CLUSTER

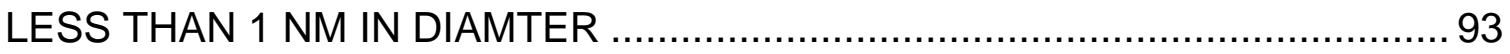

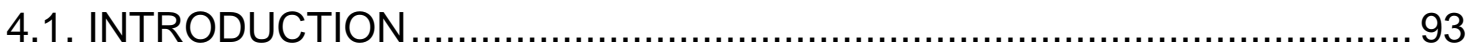




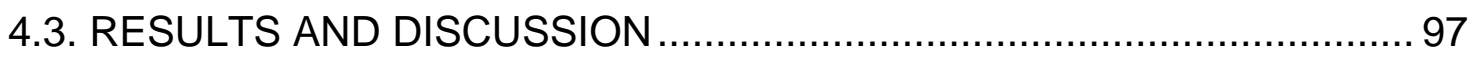

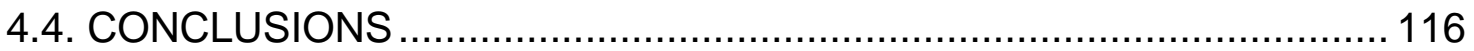

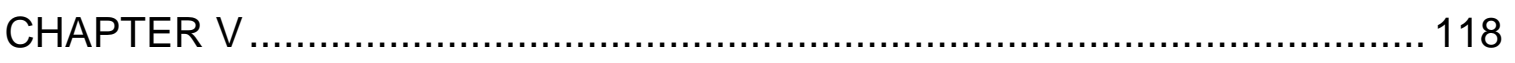

NANOPARTICLES AGgREGATE STRUCTURE EFFECT THE THERMODYNAMICS OF ELECTROCHEMICAL METAL NANOPARTICLES

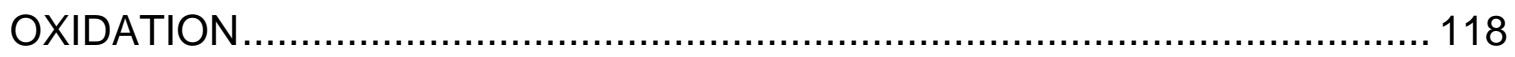

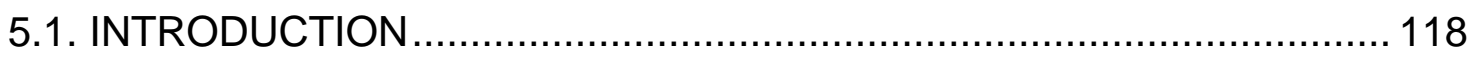

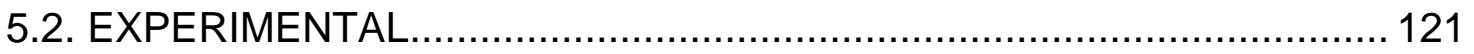

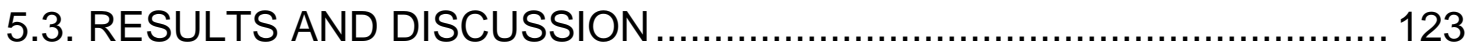

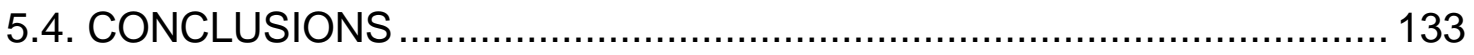

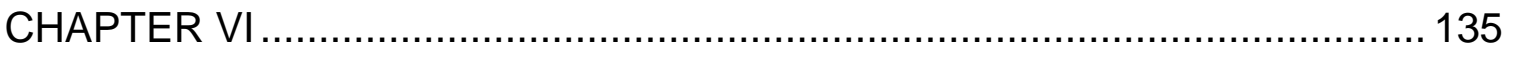

EEEFECT OF SIZE, COVERAGE, AND DISPERSITY ON THE POTENTIALCONTROLLED OSTWALD RIPENING OF METAL NANOPARTICLES .......... 135

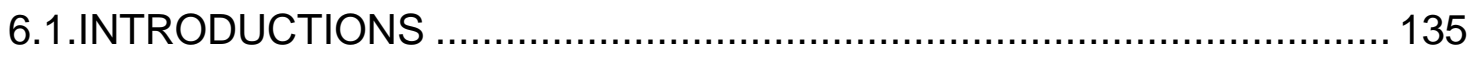

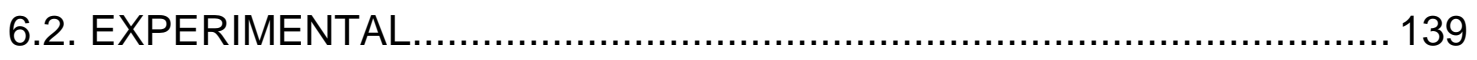

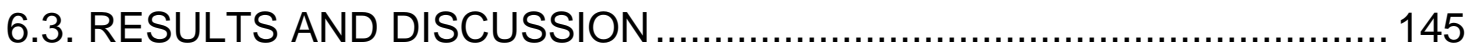

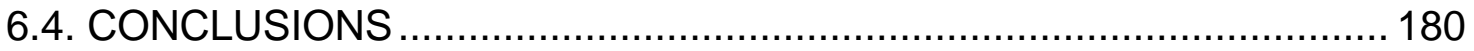

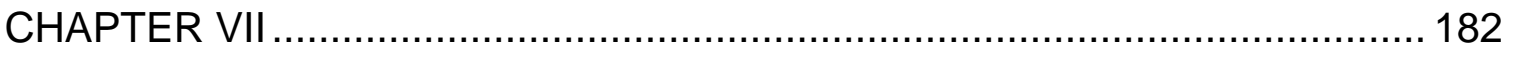

SIZE DEPENDENT GALVANIC EXCHANGE BETWEEN Au NANOPARTICLES

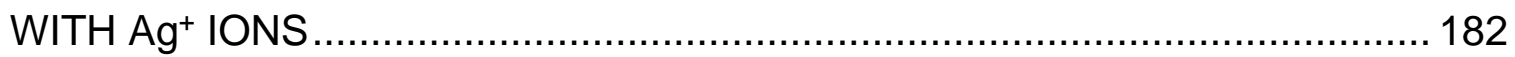


7.1. INTRODUCTIONS

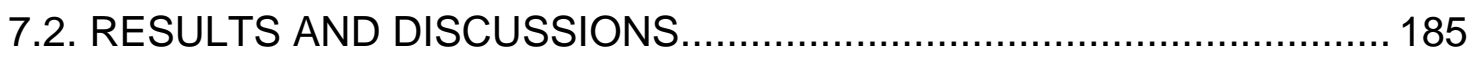

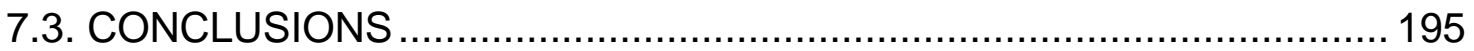

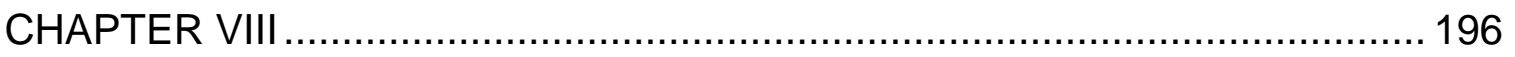

HALIDE-DEPENDENT DEALLOYING OF Cux/Auy CORE/SHELL NANOPARTICLES FOR COMPOSITION ANALYSIS BY ANODIC STRIPPING

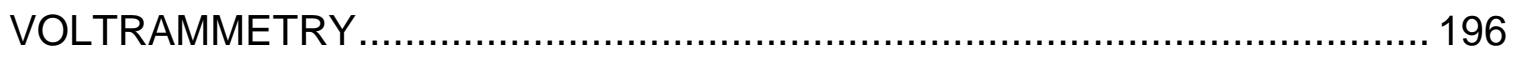

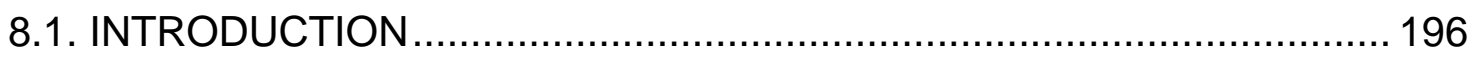

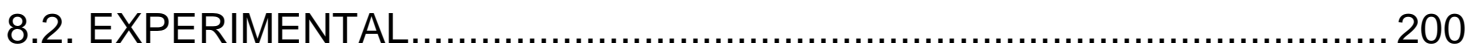

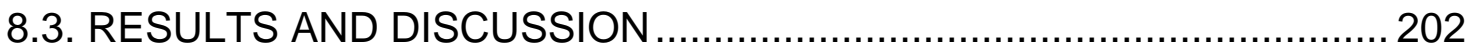

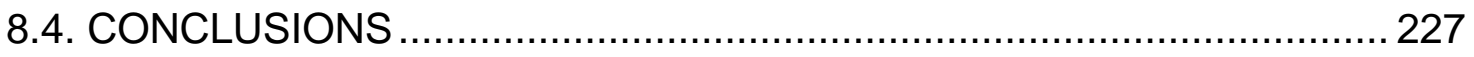

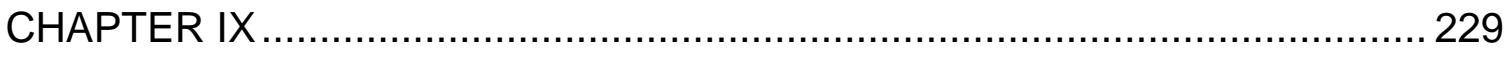

THE USE OF ANODIC STRIPPING VOLTAMMETRY TO ANALYZE THE CHANGES IN ATOMIC ARRANGEMENTS OF AuCu BIMETALLIC

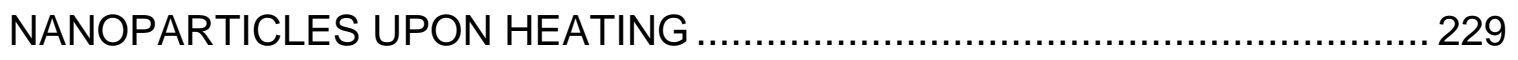

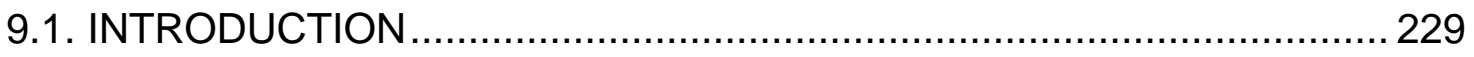

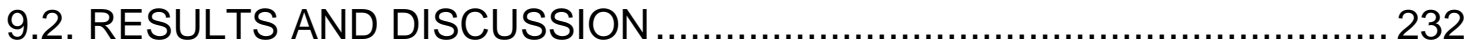

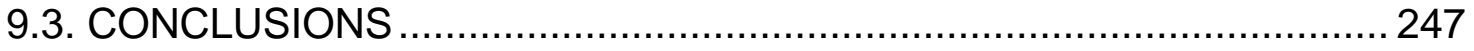

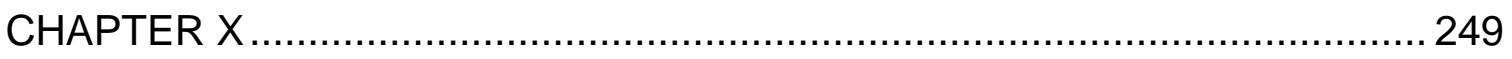

SUMMARY, CONCLUSIONS AND FUTURE DIRECTIONS …...................... 249

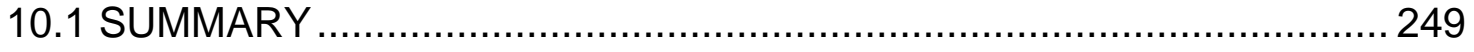


10.2. FUTURE DIRECTIONS.

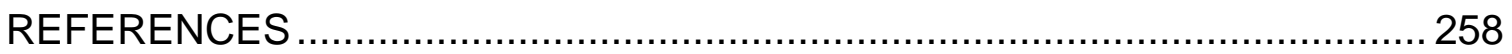

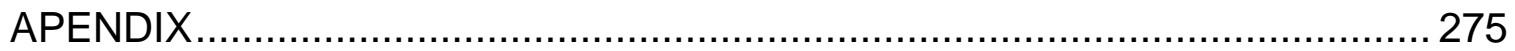

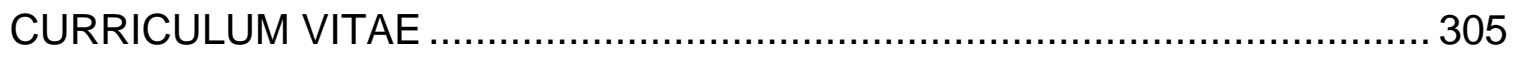




\section{LIST OF TABLES}

Table 6. 1. Microscopy measured radius and electrochemically measured surface areato-total volume ratio (SA/V) of different sized Au NPs. 161

Table 6. 2. Calculated size of NPs based on electrochemically-measured SA/V after Ostwald ripening for $0.0,35,70,105$ and $140 \min$ for 2, 4, 15 and 1:3 mixture of 4 and 15 $\mathrm{nm}$ Au NPs at different ripening potential $(0.0 \mathrm{~V}, 0.30 \mathrm{~V}$, and $0.45 \mathrm{~V})$ 162

Table 6. 3.Data of electrochemically measured NPs diameter/min and NPs diameter (\% increase per min) as a function of ripening potential for $1.6,4,15$ and mix of 4 \& $15 \mathrm{~nm} \mathrm{Au}$ NPs at $0.0,0.30$ and $0.45 \mathrm{~V}$. 166

Table 6. 4. Microscopy measured size of various Au NPs after different ripening times and different ripening potentials. 170

Table 7. 1. Integrated charge obtained from ASV peak of Ag and Au for $1.6 \mathrm{~nm}$ diameter Au NPs after 3 min of galvanic exchange with $1 \times 10^{-4} \mathrm{M} \mathrm{AgNO}_{3}$ solution. 190

Table 8. 1. Comparison of theoritical and experimetal of $\mathrm{Au} / \mathrm{Cu}$ ratio obtained from ASV in both $\mathrm{Br}$ - and $\mathrm{Cl}^{-}$containing acid electrolyte solution. 213

Table 8. 2. Composition and size information on the rearranged Cu/Au NPs following initial Cu stripping in $\mathrm{KBr}$ electrolyte. .222 


\section{LIST OF FIGURES}

Figure 1. 1. General experimental protocol for the chemical synthesis of Au NPs...........9 Figure 1.2. Representation of monolayer protected Au cluster (a) thiophenyl coated Au cluster and (b) alkane thiol coated Au cluster. 11

Figure 1. 3. Crooks method of synthesis of dendrimer coated 1-2 nm diameter Au NPs.

Figure 1. 4. (A) Duff method of synthesis of Tetrakis(hydroxymethyl)phosphonium chloride coated 1-2 nm diameter Au NPs and (B) representation of the triphenylphosphine coated Au nanocluster.......

Figure 1. 5. Theoretical oxidation peak potential shift of Au NPs based on Plieth's equation. 17

Figure 1. 6. Schematic representation of two types ripening mechanisms of metal NPs known as (A) Ostwald ripening and (B) Smoluchowski ripening. .25

Figure 2. 1. Procedure for the synthesis of $A u_{11}$ clusters stabilized with triphenylphosphine monosulfonate. $\quad 43$

Figure 2. 2. (A) Reaction involved in the Duff method of synthesis of 1-2 nm diameter Au NPs stabilized with Tetrakis(hydroxymethyl)phosphonium chloride and (B) proposed mechanism of synthesis of Au NPs with Tetrakis(hydroxymethyl)phosphonium chloride.

Figure 2. 3. Synthesis of citrate coated $4 \mathrm{~nm}$ diameter Au NPs. 46 
Figure 2. 4. Proposed mechanism of assembly and disassembly of $4 \mathrm{~nm}$ Au NPs with THPC. (i) The citrate coated $4 \mathrm{~nm}$ Au NPs immediately assemble into linear chains by reacting with THPC and slowly disassemble with time. The rapid assembly of NPs after addition of THPC due to the displacement of citrate with THPC. However, disassembly of Au NPs is only observed when the $\mathrm{pH}$ is above the pKa of the THPC (ii) The catalytic formation of pentavalent phosphorene occurred with Au NPs when the $\mathrm{pH}$ is above the pKa of the THPC ligand. (iii) These pentavalent THPOH isomers remain adsorbed to the surface of Au NPs and the excess negative charge of the ligand cannot maintain the assembly, which breaks apart into monodisperse components. .48

Figure 2. 5. Synthesis mechanism of $15 \mathrm{~nm}$ diameter Au NPs by the citrate reduction method. 50

Figure 2. 6. Proposed mechanism of seeded growth synthesis of $50 \mathrm{~nm}$ diameter Au NPs with $\mathrm{H}_{2} \mathrm{O}_{2}$. 50

Figure 2. 7. Synthesis strategy of citrate coated $\mathrm{Cu}$, core/shell $\mathrm{Cu} / \mathrm{Au}$ and alloy $\mathrm{Cu}-\mathrm{Au}$ NPs. 54

Figure 2. 8. Functionalization of Glass/ITO electrode with aminopropyltriethoxysilane (APTES). 55

Figure 2. 9. Attachment of metal NPs onto APTES functionalized glass/ITO electrode. 56 Figure 2. 10. Typical linear sweep voltammogram of glass/ITO/APTES/Au NPs ( 2 nm) at a scan rate of $0.01 \mathrm{~V} / \mathrm{s}$ in a solution of $0.01 \mathrm{M} \mathrm{KBr}$ plus $0.1 \mathrm{M} \mathrm{KClO}_{4}$ .59

Figure 2. 11. Typical cyclic voltammogram for a solution of $\mathrm{K}_{3} \mathrm{Fe}(\mathrm{CN})_{6}$ in $\mathrm{M} \mathrm{KNO}_{3} \ldots \ldots . . .60$

Figure 2. 12. (a) Scheme of electron beam and sample interactions. (b) Typical............63

Figure 2. 13. (a) Scheme of electron beam and sample interactions in TEM. .65 
Figure 3. 1. (A) ASV obtained in $10 \mathrm{mM} \mathrm{KBr}$ plus $0.1 \mathrm{M} \mathrm{KClO}_{4}$ solution at a scan rate of $0.01 \mathrm{~V} / \mathrm{s}$ of glass/ITO/APTES electrodes coated with different sized Cit Au NPs and THPC $\mathrm{Au}_{2 n m}$ NPs. (B) UV-Vis spectra of Cit Au $u_{4 n m}$ NPs (Blue) and THPC Au $u_{2 n m}$ NPs (Red). ...72

Figure 3. 2. ASV of a glass/ITO/APTES electrode containing $2 \mathrm{~nm}$ diameter thiocyanatecoated (A) and glutathione-coated (B) Au NPs. (C) ASV of a glass/ITO/APTES electrode containing THPC $\mathrm{Au}_{2 n m}$ NPs (red) and THPC $\mathrm{Au}_{2 n m}$ NPs after 30 min of soaking in $10 \mathrm{mM}$ glutathione solution (blue). All ASVs were run in $10 \mathrm{mM} \mathrm{KBr}$ plus $0.1 \mathrm{M} \mathrm{KClO}_{4}$ solution from $-0.2 \mathrm{~V}$ to $1.2 \mathrm{~V}$ at a scan rate of $0.01 \mathrm{~V} / \mathrm{s}$. 76

Figure 3. 3. (A) Linear sweep voltammograms (LSVs) of glass/ITO/APTES electrodes

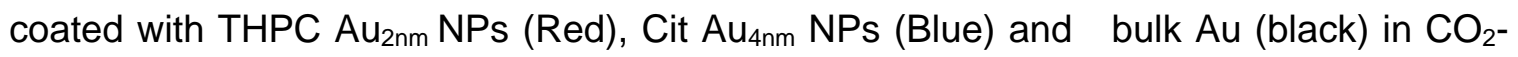
saturated $0.1 \mathrm{M} \mathrm{KHCO}_{3}$ solution. (B) Linear sweep voltammograms (LSVs) of glass/ITO/APTES electrodes coated with THPC Au $\mathrm{Anm}_{2 \mathrm{NPs}}$ in $\mathrm{CO}_{2}$-saturated $0.1 \mathrm{M} \mathrm{KHCO}_{3}$ solution (Red) and $\mathrm{N}_{2}$ purged $0.1 \mathrm{M} \mathrm{KHCO}_{3}$ solution (Blue). (C) CVs obtained in $\mathrm{N}_{2}$ saturated $0.1 \mathrm{M} \mathrm{HClO}_{4}$ solution at a scan rate of $0.01 \mathrm{~V} / \mathrm{s}$ of glass/ITO/APTES electrodes coated with Cit $\mathrm{Au}_{4 \mathrm{~nm}} \mathrm{NPs}$ (blue) and THPC Au $2 \mathrm{~nm}$ NPs (red) 77

Figure 3. 4. Linear sweep voltammograms (LSVs) of glass/ITO/APTES electrodes coated with Cit $\mathrm{Au}_{4 n \mathrm{~nm}} \mathrm{NPs}$ in $\mathrm{CO}_{2}$-saturated $0.1 \mathrm{M} \mathrm{KHCO}_{3}$ solution (Red) and $\mathrm{N}_{2}$ purged $0.1 \mathrm{M}$ $\mathrm{KHCO}_{3}$ solution (Black). 79

Figure 3. 5. Cyclic voltammogram (CVs) of glass/ITO/APTES coated with (A) Cit $A u_{4 n m}$ NPs and (C) THPC $\mathrm{Au}_{2 \mathrm{~mm}} \mathrm{NPs}$ in $0.1 \mathrm{M} \mathrm{HClO}_{4}$ solution from $0.0 \mathrm{~V}$ to $1.6 \mathrm{~V}$ at a scan rate of $0.1 \mathrm{~V} / \mathrm{s}$ (Red is the $1^{\text {st }} \mathrm{CV}$ cycle and Blue is the $2^{\text {nd }} \mathrm{CV}$ ). ASV of glass/ITO/APTES coated with (B) Cit $\mathrm{Au}_{4 \mathrm{~nm}} \mathrm{NPs}$ and (D) THPC Au $2 \mathrm{~nm}$ NPs in $10 \mathrm{mM} \mathrm{KBr}$ plus $0.1 \mathrm{M} \mathrm{KClO}_{4}$ solution from $0.0 \mathrm{~V}$ to $1.2 \mathrm{~V}$ at a scan rate of $0.01 \mathrm{~V} / \mathrm{s}$ (Red is the ASV of as-prepared Au NPs and Blue is ASV after $1 \mathrm{CV}$ cycle in $\left.0.1 \mathrm{M} \mathrm{HClO}_{4}\right)$. 82 
Figure 3. 6. ASVs of glass/ITO/APTES electrodes coated with (A) Cit Au ${ }_{4 n m}$ NPs and (B) THPC $\mathrm{Au}_{2 \mathrm{~nm}} \mathrm{NPs}$ that were untreated (red plot, $0 \mathrm{~min}$ ) or held at $0.30 \mathrm{~V}$ for the times indicated in $10 \mathrm{mM} \mathrm{KBr}$ plus $0.1 \mathrm{M} \mathrm{HClO}_{4}$ electrolyte. ASVs obtained under same conditions as in Figure 3.1. 85

Figure 3. 7. ASVs of glass/ITO/APTES electrodes coated with (A) Cit $A u_{4 n m}$ NPs and (B) THPC $\mathrm{Au}_{2 \mathrm{~nm}}$ NPs that were untreated (Red) and exposed to ozone for the different times

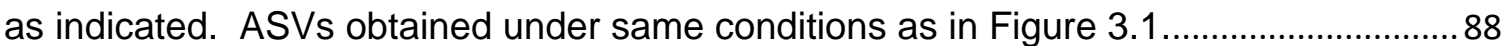

Figure 3. 8. SEM images of glass/ITO/APTES coated with THPC $A u_{2 n m}$ NPs $(A)$ asprepared, (B) after 35 min of Ostwald ripening at $0.3 \mathrm{~V}$ in $10 \mathrm{mM} \mathrm{KBr}$ plus $0.1 \mathrm{M} \mathrm{HClO}_{4}$ solution and (C) after 8 min of ozone treatment. 90

Figure 3. 9. (A) Cyclic voltammogram (CVs) of glass/ITO/APTES coated with THPC Au $u_{4 n m}$ NPs in $0.1 \mathrm{M} \mathrm{HClO}_{4}$ solution from $0.0 \mathrm{~V}$ to $1.5 \mathrm{~V}$ at a scan rate of $0.1 \mathrm{~V} / \mathrm{s}$ (Red is the $1^{\text {st }}$ $\mathrm{CV}$ and Blue is the $2^{\text {nd }} \mathrm{CV}$ ). (B) ASV of glass/ITO/APTES coated with THPC Au $\mathrm{unm}_{4 \mathrm{n}}$ NPS before (red) and after 1st CV cycle (blue). (C) ASV of glass/ITO/APTES coated with THPC $\mathrm{Au}_{4 \mathrm{~nm}} \mathrm{NPs}$ before (red) and after 35 min of ripening in $\mathrm{Br}-$ containing electrolyte at $0.3 \mathrm{~V}$ (blue). (D) ASV of glass/ITO/APTES coated with THPC Au $\mathrm{unm}_{4 \mathrm{~m}}$ NPs before (red) and after $8 \mathrm{~min}$ of ozone treatment (blue). All ASVs were obtained in $10 \mathrm{mM} \mathrm{KBr}$ plus $0.1 \mathrm{M} \mathrm{KClO}_{4}$ solution from $-0.2 \mathrm{~V}$ to $1.2 \mathrm{~V}$ at a scan rate of $0.01 \mathrm{~V} / \mathrm{s}$ 92

Figure 4. 1. (A) UV-vis spectra of TPPS $A u_{1 n m}$ (red), THPC $A u_{2 n m}$ NPs (blue), $C i t ~ A u_{4 n m}$ NPs (pink), Cit $A u_{15 n m}$ NPs (black) and Cit $A u_{50 n m}$ NPs (green). TEM image of TPPS $A u_{1 n m}$ and THPC $A u_{2 n m}$ NPs $(B, C)$. SEM image of Cit $A u_{4 n m}$, Cit $A u_{15 n m}$ NPs and Cit $A u_{50 n m}$ NPs (D, E \&F). ASV of the corresponding NPs (G). ASV obtained in $10 \mathrm{mM} \mathrm{KBr}$ plus $0.1 \mathrm{M}$ $\mathrm{KClO}_{4}$ solution at a scan rate of $0.01 \mathrm{~V} / \mathrm{s}$. 99 
Figure 4. 2. $\mathrm{CVs}$ of TPPS $A u_{1 n m}$ (red), THPC $A u_{2 n m}$ (blue) and Cit $A u_{4 n m}$ (pink) diameter Au particles at $1^{\text {st }}$ oxidation-reduction cycle $(A)$ and the same electrode in $2^{\text {nd }}$ oxidationreduction cycle (B). ASVs of TPPS $A u_{1 n m}(C)$, THPC $A u_{2 n m}(D)$ and $C i t ~ A u_{4 n m}(E)$ of NPs before and after $1^{\text {st }} \mathrm{CV}$ cycle. $\mathrm{CVs}$ were obtained in $0.1 \mathrm{M} \mathrm{HClO}_{4}$ electrolyte solution and ASVs were obtained in $10 \mathrm{mM} \mathrm{KBr}$ plus $0.1 \mathrm{~m} \mathrm{KClO}_{4}$ electrolyte solution. .103

Figure 4. 3. CVs of glass/ITO/APTES attached dendrimer coated 1-2 nm diameter Au NPs. CVs were performed in $0.1 \mathrm{~m} \mathrm{HClO}_{4}$ solution at a scan rate of $0.1 \mathrm{~V} / \mathrm{s}$ with $\mathrm{Ag} / \mathrm{AgCl}$ as a reference and $\mathrm{Pt}$ were as a counter electrode. 105

Figure 4. 4. $\mathrm{ASV}$ of $\mathrm{Cit} A u_{4 n m}$ at $\mathrm{pH} 9.0,2.4(\mathrm{~A})$, corresponding UV-Vis spectra (D) and SEM image at $\mathrm{pH}$ 2.4. ASV of THPC $\mathrm{Au}_{2 \mathrm{~nm}}$ at $\mathrm{pH} 11.1,6.1$ and $2.4(\mathrm{~A})$, corresponding UVvis spectra (E) and TEM image at pH 2.4. ASV of TPPS $A u_{1 n m}$ at $p H 11.1,6.1$ and $2.4(A)$, corresponding UV-vis spectra (F) and TEM image at pH 2.4. ASVs of Au nanocrystal were obtained in $10 \mathrm{mM} \mathrm{KBr}$ plus $0.1 \mathrm{M} \mathrm{KClO}_{4}$ solution. 106

Figure 4. 5. ASVs of glass/ITO/APTES coated TPPS $A u_{1 n m} N C s$ before and after different time of ozone exposer (A). ASVs were performed in $10 \mathrm{mM} \mathrm{KBr}$ plus $0.1 \mathrm{M} \mathrm{KClO}_{4}$ electrolyte solution. STEM images of Au particles before (B) and after 1 min of ozone exposure (C) and corresponding size histogram D (before) and E (after). 109

Figure 4. 6. ASV of THPC $A u_{2 n m}$ and TPPS $A u_{1 n m}$ NCs before (red graph), after treatment with C4 alkane thiols (pink graph) and after treatment with C4 alkane thiols followed by 30 min of ozone cleaning (blue graph). ASV were performed in $10 \mathrm{mM} \mathrm{KBr}$ plus $0.1 \mathrm{~m} \mathrm{KClO}_{4}$ electrolyte solutions. 112

Figure 4. 7. (A) CVs of glass/ITO/APTES attached THPC $A u_{2 n m}$ (blue graph), TPPS $A u_{1 n m}$ (pink graph), Cit $\mathrm{Au}_{4 \mathrm{~nm}}$ (red graph) and glass/ITO/APTES in $\mathrm{N}_{2}$ saturated $0.1 \mathrm{M} \mathrm{HClO}_{4}$ solution with a scan rate of $0.01 \mathrm{~V} / \mathrm{s}$. (B) Linear sweep voltammograms (LSVs) of 
glass/ITO/APTES electrodes attached THPC $\mathrm{Au}_{2 \mathrm{~nm}}$ NPs (blue graph), TPPS $\mathrm{Au}_{1 \mathrm{~nm}}$ (pink graph) and Cit $\mathrm{Au}_{4 \mathrm{~nm}} \mathrm{NPs}$ (blue) in $\mathrm{CO}_{2}$-saturated $0.1 \mathrm{M} \mathrm{KHCO}_{3}$ solution. 114

Figure 5. 1. General experimental procedure involved in these studies. 122

Figure 5. 2. (A) ASV of glass/ITO/APTES electrodes coated with $15 \mathrm{~nm}$ diameter Au NPs attached from solution at pH 5.8 (red graph), THPC aggregated (blue graph) and acid aggregated $\mathrm{pH} 2.6$ (black graph). All this ASV was performed in $0.1 \mathrm{M} \mathrm{KClO}_{4}+0.01 \mathrm{M}$ $\mathrm{KBr}$ from 0.0 to $+1.2 \mathrm{~V}$ at a scan rate of $10 \mathrm{mV} / \mathrm{s}$. (B) UV-vis spectra of $15 \mathrm{~nm}$ diameter $\mathrm{Au}$ NP solutions at $\mathrm{pH} 5.8$ (red graph), THPC aggregated (blue graph) and acid aggregated at $\mathrm{pH} 2.6$ (black graph). (C-D) are the STEM images of acid aggregated ( $\mathrm{pH}$ 2.60) (figure C) and THPC aggregated ( $\mathrm{pH} 5.8$ ) (figure $\mathrm{D})$ respectively. 124

Figure 5. 3. SEM image of glass/ITO/attached Au NPs (A) isolated, (B) acid aggregated, (C) THPC aggregated (Au to THPC 62.5) and (D) higher amount of THPC (Au to THPC 10.4) $15 \mathrm{~nm}$ diameter Au NPs. 126

Figure 5. 4. Pictures of solution of isolated ( $\mathrm{pH} 5.8)$, THPC aggregated $(\mathrm{pH} \mathrm{5.8)}$ and acid aggregated (pH 2.6) $15 \mathrm{~nm}$ diameter Au NPs. 127

Figure 5. 5. (A) CVs of glass/ITO/APTES electrodes coated with $15 \mathrm{~nm}$ diameter Au NPs attached from solution at $\mathrm{pH} 5.8$ (red graph), THPC aggregated (blue graph) and acid aggregated pH 2.6 (black graph). CVs was performed in $0.1 \mathrm{M} \mathrm{HClO}_{4}$ solution. (B) ASV of the same electrode after CV cycle. ASV was performed in $0.1 \mathrm{M} \mathrm{KClO}_{4}+0.01 \mathrm{M} \mathrm{KBr}$ from 0.0 to $+1.2 \mathrm{~V}$ at a scan rate of $10 \mathrm{mV} / \mathrm{s}$. 129

Figure 5. 6. (A) UV-vis spectra of $15 \mathrm{~nm}$ diameter Au NP solutions aggregated with various amount of Au : THPC ratios of 62.5 (red graph), 31.3 (blue graph) 10.4 (pink graph) and 6.3 (black graph). (B) CVs of the corresponding aggregated Au NPs. CVs were performed in $0.1 \mathrm{M} \mathrm{HClO}_{4}$ solutions. (C) ASVs of corresponding aggregated Au NPS 
performed in $0.1 \mathrm{M} \mathrm{KClO}_{4}+0.01 \mathrm{M} \mathrm{KBr}$ from 0.0 to $+1.2 \mathrm{~V}$ at a scan rate of $10 \mathrm{mV} / \mathrm{s}$. $(D, E)$ are the STEM images of THPC-aggregated NPs with the Au : THPC ratios of 62.5 and 6.3 , respectively. 131

Figure 6. 1. (A) UV-Vis spectra of THPC-stabilized $1.6 \mathrm{~nm}$ and citrate-coated 4 and 15 nm diameter Au NPs. (B) TEM image of THPC-stabilized $1.6 \mathrm{~nm}$ Au NPs. (C, D) SEM images of 4 and $15 \mathrm{~nm}$ citrate-stabilized Au NPs. (E) ASV of 1.6, 4, and $15 \mathrm{~nm}$ diameter Au NPs. ASVs were obtained in $10 \mathrm{mM} \mathrm{KBr}$ plus $0.1 \mathrm{M} \mathrm{KClO}_{4}$ electrolyte at a scan rate of $0.01 \mathrm{~V} / \mathrm{s}$. 142

Figure 6. 2. ASV of citrate coated $15 \mathrm{~nm}$ Au NPs after Ostwald ripening at 0.0 (A), 0.3 (B) and $0.45 \mathrm{~V}(\mathrm{C})$ for $0,35,70,105$ and 140 min of ripening each in $\mathrm{Br}^{-}$containing acidic electrolyte solution. ASVs were obtained in $10 \mathrm{mM} \mathrm{KBr}$ plus $0.1 \mathrm{KClO}_{4}$ electrolyte at a scan rate of $0.01 \mathrm{~V} / \mathrm{s}$. SEM images of $15 \mathrm{~nm}$ Au NPs at $0 \mathrm{~min}$ of ripening $(A)$ and after 140 min of ripening at $0.3 \vee(E)$ and $0.45(F)$. 147

Figure 6. 3. Size histogram of $15 \mathrm{~nm}$ Au NPs (A) without Ostwald ripening, (B) after 140 min of ripening at $0.3 \mathrm{~V}$ and $(\mathrm{C})$ after $140 \mathrm{~min}$ of ripening at $0.45 \mathrm{~V}$. 149

Figure 6. 4. ASV of citrate-stabilized $15 \mathrm{~nm}$ diameter Au NPs after $105 \mathrm{~min}$ of Ostwald ripening at $0.3 \mathrm{~V}(\mathrm{~A})$. ASV obtained in $10 \mathrm{mM} \mathrm{KBr}$ plus $0.1 \mathrm{M} \mathrm{KClO}_{4}$ electrolyte at a scan rate of $0.01 \mathrm{~V} / \mathrm{s}$. (B) The zoomed in portion of Figure $A$ as indicated by the dashed rectangle. 151

Figure 6. 5. ASV of citrate coated $4 \mathrm{~nm}$ Au NPs after Ostwald ripening at $0.0(\mathrm{~A}), 0.3$ (B) and $0.45 \vee(C)$ for $0,35,70,105$ and 140 min of ripening each in $\mathrm{Br}^{-}$containing acidic electrolyte solution. ASV obtained in $10 \mathrm{mM} \mathrm{KBr}$ plus $0.1 \mathrm{KClO}_{4}$ electrolyte with a scan rate $0.01 \mathrm{~V} / \mathrm{s}$. SEM images of $4 \mathrm{~nm} \mathrm{Au} \mathrm{NPs}$ at $0 \mathrm{~min}$ of ripening (A), after $140 \mathrm{~min}$ of ripening at $0.3 \mathrm{~V}(\mathrm{E})$ and after 140 min of ripening at $0.45(\mathrm{~F})$. 152 
Figure 6. 6. Size histogram of $4 \mathrm{~nm}$ Au NPs after $140 \mathrm{~min}$ of ripening at $0.3 \mathrm{~V}(\mathrm{~A})$ and after 140 min of ripening at $0.45 \mathrm{~V}(\mathrm{~B})$. 154

Figure 6. 7. ASV of THPC stabilized $1.6 \mathrm{~nm}$ Au NPs after Ostwald ripening at 0.3 (A) for 0, 35,70, 105 and 140 min of ripening each in $\mathrm{Br}^{-}$containing acidic electrolyte solution. SEM image of $1.6 \mathrm{~nm}$ Au NPs after $140 \mathrm{~min}$ of ripening at $0.30 \mathrm{~V}(\mathrm{~B})$. ASV of a $1: 3$ mixture of 4 and $15 \mathrm{~nm}$ Au NPs after Ostwald ripening at $0.30 \mathrm{~V}$ for $0,35,70,105$ and $140 \mathrm{~min}$ of ripening each in $\mathrm{Br}^{-}$containing acidic electrolyte solution (C). SEM image of 1:3 mixture of 4 and $15 \mathrm{~nm}$ Au NPs after 140 min of ripening at $0.30 \mathrm{~V}(\mathrm{D})$. All ASVs were obtained in $10 \mathrm{mM} \mathrm{KBr}$ plus $0.1 \mathrm{KClO}_{4}$ electrolyte at a scan rate of $0.01 \mathrm{~V} / \mathrm{s}$. 155

Figure 6. 8. Size histogram of $1.6 \mathrm{~nm}$ diameter Au NPs before treatment (A) and after 140 min of ripening at $0.3 \mathrm{~V}(\mathrm{~B})$. 156

Figure 6. 9. Size histogram of 1:3 mixture of 4:15 nm Au NPs after $140 \mathrm{~min}$ of ripening at $0.3 \mathrm{~V}$ 158

Figure 6. 10. (A) Cyclic voltammogram (CV) of $15 \mathrm{~nm}$ Au NPs before (0 min) and after $105 \mathrm{~min}$ of holding at $0.3 \mathrm{~V}$ vs $\mathrm{Ag} / \mathrm{AgCl}$ in $10 \mathrm{mM} \mathrm{KBr}$ plus $0.1 \mathrm{M} \mathrm{HClO}_{4}$ electrolyte solution. (B) ASV of the same electrode after 105 min of ripening at $0.3 \mathrm{~V}$. ASV was performed in $10 \mathrm{mM} \mathrm{KBr}$ plus $0.1 \mathrm{~m} \mathrm{KClO}_{4}$ solution. The $\mathrm{CV}$ coverage (electroactive surface area, SA) decreases from $7.65 \times 10^{-6} \mathrm{C}$ to $5.76 \times 10^{-6} \mathrm{C}$ and the ASV coverage (total volume of NPs, V) $3.96 \times 10^{-5} \mathrm{C}$. The SA/V value before and after were 0.19 and after 0.14 which is corresponds to 22.4 and $35.9 \mathrm{~nm}$ diameter Au NPs respectively...... 160

Figure 6. 11. Plot of electrochemically-measured surface area-to-total volume ratio (SA/V) as a function of $1 /$ radius as measured by TEM or SEM for $1.6,4,15,31,50$, and $70 \mathrm{~nm}$ diameter Au NPs. The surface area was measured by surface Au oxide formation and reduction in $0.1 \mathrm{M} \mathrm{HClO}_{4}$ and the total volume was measured by complete Au stripping in $10 \mathrm{mM} \mathrm{KBr}$ plus $0.1 \mathrm{M} \mathrm{KClO}_{4}$ electrolyte. 161 
Figure 6. 12. Electrochemically-determined average NP diameter (A-C) and relative diameter $(D-F)$ as a function of ripening time at a potential of $0.0 \vee(A, D), 0.30 \vee(B, E)$ and $0.45 \vee(C, F)$ for various sized and mixed Au NPs. 163

Figure 6. 13. Electrochemically measured NPs diameter/min (A) and \% increase of NPS diameter/min (B) as a function of ripening potential for 1.6, 4, and $15 \mathrm{~nm}$ Au NPs at 0.0, 0.30 and $0.45 \mathrm{~V}$. 167

Figure 6. 14. SEM determined NP diameter vs ripening time and $D_{\text {final }} / D_{\text {initial }}$ vs ripening time at $0.30 \mathrm{~V}(\mathrm{~A}, \mathrm{C})$ and $0.45 \mathrm{~V}(\mathrm{~B}, \mathrm{D})$ for all $\mathrm{Au}$ NPs samples. .171

Figure 6. 15. Electrochemically determined NP diameter vs ripening time (A) and $D_{\text {final }} / D_{\text {initial }}$ Vs ripening time $(B)$ at $0.30 \mathrm{~V}$ for $1: 3$ mixture of 4 and $15 \mathrm{~nm}$ Au NPs samples.

Figure 6. 16. SEM determined NP diameter vs ripening time $(A)$ and $D_{\text {final }} / D_{\text {initial }}$ Vs ripening time (B) at $0.30 \mathrm{~V}$ for $1: 3$ mixture of 4 and $15 \mathrm{~nm}$ diameter Au NPs samples. 173

Figure 6. 17. ASVs of citrate-stabilized $15 \mathrm{~nm}$ diameter Au NPs after Ostwald ripening at $0.3 \mathrm{~V}$ with the coverage of $(\mathrm{A})$ 1.1-1.3 NPs/ $/ \mathrm{m}^{2}\left(\right.$ or $(5.0-6.5) \times 10^{-6} \mathrm{C}$ ) and $(\mathrm{B}) 39.5-51.3$ $\mathrm{NPs} / \mu \mathrm{m}^{2}$ (or (2.0-2.6) x 10-4 C). ASVs were obtained in $10 \mathrm{mM} \mathrm{KBr}$ plus $0.1 \mathrm{M} \mathrm{KClO}_{4}$ electrolyte with a scan rate of $0.01 \mathrm{~V} / \mathrm{s}$ 175

Figure 6. 18. NP diameter vs ripening time $(A)$ and $D_{\text {final }} / D_{\text {initial }}$ Vs ripening time $(B)$ for 15 $\mathrm{nm}$ diameter Au NPs at $0.30 \mathrm{~V}$ for various coverage of NPs on the electrode surface as indicated. NPs diameter/min (C) and \% increase of NPs diameter/min (D) as a function of NPs coverage. 176

Figure 6. 19. Mechanism of electrochemically induced Ostwald ripening of Au NPs attached to glass/ITO electrode. 178 
Figure 7. 1. ASV of $4.1(\mathrm{~A}), 1.6(\mathrm{~B})$ and $0.9 \mathrm{~nm}(\mathrm{C})$ diameter Au NPs after 3 min of galvanic exchange with $1 \times 10^{-4} \mathrm{M} \mathrm{AgNO}_{3}$ solution. The ASV for Ag analysis was obtained in $0.5 \mathrm{M}$ $\mathrm{H}_{2} \mathrm{SO}_{4}$ electrolyte solution first and then the ASV of Au was obtained in $10 \mathrm{mM} \mathrm{KBr}$ plus $0.1 \mathrm{M} \mathrm{KClO}_{4}$ electrolyte solution, both at a scan rate of $0.01 \mathrm{~V} / \mathrm{s}$. 187

Figure 7. 2. ASV of $8 \mathrm{~nm}$ diameter $\mathrm{Ag}$ NPs in $0.5 \mathrm{M} \mathrm{H}_{2} \mathrm{SO}_{4}$ solution (black graph). ASV of AuAg alloy (50 \%-50\%) NPs in $0.5 \mathrm{M} \mathrm{H}_{2} \mathrm{SO}_{4}$ solution (red graph) and ASV of the same electrode in $10 \mathrm{mM} \mathrm{KBr}$ plus $0.1 \mathrm{~m} \mathrm{KClO}_{4}$ electrolyte solution (blue graph). All ASV obtained with a scan rate of $0.01 \mathrm{~V} / \mathrm{S}$. 189

Figure 7. 3. ASV of 4.1 (A) and 1.6 (B) nm diameter Au NPs after 3 min of galvanic exchange with $1 \times 10^{-4} \mathrm{M} \mathrm{AgNO}_{3}$ solution. ASVs for Ag were obtained in $0.5 \mathrm{M} \mathrm{H}_{2} \mathrm{SO}_{4}$ electrolyte solution and ASVs of $\mathrm{Au}$ were obtained in $10 \mathrm{mM} \mathrm{KBr}$ plus $0.1 \mathrm{M} \mathrm{KClO}_{4}$ electrolyte solution with a scan rate of $0.01 \mathrm{~V} / \mathrm{S}$. These Au NP electrodes had the same overall Au surface area. 192

Figure 7. 4. STEM (A) and STEM-EDS mapping (B) images of $4.1 \mathrm{~nm}$ diameter Au NPS after 3 min of exchange with $1 \times 10^{-4} \mathrm{M} \mathrm{AgNO}_{3}$ solution. Similarly, STEM (C) and STEMEDS mapping (D) images of $1.6 \mathrm{~nm}$ dimeter Au NPs. Images were obtained on silicon oxide coated $400 \mathrm{Ni}$ mesh TEM grids. The NPs were directly drop-cast deposited on the grid, dried in air, and then reacted by directly soaking the grid in the $\mathrm{Ag}^{+}$solution. 194

Figure 8. 1. General experimental procedure involved in this work. .202

Figure 8. 2. UV-Vis spectra of citrate coated $C u, C u_{1}-A u_{2}, C u_{1}-A u_{1}$ and $C u_{1}-A u_{0.5} N P s(A)$ and HRTEM image of $\mathrm{Cu}_{1}-\mathrm{Au}_{2}$ NPs $(B, C$ and $D)$ 204

Figure 8. 3. Anodic stripping voltammograms (ASVs) of core/shell cit-Cu $\mathrm{Cu}_{1} / \mathrm{Au}_{2} \mathrm{NPs}$ (red plot) and mixed alloy cit- $\mathrm{Cu}_{1} \mathrm{Au}_{2} \mathrm{NPs}$ (blue plot) in $10 \mathrm{mM} \mathrm{KCl}$ plus $0.1 \mathrm{M} \mathrm{HClO}_{4}$ solution. 
Figure 8. 4. Anodic stripping voltammetry (ASV) of citrate coated $\mathrm{Cu}, \mathrm{Cu}_{1} / \mathrm{Au}_{0.001}$, $\mathrm{Cu}_{1} / \mathrm{Au}_{0.01}, \mathrm{Cu}_{1} / \mathrm{Au}_{0.1} \mathrm{NPs}(\mathrm{A})$ and $\mathrm{Cu}_{1} / \mathrm{Au}_{0.2}, \mathrm{Cu}_{1} / \mathrm{Au}_{0.3}, \mathrm{Cu}_{1} / \mathrm{Au}_{0.5}, \mathrm{Cu}_{1} / \mathrm{Au}_{1}$ and $\mathrm{Cu}_{1} / \mathrm{Au}_{2} \mathrm{NPs}$ (B) in $10 \mathrm{mM} \mathrm{KCl}$ plus $0.1 \mathrm{M} \mathrm{HClO}_{4}$ solution. 208

Figure 8. 5. ASVs of $(\mathrm{A}) \mathrm{Cu}_{1} / \mathrm{Au}_{0.001}$ and $(\mathrm{B}) \mathrm{Cu}_{1} / \mathrm{Au}_{0.01}$ core/shell nanoparticles in $0.1 \mathrm{M}$ $\mathrm{HClO}_{4}$ (green), $0.01 \mathrm{M} \mathrm{KBr}+0.1 \mathrm{M} \mathrm{HClO}_{4}$ (blue), and $0.01 \mathrm{M} \mathrm{KCl}+0.1 \mathrm{M} \mathrm{HClO}_{4}$ (red) as indicated. The peaks labeled as $\mathrm{Cu}^{1}$ or $\mathrm{Cu}^{2}$ are known for sure and the peaks labeled with a number are uncertain. 209

Figure 8. 6. Anodic stripping voltammetry (ASV) of citrate coated $\mathrm{Cu}, \mathrm{Cu}_{1} / \mathrm{Au}_{0.001}$, $\mathrm{Cu}_{1} / \mathrm{Au}_{0.01}, \mathrm{Cu}_{1} / \mathrm{Au}_{0.1} \mathrm{NPs}(\mathrm{A})$ and $\mathrm{Cu}_{1} / \mathrm{Au}_{0.2}, \mathrm{Cu}_{1} / \mathrm{Au}_{0.3}, \mathrm{Cu}_{1} / \mathrm{Au}_{0.5}, \mathrm{Cu}_{1} / \mathrm{Au}_{1}$ and $\mathrm{Cu}_{1} / \mathrm{Au}_{2}$ (B) NPs in $10 \mathrm{mM} \mathrm{KBr}$ plus $0.1 \mathrm{M} \mathrm{HClO}_{4}$ solution. 215

Figure 8. 7. An illustration of the surface diffusion and rearrangement of Au atoms during the initial stages of $\mathrm{Cu}$ dealloying in the presence of $\mathrm{Br}^{-}$from $\mathrm{Cu} / \mathrm{Au}$ core/shell NPs with incomplete or thin Au shells, which leads to a more stable Cu/Au core/shell structure with a complete, passivating Au shell. 218

Figure 8. 8. (A) ASV of core/shell cit-Cu$/ \mathrm{Au}_{0.5} \mathrm{NPs}$ in $10 \mathrm{mM} \mathrm{KBr}$ plus $0.1 \mathrm{M} \mathrm{HClO}_{4}$ solution (blue plot) and $10 \mathrm{mM} \mathrm{KCl}$ plus $0.1 \mathrm{M} \mathrm{HClO}_{4}$ solution (red plot). (B) ASV of core/shell cit- $\mathrm{Cu}_{1} / \mathrm{Au}_{1} \mathrm{NPs}$ in $10 \mathrm{mM} \mathrm{KBr}$ plus $0.1 \mathrm{M} \mathrm{HClO}_{4}$ solution first (blue plot) followed by $\mathrm{ASV}$ of the same sample in $10 \mathrm{mM} \mathrm{KCl}$ plus $0.1 \mathrm{M} \mathrm{HClO}_{4}$ solution second (red plot).

Figure 8. 9. (A) ASV of cit- $\mathrm{Cu}_{1} \mathrm{Au}_{0.5}$ mixed alloy $\mathrm{NPs}$ in $10 \mathrm{mM} \mathrm{KCl}$ plus $0.1 \mathrm{M} \mathrm{HClO}_{4}$ after $15 \mathrm{~min}$ (red) and 2 hour (blue) of synthesis in the presence of $100 \mu \mathrm{L}$ of $10 \mathrm{mM} \mathrm{KBr}$. (B) Normalized UV-Vis spectra of the same alloy cit-Cu $\mathrm{Au}_{0.5} \mathrm{NPs}$ after $15 \mathrm{~min}$ (red) and 2 hour (blue) of synthesis. .226 
Figure 9. 1. (A) UV-Vis spectra of citrate stabilized core/shell $\mathrm{Cu}_{1} / \mathrm{Au}_{0.33}$, core/shell cit$\mathrm{Au}_{0.33} / \mathrm{Cu}_{1}$ and mixed $\mathrm{Cu}_{1}-\mathrm{Au}_{0.33}$ alloy NPs. TEM image of mixed $\mathrm{Cu}_{1}-\mathrm{Au}_{0.33}$ alloy (B) and core/shell cit-Auo.33/Cu 1 alloy (C) NPs. $\quad 233$

Figure 9. 2. $\mathrm{ASV}$ of citrate stabilized $\mathrm{Cu}, \mathrm{Au}$, mixed $\mathrm{Cu}_{1}-\mathrm{Au}_{0.33}$, core/shell $\mathrm{Cu}_{1} / \mathrm{Au}_{0.33}$ and core/shell $\mathrm{Au}_{0.33} / \mathrm{Cu}_{1}$ alloy NPs. ASV were performed in $10 \mathrm{mM} \mathrm{KCl}$ plus $0.1 \mathrm{M} \mathrm{HClO}_{4}$

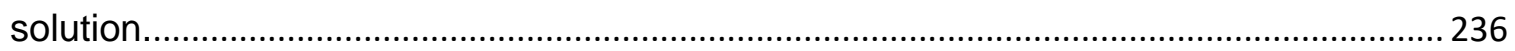

Figure 9. 3. Illustration of $\mathrm{Au}$ and $\mathrm{Cu}$ atoms arrangements in (a) mixed $\mathrm{Cu}_{1}-\mathrm{Au}_{0.33}$ alloy, (b) Core/shell $\mathrm{Au}_{0.33} / \mathrm{Cu}_{1}$ and (C) core/shell $\mathrm{Cu}_{1} / \mathrm{Au}_{0.33} \mathrm{NPs}$. 237

Figure 9. 5. ASV of citrate stabilized core/shell $\mathrm{Cu}_{1} / \mathrm{Au}_{0.33}(\mathrm{~A})$ and core/shell $\mathrm{Au}_{0.33} / \mathrm{Cu}_{1}(\mathrm{~B})$ after thermal treatment at different temperature and time as indicated in the graph. ASV were performed in $10 \mathrm{mM} \mathrm{KCl}$ plus $0.1 \mathrm{M} \mathrm{HClO}_{4}$ solution. 243

Figure 9. 6. TEM images of mixed cit- $\mathrm{Cu}_{1}-\mathrm{Au}_{0.33}$ alloy NPs before $(\mathrm{A})$ and after $(\mathrm{C})$ heating at $473 \mathrm{~K}$ for 1 hour. Size histograms of the NPs before (B) and after (D) heating at $473 \mathrm{~K}$ for 1 hour. TEM images of core/shell cit- $\mathrm{Au}_{0.33} / \mathrm{Cu}_{1}$ alloy NPs before $(\mathrm{E})$ and after $(\mathrm{G})$ heating at $473 \mathrm{~K}$ for 1 hour. Size histograms of the NPs before (F) and after (G) heating at $473 \mathrm{~K}$ for 1 hour. 246 


\section{CHAPTER I}

\section{INTRODUCTION}

\subsection{MAIN GOALS AND OVERVIEW}

The primary goal of this research was to study the ASV of controllably synthesized sub $4 \mathrm{~nm}$ diameter Au nanoparticles (NPs) coated with weak stabilizers in order to understand their size-dependent reactivity and develop ASV as a size characterization tool for small sized metal NPs. A second goal was to develop ASV for characterization of aggregate structures of Au NPs. A third goal was to characterize the composition and atomic arrangement of bimetallic $\mathrm{AuCu}$ nanoparticles (NPS). After developing ASV for characterization, we then used ASV to study the stability of small single metal and bimetallic NPs by monitoring changes in size, composition, and arrangement under various conditions. The dissertation is divided into four main sections. Chapters I and II are the first section, providing introduction to the topics and experimental details relevant to the research. Chapters III-VII make up the second section, providing the results on the characterization, reactivity, and stability of single metal Au NPs. The third section consists of Chapters VIII-IX, which deals with the analysis and reactivity of bimetallic AuCu NPs. Finally, Chapter X provides the fourth section, which is the summary and future directions of the research. 
The first section of this dissertation is made up of Chapters I and II. Chapter I provides the background information, theory, and previous research findings related to the synthesis of water-soluble ultra-small metal NPs, details about sizedependent electrooxidation of metal NPs, stability of different size NPs during various chemical and electrochemical treatments, size-dependent Ostwald ripening of Au NPs, size-dependent galvanic exchange of $\mathrm{Au} \mathrm{NPs}$ with $\mathrm{Ag}^{+}$ions, and composition and atomic arrangement analysis of alloy NPs. These are all topics relevant to the research. Chapter II describes the experimental procedures and characterization techniques used in this study.

The second section of this dissertation includes Chapters III-VII, which are dedicated to the ASV and electrochemical analysis of size, aggregate structure, stability, and reactivity of single metal Au NPs ranging from about $0.9 \mathrm{~nm}$ to $15 \mathrm{~nm}$ in diameter. Specifically, Chapter III describes the ASV characterization of $1.6 \mathrm{~nm}$ diameter Au NPs synthesized with weak stabilizers as compared to $4 \mathrm{~nm}$ diameter Au NPs, their stability and electrocatalytic activity. Chapter IV focuses on the ASV characterization, stability, and reactivity of atomically precise $\sim 0.9 \mathrm{~nm}$ diameter $\mathrm{Au}_{11}$ clusters synthesized with triphenylphosphine monosulfonate (TPPS) as a stabilizer. Chapter $\vee$ describes how the aggregate structure of Au NPs effects the thermodynamics of oxidation and therefore the ASV characterization. Chapter VI describes the effect of size, size dispersity, and coverage on the electrochemicallyinduced Ostwald ripening kinetics of electrode-attached 1.6, 4 and $15 \mathrm{~nm}$ diameter $\mathrm{Au}$ NPs. Ostwald ripening is a common process that transforms the size of $\mathrm{Au}$ NPs, making this an important type of size stability study. Chapter VII describes 
the use of ASV to study size-dependent galvanic exchange between Au NPs of different sizes and $\mathrm{Ag}^{+}$ions. This study demonstrates unique reactivity of sub 2 nm diameter Au NPs coated with weak stabilizers.

The third section of this dissertation includes Chapters VIII-IX, which are dedicated to ASV and electrochemical analysis and reactivity studies of bimetallic AuCu alloy NPs. Chapter VIII describes the effect of halide ions on the dealloying of citrate-stabilized core/shell $\mathrm{Cu}_{1} / \mathrm{Au}_{\mathrm{x}}(\mathrm{x}=0.001$ to 2$) \mathrm{NPs}$ for composition analysis by ASV. This work provides the conditions where composition analysis is possible but also describes a unique dealloying process. Chapter IX describes the use of ASV to determine the atomic arrangement of binary citrate-stabilized CuAu NPS having different synthetic arrangements and the effect of heating on their atomic arrangement.

The fourth and final section is Chapter $\mathrm{X}$, which summarizes the results of this research and provides possible future directions.

\subsection{MOTIVATION/OBJECTIVE}

The motivation behind this research was to better understand the unique electrochemical properties and chemical reactivity of ultra-small metal and metal alloy NPs. In order to do that, our goal was to develop electrochemical methods for analyzing the size, composition, and atomic arrangement of single metal and metal alloy NPs. Our findings provide valuable information regarding the effect of NP size on their standard potential $\left(\mathrm{E}^{0}\right)$ in $\mathrm{KBr}$ solution when we change the size 
of the Au NPs from 50 to $1 \mathrm{~nm}$ diameter. ${ }^{1}$ The synthesis of Au NPs in the range of 1-2 nm diameter with strongly attached stabilizers, such as thiols, is relatively straightforward and has been described in the literature. ${ }^{2}$ However, the stronglybound stabilizer does not allow one to study the electrochemical properties of the actual metal NPs because the stabilizer-metal interaction greatly alters the properties of the bare metal. Our findings provide valuable information regarding the reactivity of weakly-stabilized (assumed to behave more like bare metal) sub 2 $\mathrm{nm}$ diameter Au NPs for oxidation in $\mathrm{KBr}$ solution, electrochemical $\mathrm{CO}_{2}$ reduction, the hydrogen evolution reaction, oxidation-reduction in acidic electrolyte, ozone exposure for different times, and electrochemical Ostwald ripening in halidecontaining electrolyte. ${ }^{1}$

The synthesis and characterization of the smallest size NPs, which are about 0.8-1.0 $\mathrm{nm}$ in diameter (Au11 clusters), is very challenging. Au11 clusters have been previously characterized by mass spectrometry $(\mathrm{MS})^{3}$ and transmission electron microscopy (TEM). ${ }^{4}$ We explore electrochemical ASV as an alternative approach to determine their size and size stability under various conditions, and other size-related properties. ASV is faster, more portable than microscopic techniques, and provides the NPs size directly on the electrode surface following a variety of different electrochemical conditions and treatments. On the other hand, spectroscopic and microscopic techniques are more-costly, performed under vacuum, and need special sample preparation.

In addition to determining the size and size stability of Au NPs, we also studied the effect of NP size on electrochemical Ostwald ripening of Au NPs. Ostwald 
ripening is a common phenomenon which occurs during metal NP synthesis, different catalytic applications of NPs, and thermal treatment. Since Au NPs show size-dependent oxidation peak potentials, it is useful to monitor the size changes during Ostwald ripening by monitoring the electrochemical oxidation of the metal NPs. We also determined the rate of Ostwald ripening by monitoring the surface area-to-volume ratio (SA/V) of the metal NPs before and after Ostwald ripening.

Another motivation for this study was to understand the NP size effect on the galvanic exchange of metal NPs, where we used the exchange of Au NPs with $\mathrm{Ag}^{+}$ions as our model system. Galvanic replacement is a common method to synthesize bimetallic core/shell structures with tunable optical and electrochemical properties. Since ASV can be used to monitor the composition of Au and Ag in NPs, it is useful to quantify the amount of Ag exchange for different sized Au NPs.

One more goal for this research was to use ASV to analyze the composition and atomic arrangement of bimetallic alloy NPs. Previously, spectroscopy and microscopy techniques have been mainly used for the analysis of metal composition and atomic arrangement in some bimetallic nanostructures. Again, those techniques are costly, time consuming, and it is not possible to analyze NPs on electrode surfaces directly during dealloying of nanostructures. Therefore, direct analysis of NP composition and atomic arrangement by ASV would be highly valuable. The introduction of this dissertation was organized to describe the importance of metal NPs, various synthesis protocols available in the literature to synthesize sub $4 \mathrm{~nm}$ diameter Au NPs, and electrochemical analysis of single metal and bimetallic NPs as prepared and following various chemical and 
electrochemical treatments. The issue of NP stability is very important for application of metal NPs.

\subsection{IMPORTANCE OF METAL NANOPARTICLES RESEARCH}

Metal NPs have a higher SAN as compared to their bulk analog, which makes them useful for various catalytic applications such as $\mathrm{CO}_{2}$ reduction, ${ }^{5-6}$ oxygen reduction reaction (ORR), ${ }^{7} \mathrm{CO}$ oxidation, ${ }^{8}$ hydrogen evolution reactions (HER),${ }^{9}$ organic transformations ${ }^{10}$, photovoltaic applications, ${ }^{11-12}$ and numerous other electrocatalytic process.. Due to the higher SA/V, metal NPs have been an object of intensive studies during the past couple of decades. The optical, ${ }^{13}$ magnetic, ${ }^{14}$ thermal, ${ }^{15}$ chemical ${ }^{16}$ and electrochemical properties ${ }^{17-18}$ of metal NPs are usually very different from the bulk material and strongly related to their size, shape and morphology. Recently, NPs with various shape and structure have been controllably synthesized and used for a vast number of applications, such as catalysis, ${ }^{19}$ sensing ${ }^{20}$ nanoelectronics,${ }^{21}$ imaging, ${ }^{22}$ photothermal therapy, ${ }^{22}$ nanophotonics ${ }^{23}$ and others. Over the past few decade's researchers have developed various methods to synthesize shape- and size-controlled metal nanostructures with various stabilizers. This has led to fundamental interest and research of their size-, shape- and composition-dependent electrochemical and chemical properties of the metal.

There have been reports on the size- and shape-dependent catalytic and sensing applications of metal NPs. For example, Mistry and coworkers showed that the catalytic $\mathrm{CO}_{2}$ reduction activity of Au NPs increased with a decrease of 
size from 8 to $1 \mathrm{~nm}$ in diameter. ${ }^{5}$ Mirkin and co-workers developed DNA sensors using hybridization-induced changes in distance-dependent optical properties of Au-particle modified oligonucleotides and a scanometric DNA array based on silver amplification of hybridization events. ${ }^{24}$ Various methods such as ozone cleaning, ${ }^{25}$ thermal treatment, ${ }^{26}$ and electrochemical oxidation-reduction cycling ${ }^{27}$ have been used prior to the applications of NPs to clean their surface from stabilizer ligands. Many of these cleaning processes can change the NPs size, composition and reactivity, however.

Despite their usefulness, nanostructure characterization is one of the most important aspects in the field of nanoresearch. The most commonly-used techniques are transmission electron microscopy (TEM), ${ }^{25} \mathrm{X}$-ray photoelectron spectroscopy (XPS) ${ }^{28-29}$,energy dispersive X-ray (EDX) ${ }^{30}$ spectroscopy, and mass spectrometry, ${ }^{31}$ which provide the size and composition of metal NPs. X-ray diffraction (XRD) provides information about different crystal planes of NPs and transmission electron microscopy (TEM) gives information about the size, shape and crystal structure of the NPs. Mass spectrometry provides the total mass and size of the NPs. However, high vacuum techniques are very costly and time consuming for the analysis of metal NPs. Also, they do not provide all of the important information, such as the oxidation relevant properties. Understanding the oxidation relevant properties is very important since it determines the NP stability and reactivity during their applications. Developing a simple electrochemical method to understand the metal NP size and size relevant properties would be very useful. This dissertation focuses on the size, stability, and 
reactivity analysis of sub $4 \mathrm{~nm}$ diameter Au NPs by ASV. Also, ASV was used to determine the composition and atomic arrangements of bimetallic CuAu NPs.

\subsection{SYNTHESIS OF METAL NANOPARTICLES}

\subsubsection{Synthesis of water-soluble colloidal 4-50 $\mathrm{nm}$ diameter $\mathrm{Au}$} nanoparticles. Metal NPs are prepared in the solution phase by reducing the metal salt with an appropriate reducing reagent in the presence of some stabilizing ligand. The ligand usually controls the NPs size and shape. The surface chemistry of the NP is intrinsically related to the type of ligand used for the NPs synthesis or a desired ligand could be later incorporated by a ligand exchange approach. The ligand shell has several roles in control of the NP solubility, functionality (targeting biomolecules) and electronic properties. Various stabilizers such as citrate, ${ }^{32}$ thiols, ${ }^{33}$ amines, ${ }^{34}$ surfactants, ${ }^{35}$ biomolecules ${ }^{36}$, phosphines ${ }^{37}$ and polymers ${ }^{38}$ have been used for the synthesis of ligand protected Au NPs. Also, various reducing agents such as $\mathrm{NaBH}_{4},{ }^{32} \mathrm{H}_{2} \mathrm{O}_{2},{ }^{39}$ citrate ${ }^{40}$ and ascorbic acid ${ }^{41}$ are used for the reduction of $\mathrm{Au}$ ions to metal Au during synthesis. Figure 1.1. shows the general experimental protocol for the chemical synthesis of Au NPs. 


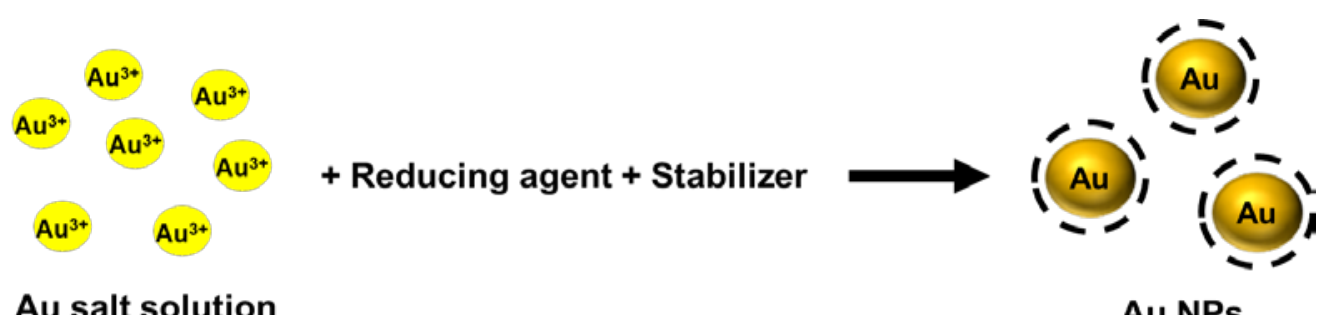

Au salt solution Au NPs

Figure 1. 1. General experimental protocol for the chemical synthesis of Au NPs.

During the last couple of decades, a significant amount of effort has been taken on synthetically controlling the size and shape of metal nanoparticles. John Turkevich published pioneering work on the synthesis of spherical Au NPs in 1951. ${ }^{40}$ He prepared citrate-stabilized spherical Au NPs by the reduction of Au salt with aqueous boiling citrate solution. Later, Frens showed the synthesis of different sized spherical Au NPs by modifying the method reported by Turkevich. ${ }^{42}$ They changed the citrate to gold salt ratios to get various size citrate-stabilized $\mathrm{Au}$ NPs. The amount of citrate relative to the Au salt controlled the nucleation and growth of the Au NPs. Higher ratios of citrate to Au salt led to smaller NPs due to faster nucleation and lower ratios of Au salt to citrate provide bigger sized Au NPs due to slower nucleation. Murphy and coworkers synthesized citrate-coated $\sim 4 \mathrm{~nm}$ diameter Au by the reduction of Au salt with sodium borohydride and extended their work on the seeded growth synthesis of up to $40 \mathrm{~nm}$ diameter Au NPs. ${ }^{32}$ Others synthesized water-soluble colloidal Au NPs with polymer stabilizers. For example, Eunkeu and coworkers reported the synthesis of colloidal Au NPs stabilized with poly(ethylene glycol) (PEG) ${ }^{43}$ The size of the Au NPs was 
controlled by the ratio of $\mathrm{HAuCl}_{4}$ to PEG stabilizer. DNA based synthesis of watersoluble Au NPs is also very popular for biological applications. ${ }^{44}$

\subsubsection{Synthesis of strong ligand protected $1-2 \mathrm{~nm}$ diameter Au NPs. In recent} days it is possible to synthesize metal NPs with atoms from thousands to just only a few atoms. The synthesis of ultra-small NPs with few atoms is possible with the help of organic ligands such as thiols, amines and polymers. The Brust method of synthesis of Au monolayer protected clusters (MPCs) are a good example of Au NPs stabilized with thiol ligands (e.g. $\mathrm{Au}_{25}$ and $\left.\mathrm{Au}_{144)}\right)^{2,45}$ The interaction between Au and thiol groups of the stabilizer ligand are considered to be very strong, which makes it possible to form very small $\mathrm{Au}$ clusters. Figure 1.2. shows a representation of Au MPCs and the gold-sulfur interface at the nanoscale level. These methods allow the synthesis of NP sizes below $1 \mathrm{~nm}$ but the main drawback of this method of Au cluster synthesis is the blocking of most of the surface Au atoms with thiols, which ultimately makes the NPs less reactive for their electrochemical and chemical applications. In fact, it was found that the Au-S bond is stronger than Au-Au bond in monolayer-protected clusters. ${ }^{46}$

Crooks and coworkers developed the synthesis protocol for few nanometer size NPs within dendrimers. ${ }^{19}$ This method of synthesis allows partial access to the metal core and makes it possible to access the surface atoms of small metal NPs. Figure 1.3. shows a representation of dendrimer encapsulated Au NPs. 


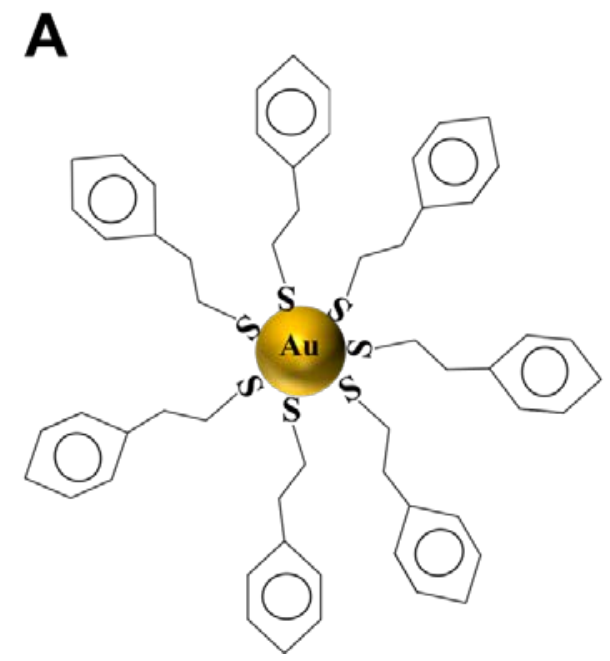

B

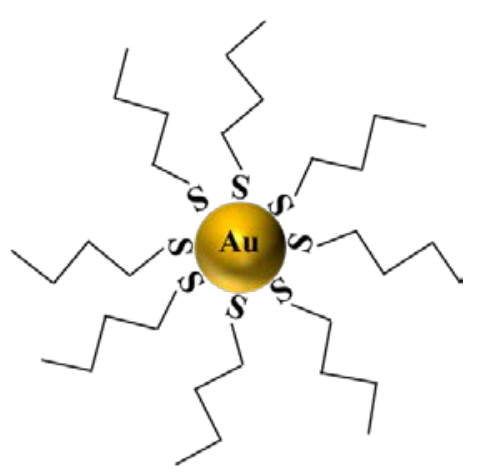

Figure 1.2. Representation of monolayer protected Au cluster (a) thiophenyl coated Au cluster and (b) alkane thiol coated Au cluster.

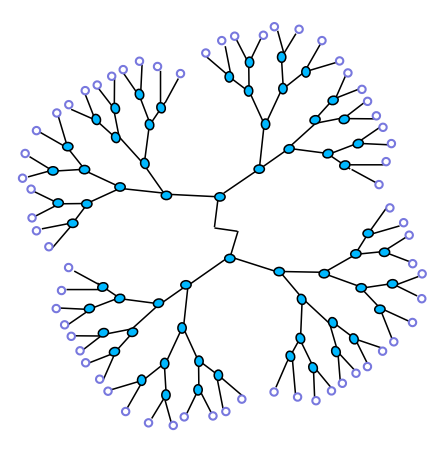

Dendrimer

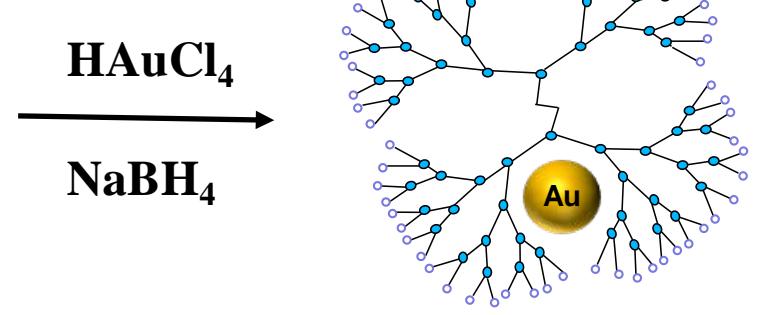

Dendrimer coated Au NPs

Figure 1. 3. Crooks method of synthesis of dendrimer coated 1-2 nm diameter Au NPs. 


\subsubsection{Synthesis of water-soluble weak ligand protected 1-2 $\mathrm{nm}$ diameter $\mathrm{Au}$}

NPs. There are a number of reports on the synthesis of water soluble 1-2 nm diameter Au nanoclusters stabilized with phosphines ligands in the literature. A potential approach to the synthesis of small Au clusters with water-soluble phosphine ligands was reported by Duff and coworkers. ${ }^{47}$ In 1993, Duff and coworkers synthesized sub $2 \mathrm{~nm}$ diameter Au NPs stabilized with tetrakis(hydroxymethyl)phosphonium chloride (THPC) ligands in water. ${ }^{47-48}$ This alkane chain phosphine ligand allows one to synthesize a very narrow size distribution of sub $2 \mathrm{~nm}$ diameter Au NPs. Figure 1.4. (A) shows the typical synthesis protocol of sub $2 \mathrm{~nm}$ Au NPs by the Duff method. In this method, the Au salt was mixed with THPC in basic conditions with a specific $A u(I I I) / T H P C$ ratio. THPC produces formaldehyde by reacting with $\mathrm{NaOH}$, which acts as a strong reducing agent to reduce the Au salt. This method of synthesis has been used for various biological and chemical applications. ${ }^{49} \quad$ Recently, different groups synthesized small Au clusters having 8-12 atoms of Au using a triphenylphosphine ligand stabilizer. For example, Yao and coworkers reported the synthesis of $A u_{11}$ clusters using triphenylphosphine monosulfonate (TPPS) as a stabilizer and sodium borohydride as a reducing agent in methanol media. ${ }^{3}$ They determined the number of atoms in the nanocluster by mass spectrometric analysis. On the other hand, other groups reported the synthesis of triphenylphosphine coated $\mathrm{Au}_{11}$ clusters by the reduction of $\mathrm{Au}$ triphenylphosphine compound $\left(\mathrm{AuPPh}_{3} \mathrm{Cl}\right){ }^{50-51}$ The synthesis of Au6 clusters by TPP ligand was also reported in the literature. For example, Huang and coworkers synthesized Au6 clusters by adjusting the ratio of 
$\mathrm{AuPPh}_{3} \mathrm{Cl}$ to $\mathrm{NaBH}_{4}$. They also found that $\mathrm{Au}_{6}$ clusters can be converted to $\mathrm{Au}_{7}$ or Aug by mixing different percentages of ammonia solution. ${ }^{51}$ Figure 1.4. (B) show the general experimental protocol for synthesis of TPP coated Au clusters.

(A)<smiles>OC[P+](CO)(CO)CO</smiles>

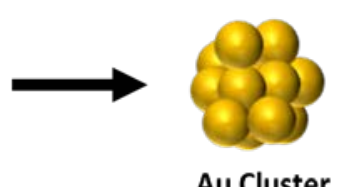

THPC

(B)<smiles>OC[P+](CO)(CO)CO</smiles>

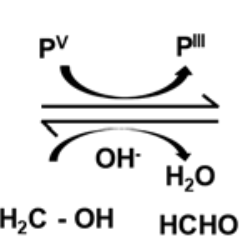<smiles>OCP(CO)CO</smiles>
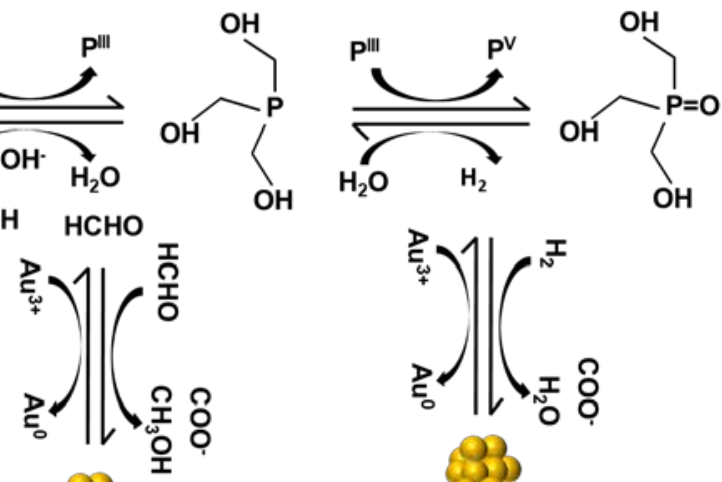

THPC

.

Au Cluster

Au Cluster

Figure 1. 4. (A) Duff method of synthesis of Tetrakis(hydroxymethyl)phosphonium chloride coated 1-2 nm diameter Au NPs and (B) representation of the triphenylphosphine coated Au nanocluster.

\subsection{ELECTROCHEMICAL STUDIES OF METAL}

\section{NANOPARTICLES}

Recently, the electrochemical analysis of metal NPs has become very popular due to its simple operation, high throughput and low cost. Various electrochemical 
studies have been reported in the literature for metal NP analysis, including electrodeposition, ${ }^{52}$ electron transfer or double layer charging, ${ }^{53}$ electrocatalysis, ${ }^{7}$ single particle collisions, ${ }^{54}$ and oxidation ${ }^{17}$. This dissertation focuses on electrooxidation studies of single metal and bimetallic NPs and describes the electrooxidation properties of metal NPs in detail in this introduction section.

1.5.1. Oxidation of metal NPs. The oxidation of metal is an important electrochemical process which happens during corrosion of a metal structure and even during the synthesis of metal NPs. Metal NPs have been widely used for different electrochemical process including electrochemical sensing, ${ }^{55}$ electrocatalysis, ${ }^{19}$ and electron transport. ${ }^{56}$ During these applications metal NPs can oxidize or corrode and ultimately lose their desirable function. Understanding metal NP oxidation properties could be useful to synthesize corrosion-protected nanomaterials. The oxidation-reduction behavior of metal NPs is directly related to the Nernst equation. The Nernst equation directly correlates the half-cell potential to the standard potential and to the activities of the electro active species. For example, a redox couple $M(O x)+n e \rightarrow M($ Red), the Nernst equation will be

$$
E=E^{O}+(0.059 / n) \log (a M(O x) / a M(R e d))
$$

Where, $\mathrm{n}$ is the number of electrons transferred during the reaction, $E$ is the half cell potential $(\mathrm{V}), E^{o}$ is the standard redox potential, $\mathrm{aM}(\mathrm{Ox})$ is the activity of the oxidized form of the metal and $a M(R e d)$ is the activity of the reduced form of the metal. The standard electrode potential is different for different metal/metal ion pairs. Tables show the standard reduction potential for metal/metal ion pairs considering the bulk metal, which significantly changes for smaller nanomaterials. 
The current tables do not consider the metal particle size effect on the standard reduction potential.

In 1977, Henglein found that small metal Ag clusters showed unusual catalytic reactivity during radiolysis and he explained the reactivity of the small metal clusters based on their standard potential. ${ }^{57} \mathrm{He}$ found that $1 \mathrm{Ag}$ atom and a 2- atom Ag cluster have a standard potential of $-1.8 \mathrm{~V}$ and $-1.0 \mathrm{~V}$, respectively, as compared to $+0.799 \mathrm{~V}$ for bulk $\mathrm{Ag}$ (vs. NHE). He calculated the standard reduction potential for different-sized Ag clusters based on the difference in sublimation energy. In 1982, Plieth published a theoretical equation that predicts the shift in oxidation potential of metal NPs as a function of size. ${ }^{58}$ For this calculation he considered an electrochemical redox cell consisting of two half-cells. One cell having a bulk metal particle (Mex ${ }^{\text {bulk}}$ ) containing more than hundred thousand atoms, which is considered a bulk size. This bulk metal particle becomes oxidized into the solvated metal ions, $\mathrm{Mex}^{\text {bulk }} \mathrm{z}^{+}$(Equation 1.2). The other cell consists of metal ions from Equation 1.2 being reduced into several smaller NPs, which are knows as being dispersed, $\mathrm{nMex}^{\text {disperse }}$ (Equation 1.3).

$$
\begin{aligned}
& \mathrm{Mex},{ }^{\text {bulk }} \rightarrow \mathrm{Mex}{ }^{\text {bulk }} \mathrm{z}^{+} \text {(solv) }+\mathrm{ze}^{-} \\
& \text {Mex, }{ }^{\text {bulk } \mathrm{z}^{+}}(\mathrm{solv})+\mathrm{ze}^{-} \rightarrow \mathrm{n} \text { Mex, }{ }^{\text {disperse }}
\end{aligned}
$$

The potential of this type of electrochemical cell can be calculated from the difference of Gibbs free energy of the dispersion process,

$$
\Delta G_{D}=G_{\text {disperse }}-G_{\text {bulk }}
$$

In terms of potential, 


$$
\Delta \mathrm{E}_{\mathrm{D}}=\mathrm{E}_{\text {disperse }}-\mathrm{E}_{\text {bulk }}=-\Delta \mathrm{GD}_{\mathrm{D}} / \mathrm{zF}
$$

Where $\Delta \mathrm{E}_{\mathrm{D}}$ is defined as the difference in the oxidation potential between NPs or clusters (Edisperse) and bulk metal (Ebulk), $\mathrm{z}$ is the number of electrons, and $\mathrm{F}$ is Faraday's constant. Plieth considered the change in surface free energy to determine the total energy change associated with this process. The change in surface free energy of a metal NP is related to the surface area (A) and the surface stress $(Y)$ as follows:

$$
d G=Y d A
$$

The surface area of a sphere is as follows:

$$
A=4 \pi r^{2}
$$

The change in surface area with respect to the radius is given by the first derivative as follows:

$$
d A=8 \pi r d r
$$

The molar volume of the metal $\left(V_{M}\right)$ is related to the radius ( $r$ )and number of moles of metal (n) as follows:

$$
V_{M}=(4 / 3) \pi r^{3} / n
$$

With rearrangement and taking the derivative of $n$ with respect to $r$, we get:

$$
\begin{gathered}
\mathrm{n}=(4 / 3) \pi r^{3} / \mathrm{V}_{\mathrm{M}} \\
\left.\mathrm{dn}=\left(4 \pi \mathrm{r}^{2}\right) / \mathrm{V}_{\mathrm{M}}\right) \mathrm{dr} \\
\mathrm{dr}=\left(\mathrm{V}_{\mathrm{M}} / 4 \pi \mathrm{r}^{2}\right) \mathrm{dn}
\end{gathered}
$$

Integration of equation 1.8 between $\mathrm{n}=0$ and $\mathrm{n}=1$ moles and combination with equations 1.6 and 1.7 gives the free surface energy of one mole of metal NPs of radius $r$. The free surface energy of the bulk can be neglected, and we obtain, 


$$
\Delta G_{D}=\left(2 Y V_{M}\right) / r
$$

Finally, by replacing $\Delta G_{D}$ value in equation 1.5 , we get the Plieth equation as follows:

$$
\Delta \mathrm{E}_{\mathrm{D}}=-2 \mathrm{Y} \mathrm{V}_{\mathrm{M}} / \mathrm{ZFr}
$$

Based on the above equation we plotted the shift in the standard reduction potential of Au NPs as a function of NP radius as shown in the Figure 1.5. We used

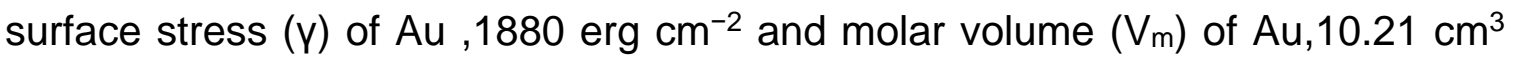
$\mathrm{mol}^{-1}$ for plotting the Figure 1.5. The potential shifts negative as the radius (or diameter) of the Au NPs decreases with a 1/radius dependence.

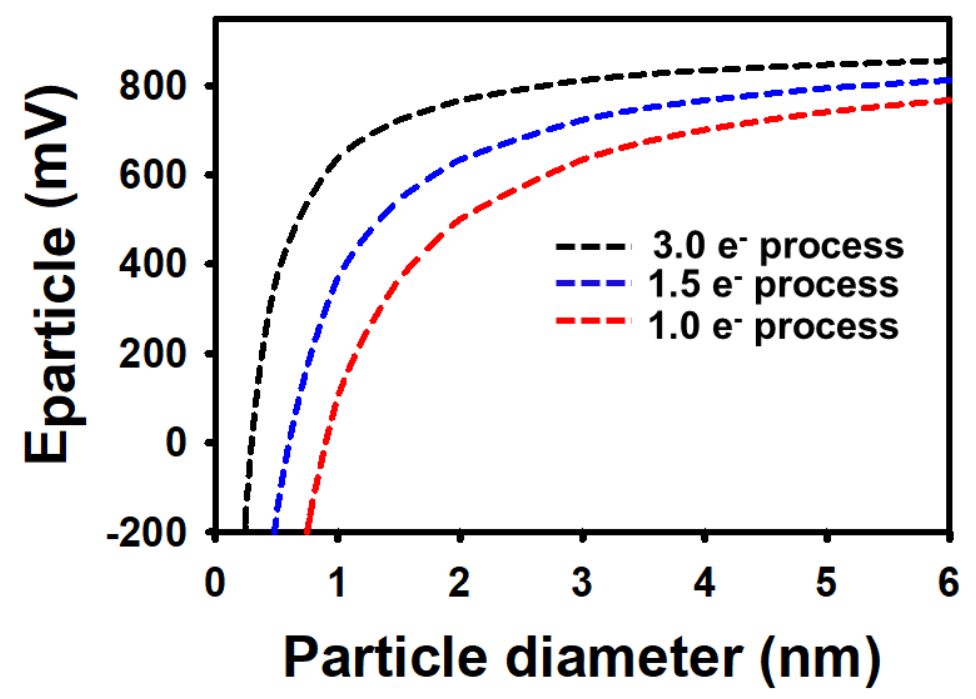

Figure 1. 5. Theoretical oxidation peak potential shift of Au NPs based on Plieth's equation.

After Plieth, ${ }^{58}$ several groups conducted experimental studies to understand the effect of NP size on their oxidation potential. For example, Schalow and 
coworkers studied the interface-controlled oxidation mechanism for Pd NPs supported on $\mathrm{Fe}_{3} \mathrm{O}_{4}$ films by molecular beam $\mathrm{CO}$ titration experiments on oxygenpre-covered Pd NPs. ${ }^{59}$ They observed a pronounced particle-size dependence of the oxidation and reduction behavior for the Pd NPs. Specifically, for small particles $(<3 \mathrm{~nm})$ there is complete oxidation of $\mathrm{Pd}$ to $\mathrm{PdO}$, whereas for intermediate particle size range $(4-10 \mathrm{~nm})$ only partial oxidation of the NPs occurred at the particle $\mathrm{Fe}_{3} \mathrm{O}_{4}$ interface by exposed $\mathrm{O}_{2}$ and $\mathrm{CO}$. In the case of larger particles (approximately $10-100 \mathrm{~nm}$ ) the formation of Pd oxide at the interface is kinetically hindered, possibly due to slow ion transport along the particle/support interface. Brus and coworkers explained the Ostwald ripening of Ag NPs based on the theoretical explanation of the Plieth equation. ${ }^{60}$ They found that Ag NPs attached to indium tin oxide (ITO)-coated glass electrodes (glass/ITO) increased in size with time when a drop of water was added to the Ag NPs on glass/ITO and it was heated. However, they did not show electrochemical results for the size dependent oxidation of Ag NPs. Compton and coworkers reported theoretically and experimentally the effect of Ag NPs size and coverage for the oxidation potential shift. ${ }^{61}$ However, in their studies they focused on diffusional effects and ignored size-dependent shifts in $\mathrm{E}^{0}$. Additionally, Compton et al. described the oxidation of hemispherical deposits of bismuth attached to a single crystal Au (111) electrode under electrochemically irreversible conditions. ${ }^{62}$ Their conclusions established that the difference in the voltammetry occurs mainly due to the morphology/orientation of deposits, leading to differences in the kinetics and thermodynamics of the process of stripping. Sieradzki and coworkers reported 
both theoretically and experimentally the size dependent stability of Pt NPs towards oxidation in acidic solution. ${ }^{63}$ They found that Pt NPs smaller than $4 \mathrm{~nm}$ were oxidized directly to $\mathrm{Pt}^{2+}$ whereas those higher than $4 \mathrm{~nm}$ in size formed a Pt oxide. They proved their theoretical explanations by confirming them with experimental observation using electrochemical scanning tunneling microscopy (ECSTM).

In 2010, Ivanova and Zamborini showed size-dependent oxidation behavior of citrate-coated Ag NPs chemically attached to glass/ITO electrodes for the first time by ASV. ${ }^{17}$ They observed a negative shift in the peak oxidation potential $\left(E_{p}\right)$ from $391 \mathrm{mV}$ to $278 \mathrm{mV}$ for a decrease in size from $\sim 45 \mathrm{~nm}$ to $\sim 8 \mathrm{~nm}$. For that work, they attached $8 \mathrm{~nm}, 16 \mathrm{~nm}, 20 \mathrm{~nm}, 26 \mathrm{~nm}, 28 \mathrm{~nm}, 38 \mathrm{~nm}$ and $45 \mathrm{~nm}$ Ag NPs to 3-aminopropyltriethoxysilane (APTES) functionalized indium tin oxide (ITO) glass electrodes. The attachment of the NPs to the electrode was due to electrostatic interactions between the negatively-charged citrate group on the NPs with the positively-charged amine group on APTES. Ag can be oxidized by the following reaction:

$$
\mathrm{Ag}^{0} \rightarrow \mathrm{Ag}^{+}+1 \mathrm{e}^{-}, \mathrm{E}^{0}=0.79 \mathrm{~V}
$$

The stripping analysis of Ag NPs was performed in $0.1 \mathrm{M} \mathrm{H}_{2} \mathrm{SO}_{4}$ and significant size dependent negative shifts in $E_{p}$ were observed for the small-sized Ag NPs. The general trend followed the expectation of the Plieth equation, but the dependence did not exactly fit the Plieth curve.

Later, Ivanova and Zamborini showed the size dependent $E_{p}$ of Au NPs synthesized both chemically (4 $\mathrm{nm}$ Au NPs) and directly electrodeposited to a 
glass/ITO electrode. ${ }^{64}$ They observed an approximately $100 \mathrm{mV}$ negative shift in $E_{p}$ for Au NPs as the size decreased from $250 \mathrm{~nm}$ to $4 \mathrm{~nm}$ in diameter. The small sized $4 \mathrm{~nm}$ Au NPs were attached electrostatically via aminopropyltriethoxy silane (APTES) while $8 \mathrm{~nm}, 13 \mathrm{~nm}, 23 \mathrm{~nm}$ and $249 \mathrm{~nm}$ were deposited directly on the electrode by applying $-0.2 \mathrm{~V}, 0.2 \mathrm{~V}, 0.4 \mathrm{~V}$ and $0.8 \mathrm{~V}$ for different times, respectively. The oxidation of Au NPs is caused by halide containing acidic electrolyte solutions as follows:

$$
\begin{aligned}
& \mathrm{Au}^{0}+4 \mathrm{Br}^{-} \longrightarrow \mathrm{AuBr}^{-}+3 \mathrm{e}^{-}\left(\mathrm{E}^{0}=0.85 \mathrm{~V}\right) \\
& \mathrm{Au}^{0}+2 \mathrm{Br}^{-} \longrightarrow \mathrm{AuBr}^{-}+\mathrm{e}^{-}\left(\mathrm{E}^{0}=0.96 \mathrm{~V}\right) \\
& 2 \mathrm{Au}^{0}+\mathrm{AuBr}^{-}+2 \mathrm{Br}^{-} \longrightarrow 3 \mathrm{AuBr}^{-} \text {(Chemically) }
\end{aligned}
$$

The amount of total Au metal was kept constant on the electrode for different size NPs in both the Ag and Au NPs analyses. The size-dependent oxidation behavior was much better correlated with the Plieth predicted size for Au than it was for Ag, which seems to be due to the effect of APTES linker for the Ag NPs. Similar to Ivanova and Zamborini, size dependent oxidation of metal NPs was also observed by Brainina and coworkers for $\mathrm{Au},{ }^{65} \mathrm{Ag}{ }^{66}$ and $\mathrm{Bi}{ }^{67}$ NPs of varying sizes. In their work, the different metal NPs were immobilized on the surface of indifferent carbon containing screen printed electrodes. Based on theoretical calculations and experimental studies they found that with decreasing size, there was a negative shift in $E_{p}$ of nanoparticle oxidation towards more negative potentials. 
The oxidation peak potential for 1-2 nm diameter Au NPs is expected to be in the range of 0.4 to $0.0 \mathrm{~V}$ vs $\mathrm{Ag} / \mathrm{AgCl}$. Masitas and Zamborini studied the negative shift in the $E_{p}$ of citrate-coated Au NPs less than $4 \mathrm{~nm}$ in diameter and observed a shift of $\sim 850 \mathrm{mV}$ from bulk $\mathrm{Au} \mathrm{E}_{\mathrm{p}}$ in acid containing halide electrolyte solution, which is in the expected range. ${ }^{68}$ They also observed that the $E_{p}$ for smaller sized (less than $4 \mathrm{~nm}$ ) Au NPs was independent of the halide ions. All of these sizedependent studies for various metal NPs were fairly well correlated to the $1 /$ radius dependence predicted by Plieth. ${ }^{58}$

Buttry and coworkers studied highly purified water-soluble 2,2'-bicinhoninic acid-capped Pd NPs using ASV in $0.1 \mathrm{M} \mathrm{HClO}_{4}$ and $10 \mathrm{mM} \mathrm{NaCl} .{ }^{69}$ They found that the $E_{p}$ also shifted negative as a function of NPs size. This shift was also in agreement with the Plieth model. ${ }^{58}$ The bulk Pd oxidized at $0.70 \mathrm{~V} v \mathrm{Ag} / \mathrm{AgCl}$, while $1 \mathrm{~nm}$ diameter Pd NPs oxidized at $0.34 \mathrm{~V}$. The positive correlation between experimental and calculated data for the electrooxidation of different metal NPs confirms the Plieth model, which is based on the increase of Gibbs surface free energy with a decrease in particle radius. Since this shift is quantitatively related to the value of Gibbs free surface energy, it is also possible to formulate the reverse problem, which is to estimate the magnitude of Gibbs free surface energy based on experimentally recorded $E_{p}$ values for electrooxidation from the stripping voltammetry.

In addition to the size determination of metal NPs based on $E_{p}$, our group recently developed a method to electrochemically analyze the metal NP size directly on the electrode surface by measuring the electroactive surface area (SA) 
of the NPs and the total volume (V) by stripping voltammetry on the same electrode. ${ }^{70-71}$ The electroactive SA was determined by measuring the surface oxidation-reduction reaction by cyclic voltammetry of Au NPs in acidic electrolyte and the total volume of the same NPs was determined by ASV in bromidecontaining electrolyte. ${ }^{72-73}$ The results correlated amazingly well with the SEM measured size of different-sized spherical Au NPs by using the relation of SA/V= 3/r, where $r$ is the radius of the spherical Au NPs. The citrate-stabilized Au NPs had to be cleaned with ozone prior to electrochemical analysis for the best results.

\subsection{SIZE STABILITY STUDY OF METAL NPS.}

1-2 nm diameter Au NPs are widely studied for different electrochemical reactions due to their unique properties. Most of these properties lead to a variety of electrochemical applications in catalysis, ${ }^{5}$ sensing ${ }^{74}$ and electronics. ${ }^{75}$ The reactivity of Au nanomaterials during these applications depend mostly on their size, their combination with other metals, stabilizer ligands and the attachment method of the NPs to the electrode surface. In each of these cases, the interactions of the NPs with the substrate surface affects their properties. Various electrochemical and chemical treatments, such as ozone cleaning, ${ }^{25}$ thermal treatment, ${ }^{76}$ electrocatalytic reactions with gas-phase molecules, ${ }^{34}$ oxidationreduction cycling in acid, ${ }^{27,77}$ and exposure to acidic or basic environments, ${ }^{60}$ can induce transformations of the NPs that lead to changes in the desirable properties of the metal NPs. These are common treatment strategies that metal NPs encounter during their applications or prior to their applications. However, during 
these treatments the size of the metal NPs usually increases, which significantly decreases their reactivity due to the dramatic decrease in SA/V. The size and structural stability of the metal NPs is critical to maintain their reactivity for different applications. In particular, NP coarsening that directly leads to a decrease in NP SA/V is highly undesirable for electrocatalysis experiments.

Two main mechanisms for coarsening have been described, which are known as Ostwald ripening ${ }^{8}$ and Smoluchowski ripening. ${ }^{78}$ In general, dissolution of small particles and redeposition of dissolved ions/species on the surface of bigger-sized particles is called Ostwald ripening. Ostwald ripening is a major process that occurs during NP synthesis and even over time in NP solutions. It is characterized by a larger average NP size and large size dispersity. ${ }^{79}$ A better understanding of the phenomenon will help to improve the size uniformity during NP synthesis and the post synthesis stability. The main driving force for Ostwald ripening of NPs is the difference in chemical potential between small-sized and bigger-sized particles. The larger interfacial area of smaller-sized particles results in larger surface free energy. The reduction in surface free energy drives them to oxidize (dissolve) and redeposit onto bigger-sized particles in the case of electrochemical Ostwald ripening. Heat-induced detachment of metal atoms from small NPs and reattachment to larger NPs can also occur, which is also a form of thermally induced Ostwald ripening. The SA/V decreases with an average increase in particles size due to Ostwald ripening, which makes them thermodynamically more stable. Ostwald ripening of metal NPs in solution can also be described based on the critical radius of NPs. ${ }^{80}$ The critical radius is the radius 
at which NPs do not grow further in size by Ostwald ripening due to the equilibrium that is established with the surrounding concentration of monomer atoms or ions. ${ }^{81}$ The critical radius is

$$
r=\left(2 Y V_{m} / R T I n S\right)
$$

Where, $\mathrm{V}_{\mathrm{m}}$ is the molar volume of monomer, $\mathrm{T}$ is the temperature, $\mathrm{Y}$ is the specific surface energy, $\mathrm{R}$ is the gas constant and $\mathrm{S}$ is the supersaturation of the monomer. $\mathrm{S}$ is the ratio of the actual monomer concentration to the monomer solubility over a flat surface. NPs below the critical radius grow in size by dissolution and redeposition onto NPs above the critical radius. The dissolution and growth of supported metal NPs was also explained by the curvature dependence on the chemical potential of NPs. ${ }^{82}$ The chemical potential of the supported metal NPs follows as

$$
\Delta \mu_{\mathrm{NP}}=2 \mathrm{~V}_{\mathrm{m}} \mathrm{Y} / \mathrm{R}
$$

Where, $V_{m}$ is the molar volume of the bulk metal atoms, $Y$ is the surface energy and $\mathrm{R}$ is the radius of curvature. Small metal NPs have high curvature and higher surface energy. Therefore, small metal NPs will disappear faster and redeposit onto a lower energy NP with higher R. 
Smoluchowski ripening is the coarsening of particles by diffusion and subsequent collision and fusion of intact particles. This kind of ripening is also very common in the field of nano research. For example, in the bottom up synthesis of metal NPs, the first step is a fast reduction of the metal ions by reducing agents. ${ }^{83}$ The metal atoms will form dimers and small clusters which marks the second step. In the final and third step, the clusters grow due to aggregation and coalescence until reaching a final particle size at which the particles are sufficiently stabilized in the presence of ligand. This method of NP growth is very popular and in most of the cases they follow Smoluchowski ripening to

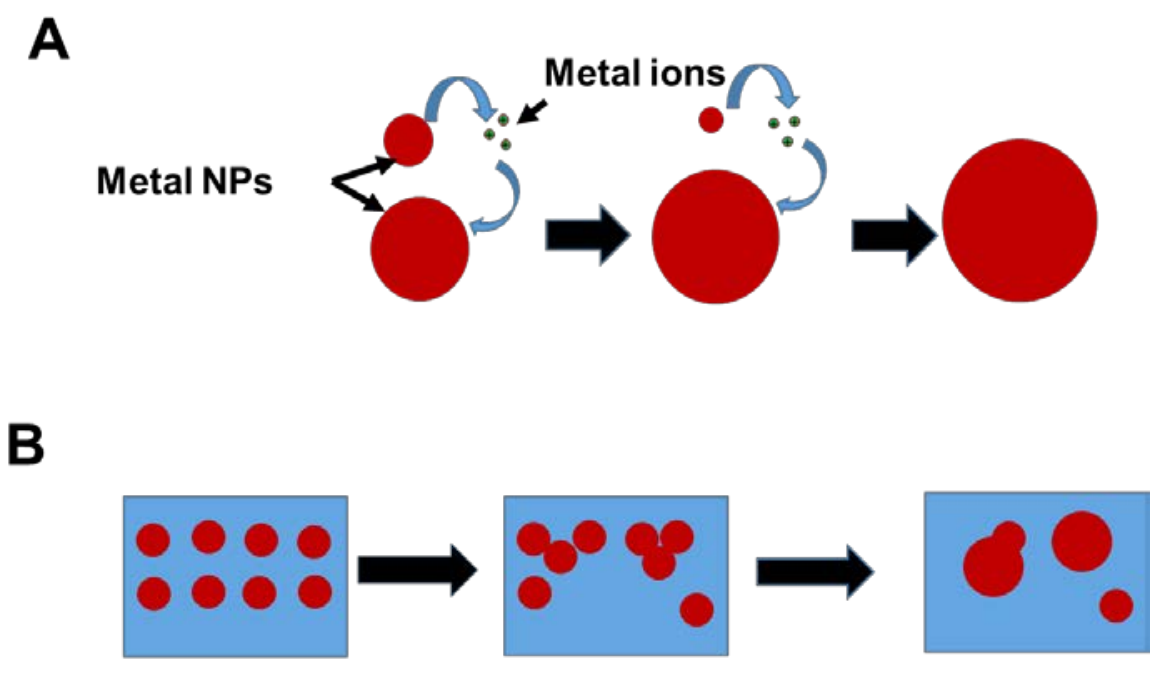

Figure 1. 6. Schematic representation of two types ripening mechanisms of metal NPs known as (A) Ostwald ripening and (B) Smoluchowski ripening. 
some degree. Both forms of ripening can occur simultaneously as was recently imaged in real time by TEM. ${ }^{84}$ Figure 1.6. illustrates the two different ripening mechanisms

There have been a significant number of reports on the ripening of metal NPs, both heat-induced ${ }^{85}$ and electrochemically-induced. ${ }^{86}$ For example, Redmond et al. studied electrochemical Ostwald ripening of colloidal Ag NPs on a conductive surface by using scanning electron microscopy. ${ }^{60}$ They deposited Ag metal NP thin films on the surface of glass/ITO and then added a couple of drops of water on the surface of the electrode. After being exposed to water, bigger $\mathrm{Ag}$ NPs were formed at the expense of smaller Ag NPs by electrochemical Ostwald ripening. Recently Liu et al. reported the electrochemical Ostwald ripening of $\mathrm{Au} / \mathrm{TiO}_{2}$ composite materials. ${ }^{87}$ They synthesized $\mathrm{TiO}_{2}$ nanoparticles first and then made a $\mathrm{TiO}_{2} / \mathrm{Au}$ composite mixture by reducing $\mathrm{HAuCl}_{4} .3 \mathrm{H}_{2} \mathrm{O}$ with ascorbic acid. After that, they attached the composite mixture onto a clean ITO-coated glass electrode and performed a few cyclic voltammetry cycles in $\mathrm{H}_{2} \mathrm{SO}_{4}$ solution. They observed the formation of bigger sized Au NPs from the smaller sized Au NPs supported by larger $\mathrm{TiO}_{2}$. Interestingly, they did not observe the Ostwald ripening phenomenon of Au NPs without $\mathrm{TiO}_{2}$. Recently, Jang and coworkers observed the growth of cetyltrimethylammonium bromide (CTAB)-stabilized Au NPs in the presence of $\mathrm{H}_{2} \mathrm{O}_{2}$ by Ostwald ripening.$^{88}$ In this case $\mathrm{Br}^{-}$from the CTAB stabilizer in combination with the oxidizing properties of $\mathrm{H}_{2} \mathrm{O}_{2}$ oxidized $\mathrm{Au}$, presumably to $\mathrm{AuBr}_{4}{ }^{-}$or $\mathrm{AuBr}_{2}{ }^{-}$, followed by redeposition to $\mathrm{Au}^{0}$ on larger $\mathrm{Au} N P s . \mathrm{H}_{2} \mathrm{O}_{2}$ sets the 
chemical potential that aids in both the oxidation and reduction processes. During this process Au NPs grow in bigger size at the expense of smaller ones.

Others reported the ripening of metal NPs during electrocatalytic applications. For example, Crooks and coworkers showed that Au NPs 1-2 nm in size are very reactive for electrochemical $\mathrm{CO}_{2}$ reduction. ${ }^{19}$ However, they found that the NPs lack long-term stability since the 1-2 nm diameter Au NPs converted to $5-6 \mathrm{~nm}$ diameter, becoming inactive for $\mathrm{CO}_{2}$ reduction. Encapsulating the $\mathrm{Au}$ NPs in dendrimers aided in keeping the size stable and maintaining their electrocatalytic activity for longer time. $\mathrm{Hu}$ and coworkers reported the size dependent ripening of $\mathrm{Au}$ nanoclusters by using high angle annular dark field scanning transmission electron microscope (HAADF-STEM) when the nanocluster was used for $\mathrm{CO}$ electrocatalysis. ${ }^{8}$ For this study the nanoclusters were attached to an amorphous carbon film. They found that small size clusters such as Au561+13 and Au923 \pm 20 grow bigger in size by direct Ostwald ripping. However, bigger sized particles such as $\mathrm{Au}_{2057 \pm 45}$ grew by Smoluchowski Ripening. Karthish and coworkers observed the dendritic assembly of Au NPs when used for $\mathrm{CO}_{2}$ reduction. ${ }^{89}$ Such an assembly was observed due to the conversion of $\mathrm{CO}_{2}$ to $\mathrm{CO}$ gas, which facilitated easy movement of particles along the substrate, leading to an aggregative Smoluchowski type of ripening. They confirmed the assembly of the particles by SEM and TEM imaging. The size changes make them difficult to use for different applications.

The stabilizers used in the synthesis of metal NPs are necessary to synthesize the NPs with size and shape control but then act as a hindrance usually 
for catalysis and other applications. Many researchers use post-synthetic treatment with ozone to remove these stabilizers in order to improve reactivity. ${ }^{90}$ However, it was found that the metal NP size changes after ozone treatment. For example, Elliott and coworkers studied the size transformation of alkanethiolatestabilized Au NPs following ozone treatment by TEM imaging. ${ }^{25}$ They found that the NP size was increased from $1.4 \mathrm{~nm}$ to $1.6 \mathrm{~nm}$ in diameter after $16 \mathrm{~min}$ of ozone treatment, but remained the same after 8 min of ozone treatment.

There are numerous studies on the thermal treatment of metal NPs prior to their application, especially in catalysis that show a significant heat-induced increase in NP size. For example, Shivhare and coworkers showed that thiolate (phenylethanethiolate, and hexanethiolate) removal from the surface of Au25 NPs took place at as low as $125{ }^{\circ} \mathrm{C}$ by extended X-ray absorption fine structure (EXAFS) spectroscopy. ${ }^{85}$ The complete removal of thiol occurred at $250{ }^{\circ} \mathrm{C}$ where the Au NPs showed the highest catalytic activity for 4-nitrophenol reduction. However, after the ligand loss, the NP size increased 1.3 to $1.9 \mathrm{~nm}$ in diameter. Geoffroy and coworkers reported the phase separation of bimetallic AuPd alloy NPs when annealed at $873 \mathrm{~K} \cdot{ }^{91}$ They observed $3.5 \mathrm{~nm}$ diameter Au rich and 25 $\mathrm{nm}$ diameter Pd rich NPs after the heat treatment. The phase separation was based on an Ostwald ripening process. Another example is the report of Li and coworkers, who showed an increase in the catalytic performance of oleyaminecoated 2-3 nm diameter Pt NPs after thermal treatment at $185^{\circ} \mathrm{C}$ for 5 hours. ${ }^{92}$ However, the method ultimately changed the NP size despite having the higher reactivity. 
Metal NP aggregation can also occur and diminish the performance of the NPs for certain applications. The aggregation of NPs is usually directed by interparticle attractions which is typically achieved by intermolecular forces such as van der Waals forces, ${ }^{93}$ л-л interactions, ${ }^{94}$ dipole-dipole interaction, ${ }^{95}$ or hydrogen bonding. ${ }^{96}$ For example, Wei and coworkers reported a photo-switchable hydrosilylation reaction mediated by dynamic aggregation of Au NPs. ${ }^{97}$ They observed that upon irradiation with UV light Au NPs aggregate and their catalytic reactivity decreases, but upon irradiation with visible light, the catalytic reactivity increased due to the NPs becoming isolated. The aggregation was monitored by dynamic light scattering (DLS) and TEM imaging. Others studied aggregated NPS for electrocatalytic oxidation of methanol, ${ }^{98}$ Glucose, ${ }^{99}$ and ethanol, ${ }^{100}$ often showing improved activity for aggregated structures. For example, Wen and coworkers showed that electrocatalytic glucose oxidation reactivity increased for 5-6 nm diameter Au aerogels synthesized with beta-cyclodexrin and aggregated using dopamine as compared to isolated, citrate-stabilized Au NPs of similar size. ${ }^{99}$ The increased reactivity of the aggregated NPs was thought to occur by the increase of porosity. Some studies have examined the effect of aggregation on the metal NP oxidation, mainly focusing on how aggregation affected the peak current. ${ }^{101-103}$ In contrast, Allen et al. was the first to study the effect of aggregation on the $E_{p}$ of oxidation. ${ }^{104}$ In that work, the aggregation of citrate-stabilized Au NPs was controlled by $\mathrm{pH}$ and characterized by ASV. It was found that after aggregation, ${ }^{105}$ the Au NP surface area decreases significantly and the $E_{p}$ shifts positive, whose magnitude depends on the aggregate size with the limit of shifting 
to the bulk Au $E_{p}$ for aggregates above a certain size where the SA/V is close to that of bulk Au.

These different studies show significant increases in the size of metal NPs during electrocatalysis or cleaning by different method, such as ozone, thermal treatment, or electrochemical cycling. In most of these cases, the aggregation or ripening of metal NPs was monitored by microscopic methods, which are costly, tedious and don't provide all the valuable information relevant to size dependent oxidation of NPs under potential control on an electrode surface. Therefore, our study of metal NP size-dependent reactivity based on their oxidation behavior is highly desirable. It is simple, cheap, fast, and offers a wealth of information on size, composition, atomic arrangement, and aggregation for NPs assembled directly on electrode surfaces in order to follow different size transformations under a variety of conditions with the ability to obtain good statistical data. This is a significant advantage. It will not completely replace electron microscopy and spectroscopic characterization but can serve as a complementary method to screen many samples initially and choose samples that need further analysis.

\subsection{SIZE DEPENDENT GALVANIC EXCHANGE REACTION OF METAL NPs.}

In recent years core/shell metal NPs with various structures, such as nanocubes, ${ }^{106}$ nanorods, ${ }^{107}$ and nanotubes, ${ }^{108}$ have gained tremendous interest in the field of nanoscience due to their potential applications in catalysis and sensing. The formation of these core/shell nanostructure has been mostly performed during 
the chemical reduction process, where ions of one metal were reduced onto the surface of another metal in the presence of some type of stabilizer. However, the synthesis of nanomaterials by this strategy takes on multiple steps, which sometimes makes it difficult to control the shape and structure. Therefore, researchers have been searching for alternative methods to synthesize nanomaterials of well-controlled structure. Recently, galvanic exchange reactions have become a promising alternative approach to make designer, well-engineered nanomaterials. The galvanic exchange reaction is driven by the difference in the standard potential of two different metals. Usually this reaction leads to the deposition of a more stable metal by the oxidation of a less stable metal. This strategy has been used recently for making various uniquely-structured nanomaterials, including hollow nanocubes, ${ }^{109}$ nanotubes, nanorattles, ${ }^{110}$ and nanocages. ${ }^{111}$ For example, Murshid and coworkers performed a galvanic exchange reaction between $\mathrm{Ag}$ decahedral NPs and an $\mathrm{Au}^{3+}$ ions to form stable core/shell Ag/Au decahedral alloy NPs. ${ }^{112}$ The following reaction is one example of a typical galvanic exchange reaction between two metals, $\mathrm{Ag}(0)$ and an $\mathrm{Au}(\mathrm{III})$ complex in this case.

$$
3 \mathrm{Ag}_{(\mathrm{s})}+\mathrm{AuCl}_{4}^{-}(\mathrm{aq}) \rightarrow \mathrm{Au}(\mathrm{s})+3 \mathrm{Ag}^{+}(\mathrm{aq})+4 \mathrm{Cl}_{(\mathrm{aq})}^{-}
$$

There are examples of non-conventional galvanic exchange between the ion of the less noble metal and the metallic form of the more noble metal, which should not occur thermodynamically. For example, galvanic exchange between thiolate-stabilized $\mathrm{Au}_{25}$ nanoclusters with $\mathrm{Ag}^{+}$and $\mathrm{Cu}^{2+}$ ions was reported first by Murray and coworkers. ${ }^{113}$ They believe that addition of metal ions on the Au25 
nanocluster was possible because thiolate ligands gain a partial negative charge after attaching to the $\mathrm{Au}$ or $\mathrm{Ag}$ surface, which assisted in $\mathrm{Ag}^{+}$ion reduction on the Au surface. Later, Wu and coworkers reported that galvanic exchange of thiolstabilized $\mathrm{Au}$ nanoclusters with $\mathrm{Ag}^{+}$ions is only possible if the $\mathrm{Au}$ nanocluster size becomes lower than $3 \mathrm{~nm}$ in diameter. ${ }^{114}$ They also believe that this reaction occurs due to the partial negative charge of thiolate ligand, which acts as a reducing agent. In 2014, Wang and coworkers reported the anti-galvanic exchange reaction of naked Au NPs. ${ }^{115}$ Ligand free naked Au NPs were obtained by the reduction of $\mathrm{Au}^{+}$ions with $\mathrm{NaBH}_{4}$ and the resultant aggregated NPs were dispersed by the laser ablation method. In this process they were able to get well disperse 1 to $19 \mathrm{~nm}$ diameter Au NPs with an average size of $13.5 \pm 0.5 \mathrm{~nm}$ in diameter. After addition of an aqueous solution of $\mathrm{AgNO}_{3}$ to the Au NPs solution they found that Ag atoms deposited on the Au NPs based on the peak in the X-ray photoelectron spectroscopy (XPS). This finding indicates that anti-galvanic exchange of Au NPs with $\mathrm{Ag}^{+}$ions is not due to the thiolate ligand but could be due to the thermodynamic negative shift of the oxidation peak potential of naked Au NPs. Yao and coworkers reported that Ag atoms do not replace Au atoms in the precursor particles, but that they instead place side by side of Au atoms by depositing on Au.116 They proved this phenomenon based on the mass spectroscopic analysis and the finding was also supported by theoretical calculations. Recently, Tian and coworkers reported that anti-galvanic exchange between Au nanoclusters and $\mathrm{Ag}^{+}$ions depends on the structure ions precursor. ${ }^{117}$ For example, galvanic exchange between aqueous $\mathrm{AgNO}_{3}$ and $\mathrm{Au}_{25}(\mathrm{PET})_{18}$, (PET 
$=\mathrm{SC}_{2} \mathrm{H}_{4} \mathrm{Ph}$ ) shows $\mathrm{Au}_{25} \mathrm{Ag}_{2}(\mathrm{PET})_{18}$ as a main product, while galvanic exchange between $\mathrm{Au}_{25}(\mathrm{PET})_{18}$ with $\mathrm{Ag}-\mathrm{DTZ}$ complexes (DTZ is dithizone) shows $\mathrm{Au}_{24} \mathrm{Ag}(\mathrm{PET})_{18}$ as a main product in the mass spectrum. Luo and coworkers reported the conversion of a 23 atom $\left[\mathrm{Au}_{23}(\mathrm{SR})_{16}\right]^{-}$nanocluster to a 21 atom $\left[\mathrm{Au}_{21}(\mathrm{SR})_{12}\left(\mathrm{Ph}_{2} \mathrm{PCH}_{2} \mathrm{PPh}_{2}\right)_{2}\right]^{+}$nanocluster by resection of two surface $\mathrm{Au}$ atoms first with two $\mathrm{Ag}^{+}$ions followed by replacement of the two $\mathrm{Ag}$ atoms by galvanic exchange with a $\mathrm{Au}(\mathrm{I})$-diphosphine complex, $\mathrm{Au}_{2} \mathrm{Cl}_{2}(\mathrm{P}-\mathrm{C}-\mathrm{P}) .{ }^{118}$ This slight surgery on the Au clusters provided a 10-fold increase in the luminescent properties of the Au nanoclusters.

Several other groups also studied galvanic exchange reactions with 2-3 nm diameter Au nanoclusters for use of the resulting alloy nanoclusters for catalytic applications. ${ }^{119-120}$ For example, Young and coworkers reported that the addition of $\mathrm{Ag}$ atoms on the surface of Au nanoclusters by galvanic exchange significantly increased the 4-electron oxygen reduction reaction (ORR) in alkaline media as compared to other Au-Ag alloy NPs. ${ }^{121}$ These studies indicate that galvanic exchange between $\mathrm{Au}$ and $\mathrm{Ag}^{+}$ions is possible, however, the mechanism of this exchange is unknown in the literature. In this dissertation we report that galvanic exchange between Au NPs and $\mathrm{Ag}^{+}$is thermodynamically favorable when the NP size becomes less than $2 \mathrm{~nm}$ in diameter due to the negative shift of the oxidation potential of Au NPs with decreasing size. Details of the size-dependent galvanic exchange between Au NPs with $\mathrm{Ag}^{+}$ions will be the focus of Chapter VII. 


\subsection{BIMETALLIC NANOPARTICLES}

The synthesis, characterization and application of alloys NPs, such as bimetallic alloy NPs, is an important area of research due to the differences in properties of alloy NPs versus single metal NPs. The properties of alloy NPs can be varied by the arrangement of different atoms, the ratio, and number of different metals used during their synthesis. Alloy NPs can be bimetallic, trimetallic, tetrametallic, and so on. Among these different varieties, bimetallic nanostructures are very popular in the field of nanoalloy research. Bimetallic NPs with different structures often show superior performance compared to their monometallic counterparts due to the combination and arrangement of two different metal atoms and metal-metal interactions. ${ }^{122-124}$ Recently, bimetallic or trimetallic particles were used for different applications in catalysis, ${ }^{125}$ sensing, ${ }^{126}$ photothermal therapy, ${ }^{127}$ nanophotonics $^{128}$ and others. ${ }^{129-132}$ Among the different types of bimetallic structures, core-shell NPs are highly popular. Core/shell NPs are composite nanomaterials constructed with a core of inner metal and shell of outer metal, both at the nanometer scale. ${ }^{133}$ Bimetallic core-shell NPs may show enhanced catalytic reactivity because combining two metals allows tuning of the electronic and geometric structures of the metal NPs.

While the development of highly active, selective, robust, low-cost and environmentally friendly catalytic systems is very challenging for researchers, there has been significant progress with the development of bimetallic alloy NPs. For example, Kim et al. successfully synthesized bimetallic CuAu alloy NPs with 
different ratios and used them to catalyze $\mathrm{CO}_{2}$ reduction. ${ }^{134}$ Citrate-coated coreshell Cu-Au NPs were synthesized by Zhang et al. for calorimetric iodine, ${ }^{126}$ sulfide anion and cysteine recognition and sensing. ${ }^{135}$ Recently, core-shell Cu-Au NPs were synthesized by Liu et al. and used to study unidirectional thermal diffusion of Au atoms from the shell to the core. ${ }^{136}$ Several groups have reported many types of core-shell NPs for various applications. However, core-shell Au-Cu NPs have received comparatively less attention because $\mathrm{Cu}$ is easily oxidized to $\mathrm{Cu}^{2+}$ or copper oxide under ambient conditions and Cu shows lower free electrons on the surface compared to other plasmonic NPs, such as Au and Ag. ${ }^{137-138}$ The catalytic activity of bimetallic particles may vary during the reaction because one metal of the core-shell nanostructure may undergo oxidation or rearrangement by diffusion from the core to the shell or vice-versa due to heat or other reaction conditions.

Recently, dealloying of one metal from the bimetallic NPs attracted tremendous attention in nanoscience research as an excellent method to prepare nanoporous metals, which ultimately increases the metal's performance in catalytic and sensing applications when compared to individual metal NPs. ${ }^{139}$ Both chemical and electrochemical methods of dealloying are used to produce nanoporous NPs. In the case of chemical dealloying, the dissolution of one metal from bimetallic alloy NPs occurs in a suitable chemical solution. For example, Li and coworkers reported nanoporous Au formation by the chemical etching of AuAg NPs with $\mathrm{Fe}\left(\mathrm{NO}_{3}\right)_{3}$ and $\mathrm{NH}_{3} \mathrm{OH}$. After the dealloying of $\mathrm{Ag}$, they found that catalytic conversion of 4-nitrophenol to 4-aminophenol increased due to the increase in $\mathrm{Au}$ surface area. ${ }^{140}$ Other groups reported the electrochemical dealloying of the less 
noble metal of an alloy NP by the appropriate applied potential in an electrolyte solution. For example, Sieradzki and coworkers reported the synthesis of nanoporous Au NPs from ATP-coated 2-6 nm diameter AuAg NPs and citratecoated $40 \mathrm{~nm}$ diameter AuAg alloy NPs by an electrochemical method. ${ }^{141}$ They observed porous Au NPs once they held the potential above $0.6 \mathrm{~V}$ in $0.1 \mathrm{M} \mathrm{HClO}_{4}$ solution, which enables dissolution of Ag. Xu and coworkers studied dealloying of Al from AuAl 2 alloy NPs in the presence of halide ions and observed a decrease in the dealloying critical potential after addition of halide ions. ${ }^{142}$ The dealloying critical overpotential decreases in the order of $\mathrm{I}^{-}<\mathrm{Br}^{-}<\mathrm{Cl}^{-}<$no halides. ${ }^{143}$ Also, addition of halide ions increases the porosity, where it was found that the porosity of the Au film increased by nine times in the presence of iodide-containing electrolyte as compared to those without the addition of iodide.

Others reported electrochemical studies on different shaped Au NPs. Since spherical Au NPs and other shapes, such as nanorods, have different crystallographic planes, mainly the low index (111), (110), and (100) surfaces, the sensitivity of those planes to particular electrochemical reactions were previously used for characterizing the Au NP structures. For example, Hernandez and coworkers specifically characterized the (111) and (110) planes of Au NPs and nanorods by lead underpotential deposition. ${ }^{144}$ They also confirmed the (111) and (110) planes of Au NPs and nanorods by high resolution transmission electron microscopy and selective area electron diffraction. Similarly, this group characterized $40 \mathrm{~nm}$ cubic Au NPs which consisted mostly of the (100) plane by analyzing the oxygen reduction reactivity of NPs in alkaline media. ${ }^{145}$ In alkaline 
media they observed mainly water as a final product for all potential ranges, which suggested that cubic Au NPs have mostly the (100) plane on its surface.

Most research reports describe the use of other non-electrochemical techniques for NP characterization. UV-visible spectroscopy analysis gives information about particle size and distribution due to the plasmonic properties. Xray photoelectron spectroscopy (XPS) and energy dispersive X-ray (EDX) spectroscopy provide the composition of particles. X-ray diffraction (XRD) provides information about different crystal planes of NPs and high-resolution transmission electron microscopy (HRTEM) gives information about the size, shape and crystal structure of the NPs. However, most of these characterization tools don't give detailed information's about both composition and atomic arrangement. In this dissertation we describe the use of ASV for the determination of composition and atomic arrangement of bimetallic Cu/Au NPs and monitor the changes in their arrangements during heating. Details of composition analysis of CuAu NPs is discussed in Chapter VIII and atomic arrangements of CuAu NPs upon thermal treatment is discussed in Chapter IX.

\subsection{SUMMARY AND ACCOMPLISHMENTS}

The middle two sections of this dissertation (Chapter III to Chapter IX) describe size characterization and stability studies of 1-2 nm diameter Au NPs, characterization of aggregate structures, galvanic exchange reaction studies of 1 to $4 \mathrm{~nm}$ diameter Au NPs with $\mathrm{Ag}^{+}$ions, and composition and atomic arrangements analysis of bimetallic CuAu NPs by ASV. 
In Chapter III, we report on the size characterization, stability, and reactivity analysis of 1-4 nm diameter Au NPs. We found that THPC coated $1.6 \mathrm{~nm}$ diameter $\mathrm{Au}$ NPs are active for both electrochemical $\mathrm{CO}_{2}$ reduction and the hydrogen evolution reaction, but that they lack thermodynamic size stability. These Au NPs show significant size transformation during one cycle of surface oxidation and reduction in acidic electrolyte, 1-2 min of ozone treatment and 10 min of holding at a potential of $0.3 \mathrm{~V}$ versus $\mathrm{Ag} / \mathrm{AgCl}$ in $\mathrm{Br}^{-}$. On the other hand, citrate-stabilized 4 nm diameter Au NPs do not show any size changes during these treatments. These results were confirmed by monitoring the positive shift in the oxidation peak potential of $1.6 \mathrm{~nm}$ diameter Au NPs from $0.46 \mathrm{~V}$ to about $0.7 \mathrm{~V} \mathrm{vs} \mathrm{Ag} / \mathrm{AgCl}$ after the various treatments. Chapter IV describes the electrochemical characterizations of TPPS stabilized atomically precise Au11 clusters that are about $0.9 \mathrm{~nm}$ in diameter. These clusters show a single oxidation peak at $0.25 \mathrm{~V} v \mathrm{Ag} / \mathrm{AgCl}$, consistent with the Plieth equation. However, they are also not stable after just one electrochemical oxidation-reduction cycle, ozone treatment, and in low $\mathrm{pH}$ environments. Furthermore, this NPs are not very active for catalytic $\mathrm{CO}_{2}$ reduction and the hydrogen evolution reaction as compared to THPC stabilized $1.6 \mathrm{~nm}$ diameter Au NPs.

In Chapter V, we report the oxidation behavior of different aggregate structures of $15 \mathrm{~nm}$ diameter Au NPs. The $E_{p}$ of $15 \mathrm{~nm}$ diameter Au NPs show a $100 \mathrm{mV}$ positive shift from that of isolated NPs when they were aggregated by acid, while the $E_{p}$ of the same NPs doesn't show any changes when they were aggregated by THPC. However, the optical signal shows plasmonic properties consistent with 
aggregated NPs. Electrochemical SA/V measurements and TEM image analysis of the aggregates provided the reason for this. THPC aggregated NPs did not show any change in their SA/V while acid aggregated NPs showed a significant decrease in SA/V as compared to isolated $15 \mathrm{~nm}$ diameter Au NPs. TEM images revealed that acid-aggregated NPs formed three dimensional structures with fused Au NPs, which is why they had a lowered SA/V. In contrast, THPC-aggregated NPs formed one dimensional or two-dimensional planar aggregates with $\sim 1 \mathrm{~nm}$ spacing between the NPs, which did not alter the SA/N. The Ep value reflected the SA/V value. These differences in aggregation structure was easily distinguishable by electrochemical measurements but not with UV-vis spectroscopy.

Chapter VI describes experiments aimed as using ASV and electrochemical SA/V measurements to study the kinetics of electrochemical Ostwald ripening as a function of electrode potential, NP size, size dispersity, and coverage for electrodeconfined Au NPs. We determined the rate of electrochemically-induced Ostwald ripening of 1.6, 4 and $15 \mathrm{~nm}$ Au NPs by measuring ASV $E_{p}$ and SA/V as a function of ripening potential and time in $\mathrm{Br}^{-}$containing acidic electrolyte solution. Since different size Au NPs oxidize at different potential and the SA/V provides the size of $\mathrm{Au} \mathrm{NPs,} \mathrm{the} \mathrm{rate} \mathrm{of} \mathrm{electrochemically-induced} \mathrm{Ostwald} \mathrm{ripening} \mathrm{can} \mathrm{be}$ determined. Our results show that the rate increases with a decrease in Au NP size for similar reactions conditions. These findings were significant, since Ostwald ripening is a very common phenomenon that occurs during the synthesis of $\mathrm{Au}$ NPs and causes size instability during electrochemical applications. Therefore, understanding the Ostwald ripening reactivity of Au by electrochemical methods 
could be helpful for designing new nanomaterials with tunable size and better stability.

Chapter VII describes the size-dependent galvanic exchange of Au NPs with $\mathrm{Ag}^{+}$ions. The main finding of this work was that TPPC coated Au NPs show complete exchange with $\mathrm{Ag}+$ ions, $1.6 \mathrm{~nm}$ diameter Au NPs show 50-60 percent exchange with $\mathrm{Ag}+$ ions, while $4 \mathrm{~nm}$ dimeter Au NPs do not show only minimal exchange with $\mathrm{Ag}^{+}$ions (<10\%). This is unique reactivity that could lead to new methods for catalyst design.

Chapter VIII describes the composition analysis of citrate stabilized core/shell $\mathrm{Cu}_{1} / \mathrm{Aux}(\mathrm{x}=0.001$ to 2$) \mathrm{NPs}$ by ASV. Our results show that the composition analysis of the NPs is possible in $\mathrm{Cl}^{-}$containing electrolyte while composition analysis becomes impossible in $\mathrm{Br}^{-}$containing acidic electrolyte due the migration of $\mathrm{Au}$ atoms and possible blocking of $\mathrm{Cu}$ oxidation by $\mathrm{Au}$ atoms during dealloying.

Chapter IX describes an analysis of the atomic arrangement of citrate stabilized CuAu bimetallic NPs having three different arrangements of $\mathrm{Cu}$ and $\mathrm{Au}$ atoms by ASV. Core/shell $\mathrm{Cu}_{1} / \mathrm{Au}_{0.33}$ or $\mathrm{Au}_{0.33} / \mathrm{Cu}_{1} \mathrm{NPs}$ display three oxidation peaks at -0.1 $\mathrm{V}$ (for bare $\mathrm{Cu}$ ), $0.25 \mathrm{~V}$ (for Cu directly in contact with $\mathrm{Au}$ ) and $0.9 \mathrm{~V}$ (Au bonded to $\mathrm{Au}$ ) while mixed $\mathrm{Cu}_{1}-\mathrm{Au}_{0.33}$ alloy NPs show only two oxidation peaks at $0.25 \mathrm{~V}$ ( $\mathrm{Cu}$ bonded to $\mathrm{Au}$ ) and $0.9 \mathrm{~V}(\mathrm{Au}$ bonded to $\mathrm{Au}$ ). However, after 1 hour at $473 \mathrm{~K}$ the oxidation peak at $0.25 \mathrm{~V}$ shifted to $-0.05 \mathrm{~V}$ for mixed $\mathrm{Cu}_{1}-\mathrm{Au}_{0.33}$ alloy NPs, indicative of segregation of $\mathrm{Cu}$ atoms. A similar trend has been observed for core/shell NPs as well. 


\section{CHAPTER II \\ EXPERIMENTAL}

\subsection{SUBSTRATE}

Indium tin oxide (tin-doped indium oxide) is made of tin (IV) oxide $\left(\mathrm{SnO}_{2}\right)$ and indium (III) oxide $\left(\ln _{2} \mathrm{O}_{3}\right)$, usually $90 \% \ln _{2} \mathrm{O}_{3}, 10 \%$ weight of $\mathrm{SnO}_{2}$. Indium Tin Oxide (ITO) coated glass (glass/ITO) is a widely used electrode for electrochemical, spectroelectrochemical, and solar cell applications because of its electrical conductivity and optical transparency. We used unpolished float (soda lime) glass coated ITO slides (Delta Technologies, LTD, Part No. CG-50IN-CUV) in our experiments as an electrode material for voltammetric analysis of NPs, for electrocatalysis experiments, and also for UV-Vis measurements. These electrodes have a resistance of 8-12 ohms. We cut the slide to a final size of $25 \mathrm{x}$ $6 \mathrm{~mm}$ using a diamond cutter and cleaned them by sonication for 30-min in ethanol, acetone, and 2-propanol. Finally, we rinsed the slides with water and dried them under $\mathrm{N}_{2}$ before use.

\subsection{CHEMICALS AND REAGENTS}

$\mathrm{HAuCl}_{4} \cdot 3 \mathrm{H}_{2} \mathrm{O}$ was synthesized from metallic $\mathrm{Au}(99.99 \%)$ in our lab. Sodium borohydride, hydrogen peroxide (30 wt.\%), (3-aminopropyl) 
triethoxysilane ( $\geq 98.0 \%$ ), 2-propanol (ACS reagent), $\mathrm{CuSO}_{4} \cdot 5 \mathrm{H}_{2} \mathrm{O}$ (ACS reagent), and potassium bicarbonate (ACS reagent) were purchased from Sigma Aldrich. Ethyl alcohol and acetone (ACS/USP grade) were purchased from PharmcoAAPER. Trisodium citrate salt, potassium perchlorate (99.0-100.5\%), potassium bromide (GR ACS), and perchlorate acid (60\%) were purchased from Bio-Rad laboratories, Beantown Chemical, EMD, and Merck, respectively. Sodium hydroxide (solid) was purchased from Fisher Scientific. Tetrakis(hydroxymethyl)phosphonium Chloride (80\% solution in water) was purchased from ACROS ORGANICS. triphenylphosphine monosulfonate (TPPS) was purchased from Tokyo Chemical Industry (TCl). $\mathrm{A} \mathrm{CO}_{2}$ gas cylinder was supplied by Welders Supply (Louisville, KY). Thiocyanate-capped $2 \mathrm{~nm}$ diameter Au NPs were purchased from BBI Solutions (Madison, WI).

\subsection{METAL NANOPARTICLES SYNTHESIS PROCEDURE}

\subsubsection{Synthesis of $0.9 \mathrm{~nm}$ Diameter TPPS-Protected $\mathrm{Au}_{11}$ Clusters.}

TPPS stabilized Au11 clusters were synthesized using the protocol reported by Yao and coworkers. ${ }^{31}$ Briefly, a mixture of $\mathrm{HAuCl}_{4}(0.5 \mathrm{mmol})$ and TPPS sodium salt $(0.75 \mathrm{mmol})$ were mixed in methanol $(11.5 \mathrm{~mL})$ under an inert atmosphere, followed by rapid addition of $3 \mathrm{~mL}$ of freshly prepared ice-cooled aqueous $\mathrm{NaBH}_{4}$ solution $(0.2 \mathrm{M})$ under vigorous stirring. After the addition of $\mathrm{NaBH}_{4}$ a brown color immediately formed in the solution, indicative of the formation of small Au clusters in the solution. Figure 2.1 shows the reaction for the synthesis of the Au11 cluster. 


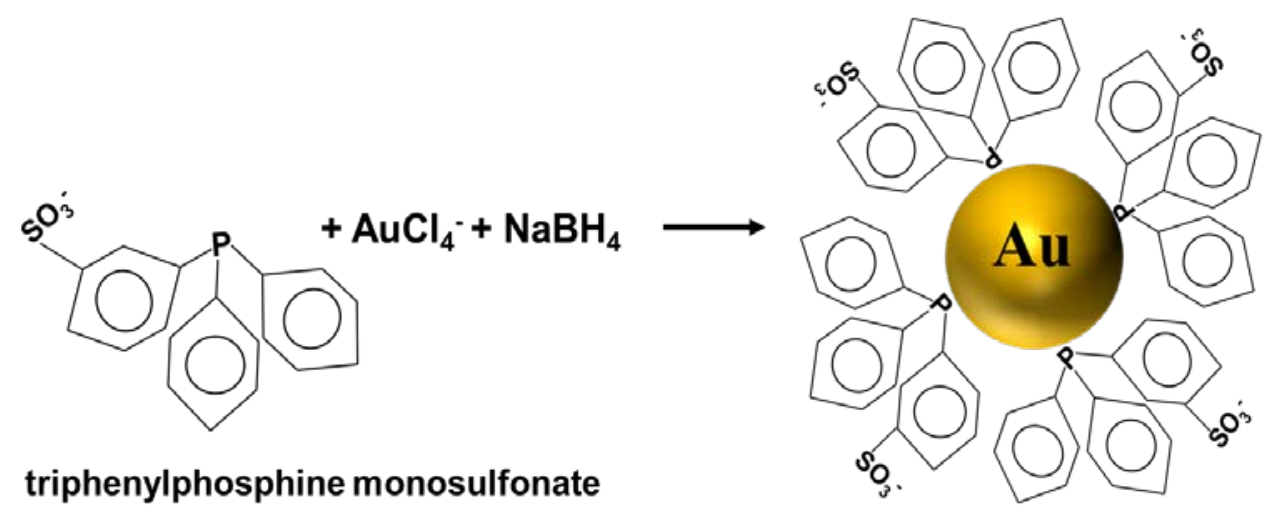

Figure 2. 1. Procedure for the synthesis of $A u_{11}$ clusters stabilized with triphenylphosphine monosulfonate.

2.3.2. Synthesis of $1.6 \mathrm{~nm}$ Diameter THPC Au NPs. THPC-stabilized $1.6 \mathrm{~nm}$ diameter Au NPs were synthesized using the procedure described by Duff and coworkers. ${ }^{47}$ Briefly, $500 \mu \mathrm{L}$ of $0.2 \mathrm{M} \mathrm{NaOH}$ solution was added to glass vials containing $15.16 \mathrm{~mL}$ of nanopure water followed by the addition of $400 \mu \mathrm{L}$ of the reducing agent THPC (200 $\mu \mathrm{L}$ of $80 \%$ THPC diluted to $16.66 \mathrm{~mL}$ of nanopure water). Finally, $660 \mu \mathrm{L}$ of $25 \mathrm{mM} \mathrm{HAuCl}{ }_{4} \cdot 3 \mathrm{H}_{2} \mathrm{O}$ was added to the vials with constant stirring. After the addition of $\mathrm{HAuCl}_{4} \cdot 3 \mathrm{H}_{2} \mathrm{O}$, immediately an orange-brown color formed in solution, indicative of small Au NPs. Figure 2.2 shows the reaction for 
the synthesis of $1.6 \mathrm{~nm}$ THPC Au NPs (Figure 2.2 A) and the proposed reaction mechanism (Figure 2.2 B)

(A)

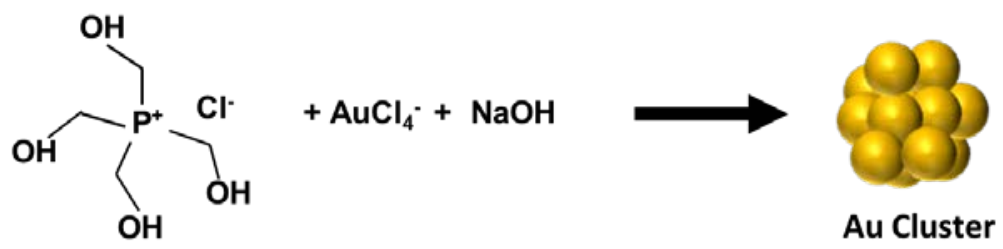

THPC

(B)<smiles>OC[P+](CO)(CO)CO</smiles>
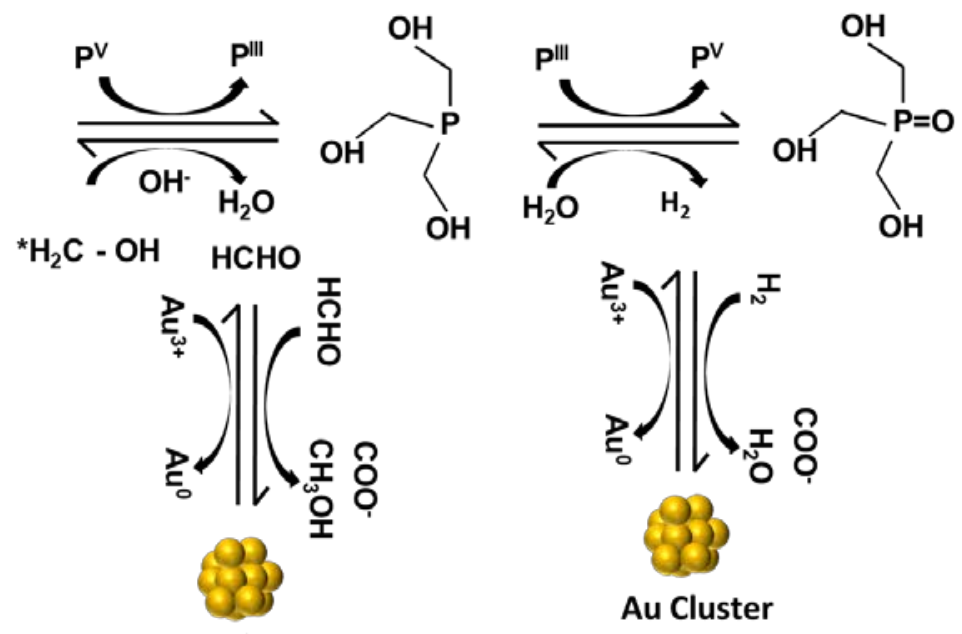

THPC

Au Cluster

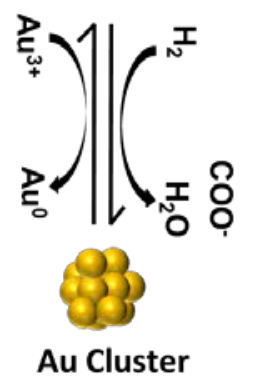

Figure 2. 2. (A) Reaction involved in the Duff method of synthesis of $1-2 \mathrm{~nm}$ diameter Au NPs stabilized with Tetrakis(hydroxymethyl)phosphonium chloride and (B) proposed mechanism of synthesis of Au NPs with Tetrakis(hydroxymethyl)phosphonium chloride.

2.3.3. Synthesis of $\sim 2 \mathrm{~nm}$ Diameter Glutathione-Capped Au NPs. Glutathionecapped Au NPs were synthesized by using the protocol reported by Negishi et al. ${ }^{146}$ Briefly, a mixture of $50 \mathrm{~mL}$ of $0.25 \mathrm{mmol}$ of $\mathrm{HAuCl}_{4}$ and $1.0 \mathrm{mmol}$ of glutathone was prepared in methanol solution. The mixture was then cooled to 0 
${ }^{\circ} \mathrm{C}$ in an ice bath for 1 hour. Then, an aqueous solution of ice cold $\mathrm{NaBH}_{4}(0.2 \mathrm{M}$, $12.5 \mathrm{~mL}$ ) was injected rapidly into this mixture under vigorous stirring. The mixture was allowed to react for another 2 hours. The resulting precipitate was spun down by centrifugation and washed with methanol to remove the starting materials. This was repeated multiple times. Finally, methanol was added to the precipitate, and the solution was sonicated for 10 min before attachment of the glutathione-capped Au NPs to a glass/ITO/APTES electrode.

\subsubsection{Synthesis of Dendrimer-Coated 1-2 $\mathrm{nm}$ Diameter Au NPs.}

Dendrimer stabilized 1-2 nm diameter Au NPs were synthesized by using the protocol reported by Crooks and coworkers. ${ }^{147}$ Briefly, $200 \mu \mathrm{L}$ of $100 \mu \mathrm{M}$ PAMAM dendrimer was added to $8.65 \mathrm{~mL}$ of nanopure water with vigorous stirring. Then, $147 \mu \mathrm{L}$ of a $20.0 \mathrm{mM} \mathrm{HAuCl}_{4}$ stock solution was added dropwise to the stirred PAMAM solution. Finally, after 2 min an $\sim 10$-fold molar excess of $\mathrm{NaBH}_{4}$ mixed with $1.0 \mathrm{~mL}$ of $0.30 \mathrm{M} \mathrm{NaOH}$ solution was added to that solution. After the addition of $\mathrm{NaBH}_{4}$, immediately a light brown color formed in the solution, indicating the formation of small Au clusters in the solution. The reaction mixture was then stirred at $25 \pm 2{ }^{\circ} \mathrm{C}$ for $12 \mathrm{~h}$ to deactivate excess $\mathrm{NaBH}_{4}$. 
2.3.5. Synthesis of Citrate Stabilized $4 \mathrm{~nm}$ Diameter Au NPs. $4 \mathrm{~nm}$ average diameter Au NPs were synthesized by using the procedure described by Murphy and co-workers. ${ }^{32}$ In this method, $0.5 \mathrm{~mL}$ of $10 \mathrm{mM} \mathrm{HAuCl}_{4} \cdot 3 \mathrm{H}_{2} \mathrm{O}$ and $0.5 \mathrm{~mL}$ of $10 \mathrm{mM}$ trisodium citrate was added to $20 \mathrm{~mL}$ of water followed by the addition of $0.6 \mathrm{~mL}$ of ice-cold $100 \mathrm{mM} \mathrm{NaBH}_{4}$ at once with rapid stirring for 2 hours. After the addition of $\mathrm{NaBH}_{4}$, the solution turned red within 5 min, indicating the formation of Au NPs. Figure 2.3 shows the reactions involved during the formation of $4 \mathrm{~nm}$ diameter Au NPs.

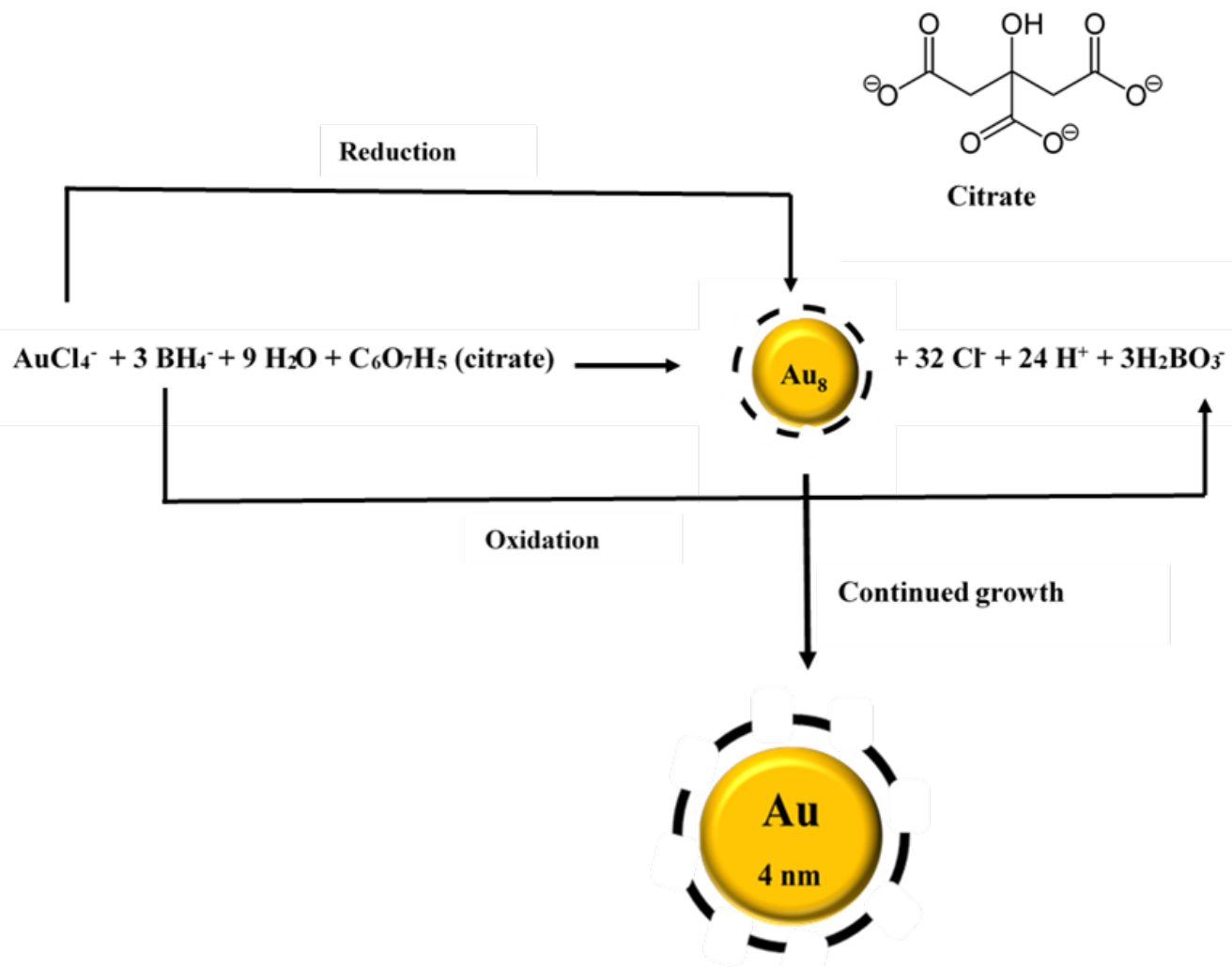

Figure 2. 3. Synthesis of citrate coated $4 \mathrm{~nm}$ diameter Au NPs. 
2.3.6. Preparation of THPC $4 \mathrm{~nm}$ Diameter Au NPs. We prepared $4 \mathrm{~nm}$ diameter THPC Au NPs by the ligand exchange method reported by Gulka et al. ${ }^{148}$ Briefly, $500 \mu \mathrm{L}$ of $100 \mu \mathrm{M}$ concentration of THPC was added to a $10 \mathrm{~mL}$ aqueous solution of citrate-coated $4 \mathrm{~nm}$ Au NPs. After addition of THPC, the Au NP solution immediately changed from red to blue as shown in Figure 2.4(i)The replacement of citrate with THPC is due to the strong interaction of the Au-P bond as compared to the Au-citrate. The NPs became red again after 24 hours of reaction. The disassembly of NPs is due to the catalytic formation of pentavalent phosphorene that occurred with Au NPs when the $\mathrm{pH}$ is above the pKa of the THPC ligand (Figure 2.4(ii)). The pentavalent THPOH isomers remain adsorbed on the surface of Au NPs and the excess negative charge of THPOH ligand causes the assembly to break apart into monodisperse components with time. After 24 hours, the NPs were attached to the glass/ITO/APTES electrode by directly soaking the electrode in the solution of THPC $4 \mathrm{~nm}$ Au NPs. 
(i)

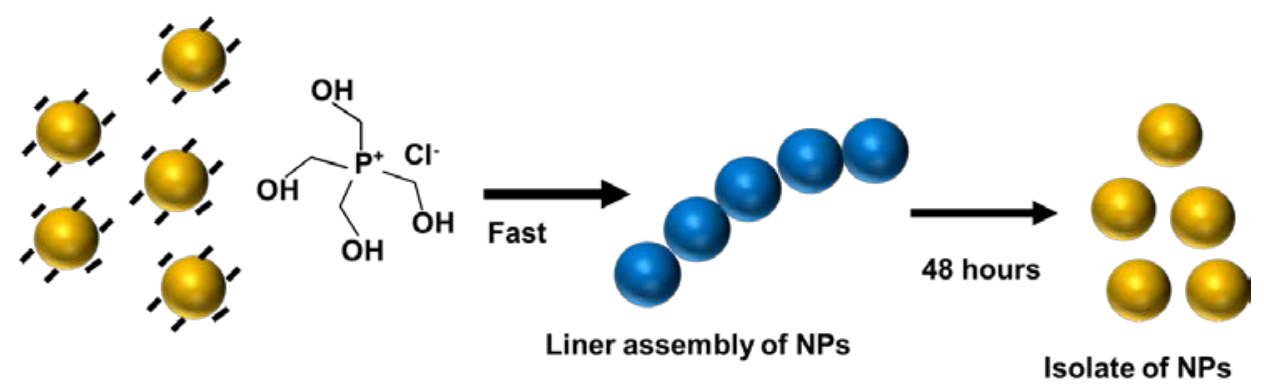

(ii)
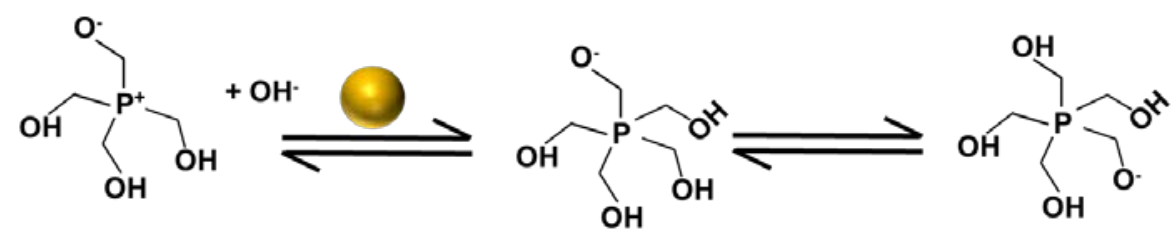

(iii)<smiles>CC[PH](CO)(CO)CO</smiles>

Figure 2. 4. Proposed mechanism of assembly and disassembly of $4 \mathrm{~nm}$ Au NPs with THPC. (i) The citrate coated $4 \mathrm{~nm}$ Au NPs immediately assemble into linear chains by reacting with THPC and slowly disassemble with time. The rapid assembly of NPs after addition of THPC due to the displacement of citrate with THPC. However, disassembly of Au NPs is only observed when the $\mathrm{pH}$ is above the pKa of the THPC (ii) The catalytic formation of pentavalent phosphorene occurred with Au NPs when the pH is above the pKa of the THPC ligand. (iii) These pentavalent THPOH isomers remain adsorbed to the surface of Au NPs and the excess negative charge of the ligand cannot maintain the assembly, which breaks apart into monodisperse components. 
2.3.7. Synthesis of $15 \mathrm{~nm}$ Average Diameter Citrate-Stabilized Au NPs. $15 \mathrm{~nm}$ average diameter Au NPs were synthesized by a modified Turkevich method. ${ }^{40}$ Briefly, $500 \mu \mathrm{L}$ of $0.01 \mathrm{M} \mathrm{HAuCl}_{4} \cdot 3 \mathrm{H}_{2} \mathrm{O}$ was added to $17.0 \mathrm{~mL}$ of nanopure water and heated to a rolling boil for $10 \mathrm{~min}$. Immediately after, $2.5 \mathrm{~mL}$ of $0.01 \mathrm{M}$ citric acid, trisodium salt solution was added and the solution was stirred for another 10 minutes. Finally, the resulting solution was stirred for another $15 \mathrm{~min}$ at room temperature and allowed to cool. At this point, the solution turned to a bright red color indicating the formation of citrate-capped $15 \mathrm{~nm}$ Au NPs. Figure 2.5 shows the procedure for the synthesis of citrate capped $15 \mathrm{~nm}$ Au NPs. 


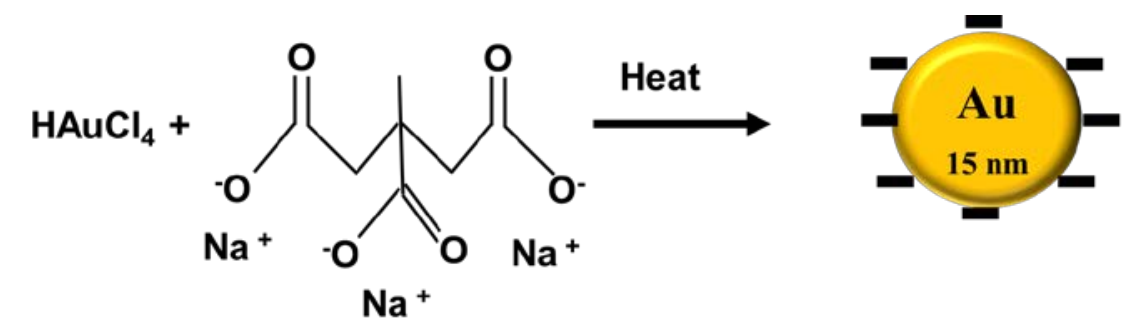

Trisodium citrate

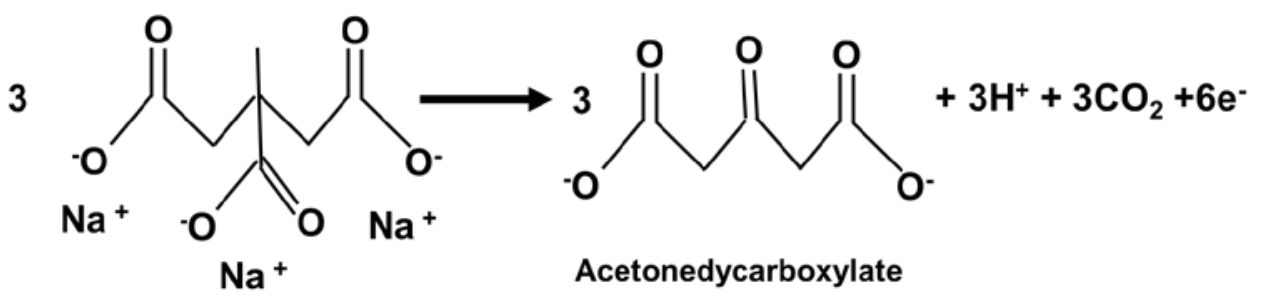

Trisodium citrate

$$
\begin{aligned}
& 2 \mathrm{AuCl}_{4}+6 \mathrm{e}^{-} \longrightarrow \text { 2Au }+8 \mathrm{Cl}^{-} \\
& \mathrm{nAu}^{\circ} \longrightarrow \text { Au nanoparticles }
\end{aligned}
$$

Figure 2. 5. Synthesis mechanism of $15 \mathrm{~nm}$ diameter Au NPs by the citrate reduction method.

$$
\mathrm{AuCl}_{4}^{-}+3 / 2 \mathrm{H}_{2} \mathrm{O}_{2} \longrightarrow \mathrm{Au}^{\circ}+4 \mathrm{Cl}^{-}+3 \mathrm{H}^{+}+3 / 2 \mathrm{O}_{2}
$$

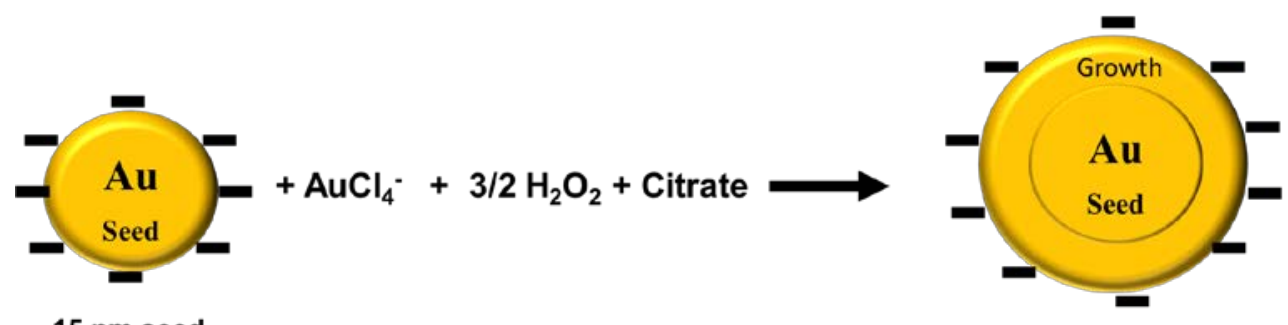

Figure 2. 6. Proposed mechanism of seeded growth synthesis of $50 \mathrm{~nm}$ diameter Au NPs with $\mathrm{H}_{2} \mathrm{O}_{2}$. 
2.3.8. Synthesis of Citrate-Stabilized $50 \mathrm{~nm}$ Diameter Au NPs. We used the seed-mediated growth method reported by Wang and coworkers ${ }^{13}$ to prepare fairly monodisperse citrate-stabilized $50 \mathrm{~nm}$ diameter Au NPs. Briefly, $500 \mu \mathrm{L}$ of $0.01 \mathrm{M}$ $\mathrm{HAuCl}_{4} \cdot 3 \mathrm{H}_{2} \mathrm{O}$ and $1.0 \mathrm{~mL}$ of $0.01 \mathrm{M}$ trisodium citrate were added to $18.0 \mathrm{~mL}$ of 30 wt $\% \mathrm{H}_{2} \mathrm{O}_{2}$ while stirring. Next, $500 \mu \mathrm{L}$ of as-prepared $15 \mathrm{~nm}$ Au NPs was added to the solution. After the addition of the $15 \mathrm{~nm}$ diameter Au seed NPs, the color of the solution changed within a few seconds to a pink red color, suggesting the formation of larger Au NPs. Reduction of $\mathrm{AuCl}_{4}^{-}$with $\mathrm{H}_{2} \mathrm{O}_{2}$ produced fairly monodisperse $50 \mathrm{~nm}$ Au NPs due to the modarate reducing power of $\mathrm{H}_{2} \mathrm{O}_{2}$, which reduces $\mathrm{AuCl}_{4}{ }^{-}$to $\mathrm{Au}^{0}$ directly onto the $15 \mathrm{~nm}$ Au NPs surface instead of producing new Au NPs. The proposed reduction reaction of $\mathrm{AuCl}_{4}^{-}$for the seeded growth synthesis of $50 \mathrm{~nm}$ diameter Au NPs proceeds as follows:

$$
\mathrm{AuCl}_{4}{ }^{-}+3 / 2 \mathrm{H}_{2} \mathrm{O}_{2} \rightarrow \mathrm{Au}^{0}+4 \mathrm{Cl}^{-}+3 \mathrm{H}^{+}+3 / 2 \mathrm{O}_{2}
$$

The final size of Au NPs can be calculated by considering that all the $\mathrm{HAuCl}_{4}$ reduced during reaction with $\mathrm{H}_{2} \mathrm{O}_{2}$ and deposited to seed, particles are spherical in shape, and reduction and growth take place without further nucleation, the final size of the NPs after seeded growth can be estimated by the following relations:

$$
d_{p}=d_{p o}\left(1+n_{m+} / n_{m} \text { seed }\right)^{1 / 3}
$$

where, $d_{p o}$ and $d_{p}$ is the initial and final diameter of Au NPs, $n_{M}=$ molarity of the metal ion in the solution and $\mathrm{n}_{\mathrm{M} \text { seed }}=$ molarity of the metal in the seed. Figure 2.6 show the procedure for the synthesis of $50 \mathrm{~nm}$ diameter Au NPs. 
2.3.9. Synthesis of Core/Shell Cu1/Aux NPs with Varied Au. Cu/Au core/shell NPs were synthesized by the using the method described by Zhang and coworkers. ${ }^{149-150}$ 148-149 147-148 146-147 145-146 144-145 143-144 142-143 141-142 140-141 139-140 In a typical synthesis, we added $50 \mu \mathrm{L}$ of $0.1 \mathrm{M} \mathrm{CuSO}_{4} \cdot 2 \mathrm{H}_{2} \mathrm{O}$ and $50 \mu \mathrm{L}$ of $0.1 \mathrm{M}$ trisodium citrate to $20 \mathrm{~mL}$ of nanopure water cooled in an ice bath with stirring. We then reduced $\mathrm{CuSO}_{4} \cdot 2 \mathrm{H}_{2} \mathrm{O}$ to metallic $\mathrm{Cu}$ NPs in the presence of the citrate stabilizer by adding $1 \mathrm{~mL}$ of freshly-prepared $0.027 \mathrm{M}$ ice cold $\mathrm{NaBH}_{4} \cdot 2 \mathrm{H}_{2} \mathrm{O}$ solution. A yellow color formed immediately in solution, consistent with the formation of Cu NPs. Finally, after 30 min of continued stirring, we added $50 \mu \mathrm{L}$ of $0.2 \mathrm{M}, 0.1 \mathrm{M}, 0.05 \mathrm{M}, 0.03 \mathrm{M}, 0.02 \mathrm{M}, 0.01 \mathrm{M}, 0.001 \mathrm{M}$, or $0.0001 \mathrm{M} \mathrm{HAuCl}_{4} \cdot 3 \mathrm{H}_{2} \mathrm{O}$ to prepare $\mathrm{Cu}_{1} / \mathrm{Au}_{2}, \mathrm{Cu}_{1} / \mathrm{Au}_{1}, \mathrm{Cu}_{1} / \mathrm{Au}_{0.5}, \mathrm{Cu}_{1} / \mathrm{Au}_{0.3}, \mathrm{Cu}_{1} / \mathrm{Au}_{0.2}, \mathrm{Cu}_{1} / \mathrm{Au}_{0.1}, \mathrm{Cu}_{1} / \mathrm{Au}_{0.01}$ and $\mathrm{Cu}_{1} / \mathrm{Au}_{0.001}$ core/shell NPs, respectively. In the case of core/shell $\mathrm{Cu}_{1} / \mathrm{Au}_{2} \mathrm{NPs}$ we added an additional $1 \mathrm{~mL}$ of $0.027 \mathrm{M} \mathrm{NaBH}_{4} .2 \mathrm{H}_{2} \mathrm{O}$ to the solution after the addition of $\mathrm{HAuCl}_{4} \cdot 3 \mathrm{H}_{2} \mathrm{O}$ to make sure all $\mathrm{HAuCl}_{4} \cdot 3 \mathrm{H}_{2} \mathrm{O}$ reduced to $\mathrm{Au}$ on the $\mathrm{Cu}$ core surface (as opposed to galvanic exchange). After addition of $\mathrm{HAuCl}_{4} \cdot 3 \mathrm{H}_{2} \mathrm{O}$ we stirred the solution for $30 \mathrm{~min}$ before attaching them to functionalized glass/ITO electrodes for ASV analysis. Pure Cu NPs were prepared using the same procedure but without adding $\mathrm{HAuCl}_{4} \cdot 3 \mathrm{H}_{2} \mathrm{O}$ (no second step). Figure 2.7. (A) illustrates the synthesis of citrate coated core/shell $\mathrm{Cu}_{1} / \mathrm{Au}_{x} \mathrm{NPs}$.

2.3.10. Synthesis of CuAu Mixed Alloy NPs. We synthesized $\mathrm{Cu}_{1} \mathrm{Au}_{2}$ mixed alloy NPs by adding $50 \mu \mathrm{L}$ of $0.1 \mathrm{M} \mathrm{CuSO}_{4} \cdot 2 \mathrm{H}_{2} \mathrm{O}, 100 \mu \mathrm{L}$ of $0.1 \mathrm{M}$ trisodium citrate and $50 \mu \mathrm{L}$ of $0.2 \mathrm{M} \mathrm{HAuCl}_{4} 3 \mathrm{H}_{2} \mathrm{O}$ to $20 \mathrm{~mL}$ of ice cold nanopure water with stirring. 
Then we co-reduced the two metals by adding $2 \mathrm{~mL}$ of freshly-prepared ice cold $0.027 \mathrm{M} \mathrm{NaBH}_{4} \cdot 2 \mathrm{H}_{2} \mathrm{O}$. After addition of ice cold $\mathrm{NaBH}_{4} \cdot 2 \mathrm{H}_{2} \mathrm{O}$, a black solution formed immediately in the solution. We continued stirring the solution for $30 \mathrm{~min}$ before attaching them to the functionalized glass/ITO electrodes for ASV analysis. Similarly, we synthesized citrate stabilized mixed $\mathrm{Cu}_{1}-\mathrm{Au}_{0.33}$ alloy NPs by adding $50 \mu \mathrm{L}$ of $0.1 \mathrm{M} \mathrm{CuSO}_{4} \cdot 2 \mathrm{H}_{2} \mathrm{O}$ and $50 \mu \mathrm{L}$ of $0.1 \mathrm{M}$ trisodium citrate to $20 \mathrm{~mL}$ of nanopure water cooled in an ice bath with stirring. Then we added $50 \mu \mathrm{L} 0.1 \mathrm{M}$ $\mathrm{HAuCl} 43 \mathrm{H}_{2} \mathrm{O}$ to prepare $\mathrm{Cu}_{3}-\mathrm{Au}_{1} \mathrm{NPs}$. Then we co-reduced the two metals by adding $2 \mathrm{~mL}$ of freshly-prepared ice cold $0.027 \mathrm{M} \mathrm{NaBH}_{4} \cdot 2 \mathrm{H}_{2} \mathrm{O}$. After addition of ice cold $\mathrm{NaBH}_{4} 2 \mathrm{H}_{2} \mathrm{O}$, a black solution formed immediately in the solution. We continued stirring the solution for 20 min before attaching them to the functionalized glass/ITO electrodes. Figure 2.7. (B) illustrates the synthesis procedure of $\mathrm{Cu}_{1}-\mathrm{Au}_{2}$ alloy NPs.

2.3.11. Synthesis of Core/Shell $\mathrm{Au}_{0.33} / \mathrm{Cu}_{1}$ NPs. We used the procedure described by Murphy and co-workers to synthesize citrate-stabilized Au NPs first. ${ }^{151}$ In a typical synthesis, we added $50 \mu \mathrm{L}$ of $0.03 \mathrm{M} \mathrm{HAuCl}_{4} \cdot 3 \mathrm{H}_{2} \mathrm{O}$ and $50 \mu \mathrm{L}$ of $0.1 \mathrm{M}$ trisodium citrate to $20 \mathrm{~mL}$ of nanopure water cooled in an ice bath with stirring. We then reduced $\mathrm{HAuCl}_{4} \cdot 3 \mathrm{H}_{2} \mathrm{O}$ to metallic Au NPs in the presence of the citrate stabilizer by adding $1 \mathrm{~mL}$ of freshly-prepared $0.027 \mathrm{M}$ ice cold $\mathrm{NaBH}_{4} \cdot 2 \mathrm{H}_{2} \mathrm{O}$ solution. A red color formed immediately in solution, consistent with the formation of Au NPs. Finally, after 2 hours of continued stirring, we added $50 \mu \mathrm{L}$ of $0.1 \mathrm{M}$ $\mathrm{CuSO}_{4} \cdot 2 \mathrm{H}_{2} \mathrm{O}$ to prepare $\mathrm{Au}_{0.33} / \mathrm{Cu}_{1}$. After $10 \mathrm{~min}$ we added $1 \mathrm{~mL}$ of $0.027 \mathrm{M}$ 
$\mathrm{NaBH}_{4} \cdot 2 \mathrm{H}_{2} \mathrm{O}$ to the solution to make sure all $\mathrm{CuSO}_{4} \cdot 2 \mathrm{H}_{2} \mathrm{O}$ reduced to $\mathrm{Cu}$ on the Au core surface. After addition of $\mathrm{CuSO}_{4} \cdot 2 \mathrm{H}_{2} \mathrm{O}$, we stirred the solution for $20 \mathrm{~min}$ before attaching them to functionalized glass/ITO electrodes.

(A)

(i)

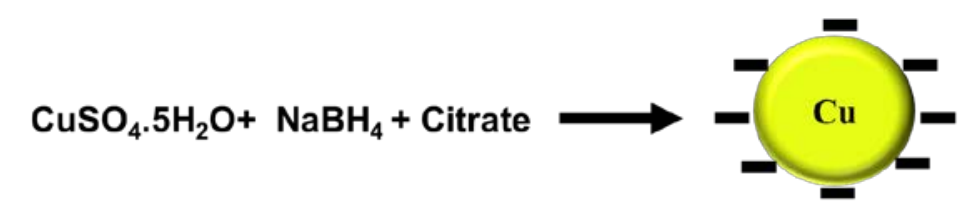

Cu NPs
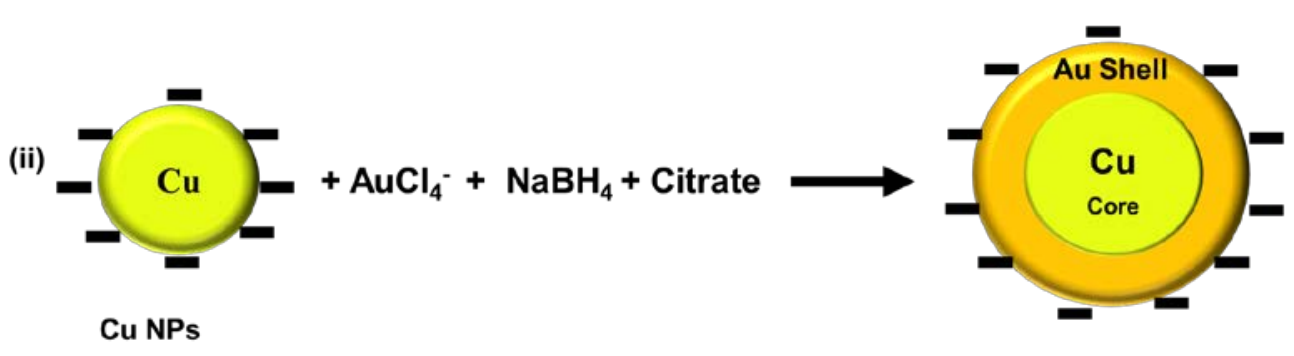

(B) $\mathrm{CuSO}_{4} \cdot 5 \mathrm{H}_{2} \mathrm{O}+\mathrm{AuCl}_{4}^{-}+\mathrm{NaBH}_{4}+$ Citrate

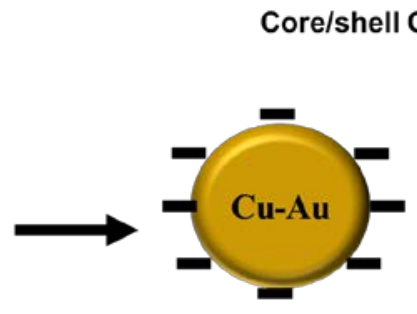

Cu-Au alloy NPs

Figure 2. 7. Synthesis strategy of citrate coated $\mathrm{Cu}$, core/shell $\mathrm{Cu} / \mathrm{Au}$ and alloy Cu-Au NPs. 


\subsection{NANOPARTICLE ATTACHMENT PROCEDURE}

2.4.1. Functionalization of Glass/ITO Electrodes with 3Aminopropyltriethoxysilane (APTES). Glass/ITO electrodes were cleaned by sonication in acetone, ethanol, and 2-propanol for $20 \mathrm{~min}$ in each solvent. The glass/ITO electrode was then functionalized with APTES by immersing into a solution containing $100 \mu \mathrm{L}$ of APTES, $10 \mathrm{~mL}$ of 2-propanol, and 2 to 3 drops of nanopore water and heating at $90^{\circ} \mathrm{C}$ for $30 \mathrm{~min}$. After $30 \mathrm{~min}$, the electrode was thoroughly rinsed with 2-propanol and dried under $\mathrm{N}_{2}$. Figure 2.8 show the step involved for the functionalization of Glass/ITO electrode.

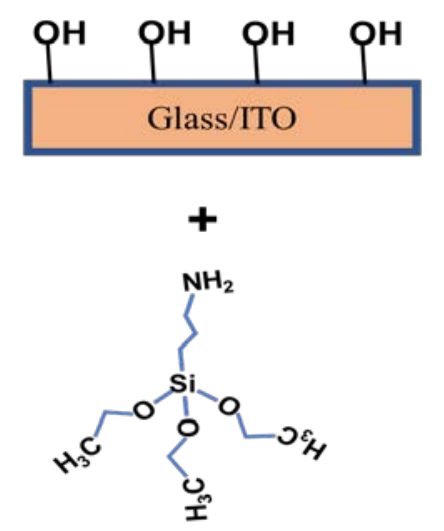

(3-aminopropyl) triethoxysilane

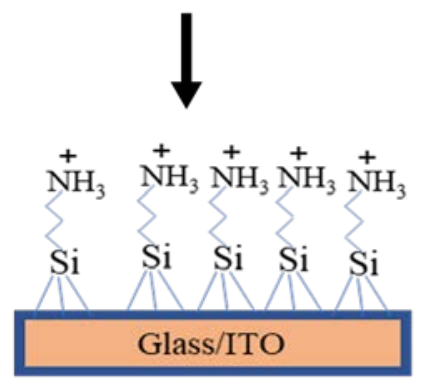

Figure 2. 8. Functionalization of Glass/ITO electrode with aminopropyltriethoxysilane (APTES). 


\subsubsection{Attachment of Citrate-Coated Nanoparticles to Functionalized}

Electrodes. The glass/ITO/APTES electrodes were soaked in the appropriate solution of NPs for $15 \mathrm{~min}$, then rinsed with nanopure water thoroughly and dried with $\mathrm{N}_{2}$ before ASV analysis. The glass/ITO electrodes were placed in NP solutions immediately after APTES functionalization and rinsing and characterized electrochemically immediately after metal NP attachment in order to reduce surface contamination and improve reproducibility. Figure 2.9 illustrates the procedure for the attachment of NPs onto electrode
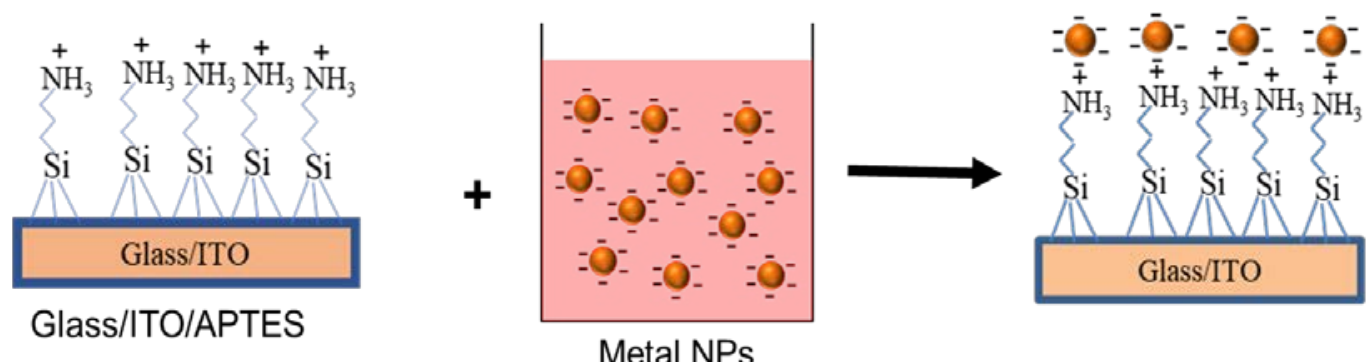

Glass/ITO/APTES

Metal NPs

Figure 2. 9. Attachment of metal NPs onto APTES functionalized glass/ITO electrode.

\subsubsection{Galvanic replacement of $\mathrm{Au}$ NPs with $\mathrm{Ag}^{+}$ions}

THPC stabilized 4.1 and $1.6 \mathrm{~nm}$ diameter Au NPs and TPPS stabilized $0.9 \mathrm{~nm}$ diameter Au NPs were attached directly on the APTES functionalized electrode surface by soaking the electrodes in the corresponding NP solution. Then, the glass/ITO/APTES/Au NPs electrode was placed in a solution containing $1 \times 10^{-4} \mathrm{M}$ 
$\mathrm{Ag}^{+}$ions for $3 \mathrm{~min}$. After $3 \mathrm{~min}$, the electrode was thoroughly rinsed with nanopure water and dried under $\mathrm{N}_{2}$ for ASV analysis.

\subsection{CHARACTERIZATION AND INSTRUMENTATIONS}

\subsubsection{Electrochemical Techniques}

Electroanalytical techniques are a branch of analytical chemistry techniques which have been developed to study the electrical and chemical changes on electroactive analytes as a function of potential, current, or charge at the interface of an electrode and electrolyte. Traditionally, electrochemical techniques have been used to determine the concentration of electroactive species. However, recently they are also developed for measuring electrochemical reactivity of the analyte. In most cases the analyte is part of an oxidation-reduction reaction which occurs at the interface of the electrolyte and an electrode. This section of the dissertation will focus on the complete electrochemical set-up and different electrochemical techniques used for this study. The main component of an electrochemical set up consists of: (i) a three-electrode cell containing a working, reference and counter electrode, (ii) a beaker with electrolyte, (iii) a potentiostat and (iv) a computer for plotting the electrochemical signal.

Electrochemical Cell. In our experiment we used a $50 \mathrm{ml}$ beaker having three electrodes (working, reference and counter electrode) as an electrochemical cell.

Working Electrode. The working electrode is the main electrode of an electrochemical system where the electrochemical reactions of interest occur. At 
the working electrode, the potential is controlled, and the current is measured. In our experiment we used glass/ITO, glass/ITO/NPs, glass/ITO/APTES, and glass/ITO/APTES/NPs as working electrodes.

Reference Electrode. The reference electrode is an electrode having a stable and well-known potential to determine and control the potential of the working electrode. In our experiment we used an $\mathrm{Ag} / \mathrm{AgCl}$ reference electrode. This electrode consists of a silver wire electrode coated with solid $\mathrm{AgCl}$ and immersed in a solution of $3 \mathrm{M} \mathrm{KCl}$ electrolyte solution.

Counter Electrode. A counter or auxiliary electrode is generally used to complete the circuit and allow charge to flow which ultimately balances the current flowing at the working electrode. The counter electrode is made from inert materials, like platinum, gold, or carbon. In our work we used a Pt wire as the counter electrode.

Potentiostat. A potentiostat is an electronic instrument which is capable of controlling the voltage difference between the reference electrode and the working electrode and measuring the current flowing between the working and counter electrode. In our studies we used $\mathrm{A} \mathrm{CH}$ Instruments model $\mathrm{CHI} 660 \mathrm{E}$ electrochemical workstation (Austin, TX).

\subsubsection{Linear Sweep Voltammetry (LSV)}

In our experiment we used LSV for studying the NPs size dependent oxidation peak potential $\left(E_{p}\right)$ of Au NPs, which has been used in our lab for 
determining the size of Au and Ag NPs. Figure 2.10. shows a typical linear sweep voltammogram of glass/ITO/Au NPs $(\sim 2 \mathrm{~nm})$ at a scan rate of $0.01 \mathrm{~V} / \mathrm{s}$ in a solution of $0.01 \mathrm{M} \mathrm{KBr}$ plus $0.1 \mathrm{M} \mathrm{KClO}_{4}$. The starting potential was $-0.2 \mathrm{~V}$ and the ending potential $1.2 \mathrm{~V}$. The peak in the plot at $\sim 450 \mathrm{mV} \mathrm{vs} \mathrm{Ag} / \mathrm{AgCl}$ results from an increase in current that corresponds to the oxidation of $\mathrm{Au}$ by $\mathrm{Br}^{-}$and formation of soluble $\mathrm{AuBr}_{4}^{-}$(mostly) and possibly $\mathrm{AuBr}_{2}{ }^{-}$complexes from the oxidation reaction. This is defined as $\mathrm{E}_{\mathrm{p}}$ as labeled in the plot.

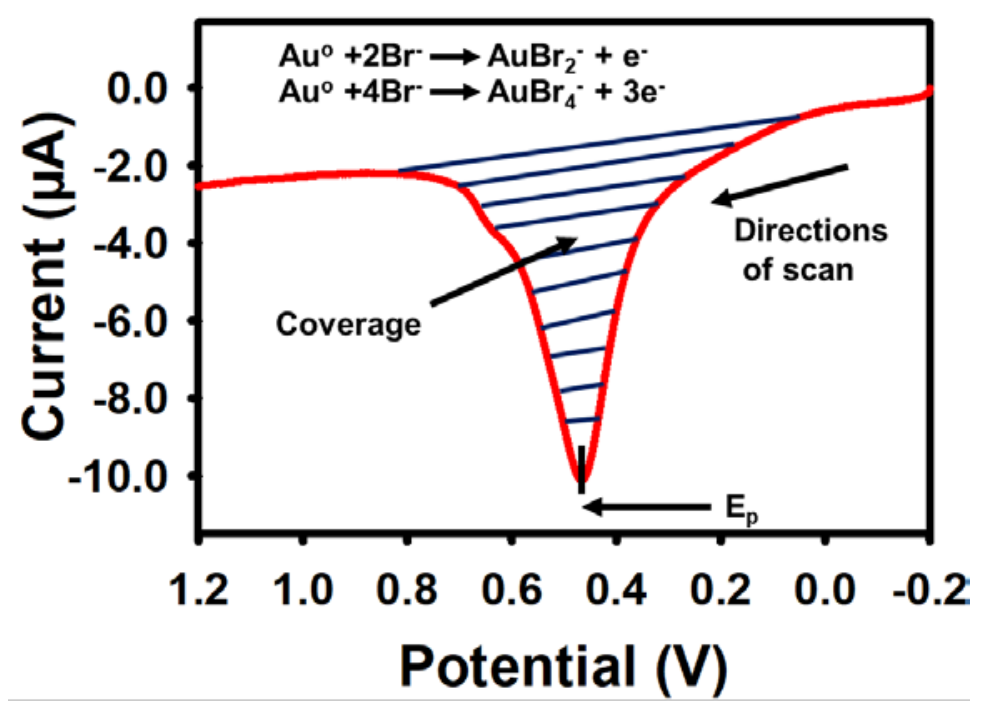

Figure 2. 10. Typical linear sweep voltammogram of glass/ITO/APTES/Au NPs $(\sim 2 \mathrm{~nm})$ at a scan rate of $0.01 \mathrm{~V} / \mathrm{s}$ in a solution of $0.01 \mathrm{M} \mathrm{KBr}$ plus $0.1 \mathrm{M} \mathrm{KClO}_{4}$.

\subsubsection{Cyclic Voltammetry (CV).}

CV is a type of potentiodynamic electrochemical method and similar to LSV. In $\mathrm{CV}$, the electrochemical response of electroactive species is recorded as current 
while a potential scan is applied to the working electrode at a constant scan rate in the forward and reverse directions. The resulting current is measured as a function of potential. The oxidation and reduction process of an electroactive species is possible to record by $\mathrm{CV}$ due to the complete cycle of this technique. Typically, the experiment begins at a potential where there is no oxidation or reduction reaction and moves to potentials where there is reduction or oxidation of the electroactive species. A typical response of a redox active species in CV is shown in Figure 2.11. In the figure, $i_{p, c}$ is the cathodic peak current, $i_{p, a}$ is anodic peak current, $E_{p, c}$ is potential of the cathodic peak and $E_{p, a}$ is potential of the anodic peak. In our studies we used CV to measure the surface Au oxidation-reduction in $0.1 \mathrm{M} \mathrm{HClO}_{4}$ electrolyte for determining the surface area (SA) of different sized $\mathrm{Au}$ NPs.

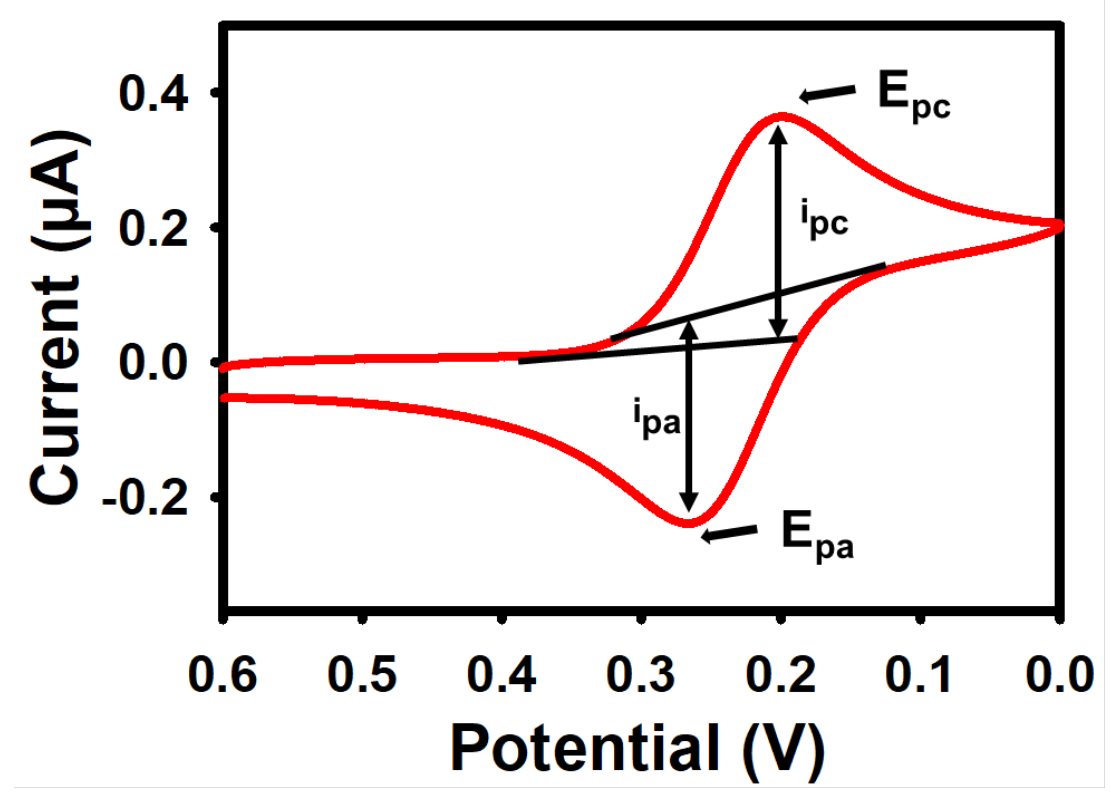

Figure 2. 11. Typical cyclic voltammogram for a solution of $\mathrm{K} 33 \mathrm{Fe}(\mathrm{CN})_{6}$ in $\mathrm{M} \mathrm{KNO}_{3}$ 


\subsubsection{Ultraviolet-Visible Spectroscopy (UV-vis)}

Ultraviolet-visible spectrophotometry (UV-Vis) was performed using a Varian instrument, Cary 50 Bio-spectrophotometer. In our experiment we measured the UV-Vis spectra of different sized Au NPs. Au NPs solution is placed inside a glass or quartz cuvette and the absorbance measured from $800 \mathrm{~nm}$ to 350 $\mathrm{nm}$. Au NPs of $4 \mathrm{~nm}$ and larger absorb strongly in the visible region due to the excitation and oscillations of conducting electrons of Au by light, which is referred to as localized surface plasmon resonance (LSPR). The LSPR band of Au NPs is size and shape dependent. The peak position shifts to longer wavelengths with an increase in NP size. However, small Au clusters (particles smaller than about 2 $\mathrm{nm}$ in dimeter) do not show a well-defined LSPR band. In our study we used UVvis spectroscopy to qualitatively determine the size of all of the Au NPs synthesized and to measure their aggregation.

\subsubsection{Scanning Electron Microscopy (SEM)}

Scanning electron microscopy is a powerful analytical tool which produces an image of a sample by scanning the surface of the sample with a focused beam of electrons. SEM images are used for analyzing the size, shape and morphology of nanomaterials. Fig 2.12 shows (a) the results of the interactions of an electron beam with a sample and (b) a typical SEM image of $50 \mathrm{~nm}$ diameter Au NPs, where the bright circular regions are the Au NPs attached to the glass/ITO electrode. The 
essential components in a SEM are the electron gun, electromagnetic lenses, apertures, a high-vacuum environment, a specimen stage, electron beam scanning coils, signal detection and a processing system that provide real time observation and image recording. The interaction of electrons with a sample produces a set of signals such as secondary electrons (SE), reflected or backscattered electrons, characteristic X-rays and transmitted electrons. Secondary electron are produced very close to the surface of interest. Therefore, SE detectors are standard equipment in all SEMs because it produces a very nice image. Backscattered electron imaging is most valuable because it can produce images by the reflected electrons from the sample by elastic scattering, which images deeper into the sample than SE. The resolution of back-scattered electron imaging is better than SE imaging.

In our studies, we used SEM to measure the size of different Au NPs before and after different treatments while attached to the glass/ITO or glass/ITO/APTES electrode. We collected SEM images with a Carl Zeiss SMT AG SUPRA 35VP field emission scanning electron microscope (FESEM) operating at an accelerating voltage of $17.00 \mathrm{kV}$ using an in-lens ion annular secondary electron detector. 

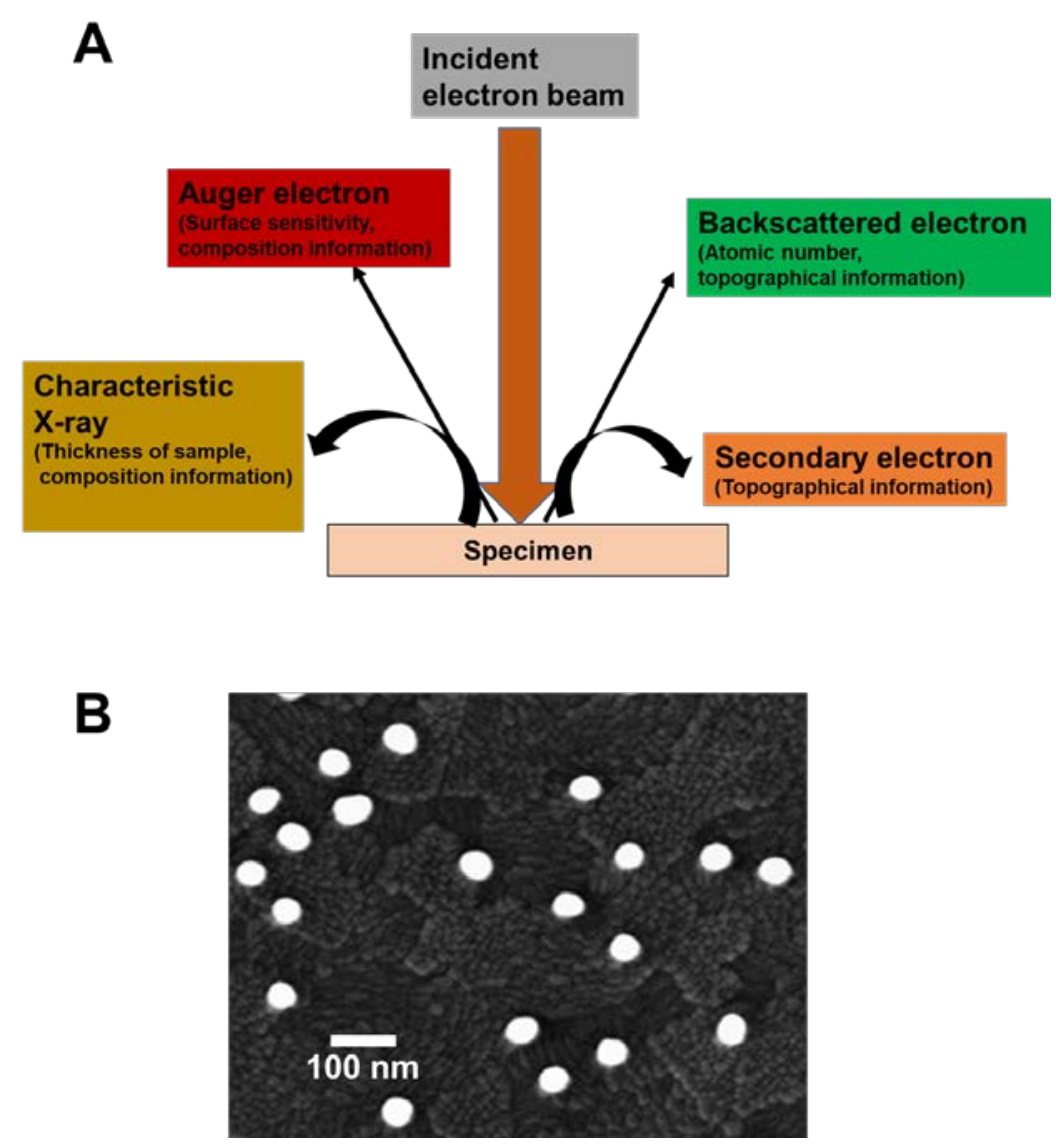

Figure 2. 12. (a) Scheme of electron beam and sample interactions. (b) Typical SEM image of $50 \mathrm{~nm}$ Au NPs attached to the surface of a glass/ITO/APTES electrode.

\subsubsection{Transmission Electron Microscope (TEM)}

The transmission electron microscope (TEM) is one of the most popular electron microscopic techniques for characterization of nanomaterials. In this electron microscopic techniques, a beam of electrons transmits through the samples to form an image. The essential parts of a TEM are similar to SEM which 
includes electron gun, acceleration tube, a vacuum system, condenser, specimen holder, objective aperture, objective lens, intermediate lens, projector lens, fluorescent screen, camera chamber, binocular and viewing port. TEM operates in a fully enclosed environment to reduce interference from outer sources and can be operated remotely. The resolution of TEM is higher than the SEM. Figure 2.13 shows the image formation process in TEM by the interaction of the electron beam and the sample.

In our study we used high resolution transmission electron microscopy (HRTEM). The images of core/shell $\mathrm{Cu}_{1} / \mathrm{Au}_{2} \mathrm{NPs}$ (Figure 2.13B) were obtained from the Electron Microscopy Center at the University of Kentucky using a FEI Talos F200X TEM operating at an accelerating voltage of $200 \mathrm{kV}$. The NPs appear as a dark feature due to them blocking the electron beam from reaching the detector. Also, several TEM images of various sized Au NPs, AuAg alloys after galvanic exchange and CuAu alloy NPs before and after thermal treatment were obtained from VINSE (Vanderbilt Institute of Nanoscale Science and Engineering) at different magnifications using a JEOL 2010F TEM operating at an accelerating voltage of $200 \mathrm{kV}$ and current density of $2.60 \mathrm{pA}$. NPs were directly drop cast deposited on a silicon dioxide-coated 400 mesh Ni TEM grid and dried under air before analysis. 

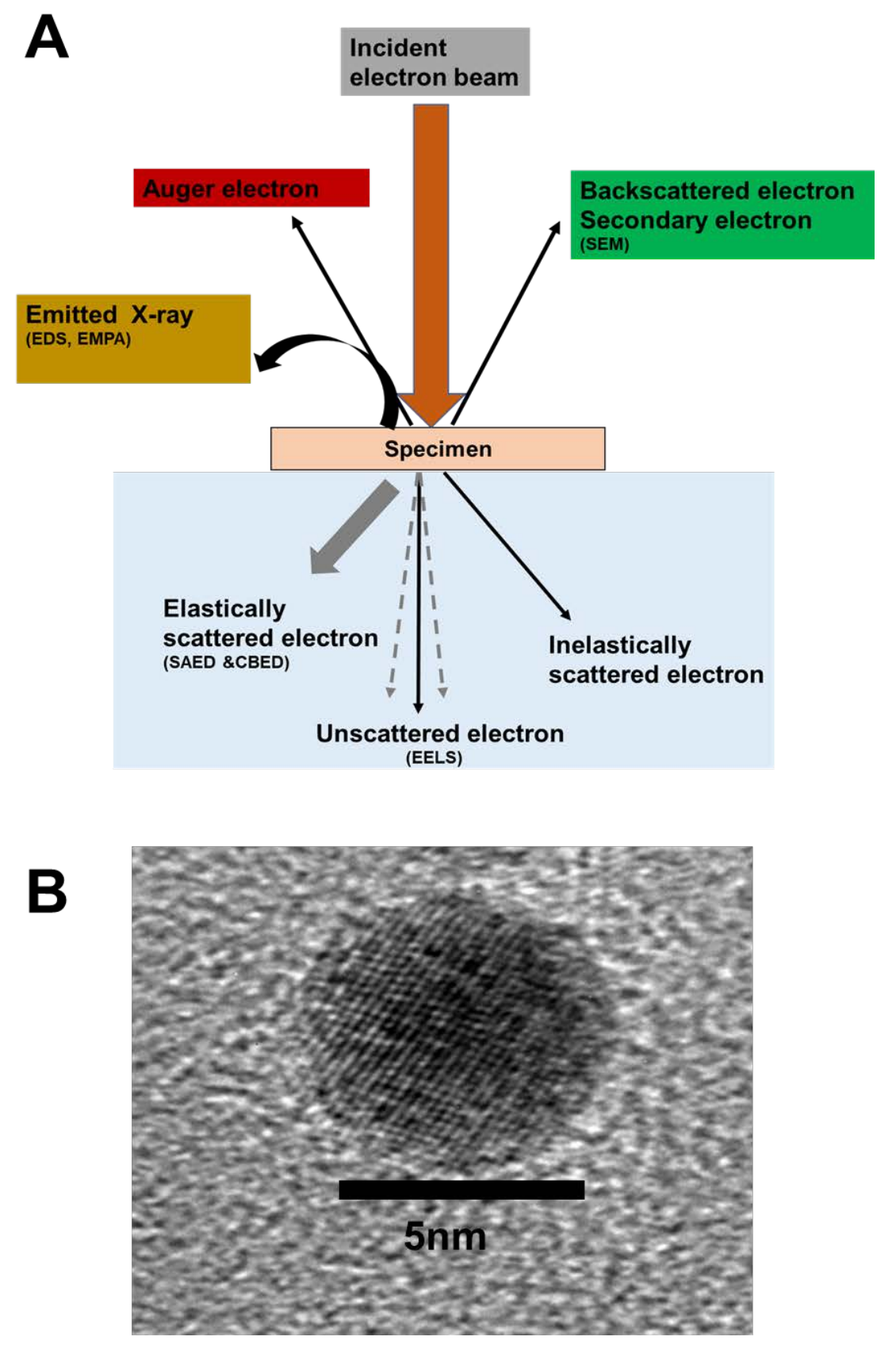

Figure 2. 13. (a) Scheme of electron beam and sample interactions in TEM.

(b) Typical TEM image of $5 \mathrm{~nm}$ average diameter of core/shell $\mathrm{Cu}_{1} / \mathrm{Au}_{2} \mathrm{NPs}$. 
2.5.5. Electrocatalysis Experiments. $\mathrm{CO}_{2}$ reduction experiments were carried out in a closed glass cell containing three electrodes (glass/ITO/APTES/Au NPs working electrode, Pt wire counter electrode, and an $\mathrm{Ag} / \mathrm{AgCl}$ reference electrode) in a $\mathrm{CO}_{2}$-saturated aqueous $0.1 \mathrm{M} \mathrm{KHCO}_{3}$ solution of $\mathrm{pH}$ 6.8. The $\mathrm{CV}$ scan ran from $0 \mathrm{~V}$ to $-0.8 \mathrm{~V}$ at a scan rate of $0.01 \mathrm{~V} / \mathrm{s}$. The ORR and HER was examined in the same set up used for $\mathrm{CO}_{2}$ reduction, but under $\mathrm{O}_{2}$ and $\mathrm{N}_{2}$ saturated $0.1 \mathrm{M}$ $\mathrm{HClO}_{4}$ solution. The potential was cycled from $0 \mathrm{~V}$ to $-0.8 \mathrm{~V}$ at a scan rate of 0.01 V/s.

2.5.6. Ozone Treatment of Au NPs Samples. Ozone treatment of different sized Au NPs were performed with a Jelight Company Inc. UVO CLEANER Model No. 42. Au NPs were first attached to a glass/ITO/APTES surface by directly soaking the electrode in the NPs solution. This electrode was then rinsed with water and dried under $\mathrm{N}_{2}$. Finally, the glass/ITO/APTES/Au NP electrodes were put in the ozone chamber for different times (typically $1 \mathrm{~min}$ to $60 \mathrm{~min}$ ).

2.5.7. Thermal Treatment of glass/ITO Attached Au NPs. Thermal treatment of glass/ITO electrodes with different sized Au NPs attached was performed in a Barnstead Thermolyne furnace (Model No. FB1315M). 


\section{CHAPTER III}

\section{A SIZE STABILITY STUDY OF CATALYTICALLY-ACTIVE SUB-2 nm DIAMETER GOLD NANOPARTICLES SYNTHESIZED WITH WEAK STABILIZER}

\subsection{INTRODUCTION}

Metal nanoparticles (NPs) exhibit unique size-dependent electrochemical and chemical reactivity, which can be potentially useful for a variety of applications. The size-dependent properties and reactivity are largely due to their increasing surface area-to-volume (SA/V) ratio with decreasing size. This leads to a material with more surface atoms, which have a lower coordination number, relative to the interior, fully coordinated atoms. Since the atoms with lower coordination number have different properties and reactivity, this can lead to NPs with vastly different properties and reactivity as the size decreases, especially below about $4 \mathrm{~nm}$ in diameter. The reactivity of NPs may not only be related to the overall surface area, however, because specific surface sites may dominate the reactivity. For example, the number of surface vertex and edge site atoms on a NP increases more dramatically than the overall SA/V with decreasing NP radius. In the case of cubic particles, for the same overall volume of material, decreasing the edge length of the cubes by a factor of 2 by breaking up the cubes increases the overall SA/V by 
a factor of 2, but increases the total number of cubes and corner vertex sites by a factor of 8 . This could potentially lead to a larger change in properties (or reactivity) than expected just based on SA/V only because corner atoms have an even lower coordination number than other surface atoms (such as terraces) and often dominate the observed reactivity. The change in reactivity would be 8 -fold based on the increase of corner sites instead of an increase of 2-fold based on the increase in SA/V.

Changes in NP size have led to dramatic changes in their electronic, ${ }^{152}$ thermal, ${ }^{153}$ and optical properties, ${ }^{13}$ sparking a number of fundamental sizedependent studies on metallic NPs and optimization of their properties for specific applications by tuning the size. Researchers have explored catalysis, ${ }^{5}$ sensing, ${ }^{74}$ melting point changes, ${ }^{153}$ changes in optical properties ${ }^{13}$ and oxidation ${ }^{154,155}$ as a function of metal NP size. During the last two decades sub $2 \mathrm{~nm}$ sized NPs (or clusters) have attracted tremendous interest in the nanotechnology field due to their unique properties, which bridge the gap between molecule-like and bulk-like behavior. For example, Au NPs transition from bulk-like properties to those exhibiting single electron double layer capacitive charging ${ }^{156}$ to those exhibiting a semiconductor like band gap or molecule-like HOMO-LUMO gap. ${ }^{157}$ Semiconductor NPs exhibit an increasing band gap with decreasing size, which leads to size-dependent photoluminescence..$^{158}$ In terms of reactivity, small NPs often exhibit increased activity as electrocatalysts. For example, ultrasmall $(<2$ $\mathrm{nm}) \mathrm{Au}$ NPs are excellent catalysts for $\mathrm{CO}_{2}$ reduction and $\mathrm{CO}$ oxidation. ${ }^{159,160}$ Also, tiny NPs provide enhanced surface area for improved sensitivity in sensing 
applications. ${ }^{161}$ Unfortunately, the smaller NPs are also more prone to oxidization, decomposition, and formation of bigger sized particles through various ripening processes, which causes the loss of their useful properties. ${ }^{19}$ Therefore, understanding the fundamental electrochemical and chemical reactivity of very small-sized NPs ( $<2 \mathrm{~nm})$ is critically important.

Our group has recently focused on the use of anodic stripping voltammetry (ASV) to study the size-dependent standard potential for the oxidation of Au and Ag NPs, which is an important parameter directly related to their stability against oxidative dissolution. In addition to providing information about their stability against oxidation, ASV can be used as an analytical tool to determine the NP size $\mathrm{e}^{154,155,162}$ and aggregation state. ${ }^{105}$ It is a simple and very low-cost method to determine NP size directly on an electrode surface. This is perfect for studying the effect of various electrochemical and chemical treatments on the size stability of NPs. The experiments offer very high throughput, allowing us to quickly determine the effect of a variety of different electrochemical conditions and treatments on the NP size while the NPs are attached to the electrode surface. In comparison, electron microscopy techniques are much more expensive and tedious. The throughput is too low for these kinds of studies and it is not possible to observe size changes directly on the electrode surface in solution unless one uses an electrochemical transmission electron microscopy (TEM) set up, which is quite challenging.

In this work, we describe the use of ASV to monitor the size stability of catalytically-active THPC Auznm NPs. We show that glass/ITO electrodes coated 
with these NPs are electrocatalytically active for $\mathrm{CO}_{2}$ reduction and the hydrogen evolution reaction (HER). However, the THPC Au2nm NPs are not stable towards surface oxidation/reduction cycling in acidic solution, low potential treatment in halide-containing electrolyte, and exposure to ozone. These are relatively mild conditions that researchers often usein standard metal NP treatment and cleaning procedures or they are often encountered in electrocatalysis applications, such as the acidic environment of fuel cells. It is important to know how detrimental these treatments are to the size and reactivity of metal NPs.

The reactivity and size stability of THPC Au2nm NPs is compared to citratestabilized $4 \mathrm{~nm}$ diameter Au NPs (Cit Au4nm NPs). THPC and Cit stabilizers are excellent for this study since they are weakly bound to Au and have open molecular structures compared to the thiols, amines, polymers, or surfactants commonly used as stabilizers. The Cit and THPC stabilizers generally do not hinder the Au NP reactivity, ensuring that the differences we observe between the different $\mathrm{Au}$ NPs are due to the metal NP size and properties, rather than the stabilizer. We chose the THPC $\mathrm{Au}_{2 n m}$ NPs and Cit Au4nm NPs for this work because these are the two smallest Au NPs that we know of reported in the literature that are stabilized with small, weakly bound molecules. There are smaller Au NPs (or clusters), such as the $<1.0 \mathrm{~nm}$ diameter Au25 clusters, but those smaller clusters are generally prepared with strongly bound thiol ligands or larger polymer/surfactant stabilizers. The small, weakly bound THPC and citrate ligands do not perturb the Au electrochemistry as much as the strongly bound or large molecule stabilizers, allowing us to better understand the size dependent behavior of the relatively 
unmodified metal. We did not include a study of larger Au NPs because their size stability is much better than $4 \mathrm{~nm}$ Au NPs and we do not have a method to synthesize Au NPs below 1.5 to $2.0 \mathrm{~nm}$ with weak stabilizers at this time. It is important to note that THPC binds relatively stronger than citrate, but we consider both to be weakly-adsorbed on $\mathrm{Au}^{163}$

\subsection{RESULTS AND DISCUSSION}

Figure 3.1 (A) shows ASVs of various-sized Au NPs attached to aminopropyltriethoxysilane (APTES)-functionalized indium-tin-oxide (ITO)-coated glass electrodes (glass/ITO/APTES) in $10 \mathrm{mM} \mathrm{KBr}$ plus $0.1 \mathrm{M} \mathrm{KClO}_{4}$ electrolyte using the same conditions reported by our group previously. ${ }^{105}$ The potential was scanned from $-0.2 \mathrm{~V}$ to $1.2 \mathrm{~V}$ versus an $\mathrm{Ag} / \mathrm{AgCl}$ reference electrode at $0.010 \mathrm{~V} / \mathrm{s}$. The anodic peaks in the scans are due to oxidation of Au by bromide to produce mostly $\mathrm{AuBr}_{4}{ }^{-}$and possibly some $\mathrm{AuBr}_{2}{ }^{-}$.The peak oxidation potential $\left(\mathrm{E}_{\mathrm{p}}\right)$ of the different sized Au NPs decreased as the diameter decreased in the order of $50 \mathrm{~nm}$ $\left(E_{p}=0.92 \mathrm{~V}\right)>15 \mathrm{~nm}\left(E_{p}=0.78 \mathrm{~V}\right)>4 \mathrm{~nm}\left(E_{p}=0.71 \mathrm{~V}\right)>2 \mathrm{~nm}\left(E_{p}=0.46 \mathrm{~V}\right)$. This is consistent with our previous work and others, showing a negative shift in oxidation potential with decreasing size for $\mathrm{Au}, \mathrm{Ag}$, and other metals. ${ }^{154,155,164}$ It is also consistent with the work of Plieth, who predicted a negative shift in the oxidation potential relative to the bulk value proportional to $1 /$ radius $^{165}$ and the work of Henglein, who calculate a negative shift in the oxidation potential of metal NPs with decreasing size based on sublimation energies. ${ }^{166}$ We previously reported on the oxidation of $250 \mathrm{~nm}$ to $4 \mathrm{~nm}$ diameter Au NPs, showing about a $200 \mathrm{mV}$ 
negative shift over this range, ${ }^{155}$ and later described the oxidation of Au NPs with average diameters in the $1-3 \mathrm{~nm}$ diameter range. ${ }^{167}$ Similar to the THPC Au2nm NPs shown here, our previous work on Au NPs in the 1-3 nm range showed $E_{p}$ values in the 0.40 to $0.50 \mathrm{~V}$ range ( $\mathrm{vs}$. $\mathrm{Ag} / \mathrm{AgCl}$ ), but also showed a population of Au NPs that oxidized at $0.7 \mathrm{~V}$ due to the presence of $4 \mathrm{~nm}$ diameter Au NPs. In our previous work, the Au NPs had very high size dispersity and low coverage of Au NPs on the glass/ITO/APTES electrode surface. The low yield and high size dispersity was not ideal in our previous work.
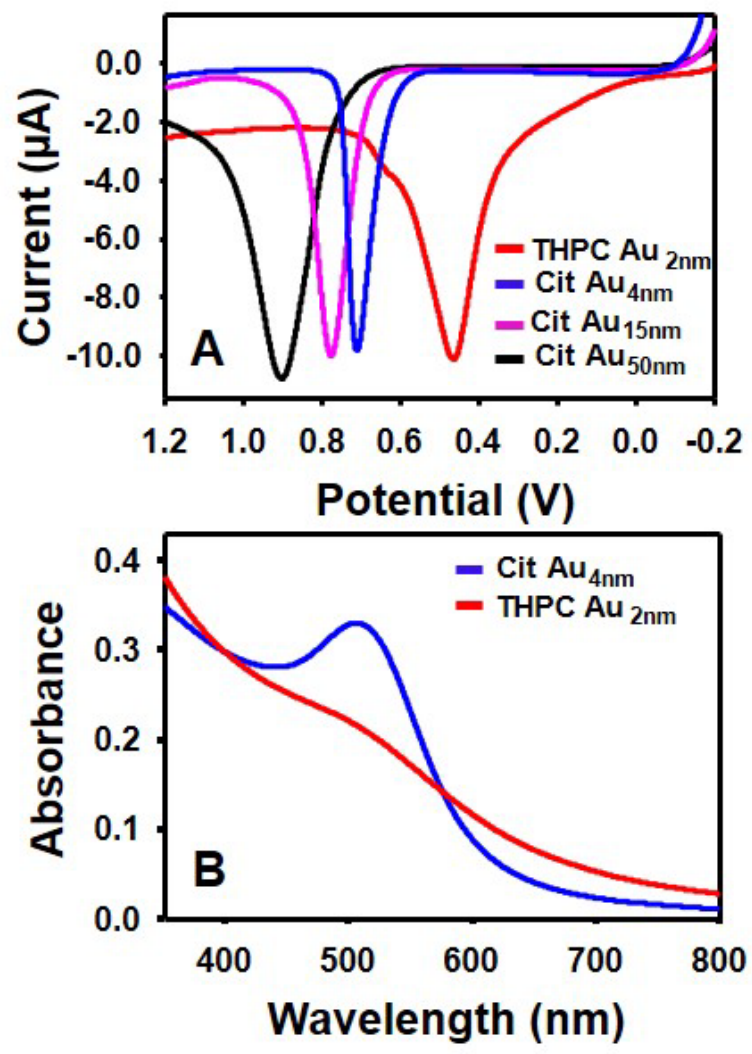

Figure 3. 1. (A) ASV obtained in $10 \mathrm{mM} \mathrm{KBr}$ plus $0.1 \mathrm{M} \mathrm{KClO}_{4}$ solution at a scan rate of $0.01 \mathrm{~V} / \mathrm{s}$ of glass/ITO/APTES electrodes coated with different sized Cit Au NPs and THPC Au2nm NPs. (B) UV-Vis spectra of Cit Autnm NPs (Blue) and THPC Au2nm NPs (Red). 
The THPC Au2nm NPs represents a significant improvement in both coverage and size dispersity compared to our previous work. Duff et al. reported their synthesis in 1993 and measured the size to be $1.5 \mathrm{~nm}$ in diameter based on transmission electron microscopy (TEM) imaging. ${ }^{47,48}$ The ASV of these THPC $\mathrm{Au}_{2 \mathrm{~nm}}$ NPs has not been reported previously and is significant here because they are stable in solution and display only one peak near $0.46 \mathrm{~V}(0.45-0.50 \mathrm{~V}$ negative of bulk $\mathrm{Au}$ ) without any additional peaks at lower or higher potentials. This means that the synthesis leads to long-lived, solution stable Au NPs near $2 \mathrm{~nm}$ average diameter with high yield and a relatively narrow size distribution compared to our previous work. This allows us to study the behavior of this size Au NP without interference from other sizes. The $E_{p}$ is clearly separated from $4 \mathrm{~nm}$ and larger $\mathrm{Au}$ NPs, making it easy to study size changes by ASV following various chemical and electrochemical treatments.

Equation 3.1 shows the predicted oxidation potential of metal NPs (Eparticles) relative to the oxidation potential of the bulk metal (Ebulk) based on the work of Plieth. ${ }^{165}$

$$
E_{\text {particles }}=\left(-\frac{2 Y V_{m}}{Z F}\right)\left(\frac{2}{d}\right)+E_{\text {bulk }}
$$

Here, $\mathrm{Y}$ is the surface stress of $\mathrm{Au}\left(1880 \mathrm{erg} \mathrm{cm}^{-2}\right), V_{m}$ is the molar volume of $\mathrm{Au}$ $\left(10.21 \mathrm{~cm}^{3} \mathrm{~mol}^{-1}\right), \mathrm{Z}$ is the number of electrons transferred per Au atom during oxidation, F is Faraday's constant, and d is the diameter of the NP. We used an Ebulk value of $0.936 \mathrm{~V}$ and $Z=1$ to fit the $50 \mathrm{~nm}$ diameter Au NPs the best, which 
was consistent with our previous bulk value..$^{155}$ Using the oxidation potential of $0.46 \mathrm{~V}$ for the THPC Au2nm NPs, equation 1 predicts an average diameter of 1.7 $\mathrm{nm}$, which is very close to the previously reported TEM value of $1.5 \mathrm{~nm} \cdot{ }^{47}$ Equation 1 predicts a size of $3.5 \mathrm{~nm}$ for the $4.0 \mathrm{~nm}$ Au NPs, which is also in excellent agreement with electron microscopy for Cit Au4nm NPs. Unfortunately, the equation predicts a diameter of $5.1 \mathrm{~nm}$ for the $15 \mathrm{~nm}$ Au NPs. We do not understand why the $15 \mathrm{~nm}$ diameter Au NPs do not fit the trend well. Based on the equation, those NPs should actually oxidize around $0.88 \mathrm{~V}$. The oxidation potential for $\sim 15 \mathrm{~nm}$ diameter Au NPs electrodeposited directly onto glass/ITO electrodes previously ${ }^{155}$ was near $0.87 \mathrm{~V}$, so it might be possible that the charge of the APTES has an effect on the oxidation potential for some Au NP sizes as described by our group previously. ${ }^{168}$ Regardless, this data shows that the THPC Au2nm NPs oxidize at drastically more negative potentials compared to $4 \mathrm{~nm}$ diameter Au NPs and larger sizes, which is a thermodynamic effect and in excellent general agreement with the prediction of Plieth. The ASV results also confirm that the THPC Au2nm NPS do not change in size during attachment to the glass/ITO/APTES electrode or throughout the ASV scan. They appear to be stable enough to make the ASV measurements. Finally, since the $E_{p}$ is more negative than Cit Autnm and larger sized Au NPs, the THPC stabilizer clearly does not significantly alter the oxidation potential of the Au. If it did, it would likely hinder Au oxidation, which would cause the $E_{p}$ value to shift positive, not negative.

The UV-vis data in Figure 3.1. (B) show the spectrum of Cit Au4nm NPs, which clearly shows a localized surface plasmon resonance (LSPR) band near $506 \mathrm{~nm}$, 
and the spectrum of THPC Au2nm NPs, which does not have a distinct LSPR band. ${ }^{47,32}$ The LSPR band at $506 \mathrm{~nm}$ is consistent with the UV-vis spectrum of Cit Au4nm NPs and it is well-known that the LSPR band disappears for NPs of about 2 nm diameter and smaller. This is consistent with the ASV data and further evidence that the THPC Au2nm NPs are below $2 \mathrm{~nm}$ in diameter.

Before exploring the size stability of the THPC Au ${ }_{2 n m}$ NPs, we wanted to confirm that they exhibited useful electrocatalytic properties. Researchers already discovered that small Au NPs are able to electrocatalyze both $\mathrm{CO}_{2}$ reduction and the hydrogen evolution reaction (HER). Both theoretical and experimental studies have been performed for $\mathrm{CO}_{2}$ reduction with different sized Au NPs. ${ }^{169,19}$ For example, Mistry and coworkers reported a drastic increase in current density for $\mathrm{CO}_{2}$ reduction when the Au NP size was decreased from $8 \mathrm{~nm}$ to $\sim 1 \mathrm{~nm}$ in size. ${ }^{5}$ In most of these electrocatalysis studies, however, the NPs contained strongly bound stabilizers, which may inhibit the interaction of $\mathrm{CO}_{2}$ with the Au surface. Also, the Au NPs were usually attached to the electrode by the drop-cast method, which can cause NP aggregation and significantly affect their reactivity. Here we control the assembly of the Au NPs onto a glass/ITO/APTES electrode by directly soaking in the Au NP solution. With our method of attachment, the NPs are well dispersed and separated on the electrode surface and they are also presumably less hindered by the Cit and THPC stabilizers compared to polymers, surfactants, and strongly-coordinated ligands (such as thiols) because they are relatively weak adsorbates and have open molecular structures. As evidence, Figure 3.2. shows a comparison between the oxidation of THPC Au2nm NPs and those stabilized with 
stronger thiocyanate and glutathione ligands, where the oxidation potential is more positive by $100-200 \mathrm{mV}$.
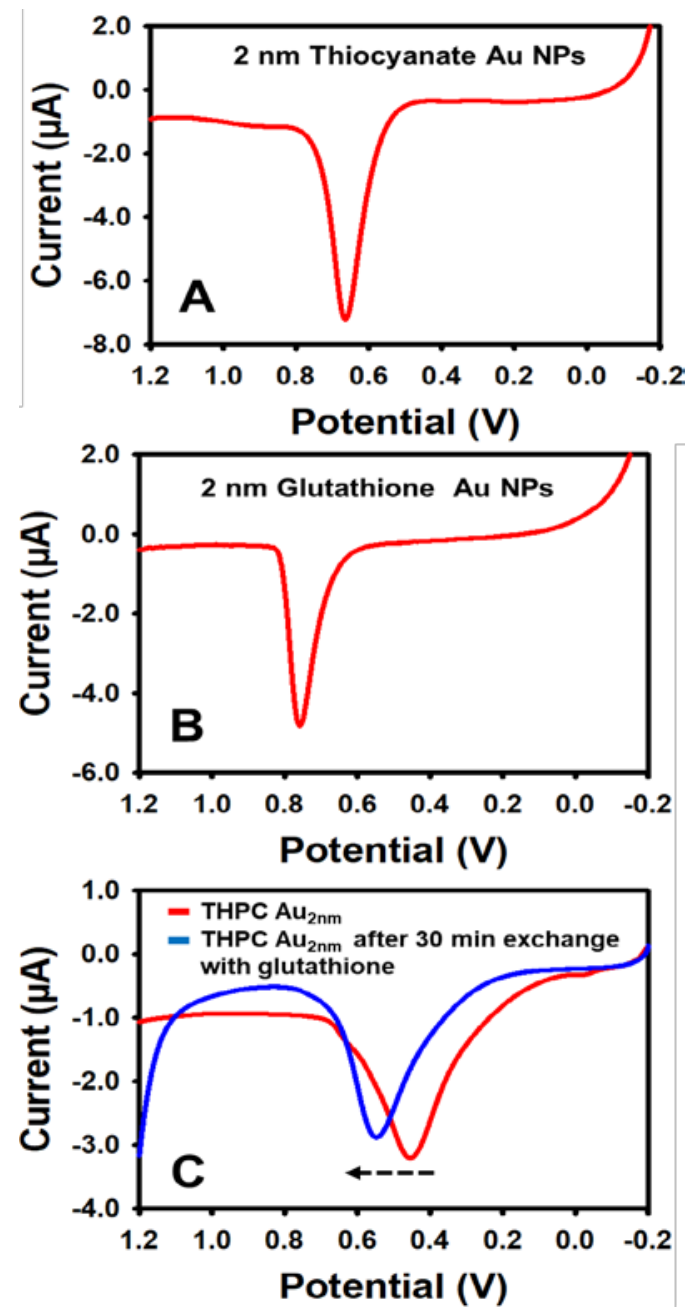

Figure 3. 2. ASV of a glass/ITO/APTES electrode containing $2 \mathrm{~nm}$ diameter thiocyanate-coated (A) and glutathione-coated (B) Au NPs. (C) ASV of a glass/ITO/APTES electrode containing THPC Au2nm NPs (red) and THPC Au2nm NPs after 30 min of soaking in $10 \mathrm{mM}$ glutathione solution (blue). All ASVs were 
run in $10 \mathrm{mM} \mathrm{KBr}$ plus $0.1 \mathrm{M} \mathrm{KClO}_{4}$ solution from $-0.2 \mathrm{~V}$ to $1.2 \mathrm{~V}$ at a scan rate of $0.01 \mathrm{~V} / \mathrm{s}$.
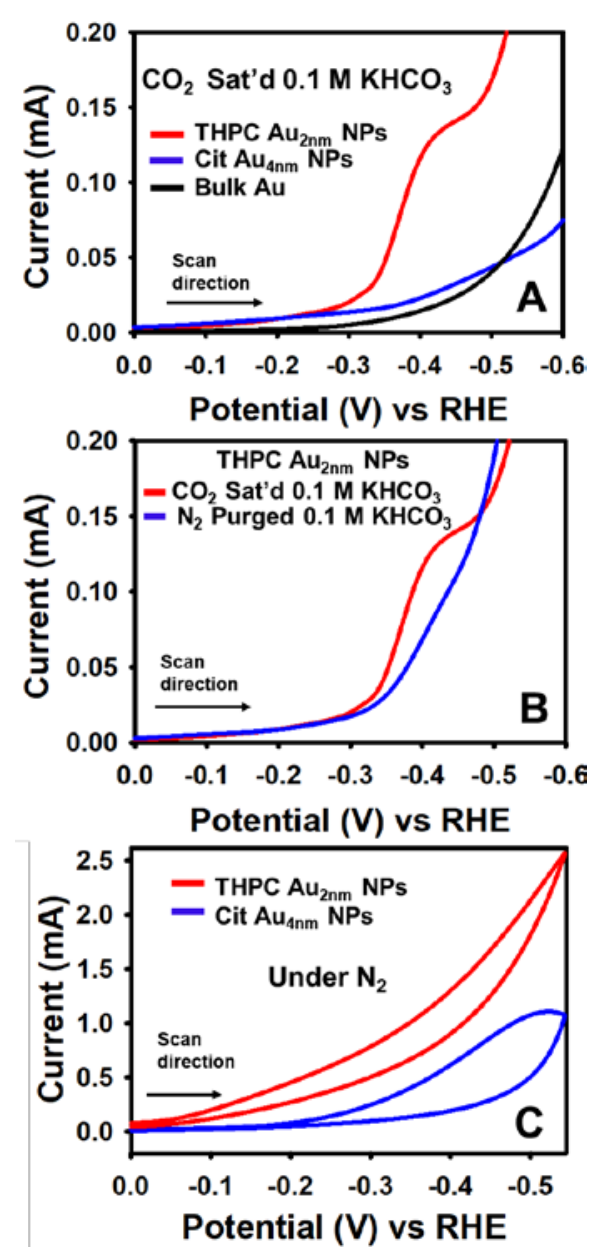

Figure 3. 3. (A) Linear sweep voltammograms (LSVs) of glass/ITO/APTES electrodes coated with THPC Au2nm NPs (Red), Cit Au4nm NPs (Blue) and bulk Au (black) in $\mathrm{CO}_{2}$-saturated $0.1 \mathrm{M} \mathrm{KHCO}_{3}$ solution. (B) Linear sweep voltammograms (LSVs) of glass/ITO/APTES electrodes coated with THPC Au2nm NPs in $\mathrm{CO}_{2-}$ saturated $0.1 \mathrm{M} \mathrm{KHCO}_{3}$ solution (Red) and $\mathrm{N}_{2}$ purged $0.1 \mathrm{M} \mathrm{KHCO}_{3}$ solution (Blue). (C) CVs obtained in $\mathrm{N}_{2}$-saturated $0.1 \mathrm{M} \mathrm{HClO}_{4}$ solution at a scan rate of $0.01 \mathrm{~V} / \mathrm{s}$ of glass/ITO/APTES electrodes coated with Cit Autnm NPs (blue) and THPC Au2nm NPs (red). 
Since the mechanism of $\mathrm{CO}_{2}$ reduction with small $\mathrm{Au}$ NPs is wellknown, ${ }^{170,171}$ this was a good electrochemical reaction to study. Figure 3.3 (A) shows $\mathrm{CVs}$ in $\mathrm{CO}_{2}$-saturated $0.1 \mathrm{M} \mathrm{KHCO}_{3}$ solution at $\mathrm{pH} 6.8$ of glass/ITO/APTES electrodes coated with Cit Au4nm NPs and THPC Au2nm NPs. During the negative scan from 0.0 to $-0.6 \mathrm{~V}$ (vs RHE) we observed a broad peak at $-0.40 \mathrm{~V}$ for the THPC Au2nm NPs with an onset potential of $\sim-0.20 \mathrm{~V}$. The CV for the Cit Au4nm NPs does not show a distinct peak, however. It instead shows a general increase in current as the potential is scanned negative, but no clear peak for $\mathrm{CO}_{2}$ reduction. This shows drastically different electrocatalytic activity for the $2 \mathrm{~nm}$ and $4 \mathrm{~nm} \mathrm{Au}$ NPs. The $\mathrm{CO}_{2}$ reduction peak was completely absent in the CV for bulk $\mathrm{Au}$, confirming that it is not active for $\mathrm{CO}_{2}$ reduction. In $\mathrm{N}_{2}$-saturated solution ( $\mathrm{pH} \sim 8.1$ ) the current density was lower and the peak was absent for the THPC Au2nm NPs (Figure 3.3.(B)), confirming that the peak is due to $\mathrm{CO}_{2}$ reduction in Figure 3.3.A. Figure 3.4 of shows the same voltammograms for Cit $\mathrm{Au}_{4 n m} \mathrm{NPs}$ in $\mathrm{CO}_{2}$ - compared to $\mathrm{N}_{2}$-saturated solutions. Our findings indicate that THPC $\mathrm{Au}_{2 n m} \mathrm{NPs}$ are more active as electrocatalysts for $\mathrm{CO}_{2}$ reduction with a lower overpotential than Cit Au4nm sized particles. This lower overpotential is due to the NP size, not the different ligands, consistent with previous work. Importantly, the THPC Au2nm NPs have an overpotential about $200-250 \mathrm{mV}$ less than thiol-stabilized $\mathrm{Au}_{25}$ nanoclusters. ${ }^{11}$ This is likely due to less hindrance from the weak, open THPC stabilizer. These could therefore be highly promising materials for $\mathrm{CO}_{2}$ reduction provided they exhibit good size stability under the reaction conditions. 


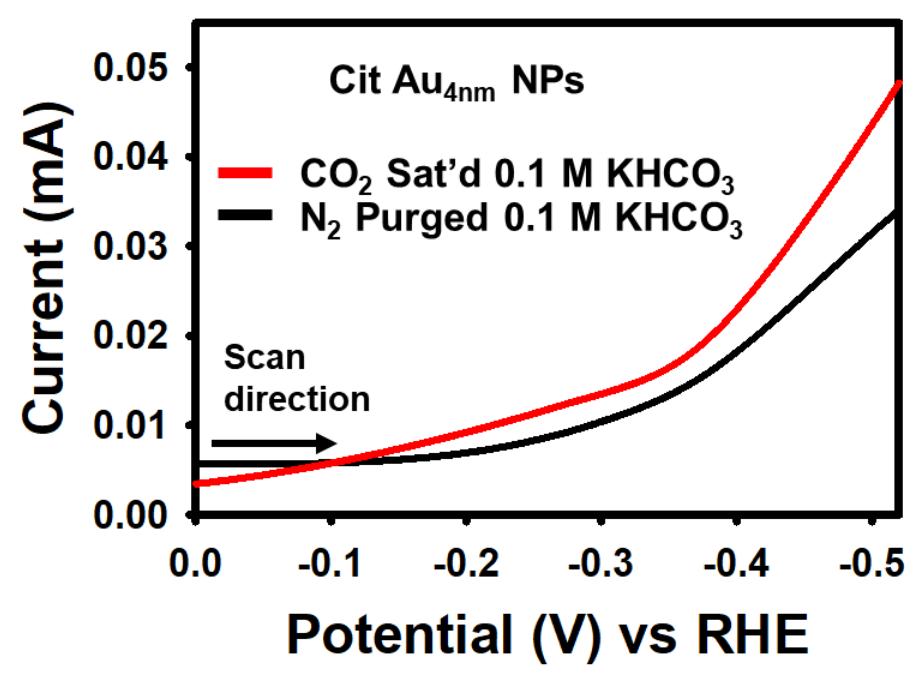

Figure 3. 4. Linear sweep voltammograms (LSVs) of glass/ITO/APTES electrodes coated with Cit Au4nm NPs in $\mathrm{CO}_{2}$-saturated $0.1 \mathrm{M} \mathrm{KHCO}_{3}$ solution (Red) and $\mathrm{N}_{2}$ purged $0.1 \mathrm{M} \mathrm{KHCO}_{3}$ solution (Black).

We also compared the Cit Au4nm NPs and THPC Au2nm NPs under $\mathrm{N}_{2}$ to explore their ability to catalyze the HER based on previous reports. ${ }^{169,172}$ Figure 3.3 . (C) shows the CVs (vs. RHE) of glass/ITO/APTES coated with Cit Au4nm NPs and THPC $\mathrm{Au}_{2 n m} \mathrm{NPs}$ in $0.1 \mathrm{M} \mathrm{HClO}_{4}$ solution under $\mathrm{N}_{2}$ saturated solution. Interestingly, we found that the $\mathrm{H}^{+}$reduction peak began at $-0.10 \mathrm{~V}$ for THPC $\mathrm{Au}_{2 \mathrm{~nm}}$ NPs, but not until about $-0.3 \vee$ (and less current) for the Cit Autnm NPs. Back and coworkers reported that small sized Au NPs are effective toward HER and $\mathrm{CO}_{2}$ reduction due to an increase in the number of edge and corner sites based on density functional theory (DFT) calculations. ${ }^{169}$ The corner sites of the Au NPs are 
more favorable towards $\mathrm{CO}_{2}$ reduction while edge sites are more active for the HER. ${ }^{169}$ Our results indicate that both $\mathrm{CO}_{2}$ reduction and the HER are favored with THPC $\mathrm{Au}_{2 n m}$ NPs as compared to Cit Au4nm NPs. Having shown good electrocatalytic activity for THPC Auznm NPs for two important electrochemical reactions relevant to renewable energy, we then explored the size stability, which is a critical issue in NP catalysis.

Our simple ASV technique for measuring the size of Au NPs synthesized with weak stabilizers (Figure 3.1) allows us to study size transformations that may occur following common treatments, such as surface oxidation/reduction cycling in acid, electrochemistry in halide-containing salt solution, and ozone treatment. Importantly, the ASV technique provides size information on the metal NPs under solution conditions directly following the particular treatment. There is no interference from placing them in a vacuum and no effect of an electron beam, which are issues that are encountered when using electron microscopy. These are important advantages of ASV for these types of measurements. ASV is also comparably much faster and can be performed directly on electrode surfaces, as opposed to a special holder as in transmission electron microscopy (TEM). We compared the behavior of the THPC Au2nm NPs to that of the Cit Au4nm NPs. The use of ASV and other electrochemical methods to monitor the dramatic difference between the size stability of these two different, but closely-sized Au NPs under mild, commonly encountered conditions is the focus of the work and described in the rest of the article. 
First, we compared the behavior of THPC Au2nm NPs to Cit Au4nm NPs during and after electrochemical cycling in $0.1 \mathrm{M} \mathrm{HClO}_{4}$ electrolyte. Figure 3.5. (A) shows the cyclic voltammogram (CV) of the Cit Au4nm NPs attached to glass/ITO/APTES in $0.1 \mathrm{M} \mathrm{HClO}_{4}$ solution at a scan rate of $0.1 \mathrm{~V} / \mathrm{s}$ in the range of $0.0 \mathrm{~V}$ to $1.6 \mathrm{~V}$ versus an $\mathrm{Ag} / \mathrm{AgCl}$ reference electrode. In the first $\mathrm{CV}$, the surface Au oxidation peak appeared at about $1.20 \mathrm{~V}$, which is due to the formation of $\mathrm{Au}_{2} \mathrm{O}_{3}$, and the subsequent reduction peak on the reverse scan, due to the reduction of $\mathrm{Au}_{2} \mathrm{O}_{3}$ back to $\mathrm{Au}$, appeared at $0.78 \mathrm{~V}$. In the $2^{\text {nd }} \mathrm{CV}$ scan, the anodic and cathodic peaks remain in the same position, suggesting that the Cit Au4nm NPs remained stable during the two oxidation and reduction cycles in acidic electrolyte. The ASVs obtained in $10 \mathrm{mM} \mathrm{KBr}$ plus $0.1 \mathrm{M} \mathrm{KClO}_{4}$ of glass/ITO/APTES coated with Cit Au4nm NPs untreated (red plot) and previously cycled once from 0.0 to 1.6 $\checkmark$ in $0.1 \mathrm{M} \mathrm{HClO}_{4}$ (blue plot) are shown in Figure 3.5. (B). Consistent with the $\mathrm{CVs}$, the ASVs show the same oxidation potential for uncycled and once-cycled Cit Au4nm NPs, showing that they are stable and unchanged during the oxidation/reduction cycle in $0.1 \mathrm{M} \mathrm{HClO}_{4}$. In fact, these Cit Au4nm NPs are stable and unchanged for at least $20 \mathrm{CV}$ oxidation/reduction cycles. 

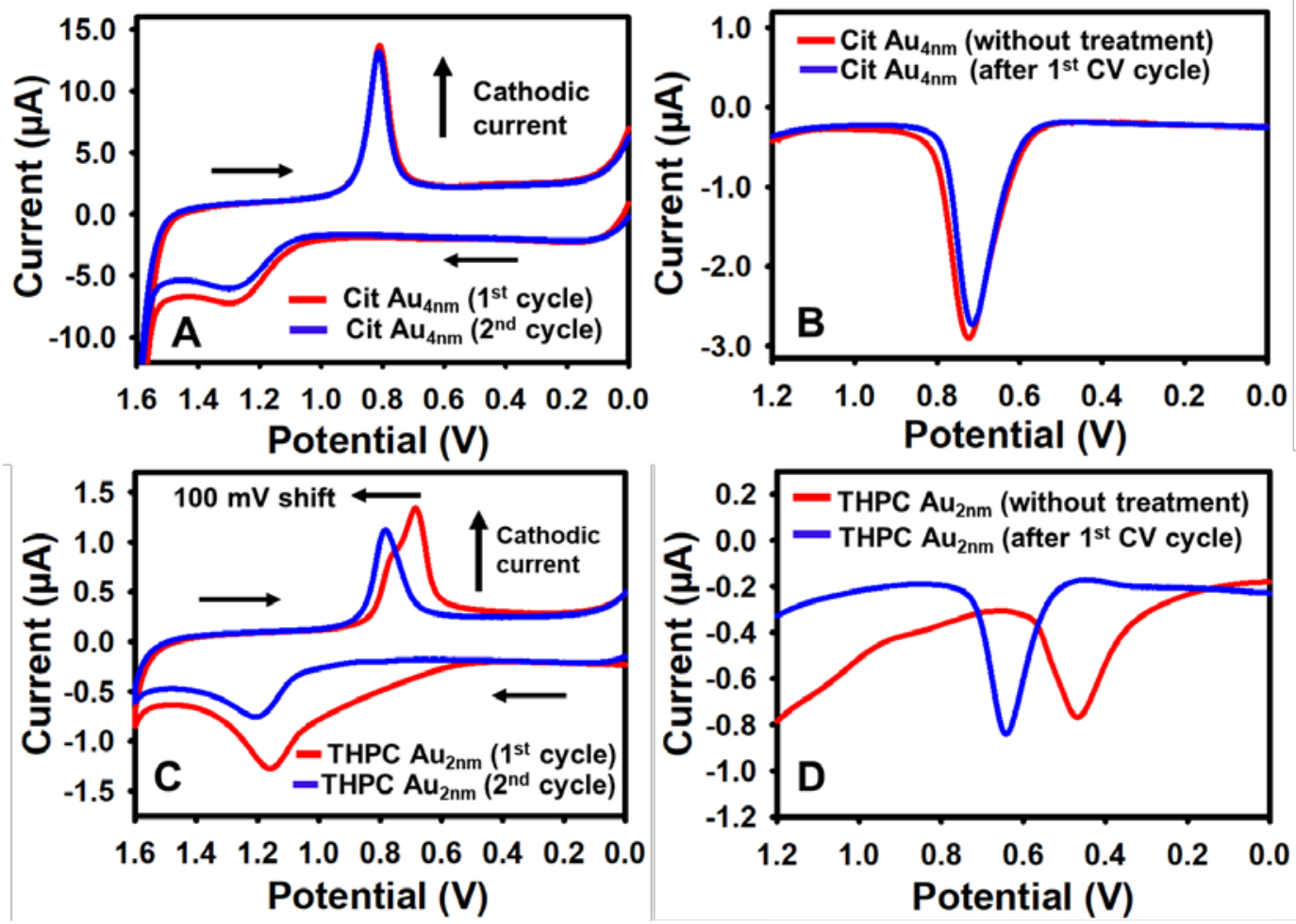

Figure 3. 5. Cyclic voltammogram (CVs) of glass/ITO/APTES coated with (A) Cit Au4nm NPs and (C) THPC Au2nm NPs in $0.1 \mathrm{M} \mathrm{HClO}_{4}$ solution from $0.0 \mathrm{~V}$ to $1.6 \mathrm{~V}$ at a scan rate of $0.1 \mathrm{~V} / \mathrm{s}$ (Red is the $1^{\text {st }} \mathrm{CV}$ cycle and Blue is the $2^{\text {nd }} \mathrm{CV}$ ). ASV of glass/ITO/APTES coated with (B) Cit Au4nm NPs and (D) THPC Auznm NPs in 10 $\mathrm{mM} \mathrm{KBr}$ plus $0.1 \mathrm{M} \mathrm{KClO}_{4}$ solution from $0.0 \mathrm{~V}$ to $1.2 \mathrm{~V}$ at a scan rate of $0.01 \mathrm{~V} / \mathrm{s}$ (Red is the ASV of as-prepared Au NPs and Blue is ASV after $1 \mathrm{CV}$ cycle in $0.1 \mathrm{M}$ $\left.\mathrm{HClO}_{4}\right)$.

Figure 3.5. (C) shows the first $\mathrm{CV}$ from 0.0 to $1.6 \mathrm{~V}$ versus $\mathrm{Ag} / \mathrm{AgCl}$ in $0.1 \mathrm{M} \mathrm{HClO}_{4}$ for glass/ITO/APTES coated with THPC Au2nm NPs. There was a significant 
shoulder from 0.6 to $1.0 \mathrm{~V}$ and major oxidation peak at $1.15 \mathrm{~V}$ and reduction peak at $0.65 \mathrm{~V}$. In the second CV, the shoulder peak disappeared, the major anodic peak shifted positive to $1.19 \mathrm{~V}$, and the cathodic peak shifted positive to $0.78 \mathrm{~V}$. The shoulder peak and more negative oxidation/reduction peaks compared to Cit Au4nm NPs on the first scan was due to the smaller size of the THPC Au2nm NPs and the shift to larger potentials on the second scan suggests that the Au NPs changed in size during the first scan. Figure 3.5. (D) shows ASVs obtained in 10 $\mathrm{mM} \mathrm{KBr}$ plus $0.1 \mathrm{M} \mathrm{KClO}_{4}$ of glass/ITO/APTES electrodes coated with THPC Au2nm NPs untreated and cycled once in $0.1 \mathrm{M} \mathrm{HClO}_{4}$. The oxidation peak for the THPC Au2nm NPs appeared at $0.46 \mathrm{~V}$ for the untreated sample and at $0.65 \mathrm{~V}$ for the sample cycled once in $0.1 \mathrm{M} \mathrm{HClO}_{4}$. This $190 \mathrm{mV}$ positive shift indicates that the small sized THPC Au2nm NPs are unstable and transformed into a more stable, larger size near about $4 \mathrm{~nm}$ in diameter after just 1 cycle in acid. This relatively mild treatment to these sub $2 \mathrm{~nm}$ Au NPs results in a dramatic size change. We performed a similar set of experiments on Cit Au4nm and THPC Au $\mathrm{u}_{2 n m}$ NPs using an Ag wire as the reference electrode to avoid possible chloride contamination from the $\mathrm{Ag} / \mathrm{AgCl}$ reference electrode. The results were the same, indicating that the size transformation occurs during surface $A u$ oxidation and reduction, not chloride-induced Au dissolution and redeposition. The exact mechanism for the change in size for the THPC Au2nm NPs size during the CV cycle is not understood at this time, but it could be due to Ostwald ripening due to Au dissolution and redeposition during Au surface oxide formation and reduction, as observed recently with carbon supported Au NPs in the 1-3 nm range, or due to the surface 
reconstruction of $\mathrm{Au}$ in acid, which is known to occur on 2D surfaces. ${ }^{173-174}$ It is important to note that this is the first time we have observed an $\sim 100 \mathrm{mV}$ negative shift of the oxidation/reduction peaks in $0.1 \mathrm{M} \mathrm{HClO}_{4}$ for small NPs. A small negative shift was observed previously for the oxidation/reduction of Au in acid for a single Au NP ${ }^{175}$ while another study suggested a positive shift occurs. ${ }^{176}$ Our data clearly supports a negative shift.

We next studied the behavior of Cit Au4nm NPs and THPC Au2nm NPs in bromide solutions at potentials below their oxidation potential to see if their size was stable. We held the potential of a glass/ITO/APTES electrode containing the Au NPs at $0.30 \mathrm{~V}$ in $10 \mathrm{mM} \mathrm{KBr}$ plus $0.1 \mathrm{M} \mathrm{HClO}_{4}$ solution for various times. This potential is well before the oxidation potential of either Au NPs, so that the Au NPs would not dissolve fully by bromide-induced oxidation. After holding for different times at $0.30 \mathrm{~V}$, we removed the electrode containing the Au NPs, rinsed with water, and dried under nitrogen before performing a full ASV in $10 \mathrm{mM} \mathrm{KBr}$ plus $0.1 \mathrm{M} \mathrm{KClO}_{4}$ solution to determine if any changes occurred. The oxidation peak in the ASV of Cit Au4nm NPs was the same for a sample untreated (0 min, red plot) and treated by holding the potential at $0.30 \mathrm{~V}$ for $35 \mathrm{~min}$ (blue plot) as shown in Figure $3.6(\mathrm{~A})$. It wasn't until $105 \mathrm{~min}$ at $0.30 \mathrm{~V}$ (Figure 3.6. (A), green plot) that a second peak appeared in the ASV at about $0.95 \mathrm{~V}$, indicating the presence of larger NPs on the electrode surface. In contrast, the ASV of THPC Au2nm NPS showed one peak at $0.46 \mathrm{~V}$ for the untreated NPs but also a second peak near 

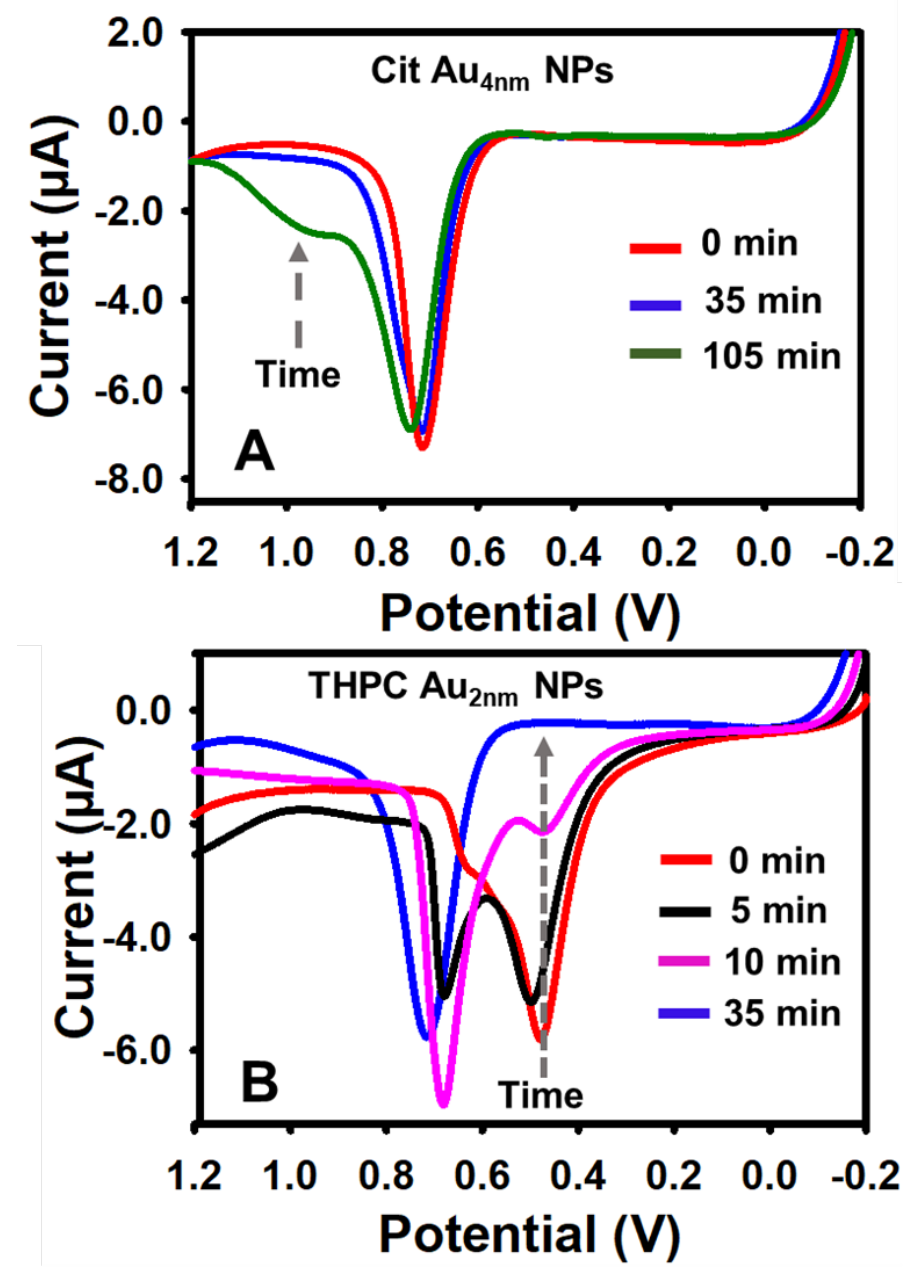

Figure 3. 6. ASVs of glass/ITO/APTES electrodes coated with (A) Cit Au4nm NPs and (B) THPC Au2nm NPs that were untreated (red plot, 0 min) or held at $0.30 \mathrm{~V}$ for the times indicated in $10 \mathrm{mM} \mathrm{KBr}$ plus $0.1 \mathrm{M} \mathrm{HClO}_{4}$ electrolyte. ASVs obtained under same conditions as in Figure 3.1.

$0.70 \mathrm{~V}$ for those held at $0.30 \mathrm{~V}$ for only $5 \mathrm{~min}$ (Figure 3.6.(B), black plot). After 10 min (pink plot), the peak at $0.46 \mathrm{~V}$ decreased further while the peak at $0.70 \mathrm{~V}$ 
increased. After $35 \mathrm{~min}$ (blue plot) the peak at $0.46 \mathrm{~V}$ was completely gone and replaced by a new peak at about $0.75 \mathrm{~V}$. This almost $300 \mathrm{mV}$ positive shift (from $0.46 \mathrm{~V}$ to $0.75 \mathrm{~V}$ ) over the $35 \mathrm{~min}$ indicates that the small-sized THPC $\mathrm{Au}_{2 \mathrm{~nm}} \mathrm{NPs}$ increased in size to about 4-10 nm during the electrochemical treatment, which we believe is due to electrochemical Ostwald ripening. ${ }^{177}$ At a potential of $0.3 \mathrm{~V}, \mathrm{Br}^{-}$ oxidized the $\mathrm{Au}$ atoms of the smallest NPs to $\mathrm{AuBr}_{4}^{-}$or $\mathrm{AuBr}_{2}^{-}$and the $\mathrm{Au}$ complexes subsequently redeposited onto slightly larger-sized NPs due to the size dispersity in the sample and the different standard potentials for the different-sized NPs. This process presumably continues for 35 min until the NPs are $>4 \mathrm{~nm}$ in diameter on average and more stable. The THPC Au2nm NPs begin to transform (ripen) into $>4 \mathrm{~nm}$ diameter Au NPs within 5 min while the Cit Au4nm NPs do not show any significant change to larger sizes until about 105 min. The rate of Ostwald ripening is related to the applied potential (Eapplied) relative to the Ep value for oxidation in the ASV (or $E^{0^{\prime}}$ ) of the two Au NPs. Since the $E_{p}$ is more negative for the THPC $\mathrm{Au}_{2 n m} \mathrm{NPs}$, they will oxidize more readily at $0.3 \mathrm{~V}$ and ripen faster compared to the larger Au NPs. The effect of NP size and applied potential on the Ostwald ripening rate will be the focus of a more detailed future report.

Nanostructures synthesized with strongly adsorbed ligands, such as thiols or certain polymers or surfactants, are typically quite stable and retain their core size under a variety of conditions. ${ }^{178}$ Unfortunately, the stabilizing ligands can block the surface of the NPs, which inhibits the catalytic activity and can introduce a barrier to electron transfer. ${ }^{179}$ Researchers have therefore used ozone treatment 
to remove these inhibiting ligands from the nanostructure surface, since ozone reacts strongly with organics. ${ }^{180,26}$

It is undesirable to change the NP size, however. Accordingly, we decided to determine the effect of ozone treatment on the two differently sized NPs synthesized in this study. We attached the Cit Au4nm NPs and THPC Au2nm NPs onto glass/ITO/APTES electrodes and treated them with ozone for different times before measuring ASVs and comparing them to the ASVs of untreated samples. Figure 3.7. (A) shows that the $E_{p}$ value for the Cit Au4nm NPs in the ASV is the same for the untreated (red plot) and ozone-treated sample after 8 min (blue plot), but that the peak shifted positive after 90 min (black plot), consistent with an increase in NP size after the longer ozone exposure to about 10-15 nm in diameter. In contrast, the peak at $0.46 \mathrm{~V}$ for the THPC Au2nm NPs (red plot) almost disappeared while a new peak appeared at about $0.68 \mathrm{~V}$ after just 1 min in ozone (blue plot) (figure 3.7.(B)). By 2 min (black plot), the peak at $0.46 \mathrm{~V}$ was completely gone and only one peak appeared at about $0.70 \mathrm{~V}$, showing that the size changed to about $4 \mathrm{~nm}$ in diameter. The ASV looked the same at $8 \mathrm{~min}$ (pink plot) as it did at 2 min. The ASV clearly shows a very rapid size transformation for the THPC Au2nm NPs relative to the Cit Au4nm NPs. Although the mechanism is not well understood at this time, there is clearly some type of ripening process occurring. This is valuable information to know since one would like to choose an ozone cleaning time that effectively removes strongly bound ligands but does not alter 
the NP size. Clearly just 1 min can significantly alter the size for the sub $2 \mathrm{~nm}$ Au NPs, which is quite surprising.
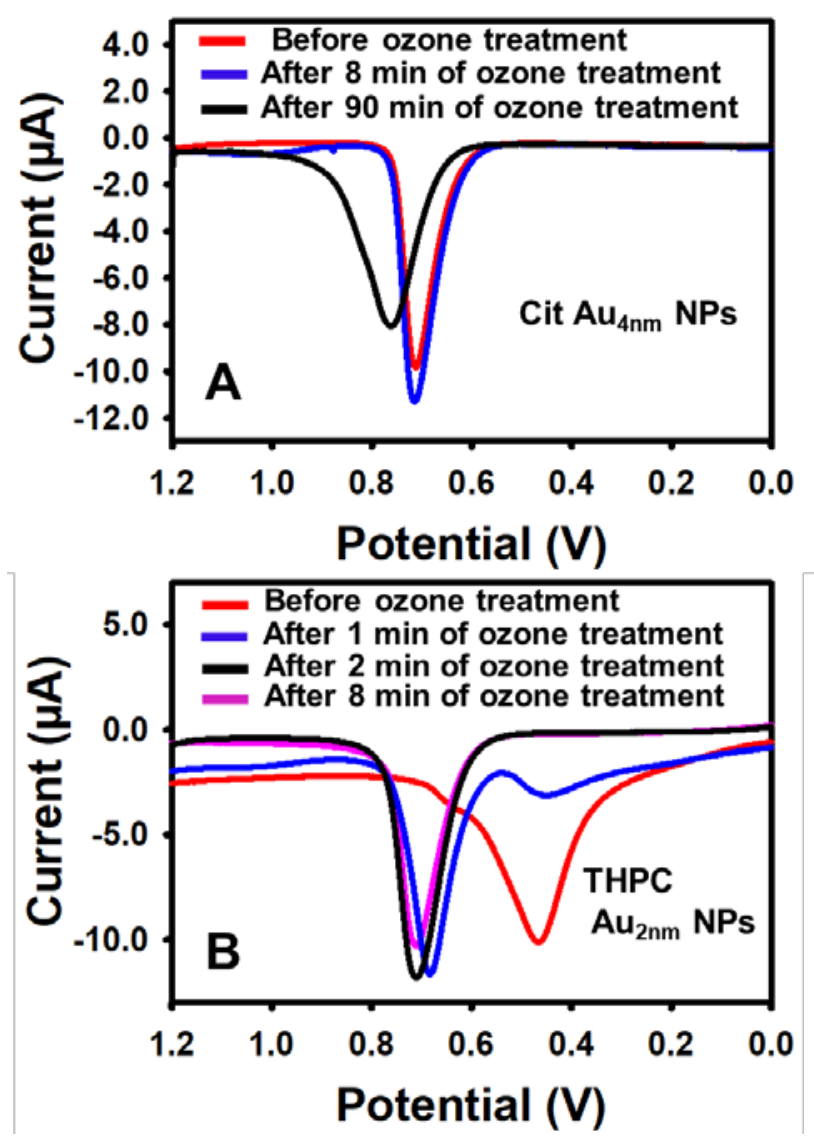

Figure 3. 7. ASVs of glass/ITO/APTES electrodes coated with (A) Cit Autnm NPs and (B) THPC Au2nm NPs that were untreated (Red) and exposed to ozone for the different times as indicated. ASVs obtained under same conditions as in Figure 3.1. 
In order to confirm the size changes due to Ostwald ripening and ozone treatment, we measured SEM images of THPC Au2nm NPs before and after the two different treatments. Figure 8 shows SEM images of as-prepared THPC $\mathrm{Au}_{2 \mathrm{~nm}}$ NPs (Figure 3.8. (A)) and THPC Au2nm NPs after 35 min of Ostwald ripening at 0.3 $\vee$ (Figure 3.8.(B)) and after 8 minutes of ozone treatment (Figure 3.8.(C)). The THPC Au ${ }_{2 n m}$ NPs are not visible in the SEM image as prepared because $1.5 \mathrm{~nm}$ to $2 \mathrm{~nm}$ is beyond the resolution of the SEM. However, after 35 min of Ostwald ripening, we were able to image the NPs because they had grown to $\sim 4-10 \mathrm{~nm}$ in diameter, a size observable by SEM. Similarly, we were able to image the NPs after ozone treatment for 8 minutes in Figure 3.8 (C) since the NPs increased in size to 3-4 $\mathrm{nm}$. The SEM images therefore confirmed what the ASVs already told us about the size transformations during these two different treatments. This again highlights the power of ASV as an analytical tool for monitoring size changes of nanostructures on electrode surfaces. 


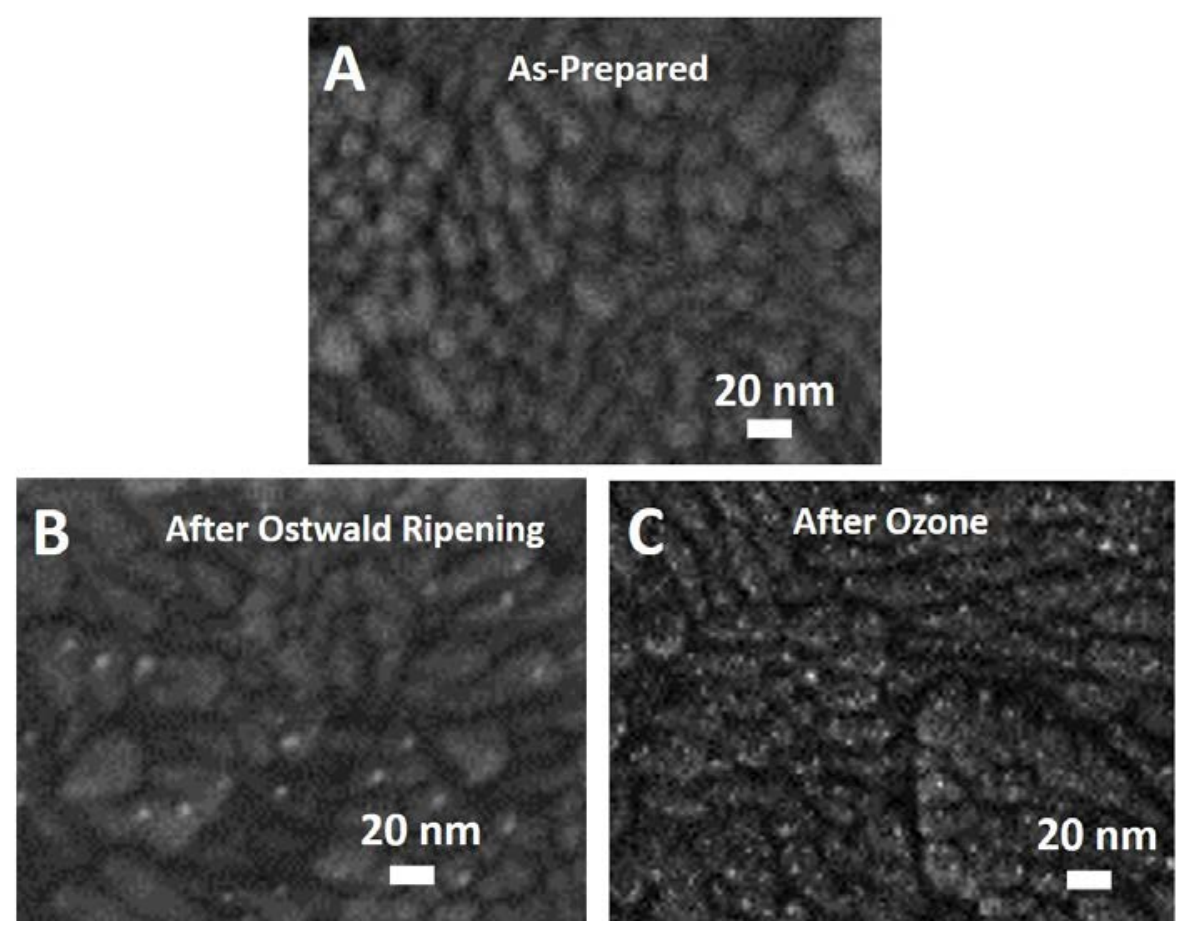

Figure 3. 8. SEM images of glass/ITO/APTES coated with THPC Au2nm NPs (A) as-prepared, (B) after $35 \mathrm{~min}$ of Ostwald ripening at $0.3 \mathrm{~V}$ in $10 \mathrm{mM} \mathrm{KBr}$ plus 0.1 $\mathrm{M} \mathrm{HClO}_{4}$ solution and (C) after 8 min of ozone treatment.

\subsection{CONCLUSIONS}

We reported the chemical reactivity and size stability of THPC Auznm NPs under a variety of conditions and compared their behavior to Cit Autnm NPs. We observed a $250 \mathrm{mV}$ negative shift in the Au oxidation potential in $\mathrm{Br}^{-}$of THPC Au2nm NPs as compared to Cit Au4nm NPs. The THPC Au2nm NPs show higher electrocatalytic activity towards $\mathrm{CO}_{2}$ reduction and the HER with a lower overpotential compared to Cit Au4nm NPs, consistent with the literature. However, 
the THPC Au2nm NPs are more active towards $\mathrm{CO}_{2}$ reduction compared to thiolprotected Au25 clusters reported in the literature likely due to the weaker interaction of THPC with Au and it's more open molecular structure. After repetitive oxidation and reduction cycles in acidic solution, the Cit Au4nm NPs remained stable for at least 20 cycles, but THPC Au2nm NPs showed an increase in size to about $4 \mathrm{~nm}$ after just one cycle. THPC Au2nm NPs are also drastically more susceptible to Ostwald ripening and a size increase during ozone treatment compared to Cit Au$4 \mathrm{~nm}$ NPs. The rates of the size transformation are at least an order of magnitude faster for the smaller Au NPs. This work importantly shows that sub $2 \mathrm{~nm}$ diameter Au NPs containing a weakly adsorbed stabilizer with an open structure have drastically different reactivity and size stability compared to a closely sized 4 nm diameter Au NP that also contains a weakly adsorbed stabilizer. These differences are due to the size difference, as opposed to the stabilizer difference, which we confirmed by performing the same studies with $4 \mathrm{~nm}$ diameter Au NPs also containing the THPC stabilizer (Figure 3.9). This provides insight into how sensitive the reactivity and size stability of a metal changes below the $4 \mathrm{~nm}$ diameter size range. Our work shows that while sub $2 \mathrm{~nm}$ metal NPs have great potential as highly active catalysts, the high reactivity also leads to low size stability under fairly mild conditions (acid, halides, ozone). The size stability is an important consideration for potential catalytic and other applications. In addition, our work shows the tremendous value of using ASV to probe size transformations of very small metal NPs attached directly to electrode surfaces. 

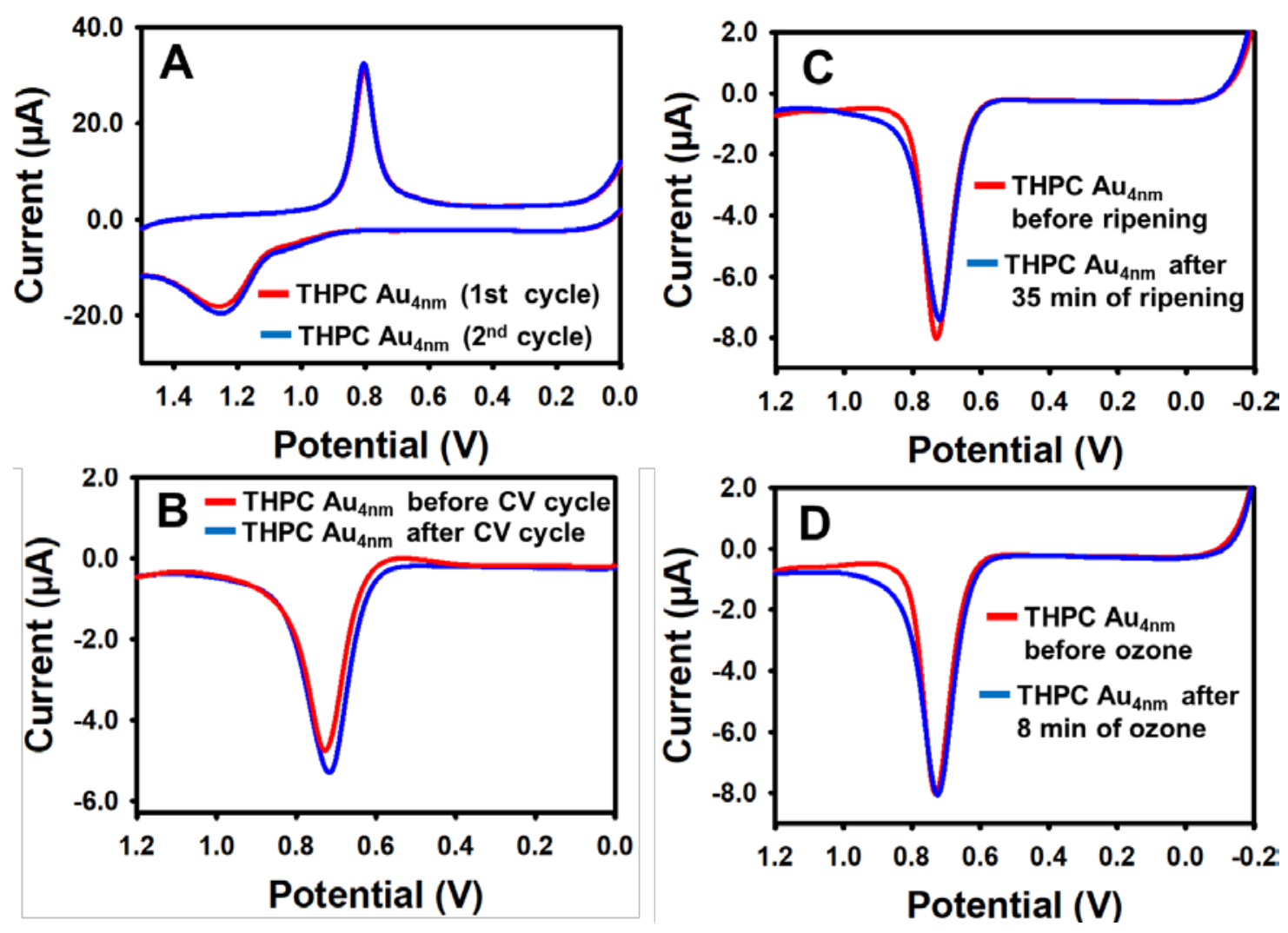

Figure 3. 9. (A) Cyclic voltammogram (CVs) of glass/ITO/APTES coated with THPC Au4nm NPs in $0.1 \mathrm{M} \mathrm{HClO}_{4}$ solution from $0.0 \mathrm{~V}$ to $1.5 \mathrm{~V}$ at a scan rate of 0.1 V/s (Red is the $1^{\text {st }} \mathrm{CV}$ and Blue is the $2^{\text {nd }} \mathrm{CV}$ ). (B) ASV of glass/ITO/APTES coated with THPC Au4nm NPs before (red) and after 1st CV cycle (blue). (C) ASV of glass/ITO/APTES coated with THPC Au4nm NPs before (red) and after 35 min of ripening in $\mathrm{Br}^{-}$containing electrolyte at $0.3 \mathrm{~V}$ (blue). (D) ASV of glass/ITO/APTES coated with THPC Au4nm NPs before (red) and after 8 min of ozone treatment (blue). All ASVs were obtained in $10 \mathrm{mM} \mathrm{KBr}$ plus $0.1 \mathrm{M} \mathrm{KClO}_{4}$ solution from -0.2 $\mathrm{V}$ to $1.2 \mathrm{~V}$ at a scan rate of $0.01 \mathrm{~V} / \mathrm{s}$ 


\section{CHAPTER IV}

\section{ELECTROXIDATION AND SIZE STABILITY OF AN 11-ATOM Au CLUSTER LESS THAN 1 NM IN DIAMTER}

\subsection{INTRODUCTION}

In recent years, the size-dependent electrochemical and chemical reactivity of metal NPs has gained a significant amount of attention due to its wide range of applications such as in catalysis, ${ }^{181}$ sensing ${ }^{182}$ and nanoelectronics devices. ${ }^{183}$ Smaller sized metal NPs often show unique electrochemical and chemical reactivity compared to bigger ones due to their higher surface free energy as compared to bigger ones. The difference in reactivity of small sized metal NPs is also due to the dramatic increase in surface area (SA) to total volume $(\mathrm{V})$ ratio and increase in number of active sites with decease in size. The electronic, ${ }^{184}$ thermal, ${ }^{185}$ optical $^{39}$ and magnetic ${ }^{186}$ properties of metal NPs also changes with the change of SA/V ratios. These changes are sometimes very useful for fundamental research on metal NPs. Researchers have explored size dependent reactivity of metal NPs towards oxidation, ${ }^{64}$ toxicity, ${ }^{187}$ catalysis, ${ }^{188}$ sensing, ${ }^{189}$ melting ${ }^{185}$ and optical behaviors. ${ }^{39}$ This sort of properties and applications of the metal NPs further depends upon the shape of the NPs and the stabilizing ligands used during the synthesis. 
In the last two-decades, $\sim 1 \mathrm{~nm}$ sized Au nanoclusters (NCs) have attracted tremendous attraction in the research field due to their distinctive molecular like properties such as quantized double layer charging, ${ }^{156}$ discrete electronic energy level $^{190}$ and photoluminescence ${ }^{191}$ which render them useful for diverse applications. However, small sized NPs are more prone to be easily oxidized ${ }^{192}$ and also undergo easy transformation to larger size by sintering, ${ }^{34}$ which causes rapid deactivation of their reactivity during applications. Therefore, understanding the electrochemical properties of these small nanocrystal will be useful to further widen their applications.

One of the major challenges to study the size dependent electrochemical and chemical reactivity of $1 \mathrm{~nm}$ diameter Au NCs is to synthesize monodisperse NCs with appropriate ligand and attach them to the electrode surface. In most of the cases, Au NCs with magic number has been synthesized by thiol (or thiolate) ligands. ${ }^{193-194}$ Thiolate ligands bridge Au centers by strong S-Au interactions and lifting the gold atoms out of the cluster surface to form staple or oligomer motifs (RS-(Au-SR)n). ${ }^{195}$ For example, Chen and coworkers synthesized different-sized glutathione-coated Au NCs and used them as photosensitizers. ${ }^{196}$ However, it is hard to understand the actual reactivity of those strong ligand protected Au NCs because the interaction between ligand and NCs surface is stronger than reactant molecules. It is also well known that the bond between Au-S is stronger than the bond between Au-Au. ${ }^{46}$ Besides thiolate, phosphine ligands have been successfully used to synthesize atomically precise Au clusters. Specially, unidentate phosphines such as triphenylphosphine $\left(\mathrm{PPh}_{3}\right)$ and its derivatives have 
been used to synthesize atomically precise Au clusters with the size range of 0.5 to $2 \mathrm{~nm}$ in diameter both in aqueous and organic media. ${ }^{31,197}$ Phosphine ligands usually prefer to coordinate surface Au atoms, yielding a simple Au-P interface which in some sense is an improvement over the Au-S bond due to their watersoluble nature and lower toxicity to living cells. However, in recent years phosphine-stabilized Au cluster have also been used for the synthesis of atomically precise thiol-stabilized Au clusters by the ligand-exchange method. For example, McKenzie and coworkers reported the solution phase synthesis of glutathione coated $\mathrm{Au}_{25}$ clusters by the ligand exchange reaction of triphenylphosphine stabilized $\mathrm{Au}_{11}$ cluster. ${ }^{198}$ This finding indicates that the Au-P bonds is weaker than the Au-S bond.

Various treatments, such as ozone cleaning, thermal treatment, or chemical and electrochemical reactions during applications can cause changes in size and eventually their reactivity. For example, Trindell and coworkers recently reported that $2 \mathrm{~nm}$ diameter Au NCs coated with thiol or dendrimers when employed for electrochemical $\mathrm{CO}_{2}$ reduction transform to around $6 \mathrm{~nm}$ in size via sintering. ${ }^{34}$ Similarly, Hu and coworkers reported that Au NCs with lower size Au561ะ 31 and Au923ะ 20 undergo size transformation by Ostwald ripening during $\mathrm{CO}$ oxidation while $\mathrm{Au}_{2057 \pm} 45$ undergo size change by cluster diffusion and coalescence as revealed by their particle size histogram. ${ }^{8}$ Therefore, it is hard to properly correlate the actual NP size and their corresponding electrocatalytic or sensing behavior when the size is not stable. Recently, we reported that sub $1.6 \mathrm{~nm}$ dimeter Au NCs coated with weak stabilizers are more reactive for $\mathrm{CO}_{2}$ reduction and the hydrogen 
evolution reaction as compared to those coated with thiols. ${ }^{199}$ However, weakly stabilized $1.6 \mathrm{~nm}$ Au NCs were unstable and converted to 4-10 nm diameter Au NPs just by one oxidation-reduction cycle, 2 min of ozone treatment and when holding at $0.30 \mathrm{~V}$ in acidic $\mathrm{KBr}$ solution. We also reported the NP size analysis, ${ }^{64}$, 154, 200 aggregation state analysis, ${ }^{104}$ surface area to volume (SA/V) analysis, ${ }^{201-202}$ and composition and atomic arrangements analysis of CuAu bimetallic nanostructures by ASV. ${ }^{203}$

In this work we focus on the electrochemical characterization and stability of weakly stabilized water-soluble $\sim 1 \mathrm{~nm}$ diameter Au NCs during various chemical and electrochemical treatment. Previous studies for size determination of less than $1 \mathrm{~nm}$ diameter Au NCs were mostly performed by mass spectrometry because microscopic techniques are not suitable for this size NCs due to lack of lattice fringes. Electrochemical methods have the advantage of simple operation, low cost and quick analysis time for the analysis of metal and metal alloy nanocrystals attached to electrode surfaces under various conditions. Contrary to this, microscopic methods are tedious, costly, and it is difficult to accurately determine the size change during electrochemical and chemical treatments of these small sized Au NCs. The microscopic techniques themselves can actually cause size transformations during the analysis of these NCs due to thermal and electronic effects generated during their operation. 


\subsection{EXPERIMENTAL}

4.2.1. Aggregation of Au Nanoparticles with Acid. The $\mathrm{pH}$ of aqueous solutions

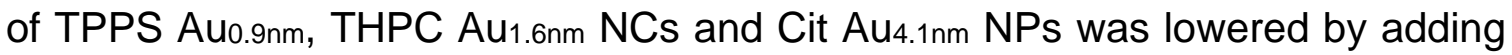
variable amounts of 2 and $8 \mathrm{M}$ perchloric acid solution depending on the desired pH. After addition of acid, the NPs were attached to the glass/ITO/APTES electrodes by directly soaking the electrode in the NP solution for variable times. Then this electrode was rinsed with water and dried under $\mathrm{N}_{2}$ for electrochemical ASV analysis.

\subsubsection{Deposition of Alkanethiol Self-assembled Monolayers (SAMs) on the} Au NPs. TPPS Au0.9nm and THPC Au1.6nm NCs were first attached on the APTES functionalized glass/ITO surface by directly soaking the electrode in the solution of NCs. Then the electrode was rinsed with water and dried under $\mathrm{N}_{2}$. Each of the electrodes were then soaked overnight in a 200 proof ethanol solution of $2 \mathrm{mM} 1$ butanethiol. Then this electrode was cleaned with nanopure water and dried under $\mathrm{N}_{2}$ or electrochemical analysis.

\subsection{RESULTS AND DISCUSSION}

We collected UV-vis spectra of TPPS Au0.9nm, THPC Au1.6nm, and Cit Au4.1nm diameter NPs as shown in Figure 4.1(A). Cit Au4.1nm NPs show a localized surface plasmon resonance (LSPR) band at $506 \mathrm{~nm}$ (pink graph, which is consistent with

those reported by our group ${ }^{201}$ and others previously. ${ }^{39}$ However, TPPS Auo.9nm and THPC Au1.6nm NPs do not display distinct LSPR bands in the UV-vis region, 
indicating that UV-vis spectra can't distinguish the NPs with sizes smaller than 2 $\mathrm{nm}$ in diameter. We then measured the size of TPPS Au0.9nm and THPC Au1.6nm by transmission electron microscopy (TEM) and scanning transmission electron microscopy (STEM). Figure 4.1 (Frames B andC) show the TEM image of TPPS Auo.9nm and THPC Au1.6nm, respectively (STEM image of TPPS Au0.9nm and THPC $\mathrm{Au}_{1.6 n m}$ are shown in inset). We used STEM images and image $\mathrm{j}$ software to determine the average size of TPPS Au0.9nm and THPC Au1.6nm NPs. The average sizes were $0.9 \pm 0.2 \mathrm{~nm}$ and $1.6 \pm 0.4 \mathrm{~nm}$, respectively, which is very close to the size reported in the literature. ${ }^{31,47}$ We measured the size of Cit Au4.1nm, Cit Au15nm and Cit Au50nm NPs by SEM as shows in Figures 4.1 (Frames D, E \& F), respectively (inset show the zoom in image of those NPs). 

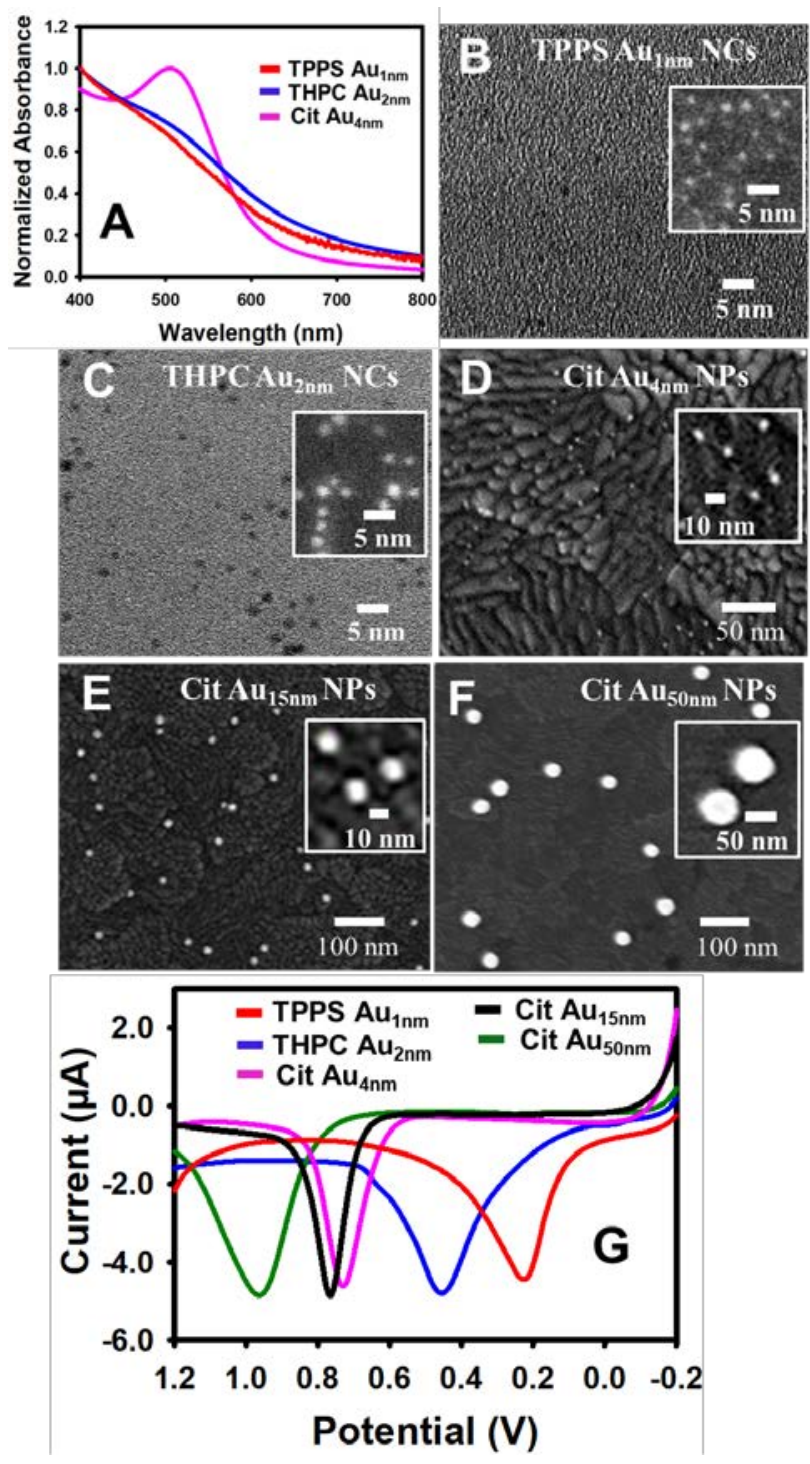

Figure 4. 1. (A) UV-vis spectra of TPPS $A u_{1 n m}$ (red), THPC Au2nm NPs (blue), Cit Au4nm NPs (pink), Cit Au15nm NPs (black) and Cit Au50nm NPs (green). TEM image of TPPS Au1nm and THPC Aunm NPs (B, C). SEM image of Cit Au4nm, Cit Au $u_{15 n m}$ NPs and Cit Au50nm NPs (D, E \&F). ASV of the corresponding NPs (G). ASV obtained in $10 \mathrm{mM} \mathrm{KBr}$ plus $0.1 \mathrm{M} \mathrm{KClO}_{4}$ solution at a scan rate of $0.01 \mathrm{~V} / \mathrm{s}$. 
Next, we monitored the oxidation behavior of these various sized Au NPs by performing $\mathrm{ASV}$ in $\mathrm{Br}^{-}$containing electrolyte solution as described by our group previously. ${ }^{192,199}$ Figure $4.1 \mathrm{G}$ shows the ASVs of glass/ITO/APTES modified with TPPS Auo.9nm (red graph), THPC Au1.6nm (blue graph), and Cit Au4.1nm (pink graph). The ASV of Cit Au5onm (green graph) NPs serves as an estimate of the bulk Au peak oxidation potential $\left(\mathrm{E}_{\mathrm{p}}\right)$. The oxidative stripping of $\mathrm{Au}$ in $0.01 \mathrm{M} \mathrm{KBr}$ plus 0.1 $\mathrm{M} \mathrm{KClO} 4$ solution produces $\mathrm{AuBr}_{4}^{-}$and possibly some $\mathrm{AuBr}_{2}-$ by the following reactions:

$$
\begin{aligned}
& \mathrm{Au}^{0}+4 \mathrm{Br}^{-} \longrightarrow \mathrm{AuBr}_{4}^{-}+3 \mathrm{e}^{-}\left(\mathrm{E}^{0}=0.85 \mathrm{~V} \text { vs. NHE }\right) \\
& \mathrm{Au}^{0}+2 \mathrm{Br} \longrightarrow \mathrm{AuBr}_{2}^{-}+\mathrm{e}^{-}\left(\mathrm{E}^{0}=0.96 \mathrm{~V} \text { vs. NHE }\right)
\end{aligned}
$$

The potential was scanned from $-0.2 \mathrm{~V}$ to $1.2 \mathrm{~V}$ versus an $\mathrm{Ag} / \mathrm{AgCl}$ reference electrode at a scan rate of $0.010 \mathrm{~V} / \mathrm{s}$. The $\mathrm{E}_{\mathrm{p}}$ of Cit Au50nm, Cit Au4.1nm, THPC Au1.6nm and TPPS Auo.9nm were found to be $0.965 \mathrm{~V}$ (green), $0.72 \mathrm{~V}$ (pink), $0.46 \mathrm{~V}$ (blue) and $0.20 \mathrm{~V}$ (red) $\mathrm{vs} \mathrm{Ag} / \mathrm{AgCl}$ respectively. The negative shift in oxidation potential with decreasing size is consistent with the experimental work for $\mathrm{Au},{ }^{64} \mathrm{Ag},{ }^{154}$ and $\mathrm{Pd}^{204}$ NPs and also with the theoretical work of Pleith ${ }^{58}$ and Henglein. ${ }^{205}$ Pleith derived a theoretical equation for the size-dependent oxidation behavior of metal nanocrystals by considering the change in surface free energy, whereas Henglein calculated a negative shift in the oxidation potential of metal nanocrystals with decreasing size based on sublimation energies.

In our previous study, we reported the oxidation behavior of 250-1.6 nm diameter Au NPs. ${ }^{64,199}$ In this study we report the oxidation behavior of atomically precise $0.9 \mathrm{~nm}$ diameter Au11 NPs (or clusters) synthesized by the method reported 
by Yao and coworkers. ${ }^{31}$ They determined the size of TPPS coated Au NPs by electrospray ionization mass spectrometry (ESI-MS) and solution-phase smallangle X-ray scattering (SAXS). According to their results, TPPS coated Au NCs show a primary peak for $\left[\mathrm{Au}_{11}(\mathrm{TPPS})_{9} \mathrm{Cl}\right]^{7-}$ (with $\mathrm{H}+; \mathrm{m} / \mathrm{z}=879$ ) and $\left[\mathrm{Au}_{11}(\mathrm{TPPS})_{7} \mathrm{Cl}_{3}\right]^{7-}(\mathrm{m} / \mathrm{z}=666)$ in ESI-MS, indicating that TPPS coated Au NPS mainly consist of 11 atoms. Also, based on the SAXS, they found the average diameter of these NCs to be $0.8 \mathrm{~nm}$ in diameter, which is also consistent with the ESI-MS results. The ASV of these TPPS Auo.9nm NPs has not been reported previously and is significant here because they are stable in solution and display only one peak near $0.20 \mathrm{~V}$. We calculated the size of the Au NPs based on the oxidation potential of metal NPs (ENP) relative to the oxidation potential of the bulk metal $\left(\mathrm{E}_{\mathrm{B}}\right)$ by the relation derived by Plieth. ${ }^{58}$

$$
\mathrm{E}_{\mathrm{NP}}=\left(-\frac{2 Y V_{m}}{Z F}\right)\left(\frac{2}{d}\right)+\mathrm{E}_{\mathrm{B}}
$$

Here, $\mathrm{Y}$ is the surface stress of $\mathrm{Au}\left(1880 \mathrm{erg} \mathrm{cm}^{-2}\right), V_{m}$ is the molar volume of $\mathrm{Au}$ $\left(10.21 \mathrm{~cm}^{3} \mathrm{~mol}^{-1}\right), \mathrm{Z}$ is the number of electrons, $\mathrm{F}$ is Faraday's constant, and $\mathrm{d}$ is the diameter of the nanocrystal. We used an $E_{в}$ value of $0.980 \mathrm{~V}$ and $Z=1$ (1 electron oxidation of $\mathrm{Au}$ ) to fit the $50 \mathrm{~nm}$ diameter Au NPs the best, which was consistent, but a little higher than our previous bulk value. Using the oxidation potential of $0.2 \mathrm{~V}$ for the TPPS Auo.9nm NPs, equation 1 predicts an average diameter of $1.0 \mathrm{~nm}$, which is very close to the STEM determined size of $0.9 \pm 0.2$ $\mathrm{nm}$ and previously reported SAXS determined size of $0.8 \mathrm{~nm}$. This result shows the potential of ASV to determine the size of metal NPs down to $\sim 1 \mathrm{~nm}$ in diameter. Similarly, equation 1 predicts a size of $1.5 \mathrm{~nm}$ and $3.1 \mathrm{~nm}$ for the THPC Au1.6nm 
and Cit Au4.1nm NPs which is also in fairly good agreement with the TEMdetermined size of $1.6 \pm 0.4$ and $4.1 \mathrm{~nm}$, respectively. As mentioned previously, ASV dramatically underestimates the size of Cit $\mathrm{Au}_{15 \mathrm{~nm}} \mathrm{NPs}$ for reasons that are not clear at this time. 

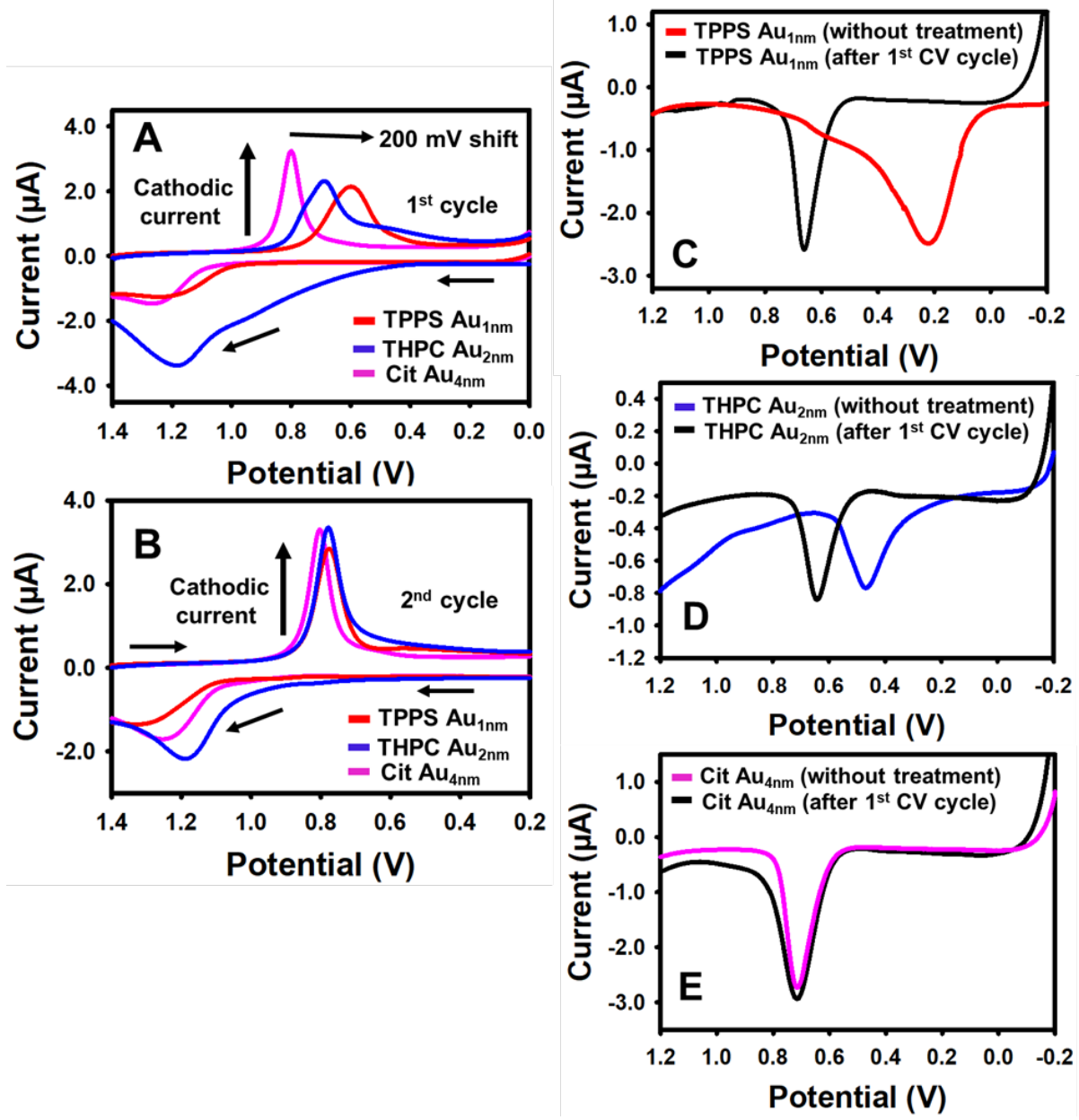

Figure 4. 2. $\mathrm{CVs}$ of TPPS $A u_{1 \mathrm{~nm}}$ (red), THPC $A u_{2 n m}$ (blue) and Cit $A u_{4 n m}$ (pink) diameter Au particles at $1^{\text {st }}$ oxidation-reduction cycle $(A)$ and the same electrode in $2^{\text {nd }}$ oxidation-reduction cycle (B). ASVs of TPPS Au $1 n$ (C), THPC Au $u_{2 n m}(D)$ and Cit Au4nm (E) of NPs before and after $1^{\text {st }}$ CV cycle. CVs were obtained in $0.1 \mathrm{M}$ $\mathrm{HClO}_{4}$ electrolyte solution and $\mathrm{ASV}$ s were obtained in $10 \mathrm{mM} \mathrm{KBr}$ plus $0.1 \mathrm{~m} \mathrm{KClO} 4$ electrolyte solution. 
Next, we studied the reactivity of TPPS Auo.9nm NPs during an electrochemical oxidation-reduction cycle of the $\mathrm{Au}$ in acid electrolyte, which normally forms a surface $\mathrm{Au}$ oxide $\left(\mathrm{Au}_{2} \mathrm{O}_{3}\right)$ as opposed to dissolving the $\mathrm{Au}$. It is interesting to determine how a Au11 cluster will behave in this type of reaction. Figure 4.2 shows the cyclic voltammogram of TPPS Auo.9nm, THPC Au1.6nm and Cit Au4.1nm diameter particles attached to glass/ITO/APTES in $0.1 \mathrm{M} \mathrm{HClO}_{4}$ solution in the potential range of $0.0 \mathrm{~V}$ to $1.4 \mathrm{~V}$ versus $\mathrm{Ag} / \mathrm{AgCl}$ with a scan rate of $0.1 \mathrm{~V} / \mathrm{s}$. In the first scan, the surface $\mathrm{Au}_{2} \mathrm{O}_{3}$ reduction peak for TPPS Au0.9nm, THPC Au1.6nm

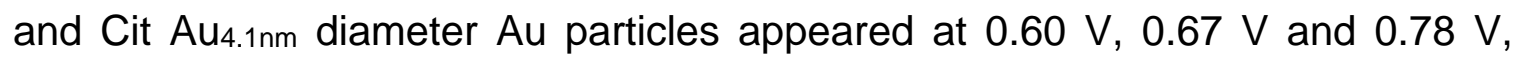
respectively. However, in the second scan the surface $\mathrm{Au}_{2} \mathrm{O}_{3}$ reduction peak shifted to higher potential $(0.78 \mathrm{~V})$ for TPPS $A u_{0.9 n m}$ and THPC $\mathrm{Au}_{1.6 \mathrm{~nm}} \mathrm{NPs}$, indicating that sub $2 \mathrm{~nm}$ diameter Au NPs are not stable during the surface $\mathrm{Au}$ oxidation-reduction cycle. One important point to mention here is that we observed a $180 \mathrm{mV}$ negative shift of the reduction peak potential of TPPS Au0.9nm NPs. In our previous work, ${ }^{199}$ we reported the $110 \mathrm{mV}$ negative shift of the surface $\mathrm{Au}$ reduction peak for THPC Au1.6nm NPs but TPPS Au0.9nm show another $\sim 70 \mathrm{mV}$ more negative shift of the reduction peak due to the smaller size of the Au, which makes it easier to reduce the Au oxide. The negative shift of the surface Au reduction peak has been observed by other groups previously for a single Au NP undergoing an oxidation-reduction cycle, ${ }^{206}$ while others observed a positive shift in potential. ${ }^{176}$ Our results clearly confirm that the surface Au reduction peak shifts negative with decreasing size down to $\sim 1 \mathrm{~nm}$ diameter NPs. The ligand does not seem to be a major factor, but more work is needed to determine the full effect of 
the ligand on this surface oxide formation and reduction reaction. To further confirm our findings, we performed oxidation-reduction cycles in acid on dendrimer coated 1-2 nm diameter Au NPs. Our results show the reduction peak of dendrimer coated 1-2 $\mathrm{nm}$ diameter Au NPs appeared at $0.6 \mathrm{~V} \mathrm{vs} \mathrm{Ag} / \mathrm{AgCl}$ which is also 180 $\mathrm{mV}$ negative of bulk Au NPs (Figure 4.3). Also, during multiple CV cycles the reduction peak shifts to positive potential, indicating that smaller sized Au NPs do not remain stable during these cycles when formed with dendrimers as well.

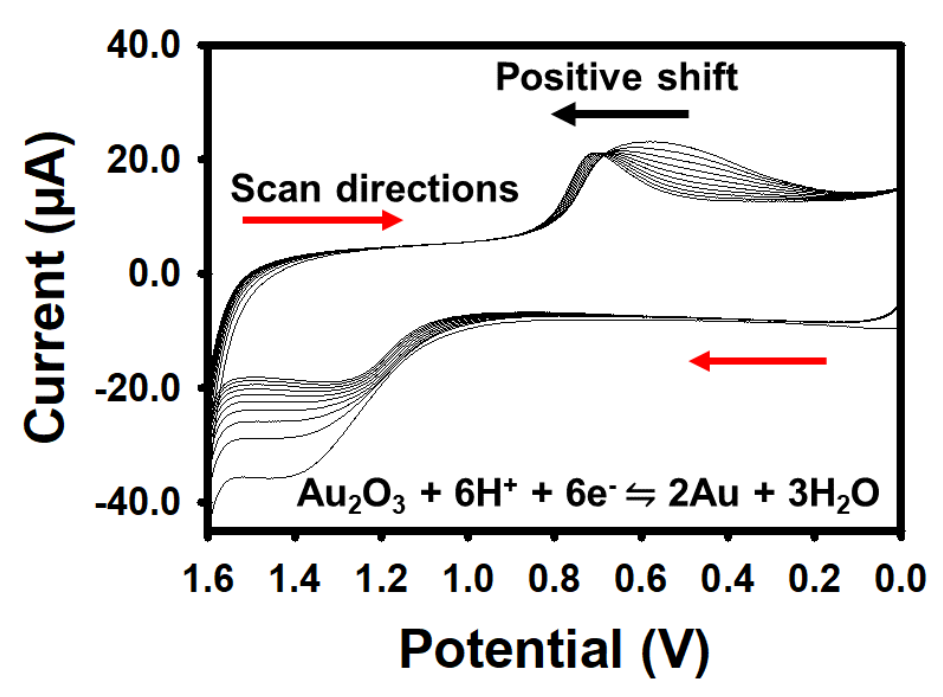

Figure 4. 3. CVs of glass/ITO/APTES attached dendrimer coated 1-2 nm diameter Au NPs. CVs were performed in $0.1 \mathrm{~m} \mathrm{HClO}_{4}$ solution at a scan rate of $0.1 \mathrm{~V} / \mathrm{s}$ with $\mathrm{Ag} / \mathrm{AgCl}$ as a reference and Pt were as a counter electrode. 

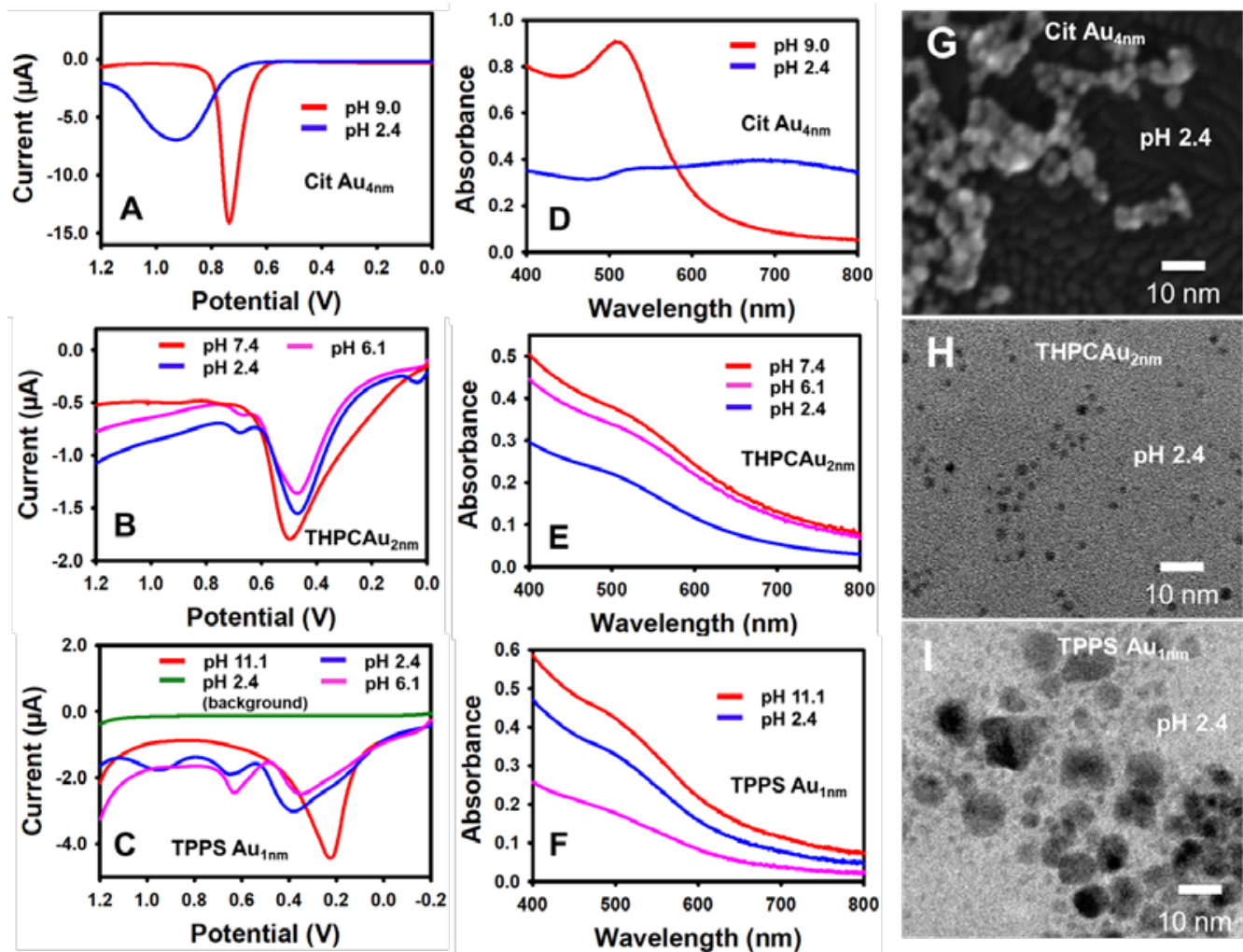

Figure 4. 4. ASV of Cit Au4nm at pH 9.0, 2.4 (A), corresponding UV-Vis spectra (D) and SEM image at $\mathrm{pH}$ 2.4. ASV of THPC $\mathrm{Au}_{2 \mathrm{~nm}}$ at $\mathrm{pH} 11.1,6.1$ and $2.4(\mathrm{~A})$, corresponding UV-vis spectra (E) and TEM image at pH 2.4. ASV of TPPS Au1nm at $\mathrm{pH}$ 11.1, 6.1 and $2.4(\mathrm{~A})$, corresponding UV-vis spectra (F) and TEM image at $\mathrm{pH}$ 2.4. ASVs of Au nanocrystal were obtained in $10 \mathrm{mM} \mathrm{KBr}$ plus $0.1 \mathrm{M} \mathrm{KClO}_{4}$ solution. 
We next studied the pH stability of TPPS Au0.9nm, THPC Au1.6nm, and Cit Au4.1nm. Our group previously studied the pH stability of citrate-stabilized Au NPs with the range of 4 to $50 \mathrm{~nm}$ in diameter. ${ }^{104}$ The ASV of aggregated NPs showed a positively-shifted oxidation peak that depended on the size of the aggregates, reaching as high as $\sim 0.9 \mathrm{~V}$, which is similar to bulk Au oxidation. We did not previously report on the $\mathrm{pH}$ stability of NPs smaller than $2 \mathrm{~nm}$ in diameter or those coated with different stabilizers than the citrate ligands. ${ }^{104}$ In this work we describe the $\mathrm{pH}$ stability of the 1-2 nm diameter Au NPs coated with the two different ligands, THPC and TPPS, as compared to the $4.1 \mathrm{~nm}$ diameter citrate Au NPs. The initial $\mathrm{pH}$ of the $\mathrm{Cit} \mathrm{Au}_{4.1 \mathrm{~nm}}$ is 9.0. At this $\mathrm{pH}$, Cit $\mathrm{Au}_{4.1 \mathrm{~nm}}$ shows only one single oxidation peak at $\sim 0.7 \mathrm{~V}$ (Figure $4.4(\mathrm{~A})$, red graph) but with the decrease of $\mathrm{pH}$ to 2.4 , the Ep shifted to $0.92 \mathrm{~V}$ (Figure 4.4(A), blue graph) due to the aggregation of NPs caused by protonation of the carboxylate groups of the citrate ligand stabilizer, which removes the needed electrostatic repulsion between NPs that keeps them stable in aqueous solution. ${ }^{104}$ The UV-vis spectra of Cit Au4.1nm NPs show a significant decrease in the LSPR band at $505 \mathrm{~nm}$ and a broad absorbance band observed around $700 \mathrm{~nm}$, which is consistent with our ASV results. SEM images of Cit Au4.1nm NPs show clear aggregation (Figure 4.4(G)), which is vastly different than the SEM images of well-isolated Cit Au4.1nm NPs as shown in Figure 4.1(D).

Figure 4.4(B) shows the ASV of THPC Au1.6nm NPs at various $\mathrm{pH}$ values. At the initial $\mathrm{pH}$ of 11.1, the ASV of THPC Au1.6nm NPs shows one single oxidation peak at $E_{p}$ of $0.45 \mathrm{~V}$ as reported by us previously. ${ }^{199}$ There is no significant change in the ASV over the $\mathrm{pH}$ range of 2.4 to 11.1, other than a small shoulder peak at 
$\sim 0.65 \mathrm{~V}$, indicating that THPC Au1.6nm NPs are very stable against aggregation in both acidic and basic aqueous solutions. The stability is likely due to the positivelycharged phosphine ligands, whose charge is not affected by the pH. UV-vis spectra show a slight decrease in the absorbance band with the decrease in $\mathrm{pH}$ (Figure 4.4(E)). Also, TEM images of THPC Au1.6nm at pH 2.4 show that the NPs were clearly dispersed, consistent with our ASV and UV-vis results.

Finally, we studied the pH stability of TPPS Auo.9nm NPs. At pH 7.4, the ASV shows only one single oxidation peak at $\sim 0.2 \mathrm{~V}$ vs $\mathrm{Ag} / \mathrm{AgCl}$. However, at $\mathrm{pH} 6.4$, the ASV consists of multiple oxidation peaks, including those at $0.35 \mathrm{~V}$ and 0.65 $\mathrm{V}$, which corresponds to $\sim 1.3$ and $\sim 3.4 \mathrm{~nm}$ diameter Au NPs based on the Pleith equation. ${ }^{58}$ We believe this indicates partial NP aggregation in solution (dimers, trimers, etc.). At pH 2.4, the ASV of TPPS Au0.9nm shows three distinct oxidation peaks at $0.35 \vee, 0.65 \mathrm{~V}$ and $0.90 \mathrm{~V}$. The peak at $0.9 \mathrm{~V}$ represents larger aggregates of the NPs, leading to an oxidation potential similar to that of bulk. Interestingly, the UV-vis spectra of TPPS Auo.9nm NPs don't show significant changes at the different $\mathrm{pH}$, except for a decrease in the absorbance intensity (Figure 4.4(F)), which is similar to THPC Au1.6nm NPs. Plasmon coupling between small Au NPs does not occur when there is no plasmon band on the initial Au NP, due to the small size. The TEM of TPPS Auo.9nm NPs shows clearly a huge size variation in NP sizes including aggregation to larger size NPs (sizes larger than 10 $\mathrm{nm}$ in diameter) with average size of $3.0 \pm 2.7 \mathrm{~nm}$ in diameter. The aggregation of TPPS Auo.9nm NPs in solution is likely due to the partial protonation of the sulfonate group of the TPPS ligand in acid which causes a decrease in the electrostatic 
repulsion between NPs, leading to partial aggregation in solution. While aggregation of these sub $2 \mathrm{~nm}$ Au NPs cannot be detected by UV-vis absorbance spectroscopy, it can clearly be detected by ASV measurements and confirmed by TEM imaging.

We next studied the stability of the 1-4 nm diameter Au NPs to ozone treatment. Ozone cleaning is a common method researchers use for removing ligands from the surface of the NPs to make them more active for catalysis and other applications. ${ }^{25}$ The goal is to cleanly remove the ligands from the NP surface without altering the NP size and structure, but this is not always successful.
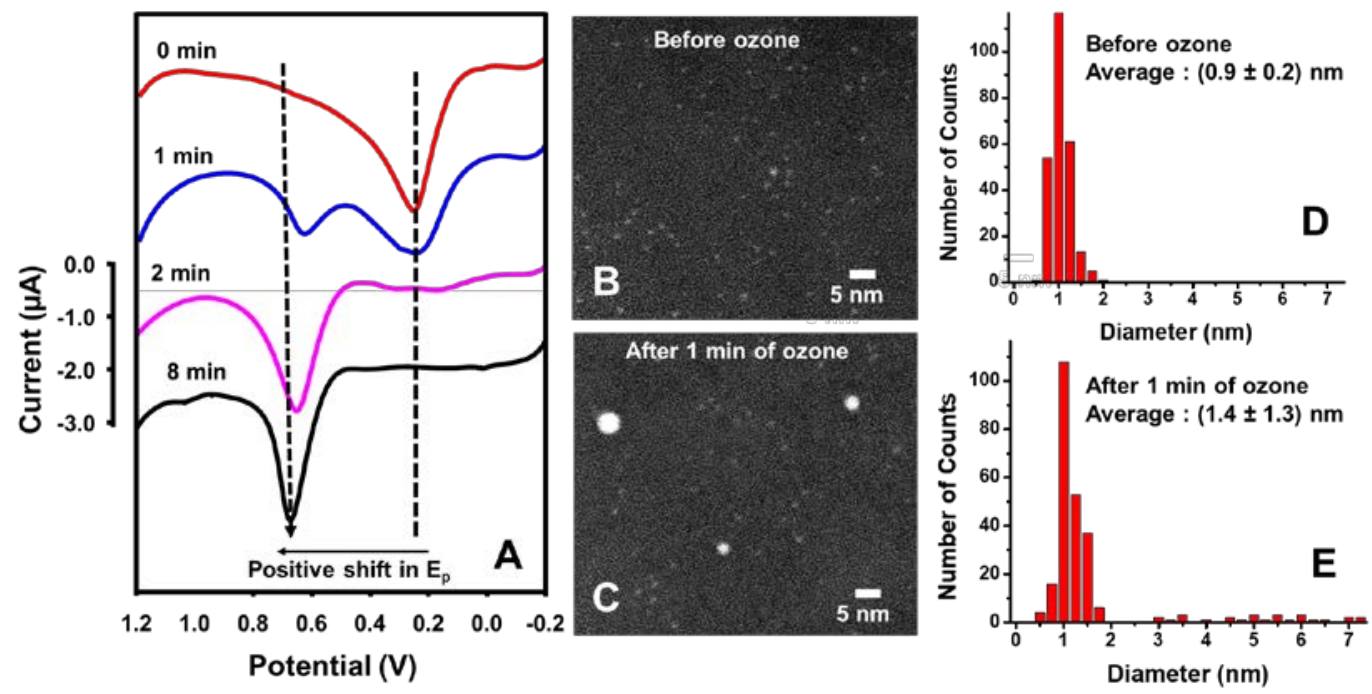

Figure 4. 5. ASVs of glass/ITO/APTES coated TPPS Aunn NCs before and after different time of ozone exposer (A). ASVs were performed in $10 \mathrm{mM} \mathrm{KBr}$ plus 0.1 $\mathrm{M} \mathrm{KClO}_{4}$ electrolyte solution. STEM images of Au particles before (B) and after 1 
min of ozone exposure $(C)$ and corresponding size histogram $D$ (before) and $E$ (after).

For example, Elliot and coworkers reported that $1.4 \mathrm{~nm}$ diameter Au NPs increased in size after 16 min of ozone cleaning, while the size didn't change significantly for up to 8 min of ozone cleaning. ${ }^{25}$ We performed similar experiments on the NPs studied in this work and monitored size changes by ASV. Figure 4.5. (A) shows the ASVs of TPPS Aunm NPs before and after 1, 2 and 8 min of ozone treatment. ASVs of TPPS Au $u_{1 n m}$ NPs before treatment shows a single oxidation peak at $0.2 \mathrm{~V}$ as discussed previously, but after 1 min of ozone exposure two distinct oxidation peaks at $0.2 \mathrm{~V}$ and $0.65 \mathrm{~V}$ appeared in the ASV (Figure 4.5. (A), blue graph). The peak at $0.65 \mathrm{~V}$ corresponds to $4-5 \mathrm{~nm}$ diameter Au particles and is still the minority population after this treatment. After 2 min of ozone, the ASV of TPPS Au1nm NPs shows mostly one single peak at $0.65-0.70 \mathrm{~V}$ with very little shouldering peak at 0.2-0.3 $\mathrm{V}$ region (Figure 4.5. (A), pink graph), indicating that almost all of the NPs converted their size from $1 \mathrm{~nm}$ to $4-5 \mathrm{~nm}$ in diameter by some type of sintering process. After 8 min of ozone, the peak at $0.2 \mathrm{~V}$ completely disappeared with one single peak at $0.7 \vee$ (Figure 4.5. (A), black graph). These findings were also confirmed by STEM imaging of TPPS Aunn NPs before (Figure 4.5. (B)) and after 1 min of ozone exposure (Figure 4.5.(C)). Before exposure to ozone we observed small size NCs with the average size of $0.9 \pm 0.2 \mathrm{~nm}$ and the size histogram show narrow size distribution (figure 4.5.(D)). However, after 1 min of ozone we found significant size variation with the average size of $1.4 \pm 1.3 \mathrm{~nm}$, include $4-5 \mathrm{~nm}$ in 
diameter NPs in the image as a well. The size histogram (figure 4.5.(E)) show wide size distribution of NPs, indicating that NCs are growing in size by Ostwald ripening mechanism. We reported a similar trend for THPC $\mathrm{Au}_{1.6 n m} \mathrm{NCs}$ before and after ozone treatment previously. ${ }^{199}$ Despite the different molecular structure of the stabilizer ligands and different NP size, the results show a similar trend of NP size transformation under the presence of ozone. It seems to be an Ostwald ripening mechanism, but the details are unclear at this time.

We next studied the stability of NPs after exchange of the TPPS ligands and THPC ligands on Au0.9nm and Au1.6nm, respectively, with 1-butanethiol. Thiol place exchange is a common synthetic strategy to replace one type of thiol coating Au NPs with new thiols in order to impart new functionality ${ }^{207-210}$ and it can also be used to completely replace other more weakly-bound ligand stabilizers to improve stability, add functionality, or alter properties, such as solubility. ${ }^{211-212}$ In this work we soaked TPPS Au0.9nm-and THPC Au1.6nm-coated glass/ITO/APTES electrodes in a $2 \mathrm{mM}$ ethanolic solution of 1-butanethiol overnight to determine the ability of the short alkanethiol to replace the TPPS or THPC ligand on these NPs, with the goal of probing potential size and ligand effects on the ability for thiol displacement. ${ }^{213-214}$ We compared the ASV of the Au NPs before and after thiol soaking. Figure 4.6A shows the ASVs of THPC Au1.6nm NPs untreated (red graph), after overnight soaking in 1-butanethiol (pink graph), and after overnight soaking with 1-butanethiol followed by ozone cleaning for $30 \mathrm{~min}$ (blue graph). The $E_{p}$ of untreated THPC Au1.6nm NPs appeared at $0.46 \mathrm{~V}(\mathrm{vs} \mathrm{Ag} / \mathrm{AgCl})$ as before, while there was no well-defined peak following thiol exposure (2 broad, low current 
peaks). We attribute this to the blocking of the Au NP surface by the more strongly bound butane thiolate, which has a strong Au-S bond. A second possibility is that treatment with 1-butanethiol caused removal of the Au NPs physically or chemically, or by becoming soluble.
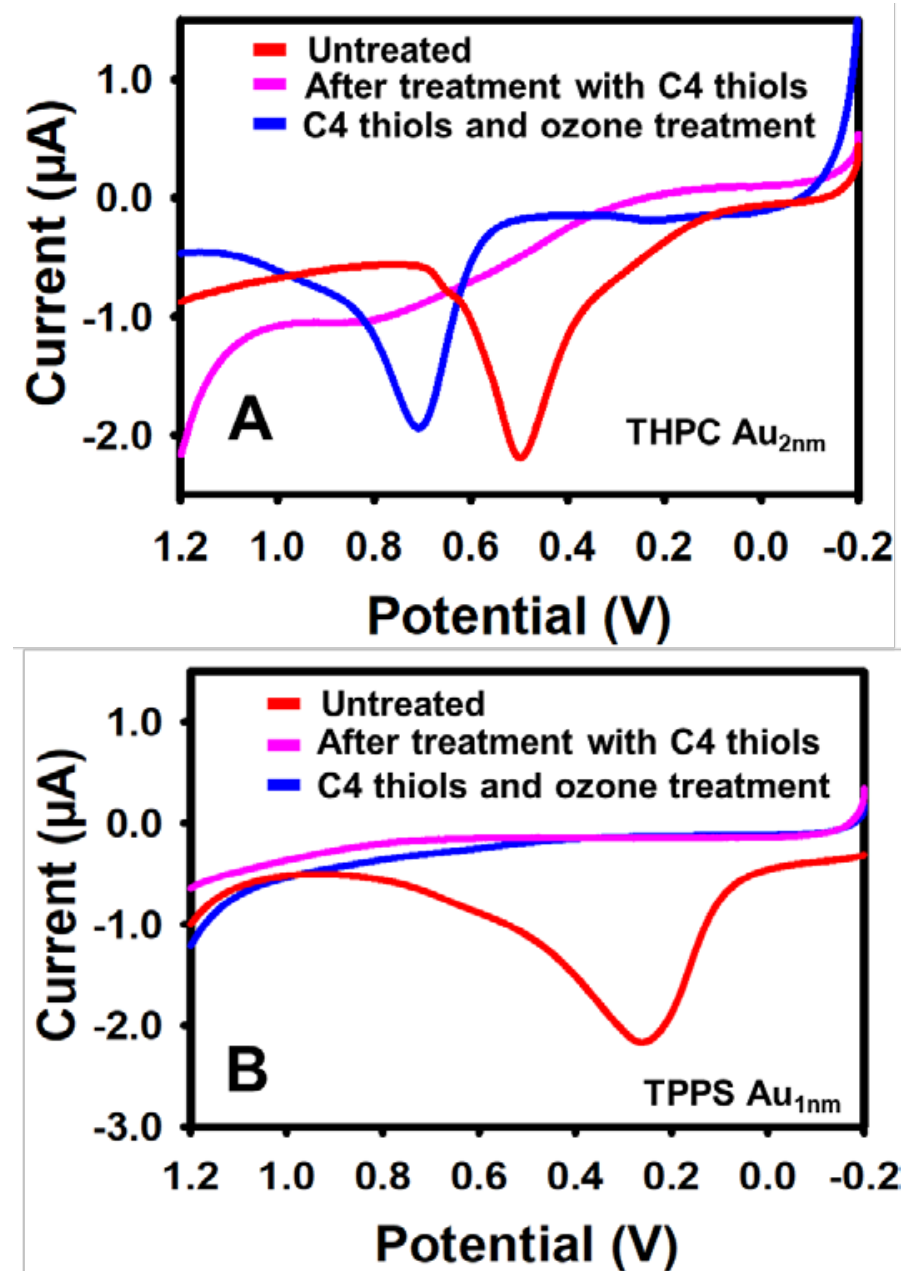

Figure 4. 6. ASV of THPC Au2nm and TPPS Au1nm NCs before (red graph), after treatment with C4 alkane thiols (pink graph) and after treatment with C4 alkane thiols followed by 30 min of ozone cleaning (blue graph). ASV were performed in $10 \mathrm{mM} \mathrm{KBr}$ plus $0.1 \mathrm{~m} \mathrm{KClO}_{4}$ electrolyte solutions. 
To test that, THPC Au1.6nm-coated glass/ITO/APTES was soaked first in 1butanthiol solution overnight, removed, rinsed with ethanol, dried under $\mathrm{N}_{2}$, and finally treated with $30 \mathrm{~min}$ of ozone cleaning in order to remove the 1-butanethiol. If the Au NPs remained on the surface, then we should observe the Au peak in the ASV after ozone cleaning. Figure 4.6.(A) (blue plot) shows the ASV after this treatment. The peak for Au oxidation appeared after ozone cleaning, indicating that the Au NPs remained on the surface after the 1-butanethiol soaking. The Ep for the peak was at $0.7 \vee$ due to the size increase caused by ozone. Ozone both removed the 1-butanethiol and increased the Au NP size. We performed the same set of experiments on the TPPS Au1nm NPs. In this case, the $E_{p}$ for Au oxidation was at $0.2 \mathrm{~V}$ for as-prepared Au NPs, while after treatment with 1butanethiol there is no noticeable peak observed (figure 4.6. (B). Following ozone cleaning of separate sample first treated with 1-butanethiol, there was also not peak observed in the ASV. This indicates that replacement of TPSS with 1butanethiol leads to removal of the Au NPs from the glass/ITO/APTES surface. This could be due to dissolution of Au as Au-thiolates or removal of the Au NPs through solubility in the solution. The different behavior for TPPS Au1nm versus THPC Au1.6nm is likely due to the Au NP size rather than the nature of the ligand. 

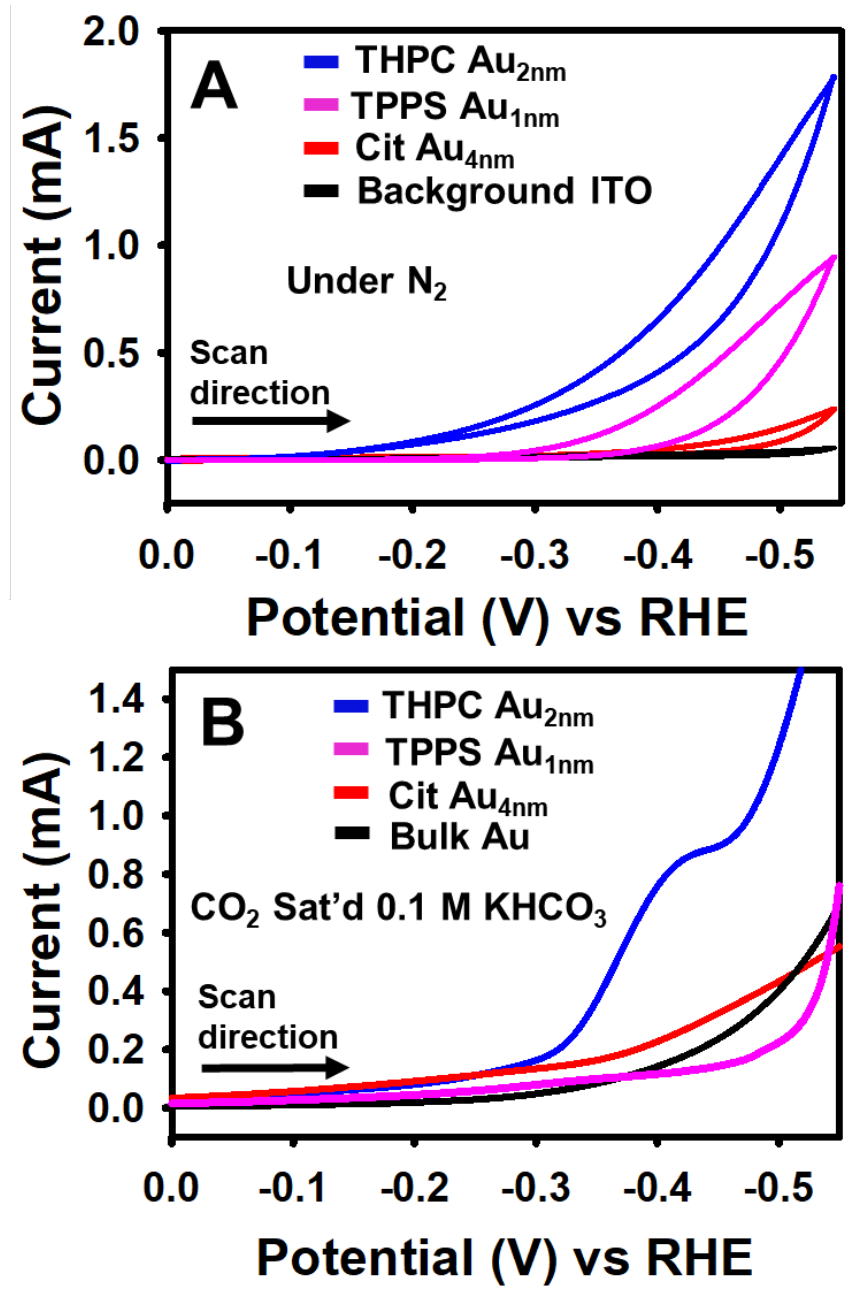

Figure 4. 7. (A) CVs of glass/ITO/APTES attached THPC Auznm (blue graph), TPPS Au1nm (pink graph), Cit Au4nm (red graph) and glass/ITO/APTES in $\mathrm{N}_{2}$ saturated $0.1 \mathrm{M} \mathrm{HClO}_{4}$ solution with a scan rate of $0.01 \mathrm{~V} / \mathrm{s}$. (B) Linear sweep voltammograms (LSVs) of glass/ITO/APTES electrodes attached THPC $\mathrm{Au}_{2 n m} \mathrm{NPs}$ (blue graph), TPPS Au1nm (pink graph) and Cit Au4nm NPs (blue) in $\mathrm{CO}_{2-}$ saturated $0.1 \mathrm{M} \mathrm{KHCO}_{3}$ solution. 
We studied the TPPS Auo.9nm NPs as a catalyst for the hydrogen evolution reaction (HER) and $\mathrm{CO}_{2}$ reduction reaction and compared those with THPC Au1.6nm and $\mathrm{Cit} \mathrm{Au}_{4.1 \mathrm{~nm}} \mathrm{NPs}$. $\mathrm{CO}_{2}$ reduction and $\mathrm{HER}$ was performed in $\mathrm{CO}_{2}$-saturated 0.1 $\mathrm{M} \mathrm{KHCO} 3\left(\mathrm{pH}\right.$ 6.8) and $\mathrm{N}_{2}$-saturated $0.1 \mathrm{M} \mathrm{HClO}_{4}$ electrolyte solution, respectively. It is well known that small-sized Au NPs are good catalysts for HER in acidic media. ${ }^{215-216}$ Back and coworkers reported that small-sized Au NPs are effective toward HER due to an increase in the number of edge and corner sites based on density functional theory (DFT) calculations. ${ }^{217}$ The edge sites are more active for the HER and corner sites are more favorable towards $\mathrm{CO}_{2}$ reduction. Recently, we also reported that THPC Au1.6nm NPs are good catalysts for the HER in acidic electrolyte. ${ }^{199}$ Figure 4.7 shows $\mathrm{CVs}$ in $0.1 \mathrm{M} \mathrm{HClO}_{4}$ of TPPS Auo.9nm NPs (red graph) and THPC Au1.6nm (blue graph) NPs attached to glass/ITO/APTES. The onset potential for the HER was found to be -0.1 to $-0.2 \mathrm{~V}$ vs RHE for THPC Au1.6nm and -0.25 to $-0.30 \mathrm{~V}$ vs RHE for TPPS Auo.9nm NPs, indicating that it is relatively less catalytic for HER compared to THPC Au1.6nm. We also studied $\mathrm{CO}_{2}$ reduction with TPPS Auo.9nm in a $\mathrm{CO}_{2}$ saturated $0.1 \mathrm{M} \mathrm{KHCO}_{3}$ solution and compared to that with THPC Au1.6nm and Cit Au4.1nm NPs. THPC Au1.6nm NPs show a broad peak at $-0.4 \mathrm{~V}$ vs RHE as reported by us in our recent work. ${ }^{199}$ TPPS Auo.9nm and Cit Au4.1nm NPs do not show any peak in the CVs, indicating that both of these particles are not catalytic for the $\mathrm{CO}_{2}$ reduction reaction. There are several examples in the literature of a NP actually losing its catalytic activity below a certain size. For example, Bard and coworkers recently showed that the catalytic activity of Pt NPs for the HER increased with increasing Pt NP size from $0.25 \mathrm{~nm}$ 
to $0.75 \mathrm{~nm}$, indicating that there is an optimum size of NPs for their specific catalytic applications. ${ }^{9}$ Our results indicate that the HER is favored with THPC Au1.6nm NPs and Cit Au4.1nm NPs but not favored for TPPS Au0.9nm NPs. The lower reactivity of TPPS Au0.9nm NPs for the HER could be due to the zero number of edge site ${ }^{218}$ type of atoms, which is the main active site for catalytic HER with Au NPs ${ }^{217}$ and also could be due to the instability ${ }^{9}$ of NPs during applications.

\subsection{CONCLUSIONS}

In conclusion, we have described the electrooxidation and size stability of TPPS-coated Auo.9nm NPs under a variety of conditions and compared those results with THPC $\mathrm{Au}_{1.6 \mathrm{~nm}}$ NPs and Cit Au4.1nm NPs. We found that TPPS Au0.9nm NCs oxidized at $0.20 \mathrm{~V}$ as compared to $0.46 \mathrm{~V}, 0.70 \mathrm{~V}$, and $0.97 \mathrm{~V}$ for THPC $\mathrm{Au}_{1.6 \mathrm{~nm}}$, Cit Au4.1nm, and bulk Au, respectively. TPPS Auo.9nm NPs and THPC Au1.6nm have very low size stability towards electrochemical surface $\mathrm{Au}$ oxidation/reduction cycles in acid electrolyte, converting to $4 \mathrm{~nm}$ or larger-sized $\mathrm{Au}$ NPs after just one oxidation/reduction cycle. Also, TPPS Au0.9nm NPs are not stable in acidic environment, possibly due to the protonation of the TPPS ligand, whereas the positively-charged THPC $\mathrm{Au}_{1.6 \mathrm{~nm}} \mathrm{NPs}$ are stable in both acidic and basic environments. TPPS Au0.9nm NPs increase in size after just 1 min of ozone treatment, similar to THPC Au1.6nm NPs. Also, TPPS Au0.9nm NPs either dissolved completely in solution or were somehow removed from the electrode surface during replacement of their ligands with 1-butanethiol by simple soaking. On the 
other hand, the oxidation peak potential was largely blocked for THPC Au1.6nm NPs following soaking with 1-butanethiol. With THPC Au1.6nm NPs, the butanethiol strongly adsorbs to the Au NP surface and passivates it from oxidation in $\mathrm{KBr}$. With TPPS Auo.9nm NPs, the assembly of 1-butanethiol causes the Au NPs to be removed from the surface somehow, which we believe is due to dissolution of the Au. THPC $A u_{1.6 n m} N P s$ are more reactive for $\mathrm{HER}$ and $\mathrm{CO}_{2}$ reduction reactions as compared to TPPS Auo.9nm NPs, which is likely due to the $1.6 \mathrm{~nm}$ diameter size having a large number of active corner and vertex sites, whereas the $0.9 \mathrm{~nm}$ diameter size has a completely different NP structure and electronic structure due to the much fewer $\mathrm{Au}$ atoms. In that case the active Au atoms no longer exist. Overall, this work demonstrates the effective implementation of ASV for revealing interesting NP size-dependent reactivity and size stability under a variety of conditions common to their treatment and use. 


\section{CHAPTER V}

\section{NANOPARTICLES AGGREGATE STRUCTURE EFFECT THE THERMODYNAMICS OF ELECTROCHEMICAL METAL NANOPARTICLES OXIDATION}

\subsection{INTRODUCTION}

The aggregation of metal nanoparticles (NPs) has been studied for several years, mainly because of the fascinating changes in optical properties upon aggregation of metal NPs. The unique properties of aggregated metal NPs is mainly due to electronic coupling between adjacent particles which leads to many distinct properties of nanostructures. For example, electronic coupling between adjacent particles ( $\mathrm{Au}, \mathrm{Ag}$ ) is widely considered as a hot spot for surface-enhanced Raman spectroscopy (SERS). ${ }^{219-221}$ However, aggregated NPs display a fewer number of surface atoms (SA) as compared to total number of atoms, or volume (V), which is an important parameter to determine the NP stability and reactivity. In general, small metal NPs show higher SA/V value as compared to the bulk metal, which ultimately changes their electronic structure. Usually small metal NPs are highly unstable,,$^{1,68}$ show toxicity, ${ }^{222-223}$ and oxidize, ${ }^{64,200,202-203}$ sinter, $^{25,147}$ or aggregate easily, ${ }^{103}, 105,224$ which causes a change in their reactivity during applications. ${ }^{225}$ Aggregation of NPs is a very common phenomenon which plays 
an important role even in the synthesis of NP structures. It causes the dramatic decrease of surface area and ultimately NPs behave like bulk metal. ${ }^{105}$ Various methods have been developed to synthesize and understand the properties of aggregated NPs. In most cases, aggregation of NPs was achieved by template, ${ }^{226}$ polymer, ${ }^{227}$ pulse laser, ${ }^{228}$ the electron beam of a microscope, ${ }^{229}$ molecular linker, or ligand. ${ }^{230}$ The aggregation of NPs is usually directed by the interparticle attractions, which is typically achieved by intermolecular van der Waals forces, ${ }^{93}$ л-л interactions, ${ }^{94}$ dipole-dipole interactions, ${ }^{95}$ or hydrogen bonding.${ }^{96}$

Aggregation has mainly been studied by optical and imaging methods and is useful for sensing and catalytic applications. For example, Wei and coworkers reported on a photo-switchable hydrosilylation reaction mediated by dynamic aggregation of Au NPs. ${ }^{97}$ They observed that upon irradiation with UV light $\mathrm{Au}$ NPs aggregate and catalytic reactivity decreases, but upon irradiation with visible light catalytic reactivity increased due to the NPs becoming isolated. This aggregation of NPs was monitored by dynamic light scattering (DLS) and TEM imaging. Others used aggregated NPs for electrocatalytic oxidation of methanol, ${ }^{98}$ glucose ${ }^{99}$ and ethanol. ${ }^{100}$ For example, Wen and coworkers showed that electrocatalytic glucose oxidation reactivity increased for a dopamine induced, aggregated 5-6 nm diameter Au aerogel synthesized with beta-cyclodexrin as compared to citrate stabilized isolated NPs of similar size. ${ }^{99}$ The increased reactivity was thought to be caused by the increase of porosity in aggregated NPs. However, there are very few studies on the electrochemical monitoring of the aggregation process on metal NPs. Some studies have looked at the effect of 
aggregation on the metal NP oxidation current, ${ }^{101-103}$ but Allen was the first to study the effect of aggregation on peak oxidation potential $\left(E_{p}\right)$. In that work, aggregation of NPs was controlled by $\mathrm{pH}$ and characterized by ASV. It was found that after aggregation, ${ }^{105}$ the Au NP surface area decreases dramatically and they oxidize at larger potentials for small aggregates and the potential of bulk metal for larger aggregates.

In this work we focus on the electrochemical behavior of assembly-aggregated $\mathrm{Au}$ NPs and compare this result with the $\mathrm{pH}$-induced aggregated Au NPs. These studies are important for several reasons. First, aggregated NPs behave like a bulk metal and they oxidize at higher potentials ${ }^{105}$ because they lose surface area after aggregation. For example, the SA/V value of $4 \mathrm{~nm}$ diameter Au NPs decreases by roughly a factor of 7 when they are aggregated as compared to isolated NPs. ${ }^{105}$ The trend of losing surface area is higher for smaller sized NPs as compared to larger sized NPs. However, small sized NPs are more reactive for catalytic applications. Therefore, it is important to know how different types of NP assemblies (or aggregates) change their SA/V value and $E_{p}$. Second, assemblyaggregated NPs will be connected by a molecular linker. Therefore, the distance between the nearest NP will be in the sub-nanometer level. At this distance, NPs should be able to participate in electronic coupling between adjacent particles but not necessarily lose much surface area. It is important to determine if the oxidation potential of NPs is affected by their electronic coupling or change in SA/V. Third, the study of aggregation behavior of NPs by electrochemical methods is interesting fundamentally (related to $S A / V$ ) and could be useful for sensing applications. It 
may be more sensitive than optical and imaging methods and it does not require plasmonic NPs of a certain size.

\subsection{EXPERIMENTAL}

\subsubsection{Aggregation of $15 \mathrm{~nm}$ Average Diameter Au NPs with THPC.}

Aggregation of Au NPs with THPC was obtained by the method reported by Gulka and coworkers. ${ }^{148}$ Briefly, we added $1 \mu \mathrm{L}$ of $100 \mu \mathrm{M}, 400 \mu \mathrm{M}, 600 \mu \mathrm{M}$ and $1 \mathrm{mM}$ of THPC to the $5 \mathrm{~mL}$ aqueous solution of citrate coated $15 \mathrm{~nm}$ diameter Au NPs, which gave Au to THPC ratios of $62.5,31.3,10.4$ and 6.3. Immediately after addition of THPC, the color of the Au NPs changed from red to blue, indicating that the NPs were aggregated in solution. After 5 min of THPC addition, we directly attached aggregated Au NPs to a glass/ITO/APTES electrode by soaking for 3-5 $\min$.

5.2.2. Aggregation of $15 \mathrm{~nm}$ Average Diameter Au NPs with Acid. The $\mathrm{pH}$ of an aqueous solution of $15 \mathrm{~nm}$ diameter Au NPs was lowered by adding 10 $\mu \mathrm{L}$ of $8 \mathrm{M}$ perchloric acid solution. After addition of acid, the NPs began to precipitate at this $\mathrm{pH}$ condition. Stripping voltammetry of the aggregated NPs was obtained of the aggregated Au NPs attached to the glass/ITO/APTES surface. 


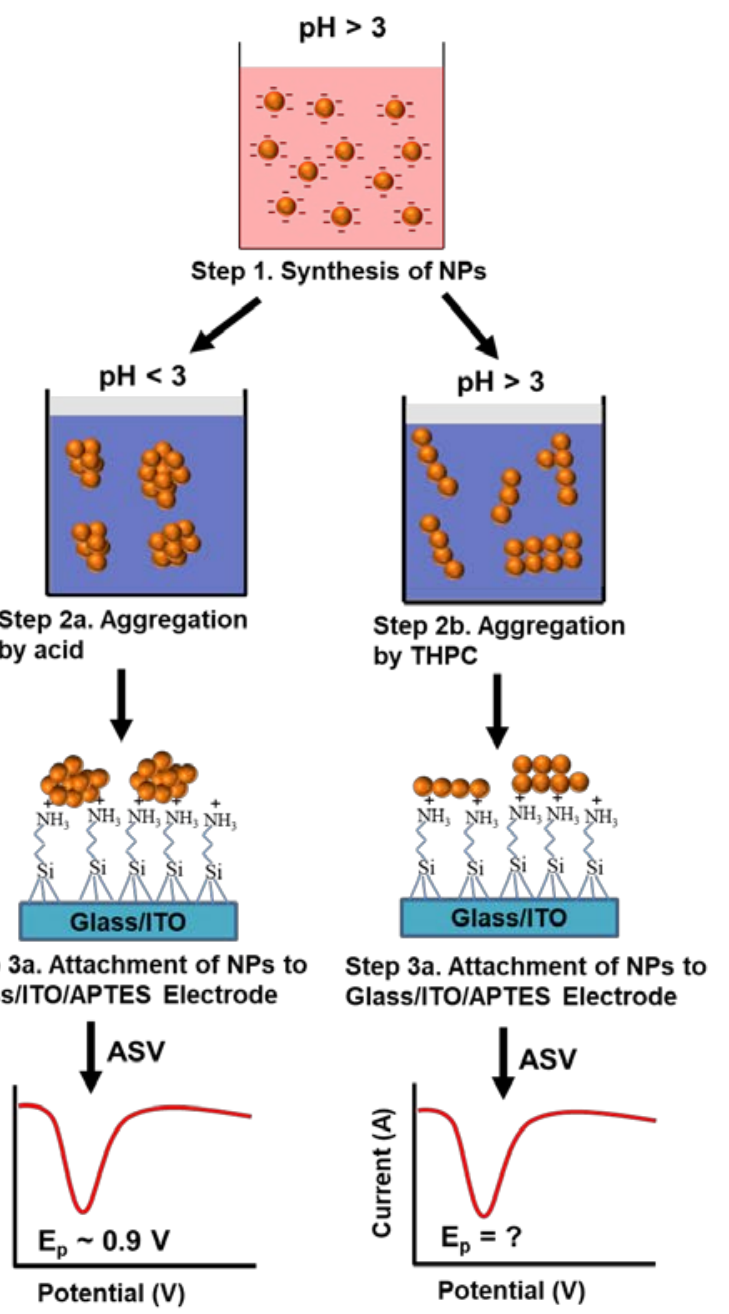

Figure 5. 1. General experimental procedure involved in these studies. 


\subsection{RESULTS AND DISCUSSION}

Figure 5.1 shows the general experimental procedure involved in these studies. Step 1 is the synthesis of citrate-coated $15 \mathrm{~nm}$ dimeter Au NPs. We synthesized $15 \mathrm{~nm}$ diameter Au NPs by the procedure reported by Turkevich ${ }^{40}$ and the initial $\mathrm{pH}$ of the solution was $\sim 5.8$. In step $2 \mathrm{a}$ we lowered the $\mathrm{pH}$ from 5.8 to 2.6 by adding $1 \mu \mathrm{L}$ of concentrated perchloric acid in order to aggregate the NPs as described by Allen et al. previously. ${ }^{105}$ In step $2 \mathrm{~b}$ we added THPC instead of acid in order to get 1D and 2D aggregated assemblies of NPs. ${ }^{148}$ In the case of acid aggregation, the carboxylic acid group of the citrate ligand became protonated, which decreases the electrostatic repulsion between the NPs and ultimately the NPs became aggregated as reported by our group and others. However, in the case of THPC aggregation, it has been shown that THPC rapidly replaces citrate from the Au NPs surface and produces a temporary dipole moment on each NP, which causes the NPs to move into a liner or 2D assemblies. ${ }^{148,231}$ Step 3 is the attachment of aggregated (both acid and THPC) NPs to aminopropyltriethoxysilane-functionalized glass/ITO electrodes (glass/ITO/APTES) by directly soaking the functionalized electrodes in the aggregated (or non-aggregated for comparison) Au NP solutions.

Figure 5.2.(A) shows UV-vis spectra of citrate-coated $15 \mathrm{~nm}$ diameter $\mathrm{Au}$ NPs in an isolated ( $\mathrm{pH} 5.8)$, acid aggregated ( $\mathrm{pH} 2.6)$, and THPC aggregated $(\mathrm{pH}$ 5.8) (Au : THPC $=62.5)$ states. UV-visible spectra were performed directly in the solution. Isolated $15 \mathrm{~nm}$ diameter Au NPs show an LSPR band at $518 \mathrm{~nm}$ (Figure 
5.2.(A), red plot), which is consistent with the LSPR band position of $15 \mathrm{~nm} \mathrm{Au}$ NPs reported previously by our group ${ }^{105}$ and
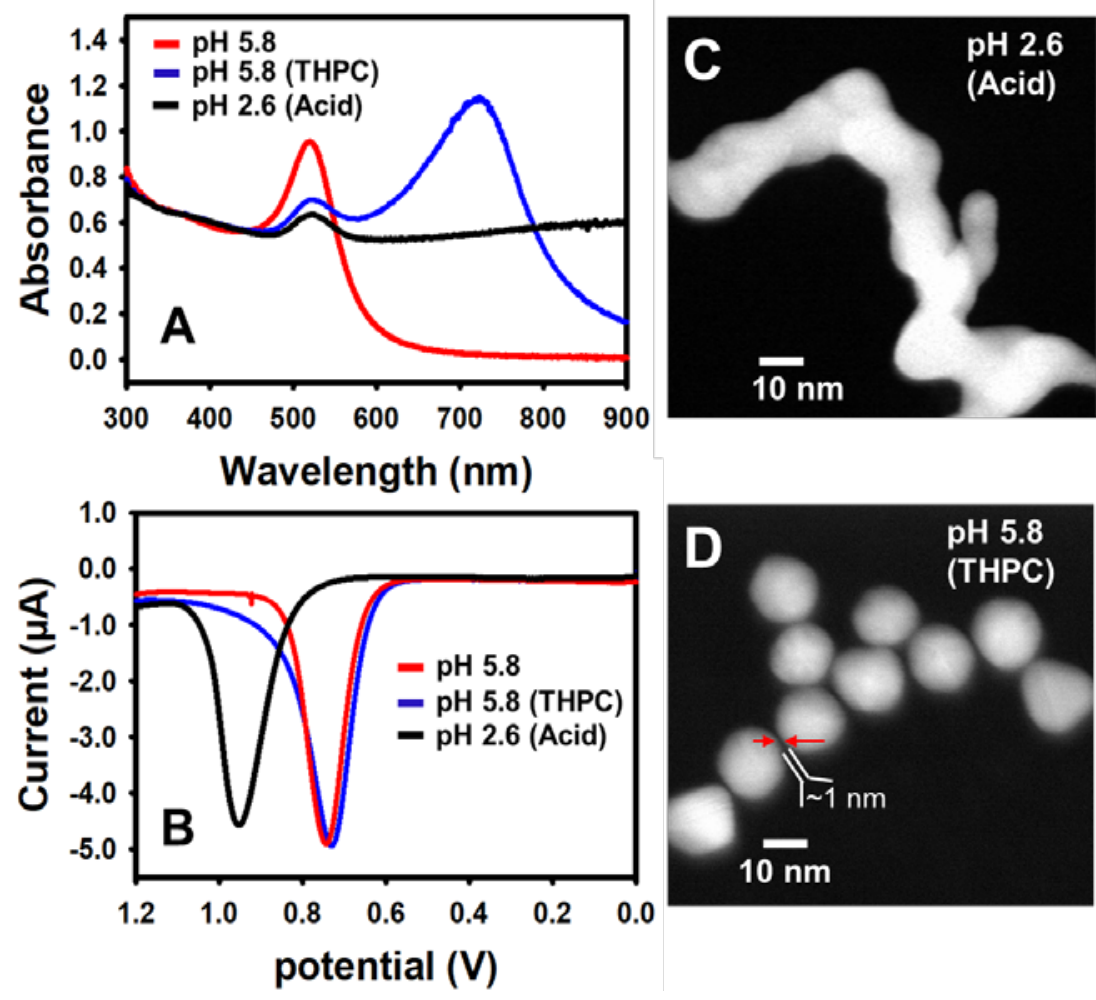

Figure 5. 2. (A) ASV of glass/ITO/APTES electrodes coated with $15 \mathrm{~nm}$ diameter Au NPs attached from solution at pH 5.8 (red graph), THPC aggregated (blue graph) and acid aggregated $\mathrm{pH} 2.6$ (black graph). All this ASV was performed in $0.1 \mathrm{M} \mathrm{KClO}_{4}+0.01 \mathrm{M} \mathrm{KBr}$ from 0.0 to $+1.2 \mathrm{~V}$ at a scan rate of $10 \mathrm{mV} / \mathrm{s}$. (B) UV-vis spectra of $15 \mathrm{~nm}$ diameter Au NP solutions at pH 5.8 (red graph), THPC aggregated (blue graph) and acid aggregated at pH 2.6 (black graph). (C-D) are the STEM images of acid aggregated ( $\mathrm{pH} 2.60$ ) (figure C) and THPC aggregated (pH 5.8) (figure D) respectively. 
others. ${ }^{39}$ ASV of isolated NPs show a single oxidation peak at $0.73 \mathrm{Vvs} \mathrm{Ag} / \mathrm{AgCl}$ (Figure 5.2.(B), red plot), which also supports the results obtained from the UV-vis spectra. We also obtained scanning electron microscope (SEM) image of those isolated NPs on the glass/ITO/APTES electrode and found that NPs were isolated as showed in Figure 5.3. However, after addition of acid ( $\mathrm{pH} 2.6)$ the solution color change red to blue immediately as shown in figure 5.4 The UV-vis spectra shows a slight red-shift from 518 to $523 \mathrm{~nm}$ (Figure 5.2.(A), black plot) and a significant increase of the baseline absorbance value at higher wavelengths as reported by our group previously. ${ }^{105}$ This indicates that NPs were aggregated in the solution. The ASV of acid-aggregated NPs showed the $E_{p, o x}$ at $0.9 \mathrm{~V}$ (Figure 5.2.(B), black plot), indicating that the NPs were aggregated in solution, consistent with the UVvis spectra (Figure 5.2.(A), black plot). The corresponding STEM image shows clear 3D aggregation and fusion of NPs after addition of acid as show in Figure 5.2.(C) (SEM image of pH-aggregated NPs are shown in Figure 5.3.B). We then analyzed THPC-aggregated NPs by obtaining UV-vis spectra, ASV, and STEM images. After the addition of THPC, the color of the solution immediately changes from red to blue as shown in Figure 5.4. The LSPR band at $518 \mathrm{~nm}$ decreased significantly and a new plasmon band appears at $710 \mathrm{~nm}$ (Figure 5.2.(A), blue plot). The color of the solution turned to a blue/purple color immediately, which indicates that the NPs were aggregated in the solution. Interestingly, the ASV of THPC-aggregated NPs showed a single oxidation peak at $0.73 \mathrm{~V}$ (Figure 5.2.(B), blue plot), identical to the ASV of isolated Au NPs. This was puzzling since we expected the $\mathrm{E}_{\mathrm{p}, \mathrm{ox}}$ in increase upon aggregation as with the $\mathrm{pH}$-induced 

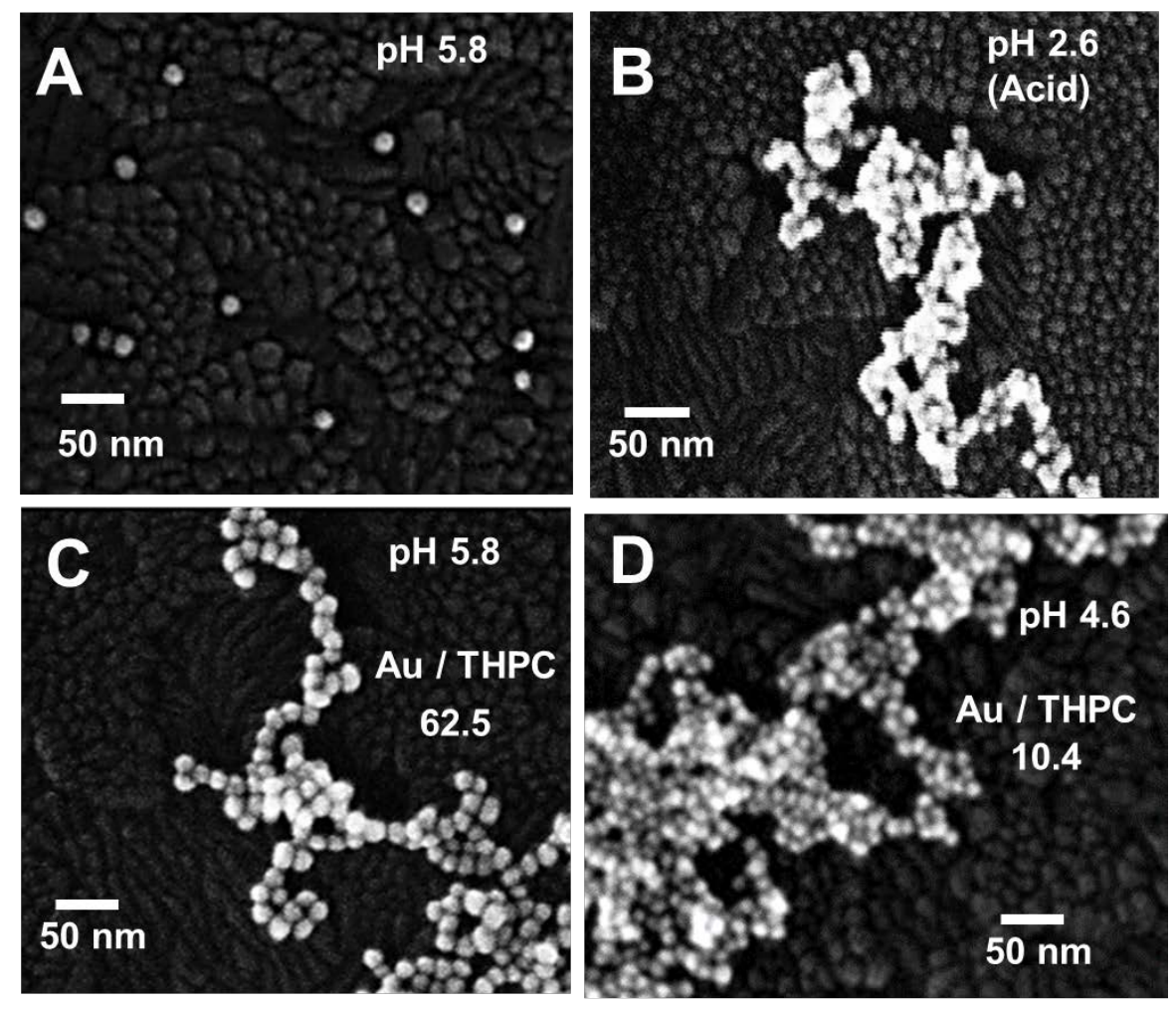

Figure 5. 3. SEM image of glass/ITO/attached Au NPs (A) isolated, (B) acid aggregated, (C) THPC aggregated (Au to THPC 62.5) and (D) higher amount of THPC (Au to THPC 10.4) $15 \mathrm{~nm}$ diameter Au NPs.

aggregated NPs. We then obtained an STEM image of the THPC-aggregated NPS (Figure 5.2.(D)), which revealed a very different aggregated structure compared to the $\mathrm{pH}$-aggregated NPs. The NPs remained spaced out and there was no apparent fusion of the NPs. This is interesting since the Au NPs are close enough to exhibit strong plasmon coupling, which altered the UV-vis spectrum, but they are not close enough to alter the $E_{p, o x}$, which depends on a change in the surface 
area-to-volume ratio (SA/V). The SA/V is not significantly altered by aggregation due to the open space between the NPs.

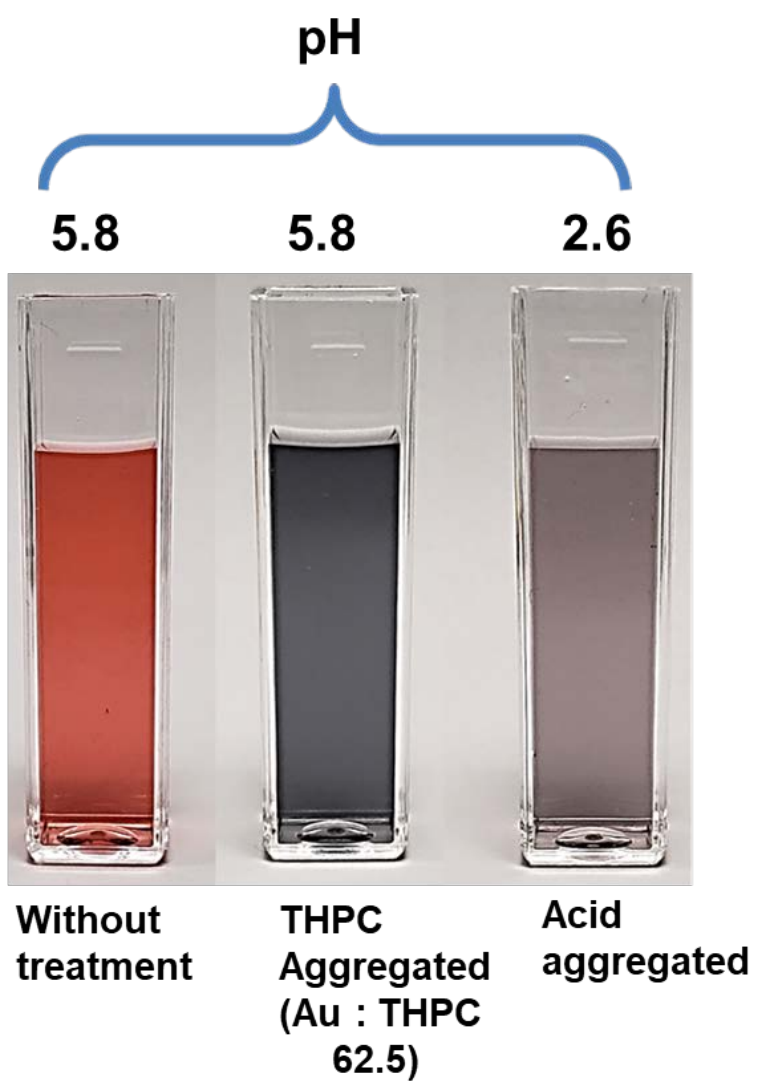

Figure 5. 4. Pictures of solution of isolated ( $\mathrm{pH} 5.8)$, THPC aggregated ( $\mathrm{pH} 5.8)$ and acid aggregated (pH 2.6) $15 \mathrm{~nm}$ diameter Au NPs.

To confirm our belief that the $E_{p, o x}$ remained the same due to no change in SA/V of the NPs after aggregation, we performed electrochemical SA/V measurements as reported by our group previously for the size analysis of isolated Au NPs ${ }^{201}$ and aggregated NPs. ${ }^{105}$ The SA was obtained by performing cyclic voltammetry (CV) 
of Au NPs attached to glass/ITO/APTES in $0.1 \mathrm{M} \mathrm{HClO}_{4}$ solution and the total volume of the same electrode was obtained from ASV in a $\mathrm{Br}^{-}$containing $\mathrm{KClO}_{4}$ electrolyte solution. Figure 5.5.(B) shows the ASVs of isolated, THPC aggregated, and acid aggregated $15 \mathrm{~nm}$ diameter Au NPs having coverage of $2.03 \times 10^{-5} \mathrm{C}$, $2.31 \times 10^{-5} \mathrm{C}$, and $2.25 \times 10^{-5} \mathrm{C}$, respectively. Despite having similar ASV coverages, their SA was found to be $9.42 \times 10^{-6} \mathrm{C}, 8.66 \times 10^{-6} \mathrm{C}$, and $1.19 \times 10^{-6}$ $\mathrm{C}$ for the isolated, THPC-aggregated, and $\mathrm{pH}$-aggregated NPs respectively (figure 5.5.(A)). The average SA/V ratio of isolated, THPC-aggregated, and $\mathrm{pH}-$ aggregated NPs from 3 samples was $0.40 \pm 0.04,0.36 \pm 0.02$ and $0.046 \pm 0.005$, respectively. The measured geometric size considering that the diameter of a sphere is equal to $6 \mathrm{~V} / \mathrm{SA}$ corresponds to a diameter of $15.0 \pm 1.4 \mathrm{~nm}, 16.9 \pm 1.0$ $\mathrm{nm}$, and $133 \pm 15 \mathrm{~nm}$, respectively. This result indicates that THPC-aggregated NPs barely lose their electroactive surface area while $\mathrm{pH}$-aggregated NPs drop by almost a factor of 10 . The dramatic decrease in $S A / V$ is the reason for the large shift in $E_{p, o x}$ for the $\mathrm{pH}$-aggregated NPs. The THPC-aggregated NPs only show a slight shouldering in the oxidation peak from 0.8 to $0.95 \mathrm{~V}$ (Figure 5.5.(B), blue plot) since the SA/V only changes minimally. The large decrease in SA/V for $\mathrm{pH}$ aggregated NPs is likely due to the 3D nature of the aggregates and apparent fusion of the NPs in the STEM images. In contrast, the THPC-aggregated NPS were aggregated low dimensionally (1D and 2D) with significant spacing between the NPs. It is remarkable that the ASV can distinguish between these different types of aggregation, showing the sensitivity of ASV to not only NPs size and aggregation, but specific aggregation structure. 

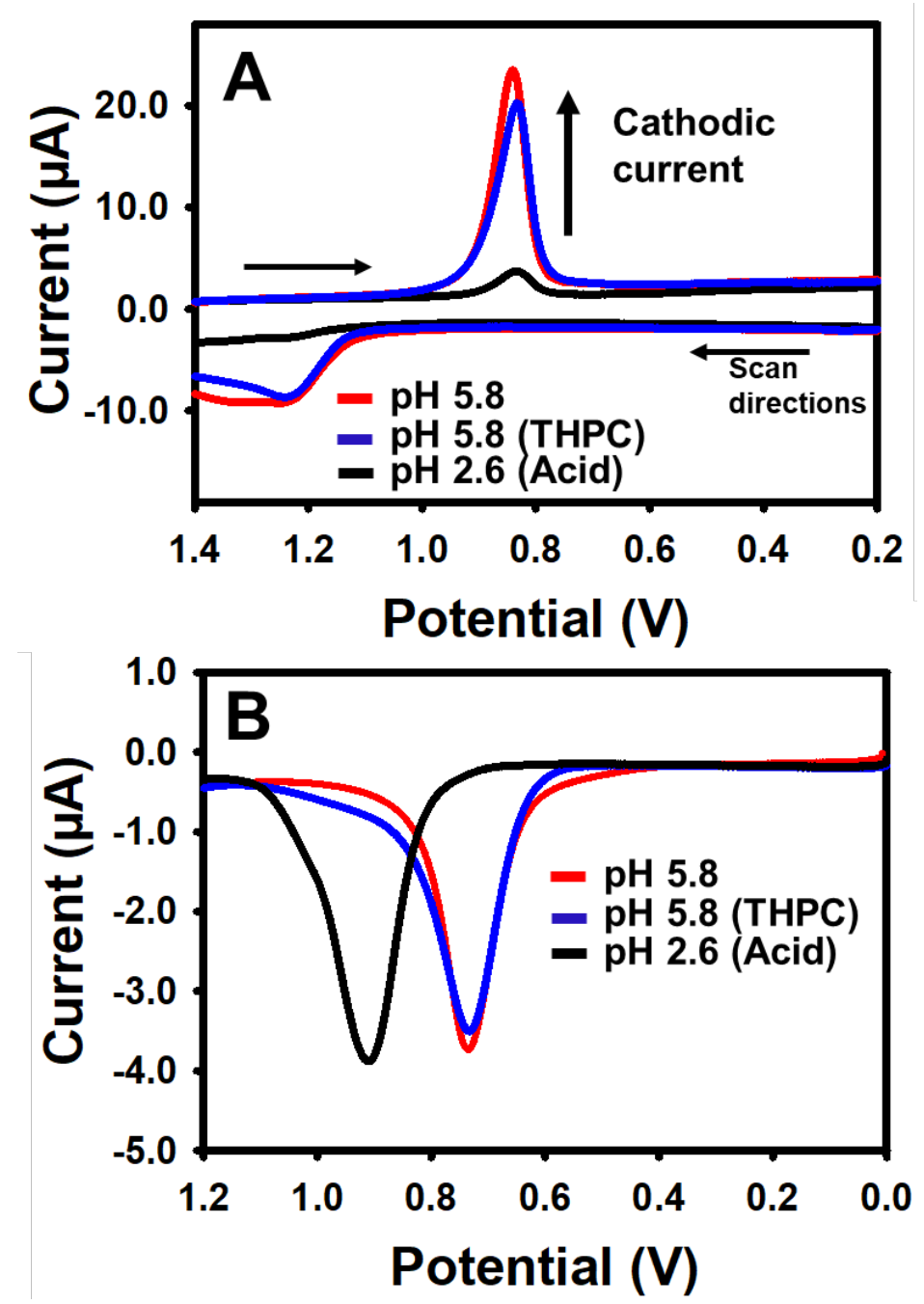

Figure 5. 5. (A) CVs of glass/ITO/APTES electrodes coated with $15 \mathrm{~nm}$ diameter Au NPs attached from solution at pH 5.8 (red graph), THPC aggregated (blue graph) and acid aggregated pH 2.6 (black graph). CVs was performed in $0.1 \mathrm{M}$ $\mathrm{HClO}_{4}$ solution. (B) ASV of the same electrode after CV cycle. ASV was performed in $0.1 \mathrm{M} \mathrm{KClO}_{4}+0.01 \mathrm{M} \mathrm{KBr}$ from 0.0 to $+1.2 \mathrm{~V}$ at a scan rate of $10 \mathrm{mV} / \mathrm{s}$. 
We also studied the effect of higher THPC concentration on the $E_{p, o x}$ and the structure of the NP aggregates. Figure 5.6.(A) shows the UV-vis spectra of Au NPs with Au:THPC ratios of $62.5,31.3,10.4$, and 6.3. The results show minimal change in the LSPR band position with increasing THPC concentration by up to a factor of 10. The absorbance at $710 \mathrm{~nm}$ slightly decreased, indicating the potential start of NP precipitation, but is otherwise very similar. We also obtained ASVs of glass/ITO/APTES containing the Au NPs with the same THPC : Au ratios used in the UV-vis study. With ASV, the $E_{p, o x}$ shifted by $\sim 150 \mathrm{mV}$ from the highest to lowest ratio. The $E_{p, o x}$ values for the various ratios were $0.733 \pm 0.002 \mathrm{~V}, 0.781 \pm 0.010$ $\mathrm{V}, 0.819 \pm 0.010 \mathrm{~V}$, and $0.882 \pm 0.005 \mathrm{~V}$ vs. $\mathrm{Ag} / \mathrm{AgCl}$ for the $\mathrm{Au}: \mathrm{THPC}$ ratios of 62.5, 31.310 .4 and 6.3, respectively, as show in Figure 5.6.(B). The positive shift in oxidation potential indicates that the NPs were aggregated in a similar fashion to the 3D assembly with possible NP fusion for the higher amounts of THPC. In order to compare their SA/V ratios, we compared the SA values for samples that had a very similar ASV coverage as shown in Figure 5.6.(C). The electroactive surface area was found to decrease with a decrease in Au : THPC ratio. The SA/V ratio was found to be $0.36 \pm 0.02,0.19 \pm 0.01,0.15 \pm 0.02$ and $0.11 \pm 0.01$ for $\mathrm{Au}$ : THPC ratios of $62.5,31.3,10.4$, and 6.3 ratios, respectively. This finding shows that the NPs aggregated into a 3D assembly with some fusion of NPs as the THPC concentration increased. The ASV signature correlates very well with the STEM images of aggregated NPs. For Au : THPC ratio of 62.5 , we can clearly see NPs were aggregated into 1D/2D structures and with clear gaps between the NPs (Figure 5.6.(D)). However, for Au : THPC ratio of 6.3, most of the NPs were 
assembled into 3D structures as shown by the bright regions and were also interconnected, or fused, as shown by the arrows (Figure 5.6.(E)). It is not clear at this time why the larger amount of THPC leads to interconnected, or fused, Au NPs. The interconnection leads to a decrease in electroactive surface area and more positive $\mathrm{E}_{\mathrm{p}, \mathrm{ox}}$ values, similar to $\mathrm{pH}$-aggregated Au NPs.
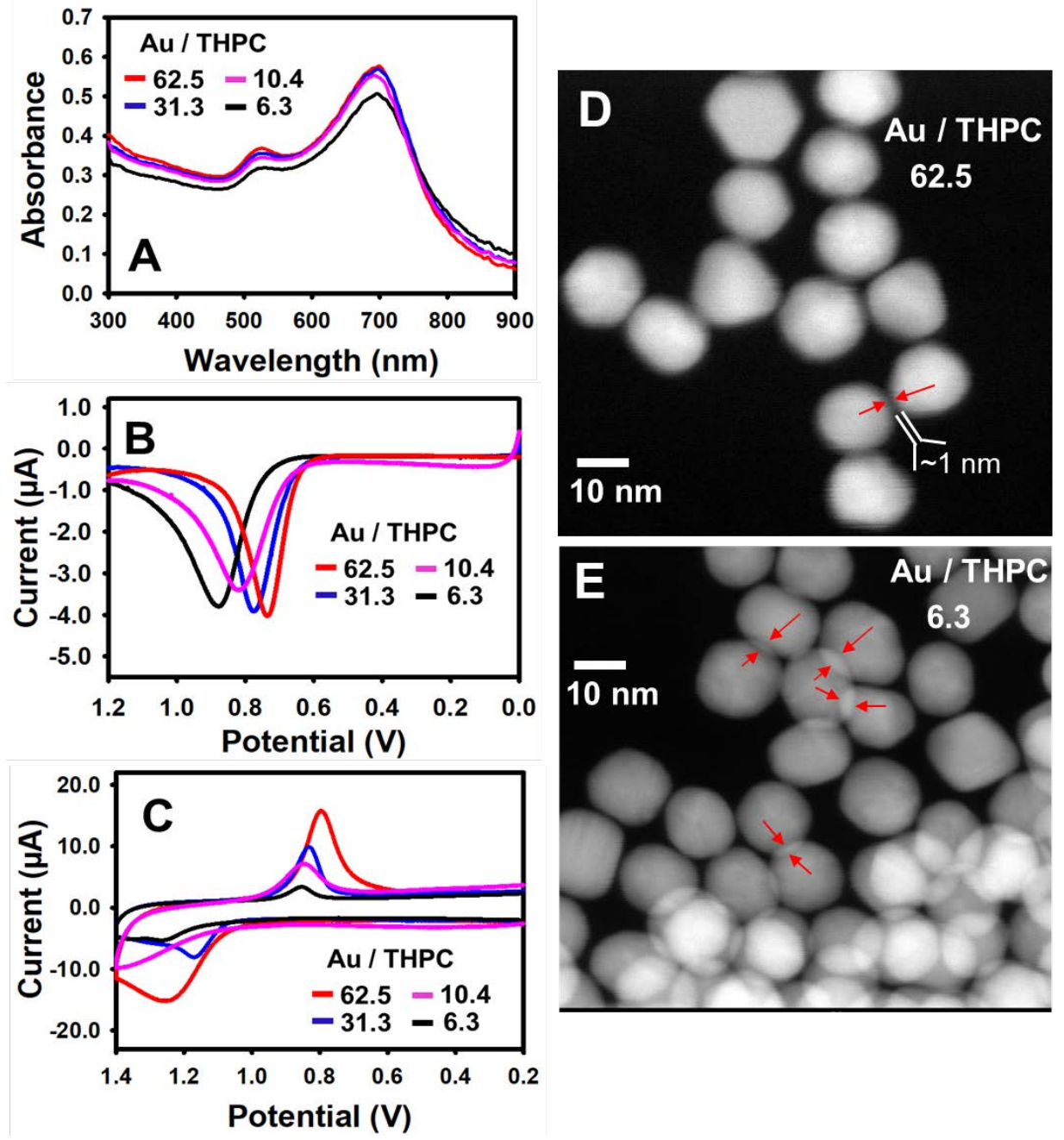

Figure 5. 6. (A) UV-vis spectra of $15 \mathrm{~nm}$ diameter Au NP solutions aggregated with various amount of Au : THPC ratios of 62.5 (red graph), 31.3 (blue graph) 10.4 
(pink graph) and 6.3 (black graph). (B) CVs of the corresponding aggregated Au NPs. CVs were performed in $0.1 \mathrm{M} \mathrm{HClO}_{4}$ solutions. (C) ASVs of corresponding aggregated Au NPs performed in $0.1 \mathrm{M} \mathrm{KClO}_{4}+0.01 \mathrm{M} \mathrm{KBr}$ from 0.0 to $+1.2 \mathrm{~V}$ at a scan rate of $10 \mathrm{mV} / \mathrm{s}$. (D,E) are the STEM images of THPC-aggregated NPs with the Au : THPC ratios of 62.5 and 6.3 , respectively.

We also measured the surface charge of NPs by monitoring the zeta potential of isolated NPs, pH-aggregated NPs, and THPC-aggregated Au NPs with various Au:THPC ratios. The zeta potential was $-25 \pm 2$, and $16 \pm 1 \mathrm{mV}$ for isolated and $\mathrm{pH}$-aggregated NPs, consistent with previous reports in literature. ${ }^{148,232}$ The zeta potential was $-46.8 \pm 0.6,-42.1 \pm 1.5,-35.9 \pm 3.7$, and $-22 \pm 4 \mathrm{mV}$ for Au:THPC ratios of $62.5,31.3,10.4$, and 6.3 , respectively. The $\mathrm{pH}$ of the corresponding solutions was 5.8, 5.0, 4.6 and 3.6, respectively. The initial decrease in the zeta potential for the Au:THPC ratio of 62.5 is due to the rapid displacement of citrate ions, which causes the slowdown of NPs electrophoretic mobility due to the assembly of NPs. ${ }^{148}$ Also, Au NPs act as a catalyst to oxidize THPC to tri(hydroxymethyl) phosphine oxide (THPO). However, in this conversion process THPC forms an intermediate compound of THPC hydroxide (THPOH) as reported by Gulka ${ }^{148}$ and observed by others. ${ }^{233}$ The THPOH, having negative charge, decrease the initial zeta potential. However, with the increase of THPC, the zeta potential eventually increases, and the $\mathrm{pH}$ of the solution also decreases, indicating that attractive force being dominant over repulsion force between particles and ultimately NPs were fused. The decrease of $\mathrm{pH}$ could be due to the 
excess amount of positive charge ligand (THPC) which is not directly binding to the NPs surface but placed in between NPs as shown in the STEM image (Figure 5.6E). The excess THPC decreases the electrostatic repulsion between particles and as a results NPs became fused.

\subsection{CONCLUSIONS}

In conclusion, we showed here that the oxidation potential $\left(E_{p, o x}\right)$ of $15 \mathrm{~nm}$ diameter citrate-stabilized Au NPs depends on whether they are isolated or in a 1D/2D aggregated assembly with space between the NPs, or in a 3D aggregate with the NPs interconnected, or fused. The addition of low amount of THPC (Au:THPC $=62$ ) led to $1 \mathrm{D} / 2 \mathrm{D}$ assemblies with gaps between the aggregated NPs. The addition of acid to lower the NP solution $\mathrm{pH}$ below 3 or addition of large amounts of THPC (Au:THPC $=6$ ) to the Au NPs led to 3D assemblies of interconnected Au NPs. In all 3 cases, the solutions turned purple/blue and the UV-vis showed significant absorbance at higher wavelengths, indicative of plasmonic coupling between the aggregated NPs in the assembly. Interestingly, the $E_{p, o x}$ of the Au NPs shifted positive relative to isolated Au NPs at low pH and with high amounts of THPC, where the NPs were in a 3D assembly with interconnected NPs. The Ep,ox did not shift with low amounts of THPC, where the aggregated NPs were in a 1D/2D assembly with gaps between the NPs. The 3D

interconnected assemblies showed a significantly lower electrochemicallymeasured SA/V compared to isolated or 1D/2D NP assemblies with low THPC. 
The measured SA/V was consistent with the measured $E_{p, o x}$, where a lower SA/V led to a higher $\mathrm{E}_{\mathrm{p}, \mathrm{ox}}$. Zeta potential measurements were consistent with $\mathrm{pH}$ neutralization of the NPs or replacement of citrate with THPC during aggregation. Studying the electrochemical properties of aggregated metal nanostructures is important for gaining a better fundamental understanding of metal NP reactivity and for applications in aggregation-based sensors and electrocatalysis. 


\section{CHAPTER VI}

\section{EEEFECT OF SIZE, COVERAGE, AND DISPERSITY ON THE POTENTIAL-CONTROLLED OSTWALD RIPENING OF METAL NANOPARTICLES}

\subsection{INTRODUCTIONS}

Ultra-small metallic nanoparticles (NPs) are widely used for catalytic and sensing applications due to their higher surface area-to-volume ratio (SA/V) and increased reactivity as compared to their bulk counterpart. ${ }^{181,188,234-236}$ For different catalytic and sensing applications, NPs are usually used at relatively high temperature, ${ }^{78}$ high electrochemical potential, ${ }^{237}$ or under gaseous conditions. ${ }^{225}$ The structural stability of the metal NPs is critical to maintain their reactivity for their particular application. Coarsening (size increase) is an undesirable process that directly leads to a decrease in the SA/V of the NP catalyst, resulting in loss in surface activity, by different mechanisms. Two common mechanisms are known as Smoluchowski ripening ${ }^{238}$ and Ostwald ripening. ${ }^{91}$ The growth of bigger metal NPs at the expense of smaller metal NPs is called Ostwald ripening. Smoluchowski ripening involves the coarsening of particles by diffusion, subsequent collision, and fusion of intact particles. 
Ostwald ripening is a major process that occurs during NP synthesis and even over time in a solution of metallic NPs. A better understanding of Ostwald ripening is needed to improve NP synthesis and size stability. The main driving force for the Ostwald ripening mechanism is the chemical potential difference between small and large NPs brought about by the larger surface free energy for the smaller NPs, which drives them to become larger in size in order to reduce their SA/V. ${ }^{58}$ The larger interfacial area of the smaller-sized particles results in larger surface free energy, which drives them to become oxidized and redeposit onto larger-sized particles in the case of electrochemical Ostwald ripening. The thermodynamic NP stability against oxidation depends on the particles size. ${ }^{192,} 202-203,239$ Various methods, such as ozone treatment, ${ }^{25}$ thermal treatment, ${ }^{26}$ or electrochemical ${ }^{27}$ and chemical treatment ${ }^{240}$ have been used prior to applications of NPs in catalysis and sensing, which can change their size by Ostwald ripening. This negatively effects their performance in the catalytic or sensing application.

There have been previous studies on Ostwald ripening of metal NPs both in the solution phase ${ }^{241}$ and on conductive substrates. ${ }^{60}$ Some studies reported ripening of NPs during catalytic applications, ${ }^{34,242}$ but others observed it during the physiochemical treatment. ${ }^{25,76}$ The size change of NPs after Ostwald ripening has mainly been monitored by microscopic methods. For example, Hu and coworkers reported the size-dependent ripening of Au nanoclusters by using a high angle annular dark field scanning transmission electron microscope (HAADF-STEM) while the nanocluster was being used as a $\mathrm{CO}$ oxidation catalyst. ${ }^{8}$ Nanoclusters were attached to an amorphous carbon film. Based on the microscopic size 


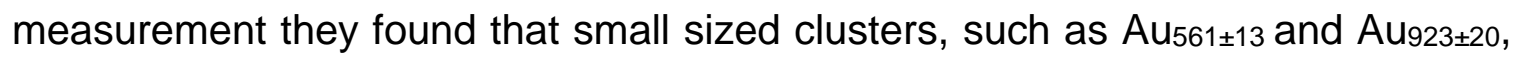
grew bigger in size by Ostwald ripening. However, bigger sized particles, such as $\mathrm{Au}_{2057 \pm 45}$ grew bigger in size by Smoluchowski ripening. Recently, Crooks and coworkers reported that the size of citrate-coated $2 \mathrm{~nm}$ diameter Au NPs increased to 6-7 $\mathrm{nm}$ by ripening after $\mathrm{CO}_{2}$ reduction based on STEM size measurements. ${ }^{19}$ However, they found that similar-sized dendrimer-capped $2 \mathrm{~nm}$ Au NPs almost remained the same size after the same catalytic cycle. Challa and coworkers reported the in-situ monitoring of $\mathrm{Ni} N P s$ (attached to an $\mathrm{MgAl}_{2} \mathrm{O}_{4}$ substrate) during exposure to an equimolar mixture of $\mathrm{H}_{2}$ and $\mathrm{H}_{2} \mathrm{O}$ at $750{ }^{\circ} \mathrm{C}$ (conditions relevant to methane stream reforming) by transmission electron microscopy (TEM). ${ }^{243}$ They found that smaller Ni NPs remained immobile and shrunk in size at a steady rate and finally disappeared. However, the size of bigger Ni NPs increased within a few seconds. This study clearly showed the NP sizes increased by Ostwald ripening.

Besides these studies, there have been reports on chemically- and electrochemically-induced Ostwald ripening of metal NPs attached to conductive surfaces. For example, Brus and co-workers studied electrochemical Ostwald ripening of colloidal Ag NPs on a conductive surface using scanning electron microscopy (SEM) ${ }^{60}$ They deposited thin films of Ag metal on the surface of ITO and then added a couple of drops of water on the surface of the electrode. After being exposed to water, bigger Ag NPs formed on the ITO over time at the expense of smaller NPs, which they attributed to an Ostwald ripening mechanism. Recently, Lee and coworkers showed the applications of electrochemical Ostwald 
ripening towards the welding of $\mathrm{Ag}$ nanowires (Ag NWs) by spontaneous deposition of Ag NPs on the surface of Ag NWs. ${ }^{244}$ The as-synthesized Ag NWs were dispersed onto a glass/ITO electrode followed by electron beam evaporation of Ag NPs. Addition of a drop of water to the electrode surface led to the welding of the $\mathrm{Ag} \mathrm{NWs}$ for hours by the oxidation of $\mathrm{Ag}$ to $\mathrm{Ag}^{+}$and redeposition onto the Ag NWs. This occurred due to the potential difference between the Ag NPs and Ag NWs and improved the stretchability of Ag NWs. Recently, Steven and coworkers studied the stability of different sized carbon-supported Au NPs in acidic electrolyte and found that the NPs size increased by Ostwald ripening during the acidic oxidation cycle based on the SEM-determined size of those NPs. ${ }^{173}$.

To the best of our knowledge there is no report on the electrochemical determination of the NP size transformation rate during Ostwald ripening. Also, details of the ripening mechanism remains undiscovered, especially under potential control. There have not been systematic studies on the effect of NP size, electrode potential, NP coverage and NP size dispersity on the Ostwald ripening rate of metal NPs. Therefore, exploring the electrochemically-induced Ostwald ripening kinetics of electrode-immobilized metal NPs is highly desirable. In this work we describe the use of electrochemical methods, ASV and electrochemicallymeasured SA/V, to study the electrochemically-controlled Ostwald ripening rate of 1.6, $4,15 \mathrm{~nm}$, and mixed $4 \mathrm{~nm} / 15 \mathrm{~nm}$-sized Au NPs attached to aminefunctionalized glass/ITO electrodes. We recently described the use of electrochemical ASV and SA/V measurements for the simple, fast, and low cost analysis of Au NP sizes from sub $2 \mathrm{~nm}$ up to $250 \mathrm{~nm}$ in diameter. ${ }^{200-201,245-246}$ 
Electrochemistry is ideal for this study because it would be costly and tedious to measure NP sizes from multiple samples under multiple conditions by electron microscopy or scanning probe techniques. Our electrochemical approach is fast, low cost, and simple, allowing high throughput and statistical analysis of the results. Another unique aspect of this work is that the Ostwald ripening process is measured under potential control. This allows us to better understand the effect of electrode potential on the ripening rate. Most studies are performed at an unknown open circuit potential but the applications involving electrocatalysis are under potential control. It is therefore important to control and better understand the effect of potential and other factors (NP size, NP coverage, and NP size dispersity) on the Ostwald ripening rate.

\subsection{EXPERIMENTAL}

6.2.1. UV-Vis Characterization. UV-vis spectra of as synthesized 1.6, 4 and 15 nm diameter Au NPs are shown in Figure 6.1.(A). The UV-vis spectra were normalized for absorbance of the different sized Au. The localized surface plasmon resonance (LSPR) band of citrate-coated 4 and $15 \mathrm{~nm}$ diameter Au NPs appeared at 506 and $518 \mathrm{~nm}$, respectively, which is consistent with the plasmon band position of 4 and $15 \mathrm{~nm}$ Au NPs reported previously by our group ${ }^{201}$ and others. ${ }^{39}$ However, THPC-coated $1.6 \mathrm{~nm}$ diameter Au NPs did not show a plasmon band over this wavelength range. It is widely-known that the LSPR band disappears for NPs of about $2 \mathrm{~nm}$ in diameter or smaller. ${ }^{47,239,247}$ 
6.2.2. Microscopic Characterization. The as-synthesized Au NPs were attached to aminopropyl triethoxysilane (APTES)-functionalized glass/ITO (glass/ITO/APTES) electrodes by directly soaking the electrodes into solutions of the NPs. We monitored the successful assembly of 4 and $15 \mathrm{~nm}$ Au NPs on the glass/ITO/APTES electrode by scanning electron microscopy (SEM) imaging (Figure 6.1. (C) and 6.1. (D). Statistical analysis showed that the $15 \mathrm{~nm}$ Au NPs were $15.1 \pm 1.6 \mathrm{~nm}$ in diameter. The $4 \mathrm{~nm}$ Au NPs were difficult to analyze accurately by SEM imaging but had previously been determined by our group to be $4.1 \pm 0.7 \mathrm{~nm}$ by transmission electron microscopy (TEM) imaging. ${ }^{192}$ TEM images of THPC-coated $1.6 \mathrm{~nm}$ diameter Au NPs were obtained with a $200 \mathrm{kV}$ FEI Tecnai F20 operated in TEM mode. First, we functionalized silicon oxide coated 400 mesh Au grids (SPI supplies, West Chester, PA) with APTES using the same procedure as we used for the functionalizing the glass/ITO electrode. NPs were attached to grids by directly soaking APTES-functionalized grids in the NP solution for 4-5 min. After that, the grids were rinse with water and dried gently under $\mathrm{N}_{2}$. TEM images of the $1.6 \mathrm{~nm}$ Au NPs showed them to be $1.6 \pm 0.4 \mathrm{~nm}$ in diameter (Figure 6.1.B), which is consistent with Duff et al., who reported their size to be 1.5 $\mathrm{nm}$ in diameter based on TEM. ${ }^{47}$

\subsubsection{Au NP Size Analysis by Anodic Stripping Voltammetry (ASV). A CH} Instruments (Austin, TX) model CHI 660E electrochemical cell consisting of a 3electrode set-up with the $\mathrm{Ag} / \mathrm{AgCl}(3 \mathrm{M} \mathrm{KCl})$ as a reference electrode, a Pt wire counter electrode and glass/ITO/APTES/Au NPs as the working electrode, was 
used to perform ASV measurements. We measured the peak oxidation potential $\left(E_{p}\right)$ of the Au NPs by scanning from -0.2 to $1.6 \mathrm{~V}$ (vs. $\left.\mathrm{Ag} / \mathrm{AgCl}\right)$ in $10 \mathrm{mM} \mathrm{KBr}$ plus $0.1 \mathrm{M} \mathrm{KClO}_{4}$ electrolyte at $0.01 \mathrm{~V} / \mathrm{s}$ as shown in Figure 6.1.(E). The downward anodic peaks in the scans are due to oxidation of $\mathrm{Au}$ by bromide as shown in equations below:

$$
\begin{aligned}
& \mathrm{Au}^{0}+4 \mathrm{Br}^{-} \longrightarrow \mathrm{AuBr}_{4}^{-}+3 \mathrm{e}^{-}\left(\mathrm{E}^{0}=0.85 \mathrm{~V} \text { vs. } \mathrm{NHE}\right) \\
& \mathrm{Au}^{0}+2 \mathrm{Br}^{-} \longrightarrow \mathrm{AuBr}^{-}+\mathrm{e}^{-}\left(\mathrm{E}^{0}=0.96 \mathrm{~V} \text { vs. } \mathrm{NHE}\right)
\end{aligned}
$$

The $E_{p}$ of 1.6, 4 and $15 \mathrm{~nm}$ diameter Au NPs decreased as the size of the NPs decreased in the order of $1.6 \mathrm{~nm}\left(E_{p}=0.45 \mathrm{~V}\right)<4 \mathrm{~nm}\left(\mathrm{E}_{p}=0.69 \mathrm{~V}\right)<15 \mathrm{~nm}\left(\mathrm{E}_{p}=\right.$ $0.77 \mathrm{~V})$. This is consistent with our previous work ${ }^{192,239,246}$ and others, ${ }^{248}$ showing a negative shift in $\mathrm{E}_{\mathrm{p}}\left(\right.$ and $\mathrm{E}^{0}$ ) with decreasing size. The size-dependent oxidation of metal NPs is also consistent with the theoretical work of Plieth, who predicted a negative shift in the oxidation potential relative to the bulk value proportional to $1 /$ radius. ${ }^{58}$ Henglein calculated a negative shift in the oxidation potential of metal NPs with decreasing size based on sublimation energies. ${ }^{205}$ 

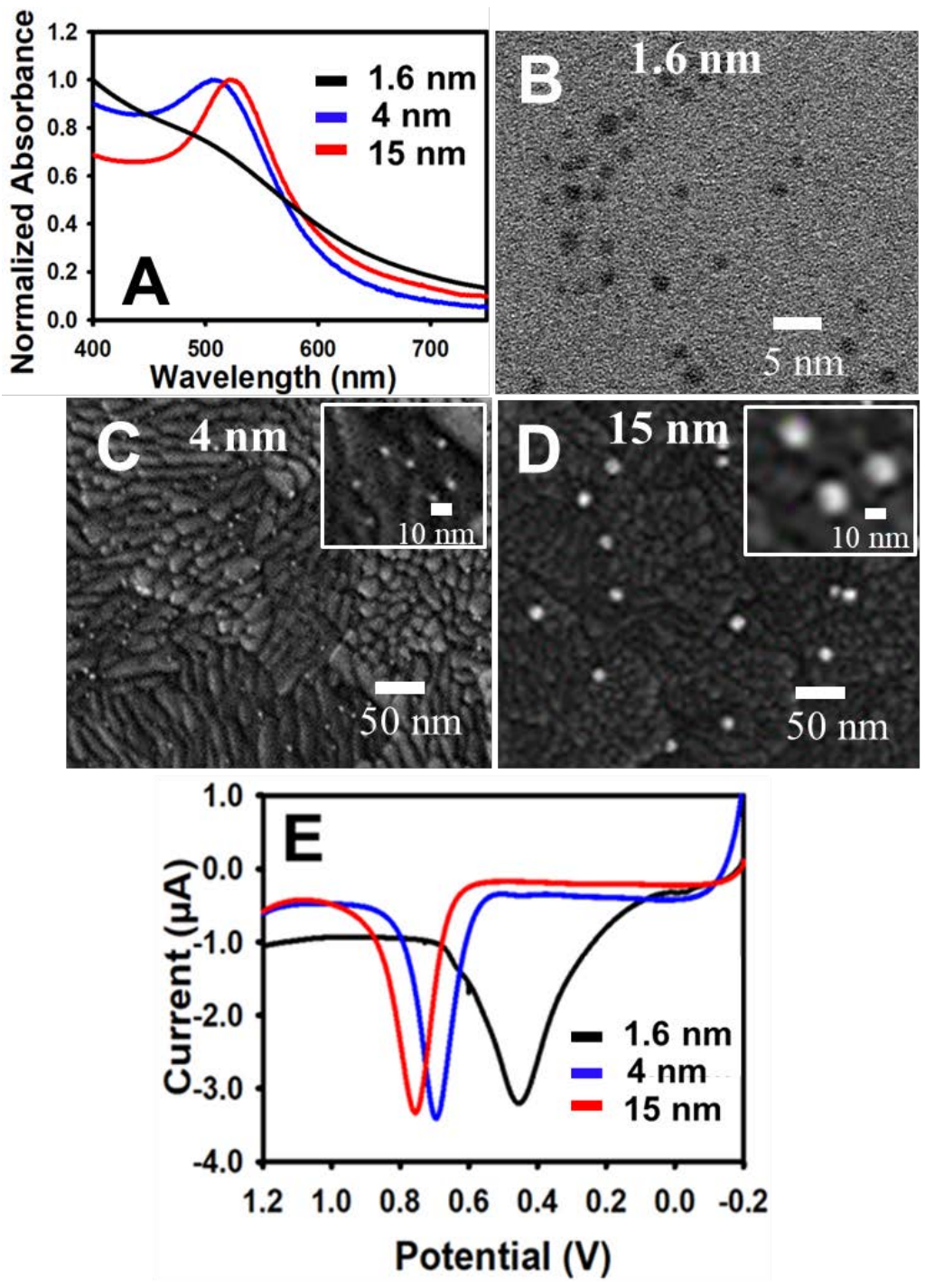

Figure 6. 1. (A) UV-Vis spectra of THPC-stabilized $1.6 \mathrm{~nm}$ and citrate-coated 4 and $15 \mathrm{~nm}$ diameter Au NPs. (B) TEM image of THPC-stabilized $1.6 \mathrm{~nm}$ Au NPs. (C, D) SEM images of 4 and $15 \mathrm{~nm}$ citrate-stabilized Au NPs. (E) ASV of 1.6, 4, and $15 \mathrm{~nm}$ diameter Au NPs. ASVs were obtained in $10 \mathrm{mM} \mathrm{KBr}$ plus $0.1 \mathrm{M} \mathrm{KClO}_{4}$ electrolyte at a scan rate of $0.01 \mathrm{~V} / \mathrm{s}$. 


\subsubsection{Au NP Size Analysis by Electrochemical Surface Area-to-Volume} (SA/V) Measurement. Cyclic voltammetry was performed on Au NP-coated glass/ITO/APTES electrodes by scanning from $-0.2 \mathrm{~V}$ to $1.6 \mathrm{~V}$ and back in $0.1 \mathrm{M}$ $\mathrm{HClO}_{4}$ at a scan rate of $0.1 \mathrm{~V} / \mathrm{s}$ to measure the electroactive surface area (SA) of the $\mathrm{Au}$ NPs from the Au oxide reduction peak as described by our group previously. ${ }^{201}$ The oxidation/reduction reaction of the Au NP surface in $0.1 \mathrm{M}$ $\mathrm{HClO}_{4}$ occurs by the following reversible reaction as shown in equation below.

$$
\mathrm{Au}_{2} \mathrm{O}_{3}+6 \mathrm{H}^{+}+6 \mathrm{e}^{-} \leftrightharpoons 2 \mathrm{Au}+3 \mathrm{H}_{2} \mathrm{O}
$$

The Au oxide layer only forms on the Au NP surface. The integrated charge of the peak corresponding to the oxide reduction corresponds to the electrochemical $\mathrm{Au}$ NP surface area (SA). The oxidation peak in the ASV from reactions 1 and 2 discussed earlier corresponds to the dissolution of all of the Au from the Au NPs and therefore reflects the total volume $(\mathrm{V})$ of the sample of the Au NPs. SA/V is then determined by dividing the Coulombs of charge from the reduction of Au oxide by the Coulombs of charge from full stripping of the Au NPs and that further gives the radius of the spherical NP, which is equal to $3 S A / V$. This is a useful method to electrochemically determine the average size of the Au NPs on the electrode surface. $^{201}$

6.2.5. Ostwald Ripening Studies. We kept a similar total volume (total amount of $\mathrm{Au}$ ) of different sized Au NPs on the glass/ITO/APTES surface for all of our Ostwald ripening studies in order to relate the Ostwald ripening rate to size as opposed to Au coverage effects. In order to keep the coverage same, we obtained CVs of the glass/ITO/APTES/Au NPs in $0.1 \mathrm{M} \mathrm{HClO}_{4}$ in order to determine their 
electroactive surface area (SA). Since we know the Au NP size, we then estimated the volume $(V)$ since $V=\left(S A^{*} r\right) / 3$. The $C V$ coverage was $2.0-2.7 \times 10^{-5} \mathrm{C}$ and $0.7-$ $1.0 \times 10^{-5} \mathrm{C}$ for 4 and $15 \mathrm{~nm}$ Au NPs, respectively, which gives a total volume of Au in the range of $3.25 \times 10^{-5}$ to $4.25 \times 10^{-5} \mathrm{C}$. We unfortunately could not directly measure the electroactive surface area of $1.6 \mathrm{~nm}$ Au NPs because they are unstable and convert to $4 \mathrm{~nm}$ Au NPs in just one CV cycle. ${ }^{239}$ In this case, we obtained the coverage by performing the ASV after 3 min of soaking the glass/ITO/APTES in a solution of 10-fold diluted $1.6 \mathrm{~nm}$ diameter THPC-coated Au NPs. The reproducibility of the soaking conditions was sufficient to obtain good statistics on the Ostwald ripening rate of different-sized Au NPs, independent of the Au coverage.

After we measured the surface area of glass/ITO/APTES/Au NPs (or assumed it in the case of $1.6 \mathrm{~nm}$ Au NPs), we held the potential of the various Au NP-coated electrodes at $0.0 \mathrm{~V}, 0.30 \mathrm{~V}$ and $0.45 \mathrm{~V}$ for $35,70,105$ or $140 \mathrm{~min}$ in $10 \mathrm{mM} \mathrm{KBr}$ plus $0.1 \mathrm{M} \mathrm{HClO}_{4}$ electrolyte solution. We skipped $0.45 \mathrm{~V}$ for the $1.6 \mathrm{~nm}$ Au NPs for reasons discussed later. Then we removed the electrode from the cell, rinsed with nanopore water, and dried under $\mathrm{N}_{2}$. The same electrode was then used to measure the SA in $0.1 \mathrm{M} \mathrm{HClO}_{4}$ electrolyte solution and total $\mathrm{V}$ in $10 \mathrm{mM} \mathrm{KBr}$ plus $0.1 \mathrm{M} \mathrm{KClO}_{4}$ electrolyte solution. Finally, the measured SA/ $\mathrm{V}$ was converted to NP diameter for the different ripening times, which allowed the determination of the rate of electrochemical Ostwald ripening. The experiments were performed similarly for studies that varied the Au NP coverage and size dispersity. In those 
studies, we kept the size constant but varied the coverage or we changed from one size NP to a mixture of $4 \mathrm{~nm}$ and $15 \mathrm{~nm}$ Au NPs to introduce size dispersity.

\subsection{RESULTS AND DISCUSSION}

\subsubsection{Monitoring Ostwald Ripening as a Function of Nanoparticle Size} and Electrode Potential by Anodic Stripping Voltammetry (ASV). We monitored the effect of potential and NP size on the electrochemically-controlled Ostwald ripening by using ASV. ASV is a powerful technique to monitor NP size changes on the electrode surface due to the dependence of the peak oxidation potential $\left(E_{p}\right)$ on the NP size (Figure 6.1). ${ }^{239}$ Figure 6.2 shows ASVs of glass/ITO/APTES/15 nm Au NP electrodes held at $0.0 \mathrm{~V}$ (Figure 6.2. (A)), $0.30 \mathrm{~V}$ (Figure 6.2.(B)) and $0.45 \mathrm{~V}$ (Figure 6.2. (C)) for 0, 35, 70, 105 and 140 min in 10 $\mathrm{mM} \mathrm{KBr}$ plus $0.1 \mathrm{M} \mathrm{HClO}_{4}$ electrolyte. There is no shift in $\mathrm{Ep}_{\mathrm{p}}$ for up to $105 \mathrm{~min}$ at $0.0 \mathrm{~V}$ (vs Ag/AgCl), but a small shift of about $40-50 \mathrm{mV}$ after holding for $140 \mathrm{~min}$ in the $\mathrm{Br}^{-}$-containing electrolyte (Figure 6.2.(A)). However, the $E_{p}$ starts to show significant changes after $35 \mathrm{~min}$ of ripening at $0.30 \mathrm{~V}$ (Figure 6.2. (B), blue plot). We observed a new shoulder peak at $0.95 \mathrm{~V}$ after $70 \mathrm{~min}$ of holding the potential at $0.30 \mathrm{~V}$, indicating an increase in the NP size. The shoulder peak at $0.95 \mathrm{~V}$ increased with potential holding time and became a clearly distinct peak after 140 min of Ostwald ripening (Figure 6.2.(B), brown plot). Based on the theoretical work by Pleith ${ }^{58}$ and experimental work by our group, ${ }^{245}$ the new peak at $0.95 \mathrm{~V}$ indicates that some of the NPs increased to sizes larger than $20 \mathrm{~nm}$ during ripening. Also, the peak at $0.76 \mathrm{~V}$ indicates that some particles remained at $\sim 15$ 
$\mathrm{nm}$ in size. There are also some very small peaks at potentials between 0.0 and $0.4 \mathrm{~V}$, which are likely very small NPs that did not fully dissolve during ripening. This inhomogeneous size distribution clearly indicates that a significant fraction of NPs grew in size by Ostwald ripening. 

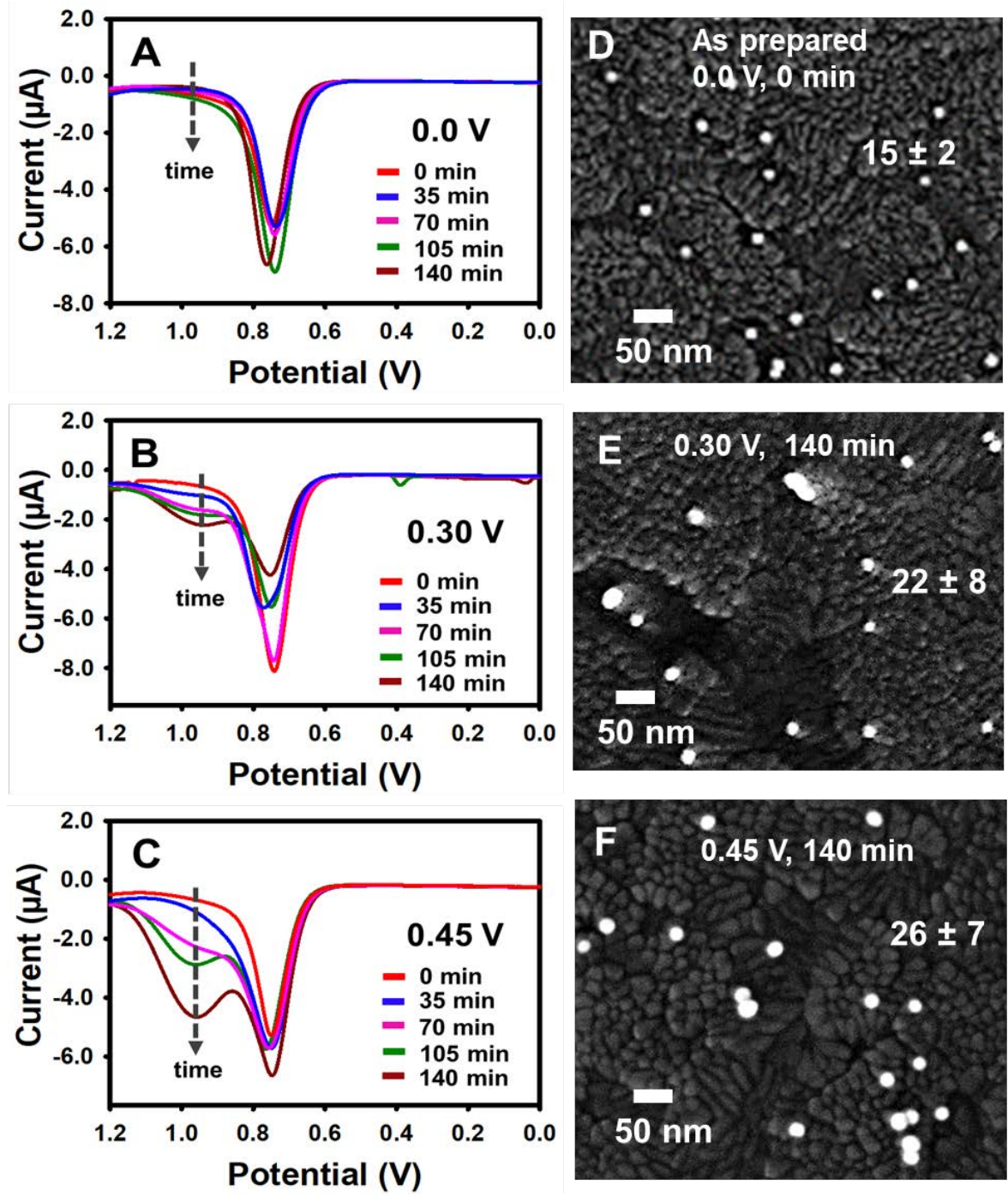

Figure 6. 2. ASV of citrate coated $15 \mathrm{~nm}$ Au NPs after Ostwald ripening at $0.0(\mathrm{~A})$, 0.3 (B) and $0.45 \vee(C)$ for $0,35,70,105$ and 140 min of ripening each in $\mathrm{Br}^{-}$ containing acidic electrolyte solution. ASVs were obtained in $10 \mathrm{mM} \mathrm{KBr}$ plus 0.1 $\mathrm{KClO}_{4}$ electrolyte at a scan rate of $0.01 \mathrm{~V} / \mathrm{s}$. SEM images of $15 \mathrm{~nm}$ Au NPs at 0 min of ripening (A) and after 140 min of ripening at $0.3 \mathrm{~V}(\mathrm{E})$ and $0.45(\mathrm{~F})$. 
To confirm our findings, we obtained SEM images of $15 \mathrm{~nm}$ Au NPs after $140 \mathrm{~min}$ of ripening (Figure 6.2.(E)) and compared to that for no ripening (Figure 6.2.(D)). The average NP size increased from $15 \pm 2 \mathrm{~nm}$ to $22 \pm 8 \mathrm{~nm}$ after $140 \mathrm{~min}$ of ripening at $0.30 \mathrm{~V}$. The larger standard deviation after ripening is due to the inhomogeneous size distribution of the NPs, consistent with the ASV. We determined the size histogram of $15 \mathrm{~nm}$ Au NPs before (Figure 6.3. (A)) and after 140 min of ripening at $0.30 \mathrm{~V}$ (Figure 6.3.(B)). In the size histogram we found more NPs in the size range of 20-24 nm. The size histogram further supports our ASV finding that NPs grew by potential-controlled Ostwald ripening. 

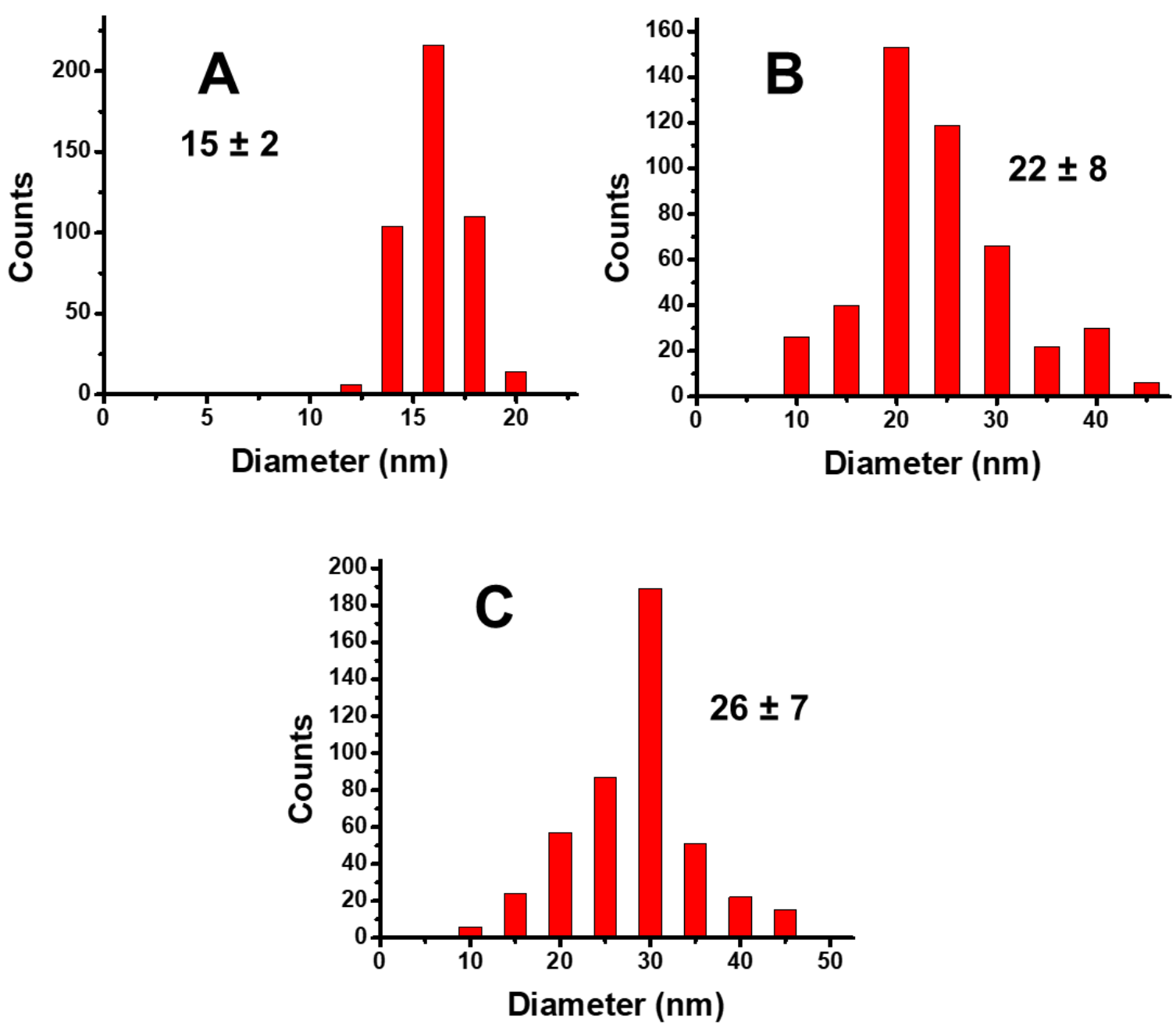

Figure 6. 3. Size histogram of $15 \mathrm{~nm}$ Au NPs (A) without Ostwald ripening, (B) after 140 min of ripening at $0.3 \mathrm{~V}$ and (C) after $140 \mathrm{~min}$ of ripening at $0.45 \mathrm{~V}$. 
Next, we held the potential at $0.45 \mathrm{~V} v \mathrm{Ag} / \mathrm{AgCl}$ in the same electrochemical cell as we just discussed. In this case we found that the shoulder peak at $0.95 \mathrm{~V}$ appeared within $35 \mathrm{~min}$ (Figure 6.2.(C), blue plot). We observed that the peak at $0.95 \mathrm{~V}$ increased relatively faster than for the same peak at $0.30 \mathrm{~V}$. After $140 \mathrm{~min}$ at $0.45 \mathrm{~V}$ we observed two well distinct oxidation peaks for $\mathrm{Au}$ at 0.76 and $0.95 \mathrm{~V}$, representing two different size populations. This clearly indicates that the rate of NP size transformation of $15 \mathrm{~nm}$ Au NPs is potential dependent, and it is faster at higher potentials. The final average size of the Au NPs was found to be $26 \pm 7 \mathrm{~nm}$ based on SEM images. The size histogram of the Au NPs after 140 min at $0.45 \mathrm{~V}$ is shown in Figure 6.3.(C). We observed a higher population of $26-29 \mathrm{~nm}$ diameter Au NPs, consistent with the ASV.

We were able to successfully monitor the average NP size change following ripening by ASV as discussed previously. However, we were not able to observe a small population of 1-2 nm diameter Au NPs at any stage of Ostwald ripening in the SEM images because that is beyond the resolution. Small Au NPs would be expected to appear in samples due to NPs not being fully oxidized. As the NPs dissolve, the dissolution rate increases, but it is common to observe NPs smaller than the original population in Ostwald Ripening studies. ${ }^{8}$ In some samples we did observe peaks at lower oxidation potentials in the ASV of $15 \mathrm{~nm}$ Au NPs in the potential range of $0.0-0.45 \mathrm{~V}$ after ripening for $105 \mathrm{~min}$ at $0.30 \mathrm{~V}$. Figure 6.4.(A) shows an $E_{p}$ at $0.75 \mathrm{~V}$ and a small shoulder at $0.95 \mathrm{~V}$ as discussed previously. Figure 6.4.(B) shows the same ASV zoomed in from 0.0-0.65 V, where it is clear there is a series of small peaks in the 0.10 to $0.55 \mathrm{~V}$ range. The peaks in this 
region correspond to the oxidation of Au NPs which were in the range of 1-2 nm diameter based on the Plieth
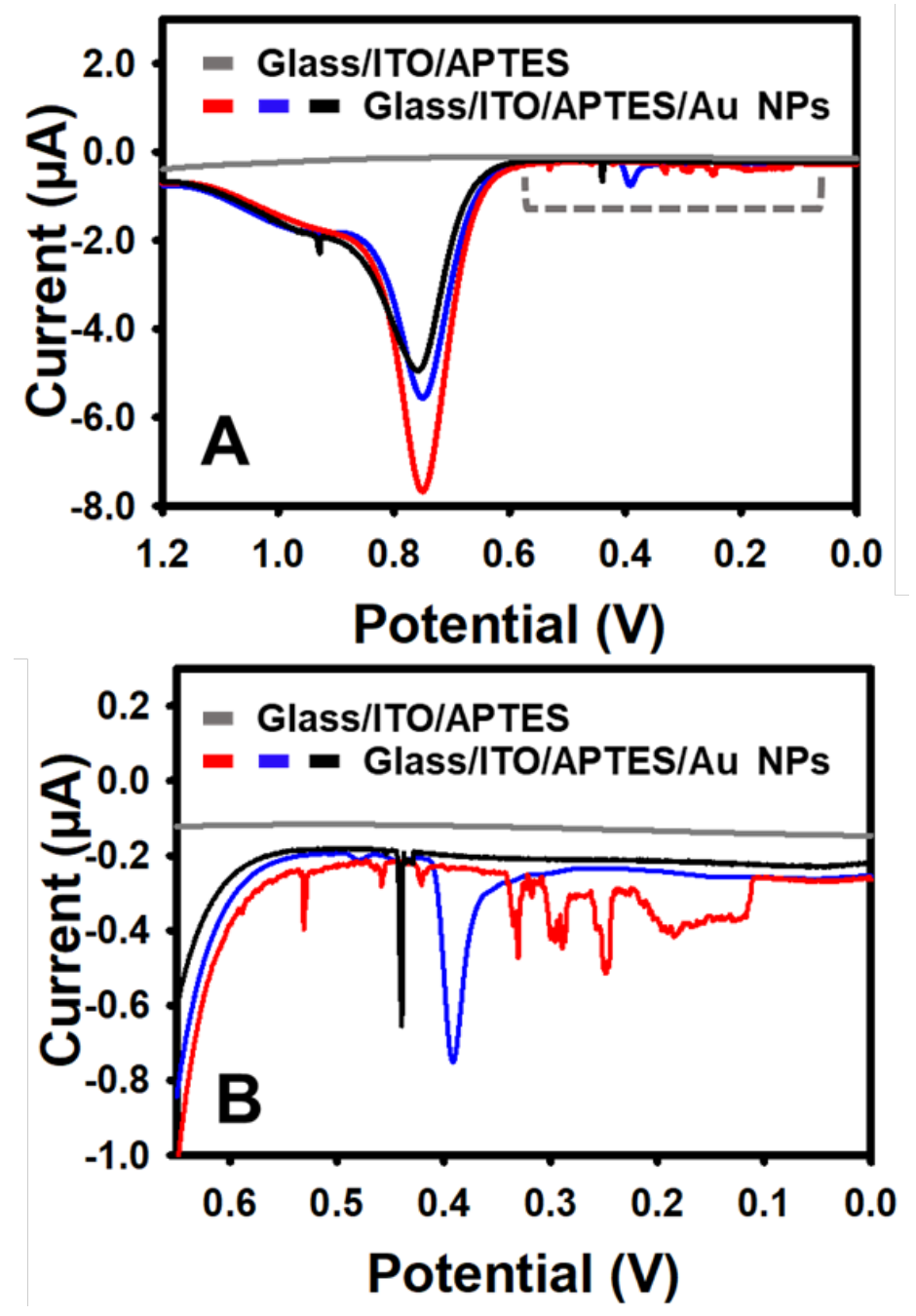

Figure 6. 4. ASV of citrate-stabilized $15 \mathrm{~nm}$ diameter Au NPs after $105 \mathrm{~min}$ of Ostwald ripening at $0.3 \mathrm{~V}(\mathrm{~A})$. ASV obtained in $10 \mathrm{mM} \mathrm{KBr}$ plus $0.1 \mathrm{M} \mathrm{KClO}_{4}$ electrolyte at a scan rate of $0.01 \mathrm{~V} / \mathrm{s}$ (B) The zoomed in portion of Figure A as indicated by the dashed rectangle. 

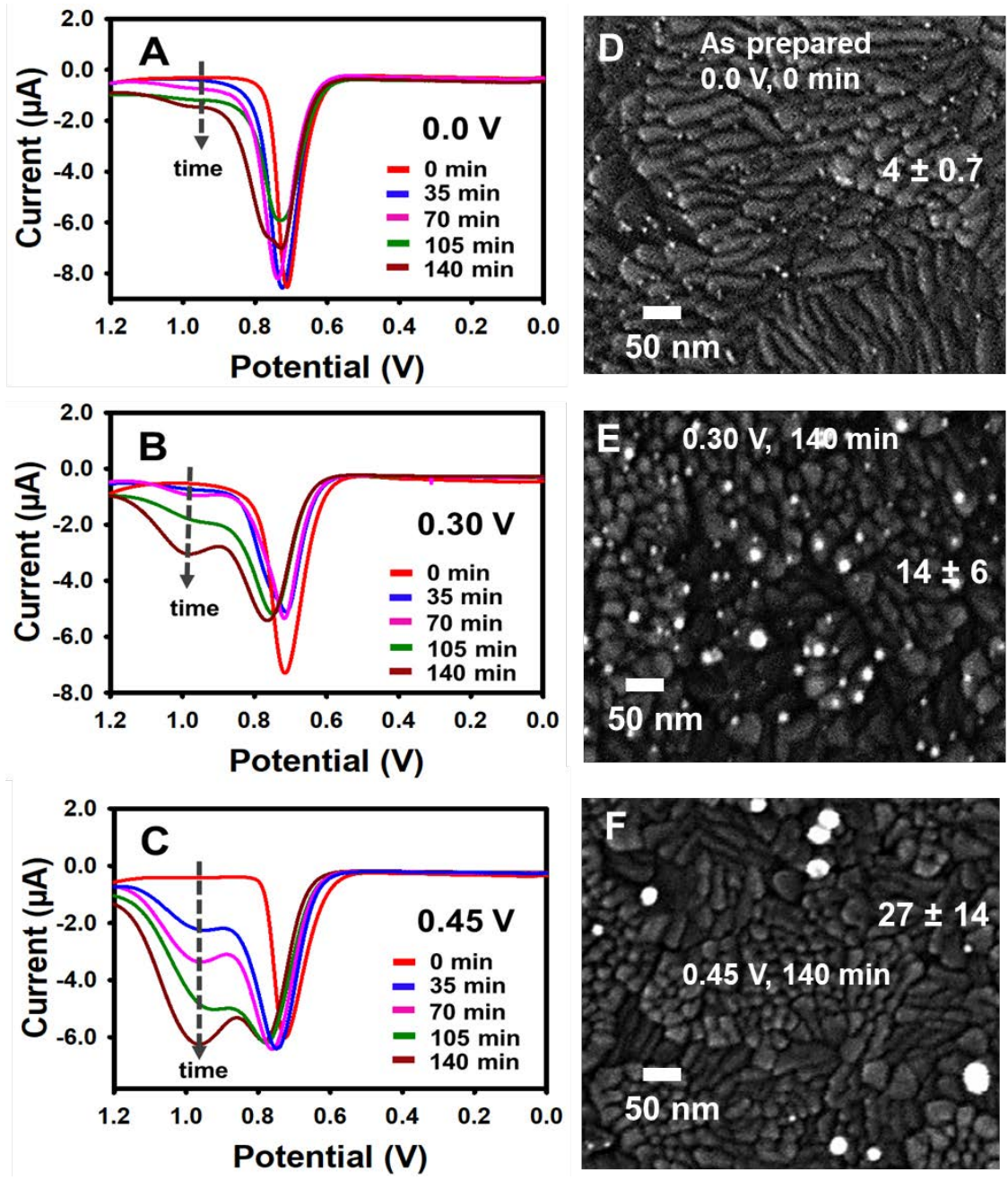

Figure 6. 5. ASV of citrate coated $4 \mathrm{~nm}$ Au NPs after Ostwald ripening at $0.0(\mathrm{~A})$, 0.3 (B) and $0.45 \mathrm{~V}(\mathrm{C})$ for $0,35,70,105$ and 140 min of ripening each in $\mathrm{Br}^{-}$ containing acidic electrolyte solution. ASV obtained in $10 \mathrm{mM} \mathrm{KBr}$ plus $0.1 \mathrm{KClO}_{4}$ electrolyte with a scan rate $0.01 \mathrm{~V} / \mathrm{s}$. SEM images of $4 \mathrm{~nm}$ Au NPs at 0 min of ripening $(A)$, after $140 \mathrm{~min}$ of ripening at $0.3 \mathrm{~V}(\mathrm{E})$ and after $140 \mathrm{~min}$ of ripening at $0.45(F)$. 
prediction ${ }^{58}$ and earlier reports by our group. ${ }^{192,239}$ These small Au NPs come from Au NPs that dissolved significantly, but didn't quite fully dissolve. It is typically very hard to catch these smaller Au NPs after dissolving/ripening, but they occasionally appear in the ASV (25-30\% frequency) as shown in Figure 6.2.(B) and Figure 6.4.

Figure 6.5. shows the effect of potential on the Ostwald ripening of $4 \mathrm{~nm}$ diameter Au NPs under the same conditions as shown for $15 \mathrm{~nm}$ diameter Au NPs in Figure 6.2 in order to understand the effect of size on the Ostwald ripening of Au NPs. Figure 6.5.(A) shows the ASV of $4 \mathrm{~nm}$ Au NPs after 0, 35, 70, 105 and 140 min of ripening at $0.0 \mathrm{~V} v \mathrm{vg} / \mathrm{AgCl}$. The $\mathrm{E}_{\mathrm{p}}$ does not show any significant change up to 105 min. However, after 140 min we observe the main peak becoming a doublet and a small peak also appeared around $0.95 \mathrm{~V}$ (Figure 6.5.(A), brown plot). The doublet and new peak at higher potential clearly indicates a size increase at $0.0 \mathrm{~V}$ for $4 \mathrm{~nm}$ diameter Au NPs.

After holding at $0.30 \mathrm{~V}$ the peak at $0.95 \mathrm{~V}$ became much more distinct from the peak at $0.69 \mathrm{~V}$ after $140 \mathrm{~min}$ of ripening compared to the same time at $0.0 \mathrm{~V}$ (Figure 6.5.(B), brown plot). We obtained SEM images after 140 min of ripening at $0.3 \mathrm{~V}$ (Figure 6.5.(E)) and found the average diameter increased to $14 \pm 6 \mathrm{~nm}$. The average size of the NPs is larger and there is also greater size dispersity. The size histogram in Figure 6.6 of shows that most of the NPs were larger than $8 \mathrm{~nm}$ in diameter. We don't have size histograms of $4 \mathrm{~nm}$ Au NPs without ripening to directly compare our results because this is near the lower limit of resolution of our 
SEM instrument. However, our group previously measured the size of $4 \mathrm{~nm} \mathrm{Au}$ NPs by transmission electron microscopy (TEM) and found them to be $4.1 \pm 0.7$ $n m .{ }^{167}$ Based on SEM images of $4 \mathrm{~nm}$ Au NPs before and after ripening and the size histogram after ripening (Figure 6.6), we can clearly see increased size and increased inhomogeneity of the NPs. This confirms that the NPs are growing by electrochemical Ostwald ripening. The ripening of $4 \mathrm{~nm}$ Au NPs at $0.45 \mathrm{~V}$ was faster compared to $0.30 \mathrm{~V}$ (Figure 6.5.(C). At this potential the oxidation peak at $0.95 \mathrm{~V}$ was more prominent than the peak at $0.69 \mathrm{~V}$, indicating that most of the smaller NPs are gone. The average size after $140 \mathrm{~min}$ of ripening at $0.45 \mathrm{~V}$ was $27 \pm 14 \mathrm{~nm}$ based on the SEM images. The size range was 5 to $60 \mathrm{~nm}$ (Figure 6.5.(F) and Figure 6.6.(B).
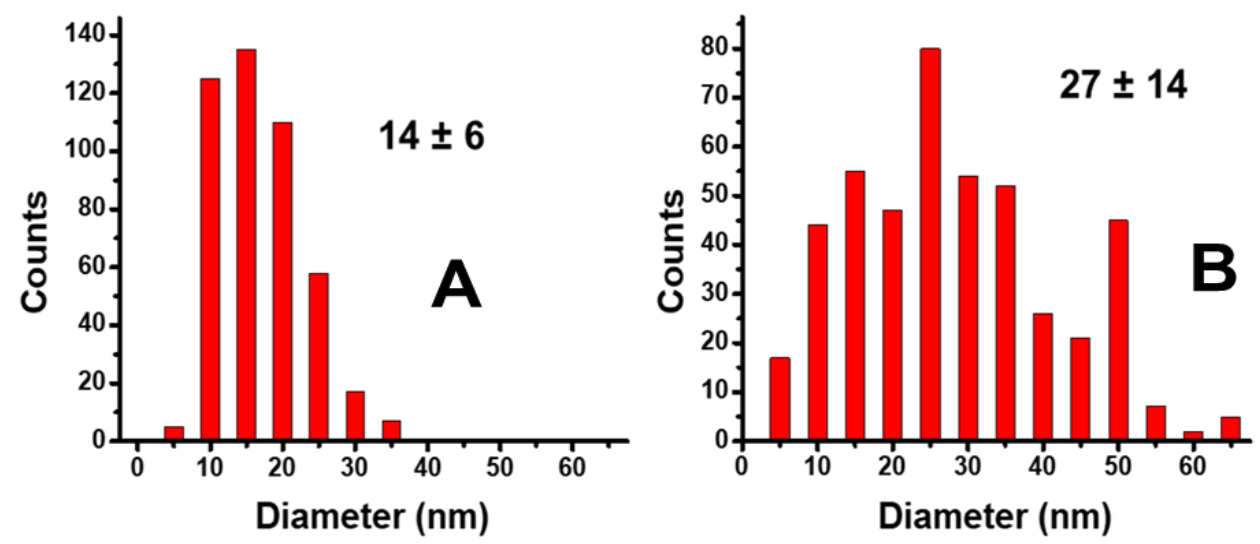

Figure 6. 6. Size histogram of $4 \mathrm{~nm}$ Au NPs after $140 \mathrm{~min}$ of ripening at $0.3 \mathrm{~V}(\mathrm{~A})$ and after $140 \mathrm{~min}$ of ripening at $0.45 \mathrm{~V}(\mathrm{~B})$. 

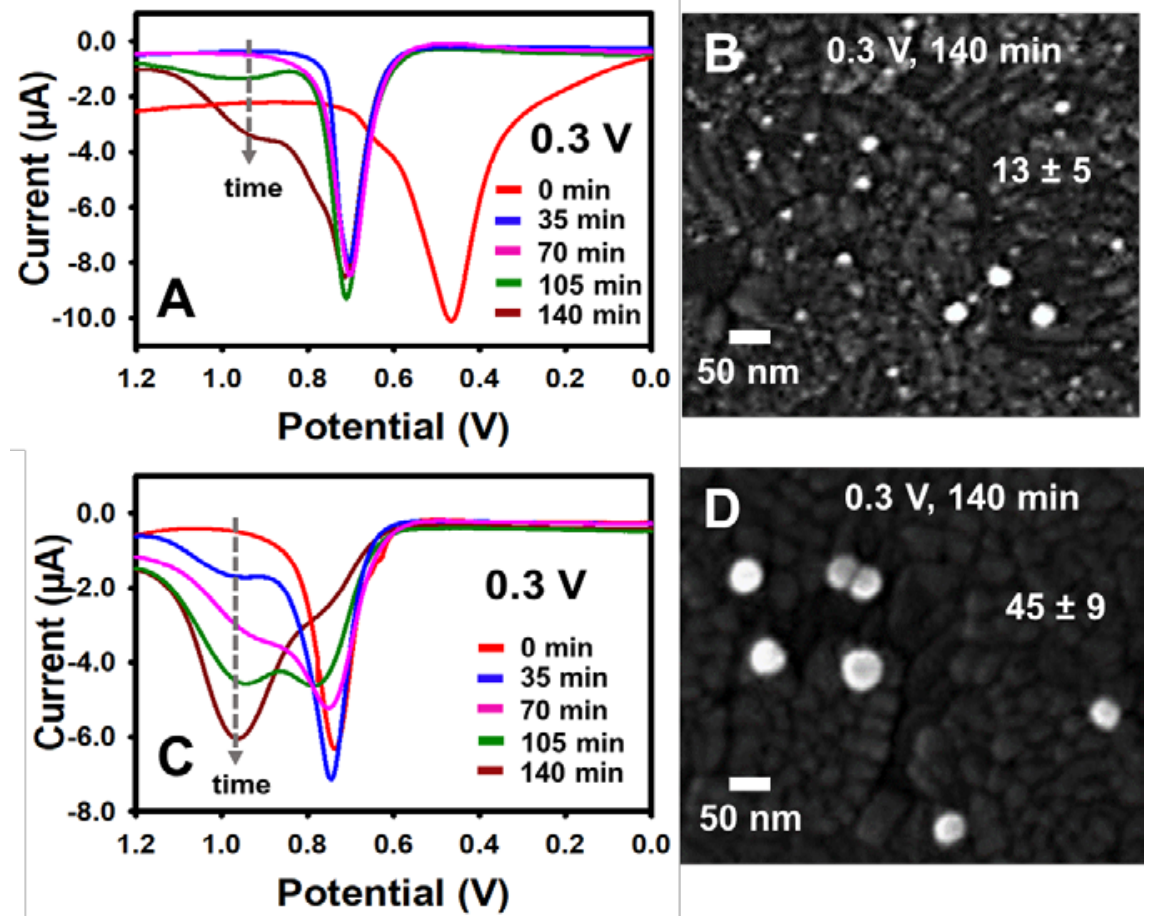

Figure 6. 7. ASV of THPC stabilized $1.6 \mathrm{~nm}$ Au NPs after Ostwald ripening at 0.3 (A) for $0,35,70,105$ and 140 min of ripening each in $\mathrm{Br}^{-}$containing acidic electrolyte solution. SEM image of $1.6 \mathrm{~nm}$ Au NPs after $140 \mathrm{~min}$ of ripening at 0.30 V (B). ASV of a 1:3 mixture of 4 and $15 \mathrm{~nm}$ Au NPs after Ostwald ripening at 0.30 $\checkmark$ for $0,35,70,105$ and 140 min of ripening each in $\mathrm{Br}^{-}$containing acidic electrolyte solution (C). SEM image of 1:3 mixture of 4 and $15 \mathrm{~nm}$ Au NPs after $140 \mathrm{~min}$ of ripening at $0.30 \mathrm{~V}(\mathrm{D})$. All ASVs were obtained in $10 \mathrm{mM} \mathrm{KBr}$ plus $0.1 \mathrm{KClO}_{4}$ electrolyte at a scan rate of $0.01 \mathrm{~V} / \mathrm{s}$. 
The ripening of $1.6 \mathrm{~nm}$ diameter Au NPs under the same reaction conditions at $0.30 \mathrm{~V}$ occurred very fast as reported in our recent article. ${ }^{239}$ In our previous work we didn't determine the rate of transformation of NPs and only showed the data for 35 min of ripening. Here we held the potential for 35, 70, 105 and $140 \mathrm{~min}$ and compared to the $4 \mathrm{~nm}$ and $15 \mathrm{~nm}$ Au NPs. Figure 6.7.(A) shows the ASV signature of $1.6 \mathrm{~nm}$ Au NPs before $(0 \mathrm{~min})$ and after ripening at $0.30 \mathrm{~V}$ for the different times. The $\mathrm{E}_{\mathrm{p}}$ shifted from $0.45 \mathrm{~V}$ to $0.70 \mathrm{~V}$ after $35 \mathrm{~min}$ of ripening and after 105 and 140 min we found a very distinct peak appeared at $0.95 \mathrm{~V}$ (Figure 6.7.(A)). After $35 \mathrm{~min}$ all of the $1.6 \mathrm{~nm}$ sized Au NPs transformed to $4-5 \mathrm{~nm}$ diameter Au NPs and some of those particles ripened further to form even larger NPs with time. SEM images (Figure 6.7.(B)) showed the Au NPs to be $13 \pm 5 \mathrm{~nm}$.
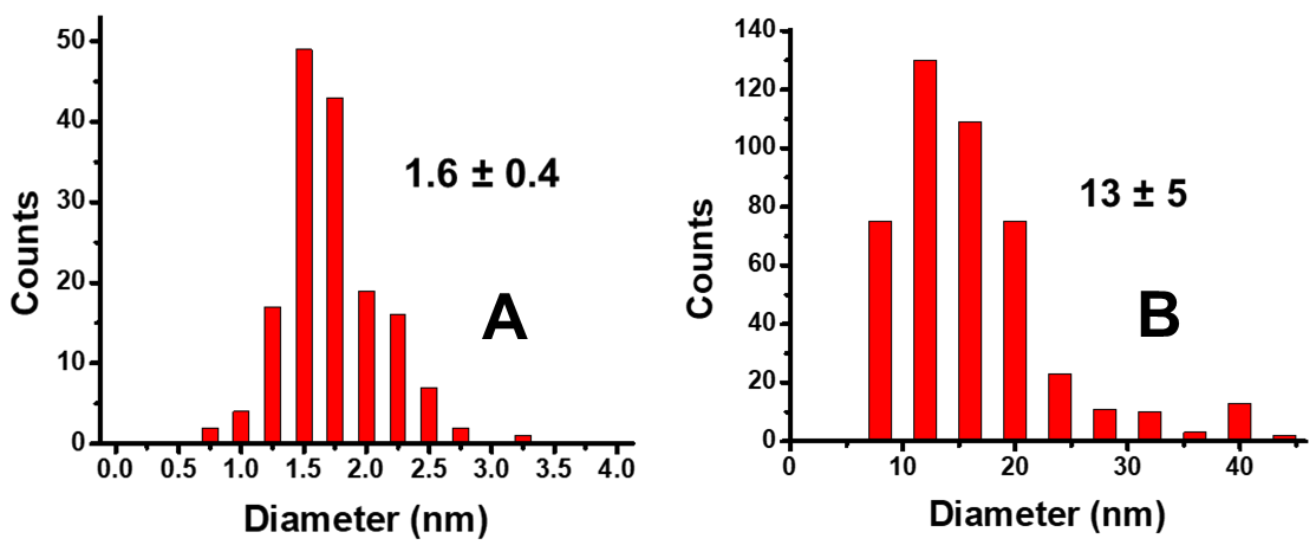

Figure 6. 8. Size histogram of $1.6 \mathrm{~nm}$ diameter Au NPs before treatment (A) and after 140 min of ripening at $0.3 \mathrm{~V}(\mathrm{~B})$. 


\subsubsection{Monitoring Ostwald Ripening as a Function of Size Dispersity by}

Anodic Stripping Voltammetry. In order to study the effect of size dispersity on the rate of Ostwald ripening, we attached a mixture of $4 \mathrm{~nm}$ and $15 \mathrm{~nm}$ Au NPs to the glass/ITO/APTES electrode. We hypothesized that the ripening rate would be higher for the mixture of two different size NPs due to the higher potential difference $\left(\Delta \mathrm{E}_{\mathrm{p}}\right.$ or $\left.\Delta \mathrm{E}^{0}\right)$ between the NPs, which partly drives the ripening process. The glass/ITO/APTES was soaked in a 1:3 mixture of 4 and $15 \mathrm{~nm}$ Au NPs and we held the potential of the electrode at $0.30 \mathrm{~V} v \mathrm{vs} . \mathrm{Ag} / \mathrm{AgCl}$ for $0,35,70,105$ and 140 min. Figure 6.7.(C) shows the ASV signature of the mixture of NPs with no ripening ( $0 \mathrm{~min})$ and after ripening for the indicated times. The ASV at 0 min has a dominant peak at $0.75 \mathrm{~V}$, near the $\mathrm{E}_{\mathrm{p}}$ of the $15 \mathrm{~nm}$ Au NPs. This is due to the larger fraction of $15 \mathrm{~nm}$ Au NPs in the sample. This sample should be compared to $15 \mathrm{~nm}$ Au NPs without $4 \mathrm{~nm}$ Au NPs in Figure 6.2. In this case we found that 140 min of ripening completely shifted the $E_{p}$ to $0.95 \mathrm{~V}$ with very little shouldering at $0.75 \mathrm{~V}$. This is dramatically different than $15 \mathrm{~nm}$ Au NPs without $4 \mathrm{~nm}$ Au NPs at $0.30 \mathrm{~V}$ after $140 \mathrm{~min}$ (Figure 6.2.(B), where there was only a very small peak at $0.95 \mathrm{~V}$. This result clearly shows that the ripening rate of NPs is significantly higher when there is a large size distribution of the NPs. In this case, the ripening rate of the $15 \mathrm{~nm}$ Au NPs increased dramatically by adding $4 \mathrm{~nm}$ Au NPs to the electrode. A 1:3 $4 \mathrm{~nm}: 15 \mathrm{~nm}$ Au NP ratio amounts to about $25 \%$ of the NPs being $4 \mathrm{~nm}$ but $>99 \%$ of the total Au on the electrode resides within the $15 \mathrm{~nm}$ Au NPs because of the large different in the total number of $\mathrm{Au}$ atoms per NP, which is proportional to (radius) $^{3}$. A similar effect of NP size dispersity on Ostwald ripening in solution has 
been reported by others ${ }^{249-250}$ but our studies show direct evidence of the effect of size dispersity on Ostwald ripening of electrode surface-attached NPs under potential control. SEM images (Figure 6.7.(D)) found the NPs to be $45 \pm 9 \mathrm{~nm}$, which is about twice as large as the $22 \mathrm{~nm}$ size at this potential without $4 \mathrm{~nm} \mathrm{Au}$ NPs (Figure 6.2.(E)). The size histogram (Figure 6.9) shows the maximum number of particles remains in the size range of $40-50 \mathrm{~nm}$ in diameter. The size based on the $E_{p}$ values in ASV correlated well with the SEM images of the NPs after ripening.

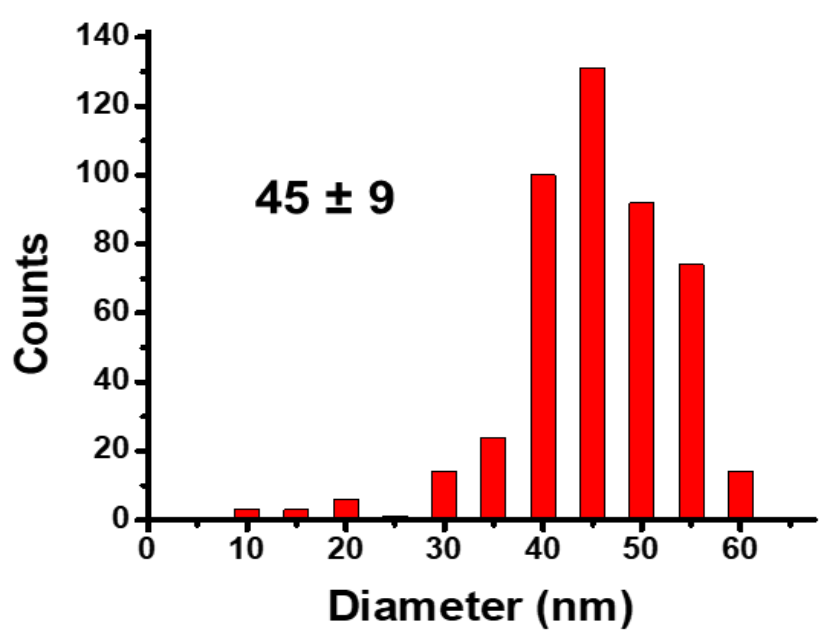

Figure 6. 9. Size histogram of 1:3 mixture of $4: 15 \mathrm{~nm}$ Au NPs after 140 min of ripening at $0.3 \mathrm{~V}$. 


\subsubsection{Monitoring Ostwald Ripening as a Function of Size by Electrochemical}

SA/V Measurements. The surface area-to-volume ratio (SA/V) is equal to 3/radius for spherical-shaped NPs. It is therefore a good method to measure NP size $($ radius $=3 \mathrm{~V} / \mathrm{SA})$. Measuring SA $/ \mathrm{V}$ as a function of time at a given Ostwald ripening potential allowed us to determine the average NP size as a function of ripening time. This allowed us to measure the average rate of ripening at different electrode potential for different Au NP sizes, dispersity, and coverage in order to better understand Ostwald ripening. Figure 6.10.(A) shows a CV of $15 \mathrm{~nm}$ Au NPs before (0 min) and after (105 min) of Ostwald ripening at $0.3 \mathrm{~V}$ in $0.1 \mathrm{M} \mathrm{HClO}_{4}$. The electroactive surface as determined by the integrated area of the reduction peak at $0.8 \mathrm{~V}$ decreased from $7.65 \times 10^{-6} \mathrm{C}$ to $5.76 \times 10^{-6} \mathrm{C}$ during that time. The decrease in SA is consistent with an increasing size of the Au NPs due to Ostwald ripening. A similar trend was observed for all sizes of Au NPs studied in this work. The volume (V) of the same sample was measured by perfming ASV in $\mathrm{Br}^{-}$ contianing electrolyte which gave the total intergrated charge of $3.96 \times 10^{-5} \mathrm{C}$ (Figure 6.10.(B)). The SA/V value for this sample was 0.145 , determined by dividing the charge for the peak at $0.8 \mathrm{~V}$ from the $\mathrm{CV}$ by the charge for the stripping peak in ASV $\left(5.76 \times 10^{-6} / 3.96 \times 10^{-5}=0.145\right)$. Similarly, we measured the SA/V ratios for all the sample we studied quantatvely for determinig the NPs size after Ostwald ripening. Table A1-A10 (apendex) show all data of SA/V obtained for different sized Au NPs for various conditions. 

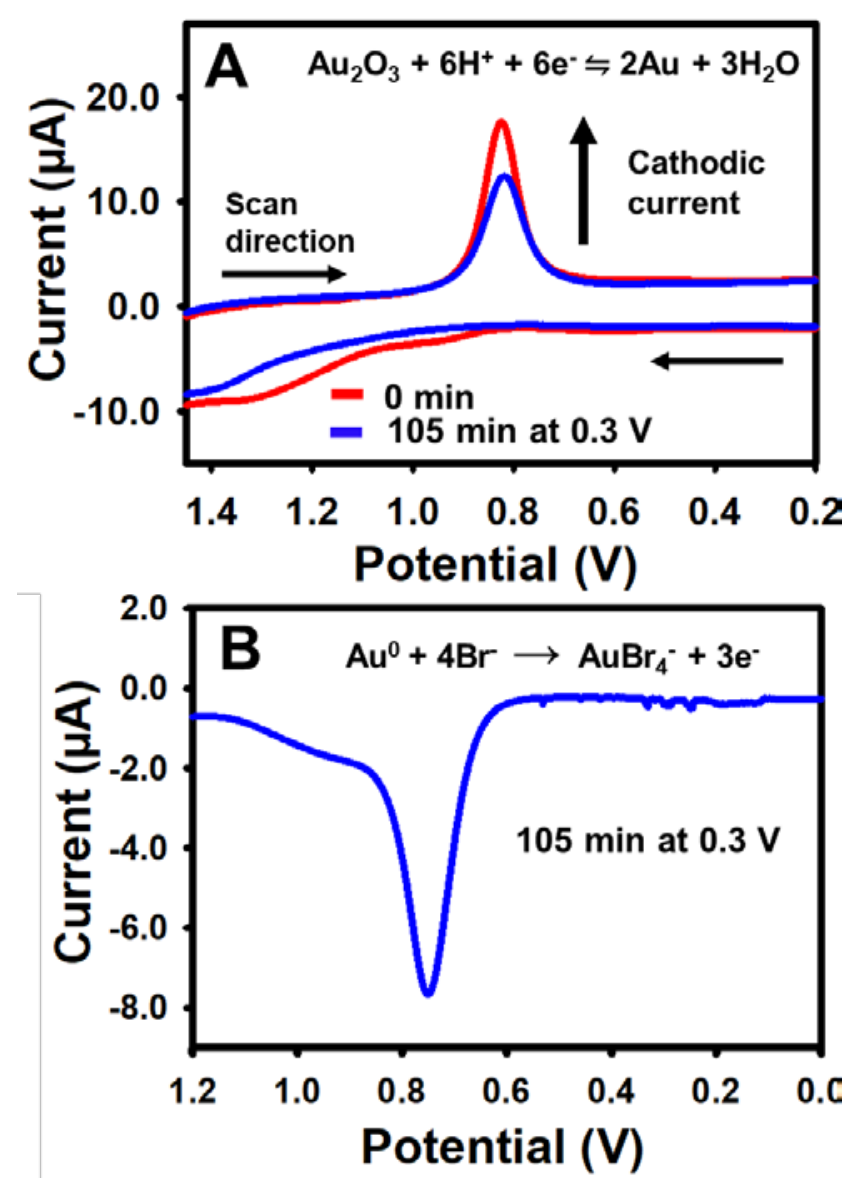

Figure 6. 10. (A) Cyclic voltammogram (CV) of $15 \mathrm{~nm}$ Au NPs before (0 min) and after $105 \mathrm{~min}$ of holding at $0.3 \mathrm{~V}$ vs $\mathrm{Ag} / \mathrm{AgCl}$ in $10 \mathrm{mM} \mathrm{KBr}$ plus $0.1 \mathrm{M} \mathrm{HClO}_{4}$ electrolyte solution. (B) ASV of the same electrode after 105 min of ripening at 0.3 V. ASV was performed in $10 \mathrm{mM} \mathrm{KBr}$ plus $0.1 \mathrm{~m} \mathrm{KClO}_{4}$ solution. The $\mathrm{CV}$ coverage (electroactive surface area, SA) decreases from $7.65 \times 10^{-6} \mathrm{C}$ to $5.76 \times 10^{-6} \mathrm{C}$ and the ASV coverage (total volume of NPs, V) $3.96 \times 10^{-5} \mathrm{C}$. The SA/V value before and after were 0.19 and after 0.14 which is corresponds to 22.4 and $35.9 \mathrm{~nm}$ diameter Au NPs respectively 
Table 6. 1. Microscopy measured radius and electrochemically measured surface area-to-total volume ratio (SA/V) of different sized Au NPs.

\begin{tabular}{|c|c|c|}
\hline $\begin{array}{c}\text { Number of } \\
\text { trials }\end{array}$ & $\begin{array}{c}\text { Microscopy } \\
\text { measured } \\
\text { radius }(\mathrm{nm})\end{array}$ & $\begin{array}{c}\text { Average } \\
\text { SA/V }\end{array}$ \\
\hline 4 & $0.75 \pm 0.2$ & $2.34 \pm 0.07$ \\
\hline 4 & $2.0 \pm 0.4$ & $0.64 \pm 0.03$ \\
\hline 4 & $7.5 \pm 0.8$ & $0.23 \pm 0.08$ \\
\hline 4 & $15.6 \pm 0.9$ & $0.10 \pm 0.01$ \\
\hline 4 & $25.1 \pm 1.5$ & $0.06 \pm 0.01$ \\
\hline 4 & $35.4 \pm 1.4$ & $0.04 \pm 0.01$ \\
\hline
\end{tabular}

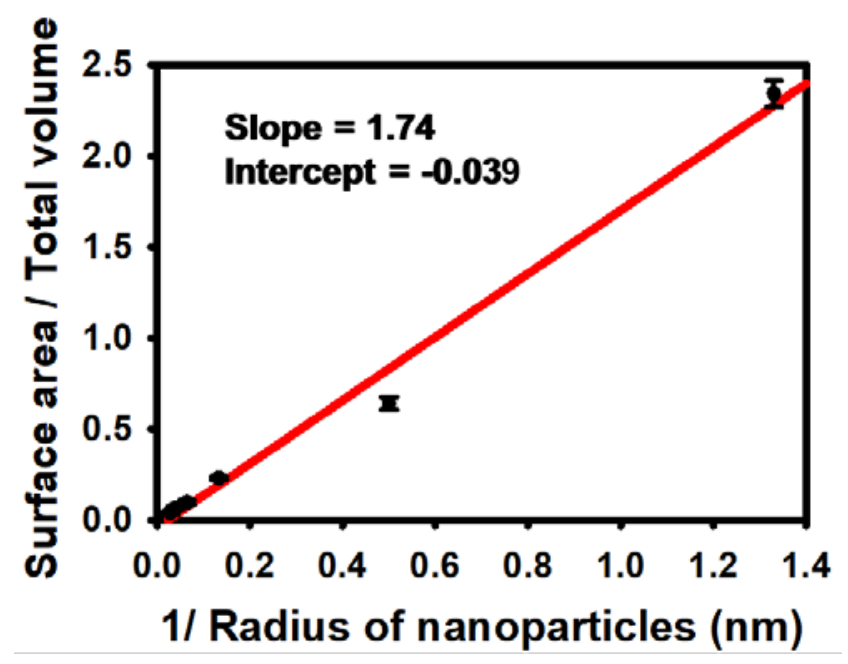

Figure 6. 11. Plot of electrochemically-measured surface area-to-total volume ratio (SA/V) as a function of 1 /radius as measured by TEM or SEM for 1.6, 4, 15, 31, 50, and $70 \mathrm{~nm}$ diameter Au NPs. The surface area was measured by surface $\mathrm{Au}$ oxide formation and reduction in $0.1 \mathrm{M} \mathrm{HClO}_{4}$ and the total volume was measured by complete Au stripping in $10 \mathrm{mM} \mathrm{KBr}$ plus $0.1 \mathrm{M} \mathrm{KClO}_{4}$ electrolyte. 
Table 6. 2. Calculated size of NPs based on electrochemically-measured SA/V

\begin{tabular}{|c|c|c|c|c|c|c|c|}
\hline $\begin{array}{c}\text { NPs } \\
\text { size }(n m)\end{array}$ & $\begin{array}{c}\text { Time of } \\
\text { ripening } \\
\text { (min) }\end{array}$ & $\begin{array}{l}\text { Calculated } \\
\text { sized of } \\
\text { NPs after } \\
\text { ripening at } \\
0.0 \mathrm{~V}\end{array}$ & \begin{tabular}{|c} 
Diameter \\
(final)/ \\
Diameter \\
(initial)
\end{tabular} & $\begin{array}{l}\text { Calculated } \\
\text { sized of } \\
\text { NPs after } \\
\text { ripening at } \\
0.30 \mathrm{~V}\end{array}$ & \begin{tabular}{|c|} 
Diameter \\
(final)/ \\
Diameter \\
(initial)
\end{tabular} & $\begin{array}{l}\text { Calculated } \\
\text { sized of } \\
\text { NPs after } \\
\text { ripening at } \\
0.45 \mathrm{~V}\end{array}$ & \begin{tabular}{|c|} 
Diameter \\
(final)/ \\
Diameter \\
(initial)
\end{tabular} \\
\hline \multirow{5}{*}{1.6} & 0 & & & $1.5 \pm 0.1$ & $1.00 \pm 0.1$ & & \\
\hline & 35 & & & $3.7 \pm 0.6$ & $2.47 \pm 0.4$ & & \\
\hline & 70 & & & $5.4 \pm 0.3$ & $3.56 \pm 0.3$ & & \\
\hline & 105 & & & $6.3 \pm 0.3$ & $4.51 \pm 0.4$ & & \\
\hline & 140 & & & $11.9 \pm 0.6$ & $6.11 \pm 0.5$ & & \\
\hline \multirow{5}{*}{4} & 0 & $\overline{5.6 \pm 0.2}$ & $1.00 \pm 0.05$ & $5.6 \pm 0.2$ & $1.00 \pm 0.05$ & $5.6 \pm 0.2$ & $1.00 \pm 0.05$ \\
\hline & 35 & $6.1 \pm 0.6$ & $1.09 \pm 0.1$ & $6.5 \pm 0.4$ & $1.16 \pm 0.08$ & $11.7 \pm 1.6$ & $2.09 \pm 0.3$ \\
\hline & 70 & $6.4 \pm 0.3$ & $1.14 \pm 0.07$ & $8.1 \pm 0.4$ & $1.44 \pm 0.09$ & $23.1 \pm 4.9$ & $4.13 \pm 0.9$ \\
\hline & 105 & $6.7 \pm 0.3$ & $1.20 \pm 0.07$ & $10.5 \pm 0.5$ & $1.88 \pm 0.1$ & $34.2 \pm 4.9$ & $6.11 \pm 0.9$ \\
\hline & 140 & $7.6 \pm 0.6$ & $1.35 \pm 0.1$ & $14.1 \pm 2.7$ & $2.52 \pm 0.5$ & $70.8 \pm 7.9$ & $12.64 \pm 1.5$ \\
\hline \multirow{5}{*}{15} & 0 & $18.0 \pm 1.0$ & $1.00 \pm 0.08$ & $18.0 \pm 1.0$ & $1.00 \pm 0.08$ & $18.0 \pm 1.0$ & $1.00 \pm 0.08$ \\
\hline & 35 & $19.9 \pm 2.7$ & $1.10 \pm 0.2$ & $22.5 \pm 2.9$ & $1.25 \pm 0.2$ & $25.3 \pm 3.2$ & $1.41 \pm 0.2$ \\
\hline & 70 & $21.2 \pm 0.5$ & $1.18 \pm 0.07$ & $28.1 \pm 1.0$ & $1.56 \pm 0.1$ & $34.5 \pm 0.9$ & $1.92 \pm 0.1$ \\
\hline & 105 & $21.9 \pm 0.6$ & $1.21 \pm 0.07$ & $32.8 \pm 2.3$ & $1.82 \pm 0.2$ & $43.6 \pm 3.6$ & $2.42 \pm 0.2$ \\
\hline & 140 & $22.2 \pm 1.6$ & $1.23 \pm 0.1$ & $40.3 \pm 5.0$ & $2.24 \pm 0.3$ & $60.3 \pm 9.0$ & $3.35 \pm 0.5$ \\
\hline \multirow{5}{*}{$\begin{array}{l}\text { Mix. of } \\
4 \& 15\end{array}$} & 0 & & & $14.4 \pm 1.3$ & $1.00 \pm 0.1$ & & \\
\hline & 35 & & & $23.5 \pm 1.5$ & $1.63 \pm 0.2$ & & \\
\hline & 70 & & & $33.7 \pm 0.7$ & $2.34 \pm 0.2$ & & \\
\hline & 105 & & & $53.8 \pm 9.3$ & $3.72 \pm 0.7$ & & \\
\hline & 140 & & & $115 \pm 36$ & $7.95 \pm 2.6$ & & \\
\hline
\end{tabular}


after Ostwald ripening for 0.0, 35, 70, 105 and $140 \min$ for 2, 4, 15 and 1:3

mixture of 4 and $15 \mathrm{~nm}$ Au NPs at different ripening potential $(0.0 \mathrm{~V}, 0.30 \mathrm{~V}$, and $0.45 \mathrm{~V})$.

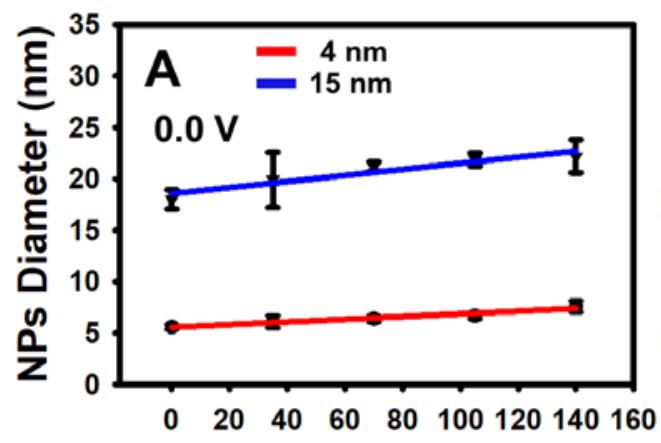

Ripening Time (min)

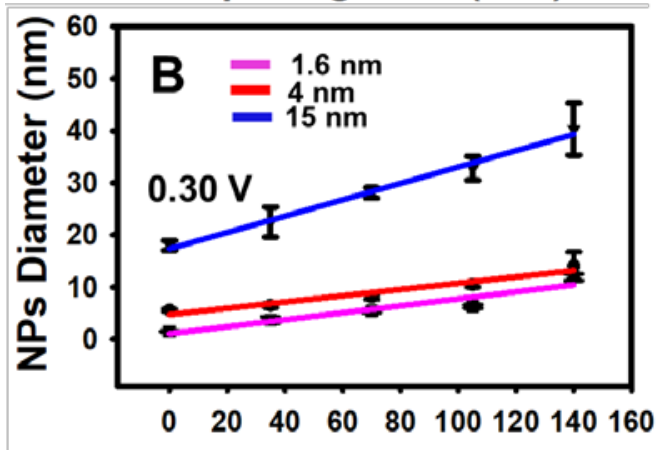

Ripening Time ( $\mathrm{min})$

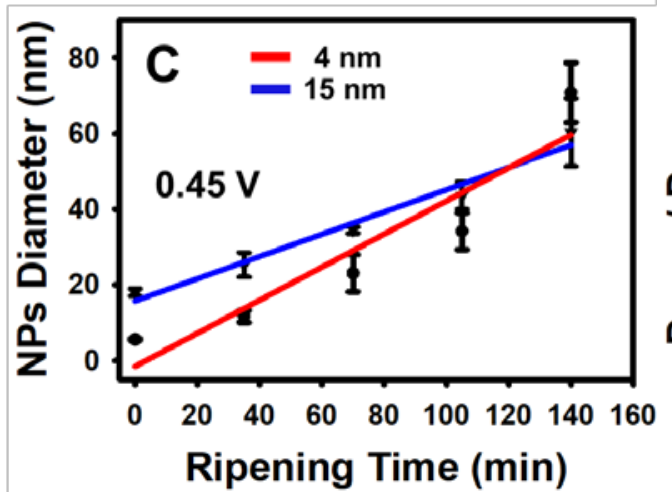

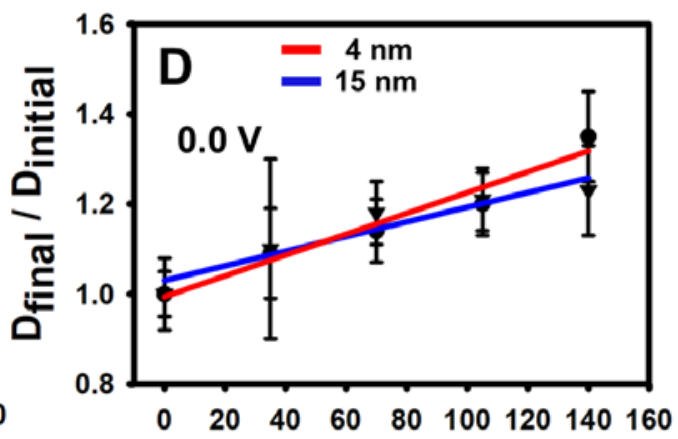

Ripening Time (min)

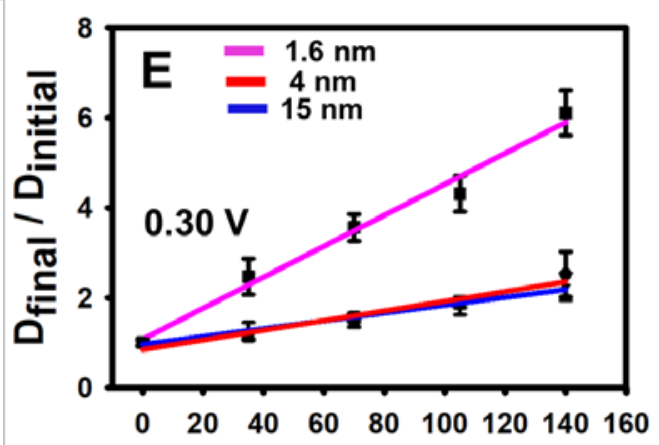

Ripening Time (min)

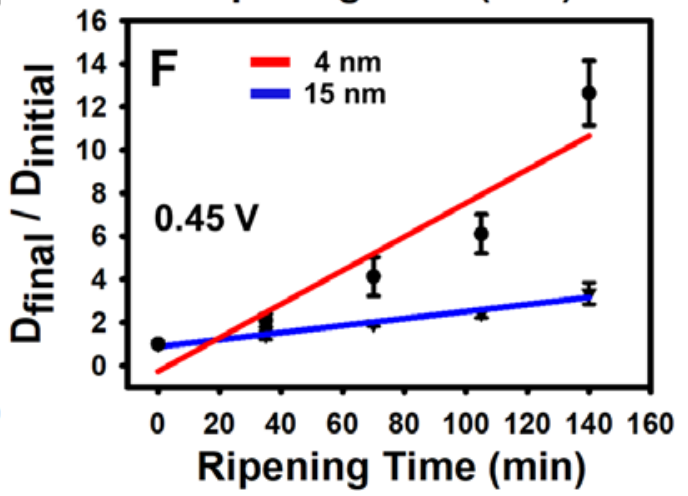

Figure 6. 12. Electrochemically-determined average NP diameter (A-C) and relative diameter (D-F) as a function of ripening time at a potential of $0.0 \vee(A, D)$, $0.30 \vee(B, E)$ and $0.45 \vee(C, F)$ for various sized and mixed Au NPs. 
We did not use $E_{p}$ values to determine the NP average size (and rate) because $E_{p}$ values can't accurately determine the size if the sample has high size dispersity and it does not distinguish sizes very well above about $30 \mathrm{~nm}$ in diameter. We converted the electrochemically-measured SA/V to NP diameter using the relation of $d=6$ VISA (where, $d$ is the diameter of NPs) for all sized NPs. Unfortunately, the SA/V ratio did not match well with the SEM measured size without first cleaning with ozone as reported by our group recently. ${ }^{201}$ In this work, we could not clean the NPs with ozone for Ostwald ripening studies because ozone causes NP size changes as reported by us $^{245}$ and others, ${ }^{25}$ especially for smaller $1.6 \mathrm{~nm}$ diameter Au NPs.

We instead used a calibration curve to determine the size of the NPs. The SA/V values for different sized Au NP standards is shown in Table 6.1. Figure 6.11 shows the SA/V as a function of $1 /$ radius of NPs for $1.6,4,15,32,50$ and $70 \mathrm{~nm}$ diameter Au NPs. The line of best fit was used to approximate the size of the Au NPs after different Ostwald ripening experiments. The size measured after Ostwald ripening by SA/V values was found to be very close to the SEM measured size of NPs. For example, based on the calibration curve the SA/V measured size of $1.6 \mathrm{~nm}$ diameter Au NPs after $140 \mathrm{~min}$ of ripening at $0.30 \mathrm{~V}$ was $11.9 \pm 0.6 \mathrm{~nm}$, which is very close to the SEM measured size of $13.4 \pm 4.5 \mathrm{~nm}$, indicating that SA/V measurements are an excellent method for determining the average NP size. Table 6.2. shows the electrochemically-measured size of NPs and the relative 
change in diameter (final diameter divided by initial diameter , Dinal / Dinitial) based on the SA/V measurements and the calibration curve for various ripening potentials and time.

Figure 6.12 shows the NP diameter and relative diameter, respectively, as a function of ripening time at $0.0 \mathrm{~V}$ (Figure 6.12.( $(A, D)), 0.30 \mathrm{~V}($ Figure 6.12.(B,E)), and $0.45 \mathrm{~V}$ (Figure 6.12. (C,F)) for 4 and15 nm Au NPs. At $0.0 \mathrm{~V}, 4 \mathrm{~nm}$ Au NPs showed about a 35\% increase in size after $140 \mathrm{~min}$, while the $15 \mathrm{~nm}$ Au NPs showed about $20 \%$ size increase. At $0.30 \mathrm{~V}$ (Figure 6.12. (B,E)) all samples changed more significantly with increasing ripening time. 4 and $15 \mathrm{~nm}$ diameter Au NPs increased by about a factor of 2 to 2.5 . The final diameter was larger for the $15 \mathrm{~nm}$ diameter due to the initially larger size, but the $4 \mathrm{~nm}$ diameter Au NPs increased by a higher percentage. The $1.6 \mathrm{~nm}$ diameter Au NPs increased to a similar final diameter as $4 \mathrm{~nm}$ but had a larger factor of 8 increase due to the initially smaller size. Figure 6.12. (C,F) shows the NP diameter as a function of ripening time at a potential $0.45 \mathrm{~V}$ for 4 and $15 \mathrm{~nm}$ Au NPs. Both sizes grew to a final 60$70 \mathrm{~nm}$ diameter size while the $4 \mathrm{~nm}$ Au NPs showed a higher relative change, growing by a factor of 13-14 as compared to 3-4 for the $15 \mathrm{~nm}$ Au NPs. 
Table 6. 3.Data of electrochemically measured NPs diameter/min and NPs diameter (\% increase per min) as a function of ripening potential for 1.6, 4,15 and mix of $4 \& 15 \mathrm{~nm}$ Au NPs at $0.0,0.30$ and $0.45 \mathrm{~V}$.

\begin{tabular}{|c|c|c|c|}
\hline $\begin{array}{c}\text { NPs } \\
\text { size } \\
(\mathrm{nm})\end{array}$ & $\begin{array}{c}\text { Ripening } \\
\text { potential } \\
(\mathrm{V})\end{array}$ & $\begin{array}{c}\text { NPs } \\
\text { diameter } \\
(\mathrm{nm} / \mathrm{min})\end{array}$ & $\begin{array}{c}\text { NPs diameter } \\
\text { (\%increase/min) }\end{array}$ \\
\hline \multirow{2}{*}{1.6} & 0.30 & 0.07 & 3.5 \\
\hline \multirow{3}{*}{4} & 0.00 & 0.01 & 0.23 \\
\cline { 2 - 4 } & 0.30 & 0.06 & 1.07 \\
\cline { 2 - 4 } & 0.45 & 0.42 & 7.8 \\
\hline \multirow{3}{*}{15} & 0.00 & 0.029 & 0.16 \\
\cline { 2 - 4 } & 0.30 & 0.16 & 0.87 \\
\cline { 2 - 4 } & 0.45 & 0.29 & 1.63 \\
\hline \multirow{2}{*}{ mix } & 0.30 & 0.67 & 4.57 \\
\hline
\end{tabular}



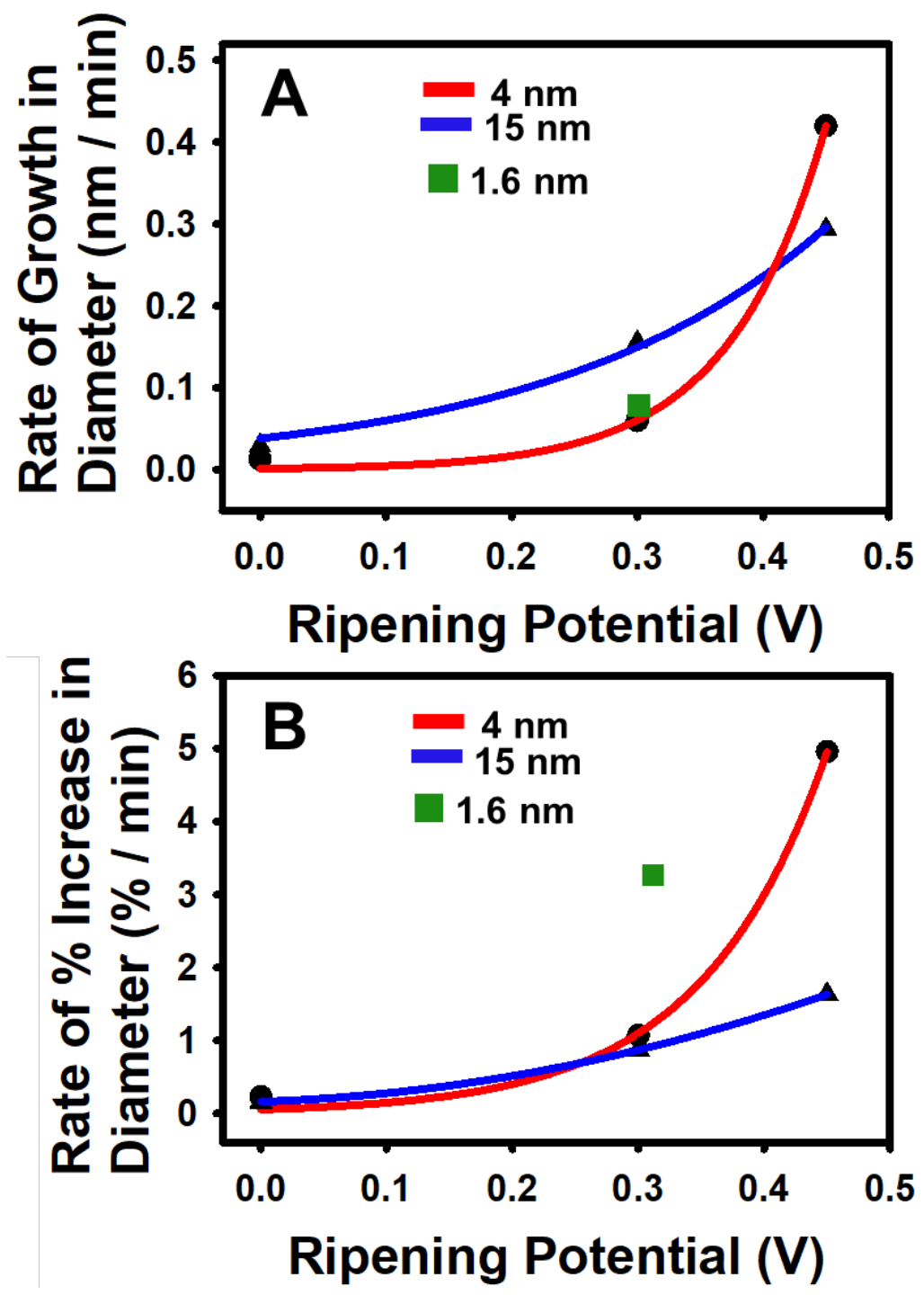

Figure 6. 13. Electrochemically measured NPs diameter/min (A) and \% increase of NPs diameter/min (B) as a function of ripening potential for 1.6, 4, and $15 \mathrm{~nm}$ Au NPs at $0.0,0.30$ and $0.45 \mathrm{~V}$. 
Next, we determined the slope of the best fit line of NPs diameter vs ripening time and Dfina/ $/ \mathrm{D}$ initial vs ripening time plot for all sized NPs and potential. The $\mathrm{R}$ value of the best fit line was higher than 0.92 indicating that our data fitted well with the linear lines. Table 6.3 shows the slope for NP diameter vs time plot (Table 6.3, column 3) and slope of $D_{\text {final/ }} / D_{\text {initial }}$ vs time plot (Table 6.3, column 4). We multiplied the slope of $D_{\text {final }} / D_{\text {initial }}$ vs time plot to show the percent increase. Figures 6.13. shows the plot of NPs diameter/min vs ripening potential (Figure 6.13.(A)) and \% increase of NPs diameter/min vs ripening potential (Figure 6.13. (B)) for all sized Au NPs. The ripening rate increased with an increase in ripening potential for all Au NPs studied. At $0.0 \mathrm{~V}$ the rate is low, but it starts to show an exponential increase for $4 \mathrm{~nm}$ Au NPs. We don't have ripening data at $0.0 \mathrm{~V}$ and $0.45 \mathrm{~V}$ for 1.6 $\mathrm{nm}$ Au NPs. However, we put the slope of best fit line of $1.6 \mathrm{~nm}$ Au NPs as shown in the green square box at $0.30 \mathrm{~V}$. It clearly shows that the $\%$ increase of NP diameter is very high for $1.6 \mathrm{~nm}$ Au NPs compared to individual 4 and $15 \mathrm{~nm} \mathrm{Au}$ NPs at $0.30 \mathrm{~V}$. We also measured the microscopy size of Au NPs at different time of ripening for $0.30 \mathrm{~V}$ and $0.45 \mathrm{~V}$. The average size of NPs before and after ripening are shown in table 6.4. We then plotted microscopically measured NP diameter vs ripening time and $D_{\text {final }} / D_{\text {initial }}$ vs ripening time for all Au NPs samples at 0.30 and $0.45 \mathrm{~V}$ (Figure 6.14). The results were found to be very close to the electrochemically-determined sizes. For example, $1.6 \mathrm{~nm}$ diameter Au NPs increased by a factor of 8 based on the microscopically measured size which is also a factor of 8 for the electrochemically-measured size. Similarly, $4 \mathrm{~nm}$ diameter Au NPs increase by a factor of 3.4 which is close to the electrochemically 
measured value of 2.5 for $4 \mathrm{~nm}$ Au NPs. This is indicating that our electrochemical measured size is very closely matches with the microscopically measured size of NPs. 
Table 6. 4. Microscopy measured size of various Au NPs after different ripening times and different ripening potentials.

\begin{tabular}{|c|c|c|c|c|c|}
\hline $\begin{array}{l}\text { NPs } \\
\text { size } \\
(n m)\end{array}$ & $\begin{array}{c}\text { Time of } \\
\text { ripening } \\
\text { (min) }\end{array}$ & $\begin{array}{c}\text { Microscopic } \\
\text { sizes }(\mathrm{nm}) \\
\text { of NP after } \\
\text { ripening at } \\
0.30 \mathrm{~V}\end{array}$ & $\begin{array}{c}\text { Diameter } \\
\text { (final)/ } \\
\text { Diameter } \\
\text { (initial) at } \\
0.30 \mathrm{~V}\end{array}$ & $\begin{array}{c}\text { Microscopic } \\
\text { sizes (nm) } \\
\text { of NPs after } \\
\text { ripening at } \\
0.45 \mathrm{~V}\end{array}$ & $\begin{array}{l}\text { Diameter } \\
\text { (final)/ } \\
\text { Diameter } \\
\text { (initial) at } \\
0.45 \mathrm{~V}\end{array}$ \\
\hline \multirow{5}{*}{1.6} & 0 & $1.6 \pm 0.4$ & $1.0 \pm 0.3$ & & \\
\hline & 35 & & & & \\
\hline & 70 & $7.15 \pm 3.6$ & $4.5 \pm 2.5$ & & \\
\hline & 105 & & & & \\
\hline & 140 & $13.4 \pm 4.5$ & $8.4 \pm 3.5$ & & \\
\hline \multirow{5}{*}{4} & 0 & $4.1 \pm 0.7$ & $1.0 \pm 0.2$ & $4.1 \pm 0.7$ & $1.0 \pm 0.2$ \\
\hline & 35 & $7.1 \pm 1.5$ & $1.7 \pm 0.5$ & $10.2 \pm 3.4$ & $2.5 \pm 0.9$ \\
\hline & 70 & $8.3 \pm 4.7$ & $2.0 \pm 1.1$ & $13.3 \pm 5.1$ & $3.2 \pm 1.3$ \\
\hline & 105 & $9.9 \pm 3.8$ & $2.4 \pm 1.0$ & $17.0 \pm 7.0$ & $4.1 \pm 1.8$ \\
\hline & 140 & $14.4 \pm 6.0$ & $3.5 \pm 1.6$ & $26.5 \pm 14$ & $6.5 \pm 3.6$ \\
\hline \multirow{5}{*}{15} & 0 & $15.1 \pm 1.6$ & $1.0 \pm 0.1$ & $15.1 \pm 1.6$ & $1.0 \pm 0.1$ \\
\hline & 35 & $16.1 \pm 2.8$ & $1.1 \pm 0.2$ & $17.9 \pm 3.2$ & $1.2 \pm 0.2$ \\
\hline & 70 & $17.6 \pm 3.7$ & $1.2 \pm 0.3$ & $19.0 \pm 4.7$ & $1.3 \pm 0.4$ \\
\hline & 105 & $19.3 \pm 4.5$ & $1.3 \pm 0.3$ & $21.3 \pm 5.1$ & $1.4 \pm 0.4$ \\
\hline & 140 & $22.0 \pm 7.5$ & $1.5 \pm 0.5$ & $26.1 \pm 7.0$ & $1.7 \pm 0.5$ \\
\hline \multirow{5}{*}{$\begin{array}{c}\text { Mix. } 4 \& \\
15\end{array}$} & 0.0 & $11.0 \pm 4.0$ & $1.0 \pm 0.5$ & & \\
\hline & 35 & $17 \pm 5.0$ & $1.5 \pm 0.7$ & & \\
\hline & 70 & $24 \pm 6.0$ & $2.2 \pm 1.0$ & & \\
\hline & 105 & $31 \pm 7.5$ & $2.8 \pm 1.2$ & & \\
\hline & 140 & $45.5 \pm 9.0$ & $4.1 \pm 1.7$ & & \\
\hline
\end{tabular}



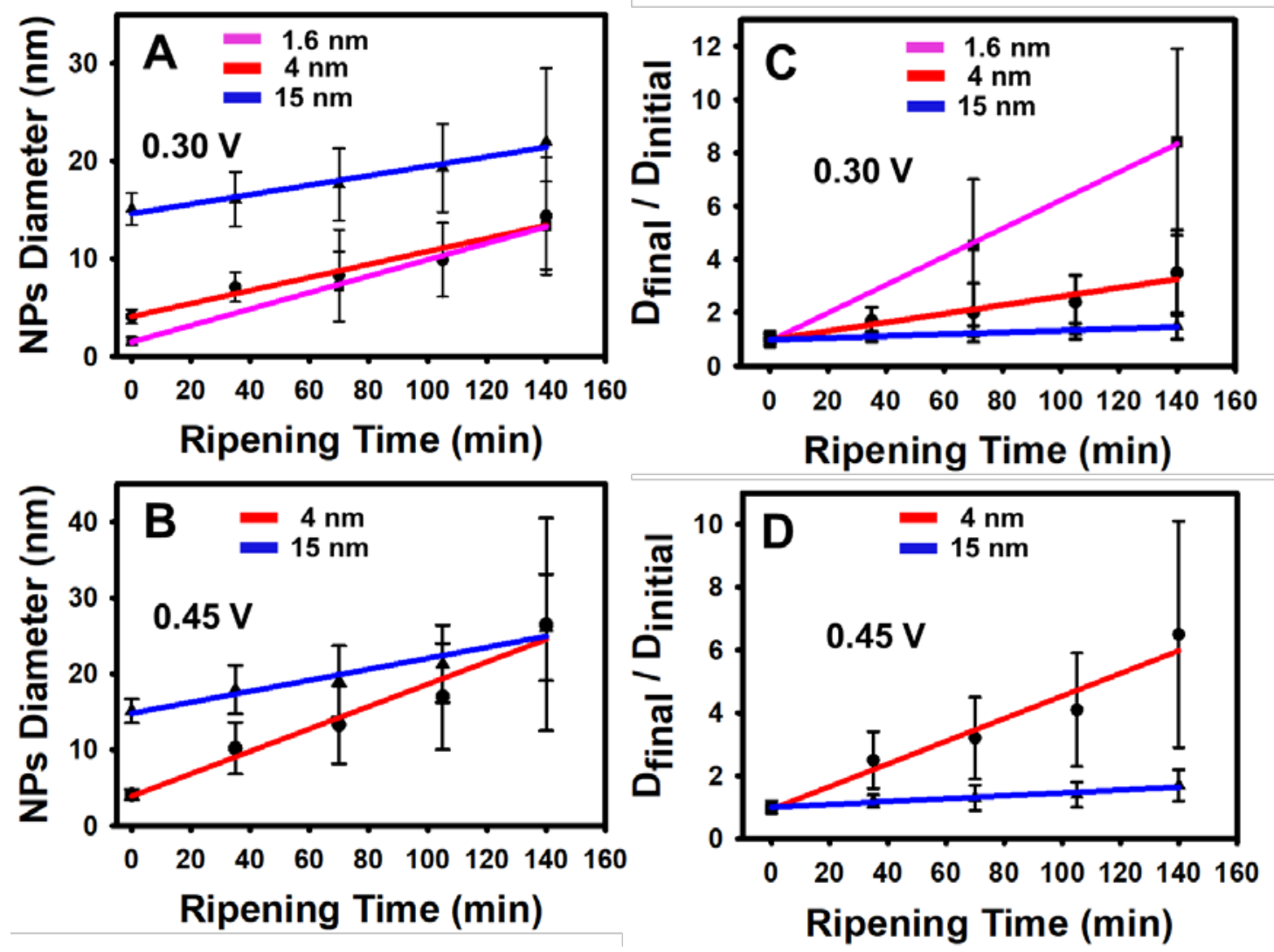

Figure 6. 14. SEM determined NP diameter vs ripening time and $D_{\text {final }} / D_{\text {initial }}$ vs ripening time at $0.30 \mathrm{~V}(\mathrm{~A}, \mathrm{C})$ and $0.45 \mathrm{~V}(\mathrm{~B}, \mathrm{D})$ for all Au NPs samples. 

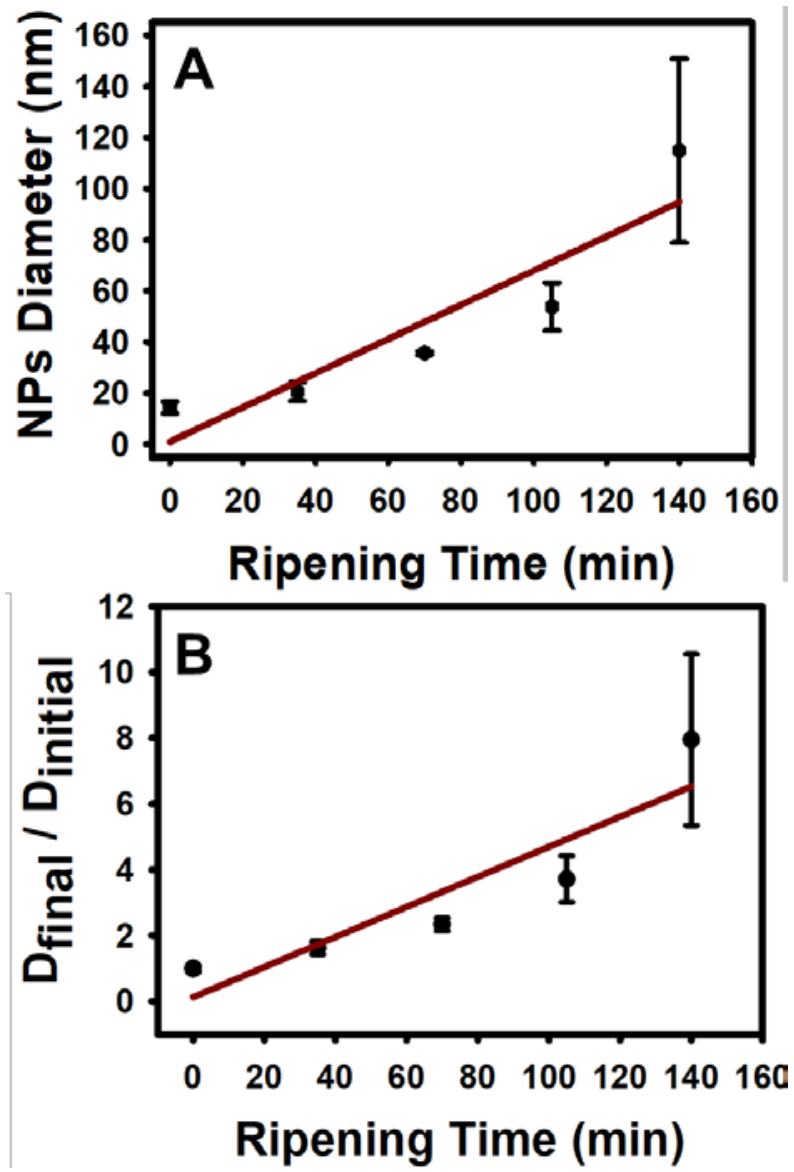

Figure 6. 15. Electrochemically determined NP diameter vs ripening time $(A)$ and $D_{\text {final }} / \mathrm{D}_{\text {initial }}$ Vs ripening time $(\mathrm{B})$ at $0.30 \mathrm{~V}$ for $1: 3$ mixture of 4 and $15 \mathrm{~nm}$ Au NPs samples. 

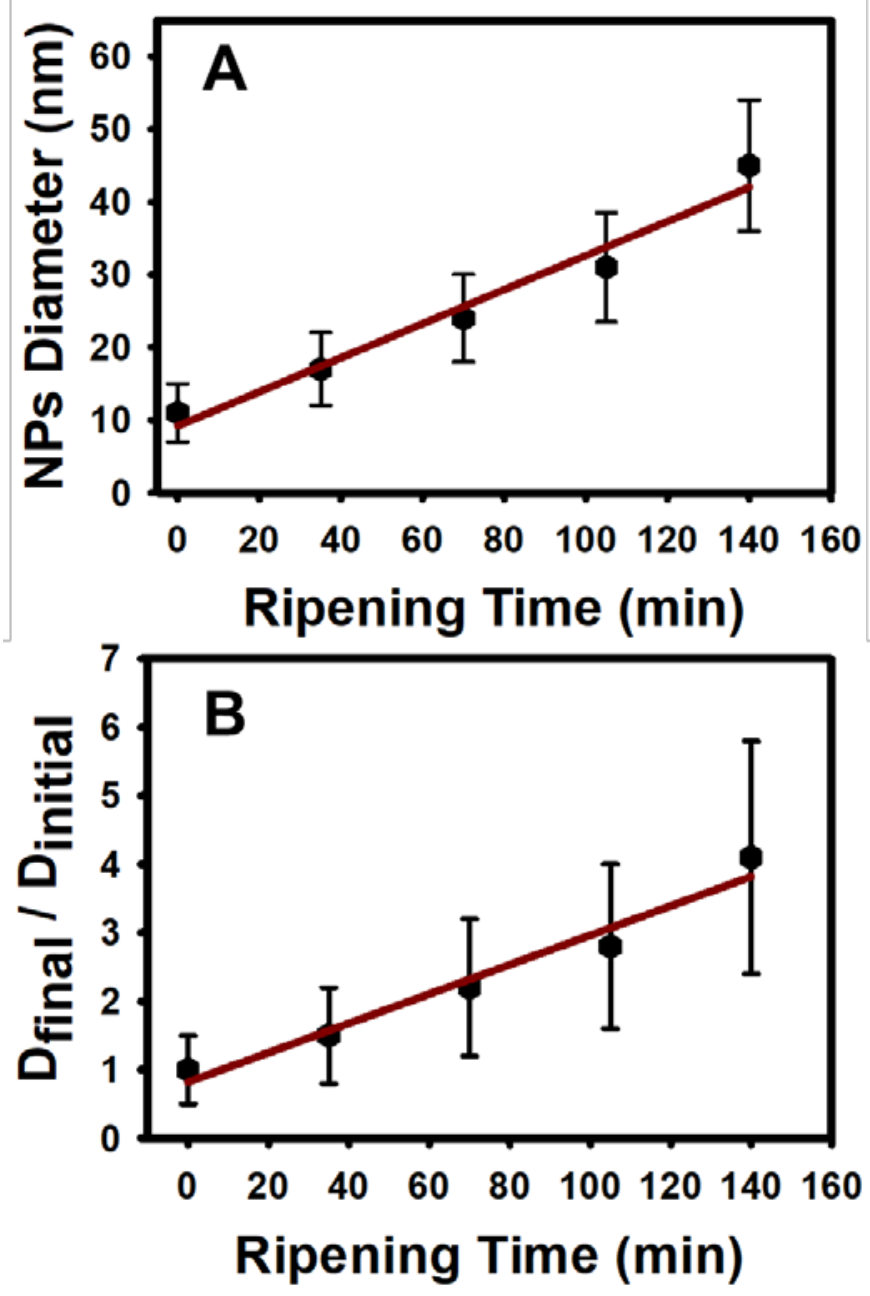

Figure 6. 16. SEM determined NP diameter vs ripening time $(A)$ and $D_{\text {final }} / D_{\text {initial }}$ vs ripening time (B) at $0.30 \mathrm{~V}$ for $1: 3$ mixture of 4 and $15 \mathrm{~nm}$ diameter Au NPs samples. 


\subsubsection{Monitoring Ostwald Ripening as a Function of NP Size Dispersity and}

Coverage by Electrochemical SA/V. We monitored the effect of NPS size dispersity and coverage on the Ostwald ripening of NPs. Figure 6.15. (A\&B) show the NP diameter and relative diameter respectively, as a function of ripening time at $0.3 \mathrm{~V}$ for $1: 3$ mixture of 4 and $15 \mathrm{~nm}$ Au NPs. The 4 and $15 \mathrm{~nm}$ diameter mixed sample showed an extremely large ripening rate in terms of total final diameter $(\sim 120 \mathrm{~nm})$ and size increase relative to the initial $15 \mathrm{~nm}$ diameter NPs ( $800 \%)$ for electrochemical measured NPs diameter. Size measured by microscopic method showed the $-300 \%$ increase in size relative to the initial size of $15 \mathrm{~nm}$ (figure 6.16.( A\&B)). We also determined the slope of the best fit line of NPs diameter vs ripening time and $D_{\text {final }} / \mathrm{D}_{\text {initial }}$ Vs ripening time plot. The slope of found to be 0.67 $\mathrm{nm} / \mathrm{min}$ and 4.63 diameter $\%$ increase per min as shown in table 6.3. The slope value (rate) is significantly higher than individual 4 and $15 \mathrm{~nm}$ Au NPs, indicates that the dispersity of NPs significantly effects the Ostwald ripening rate.

Table 6.5. Data of electrochemically measured NPs diameter/min and NPs diameter (\% increase per min) as a function of coverage for $15 \mathrm{~nm}$ Au NPs at 0.30 V.

\begin{tabular}{|c|c|c|}
\hline $\begin{array}{c}\text { NPs } \\
\text { coverage } \\
\text { (NPs/ } / \mu \mathrm{m} 2)\end{array}$ & $\begin{array}{c}\text { NPs } \\
\text { diameter } \\
\text { (nm/min) }\end{array}$ & $\begin{array}{c}\text { NPs diameter } \\
\text { (\%increase/min) }\end{array}$ \\
\hline $1.0-1.3$ & 0.0814 & 0.43 \\
\hline $7.0-8.0$ & 0.159 & 0.89 \\
\hline $40-51$ & 1.136 & 4.36 \\
\hline
\end{tabular}



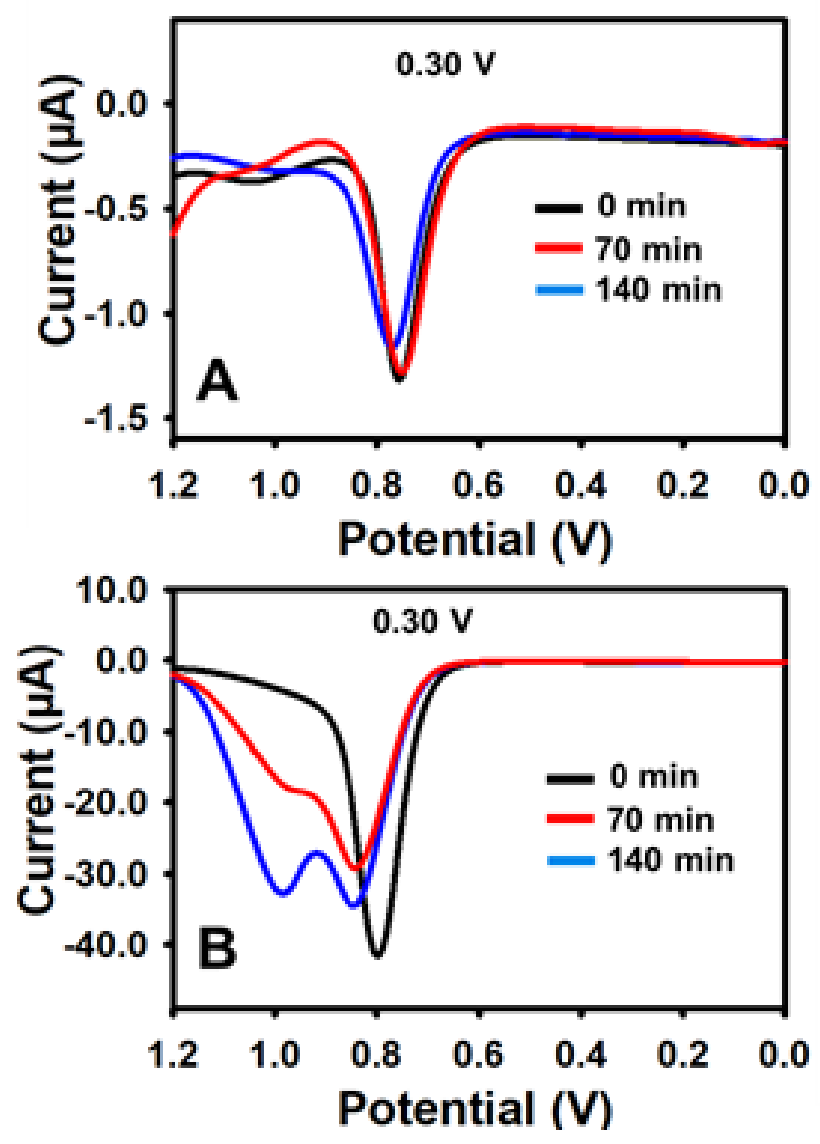

Figure 6. 17. ASVs of citrate-stabilized $15 \mathrm{~nm}$ diameter Au NPs after Ostwald ripening at $0.3 \mathrm{~V}$ with the coverage of $(\mathrm{A}) 1.1-1.3 \mathrm{NPs} / \mu^{2}$ (or $(5.0-6.5) \times 10^{-6} \mathrm{C}$ ) and (B) 39.5-51.3 NPs $/ \mu^{2}$ (or (2.0-2.6) $\times 10^{-4} \mathrm{C}$ ). ASVs were obtained in $10 \mathrm{mM}$ $\mathrm{KBr}$ plus $0.1 \mathrm{M} \mathrm{KClO}_{4}$ electrolyte with a scan rate of $0.01 \mathrm{~V} / \mathrm{s}$. 

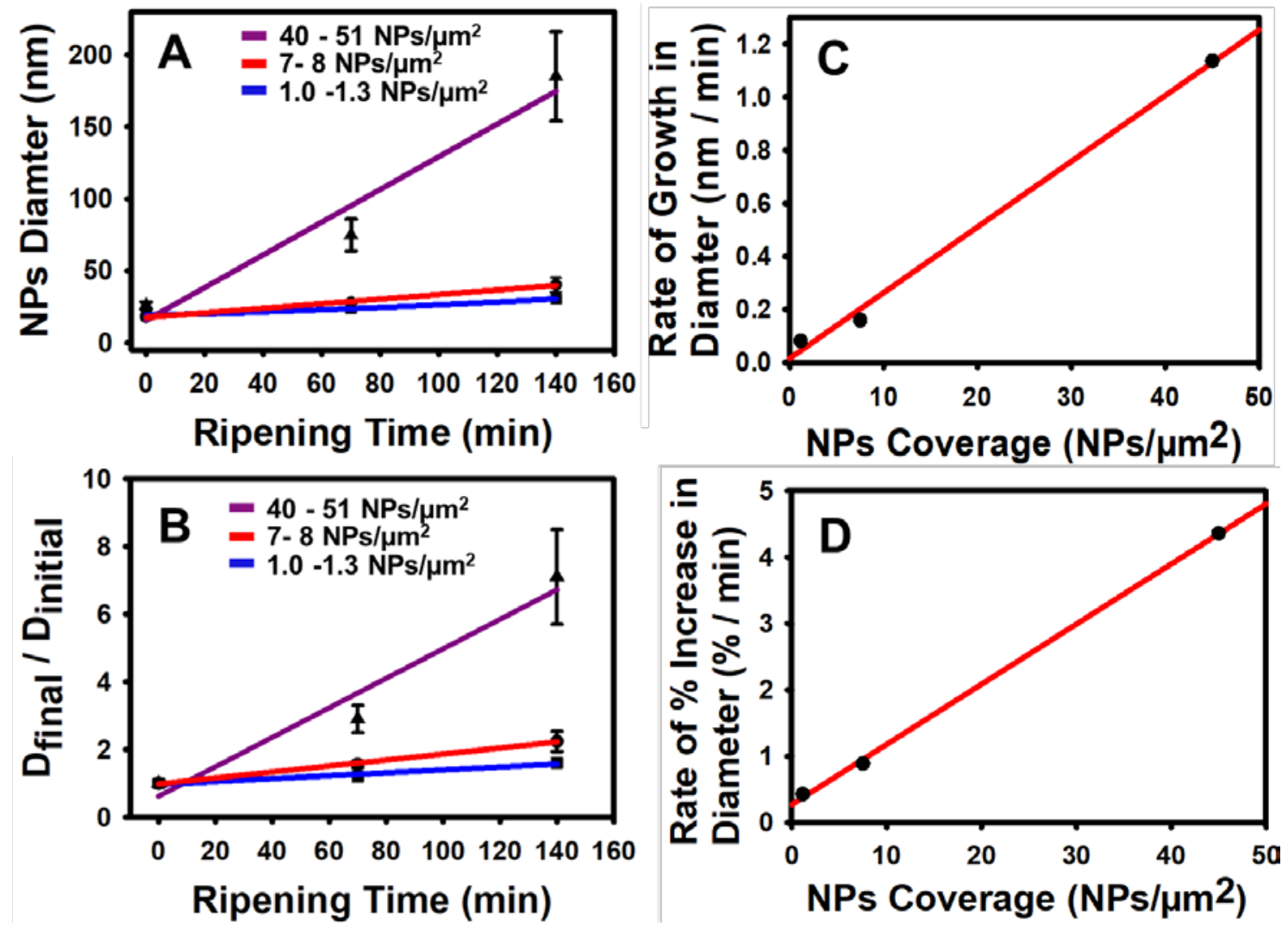

Figure 6. 18. NP diameter vs ripening time $(A)$ and $D_{\text {final }} / D_{\text {initial }}$ vs ripening time (B) for $15 \mathrm{~nm}$ diameter Au NPs at $0.30 \mathrm{~V}$ for various coverage of NPs on the electrode surface as indicated. NPs diameter/min (C) and \% increase of NPs diameter/min (D) as a function of NPs coverage. 
Also, we hypothesized that the coverage of NPs on the electrode surface would also play a significant role on the rate of Ostwald ripening since the $\mathrm{AuBr}_{\mathrm{x}}{ }^{-}$ ions from a smaller dissolving Au NP has to diffuse to a larger Au NP before deposition. Close proximity of the Au NPs should increase the ripening rate and allow greater collection of the $\mathrm{AuBr}_{\mathrm{x}}{ }^{-}$ions at the larger NPs. Accordingly, we studied the effect of coverage on the ripening rate for $15 \mathrm{~nm}$ Au NPs at a potential of $0.30 \mathrm{~V}$ vs Ag/AgCl. The coverage was determined from the ASV of the $15 \mathrm{~nm}$ Au NPs, which was (5.0-6.5) $\times 10^{-6} \mathrm{C}$ and (2.0-2.6) $\times 10^{-4} \mathrm{C}$ for low and high coverage, respectively. This corresponds to about 1 and $45 \mathrm{NPs} / \mu \mathrm{m}^{2}$, respectively. The $E_{p}$ and SA/V ratio of $15 \mathrm{~nm}$ Au NPs at low coverage did not show any significant change with ripening time as shown in Figure 6.17.(A). However, the $E_{p}$ and overall SA/ $V$ ratio changed significantly for higher coverage as shown in Figure 6.17. (B), showing that the average NP size increased due to significant ripening. We plotted the NP diameter vs ripening time and $D_{\text {final }} / D_{\text {initial }}$ vs ripening time for the coverage range of $(5.0-6.5) \times 10^{-6} \mathrm{C},(3.5-4.2) \times 10^{-5} \mathrm{C}$ and $(2.0-2.6) \times$ $10^{-4} \mathrm{C}$ at a ripening potential of $0.30 \mathrm{~V}$ as shown in the Figure 5.18.( $\mathrm{A}$ and $\left.\mathrm{B}\right)$. For

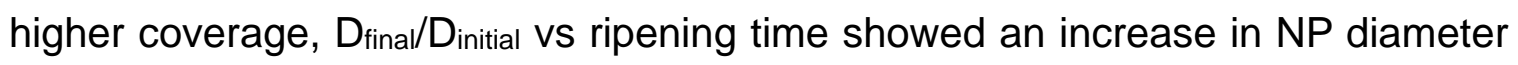
by about 8 times but for the lower two coverages it showed an increase of only 2 and 1.3 times, respectively. These results clearly confirm that the rate of Ostwald ripening increases with an increase in NP coverage on the electrode surface. Furthermore, we determined the slope of the best fit line of NPs diameter vs ripening time and $D_{\text {tinal }} / \mathrm{D}_{\text {initial }}$ vs ripening time plot of figure 6.18. (A) and 6.18. (B) respectively for various coverage of $15 \mathrm{~nm}$ diameter Au NPs at potential $0.3 \mathrm{~V}$. 
Table 6.5 shows the slope for NP diameter vs ripening time plot (Table 6.5, column 2) and slope of $D_{\text {final }} / D_{\text {initial }}$ vs time plot (Table 6.5 , column 3) for three different coverage of $15 \mathrm{~nm}$ Au NPs as indicated in table 6.5, column 1. We multiplied the slope of $D_{\text {final }} / D_{\text {initial }} v s$ ripening time plot to show the percent increase. Figures 6.18.( C\& D) shows the plot of NPs diameter/min vs NPs coverage (Figure 6.18.(C)) and \% increase of NPs diameter/min vs NPs coverage (Figure 6.18.(D)) for $15 \mathrm{~nm}$ diameter Au NPs studied at potential 0.3 V. Figure 6.18. (C \& D) clearly show that ripening rate increased linearly with an increase of NPs coverage.

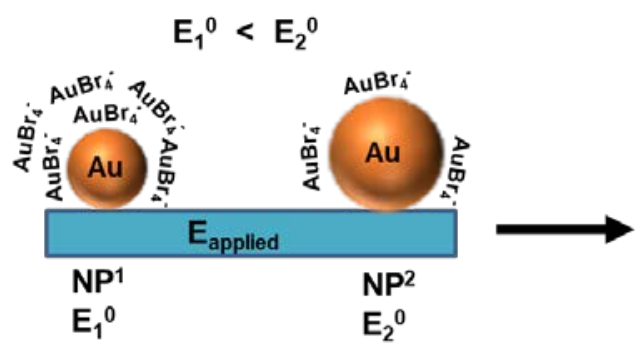

Step 1: Dissolution of $\mathrm{Au}$

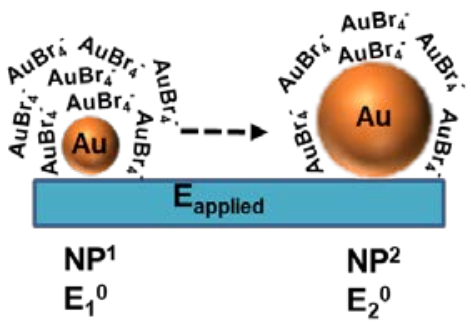

Step 2: Diffusion of Au

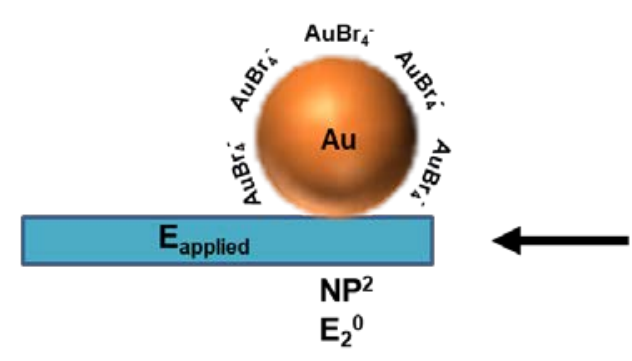

Step 4: Equilibrium Au

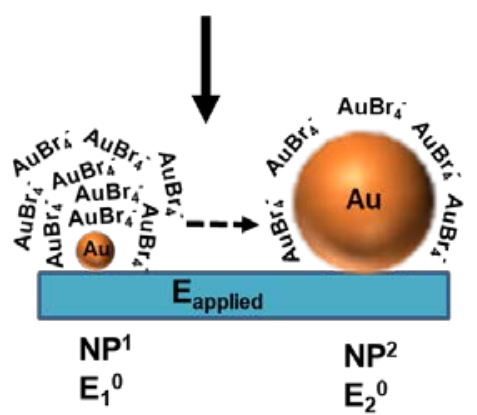

Step 3: Deposition of $\mathrm{Au}$

Figure 6. 19. Mechanism of electrochemically induced Ostwald ripening of Au NPs attached to glass/ITO electrode. 
Based on our work, Ostwald ripening of Au NPs occurs by multistep reactions and several factors controlled the rate of ripening as shown in figure 6.19. Oxidation is the first step of Ostwald ripening. The smaller Au NPs dissolve at lower electrochemical potentials and with faster rates. At potentials below the $E_{p}$ value, the particles are not completely dissolved, but a small amount of dissolution from the NPs that are much smaller than the average size occurs based on the following Nernst equation, assuming $3 \mathrm{e}^{-}$oxidation of $\mathrm{Au}$ is with $\mathrm{Br}^{-}$dominant electrochemical reaction:

$$
E=E^{0}+(0.0592 / 3) \log \left(\left[\mathrm{AuBr}_{4}^{-}\right]\left[\mathrm{Br}^{-}\right]^{4}\right)
$$

Solving for the concentration of dissolved Au in terms of $\mathrm{AuBr}_{4}{ }^{-}$in the Nernst equation gives:

\section{$\left[\mathrm{AuBr}_{4}{ }^{-}\right]=[\mathrm{Br}]^{4} 10^{(3(\mathrm{E}-\mathrm{E} 0) / 0.0592)}$}

Where, $E$ is the applied potetnial and $E^{0}$ is the size-dependent standard potential for the electrochemical half reaction of Au oxidation in $\mathrm{Br}^{-}$. Since $[\mathrm{Br}-]$ is constant (in excess), $\left[\mathrm{AuBr}_{4}^{-}\right]$next to the Au NPs depends exponentially on how large $E$ is relative to $\mathrm{E}^{0}$, or the magnitude of $\mathrm{E}-\mathrm{E}^{0}$. Since $\mathrm{E}$ is constant, but $\mathrm{E}^{0}$ is smaller for smaller Au NPs, this means that there will be a larger [ $\left.\mathrm{AuBr}_{4}{ }^{-}\right]$next to smaller $\mathrm{Au}$ NPs and smaller [AuBr4$\left.{ }^{-}\right]$next to larger Au NPs as shown in figure 6.19, step 2. The concentration gradient leads to diffusion of $\mathrm{AuBr}_{4}{ }^{-}$from the small NPs to the large NPs. This causes $\left[\mathrm{AuBr}_{4}{ }^{-}\right]$to be too large according to the Nernst equation at the large Au NPs, leading to Au deposition on the larger Au NPs (figure 6.19, step 3). As the larger Au NPs become even larger, their $E^{0}$ value shifts more positive, which further drives reduction of $\mathrm{AuBr}_{4}{ }^{-}$in solution by deposition onto the 
Au NPs. This causes those NPs to grow larger until equilibrium is established with $\mathrm{AuBr}_{4}{ }^{-}$in solution (figure 6.19, step 4). In contrast, the $\mathrm{E}^{0}$ shifts more negative as the smaller Au NPs dissolve. The combination of this and diffusion of $\mathrm{AuBr}_{4}{ }^{-}$away from the small NPs leads to further dissolution of those Au NPs until they eventually fully dissolve. The variation of the $E^{0}$ values for different-sized Au NPs leads to different local $\left[\mathrm{AuBr}_{4}^{-}\right]$at the different-sized Au NPs, and then diffusion and the changing $\mathrm{E}^{0}$ values as large NPs grow and small NPs dissolve lead to the Ostwald ripeneing process. Based on this description, the ripening rate depends on the value of $E$ relative to the average $E^{0}$ of the sample, the variation of $E^{0}$ values within the sample, and the distance between Au NPs. The variation of $E^{0}$ within the sampe and distance between the Au NPs control the diffusion gradient and flux caused by diffusion. The value of $E$ relative to the average value of $E^{0}$ determines how much dissolution and overall $\left[\mathrm{AuBr}_{4}{ }^{-}\right]$appears in the solution. Our data on potential, size, size dispersity, and coverage are consistent with the Ostwald ripening model.

\subsection{CONCLUSIONS}

We report the size dependent Ostwald ripening reactivity of 1.6, 4 and 15 $\mathrm{nm}$ diameter Au NPs under control potential. The size change before and after ripening at different potential and time were monitored by both electrochemical and microscopic methods. Our results show smaller sized Au NPs display a higher rate of Ostwald ripening as compared to larger size Au NPs under similar electrochemical conditions. The rate of Ostwald ripening also depends on the 
dispersity of NPs. A mixture of two different sized NPs displayed a higher rate of ripening as compared to the individual NPs under the same reaction conditions due to the larger variation in $\mathrm{E}-\mathrm{E}^{0}$ at different NPs. The mechanism of Ostwald ripening was confirmed from the SEM measured size and size histogram of NPS before and after ripening. The size histogram shows larger size variation after ripening, indicating that NPs grew bigger in size at the expense of others that reduced in size. The Ostwald ripening of Au NPs, as confirmed by ASV, showed two distinct oxidation peaks at 0.75 and $0.95 \mathrm{~V}$ for two different NP populations after ripening, but also showed multiple small oxidation peaks in the potential range of $0.0-0.45 \mathrm{~V}$ vs Ag/AgCl. These peaks represent small Au NPs which were formed during the dissolution of bigger-sized Au NPs, also confirming Ostwald ripening. The rate of Ostwald ripening rate increased dramatically with the increasing NP coverage, showing the importance of diffusion of $\mathrm{AuBr}_{x}{ }^{-}$species from small to larger NPs. The rate of Ostwald ripening determined from the electrochemicallymeasured SA/V of Au NPs was comparable to the rate measured by SEM, but much faster and simpler. Our work clearly shows the importance of electrochemical methods as analytical tools to understand the fundamental reactivity and size stability of metals on the nanoscale. This work may lead to improved NP size stability but also new electrochemical methods to control NP sizes on electrodes surfaces. 


\section{CHAPTER VII}

\section{SIZE DEPENDENT GALVANIC EXCHANGE BETWEEN Au NANOPARTICLES WITH $\mathrm{Ag}^{+}$IONS}

\subsection{INTRODUCTIONS}

Recently, the formation of bimetallic nanostructures by a galvanic exchange reaction has become very popular as it provides enormous opportunities to engineer new nanomaterials for catalytic and sensing applications. Galvanic exchange is a simple oxidation/reduction reaction which involves the spontaneous reduction of a noble-metal cation by a less noble metal in a solution driven by the difference in standard potential of the two metals. Galvanic replacement has been used recently for engineering various nanomaterials including hollow nanocubes, ${ }^{109}$ nanotubes, nanorattles, ${ }^{110}$ and nanocages. ${ }^{111}$ For example, Murshid and coworkers showed a galvanic exchange reaction between Ag decahedral NPs with $\mathrm{Au}(\mathrm{III})$ complexes to form stable core/shell Ag/Au decahedral alloy NPs. ${ }^{112}$ The following reaction is an example of a galvanic exchange reaction between $\mathrm{Ag}$ metal and $\mathrm{Au}(\mathrm{III})$ complex ions:

$3 \mathrm{Ag}(\mathrm{S})+\mathrm{AuCl}_{4}^{-}(\mathrm{aq}) \rightarrow \mathrm{Au}(\mathrm{s})+\mathrm{Ag}+(\mathrm{aq})+4 \mathrm{Cl}^{-}(\mathrm{aq})$

Other than the traditional galvanic exchange reaction there have been reports on thermodynamically unfavorable galvanic exchange reactions, which 
have been termed anti-galvanic exchange. The occur in the opposite direction of the thermodynamically favorable reaction. Reactions between Au clusters and metal ions of $\mathrm{Ag}, \mathrm{Hg}, \mathrm{Cd}$, and $\mathrm{Cu}$ fall into this category. Murray and coworkers observed anti-galvanic exchange between thiolate stabilized $\mathrm{Au}_{25}$ nanoclusters and $\mathrm{Ag}^{+}$and $\mathrm{Cu}^{2+}$ ions for the first time. ${ }^{113}$ They reported that the addition of $\mathrm{Ag}^{+}$ ions onto the $\mathrm{Au}_{25}$ nanocluster was possible because of the thiolate ligands, which gain a partial negative charge after attaching to the Au surface. This negative charge assists in the reduction of $\mathrm{Ag}^{+}$ions on the surface of the Au cluster. Later, Wu and coworkers reported that anti-galvanic exchange between $\mathrm{Ag}^{+}$ions and a thiol stabilized neutral Au nanoclusters is only possible if the Au nanocluster size is lower than $3 \mathrm{~nm}$ in diameter. ${ }^{114}$ These findings indicated that the negative charge of thiolate ligands do not affect the anti-galvanic exchange reaction. Wang and coworkers reported the anti-galvanic exchange reaction of naked Au NPs. ${ }^{115}$ Ligand-free naked Au NPs were obtained by the reduction of $\mathrm{Au}^{+}$ions with $\mathrm{NaBH}_{4}$ and the resultant aggregated NPs were dispersed by the laser ablation method. In this process they were able to get well dispersed Au NPs with the average size of $13.5 \pm 0.5 \mathrm{~nm}$ in diameter. After the addition of an aqueous solution of $\mathrm{AgNO}_{3}$ to the Au NPs solution they found that Ag atoms were deposited on the Au NPs based on the Ag peak in the X-ray photoelectron spectroscopy (XPS). This finding further suggested that anti galvanic exchange of $\mathrm{Au}$ NPs with $\mathrm{Ag}^{+}$ions is not due to the thiolate ligand, but could be due to the thermodynamic negative shift of the oxidation peak potential of naked Au NPs. Yao and coworkers reported that Ag atoms do not replace Au atoms in the precursor particles, but instead are placed 
side by side with the $\mathrm{Au}$ atoms (deposited on them). ${ }^{116}$ They proved this phenomenon with UV-visible spectroscopy and mass spectrometry. Their finding was also supported by theoretical calculations. Recently, Tian and coworkers reported that anti galvanic exchange between $\mathrm{Au}$ nanoclusters and $\mathrm{Ag}^{+}$ions depends on the structure of the precursor ions. ${ }^{117}$ For example, galvanic exchange between aqueous $\mathrm{AgNO}_{3}$ and $\mathrm{Au}_{25}(\mathrm{PET})_{18}$, $\left(\mathrm{PET}=\mathrm{SC}_{2} \mathrm{H}_{4} \mathrm{Ph}\right)$ results in $\operatorname{Au}_{25} \mathrm{Ag}_{2}(\mathrm{PET})_{18}$ as the main product while galvanic exchange between $\mathrm{Au}_{25}(\mathrm{PET})_{18}$ and $\mathrm{Ag}-\mathrm{DTZ}$ complexes (DTZ is dithizone) produced $\mathrm{Au}_{24 \mathrm{Ag}}(\mathrm{PET})_{18}$ as a main product in the mass spectrum. Li and coworkers reported the formation of heavily doped $\mathrm{Au}_{25-\mathrm{x}} \mathrm{Ag}_{\mathrm{x}}\left(\mathrm{SC}_{6} \mathrm{H}_{11}\right)_{18}$ nanoclusters (where $\mathrm{x}=9$ ) by reacting $\mathrm{Au}_{23}(\mathrm{SR})_{16}{ }^{-}$with a $\mathrm{Ag}(\mathrm{I})$-thiolate complex. Based on the X-ray crystallographic analysis they reported that $\mathrm{Ag}$ atoms not only go into the core of the Au clusters, but they also incorporate into the surface motif. Luo and coworkers reported conversion of the 23-atom $\left[\mathrm{Au}_{23}(\mathrm{SR})_{16}\right]^{-}$nanocluster to a 21-atom $\left[\mathrm{Au}_{21}(\mathrm{SR})_{12}\left(\mathrm{Ph}_{2} \mathrm{PCH}_{2} \mathrm{PPh}_{2}\right)_{2}\right]^{+}$nanocluster by the resection of two surface $\mathrm{Au}$ atoms first with $\mathrm{Ag}^{+}$ions followed by replacement of two $\mathrm{Ag}$ atoms by galvanic exchange with a $\mathrm{Au}(\mathrm{I})$-diphosphine complex, $\mathrm{Au}_{2} \mathrm{Cl}_{2}(\mathrm{P}-\mathrm{C}-\mathrm{P}) .{ }^{118}$ This slight modification on the Au clusters provided a 10 fold increase in the luminescent properties of Au nanoclusters.

Several others also studied galvanic exchange with Au nanoclusters in the 2-3 nm diameter range and used those alloy nanoclusters for catalytic applications. ${ }^{119-120}$ For example, Young and coworkers reported that galvanic exchange addition of $\mathrm{Ag}$ atoms on the surface of Au nanoclusters significantly 
increased the 4-electron oxygen reduction reaction (ORR) electrocatalytic activity in alkaline media as compared to other Au-Ag alloy NPs. ${ }^{121}$ All these studies indicate that galvanic exchange between $\mathrm{Au}$ and $\mathrm{Ag}^{+}$ions is possible, however the mechanism of this exchange is still unclear in the literature. To the best of our knowledge no literature report has correlated the anti-galvanic exchange reaction of Au NPs with the size-dependent oxidation peak potential of Au NPs and used ASV to monitor the exchange process. Our studies show that the anti-galvanic exchange reaction is thermodynamically favorable when the NP size becomes less than $4 \mathrm{~nm}$ in diameter, which we believe is due to the size-dependent negative shift of the oxidation peak potential of Au NPs.

\subsection{RESULTS AND DISCUSSIONS}

We synthesized THPC-stabilized 4.1, 1.6 and TPPS-stabilized $0.9 \mathrm{~nm}$ diameter Au NPs as described in the experimental Chapter II. Figure 4.1. (A) shows the UV-visible spectra of 4.1, 1.6 and $0.9 \mathrm{~nm}$ diameter Au NPs. 1.6 and 0.9 nm diameter Au NPs do not display any localized surface plasmon resonance band (LSPR) in the UV-vis range. It is well known that the LSPR band disappears for Au NP sizes of about $2 \mathrm{~nm}$ or smaller in diameter. The LSPR of $4 \mathrm{~nm}$ diameter Au NPs shows a peak at $507 \mathrm{~nm}$ which is consist with the literature reported value for $4 \mathrm{~nm}$ diameter Au NPs, indicating that we were able to successfully synthesize 4 $\mathrm{nm}$ diameter Au NPs. For further confirmation of size, we measured the TEM images of 1.6 and $1 \mathrm{~nm}$ diameter Au NPs and found NPs would be $1.6 \pm 0.4 \mathrm{~nm}$ and $0.9 \pm 0.2 \mathrm{~nm}$ respectively (Figure 4.1), which is also consist with the literature 
reported sizes of corresponding NPs. ${ }^{31,47}$ We do not have TEM image of $4 \mathrm{~nm}$ diameter Au NPs, however sizes of $4 \mathrm{~nm}$ diameter Au NPs previously measured by our group ${ }^{192}$ from TEM images and found to be $4.1 \pm 0.7 \mathrm{~nm}$ in diameter. Next, we attached those NPs on the glass/ITO/APTES electrode surface by directly soaking glass/ITO/APTES electrode to the NPs solution. Electrode attached Au NPs were then analyze by monitoring their oxidation peak potential. Oxidation peak potential of $0.9,1.6$ and $4 \mathrm{~nm}$ diameter Au NPs appeared at 0.2, 0.45 and $0.71 \mathrm{~V} v \mathrm{~s} \mathrm{Ag} / \mathrm{AgCl}$ respectively as shown in figure 4.1. We determined the size of 0.9, 1.6 and $4 \mathrm{~nm}$ diameter Au NPs based on the Pleith equation ${ }^{58}$ and it was found to be 1.01 .6 and $3.1 \mathrm{~nm}$ in diameter respectively.

We monitored the size dependent galvanic exchange of Au NPs with $\mathrm{Ag}^{+}$ ions by ASV. Figure 7.1 shows the ASV signature of glass/ITO/APTES coated with $0.9,1.6$ and $4.1 \mathrm{~nm}$ diameter Au NPs after 3 min of exchange with a $1 \times 10^{4} \mathrm{M}$ solution of $\mathrm{Ag}^{+}$ions. We kept a similar total coverage of all sized Au NPs in order to determine the size dependent reactivity of $\mathrm{Au}$ NPs with $\mathrm{Ag}^{+}$ions. After galvanic exchange, ASV were performed first in $0.5 \mathrm{M} \mathrm{H}_{2} \mathrm{SO}_{4}$ in order to determine the amount of Ag incorporated into the Au NPs. The same electrode was then rinsed with water, dried under $\mathrm{N}_{2}$ and used to perform ASV in $10 \mathrm{mM} \mathrm{KBr}$ plus $0.1 \mathrm{M}$ $\mathrm{KClO}_{4}$ electrolyte solution to determine the amount of $\mathrm{Au}$ in the NPs relative to the amount of Ag. Olga and coworkers showed that Ag NPs oxidize 

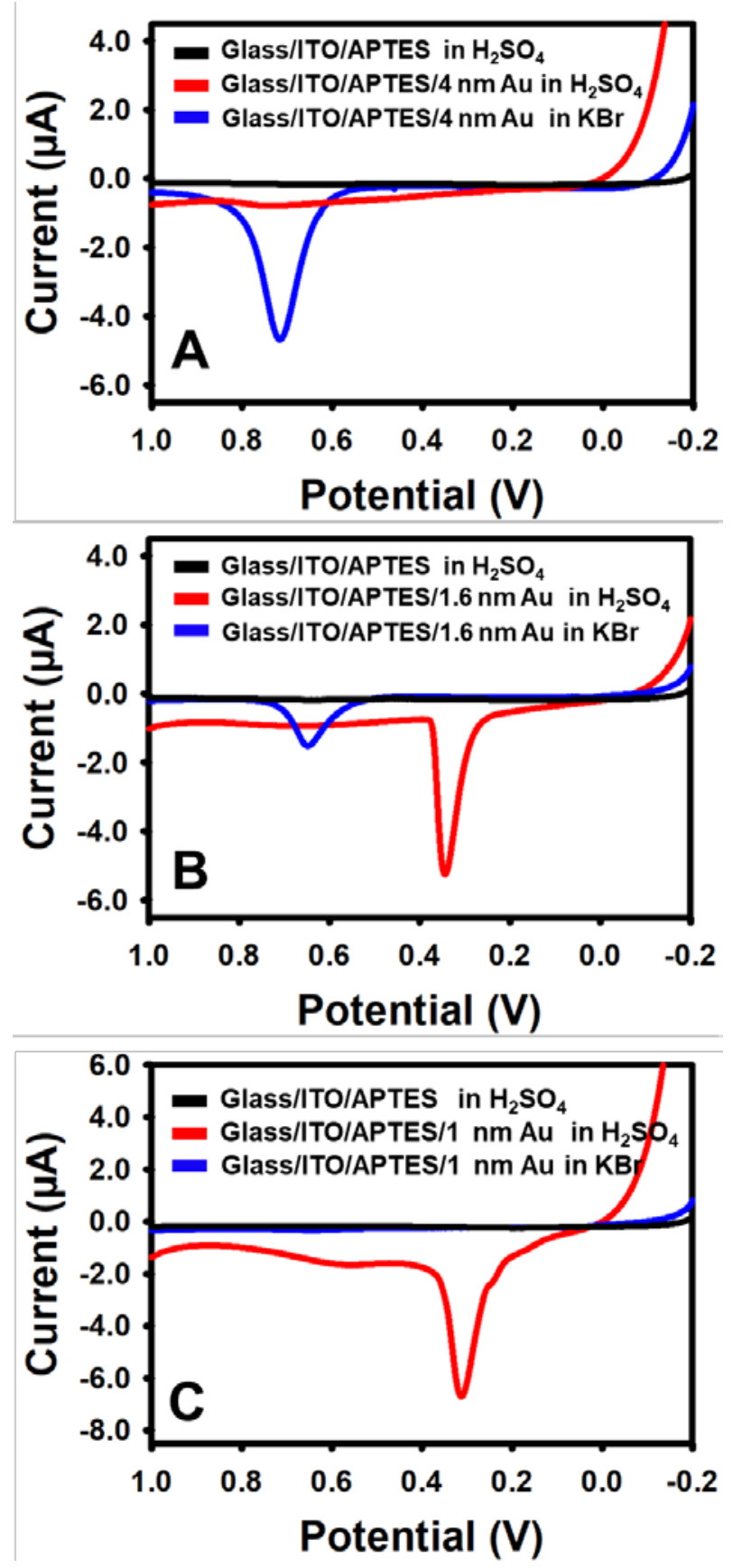

Figure 7. 1. ASV of 4.1 (A), 1.6 (B) and $0.9 \mathrm{~nm}(\mathrm{C})$ diameter Au NPs after $3 \mathrm{~min}$ of galvanic exchange with $1 \times 10^{-4} \mathrm{M} \mathrm{AgNO}_{3}$ solution. The ASV for $\mathrm{Ag}$ analysis was obtained in $0.5 \mathrm{M} \mathrm{H}_{2} \mathrm{SO}_{4}$ electrolyte solution first and then the ASV of Au was obtained in $10 \mathrm{mM} \mathrm{KBr}$ plus $0.1 \mathrm{M} \mathrm{KClO}_{4}$ electrolyte solution, both at a scan rate of $0.01 \mathrm{~V} / \mathrm{s}$. 
at $0.27-0.45 \mathrm{~V}$ in $0.5 \mathrm{M} \mathrm{H}_{2} \mathrm{SO}_{4}$ solution based on the size of Ag NPs. ${ }^{154}$ Citrate stabilized $8 \mathrm{~nm}$ diameter $\mathrm{Ag} \mathrm{NPs}$ oxidized at $0.27 \mathrm{~V} \mathrm{vs} \mathrm{Ag} / \mathrm{AgCl}$. The ASV of glass/ITO/APTES in $0.5 \mathrm{M} \mathrm{H}_{2} \mathrm{SO}_{4}$ after soaking in $\mathrm{Ag}^{+}$ions does not show any oxidation peak for $\mathrm{Ag}$ (Figure7.1A, black graph), indicating that the $\mathrm{Ag}^{+}$ions do not directly attach to the electrode surface. This important control experiment confirms that any Ag observed will be due to exchange with the Au NPs.

Next, we performed ASV of the glass/ITO/APTES/Au4.1nm NPs in $0.5 \mathrm{M}$ $\mathrm{H}_{2} \mathrm{SO}_{4}$ after a 3 min exposure to $\mathrm{Ag}^{+}$ions. As shown in Figure 7.1A (red plot), there was no oxidation peak for $\mathrm{Ag}$ in the potential range of 0 to $0.6 \mathrm{~V}$, indicating that 4.1 nm Au NPs do not exchange appreciably with $\mathrm{Ag}^{+}$ions under these conditions. The ASV obtained in $\mathrm{KBr}$ show a single oxidation peak for $\mathrm{Au}$ at $0.71 \mathrm{~V}$, indicating the presence of the $4.1 \mathrm{~nm}$ diameter Au NPs. Figure 7.1B shows the same set of ASVs after exposing glass/ITO/APTES/Au1.6nm NPs to $\mathrm{Ag}^{+}$for the same amount of time. The ASV in $0.5 \mathrm{M} \mathrm{H}_{2} \mathrm{SO}_{4}$ now shows a sharp oxidation peak at $0.35 \mathrm{~V}$ due to the oxidation of $\mathrm{Ag}$ (red plot). ${ }^{154} \mathrm{The} \mathrm{E}_{\mathrm{p}}$ for $\mathrm{Ag}$ is $\sim 80 \mathrm{mV}$ positive of the value for $8 \mathrm{~nm}$ diameter Ag NPs. It is likely that it is more positive after exchange due to the interaction with $\mathrm{Au}$, which has been observed previously by $\mathrm{us}^{203}$ and others. $^{204,251}$

The ASV in $\mathrm{KBr}$ electrolyte shows one oxidation peak for $\mathrm{Au}$ at $0.65 \mathrm{~V}$, which is consistent with Au NPs, but the $E_{p}$ is about $200 \mathrm{mV}$ positive from the $E_{p}$ of $0.45 \mathrm{~V}$ for the original $1.6 \mathrm{~nm}$ diameter Au NPs. This increased $\mathrm{E}_{\mathrm{p}}$ is either due to the first ASV scan in $\mathrm{H}_{2} \mathrm{SO}_{4}$, which leads to a particle size increase, or due to a a size change in the Au NPs during exchange with $\mathrm{Ag}^{+} .{ }^{199}$ Figure $7.1 \mathrm{C}$ shows the ASV 
analysis of glass/ITO/APTES/Auo.9nm NPs after monitored the exposure to with $\mathrm{Ag}^{+}$ions. There is one sharp oxidation peak at $0.28 \mathrm{~V}$ for the ASV in $0.5 \mathrm{M} \mathrm{H}_{2} \mathrm{SO}_{4}$ (red plot) but no noticeable peak for Au oxidation in the ASV in $\mathrm{KBr}$ electrolyte (blue plot). This indicates that $\mathrm{Ag}^{+}$ions completely, or near completely, exchanged with all of the Au atoms for these smallest size NPs. Taken together, the results of Figure 7.1 indicate that for the same amount of total Au on the electrode surface, the amount of exchange with $\mathrm{Ag}^{+}$ions increases as the size of the Au NPs decreases.

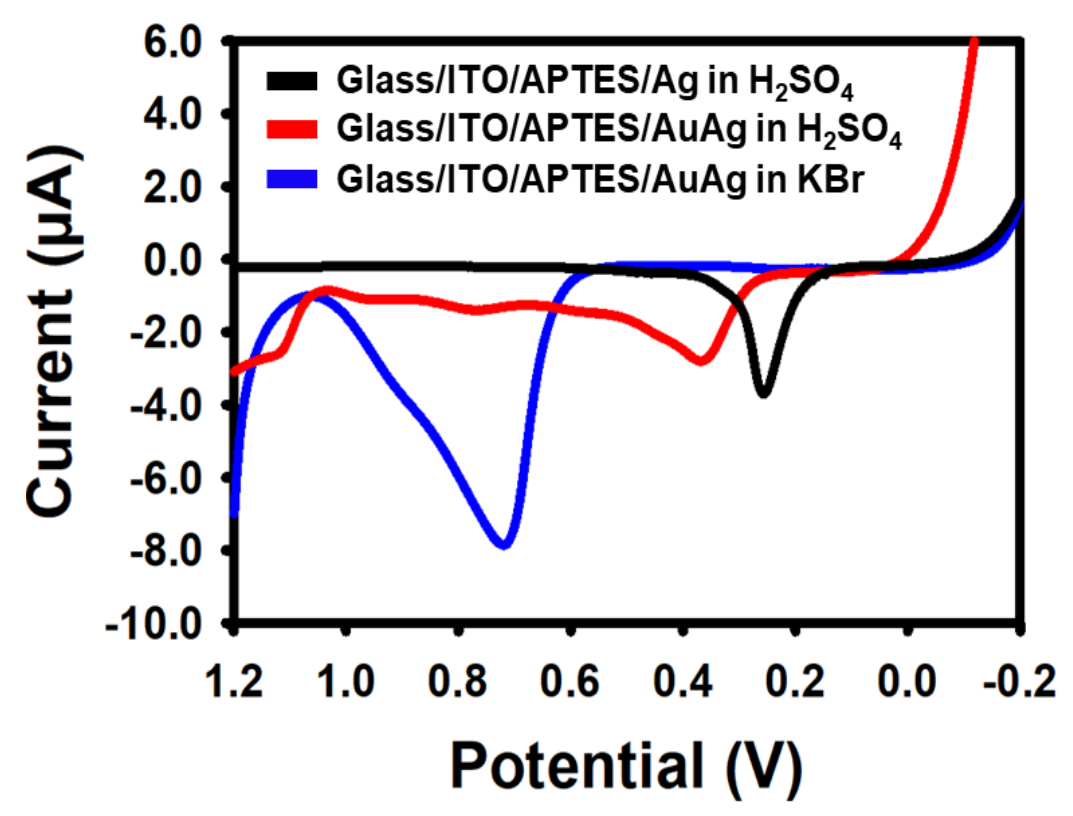

Figure 7. 2. ASV of $8 \mathrm{~nm}$ diameter Ag NPs in $0.5 \mathrm{M} \mathrm{H}_{2} \mathrm{SO}_{4}$ solution (black graph). ASV of AuAg alloy (50 \%-50\%) NPs in $0.5 \mathrm{M} \mathrm{H}_{2} \mathrm{SO}_{4}$ solution (red graph) and ASV of the same electrode in $10 \mathrm{mM} \mathrm{KBr}$ plus $0.1 \mathrm{~m} \mathrm{KClO}_{4}$ electrolyte solution (blue graph). All ASV obtained with a scan rate of $0.01 \mathrm{~V} / \mathrm{S}$. 
Table 7. 1. Integrated charge obtained from ASV peak of Ag and Au for $1.6 \mathrm{~nm}$ diameter Au NPs after 3 min of galvanic exchange with $1 \times 10^{-4} \mathrm{M} \mathrm{AgNO}_{3}$ solution.

\begin{tabular}{|c|c|c|c|c|c|c|c|}
\hline Trial & $\begin{array}{c}\mathrm{Ag} \\
\text { Coverage } \\
(\mathrm{C})\end{array}$ & $\mathrm{Ag} \mathrm{E}_{\mathrm{p}}$ & $\begin{array}{c}\text { Number } \\
\text { of } \mathrm{Ag} \\
\text { atoms } \\
\text { from } \mathrm{Ag} \\
\text { coverage }\end{array}$ & $\begin{array}{c}\mathrm{Au} \\
\text { coverage } \\
(\mathrm{C})\end{array}$ & $\begin{array}{c}\text { Number } \\
\text { of } \mathrm{Au} \\
\text { atoms }\end{array}$ & $\% \mathrm{Au}$ & $\% \mathrm{Ag}$ \\
\hline 1 & $1.65 \times 10^{-5}$ & 0.340 & $1.03 \times 10^{14}$ & $8.50 \times 10^{-6}$ & $1.77 \times 10^{13}$ & 14.7 & 85.3 \\
\hline 2 & $1.45 \times 10^{-5}$ & 0.342 & $9.06 \times 10^{13}$ & $9.57 \times 10^{-6}$ & $1.99 \times 10^{13}$ & 18.0 & 82.0 \\
\hline 3 & $2.08 \times 10^{-5}$ & 0.343 & $1.30 \times 10^{14}$ & $1.57 \times 10^{-5}$ & $3.27 \times 10^{13}$ & 20.1 & 79.9 \\
\hline 4 & $1.88 \times 10^{-5}$ & 0.392 & $1.17 \times 10^{14}$ & $1.42 \times 10^{-5}$ & $2.95 \times 10^{13}$ & 20.1 & 79.9 \\
\hline
\end{tabular}

To verify the $E_{p}$ for $\mathrm{Ag}$ within AuAg alloy NPs and confirm that we can quantitatively determine the amount of Au and Ag in AuAg alloy NPs, we performed ASV analysis of chemically-synthesized AuAg alloy NPs with a Au:Ag 1:1 composition and compared that to the ASV of pure $8 \mathrm{~nm}$ diameter Ag NPs. As shown in Figure 7.2, the $\mathrm{E}_{\mathrm{p}}$ for $\mathrm{Ag}$ in the alloy is at about $0.35 \mathrm{~V}$ (red plot) as compared to it being at $0.25 \mathrm{~V}$ (black plot) for pure Ag. The ASV of the alloy in $\mathrm{H}_{2} \mathrm{SO}_{4}$ even has some peaks in the 0.7 to $0.9 \mathrm{~V}$ range, which could be due to even more stable forms of $\mathrm{Ag}$ in the alloy. This is consistent with the $\mathrm{Ag} \mathrm{E}_{\mathrm{p}}$ values in Figure 7.1. The ASV in $\mathrm{KBr}$ (blue plot) shows the Au oxidation peak at $0.7 \mathrm{~V}$ as expected. Integration of the $\mathrm{Ag}$ and Au in Figure 7.2 gave a Au:Ag ratio of $3.2 \pm$ 0.2 , which is close to the expected 1:1 value considering that Au oxidizes with 3 electrons and Ag with 1 electron. This validates our ASV analysis of AuAg alloy NPs, showing that it indicates the presence of $\mathrm{Au}$ and $\mathrm{Ag}$ and gives an accurate composition. 
Confirming that the ASV analysis is quantitative, we next determined the composition of $\mathrm{Ag}$ and $\mathrm{Au}$ after exchange based on the total integrated Coulombs of charge from the Ag and Au peaks in the ASVs. Table 7.1 shows the integrated charge and calculated \%Ag and \%Au from four samples of $1.6 \mathrm{~nm}$ diameter $\mathrm{Au}$ NPs after exchange. The AuAg alloy NPs contained on average $18.2 \% \mathrm{Au}$ and 81.8\% Ag following exchange. Since the loss of $1 \mathrm{Au}$ atom leads to the deposition of $3 \mathrm{Ag}$ atoms, this means that about $60 \%$ of the Au from the original Au NPs were exchanged to produce the new composition. $40 \%$ of the original Au atoms are still in the NPs. We did not quantitatively analyze the $4.1 \mathrm{~nm}$ Au NPs or the $0.9 \mathrm{~m} \mathrm{Au}$ NPs because there was no clearly distinguishable Ag peak or Au peak in the ASVs, respectively.

The extent of the galvanic exchange reaction between $\mathrm{Ag}^{+}$ions and the $\mathrm{Au}$ NPs could be related to the available surface area of the Au as opposed to the energetics of the Au NPs. For the same volume of $\mathrm{Au}$, the overall available surface area is much higher for the smaller Au NPs since the SA is equal to $3 \mathrm{~V} / \mathrm{r}$. To make sure the reactivity differences were not simply due to surface area differences, we compared the reactivity of $\mathrm{Ag}^{+}$ions with 1.6 and $4.1 \mathrm{~nm}$ diameter Au NPs that had the same overall surface area by increasing the number of $4.1 \mathrm{~nm}$ diameter $\mathrm{Au}$ NPs on the electrode. The geometric surface area of $1.6 \mathrm{~nm}$ diameter Au NPs is -2.6 time higher than $4.1 \mathrm{~nm}$ diameter Au NPs. Figure 7.3A shows the ASV of 4.1 $\mathrm{nm}$ Au NPs after exchange with $\mathrm{Ag}^{+}$ions. The ASV again does not show any oxidation peak for $\mathrm{Ag}$ in acidic electrolyte, indicating that the $4.1 \mathrm{~nm}$ diameter $\mathrm{Au}$ NPs do not under go any exchange with $\mathrm{Ag}^{+}$ions. We performed the same 
experiment with $1.6 \mathrm{~nm}$ diameter Au NPs and a significant amount of exchange with Ag again occurred as discussed in the previous sections. This finding clearly confirms the high reactivity of the $1.6 \mathrm{~nm}$ and $0.9 \mathrm{~nm}$ Au NPs with $\mathrm{Ag}^{+}$while the 4.1 nm diameter Au NPs are highly inert to this exchange reaction.
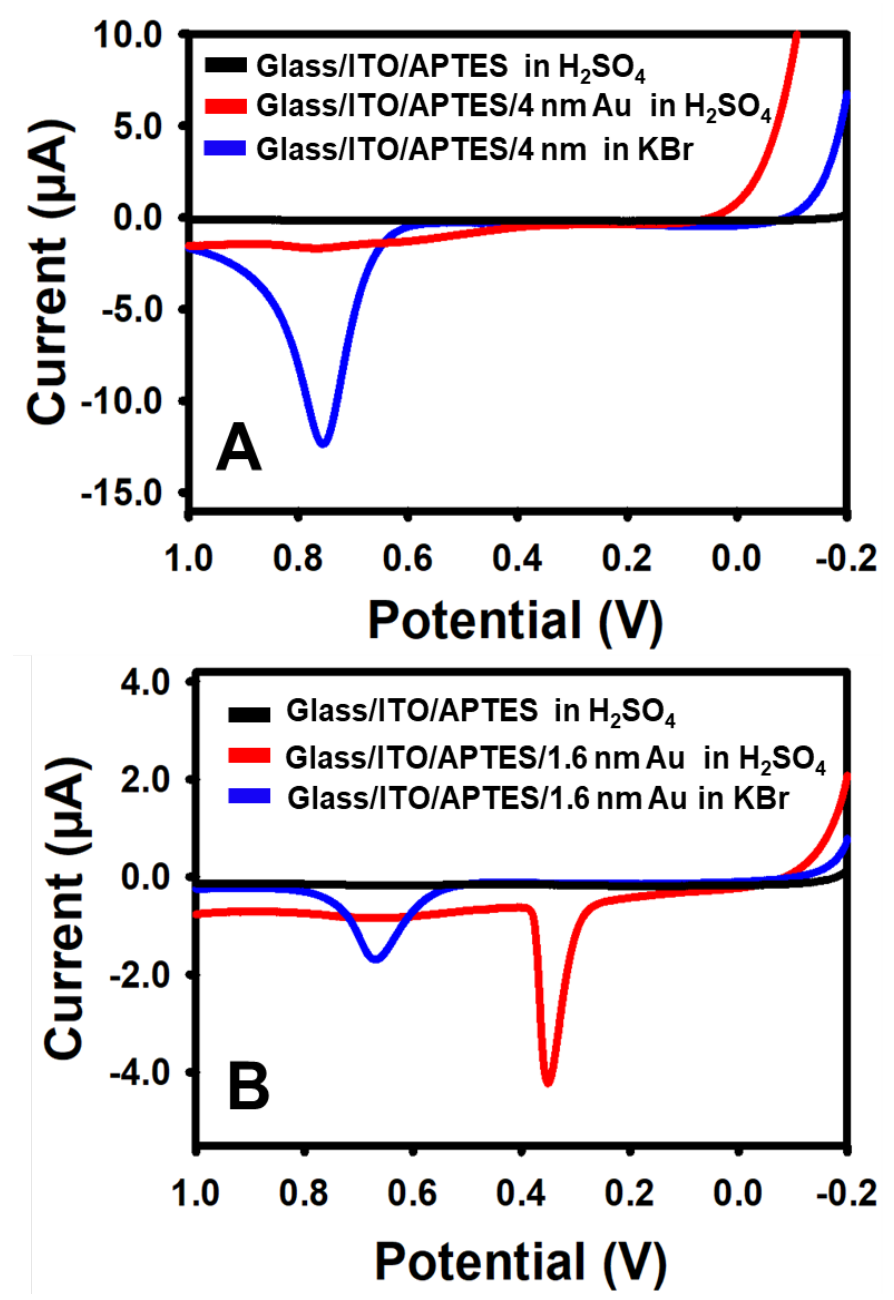

Figure 7. 3. ASV of $4.1(\mathrm{~A})$ and 1.6 (B) nm diameter Au NPs after 3 min of galvanic exchange with $1 \times 10^{-4} \mathrm{M} \mathrm{AgNO}_{3}$ solution. ASVs for $\mathrm{Ag}$ were obtained in $0.5 \mathrm{M}$ $\mathrm{H}_{2} \mathrm{SO}_{4}$ electrolyte solution and ASVs of Au were obtained in $10 \mathrm{mM} \mathrm{KBr}$ plus 0.1 
$\mathrm{M} \mathrm{KClO}_{4}$ electrolyte solution with a scan rate of $0.01 \mathrm{~V} / \mathrm{S}$. These Au NP electrodes had the same overall Au surface area.

We confirmed our findings by obtaining the composition of $\mathrm{Au}$ and $\mathrm{Ag}$ in the NPs after galvanic exchange using STEM-EDS mapping. Figure 7.4 shows the STEM images $(A, C)$ and EDS mapping $(B, D)$ of 4.1 and $1.6 \mathrm{~nm}$ diameter Au NPs having a similar overall Au surface area by drop-casting 2.6 times the amount of $4.1 \mathrm{~nm}$ diameter Au NPs. STEM-EDS mapping shows the $4.1 \mathrm{~nm}$ diameter Au NPs remain mostly red, indicating a high level of Au. The $1.6 \mathrm{~nm}$ diameter Au NPs are mostly the green color ascribed to Ag atoms. Based on the EDS mapping we obtained a Au:Ag composition of 89:11 and 10:90 for the 4.1 and $1.6 \mathrm{~nm}$ diameter Au NPs, respectively. The STEM-EDS determined value of $90 \% \mathrm{Ag}$ after exchange of the $1.6 \mathrm{~nm}$ diameter Au NPs is very close to the $82 \%$ value determined by ASV. It is also a much higher level of exchange than observed previously with thiolprotected Au clusters. This finding further confirmed that galvanic exchange of $\mathrm{Au}$ NPs with $\mathrm{Ag}^{+}$ions is possible with small sized Au NPs, which we believe is due to the size-dependent negative shift of the oxidation peak potential of Au NPs. Based on the large extent of exchange, it is likely that thiols likely hindered the exchange, as opposed to helping it, for small thiol-protected Au clusters. 


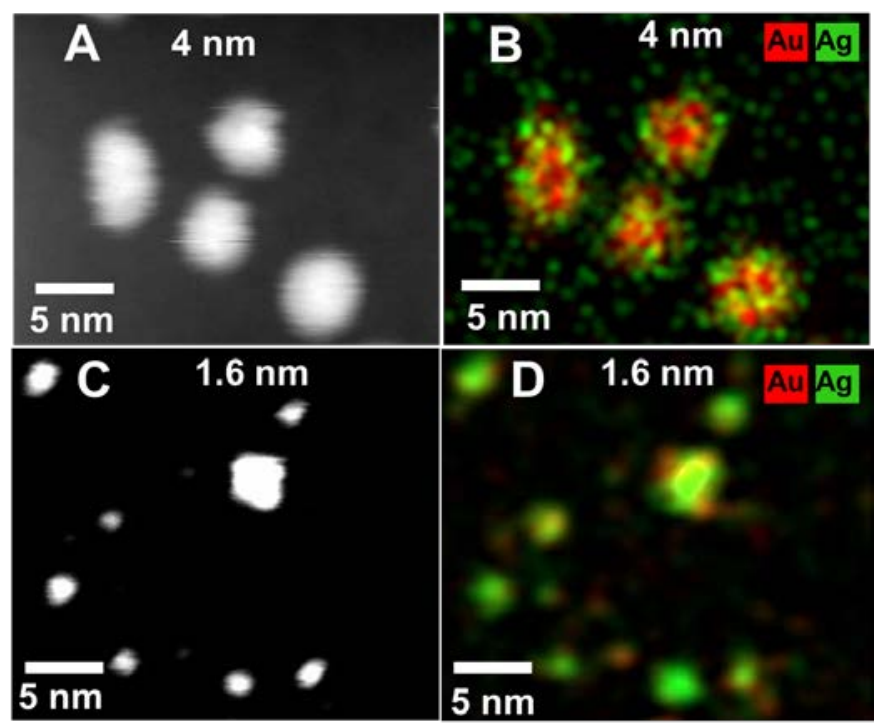

Figure 7. 4. STEM (A) and STEM-EDS mapping (B) images of $4.1 \mathrm{~nm}$ diameter Au NPs after 3 min of exchange with $1 \times 10^{-4} \mathrm{M} \mathrm{AgNO}_{3}$ solution. Similarly, STEM (C) and STEM-EDS mapping (D) images of $1.6 \mathrm{~nm}$ dimeter Au NPs. Images were obtained on silicon oxide coated $400 \mathrm{Ni}$ mesh TEM grids. The NPs were directly drop-cast deposited on the grid, dried in air, and then reacted by directly soaking the grid in the $\mathrm{Ag}^{+}$solution. 


\subsection{CONCLUSIONS}

In conclusion, we have described the size dependent galvanic exchange reaction between Au NPs of 0.9 to $4.1 \mathrm{~nm}$, and $\mathrm{Ag}^{+}$ions ${ }^{-}$. Au NPs of 0.9 $\mathrm{nm}, 1.6 \mathrm{~nm}$, and $4.1 \mathrm{~nm}$ diameter showed final Ag composition of $\sim 100 \%$ and $82 \%$, and $0 \%$, respectively, after galvanic exchange with $\mathrm{Ag}^{+}$ions as determined by ASV. STEM-EDS analysis showed good agreement of $90 \% \mathrm{Ag}$ and $11 \% \mathrm{Ag}$ for the 1.6 $\mathrm{nm}$ and $4.1 \mathrm{~nm}$ diameter Au NPs after exchange. We believe the driving force for this reaction is the decrease in the Au oxidation potential with decreasing NP size and the ability of small metal NPs to catalyze metal deposition. This fact could result in new strategies for modification of the composition of metallic NPs, especially for metal systems whose galvanic replacement is not thermodynamically favorable for bulk sizes. New alloy NPs could result with enhanced catalytic properties and other potential applications. 


\section{CHAPTER VIII}

\section{HALIDE-DEPENDENT DEALLOYING OF $\mathrm{Cu}_{x} / \mathrm{Au}_{\mathrm{y}}$ CORE/SHELL NANOPARTICLES FOR COMPOSITION ANALYSIS BY ANODIC STRIPPING VOLTRAMMETRY}

\subsection{INTRODUCTION}

Bimetallic nanoparticles (NPs) with different structures often show superior performance compared to their monometallic counterparts due to the combination and arrangement of the two different metal atoms and the metal-metal interactions. ${ }^{252}$ Recently, bimetallic NPs have been used for different applications such as catalysis, ${ }^{253-254}$ sensing, ${ }^{255-256}$ imaging, ${ }^{257}$ drug delivery, ${ }^{258}$ photothermal therapy, ${ }^{259}$ nanophotonics, ${ }^{260}$ and others. By considering the atomic arrangement, bimetallic NPs can be placed into two categories, including intermixed or intermetallic alloys, where the two metals are randomly and thoroughly mixed or

segregated with long range atomic order, respectively. ${ }^{261}$ Core/shell NPs are a type of intermetallic structure constructed with a core of inner material and a shell of outer material, both at the nanometer scale, that have become very popular for electrocatalysis applications. ${ }^{262}$

A key issue for catalysis is NP stability. ${ }^{263-267}$ There are several factors causing the low stability of mono-metal and alloy NPs including aggregation, ${ }^{105,} 268$ oxidation, ${ }^{269-272}$ and migration of atoms from core-to-shell, for example. ${ }^{273}$ 
Aggregation can be caused by a change in solution $\mathrm{pH}^{105}$ and removal of capping agents during catalysis. Metal oxidation can be an issue, especially for smallersized NPs or relatively thin shell thicknesses for the core/shell structure, which makes the metal atoms vulnerable to oxidation because the oxidation peak potential $\left(E^{0}\right)$ shifts negative as size decreases, which has been described by Plieth $^{58}$ and also known as the Gibbs-Thomson effect. ${ }^{265-266,270}$ The catalytic and sensing activity of bimetallic NPs may vary during the application because one metal undergoes rearrangement by diffusion from the core to the shell or viceversa due to heat or other reaction conditions. ${ }^{136,149,274}$ While the development of highly active, selective, robust, low-cost and environmentally friendly catalytic systems is very challenging, there has been significant progress with the development of bimetallic core/shell NPs.

Citrate-stabilized core/shell Cu/Au bimetallic NPs are the focus of this paper. These structures were previously synthesized by Yang and co-workers for the colorimetric detection of iodine, sulfide anion and cysteine. ${ }^{149-150}$ Recently Wei and coworkers $^{136}$ synthesized core/shell Cu/Au NPs and studied the unidirectional thermal diffusion of Au atoms from the shell to the core. Core/shell Au/Cu NPs have received comparatively less attention than $\mathrm{Cu} / \mathrm{Au}$ because $\mathrm{Cu}$ is easily oxidized to $\mathrm{CuO}$ and $\mathrm{Cu}_{2} \mathrm{O}$ under ambient conditions when it is in the shell and $\mathrm{Cu}$ does not exhibit the plasmonic properties of $\mathrm{Au}$ and $\mathrm{Ag} .{ }^{138,260,275} \mathrm{Cu}$ is commonly used as a sacrificial metal to synthesize stable metal NP catalysts with Au or to make nanoporous Au catalysts through galvanic place exchange reactions. ${ }^{276-277}$ 
In recent years, the composition and atomic arrangement of bimetallic core/shell or other alloy NPs have been widely characterized by using high resolution transmission electron microscopy (HRTEM), 136, 273, 278 x-ray diffraction $(\mathrm{XRD}),{ }^{273} \mathrm{x}$-ray photoelectron spectroscopy, ${ }^{279} \mathrm{x}$-ray absorption fine structure (XAFS), ${ }^{136}$ high angle annular dark field scanning transmission electron microscopy (HAADF-STEM), ${ }^{136,} 280$ energy-dispersive X-ray line spectra (EDX), ${ }^{136}$, 279 and UV-Vis absorption spectroscopy. ${ }^{136,149}$ These techniques are generally expensive and tedious or offer limited information in the case of UV-vis spectroscopy. Accordingly, our goal is to develop electrochemical methods, such as anodic stripping voltammetry (ASV), for the analysis of NPs. The benefit of electrochemistry as a method of analysis is its simplicity, speed of operation, and low cost.

While there are many spectroscopic and microscopic dealloying studies of metal alloy NPs, mostly aimed at the synthesis of porous Au NPs, ${ }^{269}$ there have only been a very few studies on the electrochemical characterization of NP composition, atomic arrangement, or general oxidation behavior by voltammetry for assemblies of metal NPs, especially for core/shell NPs. For example, Bard and co-workers electrochemically characterized core/shell $\mathrm{Ag} / \mathrm{Ag}_{2} \mathrm{O}$ NPs by cyclic voltammetry of the NPs in buffer solution. ${ }^{281}$ During the first positive scan, they did not observe any surface oxidation of Ag, which indicated that the core Ag atoms of the core/shell NPs were blocked by an $\mathrm{Ag}_{2} \mathrm{O}$ shell. After scanning to negative potentials where the reduction of $\mathrm{Ag}_{2} \mathrm{O}$ to $\mathrm{Ag}$ took place, an $\mathrm{Ag}$ surface oxidation peak then appeared, which provided structural information about the core/shell 
nature of the $\mathrm{Ag} / \mathrm{Ag}_{2} \mathrm{O}$ NPs. Tsung and coworkers showed that the reduction of $\mathrm{Au}$ is blocked during the first few cyclic voltammetry cycles of core/shell Au/Pd NPs in $\mathrm{H}_{2} \mathrm{SO}_{4}$ solution. ${ }^{273}$ After a few cycles in acid electrolyte solution, however, core $\mathrm{Au}$ atoms migrated to the shell and their oxide reduction peak became visible, providing valuable information about the initial structure of the NPs and the effect of oxidative/reductive cycling in acid. Dimitrov and coworkers compared the dealloying of $\mathrm{Cu}_{x} \mathrm{Au}_{(1-\mathrm{x})}(\mathrm{x}=0.7$ and 0.8$)$ alloy films and nanorods in acidic electrolyte. ${ }^{282}$ They observed multistage dealloying in alloy films with the critical potential shifting positive with higher Au content. For alloy nanorods about $10 \mathrm{~nm}$ wide and $20 \mathrm{~nm}$ long, the critical potential for dealloying shifted about $200 \mathrm{mV}$ negative and complete dealloying occurred. ${ }^{282}$ The shift is greater than expected based on the nanorod curvature and Gibbs-Thomson effect. Erlebacher and coworkers observed an inverse Gibbs-Thomson effect for dealloying of sub-10 nm AuAg alloy NPs, where the more noble metal passivated the less noble metal due to fluctuations in the passivating layer being too short lived to allow dissolution of the less noble metal. ${ }^{270}$ This leads to incomplete dealloying and no porosity formation. In larger NPs, the fluctuations are longer lived, allowing full dealloying and porosity formation to occur.

In this work, we aim to show that ASV can be useful to analyze the composition of core/shell citrate-stabilized Cu/Au NPs when performed in chloride containing electrolyte and at ratios of $\mathrm{Cu}_{1} / \mathrm{Au}_{\mathrm{x}}$, where $\mathrm{x} \leq 1$. Complete stripping of each metal occurs at separate potentials, allowing the composition ratio of $\mathrm{Cu}$ and $\mathrm{Au}$ to be simply obtained by peak integration of the appropriate $\mathrm{Cu}$ and $\mathrm{Au}$ oxidation peaks 
and by taking into account the number of electrons involved in the oxidation reaction. When $x \geq 2$, the Au shell is substantially thick that it prevents oxidation (dealloying) of the $\mathrm{Cu}$ core until the $\mathrm{Au}$ oxidizes. In comparison of $\mathrm{Cu}_{1} / \mathrm{Au}_{2}$ core/shell NPs to mixed (not core/shell) $\mathrm{Cu}_{1} \mathrm{Au}_{2}$ NPs reveals a big difference in the ASV due to more available surface $\mathrm{Cu}$ in the latter, providing strong electrochemical evidence of the core/shell arrangement in the former. Our results show that accurate composition analysis is not possible in bromide containing electrolyte for core/shell $\mathrm{Cu}_{1} / \mathrm{Au} \mathrm{u}_{\mathrm{x}}$ nanoparticles for any value of $\mathrm{x}$ due to a unique bromide-induced stabilization of $\mathrm{Cu}$ in the presence of $\mathrm{Au}$ at all compositions. This causes some amount of $\mathrm{Cu}$ to oxidize at the same time as Au for all compositions, preventing accurate analysis of the two elements separately. The possible reasons for this are discussed.

\subsection{EXPERIMENTAL}

8.2.1. Anodic Stripping Voltammetry (ASV). For ASV measurements, the electrochemical cell consisted of the glass/ITO/APTES electrode containing the appropriate metal NPs as the working electrode, a Pt wire counter electrode, and an $\mathrm{Ag} / \mathrm{AgCl}(3.0 \mathrm{M} \mathrm{KCl})$ reference electrode. All electrochemical measurements were performed with a $\mathrm{CH}$ Instruments model $660 \mathrm{E}$ electrochemical work station (Austin, TX) in different electrolyte solution, including $0.1 \mathrm{M} \mathrm{HClO}_{4}, 0.1 \mathrm{M} \mathrm{HClO}_{4}+10 \mathrm{~mm} \mathrm{KBr}$, and $0.1 \mathrm{M} \mathrm{HClO}_{4}+10 \mathrm{mM} \mathrm{KCl}$ solution for citrate-coated $\mathrm{Cu}, \mathrm{Cu} / \mathrm{Au}$ core/shell, and CuAu mixed alloy NP electrodes using the linear sweep voltammetry (LSV) mode in the $\mathrm{CH}$ Instruments software. The scan was started at $-0.6 \mathrm{~V}$ with the reference and counter electrodes in the 
electrolyte solution and the working electrode out of solution. The potential was scanned positive at $0.005 \mathrm{~V} / \mathrm{s}$ and paused when it reached $-0.57 \mathrm{~V}$, at which time the working electrode was immersed into the electrolyte solution under potential control. The scan was then "unpaused" and the potential scanned up to $1.6 \mathrm{~V}$ at a scan rate of $0.005 \mathrm{~V} / \mathrm{s}$. This protocol was followed so that the working electrode was introduced into the electrolyte while under potential control and therefore never experienced the open circuit potential.

Figure 8.1 shows the general experimental procedure for the synthesis and electrochemical analysis. Step 1 is the synthesis of cit-Cu NPs by reduction of $\mathrm{Cu}^{2+}$ with $\mathrm{NaBH}_{4}$ in the presence of trisodium citrate as described in the experimental section. After 30 minutes, addition of various amounts of $\mathrm{HAuCl}_{4}$. $3 \mathrm{H}_{2} \mathrm{O}$ to the solution led to the reduction of $\mathrm{Au}$ onto the $\mathrm{Cu}$ NPs to form the various core/shell $\mathrm{Cu}_{1} / \mathrm{Au}_{\times} \mathrm{NPs}$ as described in the experimental section (Step 2). After synthesis, we attached the NPs electrostatically to a glass/ITO/APTES electrode by soaking the electrode in the NP solution for 15 min (Step 3). Finally, we performed $\mathrm{ASV}$ in $10 \mathrm{mM} \mathrm{KBr}$ (or $10 \mathrm{mM} \mathrm{KCl}$ ) plus $0.1 \mathrm{M} \mathrm{HClO}_{4}$ solution (Step 4) to correlate the voltammetry with the composition and/or atomic arrangement of $\mathrm{Cu}$ and $\mathrm{Au}$ in the alloy NPs. Au and Cu oxidation in the presence of halides by the following reactions:

$$
\begin{aligned}
\mathrm{Au}^{0}+2 \mathrm{Br}^{-} & \rightarrow \mathrm{AuBr}_{2}^{-}+\mathrm{e}^{-}, \mathrm{E}^{0}=0.96 \mathrm{~V}(\text { vs. NHE}) \\
\mathrm{Au}^{0}+4 \mathrm{Br}^{-} & \rightarrow \mathrm{AuBr}_{4}^{-}+3 \mathrm{e}^{-}, \mathrm{E}^{0}=0.85 \mathrm{~V}(\text { vs. NHE) } \\
\mathrm{Au}^{0}+2 \mathrm{Cl}^{-} & \rightarrow \mathrm{AuCl}_{2}^{-}+\mathrm{e}^{-}, \mathrm{E}^{0}=1.15 \mathrm{~V}(\mathrm{vs} . \mathrm{NHE}) \\
\mathrm{Au}^{0}+4 \mathrm{Cl}^{-} & \rightarrow \mathrm{AuCl}_{4}^{-}+3 \mathrm{e}^{-}, \mathrm{E}^{0}=0.99 \mathrm{~V}(\text { vs. NHE})
\end{aligned}
$$




$$
\mathrm{Cu}^{0} \rightarrow \mathrm{Cu}^{2+}+2 \mathrm{e}^{-}, \mathrm{E}^{0}=0.34 \mathrm{~V}(\mathrm{vs} . \mathrm{NHE})
$$

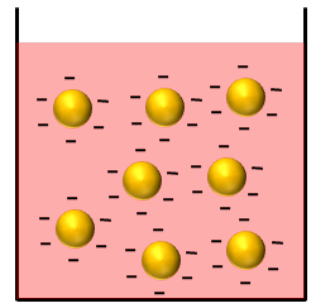

Step 1. Synthesis of $\mathrm{Cu}$ core.

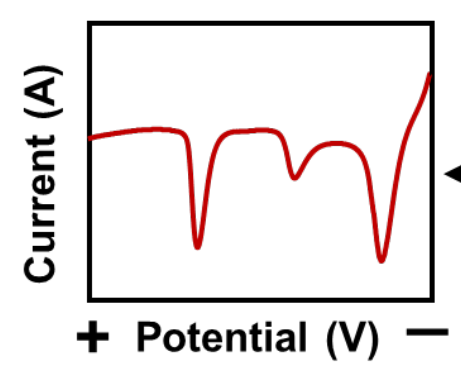

Step 4. Composition analysis by ASV.

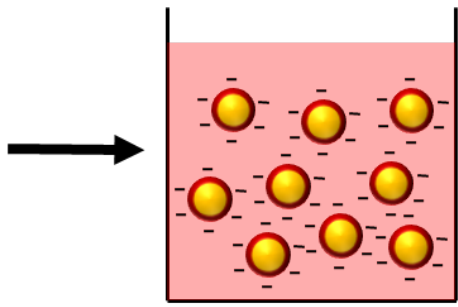

Step 2. Addition of Au shell.

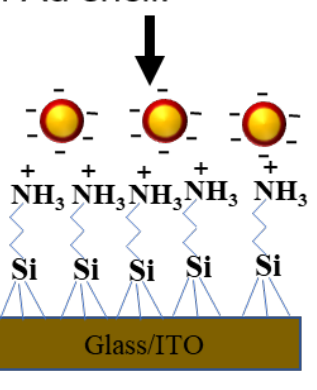

Step 3. Attachment of $\mathrm{Cu}_{1} / \mathrm{Au}_{\mathrm{x}}$ to Glass/ITO/APTES electrode.

Figure 8. 1. General experimental procedure involved in this work.

\subsection{RESULTS AND DISCUSSION}

As described in the experimental section, we synthesized citrate (cit)stabilized core/shell Cu/Au NPs with compositions of $\mathrm{Cu}_{1} / \mathrm{Au}_{\mathrm{x}}$, where $\mathrm{x}=0,0.001$, $0.01,0.1,0.3,0.5,1$, and 2 . After successfully synthesize, we used those NPs for optical, microscopic and electrochemical analysis. 


\subsubsection{Optical and Microscopic Characterization the CulAu Core/Shell}

Nanoparticles.

We collected UV-vis spectra of as-synthesized cit-Cu NPs and bimetallic core/shell cit-Cu $1 / \mathrm{Au}_{x} \mathrm{NPs}$ for qualitative assessment. Figure 8.2.(A) shows UVvis spectra of cit-stabilized $\mathrm{Cu}, \mathrm{Cu}_{1} / \mathrm{Au}_{0.5}, \mathrm{Cu}_{1} / \mathrm{Au}_{1}$, and $\mathrm{Cu}_{1} / \mathrm{Au}_{2} \mathrm{NPs}$. The assynthesized Cu NPs did not display any features in the UV-vis region other than an exponential decrease in absorbance with increasing wavelength, consistent with previous literature on the synthesis of Cu NPs prepared in this size range. ${ }^{149-}$ 150 The core/shell $\mathrm{Cu}_{1} / \mathrm{Au}_{\times} \mathrm{NPs}$ displayed a prominent peak near $510-530 \mathrm{~nm}$, which is the well-known localized surface plasmon resonance (LSPR) band for Au. The peak shifts positive and increases with increasing Au content, as expected for $\mathrm{Cu}_{1} / \mathrm{Au}_{x}$ core/shell NPs with increasing Au thickness. ${ }^{149,}{ }^{283}$ The $\mathrm{Cu}_{1} / \mathrm{Au}_{0.001}$, $\mathrm{Cu}_{1} / \mathrm{Au}_{0.01}, \mathrm{Cu}_{1} / \mathrm{Au}_{0.1}$ and $\mathrm{Cu}_{1} / \mathrm{Au}_{0.2}$ did not display any plasmon band in the UV-Vis spectrum (not shown) due to the very small amount of Au. In Figure 8.2.(A) with the higher Au concentrations, the UV-vis spectra show a more intense and redshifted Au LSPR band with increasing Au content, consistent with the expected composition of the $\mathrm{Cu}$ and $\mathrm{Au}$ based on the synthesis conditions, but the spectra do not confirm that a Cu/Au core/shell structure formed. 

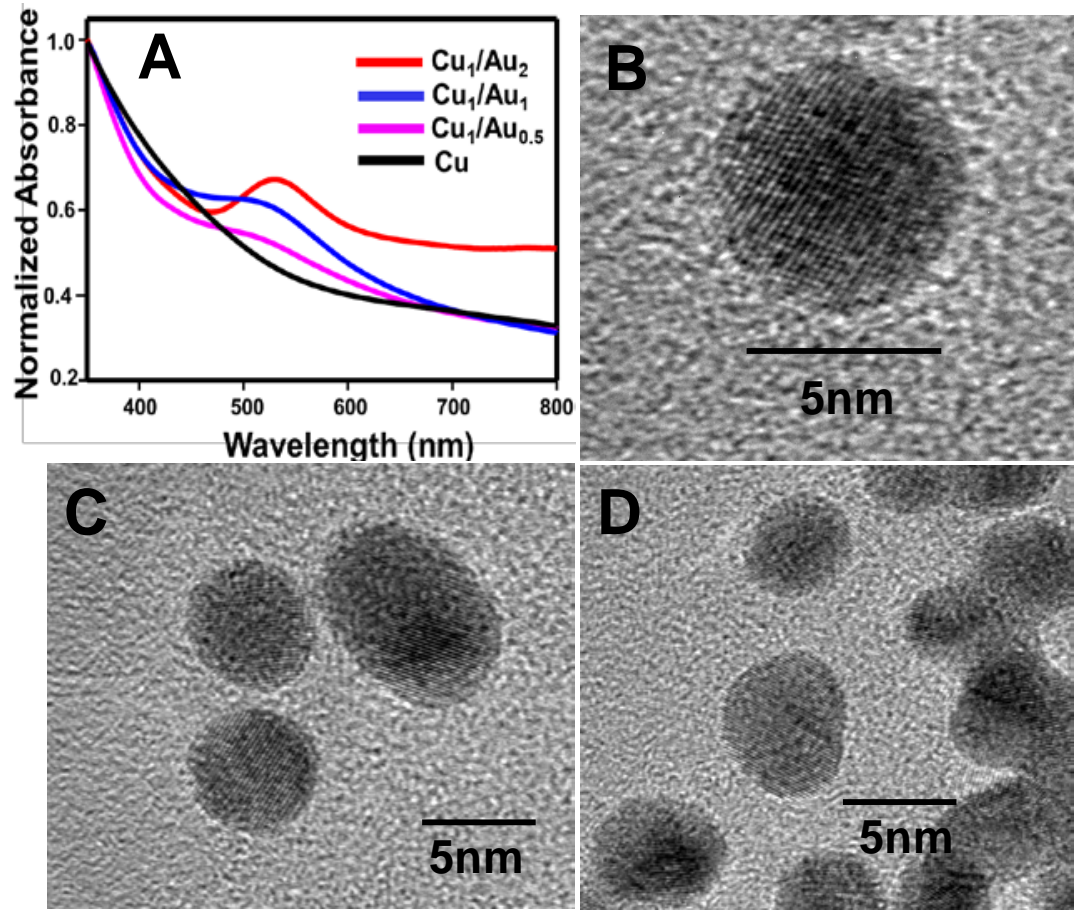

Figure 8. 2. UV-Vis spectra of citrate coated $\mathrm{Cu}, \mathrm{Cu} \mathrm{u}_{1}-\mathrm{Au}_{2}, \mathrm{Cu}_{1}-\mathrm{Au}_{1}$ and $\mathrm{Cu} \mathrm{u}_{1}-\mathrm{Au} 0.5$ NPs $(A)$ and HRTEM image of $\mathrm{Cu}_{1}-\mathrm{Au}_{2} \mathrm{NPs}(\mathrm{B}, \mathrm{C}$ and $\mathrm{D})$ 
Figure 8.2. (Frames B-D) shows high resolution transmission electron microscopy (HRTEM) images of core/shell cit $\mathrm{Cu}_{1} / \mathrm{Au}_{2}$ NPs. Size analysis of the NPs revealed an average size of $7.0 \pm 0.9 \mathrm{~nm}$, excluding NPs that were clearly aggregated. Assuming a core/shell structure, spherical shape, and $0.128 \mathrm{~nm}$ radius for $\mathrm{Cu}$ and $0.144 \mathrm{~nm}$ radius for $\mathrm{Au}$, simple geometry predicts a $\mathrm{Cu}$ core of $\sim 2.2 \mathrm{~nm}$ radius ( $\sim 4.4 \mathrm{~nm}$ diameter, $\sim 3700 \mathrm{Cu}$ atoms) and Au shell of $\sim 1.3 \mathrm{~nm}$ thick ( $7400 \mathrm{Au}$ atoms) for a Cu:Au 1:2 atomic ratio. That would be about 4-5 atomic layers of Au as the shell. Unfortunately, the TEM images could not directly confirm the $\mathrm{Cu} / \mathrm{Au}$ core/shell structure.

\subsubsection{Atomic Arrangement Determination by ASV (Core/Shell vs. Mixed} Alloy).

While UV-vis and TEM images could not definitively confirm the core/shell structure, ASV offers very solid evidence of it. Figure 8.3 shows the ASV of $\mathrm{Cu}_{1} / \mathrm{Au}_{2}$ core/shell NPs formed by reduction of $\mathrm{Cu}$ first followed by Au later, which is known to have the core/shell structure (red plot) as compared to the ASV of $\mathrm{Cu}_{1} \mathrm{Au}_{2}$ mixed alloy NPs (blue plot), which was expected to be more mixed since it was formed by the co-reduction of $\mathrm{Cu}$ and $\mathrm{Au}$ at the same time. In the latter case, the Au is more likely to be in the core due to its more positive reduction potential compared to $\mathrm{Cu}^{2+}$. Even though the amounts of $\mathrm{Cu}$ and $\mathrm{Au}$ are the same in the two different alloys, the ASVs are very different. In the core/shell $\mathrm{Cu}_{1} / \mathrm{Au}_{2}$ NPs, there was one predominant peak at $0.9 \mathrm{~V}$, which is consistent with the 
oxidation of $\mathrm{Au}$ in the presence of $\mathrm{Cl}^{-} .68$ The fact that there was not a significant oxidation peak before this peak (only a very small one at $\sim 0.2 \mathrm{~V}$ ) indicates that the Au formed a mostly closed, passivating shell over the $\mathrm{Cu}$ core, preventing oxidation of the $\mathrm{Cu}$. This positive shift of $\mathrm{Cu}$ oxidation peak is due to the direct contract of $\mathrm{Cu}$ with $\mathrm{Au}$, which has

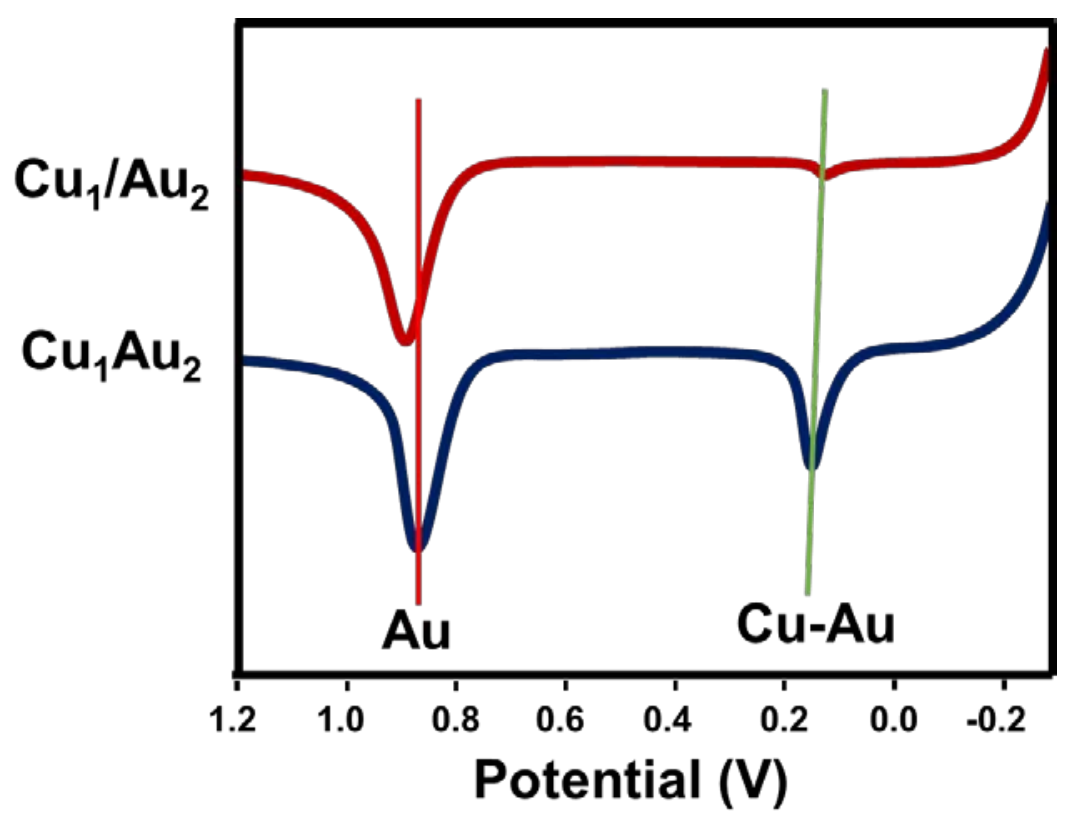

Figure 8. 3. Anodic stripping voltammograms (ASVs) of core/shell cit-Cu $/ \mathrm{Au}_{2} \mathrm{NPs}$ (red plot) and mixed alloy cit-Cu $\mathrm{Au}_{2} \mathrm{NPs}$ (blue plot) in $10 \mathrm{mM} \mathrm{KCl}$ plus $0.1 \mathrm{M}$ $\mathrm{HClO}_{4}$ solution.

been reported recently by Dimitrov group ${ }^{251}$ and others. ${ }^{284}$ The peak at $0.9 \mathrm{~V}$ therefore includes the oxidation of both $\mathrm{Au}$ and $\mathrm{Cu}$ simultaneously. In contrast, the ASV of the $\mathrm{Cu}_{1} \mathrm{Au}_{2}$ mixed alloy NPs showed two distinct peaks, one for $\mathrm{Cu}$ at 0.2 $\mathrm{V}$ and one for $\mathrm{Au}$ at $0.9 \mathrm{~V}$. The integration of the $\mathrm{Cu}$ and $\mathrm{Au}$ gives a 1:2 $\mathrm{Cu}: \mathrm{Au}$ 
ratio, taking into account that $\mathrm{Cu}$ oxidizes by 2 electrons and Au oxidizes by 3 electrons. The $\mathrm{Cu}$ is fully oxidized separately from the $\mathrm{Au}$ in this case, indicating that the $\mathrm{Cu}$ is fully accessible and dealloyed from $\mathrm{Au}$. This is expected for a CuAu mixed alloy or a reversed Au/Cu core/shell alloy, since there would be no Au shell preventing the oxidation of the $\mathrm{Cu}$. In this way, the ASV provides excellent qualitative evidence of the atomic arrangement of the two different elements in the $\mathrm{NP}$, confirming that we have successfully prepared the Cu/Au core/shell structure according to the literature..$^{149-150}$

\subsubsection{ASV Analysis of CulAu Core/Shell NPs in Acidic KCl.}

Figure 8.4.(A) shows $\mathrm{ASV}$ s versus $\mathrm{Ag} / \mathrm{AgCl}$ obtained in acidic $\mathrm{KCl}$ solution of $\mathrm{Cu}_{1} / \mathrm{Au} \mathrm{u}_{\mathrm{x}}$ core/shell NPs for $\mathrm{x}$ values ranging from 0 to 0.1 . The ASV of pure cit$\mathrm{Cu} \operatorname{NPs}(x=0)$ (blue plot) shows one oxidation peak at $-0.1 \vee$ vs an $\mathrm{Ag} / \mathrm{AgCl}$ reference electrode, which is consistent with pure $\mathrm{Cu}$ oxidation. For cit-Cu $/ \mathrm{Au}_{0.001}$ core/shell NPs (green plot), we still observed mainly one peak for $\mathrm{Cu}$ at $-0.1 \mathrm{~V}$ due to the very small amount of $\mathrm{Au}(\sim 3-4 \mathrm{Au}$ atoms per NP), but peaks also exist at 0.0, 0.35, and 0.6 V as well (Figure 8.5. (A) for expanded ASV). The signal is fairly low for these peaks and it is difficult to accurately make peak assignments, other than the $-0.1 \vee$ peak, without more information. The ASV of cit-Cu $/ \mathrm{Au}_{0.01}$ core/shell NPs (red plot) also shows the main peak at $-0.1 \mathrm{~V}$, which is broad and has a shoulder in this example, but peaks also appear at $0.25 \mathrm{~V}, 0.55 \mathrm{~V}$, and 0.75 V (figure 8.5. (B) for expanded ASV). 

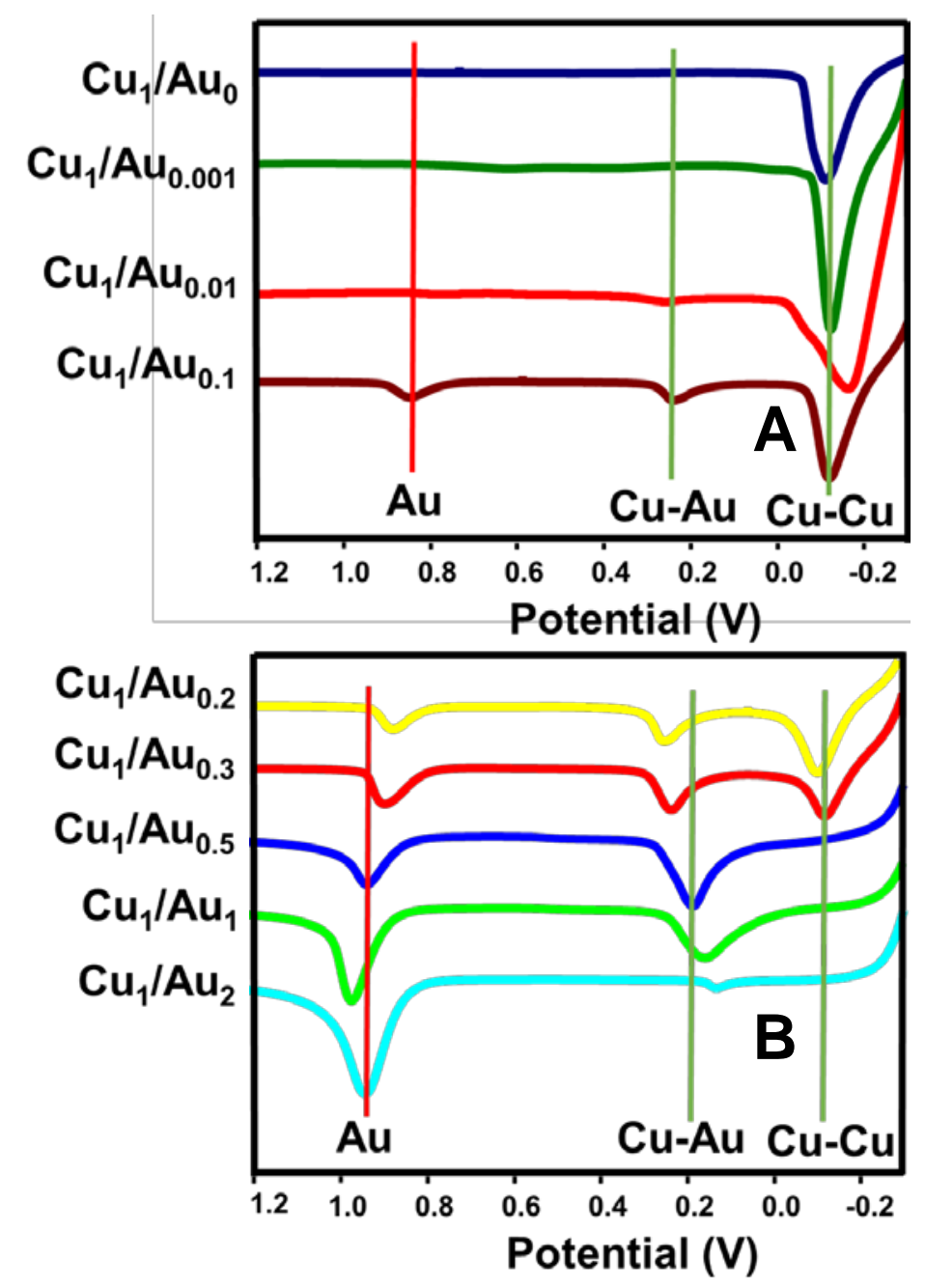

Figure 8. 4. Anodic stripping voltammetry (ASV) of citrate coated $\mathrm{Cu}, \mathrm{Cu}_{1} / \mathrm{Au}_{0.001}$, $\mathrm{Cu}_{1} / \mathrm{Au}_{0.01}, \mathrm{Cu}_{1} / \mathrm{Au}_{0.1} \mathrm{NPs}(\mathrm{A})$ and $\mathrm{Cu}_{1} / \mathrm{Au}_{0.2}, \mathrm{Cu}_{1} / \mathrm{Au}_{0.3}, \mathrm{Cu}_{1} / \mathrm{Au}_{0.5}, \mathrm{Cu}_{1} / \mathrm{Au}_{1}$ and $\mathrm{Cu}_{1} / \mathrm{Au}_{2} \mathrm{NPs}(\mathrm{B})$ in $10 \mathrm{mM} \mathrm{KCl}$ plus $0.1 \mathrm{M} \mathrm{HClO}_{4}$ solution. 

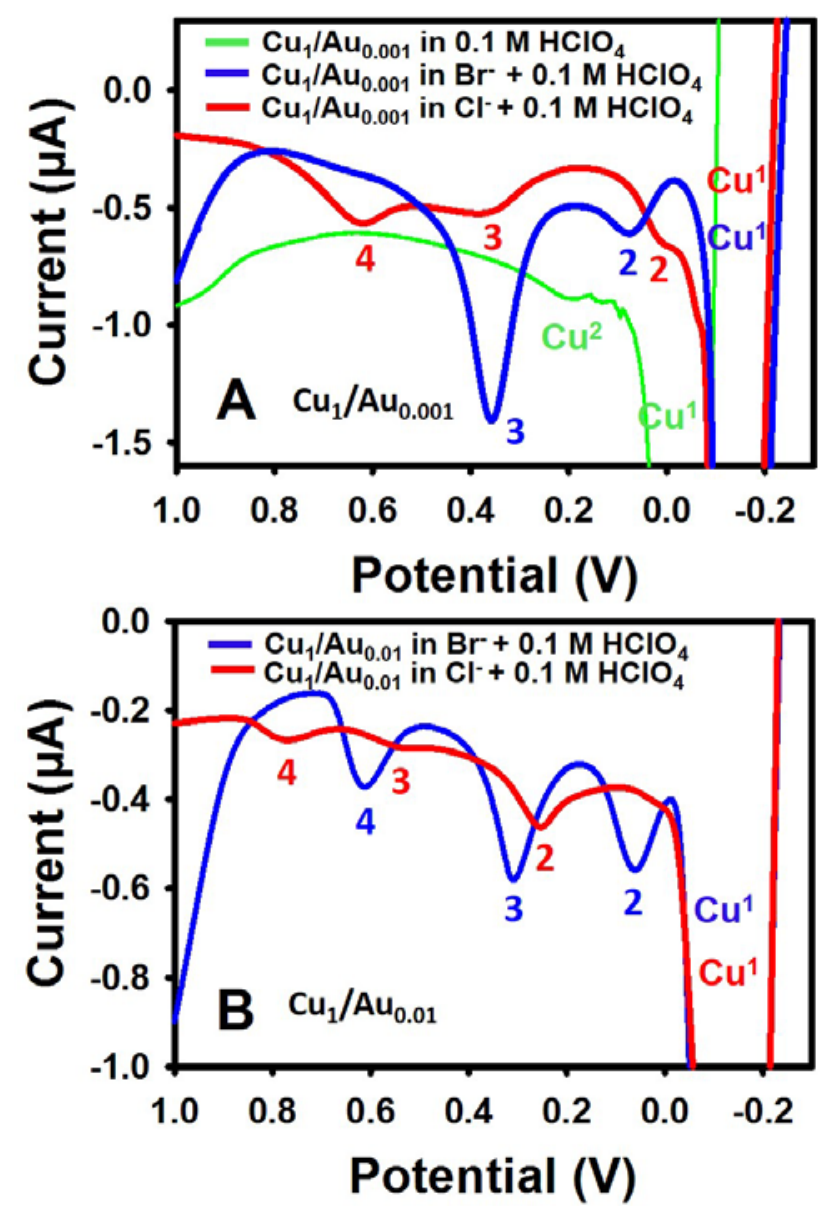

Figure 8. 5. ASVs of $(A) C u_{1} / A u_{0.001}$ and (B) $C u_{1} / A u_{0.01}$ core/shell nanoparticles in $0.1 \mathrm{M} \mathrm{HClO}_{4}$ (green), $0.01 \mathrm{M} \mathrm{KBr}+0.1 \mathrm{M} \mathrm{HClO}_{4}$ (blue), and $0.01 \mathrm{M} \mathrm{KCl}+0.1 \mathrm{M}$ $\mathrm{HClO}_{4}$ (red) as indicated. The peaks labeled as $\mathrm{Cu}^{1}$ or $\mathrm{Cu}^{2}$ are known for sure and the peaks labeled with a number are uncertain. 
Peak assignments are again difficult other than the peak at $-0.1 \mathrm{~V}$. Some of the multiple peaks are likely due to Cu bound to Au with different coordination number (atop, bridging, or three-fold hollow sites, for example). Other peaks could be due to oxidation of the $\mathrm{Au}$ that remains after dealloying the $\mathrm{Cu}$, which appear at potentials well negative of bulk Au due to the small amount of Au in these alloys (3-40 Au atoms per NP) ${ }^{64,168}$ It is interesting to think about what might happen to the remaining $\mathrm{Au}$ atoms after $\mathrm{Cu}$ removal for the $\mathrm{Cu}_{1} / \mathrm{Au}_{0.01}$ and $\mathrm{Cu}_{1} / \mathrm{Au}_{0.001}$ NPs. Do the Au atoms collapse into small spherical or irregular-shaped 3-40 atom clusters? Does the Au combine with other nearby NPs to form larger NPs in some type of ripening process? Do they retain $\mathrm{Cu}$ atoms for added stability? Studies are ongoing to address these questions.

The ASV of $\mathrm{Cu}_{1} / \mathrm{Au}_{0.1}$ core/shell NPs (figure 8.4, brown plot) is quite different from the alloys with $x=0.01$ and 0.001 . In this case there are three clearly dominant peaks in the ASV that are assignable. The first peak at $-0.1 \mathrm{~V}$ is due to Cu oxidation. Since this is where bare Cu NPs oxidize, we assign this peak to oxidation of $\mathrm{Cu}$ coordinated to other $\mathrm{Cu}$ atoms (labeled as $\mathrm{Cu}-\mathrm{Cu}$ ). The second peak is at $0.25 \mathrm{~V}$. We assign this peak to $\mathrm{Cu}$ oxidation as well, but it is due to $\mathrm{Cu}$ atoms coordinated to Au atoms that are in the shell, which shifts the oxidation potential positive (labeled as $\mathrm{Cu}-\mathrm{Au}$ ). The third peak is a clear, easily detected $\mathrm{Au}$ oxidation peak at $\sim 0.85 \mathrm{~V}$ (labeled as $\mathrm{Au}$ ). The addition of more $\mathrm{Au}$ to the outer shell compared to $x=0.01$ and 0.001 alloys led to a larger amount of $\mathrm{Cu}$ bonded to $\mathrm{Au}$, resulting in a larger peak at $0.25 \mathrm{~V}$ and smaller peak at $-0.1 \mathrm{~V}$, and a larger, 
detectable amount of Au oxidation at $0.85 \mathrm{~V}$. We are confident with these peak assignments at this level of Au in the shell.

The trend of the peaks at $0.25 \mathrm{~V}$ and $0.85 \mathrm{~V}$ growing at the expense of the peak at $-0.1 \mathrm{~V}$ continues in Figure 8.4. (B) for the $\mathrm{Cu}_{1} / \mathrm{Au}_{0.2}$ and $\mathrm{Cu}_{1} / \mathrm{Au}_{0.3}$ core/shell compositions (yellow and red plots). The Au shell oxidation peak at $0.85 \mathrm{~V}$ shifts slightly positive to $0.90 \mathrm{~V}$ due to a higher coverage of Au or larger size, which is reported by our group previously. ${ }^{155}$ In the ASV for the Cu1/Au0.5 core/shell NPS (blue plot) the peak at $-0.1 \mathrm{~V}$ disappears completely. A geometric calculation estimates we have about 1.5 layers of $\mathrm{Au}$ in the shell at this ratio. This indicates that the outer $\mathrm{Cu}$ atoms of the core are all bound to Au shell atoms and the more interior $\mathrm{Cu}$ atoms coordinated with other $\mathrm{Cu}$ atoms are not accessible for oxidation. This explains the loss of the peak at $-0.1 \mathrm{~V}$. Once the outer $\mathrm{Cu}$ atoms bound to Au begin to oxidize at $0.2 \mathrm{~V}$, the interior $\mathrm{Cu}$ atoms then become accessible for oxidation through a connected network of $\mathrm{Cu}$. As a result, all of the $\mathrm{Cu}$ atoms oxidize, but the peak oxidation is near $0.2 \mathrm{~V}$, while the peak at $-0.1 \mathrm{~V}$ is absent. This same trend occurs for the $\mathrm{Cu}_{1} / \mathrm{Au}_{1}$ core/shell NPs (green plot), where there are 2-3 layers of $\mathrm{Au}$ in the shell. All of the Cu becomes oxidized, but the peak potential is again near $0.2 \mathrm{~V}$ (no peak for bare $\mathrm{Cu}$ at $-0.1 \mathrm{~V}$ ). For the $\mathrm{Cu}_{1} / \mathrm{Au}_{2}$ core/shell NPs (light blue plot), the ASV just shows one dominant peak at the Au shell oxidation potential of $0.95 \mathrm{~V}$. This ratio corresponds to about 4-5 layers of $\mathrm{Au}$ in the shell, which completely passivates the Cu core atoms, other than a very small amount shown by the small oxidation peak at $0.2 \mathrm{~V}$. Once the Au shell oxidizes, the interior $\mathrm{Cu}$ atoms also must oxidize. ${ }^{269-271,282}$ 
We integrated the Au peak at $0.85-0.95 \mathrm{~V}$ and related it to the integrated sum of the $2 \mathrm{Cu}$ peaks at $-0.1-0.0 \mathrm{~V}$ and $0.20-0.25 \mathrm{~V}$ for all $\mathrm{Cu}_{1} / \mathrm{Au}_{\mathrm{x}}$ core/shell NPs, where $x=0.1$ to 2.0. We determined the theoretical peak ratio of $\mathrm{Au} / \mathrm{Cu}$ charge (Table 1, Column 4) from the mole ratio used in the NP synthesis and the assumption of a 3-electron oxidation for $\mathrm{Au}$ and 2 electron oxidation for $\mathrm{Cu}$. We found that the experimental $\mathrm{Au} / \mathrm{Cu}$ charge ratio matched the theoretical value for all compositions except for $\mathrm{Cu}_{1} / \mathrm{Au}_{2}$ (Table 8.1, Column 2 compared to Column 4) (individual charge obtained for different composition are show in table A11-A26 (appendix)). The $\mathrm{x}=2$ alloy is inaccurate because the Au shell passivates the $\mathrm{Cu}$ from being oxidized separately. The charge under the Au peak is due to both $\mathrm{Au}$ and $\mathrm{Cu}$ oxidation, making them indistinguishable. The good agreement between experimental and theoretical values for $x=0.1$ to 1.0 indicates that our peak assignments are correct and that ASV can be useful for accurate alloy composition analysis. 
Table 8. 1. Comparison of theoritical and experimetal of $\mathrm{Au} / \mathrm{Cu}$ ratio obtained from ASV in both $\mathrm{Br}$ - and $\mathrm{Cl}^{-}$containing acid electrolyte solution.

\begin{tabular}{|c|c|c|c|}
\hline $\begin{array}{c}\text { Synthesis } \\
\text { ratio }\end{array}$ & $\begin{array}{c}\text { Au:Cu ratio } \\
\text { obtained from } \\
\text { ASV in } \mathrm{Cl}^{-} \\
\text {containing } \\
\text { solution }\end{array}$ & $\begin{array}{c}\text { Au:Cu ratio } \\
\text { obtained from } \\
\text { ASV in } \mathrm{Br}^{-} \\
\text {containing } \\
\text { solution }\end{array}$ & $\begin{array}{c}\text { Theoretical } \\
\text { Au:Cu ratio }\end{array}$ \\
\hline $\mathrm{Cu}_{1} / \mathrm{Au}_{2}$ & $14.4 \pm 2$ & $26.3 \pm 2$ & 3.0 \\
\hline $\mathrm{Cu}_{1} / \mathrm{Au}_{1}$ & $1.56 \pm 0.1$ & $4.0 \pm 0.2$ & 1.50 \\
\hline $\mathrm{Cu}_{1} / \mathrm{Au}_{0.5}$ & $0.80 \pm 0.08$ & $2.0 \pm 0.3$ & 0.75 \\
\hline $\mathrm{Cu}_{1} / \mathrm{Au}_{0.3}$ & $0.50 \pm 0.06$ & $1.3 \pm 0.3$ & 0.45 \\
\hline $\mathrm{Cu}_{1} / \mathrm{Au}_{0.2}$ & $0.32 \pm 0.03$ & $0.80 \pm 0.03$ & 0.30 \\
\hline $\mathrm{Cu}_{1} / \mathrm{Au}_{0.1}$ & $0.15 \pm 0.01$ & $0.43 \pm 0.04$ & 0.15 \\
\hline
\end{tabular}




\subsubsection{ASV Analysis of Cu/Au Core/Shell NPs in Acidic KBr.}

We performed a similar set of ASV experiments for cit-Cu $\mathrm{Cu}_{1} / \mathrm{Au}_{\mathrm{x}}$ in $10 \mathrm{mM} \mathrm{KBr}$ plus $0.1 \mathrm{M} \mathrm{HClO}_{4}$ solution. Figure 8.6. (A) shows $\mathrm{ASV}$ versus an $\mathrm{Ag} / \mathrm{AgCl}$ reference obtained in acidic $\mathrm{KBr}$ solution of $\mathrm{Cu}_{1} / \mathrm{Au}_{\mathrm{x}}$ core/shell NPs for $\mathrm{x}$ values ranging from 0 to 0.1 . The ASV of pure cit-Cu NPs $(x=0)$ (blue plot) shows one oxidation peak at $-0.1 \mathrm{~V}$, which is consistent with pure $\mathrm{Cu}$ oxidation as we observed in acidic $\mathrm{KCl}$ electrolyte. For cit-Cu1/Aun.001 (green plot) and $\mathrm{Cu}_{1} / \mathrm{Au}_{0.01}$ (red plot) core/shell NPs, the peak at $-0.1 \mathrm{~V}$ is dominant, but there are also an additional 23 smaller peaks at more positive potentials (See Figure 8.5.(A) and 8.5.(B) for expanded ASVs). These peaks are again small and difficult to assign as with the ASVs in $\mathrm{KCl}$ because they could be due to different coordination environments for Cu with Au or due to tiny Au NPs, or clusters, that remain after Cu removal. ${ }^{68,264}$ In addition to be shifted more negative in $\mathrm{KBr}$ compared to $\mathrm{KCl}$, the intensity of the smaller peaks are larger relative to the $\mathrm{Cu}$ peak at $-0.1 \mathrm{~V}$ in $\mathrm{KBr}$ electrolyte compared to the ASV of the same composition in $\mathrm{KCl}$. It is remarkable to clearly observe peaks in the ASV resulting from 3-4 Au atoms out of $\sim 3700 \mathrm{Cu}$ atoms in the NP. It is again interesting to consider what happens to the 3-4 Au atoms when $\mathrm{x}=0.001$ and the $35-40 \mathrm{Au}$ atoms when $\mathrm{x}=0.01$ after Cu oxidation.

The ASV of $\mathrm{Cu}_{1} / \mathrm{Au}_{0.1}$ core/shell NPs (brown plot) in acidic $\mathrm{KBr}$ is similar to $\mathrm{KCl}$, except that the Au oxidation peak at $0.85 \mathrm{~V}$ in $\mathrm{KCl}$ now appeared at $0.70 \mathrm{~V}$ in $\mathrm{KBr}$ and the peak is comparatively larger relative to the two $\mathrm{Cu}$ oxidation peaks at -0.1 and $0.25 \mathrm{~V}$ compared to the ASV in $\mathrm{KCl}$ electrolyte. This larger peak indicates that 
the peak is a combination of $\mathrm{Au}$ and $\mathrm{Cu}$ oxidation. Accordingly, we assign the peak at $-0.1 \mathrm{~V}$ to $\mathrm{Cu}$ oxidation for $\mathrm{Cu}$ atoms bound to other $\mathrm{Cu}$ atoms (labeled as $\mathrm{Cu}-\mathrm{Cu}$ ). We assign the peak at $0.25 \mathrm{~V}$ to $\mathrm{Cu}$ oxidation for $\mathrm{Cu}$ atoms coordinated to $\mathrm{Au}$ atoms (labeled as $\mathrm{Cu}-\mathrm{Au}$ ). Finally, we assign the peak at $0.70 \mathrm{~V}$ to the oxidation of $\mathrm{Au}$ and $\mathrm{Cu}$ trapped by $\mathrm{Au}$ (labeled at $\mathrm{Au}-\mathrm{Cu}-\mathrm{Au}$ ).
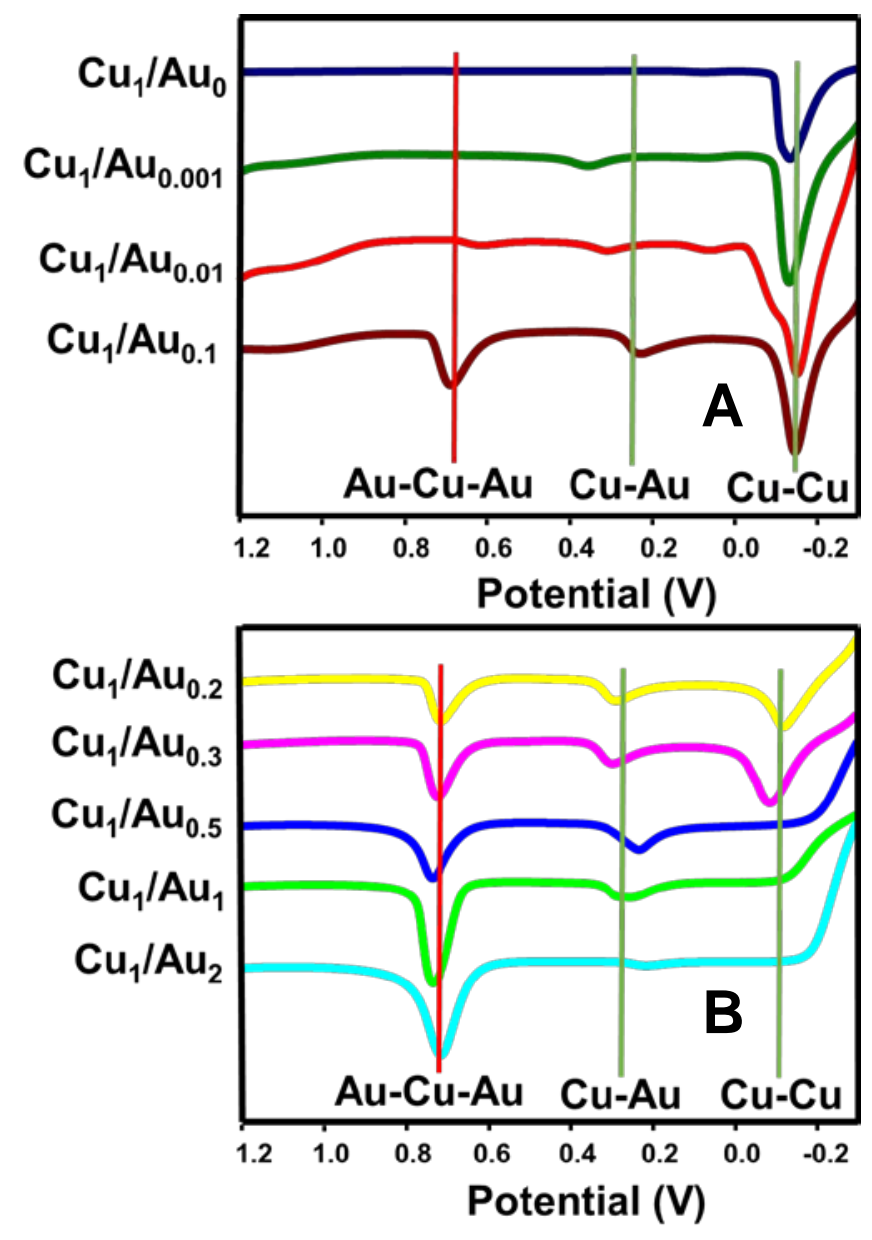

Figure 8. 6. Anodic stripping voltammetry (ASV) of citrate coated $\mathrm{Cu}, \mathrm{Cu}_{1} / \mathrm{Au}_{0.001}$, $\mathrm{Cu}_{1} / \mathrm{Au}_{0.01}, \mathrm{Cu}_{1} / \mathrm{Au}_{0.1} \mathrm{NPs}(\mathrm{A})$ and $\mathrm{Cu}_{1} / \mathrm{Au}_{0.2}, \mathrm{Cu}_{1} / \mathrm{Au}_{0.3}, \mathrm{Cu}_{1} / \mathrm{Au}_{0.5}, \mathrm{Cu}_{1} / \mathrm{Au}_{1}$ and $\mathrm{Cu}_{1} / \mathrm{Au}_{2}(\mathrm{~B}) \mathrm{NPs}$ in $10 \mathrm{mM} \mathrm{KBr}$ plus $0.1 \mathrm{M} \mathrm{HClO}_{4}$ solution. 
The trend of the peaks at $0.25 \mathrm{~V}$ and $0.70 \mathrm{~V}$ growing at the expense of the peak at $-0.1 \mathrm{~V}$ continues in Figure 8.6.(B) for the $\mathrm{Cu}_{1} / \mathrm{Au}_{0.2}$ and $\mathrm{Cu}_{1} / \mathrm{Au}_{0.3}$ core/shell compositions (yellow and pink plots) and the Au shell oxidation peak at $0.70 \mathrm{~V}$ shifts slightly positive to $0.75 \mathrm{~V}$ due to a higher coverage of $\mathrm{Au}$ and the larger size

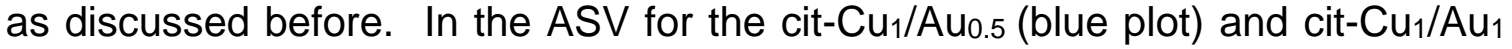
core/shell NPs (light green plot) the peak at $-0.1 \mathrm{~V}$ completely disappears. These results were similar to the oxidation behavior of core/shell NPs in $\mathrm{KCl}$ solution. Similarly, for the $\mathrm{Cu}_{1} / \mathrm{Au}_{2}$ core/shell NPs (light blue plot), the ASV shows just one main peak at about $0.75 \mathrm{~V}$ because the Au shell mostly passivates the interior $\mathrm{Cu}$ core at this composition. As mentioned earlier, geometrically an Au:Cu 2:1 ratio corresponds to about 4-5 atomic layers of Au surrounding the $\mathrm{Cu}$ core, which is apparently the amount needed to almost fully passivate the $\mathrm{Cu}$ interior from oxidation. The ASV is consistent with the geometric approximation.

As mentioned, the peak integration for $\mathrm{Au}(0.70-0.75 \mathrm{~V})$ related to the sum of the $2 \mathrm{Cu}$ peak integrations at $-0.1-0.0 \mathrm{~V}$ and $0.20-0.30 \mathrm{~V}$ did not match the theoretical $\mathrm{Au} / \mathrm{Cu}$ charge ratio based on the NP synthesis ratio, assuming a 2 electron oxidation for $\mathrm{Cu}$ and 3 electron oxidation for $\mathrm{Au}$, for any of the compositions when the ASV was performed in $\mathrm{Br}^{-}$(Table 8.1, Column 3 compared to Column 4). This is in contrast to the excellent agreement in $\mathrm{Cl}^{-}$electrolyte for $\mathrm{x}$ $=0.1$ to 1.0. All of the ASV-calculated Au/Cu ratios in $\mathrm{Br}^{-}$are much larger than the actual ratios based on the synthesis mole ratio. The only explanation for this is that some of the $\mathrm{Cu}$ oxidizes at the same time as the $\mathrm{Au}$ instead of both metals 
oxidizing independently. This would make the area of the peak at $0.70-0.75 \mathrm{~V}$ larger relative to the combined areas of the peaks at $-0.1-0.0 \mathrm{~V}$ and $0.20-0.30 \mathrm{~V}$ since the peak at $0.70-0.75 \mathrm{~V}$ would consist of both $\mathrm{Au}$ and $\mathrm{Cu}$ oxidation. This would explain the inaccurate ratios. Integrated charge of $\mathrm{Au}$ and $\mathrm{Cu}$ atoms obtained for different composition of CuAu NPs from $\mathrm{Br}^{-}$and $\mathrm{Cl}^{-}$electrolyte are shown in table 8.2-8.13.

\subsubsection{Dealloying Mechanism of CulAu Core/Shell NPs in Acidic KBr.}

Three possible explanations for $\mathrm{Cu}$ oxidizing along with $\mathrm{Au}$ instead of oxidizing separately are: (1) $\mathrm{Br}^{-}$adsorption could cause the Au atoms to diffuse on the NP surface during initial $\mathrm{Cu}$ oxidation until $\mathrm{Au}$ formed a full shell surrounding the remaining $\mathrm{Cu}$, which then does not become oxidized until the Au oxidizes at a higher potential. (2) $\mathrm{Br}^{-}$could possibly form a stable complex structure bound to both $\mathrm{Au}$ and $\mathrm{Cu}$, which makes the $\mathrm{Cu}$ oxidize only when the Au oxidation occurs. (3) Cu could oxidize by 1 electron to form $\mathrm{CuBr}$ while Au oxidizes by 3 electrons (the ratios would be closer but still too large in this case, however).

We believe the first explanation is the most plausible. There have been several studies on the potential-induced diffusion of $\mathrm{Au}$ atoms $\mathrm{by} \mathrm{Br}^{-}$in the literature. ${ }^{285-286}$ For example, Blizanac and co-workers reported a potential-induced hexagonal ("hex") to $(1 \times 1)$ transition of an Au surface, which coincides with $\mathrm{Br}^{-}$adsorption, and they found that the transition of the $\mathrm{Au}$ was faster for $\mathrm{Br}^{-}$containing acid electrolyte solution. ${ }^{286}$ They characterized the reconstruction of an Au (100) surface by cyclic voltammetry and surface X-ray scattering (SXS) measurements. 


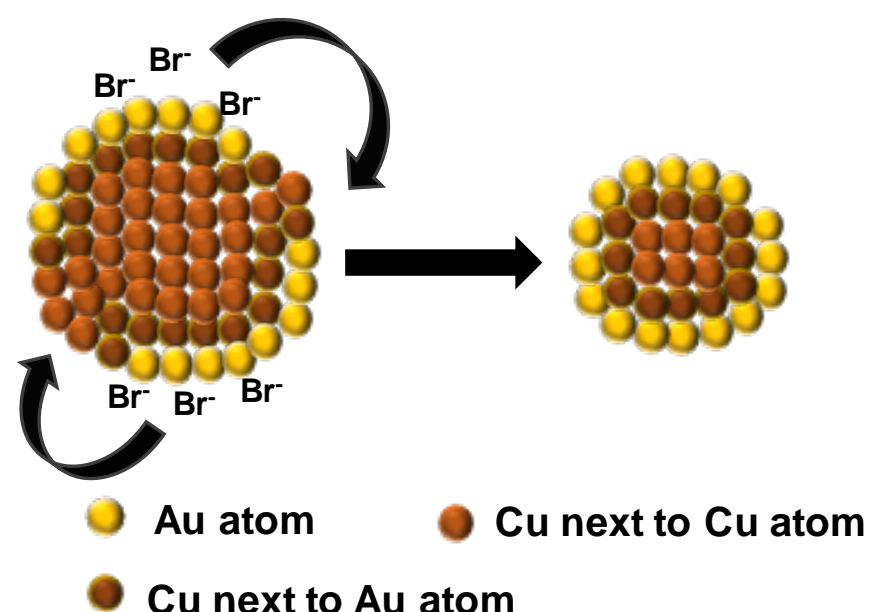

Cu next to Au atom

Figure 8. 7. An illustration of the surface diffusion and rearrangement of Au atoms during the initial stages of $\mathrm{Cu}$ dealloying in the presence of $\mathrm{Br}^{-}$from $\mathrm{Cu} / \mathrm{Au}$ core/shell NPs with incomplete or thin Au shells, which leads to a more stable $\mathrm{Cu} / \mathrm{Au}$ core/shell structure with a complete, passivating Au shell.

Au atom diffusion would be less likely to occur in $\mathrm{Cl}^{-}$containing solution since $\mathrm{Cl}^{-}$ does not adsorb as strongly to Au. Previous research on CuAu bulk alloy films showed a negative shift in the critical potential for Cu dealloying in the presence of $\mathrm{Cl}^{-}$and $\mathrm{Br}^{-}$( $\mathrm{Br}^{-}$was more negative) due to enhanced diffusion of Au surface atoms, which opened up pathways for Cu oxidation..$^{271,287-288}$ With sub-10 nm diameter NPs, however, McCue et al. showed that enhanced surface diffusion of the more 
noble alloy decreases the lifetime of these surface fluctuations, which decreases the probability of dealloying, leading to greater passivation of the less noble metal. ${ }^{270}$ We believe the latter is occurring in our system. The more strongly bound $\mathrm{Br}$ ligand leads to better corrosion protection of the $\mathrm{Cu}$ compared to $\mathrm{Cl}^{-}$or no halide due to enhanced Au surface diffusion and rearrangement, which allows a passivating Au shell to form over the remaining $\mathrm{Cu}$ after dissolution of some initial $\mathrm{Cu}$. Figure 8.7 shows an illustration of a possible $\mathrm{Br}^{-}$- induced rearrangement of the $\mathrm{Au}$ atoms during $\mathrm{Cu}$ oxidation to form a smaller $\mathrm{Cu} / \mathrm{Au}$ core/shell structure that leads to passivation of some of the $\mathrm{Cu}$ in the alloy.

Figure 8.8.(A) shows the difference in oxidation behavior of core/shell Cit$\mathrm{Cu}_{1} / \mathrm{Au}_{0.5} \mathrm{NPs}$ in $\mathrm{Br}^{-}$(blue graph) and in $\mathrm{Cl}^{-}$(red graph) electrolyte solution. For comparison we kept the second oxidation peak coverage similar to indicate how different the peak ratios are in $\mathrm{Br}^{-}$versus $\mathrm{Cl}^{-}$even though the $\mathrm{Cu}$ and $\mathrm{Au}$ composition is exactly the same in both samples. This is due to the fact that the second peak in $\mathrm{Br}^{-}$includes $\mathrm{Au}$ oxidation and trapped $\mathrm{Cu}$ oxidation (labeled as $\mathrm{Au}-$ $\mathrm{Cu}-\mathrm{Au}$, blue plot) whereas the second peak in $\mathrm{Cl}^{-}$is due to $\mathrm{Au}$ oxidation only (labeled as $\mathrm{Au}$, red plot). To test if $\mathrm{Br}^{-}$induced rearrangement of $\mathrm{Au}$ occurs, we stripped $\mathrm{Cu}$ first in $10 \mathrm{mM} \mathrm{KBr}$ plus $0.1 \mathrm{M} \mathrm{HClO}_{4}$ solution up to a potential of 0.42 $\mathrm{V}$ on a cit-Cu$/ \mathrm{Au}_{1}$ core/shell NP sample, which is beyond the observed $\mathrm{Cu}$ oxidation peak but before the Au oxidation peak (Figure 8.8B, dashed blue scan). Next, we rinsed the same electrode thoroughly with water and dried it under $\mathrm{N}_{2}$ before scanning 

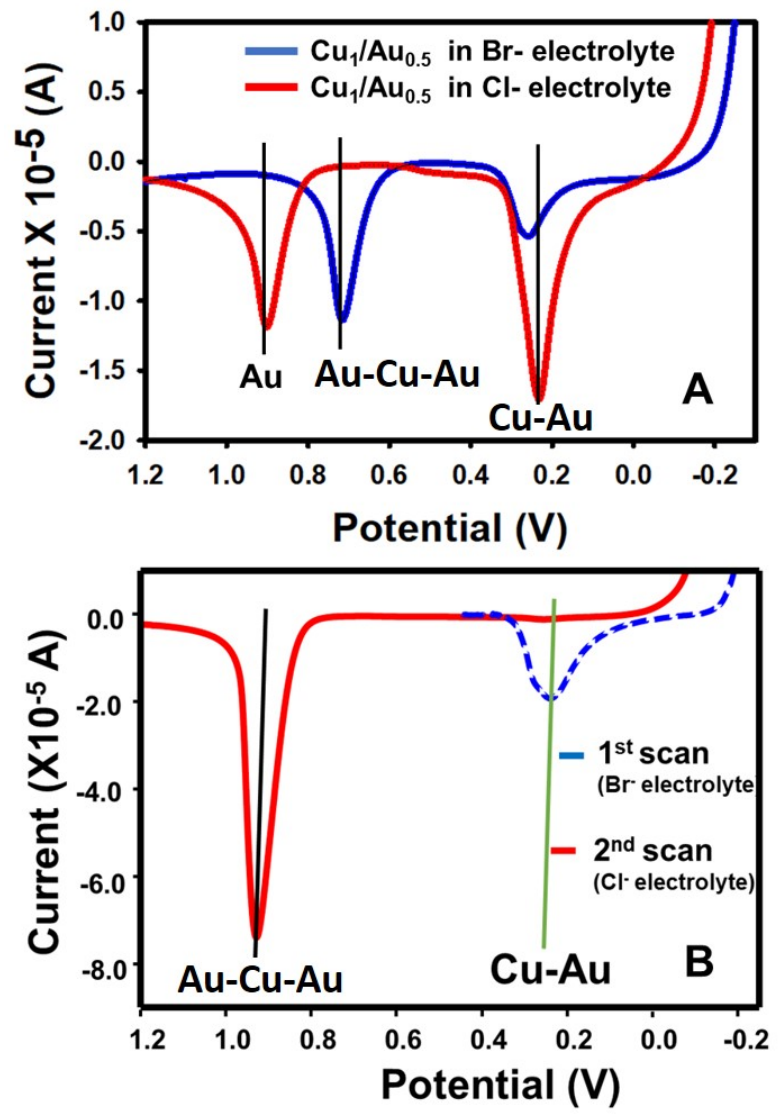

Figure 8. 8. (A) ASV of core/shell cit-Cu1/Au0.5 NPs in $10 \mathrm{mM} \mathrm{KBr}$ plus $0.1 \mathrm{M} \mathrm{HClO}_{4}$ solution (blue plot) and $10 \mathrm{mM} \mathrm{KCl}$ plus $0.1 \mathrm{M} \mathrm{HClO}_{4}$ solution (red plot). (B) ASV of core/shell cit-Cu$/ \mathrm{Au}_{1} \mathrm{NPs}$ in $10 \mathrm{mM} \mathrm{KBr}$ plus $0.1 \mathrm{M} \mathrm{HClO}_{4}$ solution first (blue plot) followed by ASV of the same sample in $10 \mathrm{mM} \mathrm{KCl}$ plus $0.1 \mathrm{M} \mathrm{HClO}_{4}$ solution second (red plot).

it from $-0.6 \mathrm{~V}$ to $1.6 \mathrm{~V}$ in $10 \mathrm{mM} \mathrm{KCl}$ plus $0.1 \mathrm{M} \mathrm{HClO}_{4}$ solution (Figure 8.8.(B), red scan). We found no significant peaks appearing in the $\mathrm{Cu}$ region in $\mathrm{KCl}$ electrolyte. Only the one peak at $0.92 \mathrm{~V}$ appeared and the large size of this peak relative to the peak near $0.2 \mathrm{~V}$ in the blue plot indicates that this peak also contains $\mathrm{Au}$ and 
trapped $\mathrm{Cu}$ oxidation (Au-Cu-Au). We integrated the charge under the peak for $\mathrm{Cu}$ in $\mathrm{Br}^{-}$electrolyte (blue plot) and the peak for $\mathrm{Au}$ in $\mathrm{Cl}^{-}$containing electrolyte solution (red plot) and found that the ratio of $\mathrm{Au} / \mathrm{Cu}$ was larger than the actual synthesis ratio $(\mathrm{Au} / \mathrm{Cu}=3.9 \pm 0.4)$ even though the ASV on the second run was in $\mathrm{Cl}^{-}$(integrated charge for individual experiment are shown in table 8.14 and 8.15

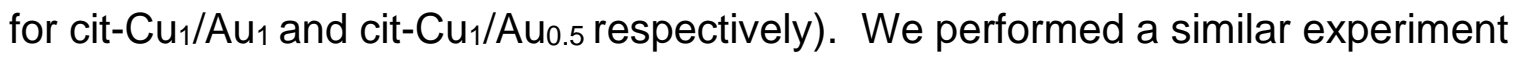
with cit-Cu1/Auo.5 and obtained the same result. In both samples, the calculated $\mathrm{Au}: \mathrm{Cu}$ ratios were the same (too large) when ASV was performed in $\mathrm{Br}^{-}$first followed by $\mathrm{Cl}^{-}$as compared to performing it in $\mathrm{Br}^{-}$only. This shows that $\mathrm{Br}^{-}$ions assist in the rearrangement of $\mathrm{Au}$ atoms during the initial oxidation of the $\mathrm{Cu}$ near $0.2 \mathrm{~V}$ and that the newly formed structure remains stable and is not reversed by removing from $\mathrm{Br}^{-}$and performing $\mathrm{ASV}$ in $\mathrm{Cl}^{-}$. Once formed the structure remains stable and the remaining $\mathrm{Cu}$ does not oxidize until the $\mathrm{Au}$ oxidizes, whether stripping occurs in $\mathrm{Br}^{-}$or $\mathrm{Cl}^{-}$. The details of the structure are not fully understood at this time, but it is likely a Cu/Au core/shell arrangement with a passivating closed $\mathrm{Au}$ shell. It could also involve adsorbed $\mathrm{Br}$, but it is not clear if the adsorbed bromide would remain on the NP surface after removal from $\mathrm{Br}$, rinsing with water, and placing it in $10 \mathrm{mM} \mathrm{Cl}^{-}$. It seems that the stability is more determined by the rearrangement of the Au during initial $\mathrm{Cu}$ oxidation in the presence of $\mathrm{Br}^{-}$and once formed, the new structure is quite stable and doesn't rely on the continued presence of $\mathrm{Br}^{-}$. 
Table 8. 2. Composition and size information on the rearranged Cu/Au NPs following initial Cu stripping in $\mathrm{KBr}$ electrolyte.

\begin{tabular}{|c|c|c|c|c|c|c|c|}
\hline $\begin{array}{c}\text { NP } \\
\text { Synthesiz } \\
\text { ed }\end{array}$ & $\begin{array}{c}\text { Au:Cu } \\
\text { Ratio } \\
\text { under the } \\
\text { Au } \\
\text { Oxidation } \\
\text { Peak }\end{array}$ & $\begin{array}{c}\% \mathrm{Cu} \\
\text { Retaine } \\
\mathrm{d}\end{array}$ & $\begin{array}{c}\text { Estimate } \\
\text { d Comp. } \\
\text { Before } \\
\text { Cu Ox. }\end{array}$ & $\begin{array}{c}\text { Estimate } \\
\text { d Comp. } \\
\text { After } \\
\text { Cu Ox. }\end{array}$ & $\begin{array}{c}\text { Core } \\
\text { Radius } \\
\text { After } \\
\text { Cu Ox. } \\
\text { (nm) }\end{array}$ & $\begin{array}{c}\text { Shell } \\
\text { Thick } \\
\text { After } \\
\mathrm{Cu} \\
\text { Ox. } \\
(\mathrm{nm})\end{array}$ & $\begin{array}{c}\text { Total } \\
\text { Radiu } \\
\text { S } \\
\text { After } \\
\text { Cu } \\
\text { Ox. } \\
(\mathrm{nm})\end{array}$ \\
\hline $\mathrm{Cu}_{1} / \mathrm{Au}_{0.1}$ & $\begin{array}{c}0.52 \pm \\
0.06\end{array}$ & $19 \pm 2$ & 37 & $\begin{array}{l}70 \\
70\end{array}$ & 1.28 & $\begin{array}{c}0.26 \\
(0.90)\end{array}$ & $\begin{array}{c}1.54 \\
(2.33)\end{array}$ \\
\hline $\mathrm{Cu}_{1}$ & & \pm & $\begin{array}{c}\mathrm{Cu}_{3700 \mathrm{Au}} \\
740\end{array}$ & $\begin{array}{c}\mathrm{Cu}_{1036} \mathrm{Au} \\
740\end{array}$ & 1.46 & $\begin{array}{l}0.38 \\
(1.3)\end{array}$ & $\begin{array}{c}1.84 \\
(2.42)\end{array}$ \\
\hline $\mathrm{Cu}_{1}$ & $\begin{array}{c}0.91 \pm \\
0.22\end{array}$ & $34 \pm 8$ & $\begin{array}{c}\mathrm{Cu}_{3700 \mathrm{Au}} \\
1110\end{array}$ & $\begin{array}{c}\mathrm{Cu}_{1259 A u} \\
1110\end{array}$ & 1.56 & $\begin{array}{l}0.48 \\
(1.7)\end{array}$ & $\begin{array}{c}2.04 \\
(2.51)\end{array}$ \\
\hline $\mathrm{Cu}_{1} / \mathrm{Au}_{0.5}$ & $\begin{array}{c}1.22 \pm \\
0.15\end{array}$ & $41 \pm 5$ & $\begin{array}{c}\mathrm{Cu}_{3700 \mathrm{Au}} \\
1850\end{array}$ & $\begin{array}{c}\mathrm{Cu}_{1518} \mathrm{Au} \\
1850\end{array}$ & 1.66 & $\begin{array}{l}0.66 \\
(2.3)\end{array}$ & $\begin{array}{c}2.32 \\
(2.67)\end{array}$ \\
\hline $\mathrm{Cu}_{1} / \mathrm{Au}_{1}$ & $\begin{array}{c}2.00 \pm \\
0.07\end{array}$ & $50 \pm 2$ & $\begin{array}{c}\mathrm{Cu}_{3700 \mathrm{Au}} \\
3700\end{array}$ & $\begin{array}{c}\mathrm{Cu}_{1851} \mathrm{Au} \\
3700\end{array}$ & 1.77 & $\begin{array}{l}1.00 \\
(3.5)\end{array}$ & $\begin{array}{c}2.77 \\
(3.00)\end{array}$ \\
\hline $\mathrm{Cu}_{1} / \mathrm{Au}_{2}$ & $\begin{array}{c}2.34 \\
\pm 0.03\end{array}$ & $85 \pm 1$ & $\begin{array}{c}\mathrm{Cu}_{3700 \mathrm{Au}} \mathrm{Au} \\
7400\end{array}$ & $\begin{array}{c}\mathrm{Cu}_{3146} \mathrm{Au} \\
7400\end{array}$ & 2.11 & $\begin{array}{l}1.34 \\
(4.7)\end{array}$ & $\begin{array}{c}3.45 \\
(3.50)\end{array}$ \\
\hline
\end{tabular}


To better understand the stabilized $\mathrm{Cu} / \mathrm{Au}$ structure formed by $\mathrm{Au}$ rearrangement during initial $\mathrm{Cu}$ oxidation in the presence of $\mathrm{Br}^{-}$, we calculated the $\mathrm{Au}: \mathrm{Cu}$ ratio under the $\mathrm{Au}$ peak at $0.7 \vee$ for the different $\mathrm{Cu}_{1} / \mathrm{Au}_{\mathrm{x}}(\mathrm{x}=0.1$ to 2$)$ compositions based on the peak integrations from the ASV and the known synthesis ratios. Table 8.2 shows the results of the calculations. The details of the calculations along with one example are in the appendix. These calculations reveal that as the amount of $\mathrm{Au}$ increases, the amount of $\mathrm{Cu}$ retained under the Au peak increases, but the overall $\mathrm{Au}$ : $\mathrm{Cu}$ ratio also increases, showing that there is actually less $\mathrm{Cu}$ retained per Au atom with increasing Au in the NP. In the NP with the least amount of $\mathrm{Au}\left(\mathrm{Cu}_{1} / \mathrm{Au}_{0.1}\right)$, for example, $19 \%$ of the $\mathrm{Cu}$ is oxidized along with the $\mathrm{Au}$, which amounts to each $\mathrm{Au}$ atom retaining a maximum of $\sim 2 \mathrm{Cu}$ atoms. Since the initial $\mathrm{Cu}$ core contained about 3700 atoms, this is an estimated

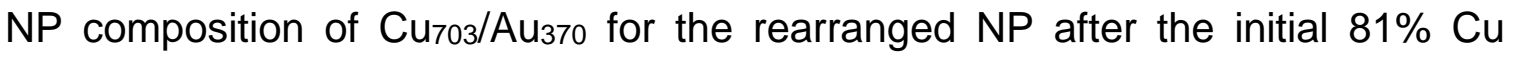
oxidation. This results in a $\sim 2.6 \mathrm{~nm}$ diameter $\mathrm{Cu}$ core with about a $0.26 \mathrm{~nm}$ thick shell for a final diameter of $\sim 3.1 \mathrm{~nm}$ (Initial diameter $\sim 4.6 \mathrm{~nm}$ ). A complete shell requires about $413 \mathrm{Au}$ atoms based on a geometric calculation with a $703 \mathrm{Cu}$ core. This is reasonably close to the $370 \mathrm{Au}$ atoms available per NP (determined by ASV) to form this type of stable core/shell structure. Table 8.16 shows the $\mathrm{Au}: \mathrm{Cu}$ ratio under the Au peak, the $\% \mathrm{Cu}$ retained, the approximate compositions of the $\mathrm{Cu} / \mathrm{Au}$ core/shell NPs before and after removal of the initial amount of $\mathrm{Cu}$ (up to $0.4 \mathrm{~V}$ ), and the radius of the core, the shell, and overall NP after initial Cu oxidation and Au rearrangement. The number in parentheses in Column 7 is the number of Au shell layers surrounding the $\mathrm{Cu}$ core for the rearranged alloy structures after 
initial $\mathrm{Cu}$ oxidation, which was determined by dividing the shell thickness by 0.288 nm. The number in parentheses in Column 8 of Table 8.16 is the initial $\mathrm{Cu} / \mathrm{Au}$ core/shell NP radius based on TEM and geometric considerations. The number of Au layers ranges from 1 to 5 layers for $x=0.1$ to 2.0 , showing that there is enough $\mathrm{Au}$ to act as a shell and passivate the $\mathrm{Cu}$ core in all of these rearranged alloy structures. A sample calculation for the atomic composition and NP dimensions is shown below.

Based on the fact that we were able to electrochemically form a stable $\mathrm{Cu} / \mathrm{Au}$ alloy NP with an $\mathrm{Au}$ :Cu ratio of 1:2 in the presence of $\mathrm{Br}$ for $\mathrm{Cu}_{1} / \mathrm{Au}_{0.1}$ after initial Cu oxidation, we attempted to directly synthesize a NP with a cit-stabilized $\mathrm{Cu}_{1} \mathrm{Au}_{0.5}$ alloy NP in the presence of $\mathrm{Br}$. The synthesis was performed the same way as the mixed alloy NPs already discussed but with the addition of $100 \mu \mathrm{L}$ of $10 \mathrm{mM} \mathrm{KBr}$ in the solution. Figure 8.9.(A) shows the ASVs of these NPs obtained in $10 \mathrm{mM} \mathrm{KCl}$ plus $0.1 \mathrm{M} \mathrm{HClO}_{4}$ solution (to avoid electrochemical rearrangement) after attachment to glass/ITO/APTES electrodes 15 minutes (red plot) and 2 hours (blue plot) after the introduction of borohydride during the synthesis. After 15 minutes of synthesis, the ASV showed both Cu and Au oxidation peaks separately with an integrated $\mathrm{Au}$ :Cu ratio of $0.74 \pm 0.11$, which is consistent with the synthesis ratio of 0.75 (in terms of Coulombs). After 2 hours of synthesis, however, there is just primarily one peak for Au oxidation. This is consistent with the passivation of $\mathrm{Cu}$ by the $\mathrm{Au}$ via $\mathrm{Br}^{-}$induced rearrangement of $\mathrm{Au}$ to form a passivating $\mathrm{Cu} / \mathrm{Au}$ core/shell structure. We performed the same synthesis of cit-Cu $u_{1} \mathrm{Au}_{0.5}$ NPs in the absence of $\mathrm{Br}^{-}$and the NPs crashed out of solution fairly quickly and were not 
stable. We believe that the synthesis initially leads to a large amount of Au in the core due to its more positive reduction potential, which leaves Cu more in the shell. In the absence of $\mathrm{Br}^{-}$, the NPs crash out due to the instability of $\mathrm{Cu}$ in the shell (it's easy to oxidize). In the presence of $\mathrm{Br}^{-}$, however, the NPs rearrange by $\mathrm{Au}$ diffusing to the shell, leading to stable $\mathrm{Cu}_{1} / \mathrm{Au}_{0.5}$ core/shell NPs. The $\mathrm{Br}$ facilitates Au rearrangement by adsorption to the NP surface. 

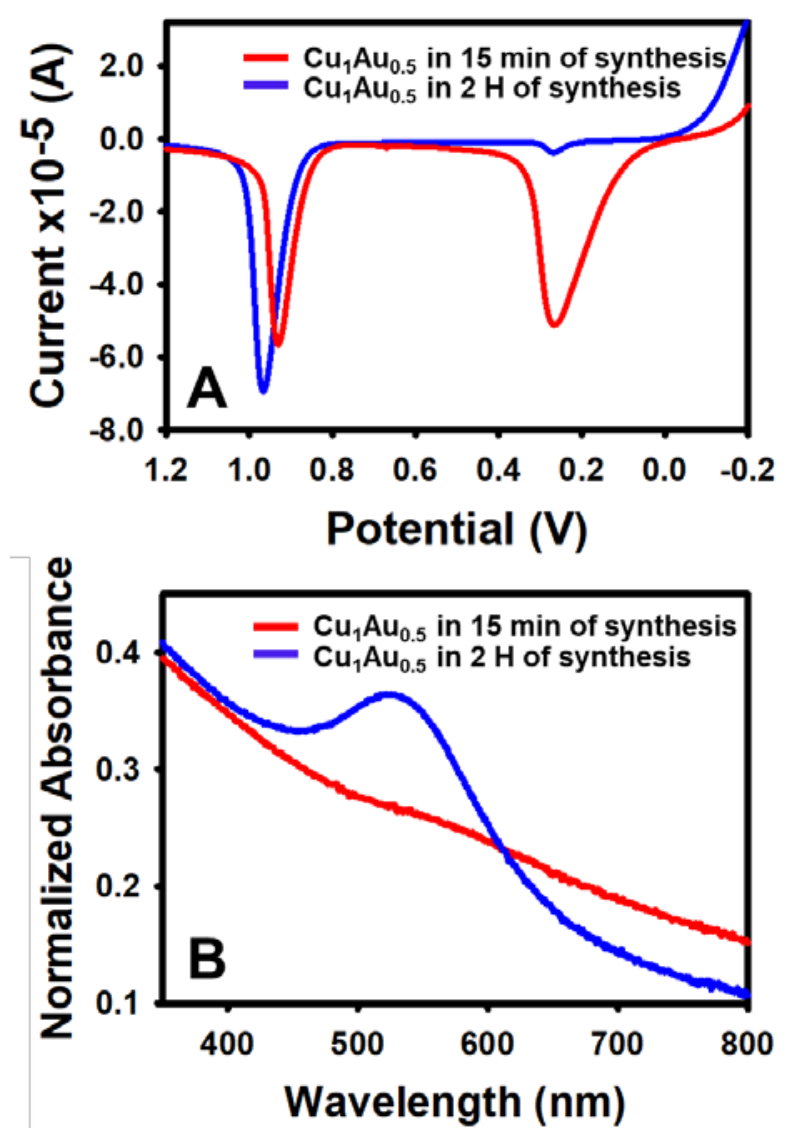

Figure 8. 9. (A) ASV of cit-Cu1Au0.5 mixed alloy NPs in $10 \mathrm{mM} \mathrm{KCl}$ plus $0.1 \mathrm{M}$ $\mathrm{HClO}_{4}$ after $15 \mathrm{~min}$ (red) and 2 hour (blue) of synthesis in the presence of $100 \mu \mathrm{L}$ of $10 \mathrm{mM} \mathrm{KBr}$. (B) Normalized UV-Vis spectra of the same alloy cit-Cu${ }_{1} \mathrm{Au}_{0.5} \mathrm{NPs}$ after 15 min (red) and 2 hour (blue) of synthesis.

Figure 8.9.(B) shows the UV-vis spectra of the Cu1Au0.5 NP solution 15 min (red) and 2 hours (blue) after borohydride reduction. The emergence of an Au LSPR peak with time is consistent with Au diffusion from the core to the shell of the alloy NP. Halide-induced transformation of Au-containing NPs have been observed 
previously and used for optical-based detection of iodide. ${ }^{149}$ Our results show $\mathrm{Cu} / \mathrm{Au}$ alloy NP transformations occur during electrochemical $\mathrm{Cu}$ dealloying of $\mathrm{Cu} / \mathrm{Au}$ core/shell NPs with incomplete or thin Au shells and during the synthesis of mixed CuAu alloy NPs in the presence of $\mathrm{Br}$. This highlights the power of ASV to track these types of transformations within alloy nanostructures.

\subsection{CONCLUSIONS}

In conclusions, we monitored the oxidation behavior of core/shell Cu/Au NPs by ASV and found consistent with the core/shell structure and varied significantly with different halides and different amounts of $\mathrm{Au}$ in the shell. The ASV in $\mathrm{KCl}$ show accurate composition analysis of $\mathrm{Cu}$ and $\mathrm{Au}$ in $\mathrm{a} \mathrm{Cu}_{1} / \mathrm{Au}_{\mathrm{x}}$ core/shell alloy NP for this range of $x=0.1$ to 1.0. The ASV in $\mathrm{KCl}$ is therefore useful for accurate composition analysis of $\mathrm{Cu}$ and $\mathrm{Au}$ in a Cu/Au core/shell alloy NP for this range of ratios. However, in $\mathrm{KBr}$ solution different $\mathrm{Cu}$ oxidation potentials appear due to different bonding environments, but the sum integration of the Cu peaks relative to Au does not correlate with the composition due to much of the $\mathrm{Cu}$ being oxidized along with the $\mathrm{Au}$. The amount of $\mathrm{Cu}$ retained with the $\mathrm{Au}$ depends upon the amount of $\mathrm{Au}$ in the shell. For the smallest amount of $\mathrm{Au}$ in the shell, there are $\sim 2$ $\mathrm{Cu}$ atoms retained for every $1 \mathrm{Au}$ atom. Our results show rearrangement of the Au atoms in the partial or thin shells occurs by surface diffusion during the initial oxidation of $\mathrm{Cu}$ in $\mathrm{KBr}$, which results in stable $\mathrm{Cu} / \mathrm{Au}$ core/shell NP structures. While the full details of the $\mathrm{Br}^{-}$- induced stabilization of the $\mathrm{Cu} / \mathrm{Au}$ core/shell NPs is not completely understood, three important discoveries have been revealed in 
this work. (1) ASV is useful to distinguish the atomic arrangement of bimetallic alloy NPs as was observed in the big difference between the ASV of Cu/Au core/shell and CuAu mixed alloy NPs with a 1:2 Cu/Au ratio. (2) ASV in the presence of $\mathrm{KCl}$ is a very useful analytical tool for the accurate composition analysis of $\mathrm{Cu}_{1} / \mathrm{Au}_{x}$ core/shell alloy NPs (and likely other atomic arrangements) over a wide composition range ( $x=0.1$ to 1.0$)$. (3) ASV is excellent for detecting and analyzing interesting NP rearrangements on the nanoscale, which in this case occurred due to the oxidation of $\mathrm{Cu}$ from Cu/Au core/shell NPs (with incomplete or thin Au shells) in $\mathrm{Br}^{-}$and during the chemical synthesis of mixed CuAu alloy NPs in the presence of $\mathrm{Br}^{-}$. 


\section{CHAPTER IX}

\section{THE USE OF ANODIC STRIPPING VOLTAMMETRY TO ANALYZE THE CHANGES IN ATOMIC ARRANGEMENTS OF AuCU BIMETALLIC NANOPARTICLES UPON HEATING}

\subsection{INTRODUCTION}

Recently bimetallic AuCu NPs with different composition and structures have been extensively studied due to their tunable optical and electrochemical properties which are useful for catalytic $289-290$ and sensing ${ }^{291-292}$ applications. The thermal stability of NPs is one of the key factors to understand the long-term reactivity of NPs during many catalytic applications. NPs are usually synthesized or often operated at high temperatures for aforementioned applications. During this, the reactivity of NPs decreases as they undergo sintering either by Ostwald ripening ${ }^{76}$ or Smoluchowski ripening. ${ }^{8}$ Sintering leads to a significant decrease in the surface area-to-volume ratio of metal NPs which further results in a remarkable decrease in their reactivity. The sintering or ripening in metal NPs has been observed by various researchers during their thermal treatment, ${ }^{76}$ ozone cleaning ${ }_{,}^{25}$ electrochemical cycling ${ }^{249}$ or by electron beam radiation on NPs. ${ }^{293}$ For example, Prevot and coworkers reported the Ostwald driven phase separation behavior of AuPd alloy NPs during thermal treatment. ${ }^{76}$ It was observed that 
bimetallic AuPd NPs segregated into individual $3.5 \mathrm{~nm}$ radius Au-rich NPs and 25 $\mathrm{nm}$ radius $\mathrm{Pd}$-rich NPs after thermal treatment at $873 \mathrm{~K}$. Very recently, Liu and coworkers studied the fusion behavior of AgCu bimetallic NPs induced by a high energy electron beam. ${ }^{293}$ The fusion phenomenon of NPs was observed by the decrease of their active surface area, increase of their sintering neck radii and decrease of neck curvature. Further, the Wei group reported unidirectional diffusion of $\mathrm{Au}$ atoms from shell to core for bimetallic core/shell Cu/Au NPs. ${ }^{294}$ They found that Au atoms start to diffuse after annealing at $423 \mathrm{~K}$. This change was monitored by in situ X-ray absorption spectroscopy and compared with theoretical simulations. In most cases, high vacuum techniques, such as transmission electron microscopy (TEM), ${ }^{295}$ high angle annular dark field scanning transmission electron microscopy (HAADF-STEM) ${ }^{294,296}$ or scanning transmission electron microscopy (STEM) with electron dispersion spectroscopy (EDS) 297,298 and X-ray absorption spectroscopy 294 have been routinely used to characterize those changes in the NPs. However, all these techniques are expensive, time consuming and cannot provide all of the necessary information, such as stability towards oxidation.

Electrochemical methods, such as anodic particle coulometry, cyclic voltammetry or anodic stripping voltammetry (ASV) are being considered as alternative approaches for analysis of NPs due to the selectivity, low-cost operation, and very short analysis time. Our group recently developed ASV for the analysis of metal NP size, ${ }^{154,199}$ aggregation state, ${ }^{299}$ and surface area-to-volume ratio (SA/V). ${ }^{201}$ Recently, we also reported the composition analysis of bimetallic 
core/shell Cu/Au NPs in one ASV. ${ }^{203}$ In this work we demonstrate the use of ASV characterize bimetallic AuCu NPs having different atomic arrangements, including core/shell $\mathrm{Cu}_{1} / \mathrm{Au}_{0.33}$, Core/shell $\mathrm{Au}_{0.33} / \mathrm{Cu}_{1}$ and mixed $\mathrm{Cu}_{1}-\mathrm{Au}_{0.33}$ alloy NPs before and after thermal treatment. Bimetallic AuCu NPs have been widely studied due to their tunable optical ${ }^{300}$ and electronic properties ${ }^{301}$ and ability to form chemically ordered structures, ${ }^{290}$ which are very important for high-density magnetic storage devices. ${ }^{302}$ One important property that needs to be better understood for longterm applications is their oxidation behavior, which often plays a significant role in the stability. ${ }^{199}$ We believe that ASV can provide information on oxidation properties but also fundamental information regarding the atomic structure of the bimetallic NPs, since the bond strength between two different metals atoms will be different when they are arranged in different ways. The different bonding should be reflected in the measurement of their oxidation potentials.

There have been previous reports on the electrochemical characterization of bimetallic NPs. For example, Brodsky and coworkers reported migration of Au atoms from well-defined core/shell Au/Pd NPs by repetitive electrochemical oxidation-reduction cycles in cyclic voltammetry (CV). ${ }^{295}$ In the first CV cycle, no significant oxidation peak for surface Au atoms was observed. However, the drastic change in core/shell structure after the $3^{\text {rd }}$ oxidation cycle was observed, where Au oxidation peaks appeared in higher coverage than from the $1^{\text {st }}$ and $2^{\text {nd }}$ cycles. However, they couldn't observe any such changes in mixed Au-Pd NPs. This study was important to understand the difference in arrangements of bimetallic NPs. Recently, Compton and coworkers reported anodic particle 
coulometry to determine the size of core/shell Au/Ag NPs. ${ }^{303}$ The size that they determined from the collision oxidation peak closely matched with the TEM determined size. This method of analysis was useful for analyzing the size of NPs but it can't show differences between different atomic arrangements. In this work, we focused on the use of ASV to analyze the atomic arrangement and thermal stability of the arrangement for bimetallic core/shell $\mathrm{Cu}_{1} / \mathrm{Au}_{0} .33$, Core/shell $\mathrm{Au} \mathbf{0}_{.33} / \mathrm{Cu}_{1}$ and mixed $\mathrm{Cu}_{1}-\mathrm{Au}_{0.33}$ alloy NPs.

\subsection{RESULTS AND DISCUSSION}

9.2.1. Synthesis and Characterizaton of AuCu Bimetallic NPs. Bimetallic AuCu NPs with three different atomic arrangements were synthesized based on the controlled reduction of two different metal ions as discussed in the experimental sections. Figure 9.1. (A) shows the UV-vis spectra of as-synthesized citrate (cit)stabilized bimetallic core/shell $\mathrm{Cu}_{1} / \mathrm{Au}_{0.33}$ (blue plot), core/shell Au0..3/ $/ \mathrm{Cu}_{1}$ (pink plot) and mixed $\mathrm{Cu}_{1}-\mathrm{Au}_{0.33}$ (red plot) alloy NPs. The core/shell cit-Cu1/Auo.33 and mixed cit-Cu1-Auo.33 NPs do not display any localized surface plasmon resonance (LSPR) band in the UV-Vis region, indicating that there is an insufficient amount of $\mathrm{Au}$ atoms to display a noticeable absorbance. The cit-Auo.33/ $\mathrm{Cu}_{1}$ core/shell NPs also do not show a LSPR band, which is due to a small amount of Au but it being in the NP core. Unfortunately, The UV-Vis spectra can't distinguish between core/shell and mixed alloy AuCu NPs. Figure 9.1.(B,C) shows transmission electron microscopy (TEM) images of mixed $\mathrm{Cu}_{1}-\mathrm{Au}_{0.3}$ alloy (B) and core/shell Au0.33/Cu1 (C) NPs, revealing an average NP size of $3.4 \pm 0.8 \mathrm{~nm}$ and $5.1 \pm 1.0 \mathrm{~nm}$ in 
diameter, respectively, for mixed $\mathrm{Cu}_{1}-\mathrm{Au}_{0.33}$ alloy and core/shell Auo.33/Cu1 NPs. TEM also does not provide details of the atomic arrangement of $\mathrm{Au}$ and $\mathrm{Cu}$.

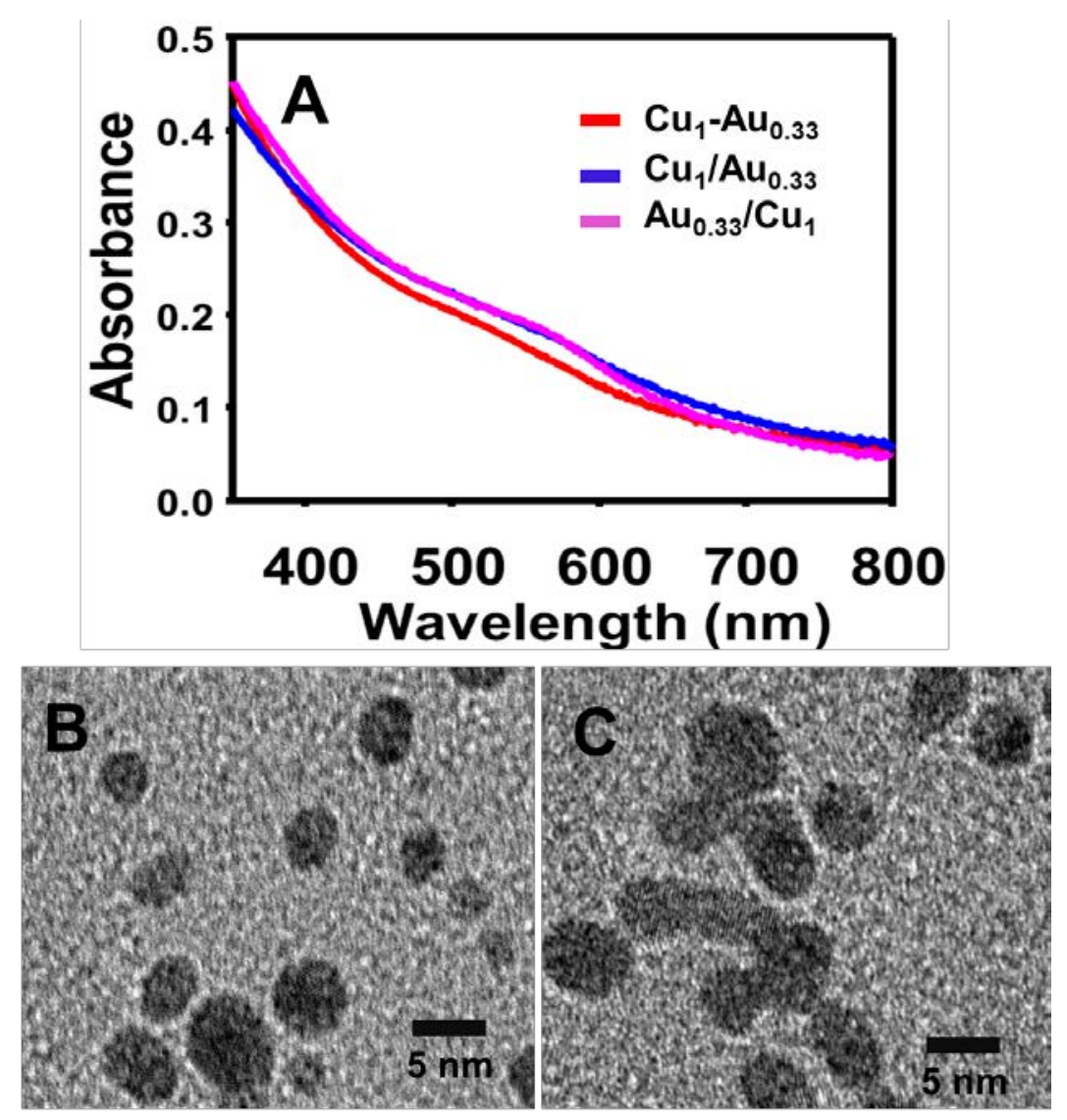

Figure 9. 1. (A) UV-Vis spectra of citrate stabilized core/shell Cu1/Auo.33, core/shell cit-Au0..3/ $\mathrm{Cu}_{1}$ and mixed $\mathrm{Cu}_{1}-\mathrm{Au}_{0.33}$ alloy NPs. TEM image of mixed $\mathrm{Cu}_{1}-\mathrm{Au}_{0.33}$ alloy (B) and core/shell cit-Au0.33/Cu alloy (C) NPs.

\subsubsection{ASV Analysis of Citrate-Stabilized AuCu Bimetallic} Nanoparticles. Figure 9.2 shows ASVs of glass/ITO/APTES electrodes coated with citrate-stabilized Cu NPs (dark green), cit-Au NPs (gray), mixed cit-Cu1-Auo.33

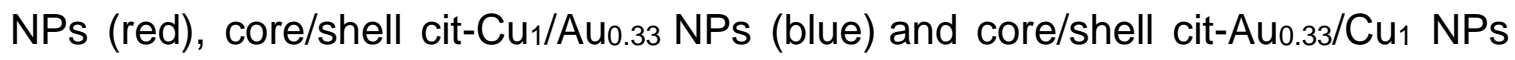


(pink). ASVs of cit-Cu and cit-Au NPs display one oxidation peak at $-0.1 \mathrm{~V}$ (dark green plot) and $0.95 \mathrm{~V}$ (gray plot) vs. an $\mathrm{Ag} / \mathrm{AgCl}$ reference electrode, respectively, which is consistent with the oxidation peak potential $\left(E_{p}\right)$ of pure $\mathrm{Cu}$ and $\mathrm{Au}$ NPs reported by our group ${ }^{203}$ and others previously. ${ }^{288}$ The ASV of mixed cit-Cu1-Aun.33 alloy NPs (red plot) displays two distinct oxidation peaks at $0.25 \mathrm{~V}$ and $0.89 \mathrm{~V}$ (red plot). The peak at $0.25 \mathrm{~V}$ is due to oxidation of $\mathrm{Cu}$ which shifted positive of pure Cu oxidation because of $\mathrm{Cu}$ bonding with $\mathrm{Au}$ atoms ( $\mathrm{Cu}-\mathrm{Au}$ bonds). The oxidation peak at $0.89 \mathrm{~V}$ is due to the oxidation of pure $\mathrm{Au}$ (Au-Au bonds) following dealloying of $\mathrm{Cu}$ at the lower potentials, as reported by our group previously. ${ }^{192,} 203$ Interestingly, the ASV of the mixed cit- $\mathrm{Cu}_{1} \mathrm{Au}_{0.33}$ does not have a peak at $-0.1 \mathrm{~V}$, which shows that there are no $\mathrm{Cu}$ atoms in the NP that are not bonded to Au atoms. Clearly having $\sim 1 \mathrm{Au}$ atom for every $3 \mathrm{Cu}$ atoms allows all $\mathrm{Cu}$ atoms to be bonded to 1 or more Au atoms. In a face-centered cubic structure, each bulk atom has 12 atoms surrounding it and each surface atom has a maximum of 9 atoms surrounding it. With a 1:3 Au:Cu ratio, each bulk $\mathrm{Cu}$ would likely have at least 3 Au atoms bound to it and each surface Cu would have at least $2 \mathrm{Au}$ atoms bonded to it based on the atomic ratio during synthesis and assuming that the atoms were mixed homogeneously. We believe the presence of 1 to $2 \mathrm{Au}$ atoms bonded to each $\mathrm{Cu}$ atom causes the 2 peak signature in the ASV. The ratio of the integrated areas of the $\mathrm{Au}$ and $\mathrm{Cu}$ peaks (Au:Cu ratio) is $0.50 \pm 0.10$, which matches very well with the theoretical $\mathrm{Au}: \mathrm{Cu}$ atomic ratio of 0.49 based on the moles of $\mathrm{Au}$ and $\mathrm{Cu}$ used during the synthesis, showing that ASV is an excellent method to determine the composition of AuCu mixed alloy bimetallic NPs. ${ }^{203}$ 
The ASV of core/shell cit-Cu1/Auo.3 NPs (Figure 9.2, blue plot) displays three distinct oxidation peaks at $-0.05 \mathrm{~V}, 0.25 \mathrm{~V}$ and $0.89 \mathrm{~V} \mathrm{vs} \mathrm{Ag} / \mathrm{AgCl}$. The main difference between this ASV and that of the mixed cit-Cu${ }_{1} \mathrm{Au}_{0.33}$ NPs is the additional oxidation peak at $-0.05 \mathrm{~V}$, which is due to the oxidation of $\mathrm{Cu}$ atoms only bonded to other $\mathrm{Cu}$ atoms. With $\mathrm{Au}$ in the outer shell of the $\mathrm{Cu}$ core instead of the Au being evenly mixed throughout creating $\mathrm{Au}-\mathrm{Cu}$ bonds for every $\mathrm{Cu}$, there are interior core $\mathrm{Cu}$ atoms not bonded to $\mathrm{Au}$ at all. There will also be some surface $\mathrm{Cu}$ atoms not bonded to Au because a 0.33 relative amount of $\mathrm{Au}$ compared to $\mathrm{Cu}$ is not enough to form a complete shell of Au over the Cu core. The Au shell is only a partial shell, leaving many surface and interior $\mathrm{Cu}$ atoms not bonded to $\mathrm{Au}$. This results in the three-peak signature in the ASV due to the $\mathrm{Cu}-\mathrm{Cu}$ bond peak at -0.05 $\mathrm{V}$. In addition, the peak at $0.25 \mathrm{~V}$ is a doublet peak instead of 1 single peak in the mixed AuCu alloy. The reasons for this are not well understood. There could be different $\mathrm{Au}-\mathrm{Cu}$ sites accounting for this. The integrated peak ratio of $\mathrm{Au}: \mathrm{Cu}$ for the core/shell cit-Cu $1 / \mathrm{Au}_{0.3} \mathrm{NPs}$ was found to be $0.49 \pm 0.11$, similar to the theoretical synthesis ratio of 0.49 .

The ASV of the core/shell Au0.33/Cu1 NPs (Figure 9.2, pink plot) shows oxidation peaks at $-0.05,0.25$ (doublet) and $0.89 \mathrm{~V}$. The ASV signature of the core/shell $\mathrm{Au}_{0.33} / \mathrm{Cu}_{1} \mathrm{NPs}$ is very similar to the core/shell $\mathrm{Cu}_{1} / \mathrm{Au}_{0.33} \mathrm{NPs}$. The main difference is the ratio between the peak for $\mathrm{Cu}$ oxidation at $-0.05 \mathrm{~V}$ relative to the Au oxidation peak at $0.95 \mathrm{~V}$. With $\mathrm{Au}$ in the core and $\mathrm{Cu}$ in the shell, there are fewer $\mathrm{Cu}-\mathrm{Au}$ bonding interactions and more $\mathrm{Cu}-\mathrm{Cu}$ interactions as compared to $\mathrm{Cu}$ in the core and Au in the shell, which makes the ASV signature similar, but still 
noticeably different for the cit-Cu1/Au0.33 NPs compared to the cit-Auo.33/Cu1 NPs. This explains the larger peak at $-0.5 \mathrm{~V}$ for $\mathrm{Cu}-\mathrm{Cu}$ interactions when $\mathrm{Cu}$ is in the shell. The overall integrated Au:Cu peak area is still very close to the actual synthesis ratio in this ASV as it was with the other atomic arrangements.

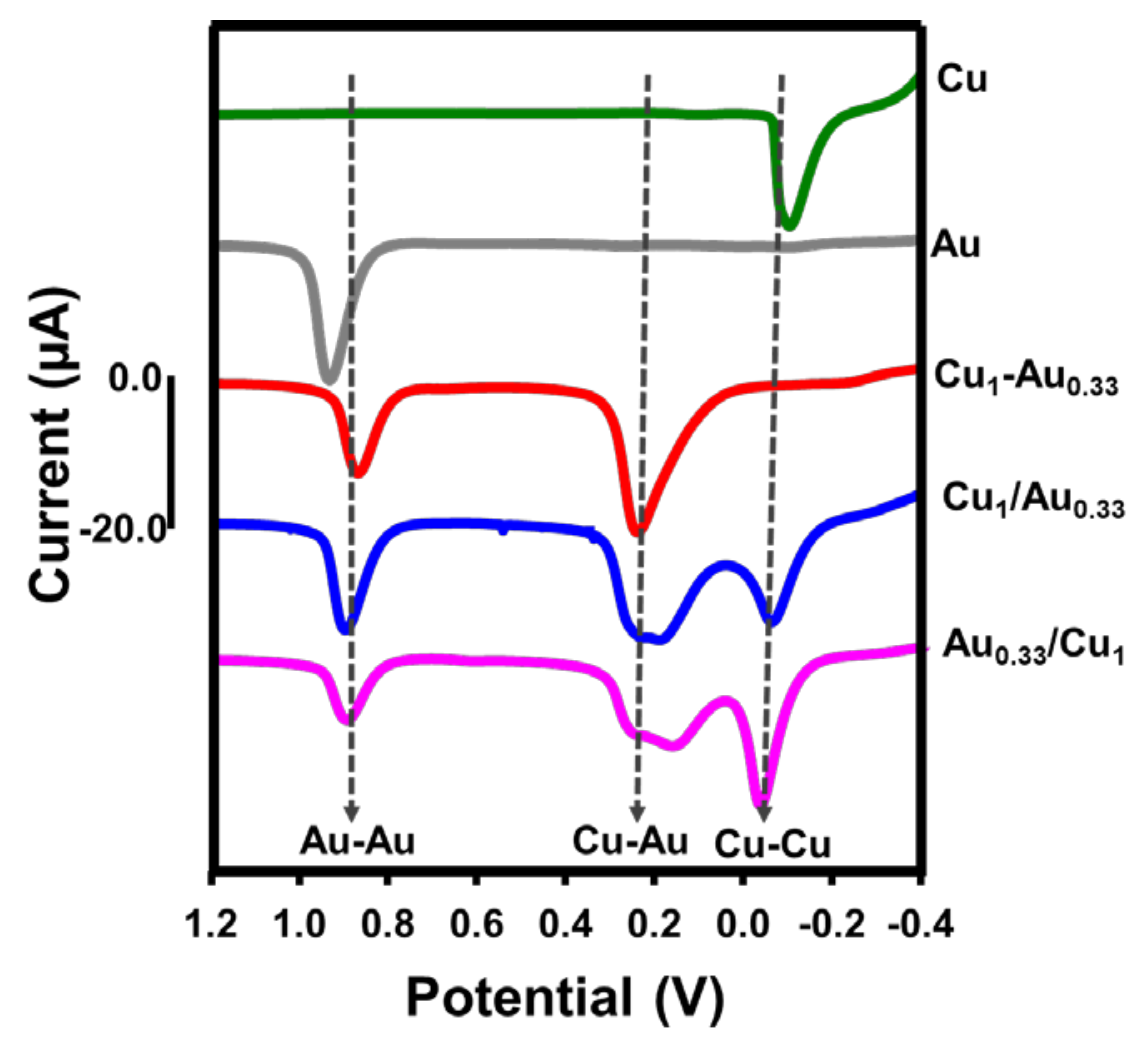

Figure 9. 2. ASV of citrate stabilized $\mathrm{Cu}, \mathrm{Au}$, mixed $\mathrm{Cu}_{1}-\mathrm{Au}_{0.33}$, core/shell $\mathrm{Cu}_{1} / \mathrm{Au}_{0.33}$ and core/shell Auo.33/Cu1 alloy NPs. ASV were performed in $10 \mathrm{mM} \mathrm{KCl}$ plus $0.1 \mathrm{M} \mathrm{HClO}_{4}$ solution.

Figure 9.3 shows the different arrangements of $\mathrm{Au}$ and $\mathrm{Cu}$ atoms in (a) mixed Cu1-Auo.33 alloy, (b) core/shell Au0.33/Cu1 and (c) core/shell Cu1/Auo.33 NPs. These three different possible arrangements of $\mathrm{Cu}$ and $\mathrm{Au}$ atoms are clearly reflected in our ASV signatures, where mixed $\mathrm{Cu}_{1}-\mathrm{Au}_{0.3}$ alloy NPs show oxidation 
peaks at $0.25 \mathrm{~V}$ and $0.90 \mathrm{~V}$ corresponding to $\mathrm{Cu}$ and $\mathrm{Au}$ atoms, indicating that $\mathrm{Au}$ atoms are mixed evenly, and every $\mathrm{Cu}$ atom is bound to at least one $\mathrm{Au}$ atom. Core/shell NPs show three distinct oxidation peak due to three different interaction of atoms (Au-Au, $\mathrm{Au}-\mathrm{Cu}$ and $\mathrm{Cu}-\mathrm{Cu}$ bonds). The difference between $\mathrm{Au} / \mathrm{Cu}$ core/shell and $\mathrm{Cu} / \mathrm{Au}$ core/shell is due to the number of $\mathrm{Au}-\mathrm{Cu}$ and $\mathrm{Cu}-\mathrm{Cu}$ bonds. As illustrated, the $\mathrm{Au} / \mathrm{Cu}$ core/shell has $14 \mathrm{Au}-\mathrm{Cu}$ bonds whereas the $\mathrm{Cu} / \mathrm{Au}$ core/shell has $10 \mathrm{Au}-\mathrm{Cu}$ bonds. This leads to a larger peak at $0.25 \mathrm{~V}$ relative to the peak at $-0.05 \mathrm{~V}$ for the $\mathrm{Au} / \mathrm{Cu}$ as compared to the $\mathrm{Cu} / \mathrm{Au}$ core/shell. The ASV signature of these bimetallic AuCu NPs with controlled arrangement along with the pure $\mathrm{Au}$ and pure $\mathrm{Cu}$ NPs provide good reference ASVs to probe atomic arrangements and changes in the atomic arrangement with various treatments, such as high temperature.

Figure 9. 3. Illustration of $\mathrm{Au}$ and $\mathrm{Cu}$ atoms arrangements in (a) mixed $\mathrm{Cu}_{1}-\mathrm{Au}_{0.33}$

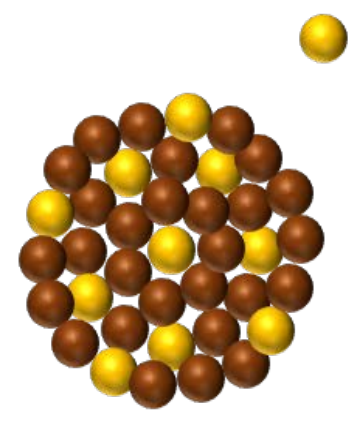

\section{Au atom}

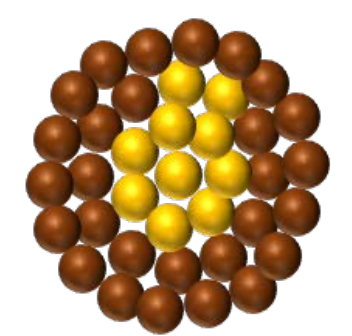

a. Mixed $\mathrm{Cu}_{1}-\mathrm{Au}_{0.3}$

b. Core/shell $\mathrm{Au}_{0.3} / \mathrm{Cu}_{1}$

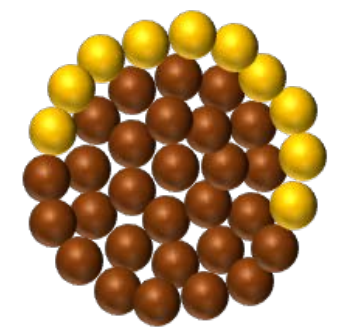

c. Core/shell $\mathrm{Cu}_{1} / \mathrm{Au}_{0.3}$

alloy, (b) Core/shell $\mathrm{Au}_{0.33} / \mathrm{Cu}_{1}$ and (C) core/shell $\mathrm{Cu}_{1} / \mathrm{Au}_{0.33} \mathrm{NPs}$. 
We next studied the thermal stability of the bimetallic AuCu NPs with the different atomic arrangements. Thermal treatment of metal NPs has been used regularly for cleaning the NPs before applications ${ }^{92}$ and also to make thin films of NPs. ${ }^{304}$ Also, NPs are often used as a catalyst under high temperature ${ }^{305}$ and gas phase conditions. ${ }^{225}$ There have been reports stating that the reactivity of bimetallic NPs decreases due to the sintering of NPs during their catalytic applications. ${ }^{306}$ Our goal here was to demonstrate the use of ASV to probe changes in atomic arrangement upon heating to better understand the thermal stability/sintering behavior of bimetallic AuCu NPs. Figure 9.4 shows the effect of thermal treatment of glass/ITO/APTES electrodes coated with mixed $\mathrm{Cu}_{1}-\mathrm{Au}_{0.33}$ alloy NPs at temperatures of $423 \mathrm{~K}$ for 1 hour (blue plot), $473 \mathrm{~K}$ for 1 hour (pink plot), and $473 \mathrm{~K}$ for 16 hour (dark red plot) as compared to the untreated NPs (red plot). The ASVs of $2 \mathrm{~nm}$ diameter Cu NPs (green plot) and $4 \mathrm{~nm}$ diameter Au NPs (black plot) after heating are also shown as references for the pure metal.

The ASV of the untreated mixed cit-Cu1-Auo.33 alloy NPs shows two distinct oxidation peaks at $0.25 \mathrm{~V}$ and $0.89 \mathrm{~V}$, which corresponds to the oxidation of $\mathrm{Cu}$ bonded to $\mathrm{Au}$ and oxidation of $\mathrm{Au}$ after dealloying of $\mathrm{Cu}$, respectively, as discussed previously. The ASV signature of mixed cit-Cu1-Auo.33 alloy NPs after 1 hour of heating at $423 \mathrm{~K}$ does not look significantly different, other than two very broad peaks appearing in the -0.2 to $0.1 \mathrm{~V}$ range and a small positive shift in the $\mathrm{Au}$ 
peaknear $0.92 \mathrm{~V}$. The ASV after 1 hour of heating at $473 \mathrm{~K}$ shows a more dramatic change, with a new peak clearly present at $0.0 \mathrm{~V}$, while the original peak at $0.25 \mathrm{~V}$ mostly disappeared. The Au peak at $0.92 \mathrm{~V}$ remained the same. The new oxidation peak at $0.0 \mathrm{~V}$ is close to the peak oxidation for pure $\mathrm{Cu}$, but is shifted positive by about $100 \mathrm{mV}$ relative to the $2 \mathrm{~nm}$ diameter Cu NPs. This could be due to the formation of larger Cu NPs upon heating, which should oxidize at potentials more positive than $-0.1 \mathrm{~V}$ due to the effect of size on oxidation peak potentials. ${ }^{64,154}$ To confirm this, we heated glass/ITO/APTES/CU2nm NPs to $473 \mathrm{~K}$ for 1 hour and found that the $\mathrm{Cu}$ oxidation peak appeared at $0.0 \mathrm{~V}$, suggesting that the peak

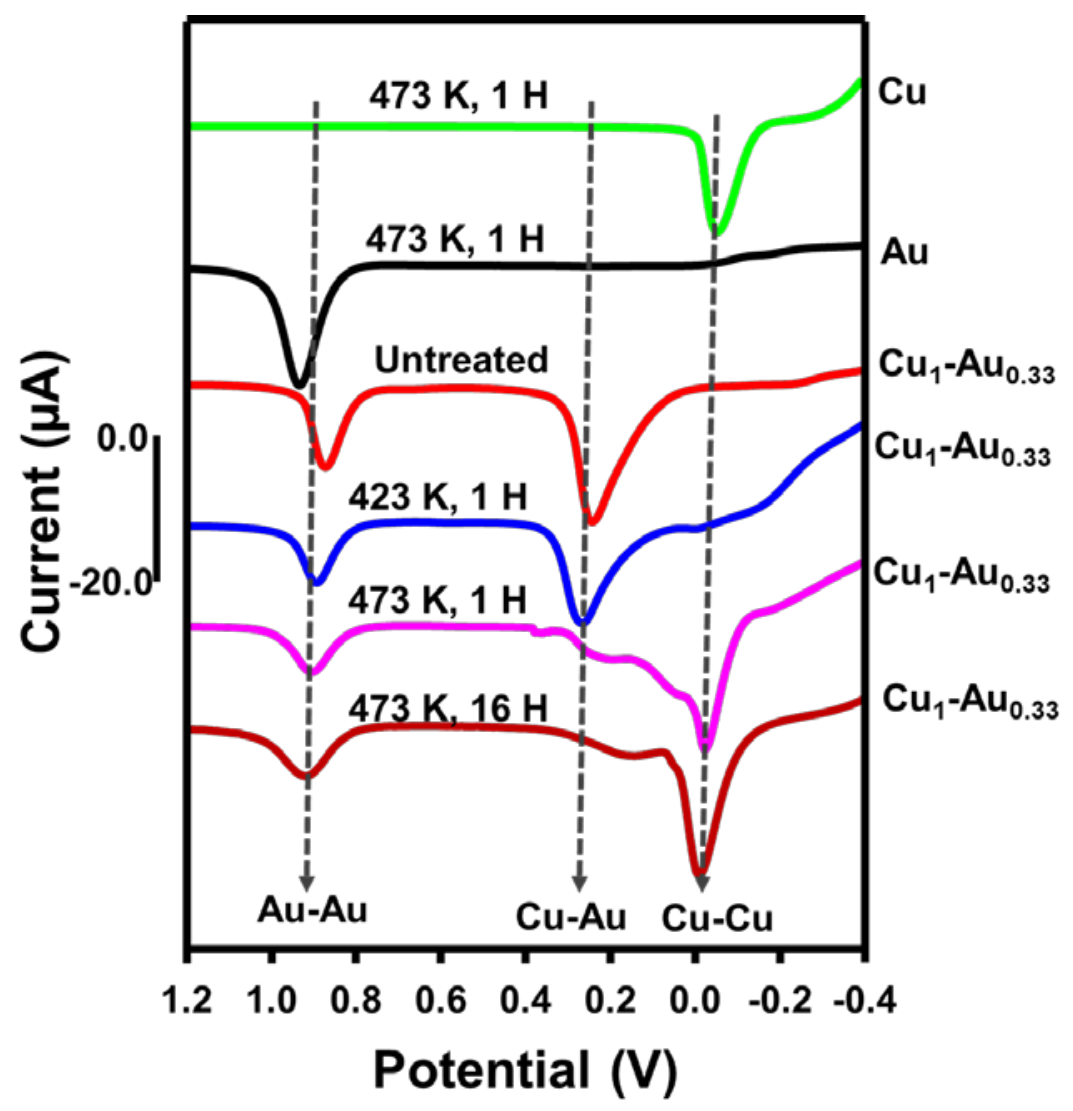

Figure 9.4. ASV of citrate stabilized $\mathrm{Cu}, \mathrm{Au}$, and mixed $\mathrm{Cu}_{1}-\mathrm{Au}_{0.33} \mathrm{NPs}$ before and after thermal treatment at different temperatures and times as indicated in the graph. ASVs were obtained in $10 \mathrm{mM} \mathrm{KCl}$ plus $0.1 \mathrm{M} \mathrm{HClO}_{4}$ solution. 
after heating the alloy is due to the oxidation of larger Cu NPs. The ASV also shows very small peaks at $0.05 \mathrm{~V}$ and $0.2 \mathrm{~V}$, which are likely $\mathrm{Cu}$ oxidation peaks for $\mathrm{Cu}$ bonded to $\mathrm{Au}$ in different arrangements. The majority of the Cu oxidizes at $0.0 \mathrm{~V}$, however. We integrated the individual peaks of $\mathrm{Cu}$ and $\mathrm{Au}$ and determined the $\mathrm{Au}: \mathrm{Cu}$ ratio to be $0.44 \pm 0.01$ which closely matches with the synthesis ratio. This shows that the same amount of $\mathrm{Au}$ and $\mathrm{Cu}$ remained on the surface after heating, but the arrangement of the atoms changed as reflected in the ASV

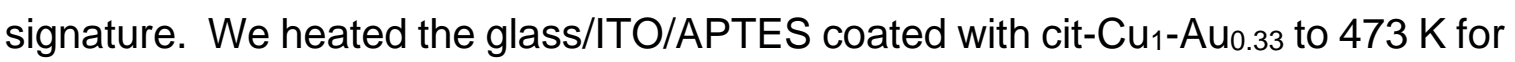
16 hours to determine whether a longer heating time had a significant effect on the atomic arrangement of the NPs. The ASV showed a similar signature as we observed after 1 hour of heating, except that the peak at $0.0 \mathrm{~V}$ became larger relative to Au and a very small broad peak remained at $0.1 \mathrm{~V}$. We believe the ASVs after heating indicate that $\mathrm{Cu}$ separates from the $\mathrm{Au}$ and becomes segregated into larger $\mathrm{Cu}$ structures or domains. This process is mostly finished after 1 hour, but small changes still occur after that at $473 \mathrm{~K}$.

The segregation of $\mathrm{Cu}$ atoms from $\sim 5 \mathrm{~nm}$ diameter $\mathrm{AuCu}$ alloy NPs supported on $\mathrm{TiO}_{2}$ during thermal treatment at $773 \mathrm{~K}$ was observed by Llorca and coworkers. ${ }^{307}$ The segregation of $\mathrm{Cu}$ atoms was determined by the expansion of lattice fringes of $\mathrm{AuCu}$ from 2.18-2.19 to $2.19-2.20$ and $2.21 \AA$ after thermal treatment at $773 \mathrm{~K}$ and $873 \mathrm{~K}$ respectively. Very recently, the segregation of $\mathrm{Cu}$ atoms from $\sim 8 \mathrm{~nm}$ diameter AuCu alloy NPs after thermal treatment at $523 \mathrm{~K}$ was reported by Zhan and coworkers. ${ }^{290}$ They monitored the surface segregation of $\mathrm{Cu}$ atoms by gradual shifting of the X-ray diffraction pattern towards low angle from 
$40.4^{\circ}$ to $38.6^{\circ}$ and $40.6^{\circ}$ to $39.2^{\circ}$ for the fcc and fct AuCu NPs after 1 hour at 473 K. Our ASV signature clearly confirms that segregation of $\mathrm{Cu}$ atoms from $\mathrm{AuCu}$ alloy NPs occurred at much lower temperature, which could depend on the support and other factors.

We performed a similar ASV temperature study for core/shell cit-Cu1/Au0.33 NPs (Figure $9.5 \mathrm{~A}$ ) and core/shell cit-Au0.33/Cu1 NPs (Figure $9.5 \mathrm{~B}$ ). The ASV of untreated core/shell $\mathrm{Cu}_{1} / \mathrm{Au}_{0.33} \mathrm{NPs}$ (Figure 9.5 A, red plot) shows three distinct oxidation peaks at $-0.05 \vee, 0.25 \mathrm{~V}$ (doublet) and $0.89 \mathrm{~V}$ before heating as discussed previously. After heating at $423 \mathrm{~K}$ for 1 hour (Figure 9.5A), blue plot), the ASV is similar except that the peak at $-0.05 \mathrm{~V}$ shifted positive to about $0.0 \mathrm{~V}$ ( $\mathrm{Cu}-\mathrm{Cu}$ bonds), the doublet at $0.25 \mathrm{~V}$ (Cu-Au bonds) split a little more, and the peak at $0.89 \mathrm{~V}$ (Au--Au bonds) shifted slightly positive. The small positive shift of the $\mathrm{Cu}$ and $\mathrm{Au}$ oxidation peaks indicates a small amount of sintering into larger $\mathrm{Cu}$ and Au NPs. It is not clear why the doublet peak splitting increased as the doublet peak in this region is not well understood, other than it is likely some form of $\mathrm{Cu}$ bonded to Au, possibly in different arrangements. The ASV indicates some small structural changes, but the details are not well understood. Next, we heated glass/ITO/APTES coated with core/shell Cu1/Auo..33 NPs to $473 \mathrm{~K}$ for 1 hour (Figure 9.5A), pink plot). The ASV shows decent sized peaks at $-0.05 \mathrm{~V}$ and $0.08 \mathrm{~V}, \mathrm{a}$ very small broad peak at $0.25 \mathrm{~V}$, and the Au oxidation peak near $0.90 \mathrm{~V}$. The peak at $0.90 \mathrm{~V}$ is again due to the Au oxidation after $\mathrm{Cu}$ dealloying. The $\mathrm{Cu}$ oxidation peak at $0.0 \mathrm{~V}$ is due to large domains of $\mathrm{Cu}-\mathrm{Cu}$ bonds and those at $0.08 \mathrm{~V}$ and $0.20 \mathrm{~V}$ are due to oxidation of $\mathrm{Cu}$ atoms bonded to $\mathrm{Au}$ atoms. The two different 
potentials could again correspond to different Au-Cu bonding arrangements. After heating at $473 \mathrm{~K}$ for $16 \mathrm{~h}$ (Figure 9.5A), maroon plot), the peak at $-0.05 \mathrm{~V}$ increased while the peak intensity at $0.08 \mathrm{~V}$ decreased significantly. We calculated the ratio of the integrated area of the peak at $-0.05 \mathrm{~V}$ to that of 0.25 before and after thermal treatment at these conditions. The ratio was $0.43 \pm 0.06$ before and $8.0 \pm 1.2$ after thermal treatment, which is an 18 fold increase. Only very small peaks existed in the 0.0 to $0.2 \mathrm{~V}$ range after heating. The main peak at $-0.05 \mathrm{~V}$ again indicates the presence of larger NP domains consisting of $\mathrm{Cu}-\mathrm{Cu}$ bonds. We determined the integrated Au:Cu peak ratio to be $0.50 \pm 0.05$, which matches with the synthesis ratio, indicating that no $\mathrm{Au}$ or $\mathrm{Cu}$ is lost during the temperature-induced atomic rearrangement.

We performed a similar ASV temperature study of core/shell Au0.33/Cu1 NPs NPs (Figure 9.5B). The ASV of untreated NPs (Figure 9.5B, red plot) again shows the three oxidation peaks at $-0.05 \mathrm{~V}, 0.25 \mathrm{~V}$ (doublet), and $0.89 \mathrm{~V}$. After heating at $423 \mathrm{~K}$ for 1 hour (Figure 9.5B, blue plot), the peak at $-0.05 \mathrm{~V}$ shifted negative a bit and had a shoulder peak near $0.05 \mathrm{~V}$, the doublet peak turned into a singlet at about $0.30 \mathrm{~V}$, and the Au peak remained unchanged. After heating at $473 \mathrm{~K}$ for 1 hour (Figure 9.5B, pink plot) and 16 hours (Figure 9.5B, maroon plot), the changes in the ASV were very similar to those for the $\mathrm{Cu}_{1} / \mathrm{Au}_{0.33}$ core/shell NPs. The peaks in the 0.05 to $0.30 \mathrm{~V}$ range disappeared and became replaced with one main peak at $0.0 \mathrm{~V}$. The peaks in the 0.05 to $0.30 \mathrm{~V}$ range are completely gone for the $\mathrm{Au} / \mathrm{Cu}$ core/shell NPs, where small peaks still remained in this range for the $\mathrm{Cu} / \mathrm{Au}$ core/shell. Since the Cu atoms appear to segregate themselves from the Au and 

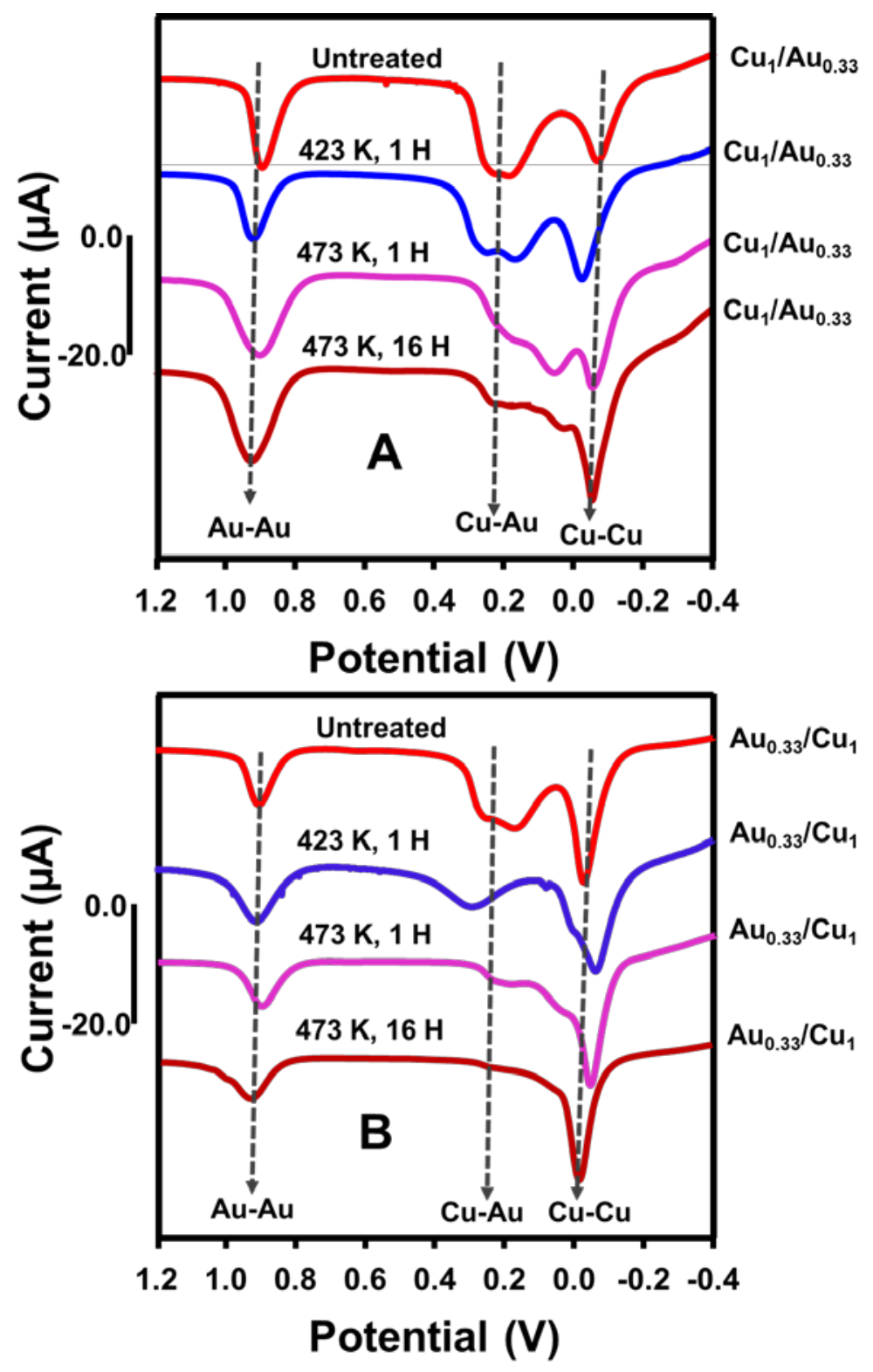

Figure 9. 4. ASV of citrate stabilized core/shell $\mathrm{Cu}_{1} / \mathrm{Au}_{0.33}(\mathrm{~A})$ and core/shell Auo.33/Cu1 (B) after thermal treatment at different temperature and time as indicated in the graph. ASV were performed in $10 \mathrm{mM} \mathrm{KCl}$ plus $0.1 \mathrm{M} \mathrm{HClO}_{4}$ solution. 
minimize Cu-Au bonding, this is likely easier to do when the $\mathrm{Cu}$ is in the shell, which is why no peaks for Au-Cu bonding remain after heating at $473 \mathrm{~K}$ for 16 hours. We again integrated all of the individual peaks for $\mathrm{Cu}$ and $\mathrm{Au}$ and determined the $\mathrm{Au}: \mathrm{Cu}$ ratio was $0.46 \pm 0.02$, which is very close to the synthesis ratio, indicating no change in atom ratio during heating, only a change in arrangement. The ratio of the Cu peak at -0.05 to $0.0 \mathrm{~V}$ relative to $0.20 \mathrm{~V}$ was 1.1 \pm 0.2 and $47 \pm 2$ before and after thermal treatment, which is a 40 fold difference. This is again due to the movement of $\mathrm{Cu}$ into segregated domains away from the $\mathrm{Au}$.

In order to confirm our interpretation of the ASV results, we obtained transmission electron microscopy (TEM) images of mixed cit-Cu1-Au0.з3 alloy and core/shell cit-Au0..3/ $/ \mathrm{Cu}_{1} \mathrm{NPs}$ after thermal treatment at $473 \mathrm{~K}$ for 1 hour and compared them to images of the untreated NPs. Figure 9.6 shows the TEM images of mixed cit-Cu1-Auo.33 alloy before (Figure 9.6(A,B)) and after heating (Figure 9.6(C,D)). The images and size histograms in Figure $5 \mathrm{C}$ and $5 \mathrm{D}$ show a significant average size increase and distribution after heating. Figure 9.6A shows a fairly uniform size distribution with an average diameter of $3.5 \mathrm{~nm}$. After heating, the average size of the NPs increases to $4.6 \mathrm{~nm}$ in diameter with high size dispersity due to significant populations of NPs near 8-9 nm, 6-7 nm, and $3 \mathrm{~nm}$. Interestingly, many of the NPs, especially the larger ones, show 2 different distinct shades of grey. We attribute the dark black shade to the heavier Au atoms, which will block the electrons more effectively, and the light gray color to the lighter $\mathrm{Cu}$ atoms. The color difference in TEM images for bimetallic NPs has been reported 
previously for the analysis of core/shell structure of bimetallic NPs. For example, Wei and coworkers reported similar color pattern for Cu-Au system as measured by HRTEM. ${ }^{294}$ Similarly, Compton and coworkers distinguish core/shell Au/Ag NPs by looking at the dark black color inside for $\mathrm{Au}$ atoms and lighter gray color for $\mathrm{Ag}$ atoms in the same NPs. ${ }^{303}$ The distinct color patterns within the larger NPs is consistent with segregation of the $\mathrm{Cu}$ and $\mathrm{Au}$ atoms, which is what the ASV results also show. In terms of the size dispersity, Granqvist and Buhrman postulated that the mechanism of ripening could be determined by measuring the size distribution of NPs in a population. ${ }^{79}$ Later, various groups validated the method to determine the ripening mechanism of metal NPs. ${ }^{8,76}$ The bimodal, or even trimodal, distribution indicates that the NPs grew in size during heating by Smoluchowski ripening. During this growth of NPs, $\mathrm{Au}$ and $\mathrm{Cu}$ atoms segregated into distinct domains. We are not certain about the composition of the large and small NPs in the sample after heating, but the large amount of dark regions suggests the larger NPs are rich in $\mathrm{Au}$, while the smaller NPs are likely rich in $\mathrm{Cu}$. We also obtained

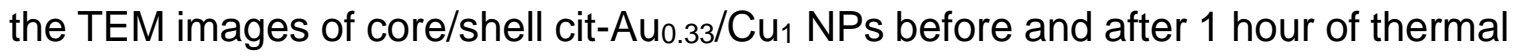
treatment at $473 \mathrm{~K}$. The TEM images show that the NPs were fairly uniform in size with an average diameter of $5.1 \pm 1.0 \mathrm{~nm}$. However, after thermal treatment, NPs increased in size to $8.1 \pm 2.9 \mathrm{~nm}$ with large size dispersity due to significant populations of NPs near 8-9 nm, 10-12 nm, and 14-15 nm in diameter. (Tables 9.1-9.12 shows the individual charge obtained for indicated experiment) 

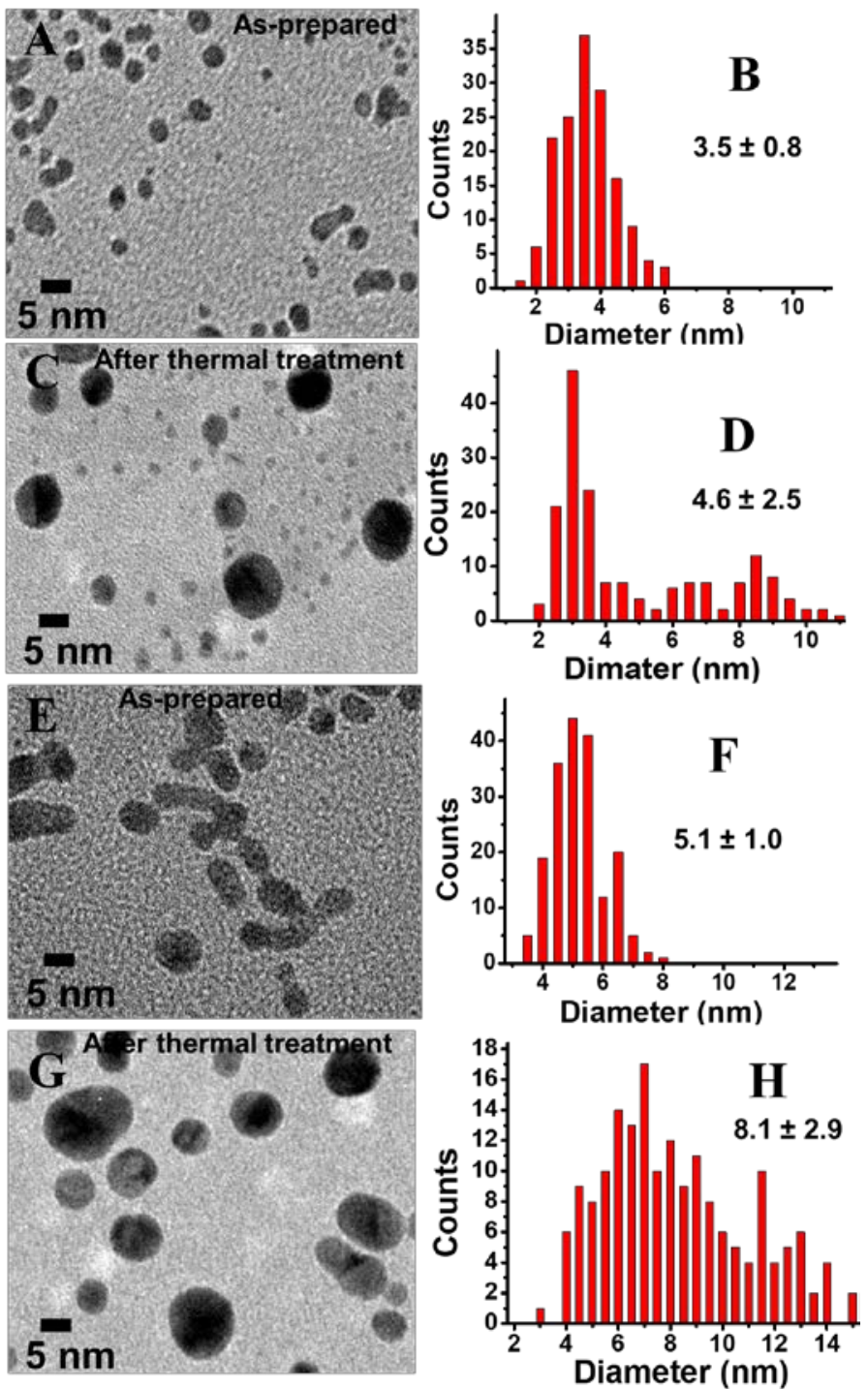

Figure 9. 5. TEM images of mixed cit-Cu1-Au0.з3 alloy NPs before (A) and after (C) heating at $473 \mathrm{~K}$ for 1 hour. Size histograms of the NPs before (B) and after (D) heating at $473 \mathrm{~K}$ for 1 hour. TEM images of core/shell cit-Auo.33/Cu 1 alloy NPs before $(E)$ and after $(G)$ heating at $473 \mathrm{~K}$ for 1 hour. Size histograms of the NPs before $(F)$ and after ( $G$ ) heating at $473 \mathrm{~K}$ for 1 hour. 


\subsection{CONCLUSIONS}

In conclusion we report the dramatic difference in the ASV signature of bimetallic mixed cit-Cu1-Auo.33 alloy NPs as compared to core/shell cit-Cu1/Au0.33

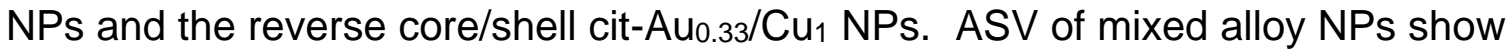
two distinct oxidation peaks where both types of core/shell NPs show three distinct peaks. This makes it easy to distinguish mixed alloy and core/shell NPs by ASV, but also the core/shell NPs were distinguishable by the peak ratio of the two $\mathrm{Cu}$ oxidation peaks. The ASV signatures for the CuAu bimetallic NPs are due to the different interactions between $\mathrm{Cu}$ and $\mathrm{Au}$ atoms in the different structures. The different arrangements led to different relative amounts of $\mathrm{Cu}-\mathrm{Au}$ bonding, which affected the size of the stripping peaks in the ASV associated with Cu bonded to Au. ASV is therefore highly promising as an analytical tool to determine approximate atomic arrangements within bimetallic NPs. In addition, ASV can determine the thermal stability of bimetallic CuAu NPs. The results show the onset of changes in atomic arrangement occurs around $423 \mathrm{~K}$. More significant changes occur at $473 \mathrm{~K}$, where upon heating for longer times the NPs sinter into populations of larger than 8-9 $\mathrm{nm}$ and $6-7 \mathrm{~nm}$ in diameter in addition to the original $3 \mathrm{~nm}$ diameter NPs. In addition, the $\mathrm{Cu}$ and Au appear to segregate from one another into specific domains, which lowers the number of $\mathrm{Cu}$-Au bonds in the NPs. The sintering and atom segregation process occurred for all of the different arrangements, indicating that the final thermodynamically most stable structure was reached independent of the original NP CuAu arrangement. More work is needed to improve the use of ASV to determine both the size and atomic 
arrangement for bimetallic NPs. Correlation of ASV with other analytical techniques will be useful in pushing the development further, where it has the benefits of being cheap, simple, and fast when compared to electron microscopy and x-ray spectroscopy methods. 


\section{CHAPTER X}

\section{SUMMARY, CONCLUSIONS AND FUTURE DIRECTIONS}

\subsection{SUMMARY}

This dissertation describes research focused on 1) the use of ASV for the characterization of size and aggregation state of Au NPs and the characterization of composition and atomic arrangement of various CuAu bimetallic alloy NPs and 2) the use of ASV and electrochemical SA/V measurements to study interesting size-dependent NP reactivity related to stability and catalysis. This includes size stability in response to ozone, heat, and electrochemical potential (Ostwald ripening). It also includes the interesting size-dependent exchange of $\mathrm{Au}$ with $\mathrm{Ag}^{+}$, unique rearrangements of CuAu upon dealloying in the presence of bromide (but not chloride), and the electrocatalytic activity of $1.6 \mathrm{~nm}$ Au NPs towards HER and $\mathrm{CO}_{2}$ reduction, but not other Au NPs sizes. All of these studies involved electrodeattached Au or CuAu NPs that would be used in electrochemical applications, including electrocatalysis, electrochemical sensing, or electrochemical energy.

One of the main goals of this research was to extend our use of ASV for Au NPs size analysis to Au NPs below $2 \mathrm{~nm}$ diameter. Accordingly, we measured the ASVs of electrode-attached-a $1.6 \mathrm{~nm}$ diameter THPC-stabilized and $0.9 \mathrm{~nm}$ 
diameter TPPS-stabilized Au NPs, determining that their peak oxidation potential $\left(\mathrm{E}_{\mathrm{p}}\right)$ was at $0.45 \mathrm{~V}$ and $0.20 \mathrm{~V}$ ( $\mathrm{vs} . \mathrm{Ag} / \mathrm{AgCl}$ reference electrode), respectively, in $\mathrm{KBr}$ electrolyte. The $\mathrm{E}_{\mathrm{p}}$ values are in line with the trend of previously-measured 2 $\mathrm{nm}$ to $250 \mathrm{~nm}$ diameter Au NPs and showed very good agreement between Plieth theory and the TEM measured diameters. This indicates the potential of ASV to determine the size of very small metal NPs directly on electrode surfaces, which is very difficult on commonly used electrodes using microscopy or other methods.

Also in the area of NP characterization, we determined the effect of different aggregation structures on the $E_{p}$ of $15 \mathrm{~nm}$ diameter citrate-stabilized Au NPs. The Au NPs aggregated at low $\mathrm{pH}$ showed and $\mathrm{E}_{\mathrm{p}}$ value of $0.90 \mathrm{~V}$ while it appeared at $0.77 \mathrm{~V}$ when aggregated by THPC. UV-vis spectra showed that both $\mathrm{pH}$ and THPC led to a high degree of aggregation. Electrochemical SA/V measurements showed a much higher SA/V for the THPC aggregated Au NPs when compared to the $\mathrm{pH}$ aggregated NPs. The higher SA/V explains the lower $E_{p}$ value, showing that SA/V is more important in determining the $E_{p}$ value than the NP size or curvature, since both aggregates are comprised of the same NPs. TEM images provide additional insight, showing that the aggregation of NPs by acid formed 3-dimensionsional structures that were highly fused, while the THPC-aggregated Au NPs formed linear 1D aggregates or planar 2D aggregates that had spacing between the NPs. The lack of 3D arrangement and fusion allowed the latter aggregates to retain their high SA/V and the same $E_{p}$ value of individual non-aggregated $15 \mathrm{~nm}$ diameter $\mathrm{Au}$ NPs. These findings clearly show that the ASV signature is highly sensitive 
towards size, but also the details of the aggregate structure. This provides additional details, especially when combined with spectroscopy data.

Our last work in the area of characterization involved the use of ASV for the composition analysis and analysis of atomic arrangement of different bimetallic citrate-stabilized CuAu NPs. The composition analysis was straight forward by measuring the peak area of $\mathrm{Cu}$ oxidation peaks and Au oxidation peaks in one ASV for citrate-stabilized core/shell Cu1/Aux,NPs when $x$ was $0.1-1.0$ and the ASV was obtained in $\mathrm{KCl}$ electrolyte. Higher $\mathrm{x}$ led to blocking of $\mathrm{Cu}$ oxidation and lower $\mathrm{x}$ made it difficult to detect the $\mathrm{Au}$. $\mathrm{KBr}$ electrolyte did not work due to $\mathrm{Cu}$ stabilization from mobile Au atoms during dealloying. ASV also allowed us to distinguish between citrate-stabilized $\mathrm{Au} / \mathrm{Cu}$ core/shell, $\mathrm{Cu} / \mathrm{Au}$ core/shell, and CuAu mixed alloy NPs from the location and relative intensities of the $\mathrm{Cu}$ and $\mathrm{Au}$ oxidation peaks in $\mathrm{KCl}$ electrolyte. The different ASV signatures are due to different number of $\mathrm{Au}-\mathrm{Au}, \mathrm{Au}-\mathrm{Cu}$, and $\mathrm{Cu}-\mathrm{Cu}$ bonds in the NPs with the different arrangements, which have $E_{p}$ values of about $0.90 \mathrm{~V}, 0.25 \mathrm{~V}$, and $-0.1 \mathrm{~V}$, respectively. CuAu mixed alloys have the largest proportion of $\mathrm{Au}-\mathrm{Cu}$ bonds and exhibit no $\mathrm{Cu}-\mathrm{Cu}$ bonding peak. $\mathrm{Cu} / \mathrm{Au}$ core/shell and $\mathrm{Au} / \mathrm{Cu}$ core/shell NPs both have a noticeable peak $\mathrm{Cu}-\mathrm{Cu}$ and $\mathrm{Cu}-\mathrm{Au}$ bonds, but the relative amount of $\mathrm{Cu}$ $\mathrm{Cu}$ bonds is greater when $\mathrm{Cu}$ is in the shell as compared to being in the core, making these two arrangements distinguishable as well. ASV is much easier than x-ray absorption methods, such as EXAFS and NEXAFS, which require synchrotron radiation sources, although they offer better information about the bonding geometry of the metals in the NPs. 
We also demonstrated the stability of weak stabilizer (THPC, TPPS) coated 1.6 and $0.9 \mathrm{~nm}$ diameter Au NPs using ASV. Our findings show that THPC stabilized $1.6 \mathrm{~nm}$ diameter Au NPs are very reactive for $\mathrm{CO}_{2}$ reduction and show $200 \mathrm{mV}$ lower over potential than literature reported value for similar sized Au NPs. Also, THPC stabilized $1.6 \mathrm{~nm}$ diameter Au NPs were found to be very active for hydrogen evolution reaction (HER) as compared to Cit Au4nm NPs. On the other hand, 0.9 nm diameter Au NPs were found to be less reactive for catalytic applications. Interestingly it was found that both 1.6 and $0.9 \mathrm{~nm}$ diameter Au NPs are unstable during oxidation reduction cycle, ozone treatment and during potential controlled ripening at lower potential than that of NPs actual oxidation peak potential. With just only one oxidation-reduction cycle they converted to $4-8 \mathrm{~nm}$ in diameter. Similarly, one min of ozone cleaning or potential holding in $\mathrm{Br}^{-}$containing acidic electrolyte solution increased their size to $4-10 \mathrm{~nm}$ in diameter. Interestingly, under all these conditions citrate stabilized $4 \mathrm{~nm}$ diameter Au NPs were found to very stable, indicating that small size NPs are very unstable despite having potential application in the field of catalysis and sensing. Additionally, TPPS stabilized 0.9 $\mathrm{nm}$ diameter Au show very less stability in acidic environments and are converted to 4 or higher than $4 \mathrm{~nm}$ diameter Au NPs but THPC stabilized $1.6 \mathrm{~nm}$ diameter Au NPs were found to be stable in both acidic and basic environment, indicating that ligand play a significant role for the NPs stability. Also, during the thiol exchange reaction, TPPS Au1nm NPs were found to be completely dissolved but THPC Au Inm $_{2 n}$ were stable and shows oxidation peak in ASV. This indicated that 1.6 
$\mathrm{nm}$ Au NPs are more stable as compared to $0.9 \mathrm{~nm}$ diameter Au NPs during thiol exchange reactions.

Finally, we demonstrated the electrochemical Ostwald ripening rate of different sized Au NPs under potential control in acidic $\mathrm{KBr}$ electrolyte. The rate of ripening was measured by the monitoring the ratio of electroactive surface area (SA) to total volume changes as a function of ripening time, ripening potential and NPs size. SA/V was then converted to NPs diameter and compared that diameter with the microscopically determined size of NPs. We found that $1.6 \mathrm{~nm}$ sized Au NPs show higher rate of Ostwald ripening as compared to 4 and $15 \mathrm{~nm}$ diameter Au NPs under similar reaction conditions due to the earlier oxidation of $1.6 \mathrm{~nm}$ diameter $\mathrm{Au}$ as compared to the other two sizes. Also, mixture of 4 \& $15 \mathrm{~nm}$ diameter Au NPs show higher rate of ripening than individual metal NPs. Coverage of NPs has a significant effect on their ripening and rate was found to be increased with the increase of NPs coverage on the electrode surface.

We also used alloy composition analysis by ASV to study the very unique anti-galvanic exchange between sub $2 \mathrm{~nm}$ diameter $\mathrm{Au}$ NPs and $\mathrm{Ag}^{+}$ions, which is thermodynamically forbidden for larger sized Au NPs. The ASV of $0.9 \mathrm{~nm}$ dimeter Au NPs after exchange with $\mathrm{Ag}^{+}$showed $\sim 100 \%$ exchange (complete absence of an Au oxidation peak) while $1.6 \mathrm{~nm}$ diameter Au NPs showed 50-60\% Ag after exchange (presence of both $\mathrm{Ag}$ and Au oxidation peaks). In contrast, the ASV of $4 \mathrm{~nm}$ diameter Au NPs showed no measurable exchange with $\mathrm{Ag}^{+}$ions (complete absence of $\mathrm{Ag}$ oxidation peak, only Au peak). These results were further confirmed by STEM EDS composition analysis, which showed that $4 \mathrm{~nm}$ 
diameter Au NPs had $\sim 10 \%$ Ag following exchange, while $1.6 \mathrm{~nm}$ diameter Au NPs were $\sim 90 \% \mathrm{Ag}$ following exchange under the same conditions. The numbers were a bit higher for STEM EDS compared to the ASV analysis but the trend was the same.

All of these studies clearly demonstrate that ASV is a powerful analytical tool for determining the size, composition, aggregate structure, and atomic arrangement of single metal or bimetallic NPs. Furthermore, it can be used to track the NP size to learn about the size stability towards ozone, temperature, and electrochemical potential (Ostwald ripening). ASV can also be useful to track the NP composition to monitor temperature-induced segregation in bimetallic CuAu alloy NPs, interesting atomic rearrangements during dealloying, and unique galvanic exchange reactions with small Au NPs that are thermodynamically forbidden with larger sizes. We also described the high electrocatalytic activity for $\mathrm{CO}_{2}$ reduction and HER that is specific for the $1.6 \mathrm{~nm}$ diameter Au NPs, but not larger or smaller sizes. An important aspect of this work is that these metal NPs are formed with weak stabilizers and the characterization is for processes occurring directly on electrode surfaces.

\subsection{FUTURE DIRECTIONS}

Future project should include the size stability study of even more atomically precise Au clusters having atomic numbers of $A u_{1}, A u_{6}$ and $A u_{8}$. Based on the theoretical prediction of Plieth, the $E_{p}$ of these cluster should be below $0.0 \mathrm{~V}$ vs $\mathrm{Ag} / \mathrm{AgCl}$. Characterization techniques for these clusters require sophisticated 
techniques such as mass spectrometry or HRTEM. However electrochemical characterization of these clusters will be very straightforward to determine their size based on $E_{p}$ values. The synthesis of $A u_{6}$ and $A u_{8}$ by a phosphine stabilizer is available in the literature. It will be interesting to understand the stability of these clusters during various electrochemical and chemical treatments, such as ozone treatment, thermal treatment, and Ostwald ripening.

Au clusters 1-2 nm in diameter are very active for catalytic $\mathrm{CO}$ and NO oxidation based on the literature. Different groups reported CO oxidation with thiolstabilized Au clusters. Thiols are considered as a strong protecting ligand and usually most of the Au surface is blocked by thiols. Since, we were able to successfully synthesize phosphine-stabilized 1-2 nm diameter Au NPs and attach them to electrode surfaces, we should expect higher catalytic activity for CO oxidation since the phosphine stabilizer is a more open molecular structure.

In our study we found that ozone causes an increase in the size of 1-2 nm diameter phosphine-coated Au clusters within 1 minute. We passed direct ozone on the glass/ITO/APTES attached Au clusters with no control of the amount of ozone. In future it will be useful to understand the effect of the amount of ozone on the ripening by diluting with inert gases, like $\mathrm{N}_{2}$ or Ar and removing any trace water. The goal would be to find the best condition where the NP size does not change but ozone removes the stabilizer ligand in order to create small, highly active metal catalysts. This study will be very important because naked Au clusters should show better catalytic and sensing reactivity as compared to ligand-protected Au cluster of similar size. Another important study of phosphine-coated clusters will be the 
design of a new stabilizer to replace the phosphine ligand without changing the cluster size. Existing ligands, like thiols or polymers, are not suitable for exposing the maximum surface of Au clusters due to the blocking of Au with these ligands.

We explored the Ostwald ripening of 1.6, 4 and $15 \mathrm{~nm}$ diameter Au NPs under potential control. The rate of Ostwald ripening was found increase with a decrease in Au NPs size. In the future I would like to continue this research with 1 nm diameter Au NPs. Based on our findings $1 \mathrm{~nm}$ diameter Au NPs should show an even higher rate of ripening as compared to $2 \mathrm{~nm}$ diameter Au NPs under similar reaction conditions. Also, it will be useful to monitor other parameters for Ostwald ripening, such as temperature, concentration of $\mathrm{KBr}$, or the effect of different stabilizer ligands on the ripening behavior of metal NPs. This will be useful in order to avoid Ostwald ripening, which leads to the dentrimental effect of forming larger NPs with lower NP density.

Chapter VIII describes the electrochemical characterization of core/shell bimetallic NPs, providing a lot of information about the potential-induced migration of surface Au atoms during ASV in acidic $\mathrm{KBr}$ electrolyte. In the future, I would like to continue this work with in-situ electrochemical TEM. I will start the potential scan from $-0.4 \mathrm{~V}$ in acidic $\mathrm{KBr}$ and monitor the NP size and shape evolution during the stripping of $\mathrm{Cu}$. In chapter VII, we studied the effect of thermal treatment on $\mathrm{Cu}_{1} \mathrm{Au}_{0.3}$ NPs. ASV showed that after 1 hour at $200^{\circ} \mathrm{C}$, the oxidation peak of $\mathrm{Cu}$ was negatively-shifted from $0.2 \mathrm{~V}$ to $-0.1 \mathrm{~V}$, which indicated that $\mathrm{Cu}$ became segregated during the thermal treatment. In the future we want to understand this process of nanostructure shape change using in-situ TEM. Lastly, our composition 
analysis of bimetallic nanostructures could be extended to other bimetallic structures, such as AuPd and AuNi. Understanding the composition and atomic arrangement of these bimetallic nanostructures would be useful since they show better electrocatalytic performance for the oxygen reduction reaction and HER.

There are clearly many possibilities for further developing ASV for size and atomic arrangement analysis and using ASV to monitor and understand processes that lead to size, composition, and atomic rearrangement for electrode-attached NPs. The technique is not envisioned to replace electron microscopy and spectroscopic analysis, but it can serve as a complementary technique. It can be especially useful for the analysis of very small metal NPs attached to electodes and as a quick screening method to determine if something interesting occurs on the electrode before further analysis by other microscopic and spectroscopic methods. The analysis should be very useful for characterizing electrode-attached metal NPs used in electrocatalysis, electrochemical sensing, and energy applications. 


\section{REFERENCES}

1. Pattadar, D. K.; Zamborini, F. P., Size Stability Study of Catalytically Active Sub-2 Nm Diameter Gold Nanoparticles Synthesized with Weak Stabilizers. J. Am. Chem. Soc. 2018, 140:, 14126-14133.

2. Dass, A.; Stevenson, A.; Dubay, G. R.; Tracy, J. B.; Murray, R. W., Nanoparticle Maldi-Tof Mass Spectrometry without Fragmentation: Au25(Sch2ch2ph)18 and Mixed Monolayer Au25(Sch2ch2ph)18-X(L)X. J. Am. Chem. Soc. 2008, 130, 5940-5946.

3. Yao, H.; Iwatsu, M., Water-Soluble Phosphine-Protected Au11 Clusters: Synthesis, Electronic Structure, and Chiral Phase Transfer in a Synergistic Fashion. Langmuir 2016, 32, 32843293.

4. Johnson, G. E.; Wang, C.; Priest, T.; Laskin, J., Monodisperse Au11 Clusters Prepared by Soft Landing of Mass Selected lons. Anal.I Chem. 2011, 83, 8069-8072.

5. Mistry, H.; Reske, R.; Zeng, Z.; Zhao, Z.-J.; Greeley, J.; Strasser, P.; Cuenya, B. R., Exceptional Size-Dependent Activity Enhancement in the Electroreduction of $\mathrm{Co} 2$ over $\mathrm{Au}$ Nanoparticles. J. Am. Chem. Soc. 2014, 136, 16473-16476.

6. Hoque Md Ariful, M. A.; Guzman, M. I., Photocatalytic Activity: Experimental Features to Report in Heterogeneous Photocatalysis. Materials 2018, 11.

7. Ostojic, N.; Duan, Z.; Galyamova, A.; Henkelman, G.; Crooks, R. M., Electrocatalytic Study of the Oxygen Reduction Reaction at Gold Nanoparticles in the Absence and Presence of Interactions with Snox Supports. J. Am. Chem. Soc. 2018, 140, 13775-13785.

8. Hu, K.-J.; Plant, S. R.; Ellis, P. R.; Brown, C. M.; Bishop, P. T.; Palmer, R. E., Atomic Resolution Observation of a Size-Dependent Change in the Ripening Modes of Mass-Selected Au Nanoclusters Involved in Co Oxidation. J. Am. Chem. Soc. 2015, 137, 15161-15168.

9. Zhou, M.; Bao, S.; Bard, A. J., Probing Size and Substrate Effects on the Hydrogen Evolution Reaction by Single Isolated Pt Atoms, Atomic Clusters, and Nanoparticles. J. Am. Chem. Soc. 2019, 141, 7327-7332.

10. Moreno, M.; Ibañez, F. J.; Jasinski, J. B.; Zamborini, F. P., Hydrogen Reactivity of Palladium Nanoparticles Coated with Mixed Monolayers of Alkyl Thiols and Alkyl Amines for Sensing and Catalysis Applications. J. Am. Chem. Soc. 2011, 133, 4389-4397.

11. Kharel, P. L.; Zamborini, F. P.; Alphenaar, B. W., Enhancing the Photovoltaic Performance of Dye-Sensitized Solar Cells with Rare-Earth Metal Oxide Nanoparticles. J. Electrochem. Soc. 2018, 165, H52-H56.

12. Kharel, P. L.; Cuillier, P. M.; Fernando, K.; Zamborini, F. P.; Alphenaar, B. W., Effect of RareEarth Metal Oxide Nanoparticles on the Conductivity of Nanocrystalline Titanium Dioxide: An Electrical and Electrochemical Approach. J. Phys. Chem. C 2018, 122, 15090-15096.

13. Liu, X.; Xu, H.; Xia, H.; Wang, D., Rapid Seeded Growth of Monodisperse, Quasi-Spherical, Citrate-Stabilized Gold Nanoparticles Via H2o2 Reduction. Langmuir 2012, 28, 13720-13726.

14. Patsula, V.; Moskvin, M.; Dutz, S.; Horák, D., Size-Dependent Magnetic Properties of Iron Oxide Nanoparticles. J. Phys. Chem. Solids 2016, 88, 24-30. 
15. Lu, H. M.; Li, P. Y.; Cao, Z. H.; Meng, X. K., Size-, Shape-, and Dimensionality-Dependent Melting Temperatures of Nanocrystals. J. Phys. Chemi. C 2009, 113, 7598-7602.

16. Zhou, X.; Xu, W.; Liu, G.; Panda, D.; Chen, P., Size-Dependent Catalytic Activity and Dynamics of Gold Nanoparticles at the Single-Molecule Level. J. Am. Chem. Soc. 2010, 132, 138146.

17. Ivanova, O. S.; Zamborini, F. P., Size-Dependent Electrochemical Oxidation of Silver Nanoparticles. J. Am. Chem. Soc. 2010, 132, 70-72.

18. Zamborini Francis, P.; Pattadar, D. K.; Sharma, J. N., Analyzing Metal Nanoparticle Transformations by Anodic Stripping Voltammetry. Abstracts of Papers, 257th ACS National Meeting Exposition, Orlando, FL, United States, Mar. 31-Apr. 4, 20192019.

19. Trindell, J. A.; Clausmeyer, J.; Crooks, R. M., Size Stability and H2/Co Selectivity for Au Nanoparticles During Electrocatalytic Co2 Reduction. J. Am. Chem. Soc. 2017, 139, 16161-16167.

20. Saha, K.; Agasti, S. S.; Kim, C.; Li, X.; Rotello, V. M., Gold Nanoparticles in Chemical and Biological Sensing. Chem. Rev. 2012, 112, 2739-2779.

21. Liu, J.; Geng, Y.; Pound, E.; Gyawali, S.; Ashton, J. R.; Hickey, J.; Woolley, A. T.; Harb, J. N., Metallization of Branched DNA Origami for Nanoelectronic Circuit Fabrication. ACS Nano 2011, 5, 2240-2247.

22. Boisselier, E.; Astruc, D., Gold Nanoparticles in Nanomedicine: Preparations, Imaging, Diagnostics, Therapies and Toxicity. Chem. Soc. Rev. 2009, 38, 1759-1782.

23. Wang, Z.-G.; Song, C.; Ding, B., Functional DNA Nanostructures for Photonic and Biomedical Applications. Small 2013, 9, 2210-2222.

24. Park, S.-J.; Taton, T. A.; Mirkin, C. A., Array-Based Electrical Detection of DNA with Nanoparticle Probes. Science 2002, 295, 1503-1506.

25. Elliott, E. W.; Glover, R. D.; Hutchison, J. E., Removal of Thiol Ligands from SurfaceConfined Nanoparticles without Particle Growth or Desorption. ACS Nano 2015, 9, 3050-3059.

26. Menard, L. D.; Xu, F.; Nuzzo, R. G.; Yang, J. C., Preparation of Tio2-Supported Au Nanoparticle Catalysts from a Au13 Cluster Precursor: Ligand Removal Using Ozone Exposure Versus a Rapid Thermal Treatment. J. Catal. 2006, 243, 64-73.

27. Yuan, M.; Zhan, S.; Zhou, X.; Liu, Y.; Feng, L.; Lin, Y.; Zhang, Z.; Hu, J., A Method for Removing Self-Assembled Monolayers on Gold. Langmuir 2008, 24, 8707-8710.

28. Yi, C. W.; Luo, K.; Wei, T.; Goodman, D. W., The Composition and Structure of Pd-Au Surfaces. J. Phy. Chem. B 2005, 109, 18535-18540.

29. Hostetler, M. J.; Zhong, C.-J.; Yen, B. K. H.; Anderegg, J.; Gross, S. M.; Evans, N. D.; Porter, M.; Murray, R. W., Stable, Monolayer-Protected Metal Alloy Clusters. J. Am. Chem. Soc. 1998, 120, 9396-9397.

30. Xu, C.; Wang, R.; Chen, M.; Zhang, Y.; Ding, Y., Dealloying to Nanoporous Au/Pt Alloys and Their Structure Sensitive Electrocatalytic Properties. Phy. Chem. Chem. Phy. 2010, 12, 239-246.

31. Yao, H.; Iwatsu, M., Water-Soluble Phosphine-Protected Au11 Clusters: Synthesis, Electronic Structure, and Chiral Phase Transfer in a Synergistic Fashion. Langmuir 2016, 32, 32843293.

32. Jana, N. R.; Gearheart, L.; Murphy, C. J., Wet Chemical Synthesis of High Aspect Ratio Cylindrical Gold Nanorods. J. Phy. Chem. B 2001, 105, 4065-4067.

33. Perala, S. R. K.; Kumar, S., On the Mechanism of Metal Nanoparticle Synthesis in the Brust-Schiffrin Method. Langmuir 2013, 29, 9863-9873.

34. Trindell, J. A.; Clausmeyer, J.; Crooks, R. M., Size Stability and H2/Co Selectivity for Au Nanoparticles During Electrocatalytic Co2 Reduction. J. Am. Chem. Soc. 2017, 139, 16161-16167.

35. Fang, A.; White, S.; Jain, P. K.; Zamborini, F. P., Regioselective Plasmonic Coupling in Metamolecular Analogs of Benzene Derivatives. Nano Lett. 2015, 15, 542-548. 
36. Hurst, S. J.; Lytton-Jean, A. K. R.; Mirkin, C. A., Maximizing DNA Loading on a Range of Gold Nanoparticle Sizes. Anal. Chem. 2006, 78, 8313-8318.

37. Shem, P. M.; Sardar, R.; Shumaker-Parry, J. S., One-Step Synthesis of Phosphine-Stabilized Gold Nanoparticles Using the Mild Reducing Agent 9-Bbn. Langmuir 2009, 25, 13279-13283.

38. Li, J.-M.; Ma, W.-F.; Wei, C.; You, L.-J.; Guo, J.; Hu, J.; Wang, C.-C., Detecting Trace Melamine in Solution by Sers Using Ag Nanoparticle Coated Poly(Styrene-Co-Acrylic Acid) Nanospheres as Novel Active Substrates. Langmuir 2011, 27, 14539-14544.

39. Liu, X.; Xu, H.; Xia, H.; Wang, D., Rapid Seeded Growth of Monodisperse, Quasi-Spherical, Citrate-Stabilized Gold Nanoparticles Via H2o2 Reduction. Langmuir 2012, 28, 13720-13726.

40. Turkevich, J.; Stevenson, P. C.; Hillier, J., A Study of the Nucleation and Growth Processes in the Synthesis of Colloidal Gold. Discuss. Faraday Soc. 1951, 11, 55-75.

41. Malassis, L.; Dreyfus, R.; Murphy, R. J.; Hough, L. A.; Donnio, B.; Murray, C. B., One-Step Green Synthesis of Gold and Silver Nanoparticles with Ascorbic Acid and Their Versatile Surface Post-Functionalization. RSC Adv. 2016, 6, 33092-33100.

42. Frens, G., Controlled Nucleation for the Regulation of the Particle Size in Monodisperse Gold Suspensions. Nat. Phy. Sci. 1973, 241, 20.

43. Oh, E.; Susumu, K.; Goswami, R.; Mattoussi, H., One-Phase Synthesis of Water-Soluble Gold Nanoparticles with Control over Size and Surface Functionalities. Langmuir 2010, 26, 76047613.

44. Chen, Q.; Kaneko, T.; Hatakeyama, R., Rapid Synthesis of Water-Soluble Gold Nanoparticles with Control of Size and Assembly Using Gas-Liquid Interfacial Discharge Plasma. Chem. Phys. Lett. 2012, 521, 113-117.

45. Li, Y.; Zaluzhna, O.; Xu, B.; Gao, Y.; Modest, J. M.; Tong, Y. J., Mechanistic Insights into the Brust-Schiffrin Two-Phase Synthesis of Organo-Chalcogenate-Protected Metal Nanoparticles. J. Am. Chem. Soc. 2011, 133, 2092-2095.

46. Xue, Y.; Li, X.; Li, H.; Zhang, W., Quantifying Thiol-Gold Interactions Towards the Efficient Strength Control. Nat. Commun. 2014, 5, 4348.

47. Duff, D. G.; Baiker, A.; Edwards, P. P., A New Hydrosol of Gold Clusters. 1. Formation and Particle Size Variation. Langmuir 1993, 9, 2301-2309.

48. Duff, D. G.; Baiker, A.; Gameson, I.; Edwards, P. P., A New Hydrosol of Gold Clusters. 2. A Comparison of Some Different Measurement Techniques. Langmuir 1993, 9, 2310-2317.

49. Shang, L.; Dong, S.; Nienhaus, G. U., Ultra-Small Fluorescent Metal Nanoclusters: Synthesis and Biological Applications. Nano Today 2011, 6, 401-418.

50. McKenzie, L. C.; Zaikova, T. O.; Hutchison, J. E., Structurally Similar TriphenylphosphineStabilized Undecagolds, Au11(Pph3)7cl3 and [Au11(Pph3)8cl2]Cl, Exhibit Distinct Ligand Exchange Pathways with Glutathione. J. Am. Chem. Soc. 2014, 136, 13426-13435.

51. Huang, T.; Huang, L.; He, W.; Song, X.; Sun, Z.; Jiang, Y.; Pan, G.; Wei, S., Ammonia-Induced Size Convergence of Atomically Monodisperse Au6 Nanoclusters. J. Phy. Chem. C 2018, 122, 64056411.

52. Gilbert, S. E.; Cavalleri, O.; Kern, K., Electrodeposition of Cu Nanoparticles on DecanethiolCovered Au(111) Surfaces: An in Situ Stm Investigation. J. Phys. Chem. 1996, 100, 12123-12130.

53. Hicks, J. F.; Miles, D. T.; Murray, R. W., Quantized Double-Layer Charging of Highly Monodisperse Metal Nanoparticles. J. Am. Chem. Soc. 2002, 124, 13322-13328.

54. Dick, J. E.; Bard, A. J., Recognizing Single Collisions of Ptcl62- at Femtomolar Concentrations on Ultramicroelectrodes by Nucleating Electrocatalytic Clusters. J. Am. Chem. Soc. 2015, 137, 13752-13755.

55. Yu, A.; Liang, Z.; Cho, J.; Caruso, F., Nanostructured Electrochemical Sensor Based on Dense Gold Nanoparticle Films. Nano Lett. 2003, 3, 1203-1207. 
56. Guo, E.; Zeng, Z.; Shi, X.; Long, X.; Wang, X., Electrical Transport Properties of Au Nanoparticles and Thin Films on Ge Probed Using a Conducting Atomic Force Microscope. Langmuir 2016, 32, 10589-10596.

57. Henglein, A., The Reactivity of Silver Atoms in Aqueous Solutions (a r-Radiolysis Study). Berichte der Bunsengesellschaft für physikalische Chemie 1977, 81, 556-561.

58. Plieth, W. J., Electrochemical Properties of Small Clusters of Metal Atoms and Their Role in the Surface Enhanced Raman Scattering. J. Phys. Chem. 1982, 86, 3166-3170.

59. Schalow, T.; Brandt, B.; Laurin, M.; Schauermann, S.; Libuda, J.; Freund, H. J., Co Oxidation on Partially Oxidized Pd Nanoparticles. J. Catal. 2006, 242, 58-70.

60. Redmond, P. L.; Hallock, A. J.; Brus, L. E., Electrochemical Ostwald Ripening of Colloidal Ag Particles on Conductive Substrates. Nano Lett. 2005, 5, 131-135.

61. Ward Jones, S. E.; Campbell, F. W.; Baron, R.; Xiao, L.; Compton, R. G., Particle Size and Surface Coverage Effects in the Stripping Voltammetry of Silver Nanoparticles: Theory and Experiment. J. Phys. Chem. C 2008, 112, 17820-17827.

62. Jones, S. E. W.; Toghill, K. E.; Zheng, S. H.; Morin, S.; Compton, R. G., The Stripping Voltammetry of Hemispherical Deposits under Electrochemically Irreversible Conditions: A Comparison of the Stripping Voltammetry of Bismuth on Boron-Doped Diamond and Au(111) Electrodes. J. Phy. Chem. C 2009, 113, 2846-2854.

63. Tang, L.; Li, X.; Cammarata, R. C.; Friesen, C.; Sieradzki, K., Electrochemical Stability of Elemental Metal Nanoparticles. J. Am. Chem. Soc. 2010, 132, 11722-11726.

64. Ivanova, O. S.; Zamborini, F. P., Electrochemical Size Discrimination of Gold Nanoparticles Attached to Glass/Indium-Tin-Oxide Electrodes by Oxidation in Bromide-Containing Electrolyte. Anal. Chem. 2010, 82, 5844-5850.

65. Brainina, K. Z.; Galperin, L. G.; Vikulova, E. V.; Stozhko, N. Y.; Murzakaev, A. M.; Timoshenkova, O. R.; Kotov, Y. A., Gold Nanoparticles Electrooxidation: Comparison of Theory and Experiment. J. Solid State Electrochem. 2011, 15, 1049-1056.

66. Brainina, K. Z.; Galperin, L. G.; Kiryuhina, T. Y.; Galperin, A. L.; Stozhko, N. Y.; Murzakaev, A. M.; Timoshenkova, O. R., Silver Nanoparticles Electrooxidation: Theory and Experiment. J. Solid State Electrochem. 2012, 16, 2365-2372.

67. Brainina, K. Z.; Galperin, L. G.; Piankova, L. A.; Stozhko, N. Y.; Myrzakaev, A. M.; Timoshenkova, O. R., Bismuth Nanoparticles Electrooxidation: Theory and Experiment. J. Solid State Chem. 2011, 15, 2469-2475.

68. Masitas, R. A.; Zamborini, F. P., Oxidation of Highly Unstable $<4 \mathrm{Nm}$ Diameter Gold Nanoparticles 850 Mv Negative of the Bulk Oxidation Potential. J. Am. Chem. Soc. 2012, 134, 50145017.

69. Kumar, A.; Buttry, D. A., Size-Dependent Anodic Dissolution of Water-Soluble Palladium Nanoparticles. J. Phys. Chem. C 2013, 117, 26783-26789.

70. Sharma, J. N.; Pattadar, D. K.; Mainali, B. P.; Zamborini, F. P., Size Determination of Metal Nanoparticles Based on Electrochemically Measured Surface-Area-to-Volume Ratios. Anal. Chem. 2018.

71. Sharma, J.; Pattadar, D. K.; Mainali Badri, P.; Zamborini Francis, P., Understanding the Role of Nanoparticle Assembly Method and Electrode Material on the Electrochemically-Determined Surface Area-to-Volume Ratio. Abstracts, 70th Southeastern Regional Meeting of the American Chemical Society, Augusta, GA, United States, October 31-November 32018.

72. Pattadar, D. K.; Sharma, J. N.; Mainali, B. P.; Zamborini, F. P., Impact of the Assembly Method on the Surface Area-to-Volume Ratio and Electrochemical Oxidation Potential of Metal Nanospheres. J. Phys. Chem. C 2019, 123, 24304-24312. 
73. Pattadar, D. K.; Zamborini, F. P., Effect of Size, Coverage, and Dispersity on the PotentialControlled Ostwald Ripening of Metal Nanoparticles. Langmuir 2019.

74. Saha, K.; Agasti, S. S.; Kim, C.; Li, X.; Rotello, V. M., Gold Nanoparticles in Chemical and Biological Sensing. Chem. Rev. 2012, 112, 2739-2779.

75. Sousa Luana, M.; Vilarinho Luana, M.; Ribeiro Gabriel, H.; Bogado André, L.; Dinelli Luís, R., An Electronic Device Based on Gold Nanoparticles and Tetraruthenated Porphyrin as an Electrochemical Sensor for Catechol. Royal Soc. Open Sci., 4, 170675.

76. Prévot, G.; Nguyen, N. T.; Alloyeau, D.; Ricolleau, C.; Nelayah, J., Ostwald-Driven Phase Separation in Bimetallic Nanoparticle Assemblies. ACS Nano 2016, 10, 4127-4133.

77. Mainali Badri, P.; Pattadar, D. K.; Zamborini Francis, P., Sintering of Metal Nanoparticles through Repetitive Electrochemical Oxidation-Reduction Cycles. Abstracts, 70th Southeastern Regional Meeting of the American Chemical Society, Augusta, GA, United States, October 31November 32018.

78. Hansen, T. W.; DeLaRiva, A. T.; Challa, S. R.; Datye, A. K., Sintering of Catalytic Nanoparticles: Particle Migration or Ostwald Ripening? Acc. Chem. Res. 2013, 46, 1720-1730.

79. Granqvist, C. G.; Buhrman, R. A., Size Distributions for Supported Metal Catalysts: Coalescence Growth Versus Ostwald Ripening. J. Catal. 1976, 42, 477-479.

80. Talapin, D. V.; Rogach, A. L.; Haase, M.; Weller, H., Evolution of an Ensemble of Nanoparticles in a Colloidal Solution: Theoretical Study. J. Phys. Chem. B 2001, 105, 12278-12285.

81. Dagtepe, P.; Chikan, V., Quantized Ostwald Ripening of Colloidal Nanoparticles. J. Phys. Chem. C 2010, 114, 16263-16269.

82. Ouyang, R.; Liu, J.-X.; Li, W.-X., Atomistic Theory of Ostwald Ripening and Disintegration of Supported Metal Particles under Reaction Conditions. J. Am. Chem. Soc. 2013, 135, 1760-1771. 83. Xu, L.; Liang, H.-W.; Yang, Y.; Yu, S.-H., Stability and Reactivity: Positive and Negative Aspects for Nanoparticle Processing. Chem. Rev. 2018, 118, 3209-3250.

84. Woehl, T. J.; Park, C.; Evans, J. E.; Arslan, I.; Ristenpart, W. D.; Browning, N. D., Direct Observation of Aggregative Nanoparticle Growth: Kinetic Modeling of the Size Distribution and Growth Rate. Nano Lett. 2014, 14, 373-378.

85. Shivhare, A.; Chevrier, D. M.; Purves, R. W.; Scott, R. W. J., Following the Thermal Activation of Au25(Sr)18 Clusters for Catalysis by X-Ray Absorption Spectroscopy. J. Phy. Chem. C 2013, 117, 20007-20016.

86. Pattadar, D.; Zamborini Francis, P., Understanding the Metal Nanoparticles Size Effect for Electrochemically Induced Ostwald Ripening: A Case Study with Au Nanoparticles. Abstracts of Papers, 257th ACS National Meeting Exposition, Orlando, FL, United States, Mar. 31-Apr. 4, 2019 2019.

87. Liu, X.; Stroppa, D. G.; Heggen, M.; Ermolenko, Y.; Offenhäusser, A.; Mourzina, Y., Electrochemically Induced Ostwald Ripening in Au/Tio2 Nanocomposite. J. Phy. Chem. C 2015, 119, 10336-10344.

88. Jang, E.; Lim, E.-K.; Choi, J.; Park, J.; Huh, Y.-J.; Suh, J.-S.; Huh, Y.-M.; Haam, S., Br-Assisted Ostwald Ripening of Au Nanoparticles under H2o2 Redox. Cryst. Growth Des. 2012, 12, 37-39.

89. Manthiram, K.; Surendranath, Y.; Alivisatos, A. P., Dendritic Assembly of Gold Nanoparticles During Fuel-Forming Electrocatalysis. J. Am. Chem. Soc. 2014, 136, 7237-7240.

90. Vidal-Iglesias, F. J.; Solla-Gullón, J.; Herrero, E.; Montiel, V.; Aldaz, A.; Feliu, J. M., Evaluating the Ozone Cleaning Treatment in Shape-Controlled Pt Nanoparticles: Evidences of Atomic Surface Disordering. Electrochem. Commun. 2011, 13, 502-505.

91. Prévot, G.; Nguyen, N. T.; Alloyeau, D.; Ricolleau, C.; Nelayah, J., Ostwald-Driven Phase Separation in Bimetallic Nanoparticle Assemblies. ACS Nano 2016, 10, 4127-4133. 
92. Li, D.; Wang, C.; Tripkovic, D.; Sun, S.; Markovic, N. M.; Stamenkovic, V. R., Surfactant Removal for Colloidal Nanoparticles from Solution Synthesis: The Effect on Catalytic Performance. ACS Catal. 2012, 2, 1358-1362.

93. Murray, C. B.; Kagan, C. R.; Bawendi, M. G., Self-Organization of Cdse Nanocrystallites into Three-Dimensional Quantum Dot Superlattices. Science 1995, 270, 1335-1338.

94. Caputo, G.; Pinna, N., Nanoparticle Self-Assembly Using ח-П Interactions. J. Mater. Chem. A 2013, 1, 2370-2378.

95. Talapin, D. V.; Shevchenko, E. V.; Murray, C. B.; Titov, A. V.; Král, P., Dipole-Dipole Interactions in Nanoparticle Superlattices. Nano Lett. 2007, 7, 1213-1219.

96. Bachelet, M.; Chen, R., Self-Assembly of Pegylated Gold Nanoparticles with Satellite Structures as Seeds. Chem. Commun. 2016, 52, 9542-9545.

97. Wei, Y.; Han, S.; Kim, J.; Soh, S.; Grzybowski, B. A., Photoswitchable Catalysis Mediated by Dynamic Aggregation of Nanoparticles. J. Am. Chem. Soc. 2010, 132, 11018-11020.

98. Lu, Q.; Huang, J.; Han, C.; Sun, L.; Yang, X., Facile Synthesis of Composition-Tunable Ptrh Nanosponges for Methanol Oxidation Reaction. Electrochim. Acta 2018, 266, 305-311.

99. Wen, D.; Liu, W.; Haubold, D.; Zhu, C.; Oschatz, M.; Holzschuh, M.; Wolf, A.; Simon, F.; Kaskel, S.; Eychmüller, A., Gold Aerogels: Three-Dimensional Assembly of Nanoparticles and Their Use as Electrocatalytic Interfaces. ACS Nano 2016, 10, 2559-2567.

100. Cai, B.; Wen, D.; Liu, W.; Herrmann, A.-K.; Benad, A.; Eychmüller, A., Function-Led Design of Aerogels: Self-Assembly of Alloyed Pdni Hollow Nanospheres for Efficient Electrocatalysis. Angew. Chem. 2015, 54, 13101-13105.

101. Cloake, S. J.; Toh, H. S.; Lee, P. T.; Salter, C.; Johnston, C.; Compton, R. G., Anodic Stripping Voltammetry of Silver Nanoparticles: Aggregation Leads to Incomplete Stripping. ChemistryOpen 2015, 4, 22-26.

102. He, D.; Bligh, M. W.; Waite, T. D., Effects of Aggregate Structure on the Dissolution Kinetics of Citrate-Stabilized Silver Nanoparticles. Environ. Sci. \& Technol. 2013, 47, 9148-9156.

103. Rees, N. V.; Zhou, Y.-G.; Compton, R. G., The Aggregation of Silver Nanoparticles in Aqueous Solution Investigated Via Anodic Particle Coulometry. ChemPhysChem 2011, 12, 16451647.

104. Allen, S. L.; Sharma, J. N.; Zamborini, F. P., Aggregation-Dependent Oxidation of Metal Nanoparticles. J. Am. Chem. Soc. 2017, 139, 12895-12898.

105. Allen, S. L.; Sharma, J. N.; Zamborini, F. P., Aggregation-Dependent Oxidation of Metal Nanoparticles. J. Am. Chem. Soc. 2017, 139, 12895-12898.

106. Yang, Y.; Liu, J.; Fu, Z.-W.; Qin, D., Galvanic Replacement-Free Deposition of Au on Ag for Core-Shell Nanocubes with Enhanced Chemical Stability and Sers Activity. J. Am. Chem. Soc. 2014, 136, 8153-8156.

107. Liu; Guyot-Sionnest, P., Synthesis and Optical Characterization of Au/Ag Core/Shell Nanorods. J. Phys. Chem. B 2004, 108, 5882-5888.

108. Guan, S.; Fu, X.; Tang, Y.; Peng, Z., Auag@Cds Double-Walled Nanotubes: Synthesis and Nonlinear Absorption Properties. Nanoscale 2017, 9, 10277-10284.

109. Zhang, W.; Yang, J.; Lu, X., Tailoring Galvanic Replacement Reaction for the Preparation of Pt/Ag Bimetallic Hollow Nanostructures with Controlled Number of Voids. ACS Nano 2012, 6, 7397-7405.

110. Sun, Y.; Wiley, B.; Li, Z.-Y.; Xia, Y., Synthesis and Optical Properties of Nanorattles and Multiple-Walled Nanoshells/Nanotubes Made of Metal Alloys. J. Am. Chem. Soc. 2004, 126, 93999406. 
111. Chen, J.; McLellan, J. M.; Siekkinen, A.; Xiong, Y.; Li, Z.-Y.; Xia, Y., Facile Synthesis of Gold-Silver Nanocages with Controllable Pores on the Surface. J. Am. Chemi. Soc. 2006, 128, 14776-14777.

112. Murshid, N.; Gourevich, I.; Coombs, N.; Kitaev, V., Gold Plating of Silver Nanoparticles for Superior Stability and Preserved Plasmonic and Sensing Properties. Chem. Commun. 2013, 49, 11355-11357.

113. Choi, J.-P.; Fields-Zinna, C. A.; Stiles, R. L.; Balasubramanian, R.; Douglas, A. D.; Crowe, M. C.; Murray, R. W., Reactivity of [Au25(Sch2ch2ph)18]1- Nanoparticles with Metal lons. J. Phys. Chem. C 2010, 114, 15890-15896.

114. Wu, Z., Anti-Galvanic Reduction of Thiolate-Protected Gold and Silver Nanoparticles. Angew. Chem. 2012, 51, 2934-2938.

115. Wang, M.; Wu, Z.; Chu, Z.; Yang, J.; Yao, C., Chemico-Physical Synthesis of Surfactant- and Ligand-Free Gold Nanoparticles and Their Anti-Galvanic Reduction Property. Chem. Asian J. 2014, 9, 1006-1010.

116. Yao, C.; Chen, J.; Li, M.-B.; Liu, L.; Yang, J.; Wu, Z., Adding Two Active Silver Atoms on Au25 Nanoparticle. Nano Lett. 2015, 15, 1281-1287.

117. Tian, S.; Yao, C.; Liao, L.; Xia, N.; Wu, Z., Ion-Precursor and Ion-Dose Dependent AntiGalvanic Reduction. Chem. Commun. 2015, 51, 11773-11776.

118. Li, Q.; Luo, T.-Y.; Taylor, M. G.; Wang, S.; Zhu, X.; Song, Y.; Mpourmpakis, G.; Rosi, N. L.; Jin, R., Molecular "Surgery" on a 23-Gold-Atom Nanoparticle. Sci. Adv. 2017, 3, e1603193.

119. Sun, J.; Wu, H.; Jin, Y., Synthesis of Thiolated Ag/Au Bimetallic Nanoclusters Exhibiting an Anti-Galvanic Reduction Mechanism and Composition-Dependent Fluorescence. Nanoscale 2014, 6, 5449-5457.

120. Li, M.-b.; Tian, S.-k.; Wu, Z., Improving the Catalytic Activity of Au25 Nanocluster by Peeling and Doping. Chin. J. Chem. 2017, 35, 567-571.

121. Young, S. L.; Hutchison, J. E., Selective Deposition of Metals onto Molecularly Tethered Gold Nanoparticles: The Influence of Silver Deposition on Oxygen Electroreduction. Chem. Mater. 2019, 31, 2750-2761.

122. Haldar, K. K.; Kundu, S.; Patra, A., Core-Size-Dependent Catalytic Properties of Bimetallic $\mathrm{Au} / \mathrm{Ag}$ Core-Shell Nanoparticles. ACS Appl. Mater. \& Interfces 2014, 6, 21946-21953.

123. Hona, R. K.; Ramezanipour, F., Remarkable Oxygen-Evolution Activity of a Perovskite Oxide from the Ca2-Xsrxfe2o6- $\Delta$ Series. Angew. Chem. Int. Ed. 2019, 58, 2060-2063.

124. Hona, R. K.; Huq, A.; Ramezanipour, F., Unraveling the Role of Structural Order in the Transformation of Electrical Conductivity in Ca2fecoo6- $\Delta$, Casrfecoo6- $\Delta$, and Sr2fecoo6- $\Delta$. Inorg. Chem. 2017, 56, 14494-14505.

125. Monzó, J.; Malewski, Y.; Kortlever, R.; Vidal-Iglesias, F. J.; Solla-Gullón, J.; Koper, M. T. M.; Rodriguez, P., Enhanced Electrocatalytic Activity of Au@Cu Core@Shell Nanoparticles Towards Co2 Reduction. J. Mater. Chem. A 2015, 3, 23690-23698.

126. Zhang, J.; Xu, X.; Yang, C.; Yang, F.; Yang, X., Colorimetric lodide Recognition and Sensing by Citrate-Stabilized Core/Shell Cu@Au Nanoparticles. Anal. Chem. 2011, 83, 3911-3917.

127. Cheng, L.-C.; Huang, J.-H.; Chen, H. M.; Lai, T.-C.; Yang, K.-Y.; Liu, R.-S.; Hsiao, M.; Chen, C.-H.; Her, L.-J.; Tsai, D. P., Seedless, Silver-Induced Synthesis of Star-Shaped Gold/Silver Bimetallic Nanoparticles as High Efficiency Photothermal Therapy Reagent. J. Mater. Chem. 2012, 22, 22442253.

128. Malasi, A.; Taz, H.; Ehrsam, M.; Goodwin, J.; Garcia, H.; Kalyanaraman, R., Enhanced and Tunable Optical Quantum Efficiencies from Plasmon Bandwidth Engineering in Bimetallic Coag Nanoparticles. APL Photonics 2016, 1, 076101. 
129. Hona, R. K.; Ramezanipour, F., Variation in Electrical Conductivity of A2fe2o5 (a = Sr, Ba): The Role of Structural Order. Mater. Res. Express 2018, 5, 076307.

130. Mulmi, S.; Hona, R. K.; Jasinski, J. B.; Ramezanipour, F., Electrical Conductivity of Sr2Xcaxfemno5 $(X=0,1,2)$. J. Solid State Electrochem. 2018, 22, 2329-2338.

131. Robinson, D. A.; Stevenson, K. J., Uniform Epitaxial Growth of Pt on Fe3o4 Nanoparticles; Synergetic Enhancement to Pt Activity for the Oxygen Reduction Reaction. J. Mater. Chem. A, 2013, 1, 13443-13453.

132. Gilroy, K. D.; Ruditskiy, A.; Peng, H.-C.; Qin, D.; Xia, Y., Bimetallic Nanocrystals: Syntheses, Properties, and Applications. Chem. Rev. 2016, 116, 10414-10472.

133. Gawande, M. B.; Goswami, A.; Asefa, T.; Guo, H.; Biradar, A. V.; Peng, D.-L.; Zboril, R.; Varma, R. S., Core-Shell Nanoparticles: Synthesis and Applications in Catalysis and Electrocatalysis. Chem. Soc. Rev. 2015, 44, 7540-7590.

134. Kim, D.; Resasco, J.; Yu, Y.; Asiri, A. M.; Yang, P., Synergistic Geometric and Electronic Effects for Electrochemical Reduction of Carbon Dioxide Using Gold-Copper Bimetallic Nanoparticles. Nat. Commun. 2014, 5, 4948.

135. Zhang, J.; Xu, X.; Yuan, Y.; Yang, C.; Yang, X., A Cu@Au Nanoparticle-Based Colorimetric Competition Assay for the Detection of Sulfide Anion and Cysteine. ACS Appl. Mater. \& Interfaces 2011, 3, 2928-2931.

136. Liu, S., et al., Unidirectional Thermal Diffusion in Bimetallic Cu@Au Nanoparticles. ACS Nano 2014, 8, 1886-1892.

137. Niu, Z., et al., Ultrathin Epitaxial Cu@Au Core-Shell Nanowires for Stable Transparent Conductors. J. Am. Chem. Soc. 2017, 139, 7348-7354.

138. Alvarez-Paneque, A. F.; Rodríguez-González, B.; Pastoriza-Santos, I.; Liz-Marzán, L. M., Shape-Templated Growth of Au@Cu Nanoparticles. J. Phys. Chem. C 2012, 117, 2474-2479.

139. Xu, C.; Liu, Y.; Wang, J.; Geng, H.; Qiu, H., Fabrication of Nanoporous Cu-Pt(Pd) Core/Shell Structure by Galvanic Replacement and Its Application in Electrocatalysis. ACS Appl. Mater. \& Interfaces 2011, 3, 4626-4632.

140. Li, W.; Kuai, L.; Chen, L.; Geng, B., "Re-Growth Etching" to Large-Sized Porous Gold Nanostructures. Nat. Sci. Rep. 2013, 3, 2377.

141. Li, X.; Chen, Q.; McCue, I.; Snyder, J.; Crozier, P.; Erlebacher, J.; Sieradzki, K., Dealloying of Noble-Metal Alloy Nanoparticles. Nano Lett. 2014, 14, 2569-2577.

142. Xu, J.; Wang, Y.; Zhang, Z., Potential and Concentration Dependent Electrochemical Dealloying of Al2au in Sodium Chloride Solutions. J. Phy. Chem. C 2012, 116, 5689-5699.

143. Dursun, A.; Pugh, D. V.; Corcoran, S. G., Dealloying of Ag-Au Alloys in Halide-Containing Electrolytes: Affect on Critical Potential and Pore Size. J. Electrochem. Soc. 2003, 150, B355-B360.

144. Hernández, J.; Solla-Gullón, J.; Herrero, E.; Aldaz, A.; Feliu, J. M., Characterization of the Surface Structure of Gold Nanoparticles and Nanorods Using Structure Sensitive Reactions. J. Phy. Chem. B 2005, 109, 12651-12654.

145. Hernández, J.; Solla-Gullón, J.; Herrero, E.; Aldaz, A.; Feliu, J. M., Electrochemistry of Shape-Controlled Catalysts: Oxygen Reduction Reaction on Cubic Gold Nanoparticles. J. Phy. Chem. C 2007, 111, 14078-14083.

146. Negishi, Y.; Nobusada, K.; Tsukuda, T., Glutathione-Protected Gold Clusters Revisited: Bridging the Gap between Gold(I)-Thiolate Complexes and Thiolate-Protected Gold Nanocrystals. J. Am. Chem. Soc. 2005, 127, 5261-5270.

147. Trindell, J. A.; Clausmeyer, J.; Crooks, R. M., J. Am. Chem. Soc. 2017, 139, 16161-16167.

148. Gulka, C. P.; Wong, A. C.; Wright, D. W., Spontaneous Self-Assembly and Disassembly of Colloidal Gold Nanoparticles Induced by Tetrakis(Hydroxymethyl) Phosphonium Chloride. Chem. Commun. 2016, 52, 1266-1269. 
149. Zhang, J.; Xu, X.; Yang, C.; Yang, F.; Yang, X., Colorimetric lodide Recognition and Sensing by Citrate-Stabilized Core/Shell Cu@Au Nanoparticles. Anal. Chem. 2011, 83, 3911-3917.

150. Zhang, J.; Xu, X.; Yuan, Y.; Yang, C.; Yang, X., A Cu@Au Nanoparticle-Based Colorimetric Competition Assay for the Detection of Sulfide Anion and Cysteine. ACS Appl. Mater. Interfaces 2011, 3, 2928-2931.

151. Jana, N. R.; Gearheart, L.; Murphy, C. J., Wet Chemical Synthesis of High Aspect Ratio Cylindrical Gold Nanorods. J. Phys. Chem. B 2001, 105, 4065-4067.

152. Behafarid, F.; Matos, J.; Hong, S.; Zhang, L.; Rahman, T. S.; Roldan Cuenya, B., Structural and Electronic Properties of Micellar Au Nanoparticles: Size and Ligand Effects. ACS Nano 2014, 8, 6671-6681.

153. Dick, K.; Dhanasekaran, T.; Zhang, Z.; Meisel, D., Size-Dependent Melting of SilicaEncapsulated Gold Nanoparticles. J. Am. Chem. Soc. 2002, 124, 2312-2317.

154. Ivanova, O. S.; Zamborini, F. P., Size-Dependent Electrochemical Oxidation of Silver Nanoparticles. J. Am. Chem. Soc. 2010, 132, 70-72.

155. Ivanova, O. S.; Zamborini, F. P., Electrochemical Size Discrimination of Gold Nanoparticles Attached to Glass/Indium-Tin-Oxide Electrodes by Oxidation in Bromide-Containing Electrolyte. Anal. Chem. 2010, 82, 5844-5850.

156. Zamborini, F. P.; Hicks, J. F.; Murray, R. W., Quantized Double Layer Charging of Nanoparticle Films Assembled Using Carboxylate/(Cu2+ or $\mathrm{Zn2+} /$ /Carboxylate Bridges. J. Am. Chem. Soc. 2000, 122, 4514-4515.

157. Chen, S.; Ingram, R. S.; Hostetler, M. J.; Pietron, J. J.; Murray, R. W.; Schaaff, T. G.; Khoury, J. T.; Alvarez, M. M.; Whetten, R. L., Gold Nanoelectrodes of Varied Size: Transition to MoleculeLike Charging. Science 1998, 280, 2098-2101.

158. Sugiuchi, M.; Maeba, J.; Okubo, N.; Iwamura, M.; Nozaki, K.; Konishi, K., AggregationInduced Fluorescence-to-Phosphorescence Switching of Molecular Gold Clusters. J. Am. Chem. Soc. 2017, 139, 17731-17734.

159. Kauffman, D. R.; Alfonso, D.; Matranga, C.; Qian, H.; Jin, R., Experimental and Computational Investigation of Au25 Clusters and Co2: A Unique Interaction and Enhanced Electrocatalytic Activity. J. Am. Chem. Soc. 2012, 134, 10237-10243.

160. Li, H.; Li, L.; Pedersen, A.; Gao, Y.; Khetrapal, N.; Jónsson, H.; Zeng, X. C., Magic-Number Gold Nanoclusters with Diameters from 1 to $3.5 \mathrm{Nm}$ : Relative Stability and Catalytic Activity for Co Oxidation. Nano Lett. 2015, 15, 682-688.

161. Chen, L.-Y.; Wang, C.-W.; Yuan, Z.; Chang, H.-T., Fluorescent Gold Nanoclusters: Recent Advances in Sensing and Imaging. Anal. Chem. 2015, 87, 216-229.

162. Sharma, J. N.; Pattadar, D. K.; Mainali, B. P.; Zamborini, F. P., Size Determination of Metal Nanoparticles Based on Electrochemically Measured Surface Area-to-Volume Ratio. Analytical Chemistry 2018, 90, 9308-9314.

163. Gulka, C. P.; Wong, A. C.; Wright, D. W., Chem. Commun. 2016, 52, 1266-1269.

164. Kumar, A.; Buttry, D. A., Size-Dependent Anodic Dissolution of Water-Soluble Palladium Nanoparticles. The Journal of Physical Chemistry C 2013, 117, 26783-26789.

165. Plieth, W. J., Electrochemical Properties of Small Clusters of Metal Atoms and Their Role in the Surface Enhanced Raman Scattering. The Journal of Physical Chemistry 1982, 86, 3166-3170.

166. Henglein, A., Physicochemical Properties of Small Metal Particles in Solution: "Microelectrode" Reactions, Chemisorption, Composite Metal Particles, and the Atom-to-Metal Transition. The Journal of Physical Chemistry 1993, 97, 5457-5471.

167. Masitas, R. A.; Zamborini, F. P., Oxidation of Highly Unstable <4 Nm Diameter Gold Nanoparticles 850 Mv Negative of the Bulk Oxidation Potential. J. Am. Chem. Soc. 2012, 134, 50145017. 
168. Masitas, R. A.; Khachian, I. V.; Bill, B. L.; Zamborini, F. P., Effect of Surface Charge and Electrode Material on the Size-Dependent Oxidation of Surface-Attached Metal Nanoparticles. Langmuir 2014, 30, 13075-13084.

169. Back, S.; Yeom, M. S.; Jung, Y., Active Sites of Au and Ag Nanoparticle Catalysts for Co2 Electroreduction to Co. ACS Catalysis 2015, 5, 5089-5096.

170. Chen, Y.; Li, C. W.; Kanan, M. W., Aqueous Co2 Reduction at Very Low Overpotential on Oxide-Derived Au Nanoparticles. J. Am. Chem. Soc. 2012, 134, 19969-19972.

171. Wuttig, A.; Yoon, Y.; Ryu, J.; Surendranath, Y., Bicarbonate Is Not a General Acid in AuCatalyzed Co2 Electroreduction. J. Am. Chem. Soc. 2017, 139, 17109-17113.

172. Tran, T. D.; Nguyen, M. T. T.; Le, H. V.; Nguyen, D. N.; Truong, Q. D.; Tran, P. D., Gold Nanoparticles as an Outstanding Catalyst for the Hydrogen Evolution Reaction. Chem. Commun. 2018, 54, 3363-3366.

173. Steven, J. T.; Golovko, V. B.; Johannessen, B.; Marshall, A. T., Electrochemical Stability of Carbon-Supported Gold Nanoparticles in Acidic Electrolyte During Cyclic Voltammetry. Electrochim. Acta 2016, 187, 593-604.

174. Yoshida, K.; Kuzume, A.; Broekmann, P.; Pobelov, I. V.; Wandlowski, T., Reconstruction and Electrochemical Oxidation of Au(110) Surface in $0.1 \mathrm{M} \mathrm{H} 2$ so4. Electrochim. Acta 2014, 139, 281-288.

175. Li, Y.; Cox, J. T.; Zhang, B., Electrochemical Responses and Electrocatalysis at Single Au Nanoparticles. J. Am. Chem. Soc. 2010, 132, 3047-3054.

176. Jude, L.; Antibe, P.; Alexander, K.; Cheng, Y.; Peng, S., Electrochemical Behaviors of Single Gold Nanoparticles. Electroanalysis 2011, 23, 2270-2274.

177. Redmond, P. L.; Hallock, A. J.; Brus, L. E., Nano Lett. 2005, 5, 131-135.

178. Ansar, S. M.; Kitchens, C. L., Impact of Gold Nanoparticle Stabilizing Ligands on the Colloidal Catalytic Reduction of 4-Nitrophenol. ACS Catalysis 2016, 6, 5553-5560.

179. Wilker, M. B.; Utterback, J. K.; Greene, S.; Brown, K. A.; Mulder, D. W.; King, P. W.; Dukovic, G., Role of Surface-Capping Ligands in Photoexcited Electron Transfer between Cds Nanorods and [Fefe] Hydrogenase and the Subsequent H2 Generation. The Journal of Physical Chemistry C 2018, 122, 741-750.

180. Elliott, E. W.; Glover, R. D.; Hutchison, J. E., Removal of Thiol Ligands from SurfaceConfined Nanoparticles without Particle Growth or Desorption. ACS Nano 2015, 9, 3050-3059.

181. Zhou, M.; Wang, D.; Mirkin, M. V., Catalytic Amplification of Au144 Nanocluster Collisions by Hydrogen Evolution Reaction. ChemElectroChem 2018, 5, 2991-2994.

182. Ibañez, F. J.; Zamborini, F. P., Chemiresistive Sensing of Volatile Organic Compounds with Films of Surfactant-Stabilized Gold and Gold-Silver Alloy Nanoparticles. ACS Nano 2008, 2, $1543-$ 1552.

183. Seitz, O.; Dai, M.; Aguirre-Tostado, F. S.; Wallace, R. M.; Chabal, Y. J., Copper-Metal Deposition on Self Assembled Monolayer for Making Top Contacts in Molecular Electronic Devices. J. Am. Chem. Soc. 2009, 131, 18159-18167.

184. Wang, H.; Gu, X.-K.; Zheng, X.; Pan, H.; Zhu, J.; Chen, S.; Cao, L.; Li, W.-X.; Lu, J., Disentangling the Size-Dependent Geometric and Electronic Effects of Palladium Nanocatalysts Beyond Selectivity. Sci. Adv. 2019, 5, eaat6413.

185. Font, F.; Myers, T. G., Spherically Symmetric Nanoparticle Melting with a Variable Phase Change Temperature. J. Nanopart. Res. 2013, 15, 2086.

186. Patsula, V.; Moskvin, M.; Dutz, S.; Horák, D., Size-Dependent Magnetic Properties of Iron Oxide Nanoparticles. J. Phys. Chem. Solids 2016, 88, 24-30.

187. Kim, T.-H.; Kim, M.; Park, H.-S.; Shin, U. S.; Gong, M.-S.; Kim, H.-W., Size-Dependent Cellular Toxicity of Silver Nanoparticles. J. Biomed. Mater. Res. A 2012, 100A, 1033-1043. 
188. Mistry, H.; Reske, R.; Zeng, Z.; Zhao, Z.-J.; Greeley, J.; Strasser, P.; Cuenya, B. R., Exceptional Size-Dependent Activity Enhancement in the Electroreduction of $\mathrm{Co} 2$ over $\mathrm{Au}$ Nanoparticles. J. Am. Chem. Soc. 2014, 136, 16473-16476.

189. Chen, H.; Kou, X.; Yang, Z.; Ni, W.; Wang, J., Shape- and Size-Dependent Refractive Index Sensitivity of Gold Nanoparticles. Langmuir 2008, 24, 5233-5237.

190. Lin, M. E.; Andres, R. P.; Reifenberger, R., Observation of the Discrete Electron Energy States of an Individual Nanometer-Size Supported Gold Cluster. Phys. Rev. Lett. 1991, 67, 477480.

191. Li, Q., et al., A Mono-Cuboctahedral Series of Gold Nanoclusters: Photoluminescence Origin, Large Enhancement, Wide Tunability, and Structure-Property Correlation. J. Am. Chem. Soc. 2019, 141, 5314-5325.

192. Masitas, R. A.; Zamborini, F. P., Oxidation of Highly Unstable $<4 \mathrm{Nm}$ Diameter Gold Nanoparticles 850 Mv Negative of the Bulk Oxidation Potential. J. Am. Chem. Soc. 2012, 134, 5014-5017.

193. Morris, D. J.; Yang, R.; Higaki, T.; Ward, M. J.; Jin, R.; Zhang, P., Core Geometry Effect on the Bonding Properties of Gold-Thiolate Nanoclusters: The Case of Hexagonal-Close-Packed Au30(Sr)18. J. Phy. Chem. C 2018, 122, 23414-23419.

194. Fields-Zinna, C. A.; Sardar, R.; Beasley, C. A.; Murray, R. W., Electrospray lonization Mass Spectrometry of Intrinsically Cationized Nanoparticles, [Au144/146(Sc11h22n(Ch2ch3)3+)X(S(Ch2)5ch3)Y]X+. J. Am. Chem. Soc. 2009, 131, 16266-16271. 195. Yao, H.; Tsubota, S.; Nobukawa, R., Water-Soluble Mixed-Phosphine-Protected Gold Clusters: Can a Single Axially Chiral Ligand Lead to Large Chiroptical Responses? J. Phy. Chem. C 2018, 122, 1299-1308.

196. Chen, Y.-S.; Kamat, P. V., Glutathione-Capped Gold Nanoclusters as Photosensitizers. Visible Light-Induced Hydrogen Generation in Neutral Water. J. Am. Chem. Soc. 2014, 136, 60756082.

197. Pettibone, J. M.; Hudgens, J. W., Gold Cluster Formation with Phosphine Ligands: Etching as a Size-Selective Synthetic Pathway for Small Clusters? ACS Nano 2011, 5, 2989-3002.

198. McKenzie, L. C.; Zaikova, T. O.; Hutchison, J. E., Structurally Similar TriphenylphosphineStabilized Undecagolds, Au11(Pph3)7cl3 and [Au11(Pph3)8cl2]Cl, Exhibit Distinct Ligand Exchange Pathways with Glutathione. J. Am. Chem. Soc. 2014, 136, 13426-13435.

199. Pattadar, D. K.; Zamborini, F. P., Size Stability Study of Catalytically Active Sub-2 Nm Diameter Gold Nanoparticles Synthesized with Weak Stabilizers. J. Am. Chem. Soc. 2018, 140, 14126-14133.

200. Pattadar, D. K.; Sharma, J. N.; Mainali, B. P.; Zamborini, F. P., Anodic Stripping Electrochemical Analysis of Metal Nanoparticles. Curr. Opin. Electrochem. 2019, 13, 147-156.

201. Sharma, J. N.; Pattadar, D. K.; Mainali, B. P.; Zamborini, F. P., Size Determination of Metal Nanoparticles Based on Electrochemically Measured Surface-Area-to-Volume Ratios. Anal. Chem. 2018, 90, 9308-9314.

202. Sharma, J. N.; Pattadar, D. K.; Mainali, B. P.; Zamborini, F. P., Electrochemical Determination of Surface Area-to-Volume Ratio for Metal Nanoparticle Size Analysis. Abstracts of Papers, 255th ACS National Meeting Exposition, New Orleans, LA, United States, March 18-22, 20182018.

203. Pattadar, D. K.; Zamborini, F. P., Halide-Dependent Dealloying of Cux/Auy Core/Shell Nanoparticles for Composition Analysis by Anodic Stripping Voltammetry. J. Phys. Chem. C 2019, 123, 9496-9505.

204. Kumar, A.; Buttry, D. A., Size-Dependent Underpotential Deposition of Copper on Palladium Nanoparticles. J. Phy. Chem. C 2015, 119, 16927-16933. 
205. Henglein, A., Physicochemical Properties of Small Metal Particles in Solution: "Microelectrode" Reactions, Chemisorption, Composite Metal Particles, and the Atom-to-Metal Transition. J. Phys. Chem. 1993, 97, 5457-5471.

206. Li, Y.; Cox, J. T.; Zhang, B., Electrochemical Responses and Electrocatalysis at Single Au Nanoparticles. J. Am. Chem. Soc. 2010, 132, 3047-3054.

207. Ionita, P.; Caragheorgheopol, A.; Gilbert, B. C.; Chechik, V., Mechanistic Study of a PlaceExchange Reaction of Au Nanoparticles with Spin-Labeled Disulfides. Langmuir 2004, 20, 1153611544.

208. Ionita, P.; Caragheorgheopol, A.; Gilbert, B. C.; Chechik, V., Epr Study of a Place-Exchange Reaction on Au Nanoparticles: Two Branches of a Disulfide Molecule Do Not Adsorb Adjacent to Each Other. J. Am. Chem. Soc. 2002, 124, 9048-9049.

209. Kassam, A.; Bremner, G.; Clark, B.; Ulibarri, G.; Lennox, R. B., Place Exchange Reactions of Alkyl Thiols on Gold Nanoparticles. J. Am. Chem. Soc. 2006, 128, 3476-3477.

210. Mainali Badri, P.; Zamborini Francis, P.; Pattadar, D., Size-Dependent Oxidation Behavior of Gold Nanoparticles Coated with Alkanethiol Self-Assembled Monolayers (Sams). Abstracts of Papers, 255th ACS National Meeting Exposition, New Orleans, LA, United States, March 18-22, 20182018.

211. Woehrle, G. H.; Brown, L. O.; Hutchison, J. E., Thiol-Functionalized, 1.5-Nm Gold Nanoparticles through Ligand Exchange Reactions: Scope and Mechanism of Ligand Exchange. J. Am. Chem. Soc. 2005, 127, 2172-2183.

212. Shichibu, Y.; Negishi, Y.; Tsukuda, T.; Teranishi, T., Large-Scale Synthesis of Thiolated Au25 Clusters Via Ligand Exchange Reactions of Phosphine-Stabilized Au11 Clusters. J. Am. Chem. Soc. 2005, 127, 13464-13465.

213. Cavalleri, O.; Gilbert, S. E.; Kern, K., Growth Manipulation in Electrodeposition with SelfAssembled Monolayers. Chem. Phys. Lett. 1997, 269, 479-484.

214. Love, J. C.; Estroff, L. A.; Kriebel, J. K.; Nuzzo, R. G.; Whitesides, G. M., Self-Assembled Monolayers of Thiolates on Metals as a Form of Nanotechnology. Chem. Rev. 2005, 105, 11031170.

215. Yang, Q.; Dong, M.; Song, H.; Cao, I.; Zhang, Y.; Wang, L.; Zhang, P.; Chen, Z., Size Dependence Electrocatalytic Activity of Gold Nanoparticles Decorated Reduced Graphene Oxide for Hydrogen Evolution Reaction. J. Mater. Sci: Mater. Electron 2017, 28, 10073-10080.

216. Wang, Y.; Laborda, E.; Tschulik, K.; Damm, C.; Molina, A.; Compton, R. G., Strong Negative Nanocatalysis: Oxygen Reduction and Hydrogen Evolution at Very Small $(2 \mathrm{Nm})$ Gold Nanoparticles. Nanoscale 2014, 6, 11024-11030.

217. Back, S.; Yeom, M. S.; Jung, Y., Active Sites of Au and Ag Nanoparticle Catalysts for Co2 Electroreduction to Co. ACS Catal. 2015, 5, 5089-5096.

218. Zhu, W.; Michalsky, R.; Metin, Ö.; Lv, H.; Guo, S.; Wright, C. J.; Sun, X.; Peterson, A. A.; Sun, S., Monodisperse Au Nanoparticles for Selective Electrocatalytic Reduction of Co2 to Co. J. Am. Chem. Soc. 2013, 135, 16833-16836.

219. Lee, A.; Andrade, G. F. S.; Ahmed, A.; Souza, M. L.; Coombs, N.; Tumarkin, E.; Liu, K.; Gordon, R.; Brolo, A. G.; Kumacheva, E., Probing Dynamic Generation of Hot-Spots in SelfAssembled Chains of Gold Nanorods by Surface-Enhanced Raman Scattering. J. Am. Chem. Soc. 2011, 133, 7563-7570.

220. Izquierdo-Lorenzo, I.; Kubackova, J.; Manchon, D.; Mosset, A.; Cottancin, E.; SanchezCortes, S., Linking Ag Nanoparticles by Aliphatic A, $\Omega$-Dithiols: A Study of the Aggregation and Formation of Interparticle Hot Spots. J. Phys. Chem. C 2013, 117, 16203-16212.

221. Dasari, R.; Zamborini, F. P., Surface Enhanced Raman Spectroscopy at Electrochemically Fabricated Silver Nanowire Junctions. Anal. Chem. 2016, 88, 675-681. 
222. Levard, C.; Hotze, E. M.; Lowry, G. V.; Brown, G. E., Environmental Transformations of Silver Nanoparticles: Impact on Stability and Toxicity. Environ. Sci. \& Technol. 2012, 46, 6900-6914. 223. Kvitek, L.; Vanickova, M.; Panacek, A.; Soukupova, J.; Dittrich, M.; Valentova, E.; Prucek, R.; Bancirova, M.; Milde, D.; Zboril, R., Initial Study on the Toxicity of Silver Nanoparticles (Nps) against Paramecium Caudatum. J. Phys. Chem. C 2009, 113, 4296-4300.

224. Nambiathan Nambiar, H.; Pattadar, D.; Zamborini Francis, P., Aggregation-Dependent Oxidation of Different-Sized Gold Nanoparticles. Abstracts, 70th Southeastern Regional Meeting of the American Chemical Society, Augusta, GA, United States, October 31-November 32018.

225. Manthiram, K.; Surendranath, Y.; Alivisatos, A. P., Dendritic Assembly of Gold Nanoparticles During Fuel-Forming Electrocatalysis. J. Am. Chem. Soc. 2014, 136, 7237-7240.

226. Zhou, Y.; Zeng, H. C., Simultaneous Synthesis and Assembly of Noble Metal Nanoclusters with Variable Micellar Templates. J. Am. Chem. Soc. 2014, 136, 13805-13817.

227. Russell, L. E.; Galyean, A. A.; Notte, S. M.; Leopold, M. C., Stable Aqueous Nanoparticle Film Assemblies with Covalent and Charged Polymer Linking Networks. Langmuir 2007, 23, 74667471.

228. González-Rubio, G.; Guerrero-Martínez, A.; Liz-Marzán, L. M., Reshaping, Fragmentation, and Assembly of Gold Nanoparticles Assisted by Pulse Lasers. Acc. Chem. Res. 2016, 49, 678-686. 229. Liu, Y.; Lin, X.-M.; Sun, Y.; Rajh, T., In Situ Visualization of Self-Assembly of Charged Gold Nanoparticles. J. Am. Chem. Soc. 2013, 135, 3764-3767.

230. Lin, G.; Chee, S. W.; Raj, S.; Král, P.; Mirsaidov, U., ACS Nano 2016, 10, 7443-7450.

231. Zhang, H.; Fung, K.-H.; Hartmann, J.; Chan, C. T.; Wang, D., Controlled Chainlike Agglomeration of Charged Gold Nanoparticles Via a Deliberate Interaction Balance. J. Phys. Chem. C 2008, 112, 16830-16839.

232. Kim, T.; Lee, C.-H.; Joo, S.-W.; Lee, K., Kinetics of Gold Nanoparticle Aggregation: Experiments and Modeling. J. Colloid Interface Sci. 2008, 318, 238-243.

233. Grayson, M., Phosphonium Compounds. lii. Mechanism of Hydroxide Cleavage of Tetrakis(Hydroxymethyl)Phosphonium Chloride. J. Am. Chem, Soc. 1963, 85, 79-83.

234. Ibañez, F. J.; Zamborini, F. P., Chemiresistive Sensing with Chemically Modified Metal and Alloy Nanoparticles. Small 2012, 8, 174-202.

235. Masitas, R. A.; Allen, S. L.; Zamborini, F. P., Size-Dependent Electrophoretic Deposition of Catalytic Gold Nanoparticles. J. Am. Chem. Soc. 2016, 138, 15295-15298.

236. Dick, J. E.; Bard, A. J., Recognizing Single Collisions of Ptcl62- at Femtomolar Concentrations on Ultramicroelectrodes by Nucleating Electrocatalytic Clusters. J. Am. Chem. Soc. 2015, 137, 13752-13755.

237. Jaramillo, T. F.; Baeck, S.-H.; Cuenya, B. R.; McFarland, E. W., Catalytic Activity of Supported Au Nanoparticles Deposited from Block Copolymer Micelles. J. Am. Chem. Soc. 2003, $125,7148-7149$.

238. Gerber, T.; Knudsen, J.; Feibelman, P. J.; Grånäs, E.; Stratmann, P.; Schulte, K.; Andersen, J. N.; Michely, T., Co-Induced Smoluchowski Ripening of Pt Cluster Arrays on the Graphene/Ir(111) Moiré. ACS Nano 2013, 7, 2020-2031.

239. Pattadar, D. K.; Zamborini, F. P., A Size Stability Study of Catalytically-Active Sub-2 Nm Diameter Gold Nanoparticles Synthesized with Weak Stabilizers. J. Am. Chem. Soc. 2018, 140, 14126-14133.

240. Ansar, S. M.; Ameer, F. S.; Hu, W.; Zou, S.; Pittman, C. U.; Zhang, D., Removal of Molecular Adsorbates on Gold Nanoparticles Using Sodium Borohydride in Water. Nano Lett. 2013, 13, 12261229.

241. Gentry, S. T.; Kendra, S. F.; Bezpalko, M. W., Ostwald Ripening in Metallic Nanoparticles: Stochastic Kinetics. J. Phys. Chem. C 2011, 115, 12736-12741. 
242. Ferreira, P. J.; la O', G. J.; Shao-Horn, Y.; Morgan, D.; Makharia, R.; Kocha, S.; Gasteiger, H. A., Instability of Pt/C Electrocatalysts in Proton Exchange Membrane Fuel Cells: A Mechanistic Investigation. J. Electrochem. Soc. 2005, 152, A2256-A2271.

243. Challa, S. R.; Delariva, A. T.; Hansen, T. W.; Helveg, S.; Sehested, J.; Hansen, P. L.; Garzon, F.; Datye, A. K., Relating Rates of Catalyst Sintering to the Disappearance of Individual Nanoparticles During Ostwald Ripening. J. Am. Chem. Soc. 2011, 133, 20672-20675.

244. Lee, H.-J.; Oh, S.; Cho, K.-Y.; Jeong, W.-L.; Lee, D.-S.; Park, S.-J., Spontaneous and Selective Nanowelding of Silver Nanowires by Electrochemical Ostwald Ripening and High Electrostatic Potential at the Junctions for High-Performance Stretchable Transparent Electrodes. ACS Appl. Mater. Interfaces 2018, 10, 14124-14131.

245. Pattadar, D. K.; Zamborini, F. P., A Size Stability Study of Catalytically-Active Sub-2 Nm Diameter Gold Nanoparticles Synthesized with Weak Stabilizers. J. Am. Chem. Soc. 2018.

246. Ivanova, O. S.; Zamborini, F. P., Electrochemical Size Discrimination of Gold Nanoparticles Attached to Glass/Indium-Tin-Oxide Electrodes by Oxidation in Bromide-Containing Electrolyte. Anal. Chem. 2010, 82, 5844-5850.

247. Sibakoti, T. R.; Stinger, C. R.; Adhihetty, P. K.; Zamborini, F. P.; Nantz, M. H., Tunable Aminooxy-Functionalized Monolayer-Protected Gold Clusters for Nonpolar and Aqueous Oximation Reactions. Part. Part. Syst. Charact. 2019, 36, 1900093

248. Han, D.; Kim, S.-S.; Kim, Y.-R.; Sohn, B.-H.; Chung, T. D., Surface Coverage and Size Effects on Electrochemical Oxidation of Uniform Gold Nanoparticles. Electrochem. Commun. 2015, 53, 11-14.

249. Liu, X.; Stroppa, D. G.; Heggen, M.; Ermolenko, Y.; Offenhäusser, A.; Mourzina, Y., Electrochemically Induced Ostwald Ripening in Au/Tio2 Nanocomposite. J. Phys. Chem. C 2015, 119, 10336-10344.

250. Polte, J.; Ahner, T. T.; Delissen, F.; Sokolov, S.; Emmerling, F.; Thünemann, A. F.; Kraehnert, R., Mechanism of Gold Nanoparticle Formation in the Classical Citrate Synthesis Method Derived from Coupled in Situ Xanes and Saxs Evaluation. J. Am. Chem. Soc. 2010, 132, 1296-1301.

251. Ambrozik, S.; Dimitrov, N., Anion Effects on the Interfacial Alloying in Successively Electrodeposited $\mathrm{Cu}$ and Au Ultrathin Films. J. Alloy. Comp. 2018, 762, 858-867.

252. Haldar, K. K.; Kundu, S.; Patra, A., Core-Size-Dependent Catalytic Properties of Bimetallic Au/Ag Core-Shell Nanoparticles. ACS Appl. Mater. Interfaces 2014, 6, 21946-21953.

253. Iyyamperumal, R.; Zhang, L.; Henkelman, G.; Crooks, R. M., Efficient Electrocatalytic Oxidation of Formic Acid Using Au@Pt Dendrimer-Encapsulated Nanoparticles. J. Am. Chem. Soc. 2013, 135, 5521-5524.

254. Zhang, F.; Defnet, P. A.; Fan, Y.; Hao, R.; Zhang, B., Transient Electrocatalytic Water Oxidation in Single-Nanoparticle Collision. J. Phys. Chem. C 2018, 122, 6447-6455.

255. Ibanez, F. J.; Zamborini, F. P., Chemiresistive Sensing with Chemically Modified Metal and Alloy Nanoparticles. Small 2012, 8, 174-202.

256. Joshi, G. K.; Deitz-McElyea, S.; Johnson, M.; Mali, S.; Korc, M.; Sardar, R., Highly Specific Plasmonic Biosensors for Ultrasensitive Microrna Detection in Plasma from Pancreatic Cancer Patients. Nano Lett. 2014, 14, 6955-6963.

257. Chen, G.; Zou, N.; Chen, B.; Sambur, J. B.; Choudhary, E.; Chen, P., Bimetallic Effect of Single Nanocatalysts Visualized by Super-Resolution Catalysis Imaging. ACS Cent. Sci. 2017, 3, 1189-1197.

258. Mishra, S. K.; Kannan, S., Doxorubicin-Conjugated Bimetallic Silver-Gadolinium Nanoalloy for Multimodal Mri-Ct-Optical Imaging and Ph-Responsive Drug Release. ACS Biomater. Sci. Eng. 2017, 3, 3607-3619. 
259. Liang, S.; Zhao, Y.; Xu, S.; Wu, X.; Chen, J.; Wu, M.; Zhao, J. X., A Silica-Gold-Silica Nanocomposite for Photothermal Therapy in the near-Infrared Region. ACS Appl. Mater. Interfaces 2015, 7, 85-93.

260. Valenti, M.; Venugopal, A.; Tordera, D.; Jonsson, M. P.; Biskos, G.; Schmidt-Ott, A.; Smith, W. A., Hot Carrier Generation and Extraction of Plasmonic Alloy Nanoparticles. ACS Photonics 2017, 4, 1146-1152.

261. Gilroy, K. D.; Ruditskiy, A.; Peng, H. C.; Qin, D.; Xia, Y., Bimetallic Nanocrystals: Syntheses, Properties, and Applications. Chem. Rev. 2016, 116, 10414-10472.

262. Gawande, M. B.; Goswami, A.; Asefa, T.; Guo, H.; Biradar, A. V.; Peng, D. L.; Zboril, R.; Varma, R. S., Core-Shell Nanoparticles: Synthesis and Applications in Catalysis and Electrocatalysis. Chem. Soc. Rev. 2015, 44, 7540-7590.

263. Holby, E. F.; Morgan, D., Application of Pt Nanoparticle Dissolution and Oxidation Modeling to Understanding Degradation in Pem Fuel Cells J. Electrochem. Soc. 2012, 159, B578B591.

264. Pattadar, D. K.; Zamborini, F. P., Size Stability Study of Catalytically Active Sub-2 Nm Diameter Gold Nanoparticles Synthesized with Weak Stabilizers. J. Am. Chem. Soc. 2018, 140, 14126-14133.

265. Tang, L.; Han, B.; Persson, K.; Friesen, C.; He, T.; Sieradzki, K.; Ceder, G., Electrochemical Stability of Nanometer-Scale Pt Particles in Acidic Environments. J. Am. Chem. Soc. 2010, 132, 596600.

266. Tang, L.; Li, X.; Cammarata, R. C.; Friesen, C.; Sieradzki, K., Electrochemical Stability of Elemental Metal Nanoparticles. J. Am. Chem. Soc. 2010, 132, 11722-11726.

267. Trindell, J. A.; Clausmeyer, J.; Crooks, R. M., Size Stability and $\mathrm{H}_{2} / \mathrm{Co}$ Selectivity for $\mathrm{Au}$ Nanoparticles During Electrocatalytic $\mathrm{Co}_{2}$ Reduction. J. Am. Chem. Soc. 2017, 139, 16161-16167.

268. Moreno, M.; Kissell, L. N.; Jasinski, J. B.; Zamborini, F. P., Selectivity and Reactivity of Alkylamine- and Alkanethiolate-Stabilized Pd and Pdag Nanoparticles for Hydrogenation and Isomerization of Allyl Alcohol. ACS Catal. 2012, 2, 2602-2613.

269. Li, X.; Chen, Q.; McCue, I.; Snyder, J.; Crozier, P.; Erlebacher, J.; Sieradzki, K., Dealloying of Noble-Metal Alloy Nanoparticles. Nano Lett. 2014, 14, 2569-2577.

270. McCue, I.; Snyder, J.; Li, X.; Chen, Q.; Sieradzki, K.; Erlebacher, J., Apparent Inverse GibbsThomson Effect in Dealloyed Nanoporous Nanoparticles. Phys. Rev. Lett. 2012, 108, 225503.

271. Moffat, T. P.; Fan, F. R. F.; Bard, A. J., Electrochemical and Scanning Tunneling Microscopic Study of Dealloying of Cu3au. J. Electrochem. Soc. 1991, 138, 3224-3235.

272. Sharma, J. N.; Pattadar, D. K.; Mainali, B. P.; Zamborini, F. P., Size Determination of Metal Nanoparticles Based on Electrochemically Measured Surface Area-to-Volume Ratio. Anal. Chem. 2018.

273. Brodsky, C. N.; Young, A. P.; Ng, K. C.; Kuo, C.-H.; Tsung, C.-K., Electrochemically Induced Surface Metal Migration in Well-Defined Core-Shell Nanoparticles and Its General Influence on Electrocatalytic Reactions. ACS Nano 2014, 8, 9368-9378.

274. Tuaev, X.; Rudi, S.; Petkov, V.; Hoell, A.; Strasser, P., In Situ Study of Atomic Structure Transformations of Pt-Ni Nanoparticle Catalysts During Electrochemical Potential Cycling. ACS Nano 2013, 7, 5666-5674.

275. Niu, Z., et al., Ultrathin Epitaxial Cu@Au Core-Shell Nanowires for Stable Transparent Conductors. J. Am. Chem. Soc. 2017, 139, 7348-7354.

276. Xia, J.; Achari, I.; Ambrozik, S.; Dimitrov, N., Synthesis, Characterization, and Testing of PtNpg Catalysts Developed by De-Alloying of Electrodeposited $\mathrm{Cu}_{\mathrm{x}} \mathrm{au}_{(1-\mathrm{x})}$ Thin Films. Mater. Res. Bull. 2017, 85, 1-9. 
277. Yancey, D. F.; Carino, E. V.; Crooks, R. M., Electrochemical Synthesis and Electrocatalytic Properties of Au@Pt Dendrimer-Encapsulated Nanoparticles. J. Am. Chem. Soc. 2010, 132, 1098810989.

278. Tschulik, K.; Ngamchuea, K.; Ziegler, C.; Beier, M. G.; Damm, C.; Eychmueller, A.; Compton, R. G., Core-Shell Nanoparticles: Characterizing Multifunctional Materials Beyond ImagingDistinguishing and Quantifying Perfect and Broken Shells. Adv. Funct. Mater. 2015, 25, 5149-5158. 279. Bonifacio, C. S.; Carenco, S.; Wu, C. H.; House, S. D.; Bluhm, H.; Yang, J. C., Thermal Stability of Core-Shell Nanoparticles: A Combined in Situ Study by Xps and Tem. Chem. Mater. 2015, 27, 6960-6968.

280. Liu, C.; Ma, Z.; Cui, M.; Zhang, Z.; Zhang, X.; Su, D.; Murray, C. B.; Wang, J. X.; Zhang, S., Favorable Core/Shell Interface within Co2p/Pt Nanorods for Oxygen Reduction Electrocatalysis. Nano Lett. 2018, 18, 7870-7875.

281. Ghilane, J.; Fan, F.-R. F.; Bard, A. J.; Dunwoody, N., Facile Electrochemical Characterization of Core/Shell Nanoparticles. Ag Core/Ag2o Shell Structures. Nano Lett. 2007, 7, 1406-1412.

282. Xia, J.; Ambrozik, S.; Crane, C. C.; Chen, J.; Dimitrov, N., Impact of Structure and Composition on the Dealloying of Cuxau(1-X) Bulk and Nanoscale Alloys. J. Phys. Chem. C 2016, 120, 2299-2308.

283. Lim, D.-K.; Kim, I.-J.; Nam, J.-M., DNA-Embedded Au/Ag Core-Shell Nanoparticles. Chem. Commun. 2008, 5312-5314.

284. Aitchison, H.; Meyerbröker, N.; Lee, T.-L.; Zegenhagen, J.; Potter, T.; Früchtl, H.; Cebula, I.; Buck, M., Underpotential Deposition of $\mathrm{Cu}$ on $\mathrm{Au}(111)$ from Neutral Chloride Containing Electrolyte. Phys. Chem. Chem. Phys. 2017, 19, 24146-24153.

285. Zou, S.; Gao, X.; Weaver, M. J., Electrochemical Adsorbate-Induced Substrate Restructuring: Gold(110) in Aqueous Bromide Electrolytes. Surf. Sci. 2000, 452, 44-57.

286. Blizanac, B. B.; Lucas, C. A.; Gallagher, M. E.; Ross, P. N.; Marković, N. M., Surface Structures and Phase Transitions at the $\mathrm{Au}(100)-\mathrm{Br}$ Interface: $\mathrm{Ph}$ and Co Effects. J. Phys. Chem. B 2004, 108, 5304-5313.

287. Renner, F. U.; Stierle, A.; Dosch, H.; Kolb, D. M.; Zegenhagen, J., The Influence of Chloride on the Initial Anodic Dissolution of Cu3au(111). Electrochem. Commun. 2007, 9, 1639-1642.

288. Ankah, G. N.; Pareek, A.; Cherevko, S.; Topalov, A. A.; Rohwerder, M.; Renner, F. U., The Influence of Halides on the Initial Selective Dissolution of Cu3au (111). Electrochim. Acta 2012, 85, 384-392.

289. Kim, D.; Xie, C.; Becknell, N.; Yu, Y.; Karamad, M.; Chan, K.; Crumlin, E. J.; Nørskov, J. K.; Yang, P., J. Am. Chem. Soc. 2017, 139, 8329-8336.

290. Zhan, W., et al., Crystal Structural Effect of Aucu Alloy Nanoparticles on Catalytic Co Oxidation. J. Am. Chem. Soc. 2017, 139, 8846-8854.

291. Tee, S. Y.; Ye, E.; Pan, P. H.; Lee, C. J. J.; Hui, H. K.; Zhang, S.-Y.; Koh, L. D.; Dong, Z.; Han, M.-Y., Fabrication of Bimetallic Cu/Au Nanotubes and Their Sensitive, Selective, Reproducible and Reusable Electrochemical Sensing of Glucose. Nanoscale 2015, 7, 11190-11198.

292. Zhang, J.; Xu, X.; Yang, C.; Yang, F.; Yang, X., Colorimetric lodide Recognition and Sensing by Citrate-Stabilized Core/Shell Cu@Au Nanoparticles. Anal. Chem. 2011, 83, 3911-3917.

293. Liu, X.; Zheng, Z.; Wang, C.; Liu, W.; An, R.; Zhang, W., Fusion Behaviour and Mechanism of Ultrafine Ag-Cu Nanoparticles Induced by Electron Beam Irradiation. J. Mater. Sci.: Mater. Electron. 2017, 28, 8206-8210.

294. Liu, S., et al., Unidirectional Thermal Diffusion in Bimetallic Cu@Au Nanoparticles. ACS Nano 2014, 8, 1886-1892. 
295. Brodsky, C. N.; Young, A. P.; Ng, K. C.; Kuo, C.-H.; Tsung, C.-K., Electrochemically Induced Surface Metal Migration in Well-Defined Core-Shell Nanoparticles and Its General Influence on Electrocatalytic Reactions. ACS Nano 2014, 8, 9368-9378.

296. Guisbiers, G.; Mejia-Rosales, S.; Khanal, S.; Ruiz-Zepeda, F.; Whetten, R. L.; José-Yacaman, M., Gold-Copper Nano-Alloy, "Tumbaga", in the Era of Nano: Phase Diagram and Segregation. Nano Lett. 2014, 14, 6718-6726.

297. Bonifacio, C. S.; Carenco, S.; Wu, C. H.; House, S. D.; Bluhm, H.; Yang, J. C., Thermal Stability of Core-Shell Nanoparticles: A Combined in Situ Study by Xps and Tem. Chem. Mater. 2015, 27, 6960-6968.

298. Lapp, A. S.; Duan, Z.; Marcella, N.; Luo, L.; Genc, A.; Ringnalda, J.; Frenkel, A. I.; Henkelman, G.; Crooks, R. M., Experimental and Theoretical Structural Investigation of Aupt Nanoparticles Synthesized Using a Direct Electrochemical Method. J. Am. Chem. Soc. 2018, 140, 6249-6259.

299. Allen, S. L.; Sharma, J. N.; Zamborini, F. P., Aggregation-Dependent Oxidation of Metal Nanoparticles. J. Am. Chem. Soc. 2017, 139, 12895-12898.

300. Xu, Z.; Lai, E.; Shao-Horn, Y.; Hamad-Schifferli, K., Compositional Dependence of the Stability of Aucu Alloy Nanoparticles. Chem. Commun. 2012, 48, 5626-5628.

301. Yin, A.; Wen, C.; Dai, W.-L.; Fan, K., Nanocasting of Cuau Alloy Nanoparticles for Methyl Glycolate Synthesis. J. Mater. Chem. 2011, 21, 8997-8999.

302. Sun, S.; Murray, C. B.; Weller, D.; Folks, L.; Moser, A., Monodisperse Fept Nanoparticles and Ferromagnetic Fept Nanocrystal Superlattices. Science 2000, 287, 1989-1992.

303. Holt, L. R.; Plowman, B. J.; Young, N. P.; Tschulik, K.; Compton, R. G., The Electrochemical Characterization of Single Core-Shell Nanoparticles. Angew. Chem. 2016, 55, 397-400.

304. King, S. R.; Gentle, A. R.; Cortie, M. B.; McDonagh, A. M., On the Development of Optical Properties During Thermal Coarsening of Gold Nanoparticle Composites. J. Phys. Chem. C 2018, 122, 12098-12105.

305. Challa, S. R.; Delariva, A. T.; Hansen, T. W.; Helveg, S.; Sehested, J.; Hansen, P. L.; Garzon, F.; Datye, A. K., Relating Rates of Catalyst Sintering to the Disappearance of Individual Nanoparticles During Ostwald Ripening. J. Am. Chem. Soc. 2011, 133, 20672-20675.

306. Hansen, T. W.; DeLaRiva, A. T.; Challa, S. R.; Datye, A. K., Sintering of Catalytic Nanoparticles: Particle Migration or Ostwald Ripening? Acc. Chem. Res. 2013, 46, 1720-1730.

307. Llorca, J.; Domínguez, M.; Ledesma, C.; Chimentão, R. J.; Medina, F.; Sueiras, J.; Angurell, I.; Seco, M.; Rossell, O., Propene Epoxidation over Tio2-Supported Au-Cu Alloy Catalysts Prepared from Thiol-Capped Nanoparticles. J. Catal. 2008, 258, 187-198. 


\section{APENDIX}

\section{Sample calculation for determining the amount of $\mathrm{Cu}$ under the Au peak from}

ASV.

Below is the calculation of the amount of $\mathrm{Au}$ and $\mathrm{Cu}$ in the alloys, including the amount of $\mathrm{Cu}$ that dealloyed separately from Au compared to the $\mathrm{Cu}$ that oxidized with the Au. This is for $\mathrm{Cu}_{1} / \mathrm{Au}_{\mathrm{x}}$ core/shell nanoparticles oxidized (stripped) in 0.01 $\mathrm{M} \mathrm{KBr}$ plus $0.1 \mathrm{M} \mathrm{HClO}_{4}$ electrolyte and is based on the integrated peak charge in Coulombs for the $\mathrm{Au}$ and $\mathrm{Cu}$ oxidation peaks and the known ratio of $\mathrm{Au}$ and $\mathrm{Cu}$ used in the synthesis.

$\mathrm{Cu}_{1} / \mathrm{Au}$ is the ratio used during the synthesis.

$\mathrm{x}=$ the amount of Au relative to the amount of $\mathrm{Cu}$.

$\mathrm{Q}_{\mathrm{cu}(\mathrm{sep})}=$ the integrated charge of $\mathrm{Cu}$ from the well-separated $\mathrm{Cu}$ peaks that appeared negative of Au oxidation. (Peaks $\left.\mathrm{Cu}^{1}+\mathrm{Cu}^{2}\right)$

$\mathrm{Q}_{\mathrm{Au}+\mathrm{Cu}}=$ the integrated charge due to $\mathrm{Au}$ and $\mathrm{Cu}$ oxidation that occurred at the $\mathrm{Au}$ oxidation potential. (Peak $\mathrm{Au}^{1}$ )

$\mathrm{Q}_{\mathrm{cu}(\text { withAu })}=$ the integrated charge due to $\mathrm{Cu}$ that oxidized along with $\mathrm{Au}$ at the $\mathrm{Au}$ oxidation potential. (Part of $\mathrm{Au}^{1}$ that is due to $\mathrm{Cu}$ oxidation) 


$$
\begin{aligned}
& \mathrm{Au} / \mathrm{Cu}=\mathrm{x} \\
& \left.\left[\left(\mathrm{Q}_{A u+C u}-\mathrm{Q}_{\mathrm{Cu}(\text { withAu })}\right) / 3\right] /\left[\left(\mathrm{Q}_{\mathrm{cu}(\mathrm{sep})}+\mathrm{Q}_{\mathrm{cu}(\text { withAu })}\right) / 2\right)\right]=\mathrm{x} \\
& \left(2 Q_{A u+C u}-2 Q_{c u}(\text { withAu })\right) /\left(3 Q_{c u(s e p)}+3 Q_{c u(w i t h A u)}\right)=x \\
& 2 \mathrm{Q}_{\mathrm{Au}+\mathrm{Cu}}-2 \mathrm{Q}_{\mathrm{Cu}(\text { withAu })}=3 \times \mathrm{Q}_{\mathrm{Cu}(\mathrm{sep})}+3 \times \mathrm{Cu}_{\mathrm{Au}} \\
& (3 \mathrm{x}+2) \mathrm{Q}_{\mathrm{Cu}(\text { withAu })}=2 \mathrm{Q}_{\mathrm{Au}}+\mathrm{Cu}-3 \mathrm{xQ} \mathrm{Qu}(\mathrm{sep}) \\
& \mathrm{Q}_{\mathrm{Cu}(\text { withAu })}=\left(2 \mathrm{Q}_{\mathrm{Au}+\mathrm{Cu}}-3 \times \mathrm{Q}_{\mathrm{Cu}(\mathrm{sep})}\right) /(3 \mathrm{x}+2)
\end{aligned}
$$

This gives the charge of $\mathrm{Cu}$ underneath the Au peak in terms of the known synthesis ratio $\mathrm{x}$ and known values from the ASVs $\left(\mathrm{Cu}^{1}, \mathrm{Cu}^{2}\right.$, and $\left.\mathrm{Au}^{1}\right)$. The remaining amount of charge under the $\mathrm{Au}^{1}$ peak is due to Au oxidation. The following equation allows one to convert the $\mathrm{Q}$ to moles of $\mathrm{Cu}$ and moles of $\mathrm{Au}$ as follows:

$\mathrm{Q} / \mathrm{nF}$, where $\mathrm{n}=$ number of electrons in the oxidation and $\mathrm{F}$ is Faraday's constant.

Example with Values: $\mathrm{Cu}_{1} / \mathrm{Au}_{1}$ core/shell. This shows how we calculated the values in Table 2 of main paper.

$\mathrm{Q}$ under the Au oxidation peak $=\mathrm{Q}_{\mathrm{Au}+\mathrm{Cu}}=6.43 \times 10^{-4}$ Coulombs

Sum of the $\mathrm{Q}$ from all Cu oxidation peaks $=\mathrm{Qcu}(\mathrm{sep})=1.60 \times 10^{-4}$ Coulombs 
$x=1$

$\mathrm{Q}_{\mathrm{Cu}(\text { withAu })}=\left(2 \mathrm{Q}_{\mathrm{Au}+\mathrm{Cu}}-3 \mathrm{x} \mathrm{Q}_{\mathrm{Cu}(\mathrm{sep}))}\right) /(3 \mathrm{x}+2)$

$\mathrm{Q}_{\mathrm{Cu}(\text { withAu })}=\left(2\left(6.43 \times 10^{-4}\right)-3\left(1.60 \times 10^{-4}\right)\right) /(5)$

$=\left(1.286 \times 10^{-3}-4.80 \times 10^{-4}\right) / 5$

$=1.61 \times 10^{-4}$ Coulombs

Total Mole Au $=\left(6.43 \times 10^{-4}\right.$ Coulombs $-1.61 \times 10^{-4}$ Coulombs $) /\left(3^{\star} 96500\right)=1.66$ $\times 10^{-9}$ mole Au

Total Mole Cu $=\left(1.61 \times 10^{-4}\right.$ Coulombs $+1.60 \times 10^{-4}$ Coulombs $) /(2 \star 96500)=1.66$ x $10^{-9}$ mole Cu

Mole Cu under $\mathrm{Au}=1.61 \times 10^{-4}$ Coulombs $/\left(2^{\star} 96500\right)=8.34 \times 10^{-10} \mathrm{~mole} \mathrm{Cu}$

$\mathrm{Au} / \mathrm{Cu}$ ratio under $\mathrm{Au}=1.66 \times 10^{-9} \mathrm{~mole} / 8.34 \times 10^{-10} \mathrm{~mole}=1.99$

$\%$ of $\mathrm{Cu}$ retained by $\mathrm{Au}=\left(8.34 \times 10^{-10} \mathrm{~mole} / 1.66 \times 10^{-9} \mathrm{~mole}\right){ }^{\star} 100 \%=50.2 \%$

Calculation of NP Composition and Size Before and After Cu Dealloying and Rearrangement

$\mathrm{Cu}_{1} / \mathrm{Au}_{0.5}$ core/shell alloy NP

The Cu core radius was determined to be $2.23 \mathrm{~nm}$ based on the average $3.5 \mathrm{~nm}$ core/shell radius of the $\mathrm{Cu}_{1} / \mathrm{Au}_{2}$ alloy NP determined by TEM. 
Using $0.128 \mathrm{~nm}$ radius for $\mathrm{Cu}$, we obtain $0.00878 \mathrm{~nm}^{3}$ for the volume of a $\mathrm{Cu}$ atom based on volume of a sphere $\left(4 / 3 \square r^{3}\right)$.

Using $0.144 \mathrm{~nm}$ radius for $\mathrm{Au}$, we obtain $0.01250 \mathrm{~nm}^{3}$ for the volume of an Au atom based on volume of a sphere $\left(4 / 3 \square r^{3}\right)$.

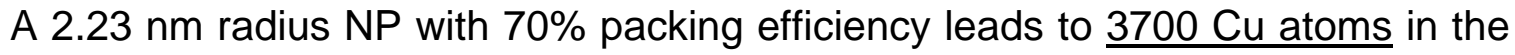
Cu core.

A total radius of $3.50 \mathrm{~nm}(2.23 \mathrm{~nm}$ core $+1.27 \mathrm{~nm}$ shell) leads to $\underline{7400 \mathrm{Au} \text { atoms}}$, giving a $\mathrm{Cu}_{1} / \mathrm{Au}_{2}$ ratio and composition of $\mathrm{Cu}_{3700 / \mathrm{Au}} \mathrm{u}_{700}$.

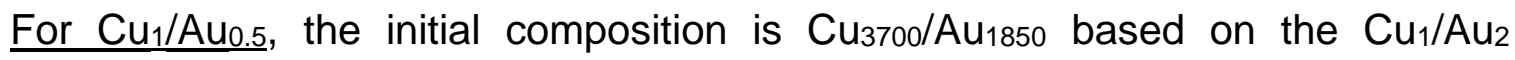
composition.

We determined that $41 \%$ of the $\mathrm{Cu}$ is retained for the $\mathrm{Cu}_{1} / \mathrm{Au} 0.5 \mathrm{NP}$ after initial $\mathrm{Cu}$ oxidation, which is equal to:

$3700 \times 0.41=1518 \mathrm{Cu}$ atoms left initial oxidation

That gives a composition of $\mathrm{Cu}_{1518} / \mathrm{Au}_{1850}$ after initial $\mathrm{Cu}$ oxidation up to $0.42 \mathrm{~V}$.

The core volume would be as follows, assuming 70\% packing efficiency:

$V_{1518 \text { Cu core }}=\left(V_{\text {Cu atom }} \times 1518\right) / 0.7=\left(0.00878 \mathrm{~nm}^{3} \times 1518\right) / 0.7=19.04 \mathrm{~nm}^{3}$

We can then solve for radius in terms of volume as follows:

$\mathrm{r}(1518 \mathrm{Cu}$ atoms $)=(3 \mathrm{~V} / 4 \square)^{1 / 3}=[(3 \times 19.04) / 4 \square]^{1 / 3}=1.66 \mathrm{~nm}$ Cu core radius after initial ox. 
To get the shell thickness we have to determine the volume of the Au from the number of atoms. This would be:

$V_{1850 \text { Au atoms }}=\left(V_{\text {Au atom }} \times 1850\right) / 0.7=\left(0.01250 \mathrm{~nm}^{3} \times 1850\right) / 0.7=33.05 \mathrm{~nm}^{3}$

The total volume of the NP is then:

$V_{T}=V_{1850}$ Au atoms $+V_{1518 \text { Cu core }}=33.05 \mathrm{~nm}^{3}+19.04 \mathrm{~nm}^{3}=52.09 \mathrm{~nm}^{3}$

The radius of the rearranged NP is then:

$r=(3 V / 4 \square)^{1 / 3}=[(3 \times 52.09) / 4 \square]^{1 / 3}=2.32 \mathrm{~nm}$ Cu/Au core/shell NP radius

$1.66 \mathrm{~nm}$ radius $\mathrm{Cu}_{1518}$ core and $0.66 \mathrm{~nm}$ thick $\mathrm{Au}_{1850}$ shell $=2.32 \mathrm{~nm}$ radius core/shell NP

$0.66 \mathrm{~nm} / 0.288 \mathrm{~nm}=2.3$ atomic Au layers

The original $\mathrm{Cu}_{1} / \mathrm{Au}_{0.5}$ core/shell NP was:

$2.23 \mathrm{~nm}$ radius $\mathrm{Cu}_{3700}$ core

$\mathrm{V}_{\text {cu3700 }}=(4 / 3) \square \mathrm{r}^{3}=(4 / 3) \square(2.23 \mathrm{~nm})^{3}=46.45 \mathrm{~nm}^{3}$

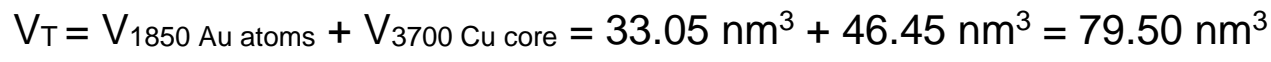

$\mathrm{r}=(3 \mathrm{~V} / 4 \square)^{1 / 3}=[(3 \times 79.50) / 4 \square]^{1 / 3}=2.67 \mathrm{~nm} \mathrm{Cu} 3700 / \mathrm{Au}_{1850}$ core/shell NP radius

$2.23 \mathrm{~nm} \mathrm{Cu} 3700$ core plus $0.44 \mathrm{~nm}$ thick $\mathrm{Au}_{1850}$ shell $=2.67 \mathrm{~nm} \mathrm{Cu} \mathrm{Cu}_{3700 / \mathrm{Au}_{1850}}$ core/shell NP

$0.44 \mathrm{~nm} / 0.288 \mathrm{~nm}=1.5$ atomic Au layers. 
The NP goes from a $2.67 \mathrm{~nm}$ radius (5.4 nm diameter) $\mathrm{Cu}_{3700 / \mathrm{Au} 1850 \mathrm{NP}}$ to a 2.32 $\mathrm{nm}$ radius (4.6 $\mathrm{nm}$ diameter) $\mathrm{Cu}_{1518} / \mathrm{Au}_{1850} \mathrm{NP}$ after $41 \% \mathrm{Cu}$ oxidation and $\mathrm{Au}$ rearrangement 


\begin{tabular}{|c|c|}
\hline Title: & $\begin{array}{l}\text { Size Stability Study of } \\
\text { Catalytically Active Sub-2 } \\
\text { nm Diameter Gold } \\
\text { Nanoparticles } \\
\text { Synthesized with Weak } \\
\text { Stabilizers }\end{array}$ \\
\hline Author: & $\begin{array}{l}\text { Dhruba K. Pattadar, } \\
\text { Francis P. Zamborini }\end{array}$ \\
\hline Publication: & $\begin{array}{l}\text { Journal of the American } \\
\text { Chemical Society }\end{array}$ \\
\hline Publisher: & $\begin{array}{l}\text { American Chemical } \\
\text { Society }\end{array}$ \\
\hline Date: & Oct 1, 2018 \\
\hline
\end{tabular}

\section{PERMISSION/LICENSE IS GRANTED FOR YOUR ORDER AT NO CHARGE}

This type of permission/license, instead of the standard Terms \& Conditions, is sent to you because no fee is being charged for your order. Please note the following:

- Permission is granted for your request in both print and electronic formats, and translations.

- If figures and/or tables were requested, they may be adapted or used in part.

- Please print this page for your records and send a copy of it to your publisher/graduate school.

- Appropriate credit for the requested material should be given as follows: "Reprinted (adapted) with permission from (COMPLETE REFERENCE CITATION). Copyright (YEAR) American Chemical Society." Insert appropriate information in place of the capitalized words.

- One-time permission is granted only for the use specified in your request. No additional uses are granted (such as derivative works or other editions). For any other uses, please submit a new request. 


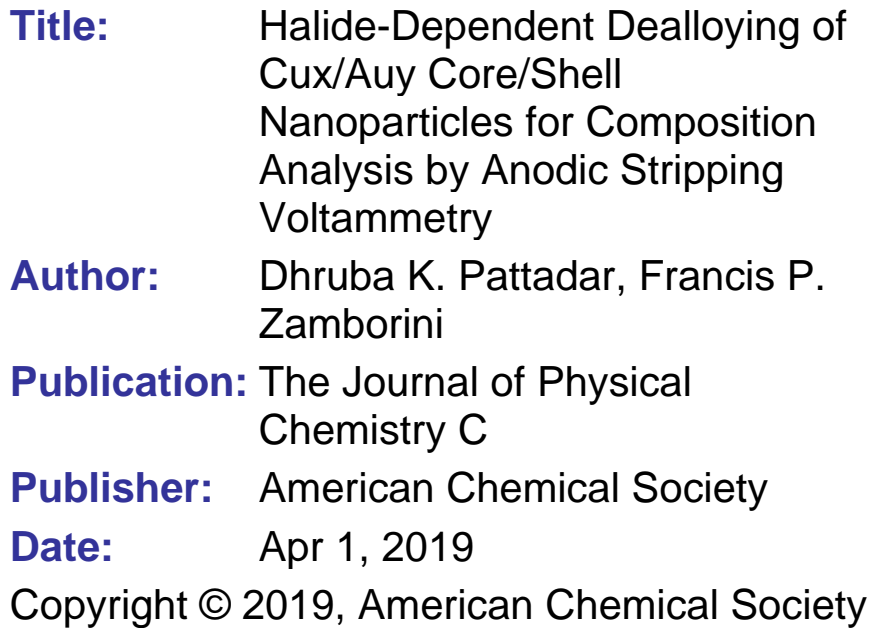

\section{PERMISSION/LICENSE IS GRANTED FOR YOUR ORDER AT NO CHARGE}

This type of permission/license, instead of the standard Terms \& Conditions, is sent to you because no fee is being charged for your order. Please note the following:

- Permission is granted for your request in both print and electronic formats, and translations.

- If figures and/or tables were requested, they may be adapted or used in part.

- Please print this page for your records and send a copy of it to your publisher/graduate school.

- Appropriate credit for the requested material should be given as follows: "Reprinted (adapted) with permission from (COMPLETE REFERENCE CITATION). Copyright (YEAR) American Chemical Society." Insert appropriate information in place of the capitalized words.

- One-time permission is granted only for the use specified in your request. No additional uses are granted (such as derivative works or other editions). For any other uses, please submit a new request.

\section{Effect of Size, Coverage, and Dispersity on the Potential-Controlled Ostwald Ripening of Metal Nanoparticles}

\section{Author:}

Dhruba K. Pattadar, Francis P. Zamborini

Publication:

Langmuir

Publisher:

American Chemical Society

Date:

Oct 1, 2019

Copyright ( 2019, American Chemical Society 


\section{PERMISSION/LICENSE IS GRANTED FOR YOUR ORDER AT NO CHARGE}

This type of permission/license, instead of the standard Terms \& Conditions, is sent to you because no fee is being charged for your order. Please note the following:

- Permission is granted for your request in both print and electronic formats, and translations.

- If figures and/or tables were requested, they may be adapted or used in part.

- Please print this page for your records and send a copy of it to your publisher/graduate school.

- Appropriate credit for the requested material should be given as follows: "Reprinted (adapted) with permission from (COMPLETE REFERENCE CITATION). Copyright (YEAR) American Chemical Society." Insert appropriate information in place of the capitalized words. - One-time permission is granted only for the use specified in your request. No additional uses are granted (such as derivative works or other editions). For any other uses, please submit a new request. 
Table A1. SA/V for $15 \mathrm{~nm}$ diameter Au NPs before and after ripening at $0.0 \mathrm{~V}$.

\begin{tabular}{|c|c|c|c|c|c|c|c|}
\hline $\begin{array}{c}\text { Trial } \\
\#\end{array}$ & $\begin{array}{c}\text { Time of } \\
\text { Ostwald } \\
\text { ripening } \\
\text { (min) }\end{array}$ & $\begin{array}{l}\text { Au oxide } \\
\text { reduction } \\
\text { charge in } \\
\text { CV (before } \\
\text { Ost.) } \\
\text { (C) }\end{array}$ & $\begin{array}{l}\text { Au oxide } \\
\text { reduction } \\
\text { charge in } \\
\text { CV (after } \\
\text { Ost.) } \\
\text { (C) }\end{array}$ & $\begin{array}{c}\text { Au } \\
\text { stripping } \\
\text { charge in } \\
\text { ASV } \\
\text { (total } \\
\text { volume) } \\
\text { (C) }\end{array}$ & $\begin{array}{l}\text { Surface } \\
\text { area } \\
\text { (SA)/ } \\
\text { Volume } \\
\text { (after } \\
\text { Ost.) } \\
\text { (V) }\end{array}$ & $\begin{array}{l}\text { Calculated } \\
\text { NP } \\
\text { diameter } \\
\text { based } \\
\text { on cal. } \\
\text { curve } \\
(\mathrm{nm})\end{array}$ & $\begin{array}{c}\text { Average } \\
\text { diameter } \\
\text { of NPs } \\
\text { based } \\
\text { on cal. } \\
\text { curve } \\
(\mathrm{nm})\end{array}$ \\
\hline 1 & 0 & $9.46 \times 10^{-6}$ & & $3.80 \times 10^{-5}$ & 0.22 & 18.8 & \multirow{4}{*}{$\begin{array}{c}18.1 \pm \\
1.0\end{array}$} \\
\hline 2 & 0 & $7.67 \times 10^{-6}$ & & $3.42 \times 10^{-5}$ & 0.23 & 18.6 & \\
\hline 3 & 0 & $1.02 \times 10^{-5}$ & & $4.39 \times 10^{-5}$ & 0.23 & 18.6 & \\
\hline 4 & 0 & $1.05 \times 10^{-5}$ & & $4.25 \times 10^{-5}$ & 0.25 & 16.6 & \\
\hline 1 & 35 & $7.91 \times 10^{-6}$ & $7.15 \times 10^{-6}$ & $3.76 \times 10^{-5}$ & 0.19 & 22.9 & \multirow{4}{*}{$\begin{array}{c}20.0 \pm \\
2.6\end{array}$} \\
\hline 2 & 35 & $7.56 \times 10^{-6}$ & $6.75 \times 10^{-6}$ & $2.82 \times 10^{-5}$ & 0.24 & 17.3 & \\
\hline 3 & 35 & $6.57 \times 10^{-6}$ & $6.38 \times 10^{-6}$ & $3.18 \times 10^{-5}$ & 0.20 & 21.5 & \\
\hline 4 & 35 & $8.08 \times 10^{-6}$ & $7.54 \times 10^{-6}$ & $3.24 \times 10^{-5}$ & 0.23 & 18.6 & \\
\hline 1 & 70 & $9.61 \times 10^{-6}$ & $7.20 \times 10^{-6}$ & $3.58 \times 10^{-5}$ & 0.20 & 21.5 & \multirow{4}{*}{$\begin{array}{c}21.3 \pm \\
0.5\end{array}$} \\
\hline 2 & 70 & $9.35 \times 10^{-6}$ & $7.84 \times 10^{-6}$ & $3.86 \times 10^{-5}$ & 0.20 & 21.5 & \\
\hline 3 & 70 & $9.48 \times 10^{-6}$ & $7.73 \times 10^{-6}$ & $3.88 \times 10^{-5}$ & 0.19 & 21.7 & \\
\hline 4 & 70 & $7.67 \times 10^{-6}$ & $5.91 \times 10^{-6}$ & $2.84 \times 10^{-5}$ & 0.21 & 20.6 & \\
\hline 1 & 105 & $9.64 \times 10^{-6}$ & $7.13 \times 10^{-6}$ & $3.57 \times 10^{-5}$ & 0.20 & 21.5 & \multirow{4}{*}{$\begin{array}{c}21.9 \pm \\
0.7 \\
\end{array}$} \\
\hline 2 & 105 & $8.21 \times 10^{-6}$ & $7.20 \times 10^{-6}$ & $3.60 \times 10^{-5}$ & 0.20 & 21.5 & \\
\hline 3 & 105 & $7.65 \times 10^{-6}$ & $6.19 \times 10^{-6}$ & $3.25 \times 10^{-5}$ & 0.19 & 22.9 & \\
\hline 4 & 105 & $7.20 \times 10^{-6}$ & $6.51 \times 10^{-6}$ & $3.25 \times 10^{-5}$ & 0.20 & 21.5 & \\
\hline 1 & 140 & $7.37 \times 10^{-6}$ & $6.23 \times 10^{-6}$ & $3.27 \times 10^{-5}$ & 0.19 & 22.9 & \multirow{4}{*}{$\begin{array}{c}22.0 \pm \\
1.1\end{array}$} \\
\hline 2 & 140 & $9.38 \times 10^{-6}$ & $7.01 \times 10^{-6}$ & $3.73 \times 10^{-5}$ & 0.19 & 22.9 & \\
\hline 3 & 140 & $7.81 \times 10^{-6}$ & $7.10 \times 10^{-6}$ & $3.45 \times 10^{-5}$ & 0.21 & 20.6 & \\
\hline 4 & 140 & $9.12 \times 10^{-6}$ & $6.30 \times 10^{-6}$ & $3.18 \times 10^{-5}$ & 0.20 & 21.5 & \\
\hline
\end{tabular}

Table A2. SA/V ratio for $15 \mathrm{~nm}$ diameter Au NPs before and after ripening at $0.3 \mathrm{~V}$. 


\begin{tabular}{|c|c|c|c|c|c|c|c|}
\hline $\begin{array}{c}\text { No. } \\
\text { of } \\
\text { trial }\end{array}$ & $\begin{array}{l}\text { Time of } \\
\text { Ostwald } \\
\text { ripping } \\
\text { (min) }\end{array}$ & $\begin{array}{l}\text { Au oxide } \\
\text { reduction } \\
\text { charge in } \\
\text { CV (before } \\
\text { Ost.) } \\
\text { (C) }\end{array}$ & $\begin{array}{l}\text { Au oxide } \\
\text { reduction } \\
\text { charge in } \\
\text { CV (after } \\
\text { Ost.) } \\
\text { (C) }\end{array}$ & $\begin{array}{c}\text { Au } \\
\text { stripping } \\
\text { charge in } \\
\text { ASV } \\
\text { (total } \\
\text { volume) } \\
\text { (C) }\end{array}$ & $\begin{array}{l}\text { Surface } \\
\text { area } \\
\text { (SA)/ } \\
\text { Volume } \\
\text { (after } \\
\text { Ost.) } \\
\text { (V) }\end{array}$ & $\begin{array}{l}\text { Calculated } \\
\text { NP } \\
\text { diameter } \\
\text { based } \\
\text { on cal. } \\
\text { curve } \\
(\mathrm{nm}) \\
\end{array}$ & $\begin{array}{c}\text { Average } \\
\text { diameter } \\
\text { of NPs } \\
\text { based } \\
\text { on cal. } \\
\text { curve } \\
(\mathrm{nm})\end{array}$ \\
\hline 1 & 0 & $9.46 \times 10^{-6}$ & & $\begin{array}{c}3.80 \times 10^{-} \\
5\end{array}$ & 0.22 & 18.8 & \multirow{4}{*}{$\begin{array}{c}18.1 \pm \\
1.0\end{array}$} \\
\hline 2 & 0 & $7.67 \times 10^{-6}$ & & $\begin{array}{c}3.42 \times 10^{-} \\
5\end{array}$ & 0.23 & 18.6 & \\
\hline 3 & 0 & $1.02 \times 10^{-5}$ & & $\begin{array}{c}4.39 \times 10^{-} \\
5\end{array}$ & 0.23 & 18.6 & \\
\hline 4 & 0 & $1.05 \times 10^{-5}$ & & $\begin{array}{c}4.25 \times 10^{-} \\
5\end{array}$ & 0.25 & 16.6 & \\
\hline 1 & 35 & $7.84 \times 10^{-6}$ & $6.34 \times 10^{-6}$ & $\begin{array}{c}3.36 \times 10^{-} \\
-\end{array}$ & 0.19 & 23.4 & \multirow{4}{*}{$\begin{array}{c}22.3 \pm \\
3.0\end{array}$} \\
\hline 2 & 35 & $1.01 \times 10^{-6}$ & $6.86 \times 10^{-6}$ & $\begin{array}{c}4.04 \times 10^{-} \\
5\end{array}$ & 0.17 & 26.2 & \\
\hline 3 & 35 & $1.02 \times 10^{-6}$ & $6.61 \times 10^{-6}$ & $\begin{array}{c}3.05 \times 10^{-} \\
-\end{array}$ & 0.22 & 18.8 & \\
\hline 4 & 35 & $9.64 \times 10^{-6}$ & $7.82 \times 10^{-6}$ & ${ }_{5}^{3.79 \times 10^{-}}$ & 0.20 & 20.9 & \\
\hline 1 & 70 & $8.46 \times 10^{-6}$ & $7.03 \times 10^{-6}$ & $\begin{array}{c}4.22 \times 10^{-} \\
5\end{array}$ & 0.17 & 27.2 & \multirow{4}{*}{$\begin{array}{c}28.0 \pm \\
1.0\end{array}$} \\
\hline 2 & 70 & $8.95 \times 10^{-6}$ & $5.42 \times 10^{-6}$ & $\begin{array}{c}3.45 \times 10^{-} \\
5\end{array}$ & 0.16 & 29.5 & \\
\hline 3 & 70 & $9.20 \times 10^{-6}$ & $6.00 \times 10^{-6}$ & $\begin{array}{c}3.68 \times 10^{-} \\
5\end{array}$ & 0.16 & 28.1 & \\
\hline 4 & 70 & $6.02 \times 10^{-6}$ & $4.66 \times 10^{-6}$ & $\underset{5}{2.83} \times 10^{-}$ & 0.17 & 27.2 & \\
\hline 1 & 105 & $8.65 \times 10^{-6}$ & $5.17 \times 10^{-6}$ & $\begin{array}{c}3.81 \times 10^{-} \\
\end{array}$ & 0.14 & 35.9 & \multirow{4}{*}{$\begin{array}{c}33.1 \pm \\
3.2\end{array}$} \\
\hline 2 & 105 & $7.67 \times 10^{-6}$ & $4.17 \times 10^{-6}$ & $\begin{array}{c}2.84 \times 10^{-} \\
5\end{array}$ & 0.15 & 30.3 & \\
\hline 3 & 105 & $7.92 \times 10^{-6}$ & $4.95 \times 10^{-6}$ & $\begin{array}{c}3.20 \times 10^{-} \\
5\end{array}$ & 0.15 & 30.3 & \\
\hline 4 & 105 & $7.65 \times 10^{-6}$ & $5.76 \times 10^{-6}$ & $\begin{array}{c}3.96 \times 10^{-} \\
5\end{array}$ & 0.14 & 35.9 & \\
\hline 1 & 140 & $9.44 \times 10^{-6}$ & $3.84 \times 10^{-6}$ & $\underset{5}{3.10 \times 10^{-}}$ & 0.12 & 40.0 & \multirow{4}{*}{$\begin{array}{c}40.3 \pm \\
5.0\end{array}$} \\
\hline 2 & 140 & $7.68 \times 10^{-6}$ & $2.67 \times 10^{-6}$ & $\begin{array}{c}2.24 \times 10^{-} \\
5\end{array}$ & 0.12 & 43.1 & \\
\hline 3 & 140 & $7.74 \times 10^{-6}$ & $4.63 \times 10^{-6}$ & $\underset{5}{3.20 \times 10^{-}}$ & 0.14 & 33.5 & \\
\hline 4 & 140 & $9.53 \times 10^{-6}$ & $3.45 \times 10^{-6}$ & $\begin{array}{c}3.01 \times 10^{-} \\
-\end{array}$ & 0.11 & 44.9 & \\
\hline
\end{tabular}


Table A3. SA/V ratio for $15 \mathrm{~nm}$ diameter Au NPs before and after ripening at $0.45 \mathrm{~V}$.

\begin{tabular}{|c|c|c|c|c|c|c|c|}
\hline $\begin{array}{c}\text { No. } \\
\text { of } \\
\text { trial }\end{array}$ & $\begin{array}{c}\text { Time of } \\
\text { Ostwald } \\
\text { ripping } \\
\text { (min) }\end{array}$ & $\begin{array}{l}\text { Au oxide } \\
\text { reduction } \\
\text { charge in } \\
\text { CV (before } \\
\text { Ost.) } \\
\text { (C) }\end{array}$ & $\begin{array}{l}\text { Au oxide } \\
\text { reduction } \\
\text { charge in } \\
\text { CV (after } \\
\text { Ost.) } \\
\text { (C) }\end{array}$ & $\begin{array}{c}\text { Au } \\
\text { stripping } \\
\text { charge in } \\
\text { ASV } \\
\text { (total } \\
\text { volume) } \\
\text { (C) }\end{array}$ & $\begin{array}{l}\text { Surface } \\
\text { area } \\
\text { (SA)/ } \\
\text { Volume } \\
\text { (after } \\
\text { Ost.) } \\
\text { (V) }\end{array}$ & $\begin{array}{c}\text { Calculated } \\
\text { NP } \\
\text { diameter } \\
\text { based } \\
\text { on cal. } \\
\text { curve } \\
(\mathrm{nm})\end{array}$ & $\begin{array}{c}\text { Average } \\
\text { diameter } \\
\text { of NPs } \\
\text { based } \\
\text { on cal. } \\
\text { curve } \\
(\mathrm{nm})\end{array}$ \\
\hline 1 & 0 & $9.46 \times 10^{-6}$ & & $3.80 \times 10^{-5}$ & 0.22 & 18.8 & \multirow{4}{*}{$\begin{array}{c}18.1 \pm \\
1.0\end{array}$} \\
\hline 2 & 0 & $7.67 \times 10^{-6}$ & & $3.42 \times 10^{-5}$ & 0.23 & 18.6 & \\
\hline 3 & 0 & $1.02 \times 10^{-5}$ & & $4.39 \times 10^{-5}$ & 0.23 & 18.6 & \\
\hline 4 & 0 & $1.05 \times 10^{-5}$ & & $4.25 \times 10^{-5}$ & 0.25 & 16.6 & \\
\hline 1 & 35 & $7.20 \times 10^{-6}$ & $4.22 \times 10^{-6}$ & $2.46 \times 10^{-5}$ & 0.17 & 26.5 & \multirow{4}{*}{$\begin{array}{c}25.0 \pm \\
3.0\end{array}$} \\
\hline 2 & 35 & $6.98 \times 10^{-6}$ & $5.32 \times 10^{-6}$ & $3.32 \times 10^{-5}$ & 0.17 & 26.5 & \\
\hline 3 & 35 & $7.81 \times 10^{-6}$ & $6.41 \times 10^{-6}$ & $3.08 \times 10^{-5}$ & 0.21 & 20.6 & \\
\hline 4 & 35 & $8.83 \times 10^{-6}$ & $6.06 \times 10^{-6}$ & $3.55 \times 10^{-5}$ & 0.17 & 26.5 & \\
\hline 1 & 70 & $6.75 \times 10^{-6}$ & $4.95 \times 10^{-6}$ & $3.52 \times 10^{-5}$ & 0.14 & 34.5 & \multirow{4}{*}{$\begin{array}{c}34.3 \pm \\
0.5\end{array}$} \\
\hline 2 & 70 & $7.66 \times 10^{-6}$ & $4.68 \times 10^{-6}$ & $3.42 \times 10^{-5}$ & 0.14 & 34.5 & \\
\hline 3 & 70 & $7.20 \times 10^{-6}$ & $4.98 \times 10^{-6}$ & $3.35 \times 10^{-5}$ & 0.15 & 33.5 & \\
\hline 4 & 70 & $6.16 \times 10^{-6}$ & $3.86 \times 10^{-6}$ & $2.68 \times 10^{-5}$ & 0.14 & 34.5 & \\
\hline 1 & 105 & $6.58 \times 10^{-6}$ & $3.99 \times 10^{-6}$ & $3.29 \times 10^{-5}$ & 0.12 & 44.2 & \multirow{4}{*}{$\begin{array}{c}43.5 \pm \\
3.7\end{array}$} \\
\hline 2 & 105 & $7.00 \times 10^{-6}$ & $4.18 \times 10^{-6}$ & $3.34 \times 10^{-5}$ & 0.13 & 40.6 & \\
\hline 3 & 105 & $7.24 \times 10^{-6}$ & $3.76 \times 10^{-6}$ & $3.38 \times 10^{-5}$ & 0.11 & 48.5 & \\
\hline 4 & 105 & $9.20 \times 10^{-6}$ & $4.36 \times 10^{-6}$ & $3.55 \times 10^{-5}$ & 0.13 & 40.6 & \\
\hline 1 & 140 & $8.08 \times 10^{-6}$ & $3.21 \times 10^{-6}$ & $3.71 \times 10^{-5}$ & 0.09 & 73.7 & \multirow{4}{*}{$\begin{array}{c}60.0 \pm \\
9.0\end{array}$} \\
\hline 2 & 140 & $6.52 \times 10^{-6}$ & $3.51 \times 10^{-6}$ & $3.39 \times 10^{-5}$ & 0.10 & 55.4 & \\
\hline 3 & 140 & $6.74 \times 10^{-6}$ & $2.78 \times 10^{-6}$ & $2.71 \times 10^{-5}$ & 0.10 & 55.4 & \\
\hline 4 & 140 & $8.09 \times 10^{-6}$ & $3.41 \times 10^{-6}$ & $3.42 \times 10^{-5}$ & 0.10 & 55.4 & \\
\hline
\end{tabular}


Table A4. SA/V determination of $4 \mathrm{~nm}$ diameter Au NPs after ripening at $0.0 \mathrm{~V}$.

\begin{tabular}{|c|c|c|c|c|c|c|c|}
\hline $\begin{array}{l}\text { No. } \\
\text { of } \\
\text { trial }\end{array}$ & $\begin{array}{c}\text { Time of } \\
\text { Ostwald } \\
\text { ripping } \\
\text { (min) }\end{array}$ & $\begin{array}{l}\text { Au oxide } \\
\text { reduction } \\
\text { charge in } \\
\text { CV (before } \\
\text { Ost.) } \\
\text { (C) }\end{array}$ & $\begin{array}{l}\text { Au oxide } \\
\text { reduction } \\
\text { charge in } \\
\text { CV (after } \\
\text { Ost.) } \\
\text { (C) }\end{array}$ & $\begin{array}{c}\text { Au } \\
\text { stripping } \\
\text { charge in } \\
\text { ASV } \\
\text { (total } \\
\text { volume) } \\
\text { (C) }\end{array}$ & $\begin{array}{c}\text { Surface } \\
\text { area } \\
\text { (SA)/ } \\
\text { Volume } \\
\text { (after } \\
\text { Ost.) } \\
\text { (V) }\end{array}$ & $\begin{array}{c}\text { Calculated } \\
\text { NP } \\
\text { diameter } \\
\text { based } \\
\text { on cal. } \\
\text { curve } \\
(\mathrm{nm})\end{array}$ & $\begin{array}{c}\text { Average } \\
\text { diameter } \\
\text { of NPs } \\
\text { based } \\
\text { on cal. } \\
\text { curve } \\
(\mathrm{nm})\end{array}$ \\
\hline 1 & 0 & $2.60 \times 10^{-5}$ & & $3.77 \times 10^{-5}$ & 0.69 & 5.3 & \multirow{4}{*}{$5.6 \pm 0.2$} \\
\hline 2 & 0 & $2.35 \times 10^{-5}$ & & $3.70 \times 10^{-5}$ & 0.63 & 5.8 & \\
\hline 3 & 0 & $2.01 \times 10^{-5}$ & & $3.10 \times 10^{-5}$ & 0.65 & 5.7 & \\
\hline 4 & 0 & $3.30 \times 10^{-5}$ & & $5.08 \times 10^{-5}$ & 0.65 & 5.7 & \\
\hline 1 & 35 & $2.15 \times 10^{-5}$ & $1.94 \times 10^{-5}$ & $3.17 \times 10^{-5}$ & 0.61 & 6.1 & \multirow{4}{*}{$6.1 \pm 0.1$} \\
\hline 2 & 35 & $2.52 \times 10^{-5}$ & $2.25 \times 10^{-5}$ & $3.73 \times 10^{-5}$ & 0.60 & 6.2 & \\
\hline 3 & 35 & $2.15 \times 10^{-5}$ & $1.94 \times 10^{-5}$ & $3.17 \times 10^{-5}$ & 0.61 & 6.1 & \\
\hline 4 & 35 & $2.71 \times 10^{-5}$ & $2.47 \times 10^{-5}$ & $4.01 \times 10^{-5}$ & 0.62 & 6.0 & \\
\hline 1 & 70 & $2.65 \times 10^{-5}$ & $2.46 \times 10^{-5}$ & $4.15 \times 10^{-5}$ & 0.59 & 6.3 & \multirow{4}{*}{$6.4 \pm 0.3$} \\
\hline 2 & 70 & $2.40 \times 10^{-5}$ & $2.04 \times 10^{-5}$ & $3.40 \times 10^{-5}$ & 0.60 & 6.2 & \\
\hline 3 & 70 & $2.73 \times 10^{-5}$ & $2.54 \times 10^{-5}$ & $4.60 \times 10^{-5}$ & 0.55 & 6.8 & \\
\hline 4 & 70 & $2.61 \times 10^{-5}$ & $2.17 \times 10^{-5}$ & $3.60 \times 10^{-5}$ & 0.60 & 6.2 & \\
\hline 1 & 105 & $2.15 \times 10^{-5}$ & $2.15 \times 10^{-5}$ & $4.03 \times 10^{-5}$ & 0.53 & 7.0 & \multirow{4}{*}{$6.7 \pm 0.3$} \\
\hline 2 & 105 & $2.60 \times 10^{-5}$ & $2.30 \times 10^{-5}$ & $4.10 \times 10^{-5}$ & 0.56 & 6.7 & \\
\hline 3 & 105 & $2.77 \times 10^{-5}$ & $1,95 \times 10^{-5}$ & $3.35 \times 10^{-5}$ & 0.58 & 6.4 & \\
\hline 4 & 105 & $2.59 \times 10^{-5}$ & $2.08 \times 10^{-5}$ & $3.62 \times 10^{-5}$ & 0.57 & 6.5 & \\
\hline 1 & 140 & $2.64 \times 10^{-5}$ & $2.07 \times 10^{-5}$ & $3.92 \times 10^{-5}$ & 0.53 & 7.0 & \multirow{4}{*}{$7.5 \pm 0.6$} \\
\hline 2 & 140 & $2.72 \times 10^{-5}$ & $2.58 \times 10^{-5}$ & $4.96 \times 10^{-5}$ & 0.52 & 7.2 & \\
\hline 3 & 140 & $2.66 \times 10^{-5}$ & $2.14 \times 10^{-5}$ & $4.31 \times 10^{-5}$ & 0.50 & 7.6 & \\
\hline 4 & 140 & $1.96 \times 10^{-5}$ & $1.80 \times 10^{-5}$ & $3.94 \times 10^{-5}$ & 0.46 & 8.3 & \\
\hline
\end{tabular}


Table A5. SA/V determination of $4 \mathrm{~nm}$ diameter Au NPs after ripening at $0.3 \mathrm{~V}$.

\begin{tabular}{|c|c|c|c|c|c|c|c|}
\hline $\begin{array}{c}\text { No. } \\
\text { of } \\
\text { trial }\end{array}$ & $\begin{array}{c}\text { Time of } \\
\text { Ostwald } \\
\text { ripping } \\
\text { (min) }\end{array}$ & $\begin{array}{l}\text { Au oxide } \\
\text { reduction } \\
\text { charge in } \\
\text { CV (before } \\
\text { Ost.) } \\
\text { (C) }\end{array}$ & $\begin{array}{l}\text { Au oxide } \\
\text { reduction } \\
\text { charge in } \\
\text { CV (after } \\
\text { Ost.) } \\
\text { (C) }\end{array}$ & $\begin{array}{c}\text { Au } \\
\text { stripping } \\
\text { charge in } \\
\text { ASV } \\
\text { (total } \\
\text { volume) } \\
\text { (C) }\end{array}$ & $\begin{array}{c}\text { Surface } \\
\text { area } \\
\text { (SA)/ } \\
\text { Volume } \\
\text { (after } \\
\text { Ost.) } \\
\text { (V) }\end{array}$ & $\begin{array}{c}\text { Calculated } \\
\text { NP } \\
\text { diameter } \\
\text { based } \\
\text { on cal. } \\
\text { curve } \\
(\mathrm{nm})\end{array}$ & $\begin{array}{c}\text { Average } \\
\text { diameter } \\
\text { of NPs } \\
\text { based on } \\
\text { cal. curve } \\
\text { (nm) }\end{array}$ \\
\hline 1 & 0 & $2.60 \times 10^{-5}$ & & $3.77 \times 10^{-5}$ & 0.69 & 5.3 & \multirow{4}{*}{$5.6 \pm 0.2$} \\
\hline 2 & 0 & $2.35 \times 10^{-5}$ & & $3.70 \times 10^{-5}$ & 0.63 & 5.8 & \\
\hline 3 & 0 & $2.01 \times 10^{-5}$ & & $3.10 \times 10^{-5}$ & 0.65 & 5.7 & \\
\hline 4 & 0 & $3.30 \times 10^{-5}$ & & $5.08 \times 10^{-5}$ & 0.65 & 5.7 & \\
\hline 1 & 35 & $2.48 \times 10^{-5}$ & $2.43 \times 10^{-5}$ & $3.93 \times 10^{-5}$ & 0.62 & 5.8 & \multirow{4}{*}{$6.5 \pm 0.4$} \\
\hline 2 & 35 & $2.30 \times 10^{-5}$ & $2.28 \times 10^{-5}$ & $4.15 \times 10^{-5}$ & 0.55 & 6.8 & \\
\hline 3 & 35 & $2.86 \times 10^{-5}$ & $2.61 \times 10^{-5}$ & $4.60 \times 10^{-5}$ & 0.57 & 6.6 & \\
\hline 4 & 35 & $2.56 \times 10^{-5}$ & $2.41 \times 10^{-5}$ & $4.27 \times 10^{-5}$ & 0.56 & 6.6 & \\
\hline 1 & 70 & $2.59 \times 10^{-5}$ & $2.10 \times 10^{-5}$ & $3.96 \times 10^{-5}$ & 0.53 & 7.5 & \multirow{4}{*}{$8.1 \pm 0.5$} \\
\hline 2 & 70 & $2.50 \times 10^{-5}$ & $1.66 \times 10^{-5}$ & $3.43 \times 10^{-5}$ & 0.48 & 7.8 & \\
\hline 3 & 70 & $1.98 \times 10^{-5}$ & $1.81 \times 10^{-5}$ & $4.03 \times 10^{-5}$ & 0.45 & 8.5 & \\
\hline 4 & 70 & $3.03 \times 10^{-5}$ & $2.58 \times 10^{-5}$ & $5.70 \times 10^{-5}$ & 0.45 & 8.4 & \\
\hline 1 & 105 & $2.21 \times 10^{-5}$ & $1.47 \times 10^{-5}$ & $4.32 \times 10^{-5}$ & 0.35 & 11.2 & \multirow{4}{*}{$\begin{array}{c}10.5 \pm \\
0.5\end{array}$} \\
\hline 2 & 105 & $2.52 \times 10^{-5}$ & $1.50 \times 10^{-5}$ & $4.23 \times 10^{-5}$ & 0.37 & 10.4 & \\
\hline 3 & 105 & $2.57 \times 10^{-5}$ & $1.58 \times 10^{-5}$ & $4.00 \times 10^{-5}$ & 0.40 & 10.2 & \\
\hline 4 & 105 & $2.63 \times 10^{-5}$ & $1.47 \times 10^{-5}$ & $3.86 \times 10^{-5}$ & 0.38 & 10.2 & \\
\hline 1 & 140 & $2.07 \times 10^{-5}$ & $1.11 \times 10^{-5}$ & $3.44 \times 10^{-5}$ & 0.32 & 12.4 & \multirow{4}{*}{$\begin{array}{c}14.1 \pm \\
2.7\end{array}$} \\
\hline 2 & 140 & $2.18 \times 10^{-5}$ & $1.10 \times 10^{-5}$ & $3.85 \times 10^{-5}$ & 0.29 & 14.1 & \\
\hline 3 & 140 & $2.38 \times 10^{-5}$ & $8.99 \times 10^{-5}$ & $4.03 \times 10^{-5}$ & 0.23 & 17.9 & \\
\hline 4 & 140 & $2.66 \times 10^{-5}$ & $1.22 \times 10^{-5}$ & $3.67 \times 10^{-5}$ & 0.33 & 11.9 & \\
\hline
\end{tabular}


Table A6. SA/V determination of $4 \mathrm{~nm}$ diameter Au NPs after ripening at $0.45 \mathrm{~V}$.

\begin{tabular}{|c|c|c|c|c|c|c|c|}
\hline $\begin{array}{l}\text { No. } \\
\text { of } \\
\text { trial }\end{array}$ & $\begin{array}{c}\text { Time of } \\
\text { Ostwald } \\
\text { ripping } \\
\text { (min) }\end{array}$ & $\begin{array}{l}\text { Au oxide } \\
\text { reduction } \\
\text { charge in } \\
\text { CV (before } \\
\text { Ost.) } \\
\text { (C) }\end{array}$ & $\begin{array}{l}\text { Au oxide } \\
\text { reduction } \\
\text { charge in } \\
\text { CV (after } \\
\text { Ost.) } \\
\text { (C) }\end{array}$ & $\begin{array}{c}\text { Au } \\
\text { stripping } \\
\text { charge in } \\
\text { ASV } \\
\text { (total } \\
\text { volume) } \\
(\mathrm{C}) \\
\end{array}$ & $\begin{array}{l}\text { Surface } \\
\text { area (SA)/ } \\
\text { Volume } \\
\text { (after } \\
\text { Ost.) } \\
\text { (V) }\end{array}$ & $\begin{array}{c}\text { Calculated } \\
\text { NP } \\
\text { diameter } \\
\text { based } \\
\text { on cal. } \\
\text { curve } \\
(\mathrm{nm}) \\
\end{array}$ & $\begin{array}{c}\text { Average } \\
\text { diameter } \\
\text { of NPs } \\
\text { based } \\
\text { on cal. } \\
\text { curve } \\
(\mathrm{nm})\end{array}$ \\
\hline 1 & 0 & $2.60 \times 10^{-5}$ & & $3.77 \times 10^{-5}$ & 0.69 & 5.3 & \multirow{4}{*}{$5.6 \pm 0.2$} \\
\hline 2 & 0 & $2.35 \times 10^{-5}$ & & $3.70 \times 10^{-5}$ & 0.63 & 5.8 & \\
\hline 3 & 0 & $2.01 \times 10^{-5}$ & & $3.10 \times 10^{-5}$ & 0.65 & 5.7 & \\
\hline 4 & 0 & $3.30 \times 10^{-5}$ & & $5.08 \times 10^{-5}$ & 0.65 & 5.7 & \\
\hline 1 & 35 & $2.90 \times 10^{-5}$ & $1.31 \times 10^{-5}$ & $3.86 \times 10^{-5}$ & 0.34 & 9.7 & \multirow{4}{*}{$\begin{array}{c}11.7 \pm \\
1.6\end{array}$} \\
\hline 2 & 35 & $2.35 \times 10^{-5}$ & $1.45 \times 10^{-6}$ & $4.13 \times 10^{-5}$ & 0.35 & 11.2 & \\
\hline 3 & 35 & $2.65 \times 10^{-5}$ & $1.21 \times 10^{-5}$ & $3.85 \times 10^{-5}$ & 0.31 & 12.7 & \\
\hline 4 & 35 & $2.71 \times 10^{-5}$ & $1.23 \times 10^{-5}$ & $4.07 \times 10^{-5}$ & 0.30 & 13.2 & \\
\hline 1 & 70 & $2.65 \times 10^{-5}$ & $1.00 \times 10^{-5}$ & $4.35 \times 10^{-5}$ & 0.23 & 18.3 & \multirow{4}{*}{$\begin{array}{c}23.1 \pm \\
4.9\end{array}$} \\
\hline 2 & 70 & $2.20 \times 10^{-5}$ & $5.14 \times 10^{-6}$ & $3.22 \times 10^{-5}$ & 0.16 & 28.8 & \\
\hline 3 & 70 & $2.68 \times 10^{-5}$ & $7.19 \times 10^{-6}$ & $4.12 \times 10^{-5}$ & 0.17 & 25.6 & \\
\hline 4 & 70 & $2.94 \times 10^{-5}$ & $1.17 \times 10^{-5}$ & $5.38 \times 10^{-5}$ & 0.22 & 19.6 & \\
\hline 1 & 105 & $2.81 \times 10^{-5}$ & $3.84 \times 10^{-6}$ & $3.10 \times 10^{-5}$ & 0.12 & 41.0 & \multirow{4}{*}{$\begin{array}{c}34.2 \pm \\
4.9\end{array}$} \\
\hline 2 & 105 & $2.77 \times 10^{-5}$ & $6.16 \times 10^{-6}$ & $3.91 \times 10^{-5}$ & 0.16 & 29.5 & \\
\hline 3 & 105 & $2.62 \times 10^{-5}$ & $5.62 \times 10^{-6}$ & $3.85 \times 10^{-5}$ & 0.15 & 32.6 & \\
\hline 4 & 105 & $2.67 \times 10^{-5}$ & $6.48 \times 10^{-6}$ & $4.52 \times 10^{-5}$ & 0.14 & 33.6 & \\
\hline 1 & 140 & $2.65 \times 10^{-5}$ & $3.15 \times 10^{-6}$ & $3.70 \times 10^{-5}$ & 0.09 & 75.9 & \multirow{4}{*}{$\begin{array}{c}70.0 \pm \\
7.1\end{array}$} \\
\hline 2 & 140 & $2.79 \times 10^{-5}$ & $4.91 \times 10^{-6}$ & $4.85 \times 10^{-5}$ & 0.10 & 59.4 & \\
\hline 3 & 140 & $2.41 \times 10^{-5}$ & $2.70 \times 10^{-6}$ & $3.44 \times 10^{-5}$ & 0.08 & 71.7 & \\
\hline 4 & 140 & $2.21 \times 10^{-5}$ & $2.00 \times 10^{-6}$ & $2.39 \times 10^{-5}$ & 0.08 & 71.7 & \\
\hline
\end{tabular}


Table A7. SA/V determination of $1.6 \mathrm{~nm}$ diameter Au NPs after ripening at $0.3 \mathrm{~V}$.

\begin{tabular}{|c|c|c|c|c|c|c|c|}
\hline $\begin{array}{l}\text { No. } \\
\text { of } \\
\text { trial }\end{array}$ & $\begin{array}{c}\text { Time of } \\
\text { Ostwald } \\
\text { ripping } \\
\text { (min) }\end{array}$ & $\begin{array}{l}\text { Au oxide } \\
\text { reduction } \\
\text { charge in } \\
\text { CV (before } \\
\text { Ost.) } \\
\text { (C) }\end{array}$ & $\begin{array}{l}\text { Au oxide } \\
\text { reduction } \\
\text { charge in } \\
\text { CV (after } \\
\text { Ost.) } \\
\text { (C) }\end{array}$ & $\begin{array}{c}\text { Au } \\
\text { stripping } \\
\text { charge in } \\
\text { ASV } \\
\text { (total } \\
\text { volume) } \\
\text { (C) }\end{array}$ & $\begin{array}{l}\text { Surface } \\
\text { area } \\
\text { (SA)/ } \\
\text { Volume } \\
\text { (after } \\
\text { Ost.) } \\
\text { (V) }\end{array}$ & $\begin{array}{c}\text { Calculated } \\
\text { NP } \\
\text { diameter } \\
\text { based } \\
\text { on cal. } \\
\text { curve } \\
(\mathrm{nm}) \\
\end{array}$ & $\begin{array}{c}\text { Average } \\
\text { diameter } \\
\text { of NPs } \\
\text { based } \\
\text { on cal. } \\
\text { curve } \\
(\mathrm{nm})\end{array}$ \\
\hline 1 & 0 & $9.85 \times 10^{-5}$ & & $4.35 \times 10^{-5}$ & 2.26 & 1.6 & \multirow{4}{*}{$1.5 \pm 0.1$} \\
\hline 2 & 0 & $5.54 \times 10^{-5}$ & & $2.36 \times 10^{-5}$ & 2.34 & 1.5 & \\
\hline 3 & 0 & $5.64 \times 10^{-5}$ & & $2.40 \times 10^{-5}$ & 2.35 & 1.5 & \\
\hline 4 & 0 & $1.98 \times 10^{-4}$ & & $8.11 \times 10^{-5}$ & 2.44 & 1.4 & \\
\hline 1 & 35 & & $3.55 \times 10^{-5}$ & $3.45 \times 10^{-5}$ & 1.01 & 3.6 & \multirow{4}{*}{$3.7 \pm 0.2$} \\
\hline 2 & 35 & & $3.48 \times 10^{-5}$ & $3.40 \times 10^{-5}$ & 1.02 & 3.5 & \\
\hline 3 & 35 & & $3.40 \times 10^{-5}$ & $3.67 \times 10^{-5}$ & 0.93 & 3.9 & \\
\hline 4 & 35 & & $4.60 \times 10^{-5}$ & $4.37 \times 10^{-5}$ & 0.95 & 3.8 & \\
\hline 1 & 70 & & $2.53 \times 10^{-5}$ & $3.61 \times 10^{-5}$ & 0.70 & 5.3 & \multirow{4}{*}{$5.4 \pm 0.3$} \\
\hline 2 & 70 & & $2.93 \times 10^{-5}$ & $3.65 \times 10^{-5}$ & 0.65 & 5.6 & \\
\hline 3 & 70 & & $3.59 \times 10^{-5}$ & $5.45 \times 10^{-5}$ & 0.66 & 5.6 & \\
\hline 4 & 70 & & $3.67 \times 10^{-5}$ & $4.94 \times 10^{-5}$ & 0.74 & 4.9 & \\
\hline 1 & 105 & & $2.85 \times 10^{-5}$ & $4.83 \times 10^{-5}$ & 0.59 & 6.3 & \multirow{4}{*}{$6.3 \pm 0.3$} \\
\hline 2 & 105 & & $2.20 \times 10^{-5}$ & $3.41 \times 10^{-5}$ & 0.64 & 5.8 & \\
\hline 3 & 105 & & $1.97 \times 10^{-5}$ & $3.38 \times 10^{-5}$ & 0.58 & 6.4 & \\
\hline 4 & 105 & & $1.98 \times 10^{-5}$ & $3.45 \times 10^{-5}$ & 0.57 & 6.5 & \\
\hline 1 & 140 & & $1.19 \times 10^{-5}$ & $3.73 \times 10^{-5}$ & 0.32 & 12.4 & \multirow{4}{*}{$\begin{array}{c}12.2 \pm \\
1.3\end{array}$} \\
\hline 2 & 140 & & $1.40 \times 10^{-5}$ & $3.98 \times 10^{-5}$ & 0.35 & 11.2 & \\
\hline 3 & 140 & & $1.62 \times 10^{-5}$ & $4.68 \times 10^{-5}$ & 0.35 & 11.4 & \\
\hline 4 & 140 & & $1.23 \times 10^{-5}$ & $4.25 \times 10^{-5}$ & 0.29 & 14.0 & \\
\hline
\end{tabular}

Table A8. SA/V determination of 1:3 mixture of 4:15 nm diameter Au NPs at $0.3 \mathrm{~V}$. 


\begin{tabular}{|c|c|c|c|c|c|c|c|}
\hline $\begin{array}{l}\text { No. } \\
\text { of } \\
\text { trial }\end{array}$ & $\begin{array}{c}\text { Time of } \\
\text { Ostwald } \\
\text { ripping } \\
\text { (min) }\end{array}$ & $\begin{array}{l}\text { Au oxide } \\
\text { reduction } \\
\text { charge in } \\
\text { CV (before } \\
\text { Ost.) } \\
\text { (C) }\end{array}$ & $\begin{array}{l}\text { Au oxide } \\
\text { reduction } \\
\text { charge in } \\
\text { CV (after } \\
\text { Ost.) } \\
\text { (C) }\end{array}$ & $\begin{array}{c}\text { Au } \\
\text { stripping } \\
\text { charge in } \\
\text { ASV } \\
\text { (total } \\
\text { volume) } \\
(\mathrm{C}) \\
\end{array}$ & $\begin{array}{l}\text { Surface } \\
\text { area } \\
\text { (SA)/ } \\
\text { Volume } \\
\text { (after } \\
\text { Ost.) } \\
\text { (V) } \\
\end{array}$ & $\begin{array}{c}\text { Calculated } \\
\text { NP } \\
\text { diameter } \\
\text { based } \\
\text { on cal. } \\
\text { curve (nm) }\end{array}$ & $\begin{array}{c}\text { Average } \\
\text { diameter } \\
\text { of NPs } \\
\text { based } \\
\text { on cal. } \\
\text { curve } \\
(\mathrm{nm}) \\
\end{array}$ \\
\hline 1 & 0 & $1.11 \times 10^{-5}$ & & $3.60 \times 10^{-5}$ & 0.31 & 12.9 & \multirow{4}{*}{$\begin{array}{c}14.9 \pm \\
1.5\end{array}$} \\
\hline 2 & 0 & $9.10 \times 10^{-6}$ & & $3.35 \times 10^{-5}$ & 0.27 & 14.9 & \\
\hline 3 & 0 & $7.45 \times 10^{-6}$ & & $2.70 \times 10^{-5}$ & 0.27 & 15.3 & \\
\hline 4 & 0 & $8.61 \times 10^{-6}$ & & $3.45 \times 10^{-5}$ & 0.25 & 16.5 & \\
\hline 1 & 35 & $7.41 \times 10^{-6}$ & $5.16 \times 10^{-6}$ & $2.73 \times 10^{-5}$ & 0.19 & 23.2 & \multirow{3}{*}{$\begin{array}{c}23.5 \pm \\
1.5 \\
\end{array}$} \\
\hline 2 & 35 & $1.01 \times 10^{-6}$ & $7.73 \times 10^{-6}$ & $3.93 \times 10^{-5}$ & 0.20 & 22.2 & \\
\hline 3 & 35 & $9.12 \times 10^{-6}$ & $6.91 \times 10^{-6}$ & $3.89 \times 10^{-5}$ & 0.19 & 25.1 & \\
\hline 1 & 70 & $7.23 \times 10^{-6}$ & $4.72 \times 10^{-6}$ & $3.35 \times 10^{-5}$ & 0.14 & 34.2 & \multirow{3}{*}{$\begin{array}{c}33.7 \pm \\
0.7\end{array}$} \\
\hline 2 & 70 & $8.61 \times 10^{-6}$ & $5.22 \times 10^{-6}$ & $3.59 \times 10^{-5}$ & 0.15 & 32.9 & \\
\hline 3 & 70 & $9.23 \times 10^{-6}$ & $5.12 \times 10^{-6}$ & $3.60 \times 10^{-5}$ & 0.14 & 33.9 & \\
\hline 1 & 105 & $9.54 \times 10^{-6}$ & $3.0 \times 10^{-6}$ & $3.21 \times 10^{-5}$ & 0.09 & 64.3 & \multirow{3}{*}{$\begin{array}{c}53.8 \pm \\
9.3\end{array}$} \\
\hline 2 & 105 & $8.63 \times 10^{-6}$ & $3.75 \times 10^{-6}$ & $3.28 \times 10^{-5}$ & 0.11 & 46.5 & \\
\hline 3 & 105 & $1.01 \times 10^{-6}$ & $3.93 \times 10^{-5}$ & $3.64 \times 10^{-5}$ & 0.11 & 50.6 & \\
\hline 1 & 140 & $8.40 \times 10^{-6}$ & $2.43 \times 10^{-6}$ & $3.23 \times 10^{-5}$ & 0.08 & 76.9 & \multirow{3}{*}{$\begin{array}{c}115.0 \pm \\
36.0\end{array}$} \\
\hline 2 & 140 & $9.50 \times 10^{-6}$ & $2.19 \times 10^{-6}$ & $3.50 \times 10^{-5}$ & 0.06 & 149.3 & \\
\hline 3 & 140 & $1.02 \times 10^{-6}$ & $2.29 \times 10^{-5}$ & $3.33 \times 10^{-5}$ & 0.07 & 117.9 & \\
\hline
\end{tabular}


Table A9. SA/V determination of lower coverage $15 \mathrm{~nm}$ diameter Au NPs at $0.3 \mathrm{~V}$.

\begin{tabular}{|c|c|c|c|c|c|c|c|c|}
\hline $\begin{array}{l}\text { No. } \\
\text { of } \\
\text { trial }\end{array}$ & $\begin{array}{c}\text { Time of } \\
\text { Ostwald } \\
\text { ripping } \\
\text { (min) }\end{array}$ & $\begin{array}{l}\text { Au oxide } \\
\text { reduction } \\
\text { charge in } \\
\text { CV } \\
\text { (before } \\
\text { Ost.) } \\
\text { (C) }\end{array}$ & $\begin{array}{l}\text { Au oxide } \\
\text { reduction } \\
\text { charge in } \\
\text { CV (after } \\
\text { Ost.) } \\
\text { (C) }\end{array}$ & $\begin{array}{c}\text { Au } \\
\text { stripping } \\
\text { charge } \\
\text { in ASV } \\
\text { (total } \\
\text { volume) } \\
\text { (C) }\end{array}$ & $\begin{array}{c}\text { Surface } \\
\text { area } \\
\text { (SA)/ } \\
\text { Volume } \\
\text { (after } \\
\text { Ost.) } \\
\text { (V) }\end{array}$ & $\begin{array}{c}\text { Calculated } \\
\text { NP } \\
\text { diameter } \\
\text { based } \\
\text { on cal. } \\
\text { curve } \\
(\mathrm{nm})\end{array}$ & $\begin{array}{c}\text { Average } \\
\text { diameter } \\
\text { of NPs } \\
\text { based } \\
\text { on cal. } \\
\text { curve } \\
(\mathrm{nm})\end{array}$ & $\begin{array}{l}\text { Diameter } \\
\text { (final)/ } \\
\text { Diameter } \\
\text { (initial) }\end{array}$ \\
\hline 1 & 0 & $\begin{array}{c}1.35 x \\
10^{-6}\end{array}$ & & $\begin{array}{c}6.25 x \\
10^{-6}\end{array}$ & 0.22 & 19.8 & \multirow{3}{*}{$\begin{array}{c}19.2 \pm \\
2.0\end{array}$} & \multirow{3}{*}{$1.0 \pm 0.1$} \\
\hline 2 & 0 & $\begin{array}{c}1.17 x \\
10^{-6}\end{array}$ & & $\begin{array}{c}5.70 x \\
10^{-6}\end{array}$ & 0.20 & 21.0 & & \\
\hline 3 & 0 & $\begin{array}{c}1.69 x \\
10^{-6}\end{array}$ & & $\begin{array}{c}6.90 x \\
10^{-6}\end{array}$ & 0.24 & 17.0 & & \\
\hline 1 & 70 & $\begin{array}{c}1.33 x \\
10^{-6}\end{array}$ & $\begin{array}{c}1.23 x \\
10^{-6}\end{array}$ & $\begin{array}{c}6.77 x \\
10^{-6}\end{array}$ & 0.18 & 24.4 & \multirow{3}{*}{$\begin{array}{c}23.7 \pm \\
0.9\end{array}$} & \multirow{3}{*}{$1.2 \pm 0.1$} \\
\hline 2 & 70 & $\begin{array}{c}1.47 x \\
10^{-6}\end{array}$ & $\begin{array}{c}1.29 \mathrm{x} \\
10^{-6}\end{array}$ & $\underset{6}{7.0} \times 1^{-}$ & 0.18 & 24.0 & & \\
\hline 3 & 70 & $\begin{array}{c}1.41 \mathrm{x} \\
10^{-6}\end{array}$ & $\begin{array}{c}1.19 x \\
10^{-6}\end{array}$ & $\begin{array}{c}6.16 x \\
10^{-6}\end{array}$ & 0.19 & 22.6 & & \\
\hline 1 & 140 & $\begin{array}{c}1.28 \mathrm{x} \\
10^{-6}\end{array}$ & $\begin{array}{c}7.44 x \\
10^{-7}\end{array}$ & $\begin{array}{c}5.15 x \\
10^{-6}\end{array}$ & 0.14 & 33.2 & \multirow{3}{*}{$\begin{array}{c}30.6 \pm \\
2.6\end{array}$} & \multirow{3}{*}{$1.6 \pm 0.1$} \\
\hline 2 & 140 & $\begin{array}{c}1.27 x \\
10^{-6}\end{array}$ & $\begin{array}{c}1.02 \mathrm{x} \\
10^{-6}\end{array}$ & $\begin{array}{c}6.65 x \\
10^{-6}\end{array}$ & 0.10 & 30.6 & & \\
\hline 3 & 140 & $\begin{array}{c}1.16 \mathrm{x} \\
10^{-6}\end{array}$ & $\begin{array}{c}9.20 x \\
10^{-7}\end{array}$ & $\begin{array}{c}5.60 x \\
10^{-6}\end{array}$ & 0.164 & 27.9 & & \\
\hline
\end{tabular}


Table A10. SA/V determination of higher coverage $15 \mathrm{~nm}$ diameter Au NPs at $0.3 \mathrm{~V}$.

\begin{tabular}{|c|c|c|c|c|c|c|c|c|}
\hline $\begin{array}{l}\text { No. } \\
\text { of } \\
\text { trial }\end{array}$ & $\begin{array}{l}\text { Time of } \\
\text { Ostwald } \\
\text { ripping } \\
\text { (min) }\end{array}$ & $\begin{array}{c}\text { Au oxide } \\
\text { reduction } \\
\text { charge in } \\
\text { CV } \\
\text { (before } \\
\text { Ost.) } \\
\text { (C) }\end{array}$ & $\begin{array}{c}\text { Au oxide } \\
\text { reduction } \\
\text { charge in } \\
\text { CV (after } \\
\text { Ost.) } \\
\text { (C) }\end{array}$ & $\begin{array}{c}\text { Au } \\
\text { stripping } \\
\text { charge } \\
\text { in ASV } \\
\text { (total } \\
\text { volume) } \\
\text { (C) }\end{array}$ & $\begin{array}{c}\text { Surface } \\
\text { area } \\
\text { (SA)/ } \\
\text { Volume } \\
\text { (after } \\
\text { Ost.) } \\
\text { (V) }\end{array}$ & $\begin{array}{l}\text { Calculated } \\
\quad N P \\
\text { diameter } \\
\quad \text { based } \\
\text { on cal. } \\
\text { curve } \\
(\mathrm{nm}) \\
\end{array}$ & $\begin{array}{l}\text { Average } \\
\text { diameter } \\
\text { of NPs } \\
\text { based } \\
\text { on cal. } \\
\text { curve } \\
(\mathrm{nm})\end{array}$ & $\begin{array}{l}\text { Diameter } \\
\text { (final)/ } \\
\text { Diameter } \\
\text { (initial) }\end{array}$ \\
\hline 1 & 0 & $\begin{array}{c}4.20 x \\
10^{-5}\end{array}$ & & $\begin{array}{c}2.20 x \\
10^{-4}\end{array}$ & 0.19 & 23.1 & \multirow{3}{*}{$\begin{array}{c}25.9 \pm \\
2.5\end{array}$} & \multirow{3}{*}{$1.0 \pm 0.1$} \\
\hline 2 & 0 & $\begin{array}{c}3.96 x \\
10^{-5}\end{array}$ & & $\begin{array}{c}2.41 \mathrm{x} \\
10^{-4}\end{array}$ & 0.16 & 27.9 & & \\
\hline 3 & 0 & $\begin{array}{c}3.38 x \\
10^{-5}\end{array}$ & & $\begin{array}{c}2.00 \mathrm{x} \\
10^{-4}\end{array}$ & 0.17 & 26.8 & & \\
\hline 1 & 70 & $\begin{array}{c}3.40 x \\
10^{-5}\end{array}$ & $\begin{array}{c}1.94 x \\
10^{-5}\end{array}$ & $\begin{array}{c}2.16 \mathrm{x} \\
10^{-4}\end{array}$ & 0.09 & 68.7 & \multirow{3}{*}{$\begin{array}{c}74.8 \pm \\
11.2\end{array}$} & \multirow{3}{*}{$2.9 \pm 0.4$} \\
\hline 2 & 70 & $\begin{array}{c}3.16 \mathrm{x} \\
10^{-5}\end{array}$ & $\begin{array}{c}1.58 \mathrm{x} \\
10^{-5}\end{array}$ & $\begin{array}{c}2.02 \mathrm{x} \\
10^{-4}\end{array}$ & 0.08 & 87.8 & & \\
\hline 3 & 70 & $\begin{array}{c}3.24 x \\
10^{-5}\end{array}$ & $\begin{array}{c}1.82 \mathrm{x} \\
10^{-5}\end{array}$ & $\begin{array}{c}2.01 x \\
10^{-4}\end{array}$ & 0.09 & 67.9 & & \\
\hline 1 & 140 & $\begin{array}{c}3.44 x \\
10^{-5}\end{array}$ & $\begin{array}{c}1.33 \mathrm{x} \\
10^{-5}\end{array}$ & $\begin{array}{c}2.17 x \\
10^{-4}\end{array}$ & 0.06 & 158.8 & \multirow{3}{*}{$\begin{array}{c}185.4 \pm \\
31.4\end{array}$} & \multirow{3}{*}{$7.1 \pm 1.4$} \\
\hline 2 & 140 & $\begin{array}{c}3.46 \mathrm{x} \\
10^{-5}\end{array}$ & $\begin{array}{c}1.18 \mathrm{x} \\
10^{-5}\end{array}$ & $\begin{array}{c}2.14 x \\
10^{-4}\end{array}$ & 0.05 & 220.1 & & \\
\hline 3 & 140 & $\begin{array}{c}4.41 x \\
10^{-5}\end{array}$ & $\begin{array}{c}1.25 x \\
10^{-5}\end{array}$ & $\begin{array}{c}2.12 \mathrm{x} \\
10^{-4}\end{array}$ & 0.06 & 177.4 & & \\
\hline
\end{tabular}


Table A11. Integrated charge under the stripping peaks of $\mathrm{Au}$ and $\mathrm{Cu}$ in $0.01 \mathrm{M} \mathrm{KBr}$ plus $0.1 \mathrm{M} \mathrm{HClO}_{4}$ electrolyte to calculate the $\mathrm{Au}$ to $\mathrm{Cu}$ ratio of $\mathrm{Cu}_{1} / \mathrm{Au}_{2}$ core/shell alloy nanoparticles.

\begin{tabular}{|c|c|c|c|c|}
\hline $\mathrm{Au}$ & $\mathrm{Cu}^{3}$ & $\mathrm{Au} / \mathrm{Cu}$ & $\begin{array}{c}\text { Average } \\
\text { Au/Cu }\end{array}$ & $\begin{array}{c}\text { Theoretical } \\
\mathrm{Au} / \mathrm{Cu}\end{array}$ \\
\hline $3.54 \times 10^{-4} \mathrm{C}$ & $1.27 \times 10^{-5} \mathrm{C}$ & 27.87 & & 3.0 \\
\hline $2.18 \times 10^{-4} \mathrm{C}$ & $9.07 \times 10^{-6} \mathrm{C}$ & 24.03 & $26 \pm 2$ & \\
\hline $1.09 \times 10^{-3} \mathrm{C}$ & $3.90 \times 10^{-5} \mathrm{C}$ & 27.95 & & \\
\hline $1.23 \times 10^{-4} \mathrm{C}$ & $4.84 \times 10^{-6} \mathrm{C}$ & 25.41 & & \\
\hline
\end{tabular}

Table A12. Integrated charge under the stripping peaks of $\mathrm{Au}$ and $\mathrm{Cu}$ in $0.01 \mathrm{M} \mathrm{KCl}$ plus $0.1 \mathrm{M} \mathrm{HClO}_{4}$ electrolyte to calculate the $\mathrm{Au}$ to $\mathrm{Cu}$ ratio of $\mathrm{Cu}_{1} / \mathrm{Au}_{2}$ core/shell alloy nanoparticles.

\begin{tabular}{|c|c|c|c|c|}
\hline $\mathrm{Au}$ & $\mathrm{Cu}^{3}$ & $\mathrm{Au} / \mathrm{Cu}$ & $\begin{array}{c}\text { Average } \\
\mathrm{Au} / \mathrm{Cu}\end{array}$ & $\begin{array}{c}\text { Theoretical } \\
\mathrm{Au} / \mathrm{Cu}\end{array}$ \\
\hline $1.78 \times 10^{-4} \mathrm{C}$ & $1.42 \times 10^{-5} \mathrm{C}$ & 12.62 & & 3.0 \\
\hline $9.83 \times 10^{-5} \mathrm{C}$ & $5.71 \times 10^{-6} \mathrm{C}$ & 17.21 & $14 \pm 2$ & \\
\hline $1.26 \times 10^{-4} \mathrm{C}$ & $1.01 \times 10^{-5} \mathrm{C}$ & 12.47 & & \\
\hline $8.20 \times 10^{-5} \mathrm{C}$ & $5.34 \times 10^{-6} \mathrm{C}$ & 15.36 & & \\
\hline
\end{tabular}

Table A13. Integrated charge under the stripping peaks of $\mathrm{Au}$ and $\mathrm{Cu}$ in $0.01 \mathrm{M} \mathrm{KBr}$ plus $0.1 \mathrm{M} \mathrm{HClO}_{4}$ electrolyte to calculate the $\mathrm{Au}$ to $\mathrm{Cu}$ ratio of $\mathrm{Cu}_{1} / \mathrm{Au}_{1}$ core/shell alloy nanoparticles.

\begin{tabular}{|c|c|c|c|c|}
\hline $\mathrm{Au}$ & $\mathrm{Cu}^{3}$ & $\mathrm{Au} / \mathrm{Cu}$ & $\begin{array}{c}\text { Average } \\
\mathrm{Au} / \mathrm{Cu}\end{array}$ & $\begin{array}{c}\text { Theoretical } \\
\mathrm{Au} / \mathrm{Cu}\end{array}$ \\
\hline $5.99 \times 10^{-4} \mathrm{C}$ & $1.40 \times 10^{-4} \mathrm{C}$ & 4.28 & & 1.5 \\
\hline $6.43 \times 10^{-4} \mathrm{C}$ & $1.60 \times 10^{-4} \mathrm{C}$ & 4.02 & $4.0 \pm 0.2$ & \\
\hline $8.40 \times 10^{-4} \mathrm{C}$ & $2.14 \times 10^{-4} \mathrm{C}$ & 3.93 & & \\
\hline $4.31 \times 10^{-4} \mathrm{C}$ & $1.12 \times 10^{-4} \mathrm{C}$ & 3.85 & & \\
\hline
\end{tabular}

Table A14. Integrated charge under the stripping peaks of $\mathrm{Au}$ and $\mathrm{Cu}$ in $0.01 \mathrm{M} \mathrm{KCl}$ plus $0.1 \mathrm{M} \mathrm{HClO}_{4}$ electrolyte to calculate the $\mathrm{Au}$ to $\mathrm{Cu}$ ratio of $\mathrm{Cu}_{1} / \mathrm{Au}_{1}$ core/shell alloy nanoparticles. 


\begin{tabular}{|c|c|c|c|c|}
\hline $\mathrm{Au}$ & $\mathrm{Cu}^{3}$ & $\mathrm{Au} / \mathrm{Cu}$ & $\begin{array}{c}\text { Average } \\
\mathrm{Au} / \mathrm{Cu}\end{array}$ & $\begin{array}{c}\text { Theoretical } \\
\mathrm{Au} / \mathrm{Cu}\end{array}$ \\
\hline $1.54 \times 10^{-4} \mathrm{C}$ & $9.30 \times 10^{-5} \mathrm{C}$ & 1.66 & & 1.5 \\
\hline $1.68 \times 10^{-4} \mathrm{C}$ & $1.25 \times 10^{-4} \mathrm{C}$ & 1.34 & $1.6 \pm 0.2$ & \\
\hline $2.38 \times 10^{-4} \mathrm{C}$ & $1.47 \times 10^{-4} \mathrm{C}$ & 1.62 & & \\
\hline $1.87 \times 10^{-4} \mathrm{C}$ & $1.17 \times 10^{-4} \mathrm{C}$ & 1.60 & & \\
\hline
\end{tabular}

Table A15. Integrated charge under the stripping peaks of $\mathrm{Au}$ and $\mathrm{Cu}$ in $0.01 \mathrm{M} \mathrm{KBr}$

plus $0.1 \mathrm{M} \mathrm{HClO}_{4}$ electrolyte to calculate the $\mathrm{Au}$ to $\mathrm{Cu}$ ratio of $\mathrm{Cu}_{1} / \mathrm{Au}_{0.5}$ core/shell alloy nanoparticles.

\begin{tabular}{|c|c|c|c|c|}
\hline $\mathrm{Au}$ & $\mathrm{Cu}^{3}$ & $\mathrm{Au} / \mathrm{Cu}$ & $\begin{array}{c}\text { Average } \\
\mathrm{Au} / \mathrm{Cu}\end{array}$ & $\begin{array}{c}\text { Theoretical } \\
\mathrm{Au} / \mathrm{Cu}\end{array}$ \\
\hline $3.18 \times 10^{-4} \mathrm{C}$ & $1.41 \times 10^{-4} \mathrm{C}$ & 2.25 & & 0.75 \\
\hline $2.09 \times 10^{-4} \mathrm{C}$ & $1.21 \times 10^{-4} \mathrm{C}$ & 1.73 & $2.0 \pm 0.3$ & \\
\hline $2.81 \times 10^{-4} \mathrm{C}$ & $1.52 \times 10^{-4} \mathrm{C}$ & 1.85 & & \\
\hline $3.52 \times 10^{-4} \mathrm{C}$ & $1.63 \times 10^{-4} \mathrm{C}$ & 2.15 & & \\
\hline
\end{tabular}

Table A16. Integrated charge under the stripping peaks of $\mathrm{Au}$ and $\mathrm{Cu}$ in $0.01 \mathrm{M} \mathrm{KCl}$ plus $0.1 \mathrm{M} \mathrm{HClO}_{4}$ electrolyte to calculate the $\mathrm{Au}$ to $\mathrm{Cu}$ ratio of $\mathrm{Cu}_{1} / \mathrm{Au}_{0.5}$ core/shell alloy nanoparticles.

\begin{tabular}{|c|c|c|c|c|}
\hline $\mathrm{Au}$ & $\mathrm{Cu}^{3}$ & $\mathrm{Au} / \mathrm{Cu}$ & $\begin{array}{c}\text { Average } \\
\mathrm{Au} / \mathrm{Cu}\end{array}$ & $\begin{array}{c}\text { Theoretical } \\
\mathrm{Au} / \mathrm{Cu}\end{array}$ \\
\hline $1.93 \times 10^{-4} \mathrm{C}$ & $2.19 \times 10^{-4} \mathrm{C}$ & 0.88 & & 0.75 \\
\hline $2.85 \times 10^{-4} \mathrm{C}$ & $4.05 \times 10^{-4} \mathrm{C}$ & 0.70 & $0.80 \pm 0.08$ & \\
\hline $1.74 \times 10^{-4} \mathrm{C}$ & $2.21 \times 10^{-4} \mathrm{C}$ & 0.77 & & \\
\hline $1.22 \times 10^{-4} \mathrm{C}$ & $1.42 \times 10^{-4} \mathrm{C}$ & 0.85 & & \\
\hline
\end{tabular}

Table A17. Integrated charge under the stripping peaks of $\mathrm{Au}$ and $\mathrm{Cu}$ in $0.01 \mathrm{M} \mathrm{KBr}$ plus $0.1 \mathrm{M} \mathrm{HClO}_{4}$ electrolyte to calculate the $\mathrm{Au}$ to $\mathrm{Cu}$ ratio of $\mathrm{Cu}_{1} / \mathrm{Au}_{0.3}$ core/shell alloy nanoparticles.

\begin{tabular}{|c|c|c|c|c|c|}
\hline $\mathrm{Au}$ & $\mathrm{Cu}^{1}$ & $\mathrm{Cu}^{3}$ & $\mathrm{Au} / \mathrm{Cu}$ & $\begin{array}{c}\text { Average } \\
\text { Au/Cu }\end{array}$ & $\begin{array}{c}\text { Theoretical } \\
\mathrm{Au} / \mathrm{Cu}\end{array}$ \\
\hline $\begin{array}{c}1.69 \times 10^{-4} \\
\mathrm{C}\end{array}$ & $\begin{array}{c}1.15 \times 10^{-4} \\
\mathrm{C}\end{array}$ & $\begin{array}{c}6.50 \times 10^{-5} \\
\mathrm{C}\end{array}$ & 0.94 & & \\
\hline $\begin{array}{c}6.21 \times 10^{-5} \\
\mathrm{C}\end{array}$ & $\begin{array}{c}2.10 \times 10^{-} \\
5\end{array}$ & $\begin{array}{c}1.90 \times 10^{-5} \\
\mathrm{C}\end{array}$ & 1.55 & $1.2 \pm 0.3$ & 0.45 \\
\hline
\end{tabular}




\begin{tabular}{|c|c|c|c|c|c|}
\hline $\begin{array}{c}1.25 \times 10^{-4} \\
\mathrm{C}\end{array}$ & $\begin{array}{c}6.01 \times 10^{-} \\
{ }^{5} \mathrm{C}\end{array}$ & $\begin{array}{c}5.13 \times 10^{-5} \\
\mathrm{C}\end{array}$ & 1.12 & & \\
\hline $\begin{array}{c}2.44 \times 10^{-} \\
{ }^{4} \mathrm{C}\end{array}$ & $\begin{array}{c}5.33 \times 10^{-} \\
{ }^{5} \mathrm{C}\end{array}$ & $\begin{array}{c}1.30 \times 10^{-} \\
{ }^{4} \mathrm{C}\end{array}$ & 1.33 & & \\
\hline
\end{tabular}

Table A18. Integrated charge under the stripping peaks of $\mathrm{Au}$ and $\mathrm{Cu}$ in $0.01 \mathrm{M} \mathrm{KCl}$ plus $0.1 \mathrm{M} \mathrm{HClO}_{4}$ electrolyte to calculate the $\mathrm{Au}$ to $\mathrm{Cu}$ ratio of $\mathrm{Cu}_{1} / \mathrm{Au}_{0.3}$ core/shell alloy nanoparticles.

\begin{tabular}{|c|c|c|c|c|c|}
\hline $\mathrm{Au}$ & $\mathrm{Cu}^{1}$ & $\mathrm{Cu}^{3}$ & $\mathrm{Au} / \mathrm{Cu}$ & $\begin{array}{c}\text { Average } \\
\text { Au/Cu }\end{array}$ & $\begin{array}{c}\text { Theoretical } \\
\text { Au/Cu }\end{array}$ \\
\hline $\begin{array}{c}1.53 \times 10^{-4} \\
\mathrm{C}\end{array}$ & $\begin{array}{c}1.57 \times 10^{-4} \\
\mathrm{C}\end{array}$ & $\begin{array}{c}1.24 \times 10^{-4} \\
\mathrm{C}\end{array}$ & 0.54 & & \\
\hline $\begin{array}{c}6.62 \times 10^{-5} \\
\mathrm{C}\end{array}$ & $\begin{array}{c}5.20 \times 10^{-5} \\
\mathrm{C}\end{array}$ & $\begin{array}{c}6.65 \times 10^{-5} \\
\mathrm{C}\end{array}$ & 0.56 & $0.50 \pm 0.06$ & 0.45 \\
\hline $\begin{array}{c}6.76 \times 10^{-5} \\
\mathrm{C}\end{array}$ & $\begin{array}{c}8.60 \times 10^{-5} \\
\mathrm{C}\end{array}$ & $\begin{array}{c}6.68 \times 10^{-5} \\
\mathrm{C}\end{array}$ & 0.44 & & \\
\hline $\begin{array}{c}1.55 \times 10^{-4} \\
\mathrm{C}\end{array}$ & $\begin{array}{c}1.22 \times 10^{-4} \\
\mathrm{C}\end{array}$ & $\begin{array}{c}2.04 \times 10^{-4} \\
\mathrm{C}\end{array}$ & 0.48 & & \\
\hline
\end{tabular}

Table A19. Integrated charge under the stripping peaks of $\mathrm{Au}$ and $\mathrm{Cu}$ in $0.01 \mathrm{M} \mathrm{KBr}$ plus $0.1 \mathrm{M} \mathrm{HClO}_{4}$ electrolyte to calculate the $\mathrm{Au}$ to $\mathrm{Cu}$ ratio of $\mathrm{Cu}_{1} / \mathrm{Au}_{0.2}$ core/shell alloy nanoparticles.

\begin{tabular}{|c|c|c|c|c|c|}
\hline $\mathrm{Au}$ & $\mathrm{Cu}^{1}$ & $\mathrm{Cu}^{3}$ & $\mathrm{Au} / \mathrm{Cu}$ & $\begin{array}{c}\text { Average } \\
\mathrm{Au} / \mathrm{Cu}\end{array}$ & $\begin{array}{c}\text { Theoretical } \\
\mathrm{Au} / \mathrm{Cu}\end{array}$ \\
\hline $\begin{array}{c}4.03 \times 10^{-5} \\
\mathrm{C}\end{array}$ & $\begin{array}{c}3.38 \times 10^{-5} \\
\mathrm{C}\end{array}$ & $\begin{array}{c}1.90 \times 10^{-5} \\
\mathrm{C}\end{array}$ & 0.76 & & \\
\hline $\begin{array}{c}1.30 \times 10^{-4} \\
\mathrm{C}\end{array}$ & $\begin{array}{c}1.26 \times 10^{-4} \\
\mathrm{C}\end{array}$ & $\begin{array}{c}3.44 \times 10^{-5} \\
\mathrm{C}\end{array}$ & 0.81 & $0.80 \pm 0.03$ & 0.30 \\
\hline $\begin{array}{c}1.37 \times 10^{-4} \\
\mathrm{C}\end{array}$ & $\begin{array}{c}1.39 \times 10^{-4} \\
\mathrm{C}\end{array}$ & $\begin{array}{c}3.65 \times 10^{-5} \\
\mathrm{C}\end{array}$ & 0.78 & & \\
\hline $\begin{array}{c}1.34 \times 10^{-4} \\
\mathrm{C}\end{array}$ & $\begin{array}{c}1.28 \times 10^{-4} \\
\mathrm{C}\end{array}$ & $\begin{array}{c}3.30 \times 10^{-5} \\
\mathrm{C}\end{array}$ & 0.83 & & \\
\hline
\end{tabular}

Table A20. Integrated charge under the stripping peaks of $\mathrm{Au}$ and $\mathrm{Cu}$ in $0.01 \mathrm{M} \mathrm{KCl}$ plus $0.1 \mathrm{M} \mathrm{HClO}_{4}$ electrolyte to calculate the $\mathrm{Au}$ to $\mathrm{Cu}$ ratio of $\mathrm{Cu}_{1} / \mathrm{Au}_{0.2}$ core/shell alloy nanoparticles. 


\begin{tabular}{|c|c|c|c|c|c|}
\hline $\mathrm{Au}$ & $\mathrm{Cu}^{1}$ & $\mathrm{Cu}^{3}$ & $\mathrm{Au} / \mathrm{Cu}$ & $\begin{array}{c}\text { Average } \\
\mathrm{Au} / \mathrm{Cu}\end{array}$ & $\begin{array}{c}\text { Theoretical } \\
\mathrm{Au} / \mathrm{Cu}\end{array}$ \\
\hline $\begin{array}{c}5.72 \times 10^{-5} \\
\mathrm{C}\end{array}$ & $\begin{array}{c}9.09 \times 10^{-5} \\
\mathrm{C}\end{array}$ & $\begin{array}{c}8.36 \times 10^{-5} \\
\mathrm{C}\end{array}$ & 0.33 & & \\
\hline $\begin{array}{c}5.69 \times 10^{-5} \\
\mathrm{C}\end{array}$ & $\begin{array}{c}1.36 \times 10^{-4} \\
\mathrm{C}\end{array}$ & $\begin{array}{c}7.58 \times 10^{-5} \\
\mathrm{C}\end{array}$ & 0.27 & $0.32 \pm 0.03$ & 0.30 \\
\hline $\begin{array}{c}8.46 \times 10^{-5} \\
\mathrm{C}\end{array}$ & $\begin{array}{c}1.53 \times 10^{-4} \\
\mathrm{C}\end{array}$ & $\begin{array}{c}9.80 \times 10^{-5} \\
\mathrm{C}\end{array}$ & 0.34 & & \\
\hline $\begin{array}{c}7.98 \times 10^{-5} \\
\mathrm{C}\end{array}$ & $\begin{array}{c}1.43 \times 10^{-4} \\
\mathrm{C}\end{array}$ & $\begin{array}{c}9.20 \times 10^{-5} \\
\mathrm{C}\end{array}$ & 0.33 & & \\
\hline
\end{tabular}

Table A21. Integrated charge under the stripping peaks of $\mathrm{Au}$ and $\mathrm{Cu}$ in $0.01 \mathrm{M} \mathrm{KBr}$ plus $0.1 \mathrm{M} \mathrm{HClO}_{4}$ electrolyte to calculate the $\mathrm{Au}$ to $\mathrm{Cu}$ ratio of $\mathrm{Cu}_{1} / \mathrm{Au}_{0.1}$ core/shell alloy nanoparticles.

\begin{tabular}{|c|c|c|c|c|c|}
\hline $\mathrm{Au}$ & $\mathrm{Cu}^{1}$ & $\mathrm{Cu}^{3}$ & $\mathrm{Au} / \mathrm{Cu}$ & $\begin{array}{c}\text { Average } \\
\mathrm{Au} / \mathrm{Cu}\end{array}$ & $\begin{array}{c}\text { Theoretical } \\
\mathrm{Au} / \mathrm{Cu}\end{array}$ \\
\hline $\begin{array}{c}2.14 \times 10^{-5} \\
\mathrm{C}\end{array}$ & $\begin{array}{c}4.14 \times 10^{-5} \\
\mathrm{C}\end{array}$ & $\begin{array}{c}7.76 \times 10^{-6} \\
\mathrm{C}\end{array}$ & 0.44 & & \\
\hline $\begin{array}{c}2.66 \times 10^{-5} \\
\mathrm{C}\end{array}$ & $\begin{array}{c}4.66 \times 10^{-5} \\
\mathrm{C}\end{array}$ & $\begin{array}{c}8.55 \times 10^{-6} \\
\mathrm{C}\end{array}$ & 0.48 & $0.43 \pm 0.04$ & 0.15 \\
\hline $\begin{array}{c}7.67 \times 10^{-5} \\
\mathrm{C}\end{array}$ & $\begin{array}{c}1.60 \times 10^{-4} \\
\mathrm{C}\end{array}$ & $\begin{array}{c}3.16 \times 10^{-5} \\
\mathrm{C}\end{array}$ & 0.40 & & \\
\hline $\begin{array}{c}6.66 \times 10^{-5} \\
\mathrm{C}\end{array}$ & $\begin{array}{c}1.38 \times 10^{-4} \\
\mathrm{C}\end{array}$ & $\begin{array}{c}3.21 \times 10^{-5} \\
\mathrm{C}\end{array}$ & 0.39 & & \\
\hline
\end{tabular}

Table A22. Integrated charge under the stripping peaks of $\mathrm{Au}$ and $\mathrm{Cu}$ in $0.01 \mathrm{M} \mathrm{KCl}$ plus $0.1 \mathrm{M} \mathrm{HClO}_{4}$ electrolyte to calculate the $\mathrm{Au}$ to $\mathrm{Cu}$ ratio of $\mathrm{Cu}_{1} / \mathrm{Au}_{0.1}$ core/shell alloy nanoparticles.

\begin{tabular}{|c|c|c|c|c|c|}
\hline $\mathrm{Au}$ & $\mathrm{Cu}^{1}$ & $\mathrm{Cu}^{3}$ & $\mathrm{Au} / \mathrm{Cu}$ & $\begin{array}{c}\text { Average } \\
\mathrm{Au} / \mathrm{Cu}\end{array}$ & $\begin{array}{c}\text { Theoretical } \\
\mathrm{Au} / \mathrm{Cu}\end{array}$ \\
\hline $\begin{array}{c}1.35 \times 10^{-5} \\
\mathrm{C}\end{array}$ & $\begin{array}{c}7.28 \times 10^{-5} \\
\mathrm{C}\end{array}$ & $\begin{array}{c}1.67 \times 10^{-5} \\
\mathrm{C}\end{array}$ & 0.15 & & \\
\hline $\begin{array}{c}2.10 \times 10^{-5} \\
\mathrm{C}\end{array}$ & $\begin{array}{c}1.06 \times 10^{-4} \\
\mathrm{C}\end{array}$ & $\begin{array}{c}2.52 \times 10^{-5} \\
\mathrm{C}\end{array}$ & 0.16 & $0.15 \pm 0.01$ & 0.15 \\
\hline $\begin{array}{c}2.51 \times 10^{-5} \\
\mathrm{C}\end{array}$ & $\begin{array}{c}1.46 \times 10^{-4} \\
\mathrm{C}\end{array}$ & $\begin{array}{c}3.15 \times 10^{-5} \\
\mathrm{C}\end{array}$ & 0.14 & & \\
\hline $\begin{array}{c}5.16 \times 10^{-5} \\
\mathrm{C}\end{array}$ & $\begin{array}{c}3.02 \times 10^{-4} \\
\mathrm{C}\end{array}$ & $\begin{array}{c}6.28 \times 10^{-5} \\
\mathrm{C}\end{array}$ & 0.14 & & \\
\hline
\end{tabular}


Table A23. Integrated charge under the stripping peaks of $\mathrm{Au}$ and $\mathrm{Cu}$ in $0.01 \mathrm{M} \mathrm{KBr}$ plus $0.1 \mathrm{M} \mathrm{HClO}_{4}$ electrolyte to calculate the $\mathrm{Au}$ to $\mathrm{Cu}$ ratio of $\mathrm{Cu}_{1} / \mathrm{Au}_{0.01}$ core/shell alloy nanoparticles.

\begin{tabular}{|c|c|c|c|c|c|c|}
\hline $\mathrm{Au}$ & $\mathrm{Cu}^{1}$ & $\mathrm{Cu}^{2}$ & $\mathrm{Cu}^{3}$ & $\mathrm{Au} / \mathrm{Cu}$ & $\begin{array}{c}\text { Average } \\
\mathrm{Au} / \mathrm{Cu}\end{array}$ & $\begin{array}{c}\text { Theoretical } \\
\mathrm{Au} / \mathrm{Cu}\end{array}$ \\
\hline $\begin{array}{c}8.58 \times 10^{-} \\
7\end{array}$ & $\begin{array}{c}2.71 \times 10^{-} \\
5\end{array}$ & $\begin{array}{c}1.01 \times \\
10^{-6}\end{array}$ & $\begin{array}{c}1.41 \times 10^{-} \\
6\end{array}$ & 0.029 & & \\
\hline $\begin{array}{c}2.05 \times 10^{-} \\
6\end{array}$ & $\begin{array}{c}3.39 \times 10^{-} \\
5\end{array}$ & $\begin{array}{c}1.57 \times \\
10^{-6}\end{array}$ & $\begin{array}{c}2.79 \times 10^{-} \\
6\end{array}$ & 0.054 & $0.06 \pm 0.03$ & 0.015 \\
\hline $\begin{array}{c}2.21 \times 10^{-} \\
6\end{array}$ & $\begin{array}{c}2.06 \times 10^{-} \\
5\end{array}$ & $\begin{array}{c}6.37 \times \\
10^{-7}\end{array}$ & $\begin{array}{c}2.49 \times 10^{-} \\
6\end{array}$ & 0.093 & & \\
\hline $\begin{array}{c}7.61 \times 10^{-} \\
7\end{array}$ & $\begin{array}{c}1.03 \times 10^{-} \\
5\end{array}$ & $\begin{array}{c}5.21 \times \\
10^{-7}\end{array}$ & $\begin{array}{c}2.15 \times 10^{-} \\
6\end{array}$ & 0.059 & & \\
\hline
\end{tabular}

Table A24. Integrated charge under the stripping peaks of $\mathrm{Au}$ and $\mathrm{Cu}$ in $0.01 \mathrm{M} \mathrm{KCl}$ plus $0.1 \mathrm{M} \mathrm{HClO}_{4}$ electrolyte to calculate the $\mathrm{Au}$ to $\mathrm{Cu}$ ratio of $\mathrm{Cu}_{1} / \mathrm{Au}_{0.01}$ core/shell alloy nanoparticles.

\begin{tabular}{|c|c|c|c|c|c|c|}
\hline $\mathrm{Au}^{1}$ & $\mathrm{Au} 2$ & $\mathrm{Cu}^{1}$ & $\mathrm{Cu}^{3}$ & $\mathrm{Au} / \mathrm{Cu}$ & $\begin{array}{c}\text { Average } \\
\mathrm{Au} / \mathrm{Cu}\end{array}$ & $\begin{array}{c}\text { Theoretical } \\
\mathrm{Au} / \mathrm{Cu}\end{array}$ \\
\hline $\begin{array}{c}2.43 \times 10^{-} \\
7\end{array}$ & $\begin{array}{c}2.38 \times 10^{-} \\
7\end{array}$ & $\begin{array}{c}1.15 \times \\
10^{-5}\end{array}$ & $\begin{array}{c}1.28 \times 10^{-} \\
6\end{array}$ & 0.037 & & \\
\hline $\begin{array}{c}4.23 \times 10^{-} \\
7\end{array}$ & $\begin{array}{c}1.08 \times 10^{-} \\
7\end{array}$ & $\begin{array}{c}1.74 \times \\
10^{-5}\end{array}$ & $\begin{array}{c}1.44 \times 10^{-} \\
6\end{array}$ & 0.028 & $0.036 \pm 0.007$ & 0.015 \\
\hline $\begin{array}{c}3.44 \times 10^{-} \\
7\end{array}$ & $\begin{array}{c}1.09 \times 10^{-} \\
7\end{array}$ & $\begin{array}{c}1.19 \times \\
10^{-5}\end{array}$ & $\begin{array}{c}1.59 \times 10^{-} \\
6\end{array}$ & 0.033 & & \\
\hline $\begin{array}{c}1.36 \times 10^{-} \\
7\end{array}$ & $\begin{array}{c}1.90 \times 10^{-} \\
7\end{array}$ & $\begin{array}{c}6.95 \times \\
10^{-6}\end{array}$ & $\begin{array}{c}3.68 \times 10^{-} \\
7\end{array}$ & 0.045 & & \\
\hline
\end{tabular}

Table A25. Integrated charge under the stripping peaks of $\mathrm{Au}$ and $\mathrm{Cu}$ in $0.01 \mathrm{M} \mathrm{KBr}$ plus $0.1 \mathrm{M} \mathrm{HClO}_{4}$ electrolyte to calculate the $\mathrm{Au}$ to $\mathrm{Cu}$ ratio of $\mathrm{Cu}_{1} / \mathrm{Au}_{0.001}$ core/shell alloy nanoparticles.

\begin{tabular}{|c|c|c|c|c|c|}
\hline $\mathrm{Au}$ & $\mathrm{Cu}^{1}$ & $\mathrm{Cu}^{2}$ & $\mathrm{Au} / \mathrm{Cu}$ & $\begin{array}{c}\text { Average of } \\
\text { Au/Cu }\end{array}$ & $\begin{array}{c}\text { Theoretical } \\
\text { Au/Cu }\end{array}$ \\
\hline $\begin{array}{c}9.40 \times 10^{-6} \\
\mathrm{C}\end{array}$ & $\begin{array}{c}1.65 \times 10^{-4} \\
\mathrm{C}\end{array}$ & $\begin{array}{c}1.59 \times 10^{-6} \\
\mathrm{C}\end{array}$ & 0.056 & & \\
\hline $\begin{array}{c}5.31 \times 10^{-6} \\
\mathrm{C}\end{array}$ & $\begin{array}{c}9.02 \times 10^{-5} \\
\mathrm{C}\end{array}$ & $\begin{array}{c}2.01 \times 10^{-6} \\
\mathrm{C}\end{array}$ & 0.057 & $0.05 \pm 0.02$ & 0.0015 \\
\hline
\end{tabular}




\begin{tabular}{|c|c|c|c|c|c|}
\hline $\begin{array}{c}3.11 \times 10^{-6} \\
\text { C }\end{array}$ & $\begin{array}{c}1.10 \times 10^{-4} \\
\text { C }\end{array}$ & $\begin{array}{c}8.59 \times 10^{-7} \\
C\end{array}$ & 0.028 & & \\
\hline $\begin{array}{c}4.28 \times 10^{-6} \\
\text { C }\end{array}$ & $\begin{array}{c}5.94 \times 10^{-6} \\
C\end{array}$ & $\begin{array}{c}1.32 \times 10^{-6} \\
C\end{array}$ & 0.070 & & \\
\hline
\end{tabular}

Table A26. Integrated charge under the stripping peaks of $\mathrm{Au}$ and $\mathrm{Cu}$ in $0.01 \mathrm{M} \mathrm{KCl}$ plus $0.1 \mathrm{M} \mathrm{HClO}_{4}$ electrolyte to calculate the $\mathrm{Au}$ to $\mathrm{Cu}$ ratio of $\mathrm{Cu}_{1} / \mathrm{Au}_{0.001}$ core/shell alloy nanoparticles.

\begin{tabular}{|c|c|c|c|c|c|}
\hline Au1 & $\mathrm{Au}^{2}$ & $\mathrm{Cu}^{1}$ & $\mathrm{Au} / \mathrm{Cu}$ & $\begin{array}{c}\text { Average of } \\
\mathrm{Au} / \mathrm{Cu}\end{array}$ & $\begin{array}{c}\text { Theoretical } \\
\mathrm{Au} / \mathrm{Cu}\end{array}$ \\
\hline $\begin{array}{c}1.07 \times 10^{-6} \\
\mathrm{C}\end{array}$ & $\begin{array}{c}8.92 \times 10^{-7} \\
\mathrm{C}\end{array}$ & $\begin{array}{c}1.03 \times 10^{-4} \\
\mathrm{C}\end{array}$ & 0.019 & & \\
\hline $\begin{array}{c}9.29 \times 10^{-7} \\
\mathrm{C}\end{array}$ & $\begin{array}{c}1.36 \times 10^{-6} \\
\mathrm{C}\end{array}$ & $\begin{array}{c}1.23 \times 10^{-4} \\
\mathrm{C}\end{array}$ & 0.018 & $0.019 \pm 0.002$ & 0.0015 \\
\hline $\begin{array}{c}3.70 \times 10^{-6} \\
\mathrm{C}\end{array}$ & $\begin{array}{c}8.50 \times 10^{-7} \\
\mathrm{C}\end{array}$ & $\begin{array}{c}7.29 \times 10^{-5} \\
\mathrm{C}\end{array}$ & 0.062 & & \\
\hline $\begin{array}{c}1.95 \times 10^{-6} \\
\mathrm{C}\end{array}$ & $\begin{array}{c}1.61 \times 10^{-6} \\
\mathrm{C}\end{array}$ & $\begin{array}{c}1.60 \times 10^{-4} \\
\mathrm{C}\end{array}$ & 0.022 & & \\
\hline
\end{tabular}

Table A27. Integrated charge under the stripping peaks of $\mathrm{Au}$ and $\mathrm{Cu}$ to calculate the $\mathrm{Au}$ to $\mathrm{Cu}$ ratio of $\mathrm{Cu}_{1} / \mathrm{Au}_{1}$ core/shell alloy nanoparticles by first stripping in $0.01 \mathrm{M} \mathrm{KBr}$ plus $0.1 \mathrm{M} \mathrm{HClO}_{4}$ solution from -0.2 to $0.4 \mathrm{~V}$ and then stripping the same electrode in $0.01 \mathrm{M}$ $\mathrm{KCl}$ plus $0.1 \mathrm{M} \mathrm{HClO}_{4}$ solution from -0.2 to $1.6 \mathrm{~V}$.

\begin{tabular}{|c|c|c|c|c|c|}
\hline $\mathrm{Au}$ & $\begin{array}{c}\mathrm{Cu}^{3} \text { (from } \\
\left.\mathrm{Br}^{-}\right)\end{array}$ & $\begin{array}{c}\mathrm{Cu}^{3} \text { (from } \mathrm{Cl}^{-} \\
\text {) }\end{array}$ & $\mathrm{Au} / \mathrm{Cu}$ & $\begin{array}{c}\text { Average } \\
\mathrm{Au} / \mathrm{Cu}\end{array}$ & $\begin{array}{c}\text { Theoretical } \\
\mathrm{Au} / \mathrm{Cu}\end{array}$ \\
\hline $8.83 \times 10^{-4}$ & $1.96 \times 10^{-4}$ & $1.38 \times 10^{-6}$ & 4.47 & & \\
\hline $7.68 \times 10^{-4}$ & $2.05 \times 10^{-4}$ & $1.29 \times 10^{-6}$ & 3.71 & $3.9 \pm 0.4$ & 1.5 \\
\hline $7.21 \times 10^{-4}$ & $1.91 \times 10^{-4}$ & $2.99 \times 10^{-6}$ & 3.71 & & \\
\hline $6.06 \times 10^{-4}$ & $1.61 \times 10^{-4}$ & $5.38 \times 10^{-7}$ & 3.75 & & \\
\hline
\end{tabular}


Table A28. Integrated charge under the stripping peaks of $\mathrm{Au}$ and $\mathrm{Cu}$ to calculate the $\mathrm{Au}$ to $\mathrm{Cu}$ ratio of $\mathrm{Cu}_{1} / \mathrm{Au}_{0.5}$ core/shell alloy nanoparticles by first stripping in $0.01 \mathrm{M} \mathrm{KBr}$ plus $0.1 \mathrm{M} \mathrm{HClO}_{4}$ solution from -0.2 to $0.4 \mathrm{~V}$ and then stripping the same electrode in $0.01 \mathrm{M}$ $\mathrm{KCl}$ plus $0.1 \mathrm{M} \mathrm{HClO}_{4}$ solution from -0.2 to $1.6 \mathrm{~V}$.

\begin{tabular}{|c|c|c|c|c|c|}
\hline $\mathrm{Au}$ & $\begin{array}{c}\mathrm{Cu}^{3} \text { (from } \\
\mathrm{Br} \text { ) }\end{array}$ & $\begin{array}{c}\mathrm{Cu}^{3} \text { (from } \mathrm{Cl}^{-} \\
\text {) }\end{array}$ & $\mathrm{Au} / \mathrm{Cu}$ & $\begin{array}{c}\text { Average } \\
\mathrm{Au} / \mathrm{Cu}\end{array}$ & $\begin{array}{c}\text { Theoretical } \\
\mathrm{Au} / \mathrm{Cu}\end{array}$ \\
\hline $2.33 \times 10^{-4}$ & $1.17 \times 10^{-4}$ & $2.44 \times 10^{-6}$ & 1.95 & & \\
\hline $2.07 \times 10^{-4}$ & $1.11 \times 10^{-4}$ & $4.33 \times 10^{-6}$ & 1.81 & $2.0 \pm 0.2$ & 0.75 \\
\hline $2.62 \times 10^{-4}$ & $1.27 \times 10^{-4}$ & $8.19 \times 10^{-7}$ & 2.02 & & \\
\hline $2.69 \times 10^{-4}$ & $1.21 \times 10^{-4}$ & $2.66 \times 10^{-6}$ & 2.17 & & \\
\hline
\end{tabular}

Table A29: Intregrated charge obtained under stripping peaks of $\mathrm{Au}$ and $\mathrm{Cu}$ in $\mathrm{Cl}^{-}$ containing acid electrolyte solution to calculate $\mathrm{Au} / \mathrm{Cu}$ ratio of mixed $\mathrm{Cu}_{3}-\mathrm{Au}_{1}$ alloy NPs.

\begin{tabular}{|l|l|l|l|l|}
\hline $\begin{array}{l}\mathrm{Au} \\
(\mathrm{C})\end{array}$ & $\begin{array}{l}\mathrm{Cu}^{2} \\
(\mathrm{C})\end{array}$ & $\mathrm{Au} / \mathrm{Cu}$ & $\begin{array}{l}\text { Average } \\
\mathrm{Au} / \mathrm{Cu}\end{array}$ & $\begin{array}{l}\text { Theoretical } \\
\mathrm{Au} / \mathrm{Cu}\end{array}$ \\
\hline $3.52 \times 10^{-5}$ & $6.92 \times 10^{-5}$ & 0.51 & & \\
\hline $4.74 \times 10^{-5}$ & $7.82 \times 10^{-5}$ & 0.60 & $0.50 \pm 0.10$ & 0.45 \\
\hline $8.26 \times 10^{-5}$ & $2.05 \times 10^{-4}$ & 0.40 & & \\
\hline
\end{tabular}

Table A30: Intregrated charge obtained under stripping peaks of $\mathrm{Au}$ and $\mathrm{Cu}$ in $\mathrm{Cl}^{-}$ containing acid electrolyte solution to calculate $\mathrm{Au} / \mathrm{Cu}$ ratio of mixed $\mathrm{Cu}_{3}-\mathrm{Au}_{1}$ alloy $\mathrm{NPs}$ after $1 \mathrm{~h}$ thermal treatment at $423^{\circ} \mathrm{K}$.

\begin{tabular}{|l|l|l|l|l|}
\hline $\begin{array}{l}\mathrm{Au} \\
(\mathrm{C})\end{array}$ & $\begin{array}{l}\mathrm{Cu}^{2} \\
(\mathrm{C})\end{array}$ & $\mathrm{Au} / \mathrm{Cu}$ & $\begin{array}{l}\text { Average } \\
\mathrm{Au} / \mathrm{Cu}\end{array}$ & $\begin{array}{l}\text { Theoretical } \\
\mathrm{Au} / \mathrm{Cu}\end{array}$ \\
\hline $1.52 \times 10^{-5}$ & $2.60 \times 10^{-4}$ & 0.57 & & \\
\hline $2.21 \times 10^{-5}$ & $4.06 \times 10^{-4}$ & 0.54 & $0.51 \pm 0.07$ & 0.45 \\
\hline $5.34 \times 10^{-5}$ & $1.22 \times 10^{-4}$ & 0.44 & & \\
\hline
\end{tabular}


Table A31: Intregrated charge obtained under stripping peaks of $\mathrm{Au}$ and $\mathrm{Cu}$ in $\mathrm{Cl}^{-}$ containing acid electrolyte solution to calculate $\mathrm{Au} / \mathrm{Cu}$ ratio of mixed $\mathrm{Cu}_{3}-\mathrm{Au}_{1}$ alloy NPs after $1 \mathrm{~h}$ thermal treatment at $473^{\circ} \mathrm{K}$.

\begin{tabular}{|l|l|l|l|l|l|}
\hline $\begin{array}{l}\mathrm{Au} \\
(\mathrm{C})\end{array}$ & $\begin{array}{l}\text { Cu1 } \\
(\mathrm{C})\end{array}$ & $\begin{array}{l}\mathrm{Cu} 2 \\
(\mathrm{C})\end{array}$ & $\mathrm{Au} / \mathrm{Cu}$ & $\begin{array}{l}\text { Average } \\
\mathrm{Au} / \mathrm{Cu}\end{array}$ & $\begin{array}{l}\text { Theoretical } \\
\mathrm{Au} / \mathrm{Cu}\end{array}$ \\
\hline $1.22 \times 10^{-4}$ & $2.42 \times 10^{-4}$ & $3.25 \times 10^{-5}$ & 0.44 & & \\
\hline $1.17 \times 10^{-4}$ & $2.03 \times 10^{-4}$ & $5.39 \times 10^{-5}$ & 0.45 & $0.44 \pm 0.01$ & 0.45 \\
\hline $4.50 \times 10^{-5}$ & $8.68 \times 10^{-5}$ & $1.43 \times 10^{-5}$ & 0.44 & & \\
\hline
\end{tabular}

Table A32: Intregrated charge obtained under stripping peaks of $\mathrm{Au}$ and $\mathrm{Cu}$ in $\mathrm{Cl}^{-}$ containing acid electrolyte solution to calculate $\mathrm{Au} / \mathrm{Cu}$ ratio of mixed $\mathrm{Cu}_{3}-\mathrm{Au}_{1}$ alloy NPs after $16 \mathrm{~h}$ thermal treatment at $473^{\circ} \mathrm{K}$.

\begin{tabular}{|l|l|l|l|l|l|}
\hline $\begin{array}{l}\mathrm{Au} \\
(\mathrm{C})\end{array}$ & $\begin{array}{l}\text { Cu1 } \\
(\mathrm{C})\end{array}$ & $\begin{array}{l}\text { Cu2 } \\
(\mathrm{C})\end{array}$ & Au/Cu & $\begin{array}{l}\text { Average } \\
\text { Au/Cu }\end{array}$ & $\begin{array}{l}\text { Theoretical } \\
\text { Au/Cu }\end{array}$ \\
\hline $7.35 \times 10^{-5}$ & $1.62 \times 10^{-4}$ & $1.41 \times 10^{-5}$ & 0.42 & & \\
\hline $5.93 \times 10^{-5}$ & $1.45 \times 10^{-4}$ & $1.42 \times 10^{-5}$ & 0.37 & $0.42 \pm 0.05$ & 0.45 \\
\hline $4.32 \times 10^{-5}$ & $8.50 \times 10^{-5}$ & $7.39 \times 10^{-6}$ & 0.47 & & \\
\hline
\end{tabular}

Table A33: Intregrated charge obtained under stripping peaks of $\mathrm{Au}$ and $\mathrm{Cu}$ in $\mathrm{Cl}^{-}$ containing acid electrolyte solution to calculate $\mathrm{Au} / \mathrm{Cu}$ ratio of core/shell $\mathrm{Cu}_{3} / \mathrm{Au}_{1}$ alloy NPs.

\begin{tabular}{|l|l|l|l|l|l|}
\hline $\begin{array}{l}\mathrm{Au} \\
(\mathrm{C})\end{array}$ & $\begin{array}{l}\text { Cu1 } \\
(\mathrm{C})\end{array}$ & $\begin{array}{l}\mathrm{Cu} 2 \\
(\mathrm{C})\end{array}$ & $\mathrm{Au} / \mathrm{Cu}$ & $\begin{array}{l}\text { Average } \\
\mathrm{Au} / \mathrm{Cu}\end{array}$ & $\begin{array}{l}\text { Theoretical } \\
\mathrm{Au} / \mathrm{Cu}\end{array}$ \\
\hline $7.76 \times 10^{-5}$ & $5.06 \times 10^{-5}$ & $1.37 \times 10^{-4}$ & 0.41 & & \\
\hline $1.51 \times 10^{-4}$ & $8.09 \times 10^{-5}$ & $1.60 \times 10^{-4}$ & 0.62 & $0.49 \pm 0.11$ & 0.45 \\
\hline $8.58 \times 10^{-5}$ & $5.61 \times 10^{-5}$ & $1.36 \times 10^{-4}$ & 0.45 & & \\
\hline
\end{tabular}


Table A34: Intregrated charge obtained under stripping peaks of $\mathrm{Au}$ and $\mathrm{Cu}$ in $\mathrm{Cl}^{-}$ containing acid electrolyte solution to calculate $\mathrm{Au} / \mathrm{Cu}$ ratio of core/shell $\mathrm{Cu}_{3} / \mathrm{Au}_{1}$ alloy NPs after thermal treatment at $423^{\circ} \mathrm{K}$ for $1 \mathrm{H}$.

\begin{tabular}{|l|l|l|l|l|l|}
\hline $\begin{array}{l}\mathrm{Au} \\
(\mathrm{C})\end{array}$ & $\begin{array}{l}\text { Cu1 } \\
(\mathrm{C})\end{array}$ & $\begin{array}{l}\mathrm{Cu} 2 \\
(\mathrm{C})\end{array}$ & $\mathrm{Au} / \mathrm{Cu}$ & $\begin{array}{l}\text { Average } \\
\mathrm{Au} / \mathrm{Cu}\end{array}$ & $\begin{array}{l}\text { Theoretical } \\
\mathrm{Au} / \mathrm{Cu}\end{array}$ \\
\hline $1.34 \times 10^{-4}$ & $9.13 \times 10^{-5}$ & $1.76 \times 10^{-4}$ & 0.50 & & \\
\hline $8.83 \times 10^{-5}$ & $6.17 \times 10^{-5}$ & $1.36 \times 10^{-4}$ & 0.45 & $0.48 \pm 0.03$ & 0.45 \\
\hline $9.06 \times 10^{-5}$ & $5.29 \times 10^{-5}$ & $1.30 \times 10^{-4}$ & 0.50 & & \\
\hline
\end{tabular}

Table A35: Intregrated charge obtained under stripping peaks of $\mathrm{Au}$ and $\mathrm{Cu}$ in $\mathrm{Cl}^{-}$ containing acid electrolyte solution to calculate $\mathrm{Au} / \mathrm{Cu}$ ratio of core/shell $\mathrm{Cu}_{3} / \mathrm{Au}_{1}$ alloy NPs after thermal treatment at $473^{\circ} \mathrm{K}$ for $1 \mathrm{H}$.

\begin{tabular}{|l|l|l|l|l|}
\hline $\begin{array}{l}\mathrm{Au} \\
(\mathrm{C})\end{array}$ & $\begin{array}{l}\text { Cu1+Cu2 } \\
(\mathrm{C})\end{array}$ & $\mathrm{Au} / \mathrm{Cu}$ & $\begin{array}{l}\text { Average } \\
\mathrm{Au} / \mathrm{Cu}\end{array}$ & $\begin{array}{l}\text { Theoretical } \\
\mathrm{Au} / \mathrm{Cu}\end{array}$ \\
\hline $8.54 \times 10^{-5}$ & $1.62 \times 10^{-4}$ & 0.53 & & \\
\hline $1.62 \times 10^{-4}$ & $3.30 \times 10^{-4}$ & 0.49 & $0.53 \pm 0.04$ & 0.45 \\
\hline $1.32 \times 10^{-4}$ & $2.25 \times 10^{-4}$ & 0.58 & & \\
\hline
\end{tabular}

Table A36: Intregrated charge obtained under stripping peaks of $\mathrm{Au}$ and $\mathrm{Cu}$ in $\mathrm{Cl}^{-}$ containing acid electrolyte solution to calculate $\mathrm{Au} / \mathrm{Cu}$ ratio of core/shell $\mathrm{Cu}_{3} / \mathrm{Au}_{1}$ alloy NPs after thermal treatment at $473^{\circ} \mathrm{K}$ for $16 \mathrm{H}$.

\begin{tabular}{|l|l|l|l|l|l|}
\hline $\begin{array}{l}\mathrm{Au} \\
(\mathrm{C})\end{array}$ & $\begin{array}{l}\text { Cu1 } \\
(\mathrm{C})\end{array}$ & $\begin{array}{l}\mathrm{Cu} 2 \\
(\mathrm{C})\end{array}$ & $\mathrm{Au} / \mathrm{Cu}$ & $\begin{array}{l}\text { Average } \\
\mathrm{Au} / \mathrm{Cu}\end{array}$ & $\begin{array}{l}\text { Theoretical } \\
\mathrm{Au} / \mathrm{Cu}\end{array}$ \\
\hline $1.08 \times 10^{-4}$ & $2.14 \times 10^{-4}$ & $2.34 \times 10^{-5}$ & 0.45 & & \\
\hline $9.41 \times 10^{-5}$ & $1.64 \times 10^{-4}$ & $2.61 \times 10^{-5}$ & 0.49 & $0.50 \pm 0.05$ & 0.45 \\
\hline $1.31 \times 10^{-4}$ & $2.21 \times 10^{-4}$ & $2.55 \times 10^{-5}$ & 0.55 & & \\
\hline
\end{tabular}


Table A37: Intregrated charge obtained under stripping peaks of $\mathrm{Au}$ and $\mathrm{Cu}$ in $\mathrm{Cl}^{-}$ containing acid electrolyte solution to calculate $\mathrm{Au} / \mathrm{Cu}$ ratio of core/shell $\mathrm{Au}_{1} / \mathrm{Cu}_{3}$ alloy NPS.

\begin{tabular}{|l|l|l|l|l|l|}
\hline $\begin{array}{l}\mathrm{Au} \\
(\mathrm{C})\end{array}$ & $\begin{array}{l}\mathrm{Cu} 1 \\
(\mathrm{C})\end{array}$ & $\begin{array}{l}\mathrm{Cu} 2 \\
(\mathrm{C})\end{array}$ & $\mathrm{Au} / \mathrm{Cu}$ & $\begin{array}{l}\text { Average } \\
\mathrm{Au} / \mathrm{Cu}\end{array}$ & $\begin{array}{l}\text { Theoretical } \\
\mathrm{Au} / \mathrm{Cu}\end{array}$ \\
\hline $1.37 \times 10^{-4}$ & $2.06 \times 10^{-4}$ & $1.63 \times 10^{-4}$ & 0.32 & & \\
\hline $1.52 \times 10^{-4}$ & $1.50 \times 10^{-4}$ & $1.38 \times 10^{-4}$ & 0.53 & $0.46 \pm 0.11$ & 0.45 \\
\hline $3.89 \times 10^{-5}$ & $3.50 \times 10^{-5}$ & $4.01 \times 10^{-5}$ & 0.52 & & \\
\hline
\end{tabular}

Table A38: Intregrated charge obtained under stripping peaks of $\mathrm{Au}$ and $\mathrm{Cu}$ in $\mathrm{Cl}^{-}$ containing acid electrolyte solution to calculate $\mathrm{Au} / \mathrm{Cu}$ ratio of core/shell $\mathrm{Au}_{1} / \mathrm{Cu}_{3}$ alloy NPs after thermal treatment at $423^{\circ} \mathrm{K}$ for $1 \mathrm{H}$

\begin{tabular}{|l|l|l|l|l|l|}
\hline $\begin{array}{l}\mathrm{Au} \\
(\mathrm{C})\end{array}$ & $\begin{array}{l}\text { Cu1 } \\
(\mathrm{C})\end{array}$ & $\begin{array}{l}\text { Cu2 } \\
(\mathrm{C})\end{array}$ & $\mathrm{Au} / \mathrm{Cu}$ & $\begin{array}{l}\text { Average } \\
\mathrm{Au} / \mathrm{Cu}\end{array}$ & $\begin{array}{l}\text { Theoretical } \\
\text { Au/Cu }\end{array}$ \\
\hline $5.86 \times 10^{-5}$ & $5.01 \times 10^{-5}$ & $4.50 \times 10^{-5}$ & 0.62 & & \\
\hline $1.84 \times 10^{-4}$ & $1.10 \times 10^{-4}$ & $1.68 \times 10^{-4}$ & 0.66 & $0.57 \pm 0.11$ & 0.45 \\
\hline $2.51 \times 10^{-5}$ & $3.80 \times 10^{-5}$ & $1.89 \times 10^{-5}$ & 0.44 & & \\
\hline
\end{tabular}

Table A39: Intregrated charge obtained under stripping peaks of $\mathrm{Au}$ and $\mathrm{Cu}$ in $\mathrm{Cl}^{-}$ containing acid electrolyte solution to calculate $\mathrm{Au} / \mathrm{Cu}$ ratio of core/shell $\mathrm{Au}_{1} / \mathrm{Cu}_{3}$ alloy NPs after thermal treatment at $473^{\circ} \mathrm{K}$ for $1 \mathrm{H}$

\begin{tabular}{|l|l|l|l|l|l|}
\hline $\begin{array}{l}\mathrm{Au} \\
(\mathrm{C})\end{array}$ & $\begin{array}{l}\text { Cu1 } \\
(\mathrm{C})\end{array}$ & $\begin{array}{l}\mathrm{Cu} 2 \\
(\mathrm{C})\end{array}$ & $\mathrm{Au} / \mathrm{Cu}$ & $\begin{array}{l}\text { Average } \\
\mathrm{Au} / \mathrm{Cu}\end{array}$ & $\begin{array}{l}\text { Theoretical } \\
\mathrm{Au} / \mathrm{Cu}\end{array}$ \\
\hline $1.27 \times 10^{-4}$ & $2.37 \times 10^{-4}$ & $2.37 \times 10^{-5}$ & 0.49 & & \\
\hline $2.75 \times 10^{-4}$ & $5.03 \times 10^{-4}$ & $2.47 \times 10^{-5}$ & 0.52 & $0.53 \pm 0.04$ & 0.45 \\
\hline $1.61 \times 10^{-4}$ & $2.42 \times 10^{-4}$ & $3.35 \times 10^{-5}$ & 0.58 & & \\
\hline
\end{tabular}


Table A40: Intregrated charge obtained under stripping peaks of $\mathrm{Au}$ and $\mathrm{Cu}$ in $\mathrm{Cl}^{-}$ containing acid electrolyte solution to calculate $\mathrm{Au} / \mathrm{Cu}$ ratio of core/shell $\mathrm{Au}_{1} / \mathrm{Cu}_{3}$ alloy NPs after thermal treatment at $473^{\circ} \mathrm{K}$ for $16 \mathrm{H}$

\begin{tabular}{|l|l|l|l|l|l|}
\hline $\begin{array}{l}\mathrm{Au} \\
(\mathrm{C})\end{array}$ & $\begin{array}{l}\text { Cu1 } \\
(\mathrm{C})\end{array}$ & $\begin{array}{l}\mathrm{Cu} 2 \\
(\mathrm{C})\end{array}$ & $\mathrm{Au} / \mathrm{Cu}$ & $\begin{array}{l}\text { Average } \\
\mathrm{Au} / \mathrm{Cu}\end{array}$ & $\begin{array}{l}\text { Theoretical } \\
\mathrm{Au} / \mathrm{Cu}\end{array}$ \\
\hline $1.85 \times 10^{-4}$ & $3.90 \times 10^{-4}$ & $8.17 \times 10^{-6}$ & 0.46 & & \\
\hline $1.87 \times 10^{-4}$ & $3.86 \times 10^{-4}$ & $8.16 \times 10^{-6}$ & 0.47 & $0.46 \pm 0.01$ & 0.45 \\
\hline $3.88 \times 10^{-4}$ & $8.41 \times 10^{-4}$ & $1.77 \times 10^{-5}$ & 0.45 & & \\
\hline
\end{tabular}




\section{CURRICULUM VITAE}

\section{Dhruba Kumar Pattadar}

2232 South Preston Street, Apt. 4, Louisville, KY, 40217, (502) 345-1829

dhrubaparradar@gmail.com

\section{SUMMARY OF QUALIFICATIONS}

- 6+ years of experience in the field of metal nanoparticle synthesis, characterization and applications related to electrocatalysis and sensing.

- Extensive experience in electrochemistry, Scanning Electron microscopy (SEM), Atomic force microscopy (AFM) and Raman spectroscopy.

- Experience in designing new nanomaterials for electrochemical and chemical applications.

- Expertise in writing and editing of scientific documents and communications for peer-reviewed research journals, conference symposia and departmental seminars.

\section{EDUCATION}

\section{$\mathrm{PhD}$ in Chemistry}

August 2019

University of Louisville, Louisville, KY, USA

Dissertation Title: "Utilization of Electrochemical Methods to Analyze Transformations, Composition and Atomic Arrangement of Single Metal and Alloy Nanostructures

Graduate Advisor: Dr. Francis P. Zamborini

\section{M.S. in Chemistry}

October 2012

University of Dhaka, Dhaka, Bangladesh

\section{B.S in Chemistry}

November 2010

University of Dhaka, Dhaka, Bangladesh 


\section{RESEARCH EXPERIENCE}

\section{Publications}

\section{Published}

- Sharma, J. N.; Pattadar, D. K.; Mainali, B. P.; Zamborini, F. P., Size Determination of Metal Nanoparticles Based on Electrochemically Measured Surface-Area-toVolume Ratios. Anal. Chem. 2018, 90, 9308-9314. (Equal first author contribution with Sharma, J.N.).

- Pattadar, D. K.; Zamborini, F. P., A Size Stability Study of Catalytically-Active Sub2 nm Diameter Gold Nanoparticles Synthesized with Weak Stabilizers. J. Am. Chem. Soc. 2018, 140, 14126-14133.

- Pattadar, D. K.; Sharma, J. N.; Mainali, B. P.; Zamborini, F. P., Anodic Stripping Electrochemical Analysis of Metal Nanoparticles. Curr. Opin. Electrochem. 2019, 13, 147-156.

- Pattadar, D. K.; Zamborini, F. P., Halide-Dependent Dealloying of $\mathrm{Cu}_{\mathrm{x}} / \mathrm{Au}_{\mathrm{y}}$ Core/Shell Nanoparticles for Composition Analysis by Anodic Stripping Voltammetry. J. Phys. Chem. C 2019, 123, 9496-9505.

- Pattadar, D. K.; Sharma, J. N.; Mainali, B. P.; Zamborini, F. P., The Impact of Assembly Method on the Surface Area-to-Volume Ratio and Electrochemical Oxidation Potential of Metal Nanoparticles. J. Phys. Chem. C 2019, 123, 2430424312.

\section{Submitted/Accepted}

1. Pattadar, D. K.; Zamborini, F. P., Effect of Size, Coverage, and Dispersity on the Potential-Controlled Ostwald Ripening of Metal Nanoparticles (Accepted to Langmuir with minor revisions).

2. Pattadar, D. K.; Mainali, B. P.; Zamborini, F. P., Electrooxidation and Size Stability of $0.9 \mathrm{~nm}$ Diameter Gold Nanoclusters Coated with a Weak Stabilizer (Submitted to Small).

\section{Manuscripts in Preparation}


1. Pattadar, D. K.; Mainali, B. P.; Sharma, J. N.; Zamborini, F. P., Understanding the Thermal Sintering Behavior of Sub $2 \mathrm{~nm}$ Diameter Gold Nanoparticles Synthesized with Weak Stabilizers.

2. Mainali, B. P., Pattadar, D. K., and Francis P. Zamborini., Sintering of Gold Nanoparticles through Repetitive Electrochemical Oxidation-Reduction Cycles.

3. Pattadar, D. K.; Mainali, B. P.; Zamborini, F. P., Atomic Arrangement Analysis of Bimetallic $\mathrm{Cu}_{1} / \mathrm{Au}_{\mathrm{x}} \mathrm{NPs}$ by Anodic Stripping Voltammetry.

4. Pattadar, D. K.; Masitas, R.A.; and Zamborini, F. P., Size-Dependent Galvanic Replacement between $\mathrm{Au}$ Nanoparticles and $\mathrm{Ag}^{+}$.

5. Mainali, B.P.; Pattadar, D. K.; and Zamborini, F. P., Size-Dependent Oxidation Behavior of Gold Nanoparticles Coated with Alkanethiol Self- Assembled Monolayers (SAMs).

6. Pattadar, D. K.; Nambiar, H. N., Allen, S. L., Zamborini, F. P., Effect of Nanoparticle Aggregate Structure on the Thermodynamics of Electrochemical Metal Nanoparticle Oxidation.

7. Sharma, J. N.; Pattadar, D.K.; Zamborini, F. P., The Surface Area-to-Volume Ratio and Oxidation Potential of Porous Au Nanostructures.

\section{Poster Presentations}

- Pattadar, D. K.; Zamborini, F. P., Size-Dependent Electrochemical Ostwald Ripening of Au NPs. Pittcon, March 18-21, 2019, Philadelphia, Pennsylvania.

- Pattadar, D. K.; Zamborini, F. P., Exploring the unique electrochemical and chemical reactivity of sub $2 \mathrm{~nm}$ diameter catalytic Au NPs. Kentucky Nano/AM Symposium, August 1-2, 2018, Louisville, Kentucky.

- Pattadar, D. K.; Zamborini, F. P., Analysis of Bimetallic Alloy Nanoparticle Composition and Atomic Arrangement by Stripping Voltammetry. Naff Symposium, March 23, 2018, University of Kentucky, Kentucky.

- Pattadar, D. K.; Zamborini, F. P., Determination of Bimetallic Alloy Nanoparticle Composition and Atomic Arrangement by Stripping Voltammetry. Gordon Research Seminar, January 6-7, 2018, Ventura, California.

- Pattadar, D. K.; Zamborini, F. P., Determination of Bimetallic Alloy Nanoparticle Composition and Atomic Arrangement by Stripping Voltammetry. Gordon Research Conference, January 8-12, 2018, Ventura, California. 
- Pattadar, D. K.; Zamborini, F. P., Stripping Voltammetry Study of Citrate-Coated Cu/Au Core/Shell Bimetallic Nanoparticles (NPs). Pittcon, March 5-9, 2017, Illinoi, Chicago.

- Sharma, J.N.; Pattadar, D.K.; Zamborini. F. P., Electrochemical determination of surface area-to-volume ratio for metal nanoparticle size analysis. National American Chemical Society Meeting, March 18-22, 2018, New Orleans, Louisiana.

- Nambiar, H.N.; Pattadar, D.K., Zamborini. F. P., Aggregation-Dependent Oxidation of Different-Sized Gold Nanoparticles. Southeast Regional Meeting of the American Chemical Society (SERMACS), October 31-November 2, 2018, Augusta, Georgia.

\section{Oral presentations}

- "Understanding the Metal Nanostructures Size Stability, Chemical Reactivity and Atomic Arrangements by Anodic Stripping Voltammetry" March 2019, Pittcon, Philadelphia, PA.

- "Size-Dependent Electrochemical Ostwald Ripening of Au NPs." Graduate Student Regional Research Conference (GSRRC), February 27-28, 2019 at the University of Louisville.

- "Exploring the unique electrochemical and chemical reactivity of sub $2 \mathrm{~nm}$ diameter catalytic Au Nanoparticles". The Southeastern Regional Meeting of American Chemical Society, October 31-November 3, Augusta, Georgia.

\section{TEACHING EXPERIENCE}

My teaching experience is divided mainly into three different parts, my experience as an instructor, as a teaching assistant (T.A.) and my mentor activities with graduate students.

- Instructor: Taught CHEM 529 (Synthesis and Analysis II) class as co-lead instructor in the Department of Chemistry at the University of Louisville. My responsibilities included preparing classes, exams, home works, lab practices, lectures as well as grading exams, homework and lab reports.

- Mentoring: Mentored fellow graduate students Badri Mainali, Hari Nambiathan and Jay Sharma. They were trained in the synthesis of 0.5 to $2 \mathrm{~nm}$ Au clusters and 
citrate-coated Au NPs from 4-50 nm in diameter, UV-vis Spectroscopy, Linear Sweep Voltammetry (LSV), Cyclic Voltammetry (CV), Chronocoulometry (CC), Chronoamperometry (CA) and Scanning Electron Microscopy (SEM). As a result, we are co-authors in many of the publications shown in my publications list.

- Teaching Assistant: Taught Introduction to Chemical Analysis I-IV (CHEM 207, CHEM 208, CHEM 209, CHEM 210) and Synthesis and Analysis II (CHEM 529) class as a teaching assistant in the Department of Chemistry at the University of Louisville. My responsibilities involved setting up labs, teaching lab practices, holding office hours to meet with students, and grading of lab reports and quizzes.

\section{AWARDS}

- Society for Electroanalytical Chemistry (SEAC) Travel Award, March 2019, Pittcon, Philadelphia, PA.

- Graduate Network in Arts and Sciences (GNAS) Travel Award, University of Louisville, January 2019.

- Southeastern Regional Meeting of the American Chemical Society (SERMACS) Travel Award for graduate students, SERMACS, October 2018.

- Graduate Student Council Travel Award (GSC), University of Louisville, January 2018. 